\title{
Investigation into the Mode of Antifungal Action of Berberine
}

By

Ghyda Hashim

A thesis submitted to the Faculty of Graduate and Postdoctoral Affairs in partial fulfillment of the requirements for the degree of

Master of Science

In Biology

Carleton University

Ottawa, Ontario

(C) 2016, Ghyda Hashim

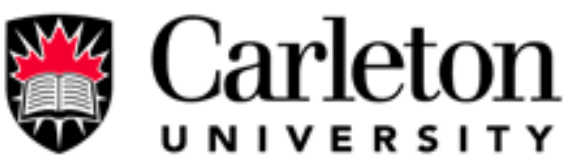




\section{Abstract:}

Berberine is an alkaloid found in several plant genera, and notably in the root bark of Barberry, Berberis vulgaris. As a natural health product, berberine has several health benefits. Berberine has broad spectrum antifungal activities, it is used in the treatment of bacterial diarrhea and against the growth of several intestinal parasites, in treating ocular trachoma infections, preventing inflammation and promoting cardiovascular health. The motivation in this research is that Berberine's antifungal mode of action is not known yet. The focus of this study is to identify the biochemical basis of yeast sensitivity to berberine (i.e. "mode of action"). The MIC of berberine with yeast was determined to be in the range of 0.156 to $0.078 \mathrm{mg} / \mathrm{ml}$. To infer berberine mode of antifungal activity, Gene Deletion Array (GDA) analysis was performed using a library of about 4700 yeast strains, each with a different gene deletion, using a berberine concentration of $0.13 \mathrm{mg} / \mathrm{ml}$. Out of the library, the 71 most sensitive yeast deletion mutant strains were identified and analyzed with GO terms. Out of the 71 sensitive strains, 3 main groups had mutations in genes that are directly involved in cytoskeletal protein binding, transferase activity and RNA and mRNA binding. In addition, 30\% of the 22 resistant strains had deletion mutations that were enriched in tubulin associated genes ( $P$ value was $<0.10)$. Finally, using berberine's natural florescence characteristic together with other histochemical stains, confocal fluorescence microscopy revealed that berberine was dynamically localized first to mitochondria and then to oil droplets within the yeast cell. 


\section{Acknowledgments}

First, I would like to express my sincere gratitude to my thesis advisor, Dr. Myron Smith for welcoming me at Carleton University to do this study and, for his support and guidance in every single step of my study. Without his patience and motivation, I would never have been successful so far. His supervision helped me in all the time of research and writing of this thesis. I could not have imagined having a better advisor and mentor for my Master study.

Also, I would like to thank the rest of my thesis committee members: Dr. Ashkan Golshani, Dr. Bill Willmore, and Dr. Tim Xing, for their academic guidance and inspiration.

I am also thankful for the Smith team for the motivating discussions and outstanding advising. My special thanks go to Imelda Galvan and Isable Cruz, who have taught me a lot during my study. I would also like to acknowledge Denise Chabot from Agriculture and Agri-Food Canada for her very valuable help in this thesis. I would also to thank my special friend Kofi Ilboudo for his daily encouragements through my master study. My special appreciations also go to Houman Moteshareie for his generous help during my study.

Last but not the least, I would like to thank my family: my parents, my brothers and sister for supporting me spiritually throughout writing this thesis and my life in general. My special appreciations to my brother Mohammed Hashim, who has always been there for me and has helped me get through my hard days for this study. None of this would have been possible without my family supports. 


\section{Table of Contents}

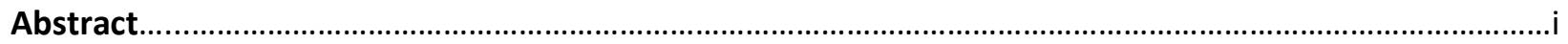

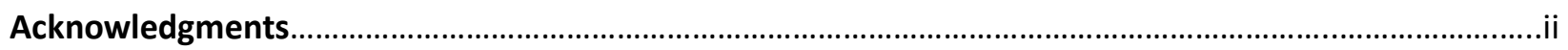

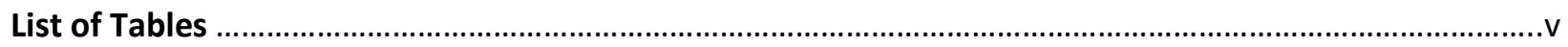

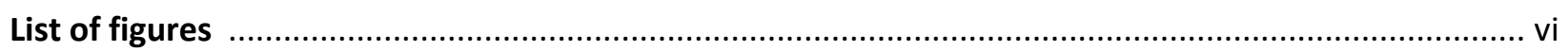

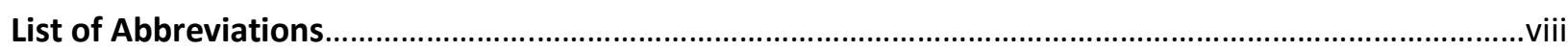

Introduction

Study Objectives

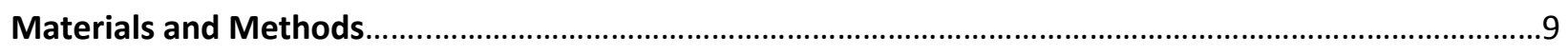

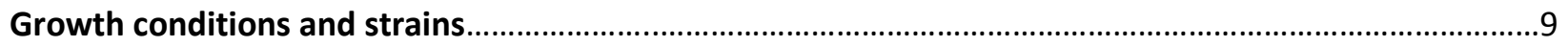

MIC

GDA

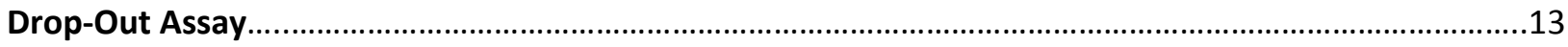

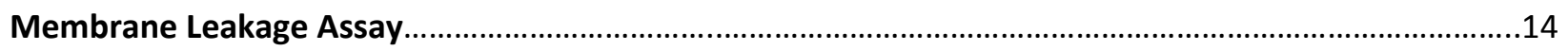

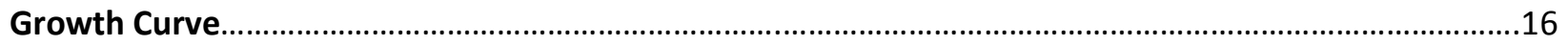

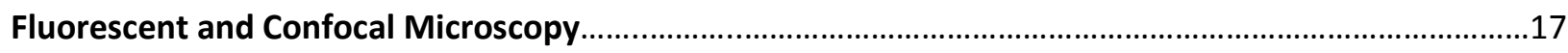

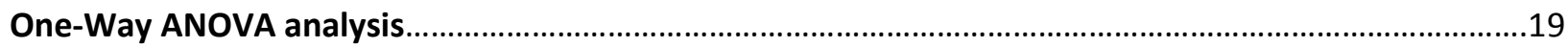

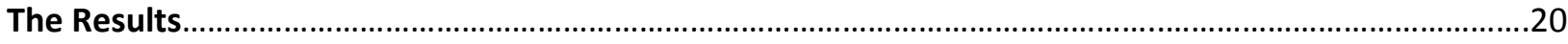

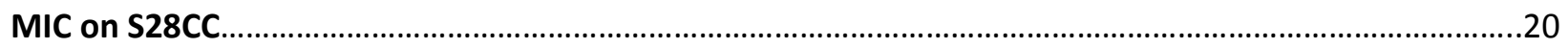

GDA

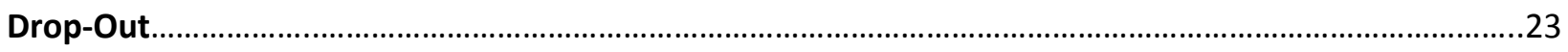

Analysis of berberine sensitivity of selected mutants based on growth curves.....................................28

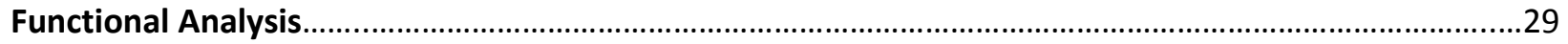

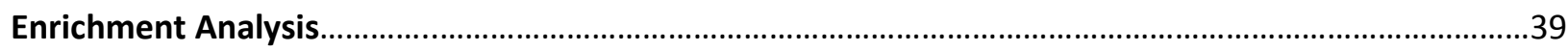

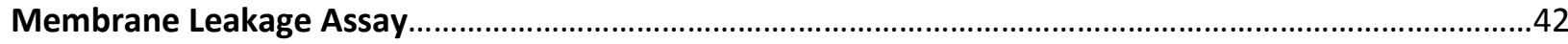

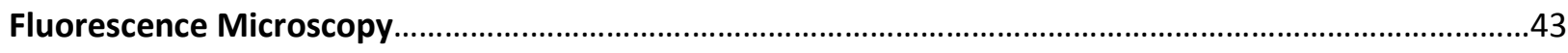

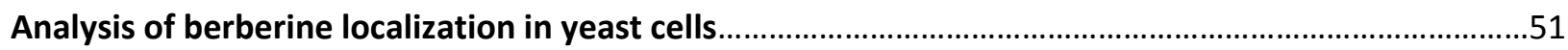

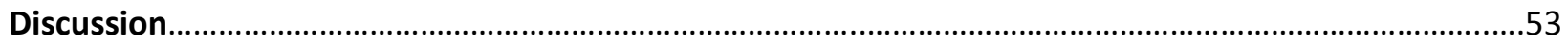

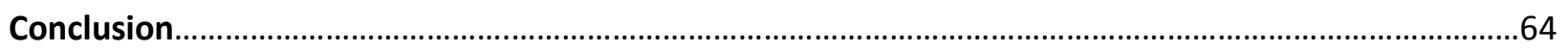




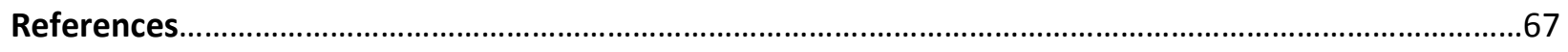

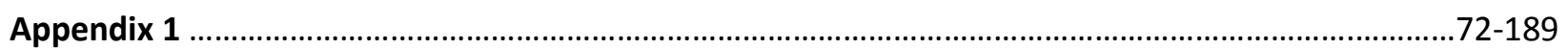

Appendix 2 


\section{List of Tables}

Table 1 (A). Berberine- sensitive deletion mutants were randomly selected from the GDA data sets for sensitivity analysis by drop-out analyses.

Table 1 (B). berberine- meduim sensitive deletion mutants were randomly selected from the GDA data sets for sensitivity analysis by drop-out analyses.

Table 2. Functional analysis of the most sensitive strains based on 3 GDA analysis. The mutants were selected based on the size reduction $\%$ between $60 \%-100 \%$

Table 3. Functional analysis of the most resistant strains based on 3 GDA analysis. The mutants were selected based on the size reduction $\leq 10 \%$.

Table 4 (A). Shows the significant enrichment $(P \leq 0.5)$ of process 'cellular polysaccharide catabolic' and 'polysaccharide catabolic' based on most sensitive mutants.

Table 4 (B). Shows the significant enrichment ( $\mathrm{P} \leq 0.5)$ of function 'GTPase activator activity' and 'DNA binding 'based on most sensitive mutants.

Table 5 (A). Shows the significant enrichment $(P \leq 0.5)$ of process 'tubulin complex assembly' and 'protein complex binding 'based on most resistant mutants.

Table 5 (B). Shows the significant enrichment ( $\mathrm{S} \leq 0.5)$ of function 'tubulin binding' and 'cytoskeletal protein binding 'based on most resistant mutants.

Table 5 (C). Shows the significant enrichment $(P \leq 0.5)$ of component 'prefoldin complex' based on most resistant mutants.

Table 6. Fluorescence microscopy of selected yeast strains treated with berberine $\geq 40 \mathrm{~min}$.

Table 7. Shows the three different staining pattern of berberine on some selected mutants. 


\section{$\underline{\text { List of Figures }}$}

Figure 1. Chemical structure of berberine. 3

Figure 2. Abbreviated biosynthesis pathways for ergosterol synthesis 4

Figure 3. shows an overview of the MIC procedure using 96-well. 10

Figure 4. shows an overview of the Membrane Leakage Assay procedure using 96-well, flat-bottom micro-titer plates. .15

Figure 5. Shown an overview of the Growth Curve experiment using 96-well, flat-bottom micro-titer plates. .16

Figure 6. Example of the GDA in Plate 3 where $(A)$ is the control plate, and (B) is the experimental plate (berberine concentration $0.13 \mathrm{mg} / \mathrm{ml}$ ). .21

Figure 7. Correlation of mean inhibition values from 2 GDA experiments done by F.Haider (Y-axis) and 3 GDA experiments done by me (G.Hashim, X-axis).

Figure 8. Comparison of berberine sensitivity of 28 selected yeast deletion mutants based on GDA and drop-out assays. .25

Figure 9. Shows drop - out assays for 8 selected sensitive deletion mutants strains. .26

Figure 10. Shows drop-out assay for 6 'medium-sensitive' gene deletion strains and the wild type. .27

Figure 11. Shows the growth rates of yeast culture with and without berberine. Berberine concentration range $0.25-0.03125 \mathrm{mg} / \mathrm{ml}$. .28

Figure 12. Functional distribution of 71 most sensitive genes deletion strains to $0.13 \mathrm{mg} / \mathrm{ml}$ berberine. Deleted genes in super sensitive strains were classified by function using Go-term. .35

Figure 13. Functional distribution of 22 most resistant genes deletion strains to $0.13 \mathrm{mg} / \mathrm{ml}$ berberine. Deleted genes in super sensitive strains were classified by function using Go-term. . .38

Figure 14. Shows that overall liposomes leakage \% is not significantly different from the carrier... .43 
Figure 15. Micrographs of (YMR119W) strain, $40 \mathrm{~min}$ exposure to $0.13 \mathrm{mg} / \mathrm{ml}$ berberine by confocal microscopy .45

Figure 16. Micrographs of (YDR388W) strain, $40 \mathrm{~min}$ exposure to $0.13 \mathrm{mg} / \mathrm{ml}$ berberine by confocal microscopy .46

Figure 17. Micrographs of (YGR078C) strain, $40 \mathrm{~min}$ exposure to $0.13 \mathrm{mg} / \mathrm{ml}$ berberine by confocal microscopy .46

Figure 18. Berberine staining patterns from first experiment with selected resistant, medium sensitive and sensitive strains.

Figure 19. Results of second experiment assaying berberine staining patterns of strains that had distinct staining patterns in the first experiment. .51

Figure 20. Live cell imaging of multi stained yeast cell (YPR154W) by confocal microscopy .52 


\section{List of Abbreviations}

\begin{tabular}{|c|c|}
\hline $\mathrm{CF}$ & Carboxyfluorescein \\
\hline DNA & deoxyribonucleic acid \\
\hline DIC & differential interference contrast \\
\hline EX & Excitation \\
\hline EM & Emission band \\
\hline Expt & experiment \\
\hline GDA & gene deletion array \\
\hline HSD & honestly significant difference \\
\hline Inhib & inhibition \\
\hline LD & lipids droplets \\
\hline LUVs & large unilamellar liposomes \\
\hline mRNA & messenger RNA \\
\hline MDRs & multidrug-resistance pumps \\
\hline MIC & minimum inhibitory concentration \\
\hline$\mu \mathrm{M}$ & Micrometer \\
\hline $\mathrm{ml}$ & milliliter \\
\hline$\mu l$ & microliter \\
\hline $\mathrm{mM}$ & millimeter \\
\hline $\mathrm{nm}$ & nanometer \\
\hline $\mathrm{mg} / \mathrm{ml}$ & Milligram per milliliter \\
\hline $\mathrm{Nacl}$ & sodium chloride \\
\hline
\end{tabular}




$\begin{array}{ll}\text { rRNA } & \text { ribosomal ribonucleic acid } \\ \text { SGA } & \text { synthetic genetic array } \\ \text { STE } & \text { steryl esters } \\ \text { 24-SMT } & \text { 24-methyl transferase } \\ \text { TGA } & \text { triacylglycerol } \\ \text { WT } & \text { wild type } \\ \text { YPD } & \text { yeast peptone dextrose }\end{array}$




\section{Introduction:}

\section{Background:}

Lewis and Ausubel (2006) explained that the secret of the relatively low amount of infectious diseases in plants is due in part to the production of antimicrobial compounds. Recently, scientific studies have been focusing more on these plant-based antimicrobial agents due to the increasing needs in the pharmaceutical industry. In the last few decades, researchers have found many beneficial plant-based antifungals; berberine is one such agent (Manske et al., 1953).

Berberine is an alkaloid compound (Figure 1) that occurs in several plant species. Most notably, and for which the molecule is named, berberine is found at high concentrations in Berberis species (Etcu and Goina, 1970). Plants from the Berberis genus are used as traditional remedies for many kinds of ailments, including infectious diseases (Monica Frele et al., 2006). Antimicrobial properties of berberine are backed up by decades of pharmacological research on berberine which has lead to its commercialization by chemical supply companies (Frele et al., 2006). Berberine has been shown to inhibit bacterial growth, as well as proliferation of viruses and fungi that can cause infections in animals, including humans (Renata Iwazaki et al., 2009). Furthermore, berberine has been used to treat diarrhea caused by bacteria (Renata Iwazaki et al., 2009), intestinal parasites (Kaneda et al., 1991), ocular trachoma infections (Babbar et al., 1982), to reduce inflammation (Wang et al., 1994) and promote cardiovascular health (MarinNeto et al., 1988). 


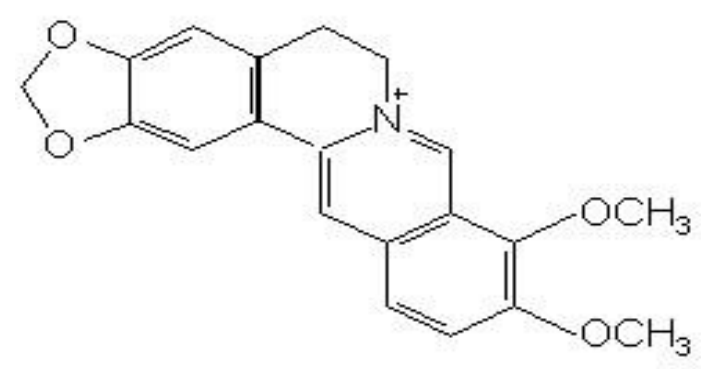

Figure 1. Chemical structure of berberine

As shown in Figure 1, berberine contains 5 heterocyclic rings attached to an incorporated nitrogen atom (Bolton et al., 2008). Berberine is slightly soluble in water, especially warm water, soluble in ethanol and very soluble in methanol (Battu et al., 2010). One of berberine's characteristics is a strong fluorescence under ultraviolet light, with an optimal excitation wavelength of $430 \mathrm{~nm}$ and an emission wavelength of $550 \mathrm{~nm}$ (Nobukazu et al., 2007; Gibbs and Seddon, 2000).

\section{Antifungal and Antibacterial Activities:}

The aim of my research project is to determine the antifungal mode of action of berberine. Preliminary results from our lab confirm that berberine is a broad spectrum antifungal agent, but its antifungal mode of action is unknown. Understanding berberine's mode of action could allow us to develop antifungal agents with enhanced efficacy. Berberine is reported to act synergistically with the antifungal fluconazole (Quan et al., 2006), and it inhibits fluconazoleresistant Candida albicans clinical isolates. This is consistent with a study that indicates that berberine interacts with an enzyme called sterol 24-methyl transferase (24-SMT; Park et al., 1999). Notably, 24-SMT is an essential enzyme in fungi because it catalyzes a chemical reaction for the biosynthesis of sterols. These fungal sterols serve a similar function in modulating 
membrane fluidity as does cholesterol in mammals (Park et al., 1999). 24-SMT transfers one methyl group to zymosterol, a precursor to ergosterol (Figure 2; Bouvier-Navé et al., 1998). Ergosterol is also synthesized from lanosterol by CYP51A1 (Figure 2). CYP51A is inhibited by azole drugs (Martel et al., 2010). Inhibiting ergosterol biosynthesis limits fungal growth, although ergosterol-deficient fungal strains are common in clinical settings since mutation in the ergosterol biosynthetic pathway confers resistance to the commonly used antifungals amphotericin B and azole drugs (Manastır et al., 2011).

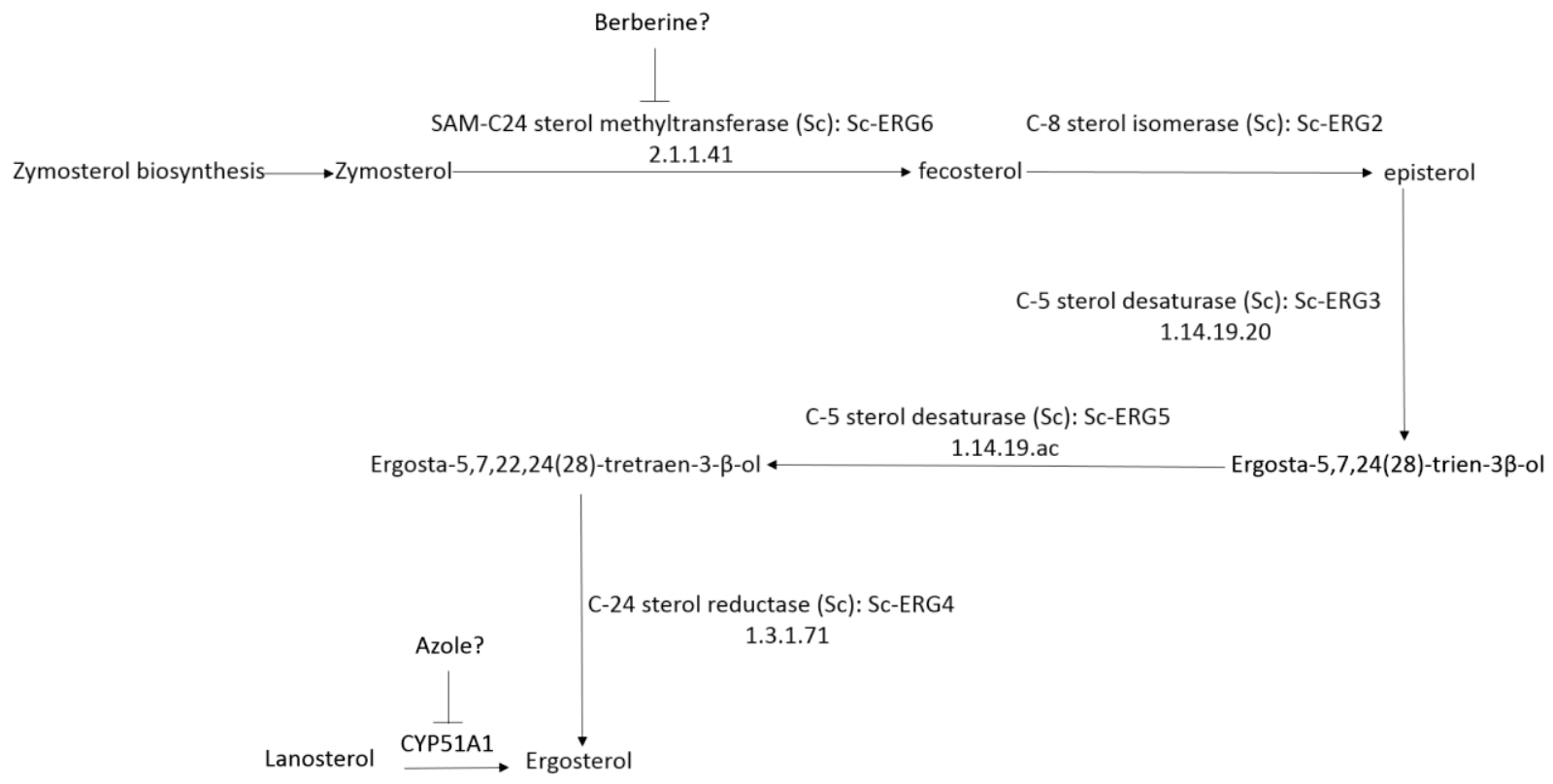

Figure 2. Abbreviated biosynthesis pathways for ergosterol synthesis (adapted from: SRI International). 
Berberine and Azoles are proposed to inhibit 2 pathways leading to ergosterol synthesis as indicated in Figure 2. Other indications of berberine's mode of action come from studies showing bacterial resistance to berberine in bacteria that have enhanced activities of multidrugresistance pumps (MDRs), which quickly remove intracellular berberine, and reduce its antimicrobial efficacy. Some cationic alkaloids such as berberine are natural substrates of MDRs (Stermitz et al., 2000). MDRs are membrane translocases that allow the bacterial cells to extrude toxins and inhibiting such pumps significantly increases berberine's efficacy against bacterial growth. Indeed, combining berberine with a drug that blocks MDRs can greatly increase berberine's toxicity towards bacteria and this aids in studies on berberine's mode of action (Ball et al., 2006). As such, greater antimicrobial activity has been observed through synergistic use of berberine with pump inhibitors 5'-methoxyhydnocarpin or INF 55 (Stermitz et al., 2000; Ball et al., 2006). Pump inhibitors are gaining the interest of researchers for their potential to counter the rise of drug-resistant microbes. Indeed, drug-resistance has often been shown to be mediated by enhanced MDR activity (Stermitz et al., 2000).

Once inside the cell, berberine's mechanism of inhibition on bacterial cells could be through binding to DNA, and continuing degradation of DNA (Stermitz et al., 2000). More recent studies have suggested that berberine inhibits the FtsZ protein which is key to bacterial cell division (Boberek et al., 2010). FtsZ coordinates septum formation during cell division, and is a homolog of tubulin in eukaryotes (Boberek et al., 2010). 


\section{Minimum Inhibitory Concentration (MIC)}

The standard way to determine the level of sensitivity of an antimicrobial drug is by using a Minimum Inhibitory Concentration (MIC) test (Andrews, 2001). The MIC method is based on a broth micro-dilution assay to determine the lowest concentration at which a compound inhibits cell growth (Lambert, 2001). This procedure is carried out by using a 96-well microtiter plate by

first doing a serial dilution of the target compound across the rows of the plate (Wiegand et al., 2008). Second, a known number of cells from overnight liquid culture is added to each well, and the plate is incubated for a specified time. It is possible to make a visual examination of wells with or without growth to get the MIC value or to use a microtiter plate reader for optical density measurements (Mann and Markham, 1998). MIC can be defined in several ways. For example, the $\mathrm{MIC}_{100}$ is the concentration that results in complete inhibition of growth. The $\mathrm{MIC}_{50}$ is a concentration that results in a $50 \%$ inhibition of growth.

\section{Gene Deletion Array (GDA)}

In this thesis, I make extensive use of a chemical-genetic interaction screening method using an S. cerevisiae Gene Deletion Array (GDA). GDA comprises a set of about 4700 mutants, each with a different non-essential gene deleted (Winzeler et al., 1999). Genes and the pathways of S. cerevisiae are functionally redundant; one gene can take over the function of a second gene and therefore a deletion mutant often has no phenotypic consequences (Memarian et al., 2007). The chemical-genetic interaction is determined by a large-scale drug sensitivity screen that monitors growth of the deletion mutants of $S$. cerevisiae when inoculated onto media with and without the inhibitor compound (Parsons 
et al., 2004). Analysis of resistant and/or highly susceptible mutants can provide us with insights as to which genes are involved in the specific process that is targeted by the inhibition. The GDA library was developed by Winzeler et al. (1999). The analysis of colony size measurements was further improved by Memarian et al. (2007), using a computerized imaging systems that automatically detects relative yeast colony size differences. This improved method for using GDA to study antifungal has been successfully used to test $S$. cerevisiae's sensitivity to chitosan (Galvan Marquez, et al., 2013), thymol (Darvishi et al.), eugenol (Darvishi et al.) and many other compounds (Alamgir et al., 2010; Parsons et al., 2006)

\section{Study Objectives.}

The first stage of my study was done by the MIC assay to determine the minimum concentration of berberine needed to inhibit the growth of yeast. This information was needed for the GDA assay. I used the MIC to approximate a berberine concentration that would slightly inhibit yeast colony growth on solid medium. Then, GDA analysis was done to create a list of deletion mutants that were sensitive and resistant to berberine. Based on the digital analysis, the change in colony size of each $S$. cerevisiae gene deletion strain was determined and about 71 most-inhibited were selected. I also selected 22 of resistant strains I verified the results of the GDA analysis using a drop-out experiment with selected sensitive and non-sensitive mutants identified by GDA analysis. Finally, sensitive strains were selected for confocal fluorescence and confocal microscopy examination. Confocal microscopy was used to see where berberine is localized within yeast cells and to provide additional insight into berberine antifungal mode-of-action. 
This research has many commercially relevant applications. We need antifungal agents that are safe to use with animals, which is a challenge since fungi and animals are sister clades (Oruc, 2010) and share many biochemical pathways. Currently, there are relatively few antifungals that do not also negatively affect mammals. Furthermore, existing antifungals that are available to consumers and patients are also becoming less useful due to an increasing number of antibiotic resistant fungal strains. The results of this study have the potential to enhance future knowledge in developing natural products to fight fungal diseases in agriculture or medicine. 


\section{Material and methods:}

\section{Culture preparations, growth conditions and strains:}

Berberine chloride powder was obtained from Sigma-Aldrich (SLPB2771V; St. Louis, MO, USA) and stored in the dark at $4^{\circ} \mathrm{C}$. To prepare a berberine stock solution, berberine was weighed and added to water in a glass vial to obtain a concentration of $0.13 \mathrm{mg} / \mathrm{ml}$. The vial was then wrapped in aluminum foil to prevent photodegradation and then incubated at $50^{\circ} \mathrm{C}$ for $15-30$ minutes or until the berberine was fully dissolved. The stock solution was kept in the fridge for future use.

Yeast strains used in this study were cultured in Yeast Peptone Dextrose (YPD) broth or agar medium. YPD medium was made of 2\% Dextrose (Batch \# 128477M029089, BDH, Toronto, Canada), 2\% Peptone (Batch \#44A32633; Bioshop, Burlington, ON, CA), and 1\% Yeast Extract (Batch \# 7199299, Bacto ${ }^{\mathrm{TM}}$, Sparks, MD, USA)] with or without 1.7\% Agar (Batch \# 3L31698, Bioshop, Burlington, ON, CA). After autoclaving, YPD medium was allowed to cool to $\sim 50{ }^{\circ} \mathrm{C}$ in a water bath before the required amount of berberine was added. After addition of berberine the medium was mixed by shaking and plates were poured.

The square plates used for the GDA tests contained $50 \mathrm{ml}$ of medium and the standard; round petri plates contained $25 \mathrm{ml}$ of medium. GDA and MIC plates were re-used following washing with water and bleach, followed by drying in a B2-level hood under UV light for 30 minutes. GDA analysis was carried out using the SGA-array-ver2-1536 Yeast Mutant Library (Fisher Scientific) which was provided by Dr. Ashkan Golshani. 


\section{MIC (Minimum Inhibitory Concentration) assays.}

An MIC assay was used to determine the minimum concentration of berberine that is needed for a GDA assay and subsequent secondary assays. The MIC method is based on a broth microdilution assay to determine the lowest concentration at which a compound inhibits cell growth (Lambert, 2001). Overnight cultures of the wild type S288C were obtained by inoculating a single colony from streak plates into $5 \mathrm{ml}$ of YPD broth and incubating at $30^{\circ} \mathrm{C}$. The absorbance of the overnight culture was determined by using the optical density where an $\mathrm{OD}_{600}$ of 1.0 is $3 \times 10^{7}$ cells $/ \mathrm{ml}$.

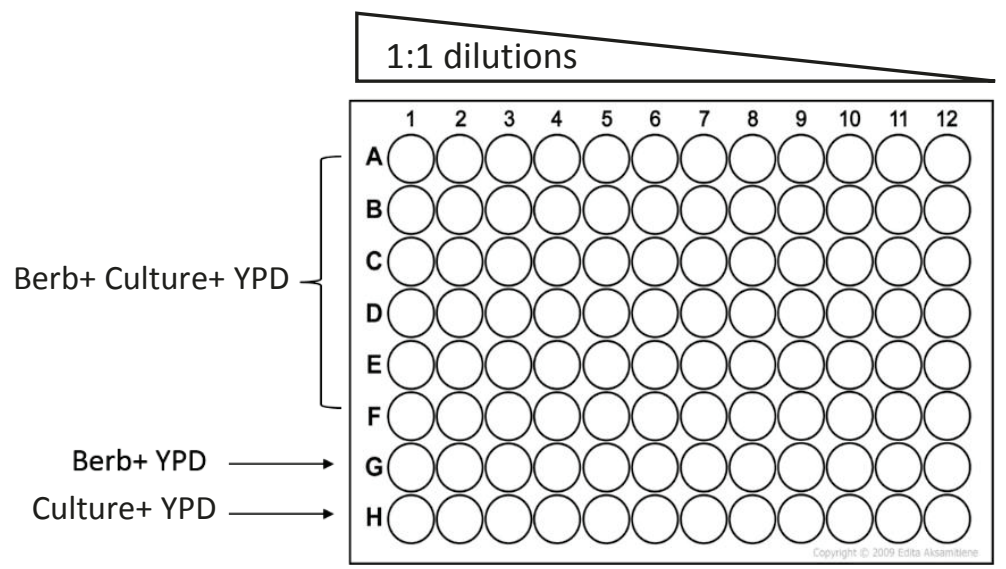

Figure 3. The figure shows an overview of the MIC procedure using 96-well, flat-bottom micro-titer plates to expose yeast cells to a range of berberine concentrations in rows $A-E$ along with positive, negative controls and the carrier in rows $\mathrm{F}-\mathrm{H}$. All wells had a final volume of $200 \mu \mathrm{l}$. The inhibition of yeast growth was examined over a range of berberine concentrations from 0.625 to $6.10 \times 10^{-4} \mathrm{mg} / \mathrm{ml}$.

As shown in Figure 3, the MIC test was carried out using 96-well micro-titer plates. In the first column of the first 6 rows (A-B-C-D-E-F), $100 \mu$ l of YPD medium was added to $100 \mu$ of berberine $(0.625 \mathrm{mg} / \mathrm{ml})$. These mixes were incrementally diluted in column 2 to column 12 by taking $100 \mu \mathrm{l}$ from the previous column and adding to $100 \mu \mathrm{l}$ of YPD in each well of the column before transferring to the next column. Finally, $100 \mu \mathrm{l}$ of diluted yeast culture containing about 
$\sim 100$ cells of the wild type strain $\mathrm{S} 288 \mathrm{C}$ was added to each well. As 'no yeast' control, well G1 was filled with $100 \mu \mathrm{l}$ of $0.625 \mathrm{mg} / \mathrm{ml}$ of berberine solution and $200 \mu \mathrm{l}$ of YPD broth in each well of row $G$ and a serial dilution was carried out in order to obtain a 1:1 ratio with a final volume at $200 \mu \mathrm{l}$. As 'no inhibition' control, $150 \mu \mathrm{l}$ of the culture and $50 \mu \mathrm{l}$ of YPD broth were added to row $\mathrm{H}$.

The wells in rows $A-F$ served as replicates whereas rows $G$ and $H$ served as the controls. The plate was incubated at $30{ }^{\circ} \mathrm{C}$ for 48 hours. After incubation, the endpoint absorbance at 600 nm was read with a Biotek ELKs 808 plate reader.

The following formula was used to determine the percent inhibition in each well:

$\%$ Inhibition $=100-\left(\left(\frac{\mathrm{Abs}_{\mathrm{DC}}-\mathrm{Abs} \mathrm{D} \mathrm{Bl}_{\mathrm{Bl}}}{\mathrm{Abs}_{\mathrm{LB}} \mathrm{C}-\mathrm{Abs_{ \textrm {LB } }}}\right) \times 100\right)$

Abs $D C=$ absorbance drug with culture

Abs $D_{B I}=$ absorbance drug blank

Abs $\angle B C=$ absorbance $L B$ with culture

Abs $\mathrm{LB}=$ absorbance LB only 


\section{GDA (Gene Deletion Array).}

S. cerevisiae GDA analysis was done with about 4700 yeast strains, each with a different, non-essential gene deleted. Using an equal amount of inoculum from each mutant strain, the strains were 'pinned' from a master plate of gene deletion strains to a plate with a sub-inhibitory concentration of berberine using a floating pin replicator. The floating pin replicator was put through a series of washes before the colonies were pinned from the library plate to the control and then the experimental plate.

The washing process took $4 \mathrm{~min}$ in total and consisted of submerging the tips of the replicator in distilled autoclaved water for 1 minute, 1 minute in bleach, $3 \times 30$ sec washes in distilled autoclaved water and then $30 \mathrm{sec}$ in 95\% ethanol. The replicator tips were then passed through a flame 2-3 times. The colonies on the replicator were printed on the control plate (without berberine) for $15-20$ seconds and then on the experimental plate $(0.13 \mathrm{mg} / \mathrm{ml}$ of berberine) for 15-20 seconds as well. Therefore, 14 plates were required to screen the whole set of 4700 mutants (set number 2 was missing and in set number 13 a small piece was contaminated). After 2 days of incubation at $30{ }^{\circ} \mathrm{C}$, colony sizes on both plates (with and without inhibitor) were determined by digital analysis. The size of the colony correlates with the rate of cell division (Memarian et al., 2007). The images were submitted through the SGAtools website (http://sgatools.ccbr.utoronto.ca/) to determine colony sizes on both control and experimental plates. The percentage inhibition of each mutant was calculated from the raw data values provided by SGAtools and values were recorded in Microsoft Excel. 
The following formula was used to determine the percentage inhibition:

Percentage Inhibition $=\left(\frac{\text { Control colony size-Experimental colony size }}{\text { Control colony size }}\right) \times 100 \%$.

Mean percentage inhibition values were calculated for each mutant and plotted against another set of values obtained from an experiment done by a previous student in the laboratory. From this plot, mutants that were consistently resistant or consistently sensitive in both data sets were chosen for functional analysis with GO Term tools. The total number of GDA experiments was five.

\section{Drop-Out Assay.}

Sensitive and non-sensitive mutants were randomly selected based on GDA results and plated on Yeast Peptone Dextrose (YPD) medium in petri dishes and incubated at $30^{\circ} \mathrm{C}$. A single colony from each mutant was then inoculated into $5 \mathrm{ml}$ of YPD broth and cultured at $30{ }^{\circ} \mathrm{C}$ for 1.5 days prior to adjusting the culture to $1 \times 10^{9}$ cells $/ \mathrm{ml}$ using spectrophotometry at $\mathrm{OD}_{600}$ and then doing a 10 -fold dilution series of the culture. Subsequently, $10 \mu \mathrm{l}$ droplets of the dilution series were administered to agar medium in a petri dish. The negative control plates contained $25 \mathrm{ml}$ of YPD-agar only. The experimental plates contained a final concentration of $0.13 \mathrm{mg} / \mathrm{ml}$ of berberine mixed with $25 \mathrm{ml}$ of YPD-agar. The droplets were allowed to dry on the surface of the agar in a laminar flow hood for a few minutes prior to incubation at $30^{\circ} \mathrm{C}$ for 48 hours. Finally, digital images were taken of the plates, and sensitivity to berberine was quantified, using the

formula: $\left(\frac{X-Y}{X}\right)$, where $X$ is colony count from control (no berberine) plate and $Y$ is colony count from the experimental (with berberine) plate. 


\section{Liposome Leakage Assay:}

A liposome leakage assay was used to see if berberine causes leakage in a cell membrane model. In this method, total-yeast-lipid liposomes containing carboxyfluorescein were prepared to test berberine for effects on membrane disruption/leakage. Large unilamellar liposomes (LUVs) were prepared by Dr. Cheetham at Carleton University. First, yeast lipids were dissolved in chloroform/methanol in a $2: 1, \mathrm{v} / \mathrm{v}$ ratio. The solvent was then removed with nitrogen gas before drying under a vacuum. $50 \mathrm{mM}$ carboxyfluorescein (CF) (Molecular Probes, OR, USA) was used to hydrate the lipid films in preparing the liposomes.

LUVs were $100 \mathrm{~nm}$ in diameter and consisted in dioleoylphosphatidylcholine (DOPC, Avanti Polar Lipids, Alabaster, AL, USA) loaded with carboxyfluorescein (CF) (Life Technologies Inc., Burlington, ON, Canada). The membrane disruption assay was carried out in a 96-well microplate (black/clear Optilux ${ }^{\mathrm{TM}}$ flat bottom; BD Bioscience, San Jose, CA, USA) at a predetermined LUV concentration. Each experimental well was measured for the threshold fluorescence intensity of the LUV suspension (excitation $492 \mathrm{~nm} / \mathrm{emission} 517 \mathrm{~nm}$ ) with an FLUOstar microplate reader (OPTIMA BMG LABTECH Inc., Durham NC, USA) to assess the fluorescence at time zero (F0). Berberine $(0.40 \mathrm{mg} / \mathrm{ml})$ was diluted in HEPES buffer (100mM Nacl 10mM HEPES, PH 7.4) before adding to the LUV suspension (1:10) to obtain final concentrations of between $1.95 \times 10^{-4}$ and $0.40 \mathrm{mg} / \mathrm{ml}$. After adding the berberine, the microplate was incubated in darkness for $1 \mathrm{~h}$ at room temperature and the fluorescent emission was measured to obtain $\mathrm{F}$ (fluorescent intensity after berberine addition). The F100 (Fluorescence intensity with 100\% leakage of CF) was read 20 min after $20 \mu \mathrm{l}$ of triton X-100 (10\% v/v) was added to each well to 
allow total lysis of liposome after the first reading was completed. The following formula was used to determine the percentage liposome lysis: \%leakage= [(F-F0)/(F100-F0)]X100.
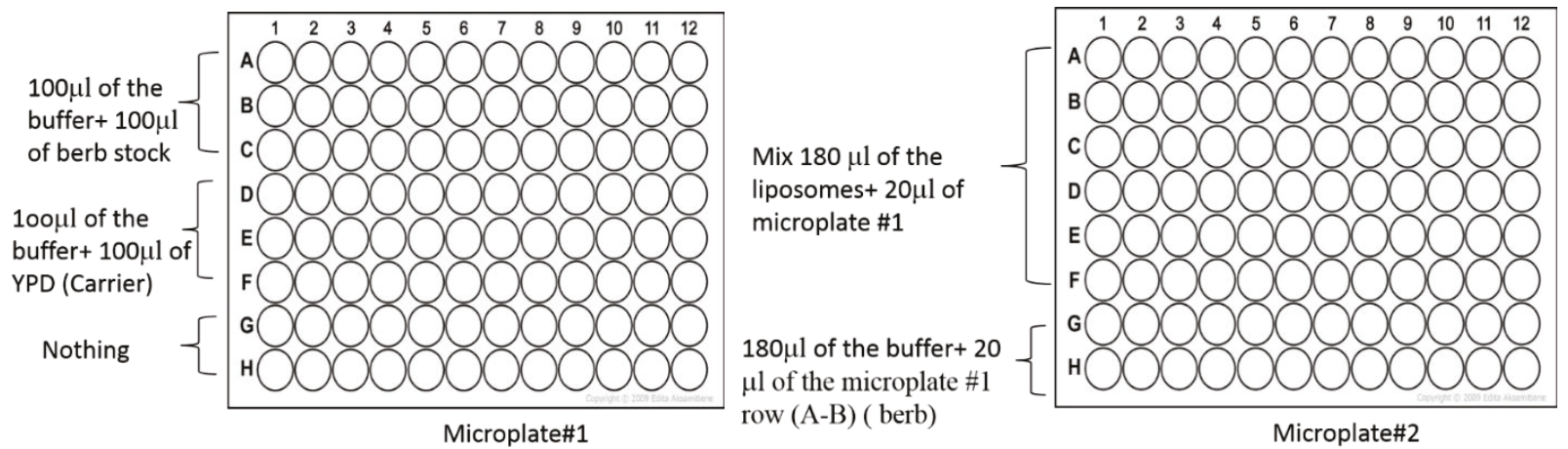

Figure 4. The figure shows an overview of the Liposome Leakage Assay 96-well, flat-bottom micro-titer plates.

As shown in Figure 4, the liposome assay was carried out by using 96-well micro- titer plates. In the first column of the first 3 rows (A-B-C) of microtiter plate \#1, $100 \mu \mathrm{l}$ of $0.40 \mathrm{mg} / \mathrm{ml}$ Berberine solution was diluted in $100 \mu$ l of the buffer (100mM Nacl $10 \mathrm{mM}$ HEPES, PH 7.4). These mixes were incrementally diluted in buffer from column 2 to column 12 by taking $100 \mu$ from the previous column and transferring it to the next one and adding $100 \mu$ of the buffer in each column. In the first column of row D, E and F of microtiter plate \# 1, $100 \mu$ l of YPD growth medium was diluted in $100 \mu \mathrm{l}$ of the buffer (100mM Nacl 10mM HEPES, PH 7.4). These mixes were incrementally diluted in the buffer from column 2 to column 12 by taking $100 \mu$ from the previous column and transferring it to the next one and adding $100 \mu \mathrm{l}$ of the buffer in each column. Finally, the last 2 rows were empty.

$20 \mu \mathrm{l}$ of each well from the first 6 rows (A-B-C-D-E-F) of microplate \#1 were transferred to the corresponding wells on microplate \#2. $180 \mu$ of the liposomes suspension was added to each 
of these wells. In rows $\mathrm{G}$ and $\mathrm{H}$ of microplate $\# 2,180 \mu \mathrm{l}$ of the buffer and $20 \mu \mathrm{l}$ of the rows $\mathrm{A}$ and D of micro plate \#1 were added to the corresponding wells (columns 1-12) of microplate \#2.

\section{Growth Curve:}

A growth curve experiment was used to determine the rate of change in cell number of particular strains that can or can not grow in the presence of berberine. In this method, eight strains were used to see the different growth rates of the cells with and without berberine. The following strains were used: YLR200W and YGR078C as resistant strains; YML008C, YPR154W and YDR388W as sensitive strains; and YMR119W, YGL086W and the wild type (S288C) were chosen as 'medium sensitive strains'.

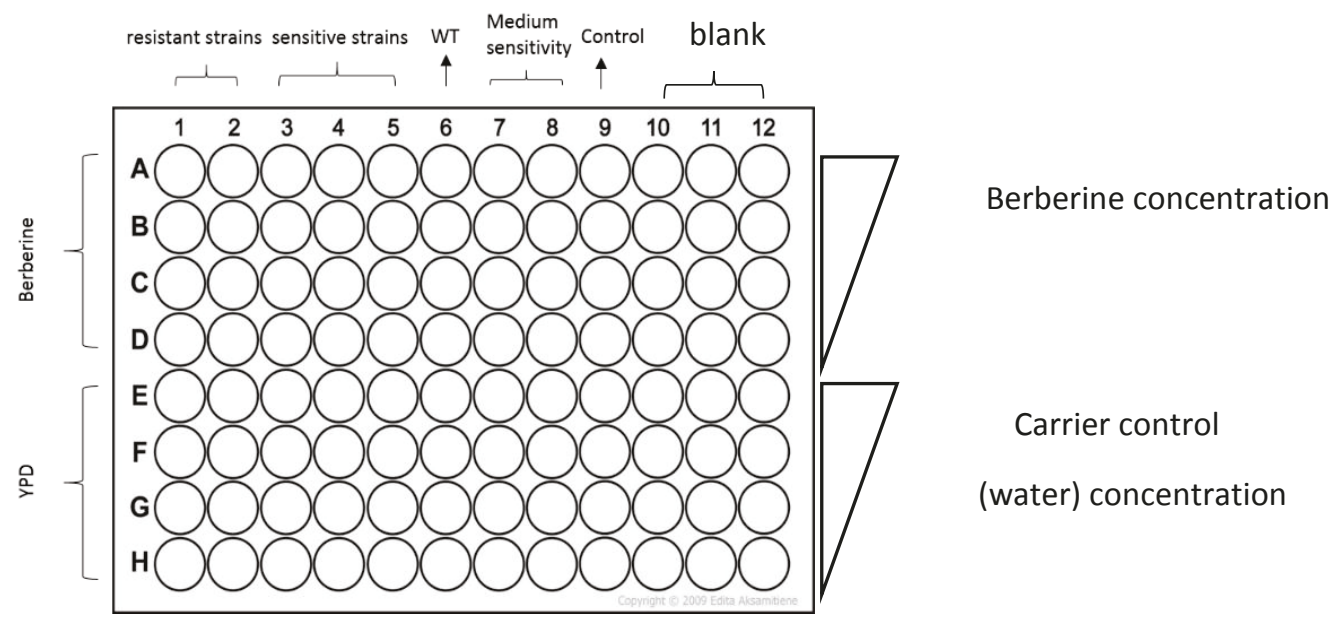

Figure 5. The figure shows an overview of the growth curve experiment using 96-well, flat-bottom microtiter plates. All wells had a final volume of $200 \mu \mathrm{l}$. The inhibitions of the mutant strain over a range of berberine concentrations from 0.25 to $0.03125 \mathrm{mg} / \mathrm{ml}$.

As shown in Figure 5, the growth curve test was carried out using 96-well micro- titer plates. In the first row (A) from well 1-8, $100 \mu$ l of YPD medium was added to $100 \mu$ l of berberine $(0.25 \mathrm{mg} / \mathrm{ml})$. These mixes were incrementally diluted from row $A$ to row $D$ by taking $100 \mu \mathrm{l}$ from 
the previous row and adding $100 \mu \mathrm{l}$ of YPD in each well of the column before transferring to the next column. Finally, $100 \mu$ l of diluted yeast culture ( 100 cells) of each yeast strain was added: resistant strains YLR200W and YGR078C to columns 1 and 2, respectively; sensitive strains YPR154W, YML008C and YDR388W were added to columns 3, 4 and 5, respectively; and for medium sensitive strains, wild type strain S288C, YMR119W and YGL086W were added to columns $6,7,8$, respectively. As 'no yeast' control, column 9 from well A-D was filled with $200 \mu \mathrm{l}$ of $0.25 \mathrm{mg} / \mathrm{ml}$ of berberine solution and $100 \mu \mathrm{l}$ of YPD broth in the first well and $200 \mu \mathrm{l}$ of YPD in the rest of the wells in this column. A serial dilution was carried by serial transfer of $100 \mu \mathrm{l}$ from row $A$ to $B$ mix by pipetting and then transfer from B to $C$, etc., down the plate. As 'no inhibition' control, $100 \mu \mathrm{l}$ of the culture and $100 \mu \mathrm{l}$ of YPD broth were added to rows E-H. The absorbance at $600 \mathrm{~nm}$ was read every hour using Biotek CYTATION5 plate reader, and the Gene 2.07 program, for a total of $12 \mathrm{hrs}$ at $30^{\circ} \mathrm{C}$.

\section{Fluorescent microscopy and confocal microscopy.}

Fluorescence microscopy was used to see where berberine localizes within yeast cells. This is possible because berberine has a natural yellow fluorescent color (Gibbs and Seddon, 2000). In this method, an Axioplan 2 Universal Microscope was used to examine yeast cells following exposure to berberine. Initially, 19 strains were selected based on GDA and drop-out results for fluorescence microscopy. In this method, liquid yeast cell culture was grown overnight as described above and diluted to $\mathrm{OD}_{600} \sim 0.550$. Cells were introduced to a sub-lethal berberine concentration of a final concentration of $0.13 \mathrm{mg} / \mathrm{ml}$. Berberine was imaged with $E x / E m=348 / 530 \mathrm{~nm}$. The following strains were examined after $40 \mathrm{~min}$ berberine exposure: medium sensitivity strains were S288C (wildtype), YGL086W and YMR119W; sensitive strains 
were YML008C, YHR030C, YBL007C, YPR154W, YGL173C, YDR388W, YNL042W, YOR153W, YMR110C, YDR378C, YDL226C, YDR289C, and YIR002C; resistant strains were YOR058C, YLR200W and YGR078C. All the selected strains were examined after $40 \mathrm{~min}$ of berberine exposure under oil immersion at 630X magnification and photographed using a Carl Zeiss Axioplan 2 imaging microscope, and the Axiovision Rel.4.6 program.

In collaboration with Denise Chabot from Agriculture and Agri-Food Canada, confocal microscopy was used to get higher resolution images that would allow identification of organelles stained by berberine. In this method, an LSM510 Zeiss confocal microscope equipped with a Zeiss Alpha Plan Apo 63X na1.46 objective and zoom factor of 2.5 and ZEN software were used to examine three strains of each category (medium sensitivity, sensitive and resistant).

For berberine staining, around $4 \mu \mathrm{l}$ of berberine-stained yeast cells were placed on a glass slide pre-treated with poly-L-lysine. These samples were then covered with high-resolution coverslips $(0.17 \mathrm{~mm})$ and sealed with nail polish. Berberine staining was imaged with $E x / E m=405 / 515-565 n m$.

For Rhodamine 123 + berberine staining, $100 \mu$ l of live yeast cells were added to $100 \mu \mathrm{l}$ of berberine solution and then the mix was incubated in the darkness for 20 minutes at room temperature. Then, an equal volume of rhodamine 123 solution $(10 \mathrm{mg} / \mathrm{ml})$ was added and the cells were incubated for 10 minutes in the dark at room temperature. The cells were resuspended twice in berberine solution, and the sample was then covered with a high-resolution coverslip $(0.17 \mathrm{~mm})$ and sealed with nail polish using $\mathrm{Ex} / \mathrm{Em}=514 / 530-600 \mathrm{~nm}$. 
For berberine and DAPI staining, about $100 \mu \mathrm{l}$ of concentrated cells (YPR154W) were mixed with $100 \mu$ l of berberine $(0.13 \mathrm{mg} / \mathrm{ml})$ and then the mixture was incubated for 40 minutes at room temperature. Then, $2 \mu$ of DAPI stock solution $(2 \mathrm{mg} / \mathrm{ml})$ was added prior to placing. $5 \mu l$ of the cell mixture on a glass slide pre-treated with poly-L-lysine and the sample was then covered with a high-resolution coverslip $(0.17 \mathrm{~mm})$ and examined under the confocal microscope using $E x / E m=405 / 420-480 n m$.

For Nile Red staining, $4 \mu \mathrm{l}$ of a stock solution of Nile Red in acetone $(2.5 \mathrm{mg} / \mathrm{ml})$ was added to $1 \mathrm{ml}$ of water for a final Nile Red concentration of $0.1 \mathrm{mg} / \mathrm{ml}$. Abot $3 \mu \mathrm{l}$ of concentrated yeast cells were mixed with $3 \mu$ of Nile Red working solution. on a glass slide pre-treated with poly-Llysine. The sample was examined under the confocal microscopy using Ex/Em=488/530-700nm

\section{One-Way ANOVA analysis.}

A 1-way ANOVA with a post-hoc Tukey Honestly Significant Difference (HSD) test with a significance level of $5 \%$ was used in this study to test for statistical differences in berberine staining patterns among selected yeast strains.

ANOVA was preformed with online tools at: http://scistatcalc.blogspot.ca/2013/11/one-wayanova-test-calculator-in.html. 


\section{$\underline{\text { Results: }}$}

\section{MIC on S. cerevisiae wild type (S288C):}

In MIC assays, the percentage inhibition of the wild-type strain was determined over a berberine concentration range of $6.10 \times 10^{-4}-0.625 \mathrm{mg} / \mathrm{ml}$. Visual examination shows that there was a great inhibition in the first 4 columns $(\geq 0.078 \mathrm{mg} / \mathrm{ml})$, but inhibition decreased abruptly in the rest of the columns $(\leq 0.039 \mathrm{mg} / \mathrm{ml})$. Concentrations of $0.156 \mathrm{mg} / \mathrm{ml}$ and $0.078 \mathrm{mg} / \mathrm{ml}$ of berberine yielded $64 \%$ to $45 \%$ inhibition, respectively. A concentration of $0.13 \mathrm{mg} / \mathrm{ml}$ was used as a semi-inhibitory concentration subsequent GDA analyses.

\section{GDA analysis:}

GDA analysis was performed on a library of $\sim 4700$ yeast strains with a concentration of $0.13 \mathrm{mg} / \mathrm{ml}$ berberine incorporated into YPD agar medium. I did three replicate GDA experiments and, in collaboration with me, an undergraduate student (Fatima Haider) did two additional GDA experiments. The GDA data presented in this thesis is, therefore, based on five replicate experiments. Yeast mutants that were sensitive to berberine were identified on the plates using digital analysis of colony sizes (Figure 6). The relative sensitivities of yeast deletion mutants were ranked, from least to most sensitive to berberine, based on replicate GDA experiments (Table 2). 
A

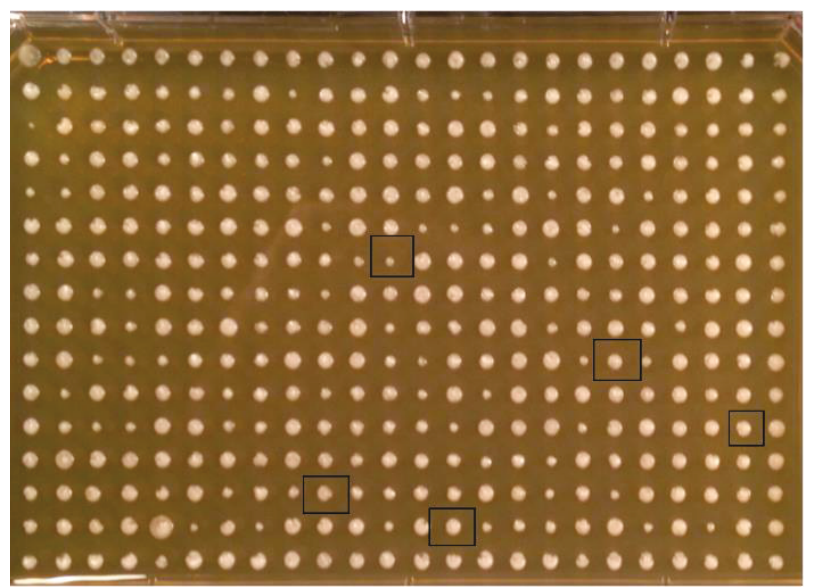

B

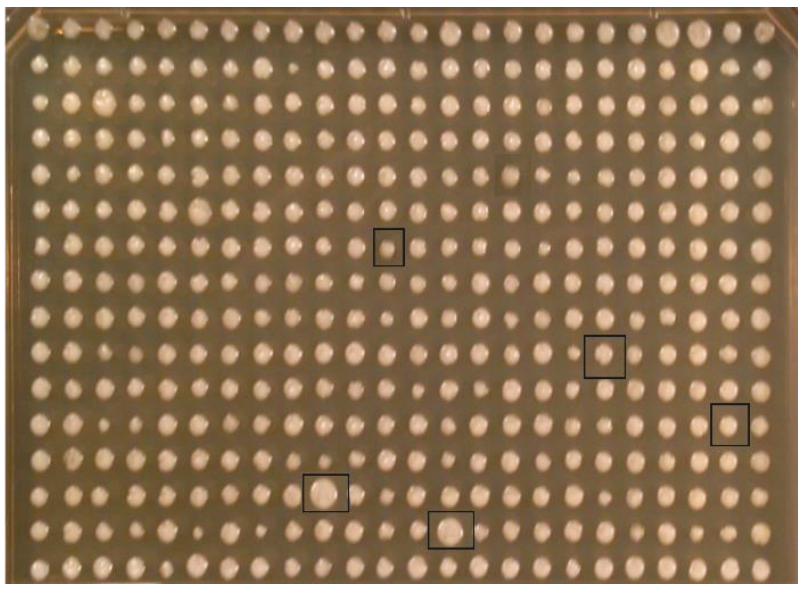

Figure 6. An example of the GDA on the mutant library in Plate 3 where $(A)$ is the experimental plate (berberine concentration $0.13 \mathrm{mg} / \mathrm{ml}$ ), and $(B)$ is the control plate (no berberine). The black squares show examples of deleted mutants that are sensitive to berberine.

The colonies indicated in the figure above represent mutant strains YDR063W (at vertical, horizontal position of 0,14$)$, YDR388W [(L, 23), RVS167], YDR378C [(J, 19), LSM6], YDR289C [(N, 10), RTT103] and YDL226C [(G, 12), GCS1] which are notably sensitive to berberine. 


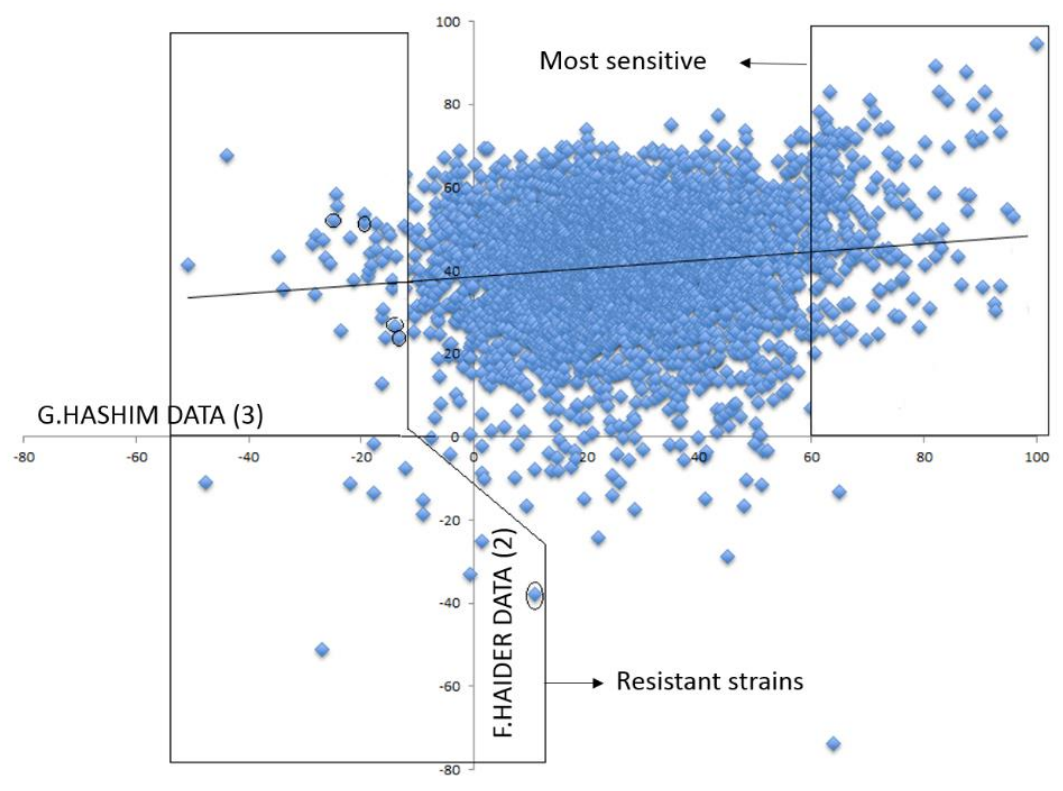

Figure 7. Correlation of mean inhibition values from 2 GDA experiments done by F.Haider (Y-axis) and 3 GDA experiments done by me (G.Hashim, $\mathrm{X}$-axis). The boxed area at left represents putatively 'resistant' mutants. The boxed area at right indicates putatively 'sensitive' mutants. The deleted genes in the boxed areas were analyzed for functional enrichment.

Figure 7. shows a correlation between the 2 GDA data sets of F. Haider and my 3 GDA data sets. The regression line has a positive slope which indicates that, in general, the relative sensitivity of the yeast deletion mutants is congruent in the two sets of experiments. An arbitrary cut-off of $\geq 60 \%$ inhibition was used to select the 71 'most sensitive' mutants. Deletion mutants that were 'resistant' were also identified. These are shown in the boxed area at the left of Figure 7 where, strains that showed the sensitivity of approximately $\leq 10 \%$ were selected for further analysis. The circled mutants formed a group that was enriched in tubulin-associated genes ( $P$ value was < 0.10). 


\section{Drop-Out analysis:}

We sought to substantiate the results of our GDA experiments using drop out assays.

Twenty-eight yeast deletion mutants were randomly selected from the 'sensitive' and 'mediumsensitive' groups, and the wild type control strain, S288C, were all tested (Table 1, Figures 8-10).

Table 1. Berberine-sensitive and medium sensitive deletion mutants were randomly selected from the GDA data sets for sensitivity analysis by drop-out analyses. A sum of GDA sensitivity values from my 3 GDA experiments and their corresponding drop out sensitivity values are presented below. List $A$ shows 17 most sensitive yeast deletion mutants based on my 3 GDA experiments. List B shows 12 randomly selected 'meduim-sensitive' yeast deletion mutants based on my 3 GDA experiments. Drop-out sensitivity ranged from a negative value (resistant) to a positive value of 1 (complete growth inhibition).

List A, 'sensitive' strains.

\begin{tabular}{|l|l|l|l|l|l|l|l|l|}
\hline Plate & Row & Column & ORF & $\begin{array}{c}\text { \% Inhib 1 } \\
\text { (GDA) }\end{array}$ & $\begin{array}{c}\text { Inhib 2 } \\
\text { (GDA) }\end{array}$ & $\begin{array}{c}\text { \% Inhib 3 } \\
\text { (GDA) }\end{array}$ & $\begin{array}{c}\text { Sum } \\
1+2+3 \\
\text { (GDA) }\end{array}$ & $\begin{array}{l}\text { Drop-out } \\
\text { sensitivity }\end{array}$ \\
\hline 6 & 15 & 23 & YHR030C & 100 & 100 & 0 & 200 & 1.0 \\
\hline 12 & 10 & 17 & YOR153W & 100 & 52.5 & 100 & 252.5 & 0.4 \\
\hline 5 & 13 & 7 & YGL173C & 72.8 & 100 & 100 & 272.8 & 0.9 \\
\hline 3 & 12 & 23 & YDR388W & 84.37 & 87.2 & 90.8 & 262.5 & 0.8 \\
\hline 14 & 4 & 8 & YNL042W & 54.6 & 66.4 & 66.3 & 187.5 & 0.6 \\
\hline 3 & 10 & 19 & YDR378C & 83.1 & 76.7 & 88.4 & 248.3 & 0.5 \\
\hline 10 & 10 & 12 & YMR110C & 60.0 & 64.6 & 65.3 & 189.10 & 1.0 \\
\hline 7 & 15 & 2 & YIR002C & 92.6 & 72.6 & & 165.3 & 0.1 \\
\hline 14 & 9 & 21 & YPR154W & 63.4 & 71.2 & 76.2 & 210.8 & 0.9 \\
\hline 3 & 14 & 10 & YDR289C & 90.0 & 87.0 & 88.9 & 266.10 & 0 \\
\hline 3 & 7 & 7 & YKL101W & 78.7 & 57.4 & 60.9 & 197.3 & 0.5 \\
\hline & 7 & 12 & YDL226C & 83.8 & 89.1 & 73.6 & 246.6 & 0.9 \\
\hline & & & & & & & & \\
\hline
\end{tabular}




\begin{tabular}{|l|l|l|l|l|l|l|l|l|}
\hline 3 & 15 & 14 & YDR063W & 62.8 & 59.1 & 75.3 & 197.5 & 0.5 \\
\hline 1 & 15 & 14 & YBL007C & 90.2 & 90.1 & 85.1 & 265.6 & 0.4 \\
\hline 1 & 2 & 14 & YBR023C & 52.8 & 74.3 & 63.4 & 190.6 & 0.5 \\
\hline 10 & 11 & 22 & YML008C & 77.1 & 71.5 & 64.6 & 213.5 & 0.9 \\
\hline 4 & 14 & 5 & YFR010W & 79.6 & 87.1 & 86.2 & 252.10 & 0.4 \\
\hline
\end{tabular}

List B, 'meduim-sensitive' strains.

\begin{tabular}{|l|l|l|l|l|l|l|l|l|}
\hline Plate & Row & Column & ORF & $\begin{array}{l}\text { \% Inhib 1 } \\
\text { (GDA })\end{array}$ & $\begin{array}{l}\text { \% Inhib 2 } \\
(\text { GDA })\end{array}$ & $\begin{array}{l}\text { \% Inhib 3 } \\
\text { (GDA) }\end{array}$ & $\begin{array}{l}\text { Sum } \\
1+2+3 \\
\text { (GDA) }\end{array}$ & $\begin{array}{l}\text { Drop-out } \\
\text { sensitivity }\end{array}$ \\
\hline 3 & 2 & 8 & YDR192C & 25.8 & 25.9 & 25.1 & 76.9 & -0.2 \\
\hline 6 & 14 & 18 & YHR151C & 5.9 & -2.9 & 0 & 3.07 & -0.5 \\
\hline 5 & 3 & 16 & YFR015C & 55.2 & -26.1 & 9.2 & 38.4 & 0 \\
\hline 5 & 5 & 19 & YGL086W & 54.3 & 40.6 & 0 & 94.9 & 0 \\
\hline 14 & 9 & 3 & YPR171W & 41.6 & 53.7 & -42.0 & 54.3 & -7.3 \\
\hline 4 & 3 & 21 & YEL003W & 0.9 & 5.6 & 26.2 & 32.8 & -0.4 \\
\hline 14 & 10 & 5 & YMR119W & 66.8 & 54.0 & 59.2 & 52.5 & 0 \\
\hline 12 & 12 & 20 & YOR058C & -37.2 & 2.9 & 17.9 & -16.3 & 0 \\
\hline 11 & 11 & 5 & YNL094W & 35.0 & -3.2 & 65.2 & 96.98 & -1 \\
\hline 5 & 6 & 23 & YGR078C & 55.8 & 24.2 & 31.6 & 111.8 & -0.5 \\
\hline 11 & 4 & 22 & YNL153C & -25.8 & 20.7 & -36.9 & -42.09 & -0.5 \\
\hline 9 & 2 & 22 & YLR200W & 7.64 & 37.4 & 44.0 & 89.16 & -0.1 \\
\hline
\end{tabular}




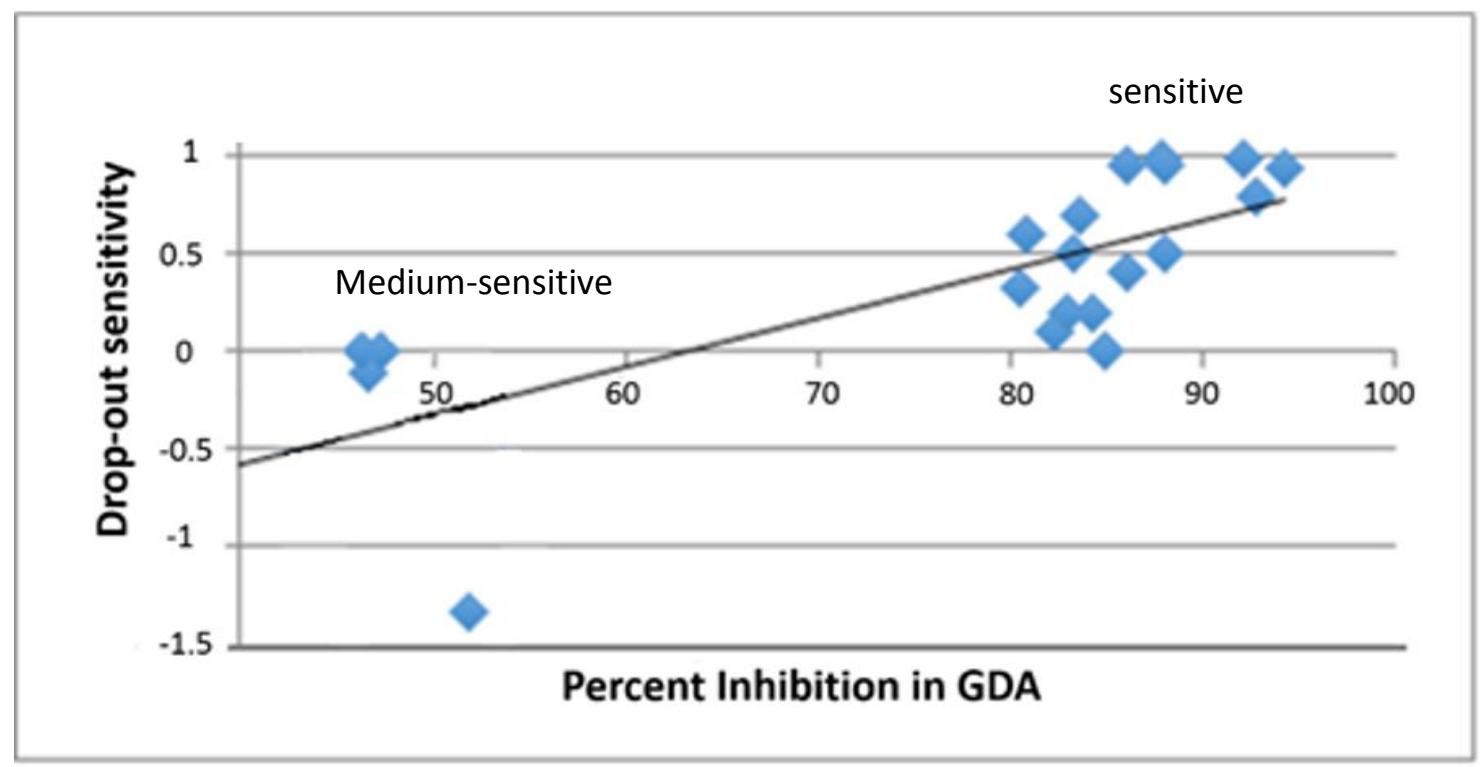

Figure 8. Berberine sensitivity of 29 randomly selected yeast deletion mutants that were sensitive or medium-sensitive in GDA data set and drop-out assays. The positive slope of the regression line indicates overall congruence in the results of sensitivity based on the two assays.

Figure 8. shows a good agreement between the drop-out sensitivity and \% inhibition from GDA experiments. In fact, the most sensitive mutants in GDA are almost all among the most sensitive mutants in drop out assays. Figure 9 and 10 show examples of drop out assays for selected sensitive and medium-sensitive deletion mutants. 

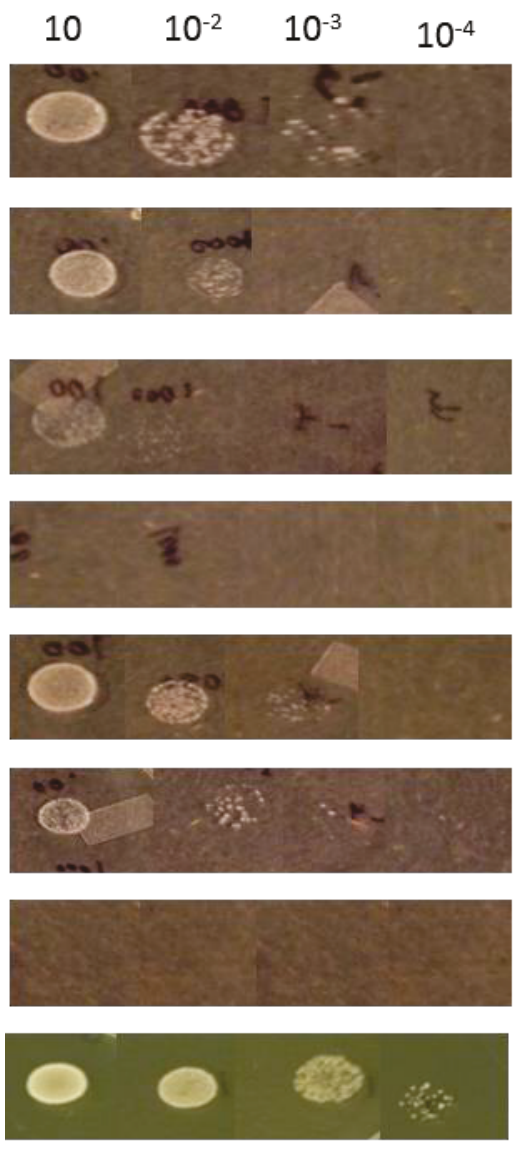

Experimental

( $0.13 \mathrm{mg} / \mathrm{mL}$ berberine)
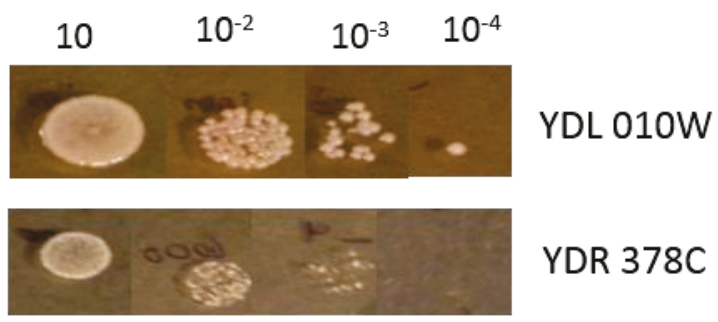

YDR 378C

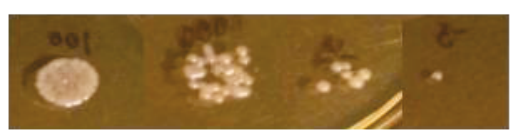

YDR 388W

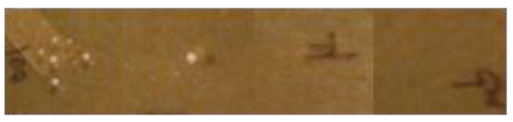

YGL 173C

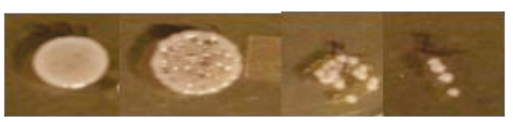

YHR 030C

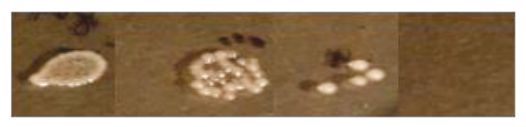

YPR 154W

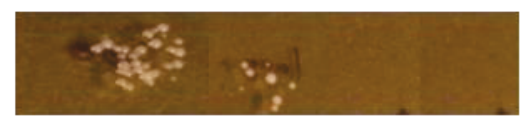

YDL 226C

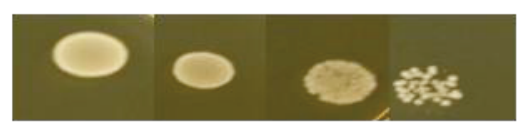

YCR 053W

\section{Control}

(No berberine)

Figure 9. Example drop -out assays for 8 selected sensitive deletion mutants strains. Dilutions are given across the top. 

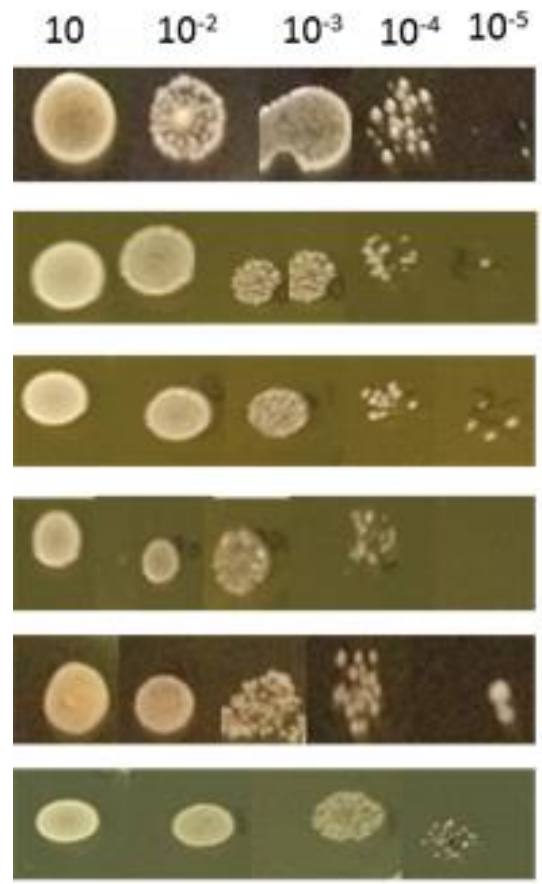

Experimental

( $0.13 \mathrm{mg} / \mathrm{mL}$ berberine)
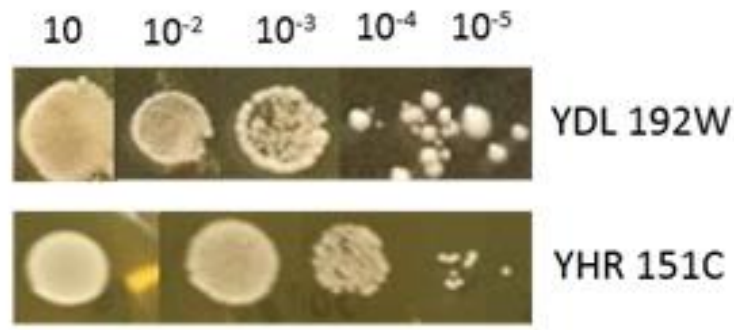

YHR 151C

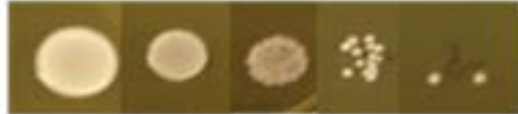

YFR 015C

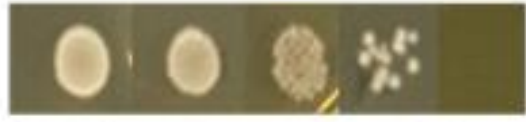

YGL 086W

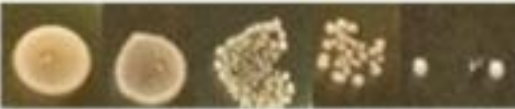

YPR $171 W$

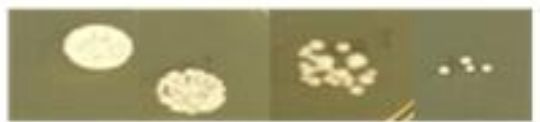

S288C

(No berberine)

Figure 10. Example drop-out assays for 6 'medium-sensitive' gene deletion strains and the wild type (S288C). Dilutions are given across the top of figure. 


\section{Analysis of berberine sensitvity of selected mutants based on growth curves.}

For this analysis, I used the Biotek CYTATION5 plate reader, and the Gene 2.07 program to track the growth of yeast cells by measuring absorbance at $600 \mathrm{~nm}$. Figure 11 presents the growth curve of yeast cells in liquid culture. Significant growth can be seen in the resistant strains (columns 1 and 2) in the presence of berberine. On the other hand, growth rate is noticeably reduced by berberine in the sensitive strains (columns 3, 4 and 5) and for medium-sensitive strains including wild type (S288C) (columns 6, 7 and 8; Figure 11). Similar to drop-out assays, this verifies GDA berberine-sensitivity assays

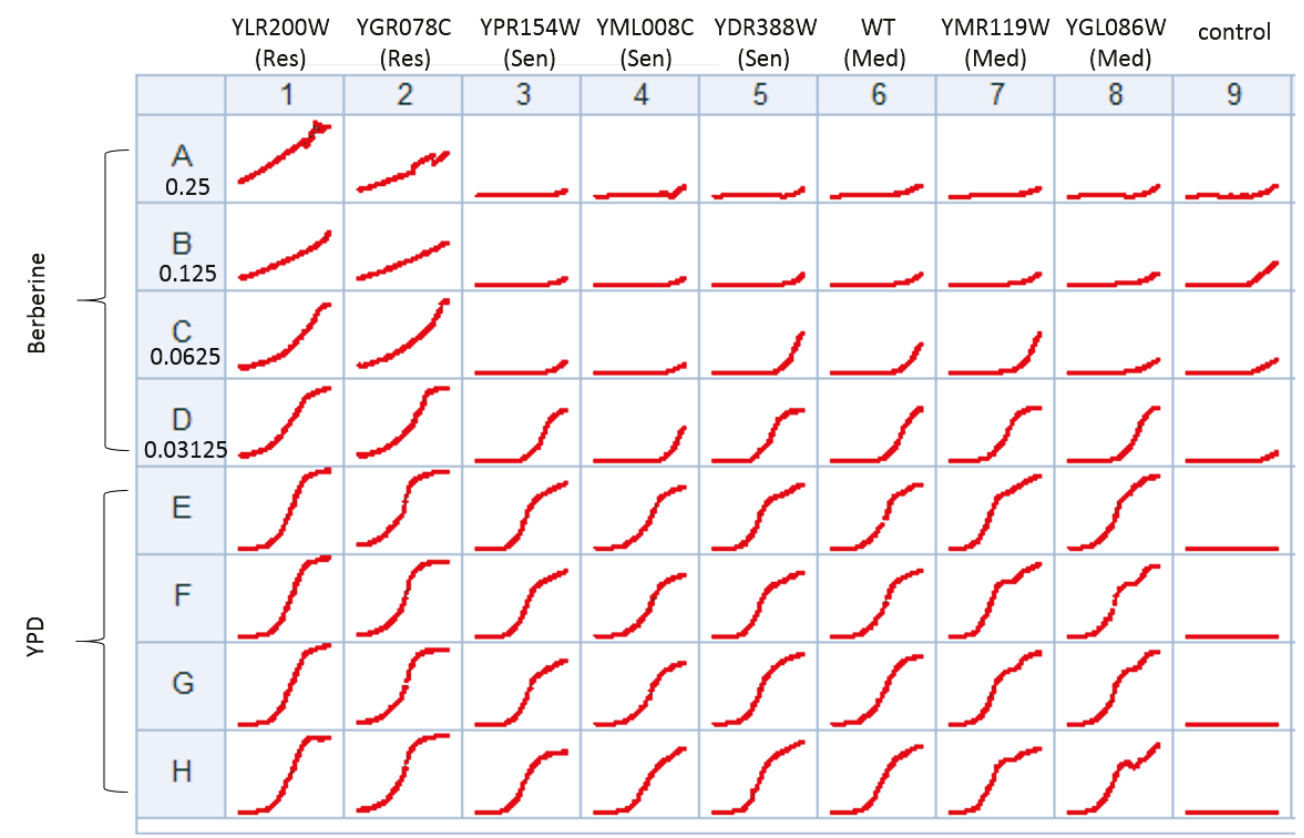

Figure 11. Shows the growth rates of yeast culture with and without berberine. Berberine concentration ranged $0.25-0.03125 \mathrm{mg} / \mathrm{ml}$. Res= resistant strains based on GDA analysis; Sen= sensitive strains based on GDA analysis; Med= medium-sensitive strains. 


\section{Functional Analysis:}

Table 2. shows the most sensitive mutants to berberine with percent inhibition greater than $60 \%$ that were used for functional analysis by Go Terms. Based on the cellular processes in which they were involved, GO-Term identified 17 different categories : transferase activity (11 mutants), DNA binding (5 mutants), enzyme regulator activity (5 mutants), RNA and mRNA binding (4 mutants), oxydoreductase activity ( 2 mutants), cytoskeletal protein binding (2 mutants), hydrolase activity (8 mutants), protein transporter activity (2 mutants), ubiquitin-like protein binding ( 2 mutants), lyase activity ( 2 mutants), structural function ( 2 mutants), other functions (10 mutants) and unknown function (16 mutants). 
Table 2. Functional analysis of the most sensitive strains based on GDA analyses. The mutants were selected based on a size reduction of between $60 \%-100 \%$ (see Figure 7 ). The deleted genes in the most sensitive strains are arranged into functional groups in the table.

\begin{tabular}{|c|c|c|c|}
\hline Gene & Strain & Gene function & $\begin{array}{l}\text { Average } \\
\text { Colony } \\
\text { size } \\
\text { reduction* }\end{array}$ \\
\hline \multicolumn{4}{|c|}{ Transferase Activity } \\
\hline SLT2 & YHR030C & $\begin{array}{l}\text { MAP kinase activity, protein serine/threonine kinase } \\
\text { activity and protein kinase activity. }\end{array}$ & $100 \%$ \\
\hline ADE8 & YDR408C & phosphoribosylglycinamide formyltransferase activity. & $93.4 \%$ \\
\hline CYT1 & YOR065W & $\begin{array}{l}\text { Electron transporter, transferring electrons within CoQH2- } \\
\text { cytochrome c reductase complex activity. }\end{array}$ & $82.8 \%$ \\
\hline GPH1 & YPR160W & Glycogen phosphorylase activity. & $79.2 \%$ \\
\hline ERG6 & YML008C & Sterol 24-C-methyltransferase activity. & $71.1 \%$ \\
\hline ARO9 & YHR137W & $\begin{array}{l}\text { Aromatic-amino-acid: 2-oxoglutarate aminotransferase } \\
\text { activity. }\end{array}$ & $70.8 \%$ \\
\hline STE14 & YDR410C & $\begin{array}{l}\text { Protein C-terminal S-isoprenylcysteine carboxyl O- } \\
\text { methyltransferase activity. }\end{array}$ & $61.3 \%$ \\
\hline RAD30 & YDR419W & DNA-directed DNA polymerase activity. & $61.1 \%$ \\
\hline TDA1 & YMR291W & Protein kinase activity. & $60.8 \%$ \\
\hline MGT1 & YDL200C & $\begin{array}{l}\text { Methylated-DNA-[protein]-cysteine S-methyltransferase } \\
\text { activity. }\end{array}$ & $60.8 \%$ \\
\hline ASI1 & YMR119W & ubiquitin-protein transferase activity. & $60.0 \%$ \\
\hline \multicolumn{4}{|c|}{ DNA Binding } \\
\hline YAP3 & YHL009C & $\begin{array}{l}\text { Transcription factor activity, sequence-specific DNA } \\
\text { binding and sequence-specific DNA binding. }\end{array}$ & $81.9 \%$ \\
\hline HMO1 & YDR174W & $\begin{array}{l}\text { DNA binding, bending, double-stranded DNA binding and } \\
\text { four-way junction DNA binding. }\end{array}$ & $77.3 \%$ \\
\hline
\end{tabular}




\begin{tabular}{|c|c|c|c|}
\hline & YPR015C & Sequence-specific DNA binding. & $75.1 \%$ \\
\hline MSN1 & YOL116W & Sequence-specific DNA binding. & $67.8 \%$ \\
\hline MIG1 & YGL035C & $\begin{array}{l}\text { RNA polymerase II core promoter proximal region } \\
\text { sequence-specific DNA binding, transcriptional repressor } \\
\text { activity, RNA polymerase II core promoter proximal region } \\
\text { sequence-specific binding and sequence-specific DNA } \\
\text { binding. }\end{array}$ & $60.9 \%$ \\
\hline \multicolumn{4}{|c|}{ Enzyme Regulator activity } \\
\hline MSB4 & YOL112W & GTPase activator activity. & $74.7 \%$ \\
\hline CLB6 & YGR109C & $\begin{array}{l}\text { cyclin-dependent protein serine/threonine kinase } \\
\text { regulator activity. }\end{array}$ & $70.5 \%$ \\
\hline NEL1 & YHR035W & GTPase activator activity. & $70.0 \%$ \\
\hline CSG2 & YBR036C & Enzyme regulator activity. & $64.1 \%$ \\
\hline SAC7 & YDR389W & GTPase activator activity. & $62.8 \%$ \\
\hline \multicolumn{4}{|c|}{ RNA Binding and mRNA binding } \\
\hline XRN1 & YGL173C & $\begin{array}{l}\text { 5'-3' exoribonuclease activity, chromatin binding and } \\
\text { mRNA binding. }\end{array}$ & $90.9 \%$ \\
\hline LSM6 & YDR378C & contributes to RNA binding. & $82.7 \%$ \\
\hline ARF3 & YOR094W & $\begin{array}{l}\text { Enzyme regulator activity, GTPase activity and mRNA } \\
\text { binding. }\end{array}$ & $71.7 \%$ \\
\hline PIN4 & YBL051C & mRNA binding. & $60.4 \%$ \\
\hline \multicolumn{4}{|c|}{ Oxidoreductase Activity } \\
\hline & YMR315W & oxidoreductase activity. & $71.1 \%$ \\
\hline HFD1 & YMR110C & $\begin{array}{l}\text { 3-chloroallyl aldehyde dehydrogenase activity and } \\
\text { carboxylate reductase activity. }\end{array}$ & $63.3 \%$ \\
\hline$y$ & $4 \mathrm{Mat}$ & nding & \\
\hline
\end{tabular}




\begin{tabular}{|c|c|c|c|}
\hline RVS167 & YDR388W & $\begin{array}{l}\text { Cytoskeletal protein binding and contributes to lipid } \\
\text { binding. }\end{array}$ & $87.4 \%$ \\
\hline GCS1 & YDL226C & actin binding and GTPase activator activity. & $82.1 \%$ \\
\hline \multicolumn{4}{|c|}{ Hydrolase Activity } \\
\hline MPH1 & YIR002C & $\begin{array}{l}\text { 3'-5' DNA helicase activity and flap-structured DNA } \\
\text { binding. }\end{array}$ & $89.9 \%$ \\
\hline UBP6 & YFR010W & ubiquitin-specific protease activity. & $84.3 \%$ \\
\hline PDR5 & YOR153W & xenobiotic-transporting ATPase activity. & $84.1 \%$ \\
\hline DOG1 & YHR044C & 2-deoxyglucose-6-phosphatase activity. & $76.1 \%$ \\
\hline NFT1 & YKR103W & macroautophagy and transport. & $73.6 \%$ \\
\hline MSH2 & YOLO9OW & $\begin{array}{l}\text { ATP binding, ATPase activity, contributes to DNA binding } \\
\text { activities. }\end{array}$ & $72.1 \%$ \\
\hline VMS1 & YDR049W & Contributes to ubiquitin-specific protease activity. & $69.4 \%$ \\
\hline HRQ1 & YDR291W & ATP-dependent 3'-5' DNA helicase activity. & $61.3 \%$ \\
\hline \multicolumn{4}{|c|}{ Protein Transporter Activity } \\
\hline MSN5 & YDR335W & $\begin{array}{l}\text { Nuclear export signal receptor activity and protein } \\
\text { binding. }\end{array}$ & $78.4 \%$ \\
\hline KAP122 & YGL016W & Protein transporter activity. & $70.9 \%$ \\
\hline \multicolumn{4}{|c|}{ Ubiquitin-like Protein Binding } \\
\hline SLA1 & YBL007C & Protein binding, bridging and ubiquitin binding. & $88.5 \%$ \\
\hline CUE2 & YKL090W & ubiquitin binding. & $74.0 \%$ \\
\hline \multicolumn{4}{|c|}{ Lyase Activity } \\
\hline HIS3 & YOR202W & imidazoleglycerol-phosphate dehydratase activity. & $73.2 \%$ \\
\hline PAD1 & YDR538W & carboxy-lyase activity and mRNA binding. & $61.2 \%$ \\
\hline \multicolumn{4}{|c|}{ Structural moleculer Activity } \\
\hline RPS11A & YDR025W & structural constituent of ribosome. & $66.1 \%$ \\
\hline CWP2 & YKL096W-A & structural constituent of cell wall. & $60.3 \%$ \\
\hline
\end{tabular}




\begin{tabular}{|c|c|c|c|}
\hline \multicolumn{4}{|c|}{ Other functions } \\
\hline RTT103 & YDR289C & RNA polymerase II core binding. & $88.6 \%$ \\
\hline CPR5 & YDR304C & Peptidyl-prolyl cis-trans isomerase activity. & $88.1 \%$ \\
\hline MBR1 & YKL093W & $\begin{array}{l}\text { Protein involved in mitochondrial functions and stress } \\
\text { response }\end{array}$ & $80.2 \%$ \\
\hline RIM8 & YGL045W & Protein involved in proteolytic activation of Rim101p. & $73.7 \%$ \\
\hline PEX11 & YOL147C & $\begin{array}{l}\text { Peroxisomal protein required for medium-chain fatty acid } \\
\text { oxidation. }\end{array}$ & $71.0 \%$ \\
\hline ADE5,7 & YGL234W & $\begin{array}{l}\text { phosphoribosylamine-glycine ligase and } \\
\text { phosphoribosylformylglycinamidine cyclo-ligase activity. }\end{array}$ & $68.2 \%$ \\
\hline ESC2 & YDR363W & $\begin{array}{l}\text { prevents accumulation of toxic intermediates during } \\
\text { replication-associated recombinational repair }\end{array}$ & $61.9 \%$ \\
\hline PUS7 & YOR243C & pseudouridine synthase activity. & $61.3 \%$ \\
\hline SUC2 & YIL162W & beta-fructofuranosidase activity and inulinase activity. & $60.2 \%$ \\
\hline SRB2 & YHR041C & $\begin{array}{l}\text { protein domain specific binding, RNA polymerase II } \\
\text { transcription activity. }\end{array}$ & $60.1 \%$ \\
\hline \multicolumn{4}{|c|}{ Unknown Function } \\
\hline & YJL120W & Unknown. & $90.2 \%$ \\
\hline & YGL024W & Unknown. & $88.9 \%$ \\
\hline BSC6 & YOL137W & Unknown. & $87.6 \%$ \\
\hline HGH1 & YGR187C & Unknown. & $78.2 \%$ \\
\hline \multirow[t]{2}{*}{ THI22 } & YPR121W & Unknown. & $74.1 \%$ \\
\hline & YDR010C & Unknown. & $73.6 \%$ \\
\hline \multirow[t]{2}{*}{ AIM6 } & YDL237W & Unknown. & $71.7 \%$ \\
\hline & YPR078C & Unknown. & $71.7 \%$ \\
\hline PIN3 & YPR154W & Unknown. & $70.3 \%$ \\
\hline
\end{tabular}




\begin{tabular}{|l|l|l|l|}
\hline PRM8 & YGL053W & Unknown. & $65.7 \%$ \\
\hline & YBL094C & Unknown. & $62.8 \%$ \\
\hline & YDR415C & Unknown. & $62.5 \%$ \\
\hline BOP3 & YNL042W & Unknown. & $62.3 \%$ \\
\hline APT1 & YML002W & Unknown. & $62.0 \%$ \\
\hline SOK1 & YDR006C & Unknown. & $61.9 \%$ \\
\hline & YDR286C & Unknown. & $60.8 \%$ \\
\hline
\end{tabular}

*The average of 3 GDA analysis for all the plates, but plate numbers 6, 7 and 13 were done only twice. 
Figure 12. Cellular processes in which the 71 most sensitive strains participate. The major groups comprise $22 \%$ with 'unknown function', $20 \%$ have a transferase activity, $15 \%$ have a hydrolase activity, 7\% DNA binding, 7\% enzyme regulator activity and 7\% RNA and mRNA binding ( $P$ value $<0.10)$. Therefore, omitting 'unknown' and 'other' functions, 5 of the categories present the majority $(\approx 53 \%)$ of the sensitive mutants.



Figure 12. Functional distribution of 71 most sensitive genes deletion strains to $0.13 \mathrm{mg} / \mathrm{ml}$ berberine. Deleted genes in most sensitive strains were classified by function using Go-terms.

Table 3. The 22 most resistant mutants to berberine (i.e. with percentage of inhibition lower than $10 \%$ ) were classified by functional analysis Go Terms. Based on the cellular processes in which they are involved, GO-Term identified 9 different categories: unknown function (4 mutants), structural constituent of ribosome (2 mutants), DNA binding (2 mutants), ATPase activity (1 mutant), transferase activity ( 2 mutants), oxydoreductase activity (1 mutant), 
transmembrane transporter activity ( 2 mutants), tubulin binding (4 mutants), enzyme regulator activity (1 mutant), and others (3 mutants).

Table 3. Functional analysis of the most resistant strains based on 3 GDA analysis. The mutants were selected based on the size reduction $\leq 10 \%$.

\begin{tabular}{|l|l|l|l|}
\hline Gene & Strain & Gene function & $\begin{array}{l}\text { Average \% } \\
\text { Colony size } \\
\text { reduction* }\end{array}$ \\
\hline
\end{tabular}

\section{Unknown Function.}

\begin{tabular}{|c|c|c|c|}
\hline BUD27 & YFL023W & Unknown. & $-47.6 \%$ \\
\hline & YDR271C & Unknown. & $-34.5 \%$ \\
\hline & YNL203C & Unknown. & $-9.0 \%$ \\
\hline & YHL005C & Unknown. & $-2.1 \%$ \\
\hline \multicolumn{4}{|c|}{ structural constituent of ribosome } \\
\hline RPL42A & YNL162W & Structural constituent of ribosome. & $-17.8 \%$ \\
\hline SWS2 & YNL081C & structural constituent of ribosome & $-8.6 \%$ \\
\hline \multicolumn{4}{|c|}{ DNA binding } \\
\hline STB4 & YMR019W & DNA binding. & $-43.7 \%$ \\
\hline PAH1 & YMR165C & $\begin{array}{l}\text { phosphatidate phosphatase activity and transcription } \\
\text { regulatory region DNA binding }\end{array}$ & $-7.6 \%$ \\
\hline \multicolumn{4}{|c|}{ ATPase activity } \\
\hline DYN2 & YDR424C & $\begin{array}{l}\text { Contributes to ATP-dependent microtubule motor } \\
\text { activity, plus-end-directed. }\end{array}$ & $-8.9 \%$ \\
\hline \multicolumn{4}{|c|}{ transferase activity } \\
\hline NQM1 & YGR043C & $\begin{array}{l}\text { sedoheptulose-7-phosphate: D-glyceraldehyde-3- } \\
\text { phosphate glyceronetransferase activity. }\end{array}$ & $-19.4 \%$ \\
\hline
\end{tabular}




\begin{tabular}{|c|c|c|c|}
\hline GAS1 & YMR307W & 1,3-beta-glucanosyltransferase activity & $-0.6 \%$ \\
\hline \multicolumn{4}{|c|}{ oxidoreductase activity } \\
\hline CAT5 & YOR125C & monooxygenase activity. & $-5.9 \%$ \\
\hline \multicolumn{4}{|c|}{ transmembrane transporter activity } \\
\hline YHB1 & YGR234W & Nitric oxide reductase activity. & $-12.4 \%$ \\
\hline YEA4 & YEL004W & $\begin{array}{l}\text { UDP-N-acetylglucosamine transmembrane } \\
\text { transporter activitY. }\end{array}$ & $-12.0 \%$ \\
\hline \multicolumn{4}{|c|}{ Tubulin binding } \\
\hline ASE1 & YOR058C & Tubulin binding. & $-5.4 \%$ \\
\hline GIM4 & YEL003W & Tubulin binding. & $-8.2 \%$ \\
\hline YKE2 & YLR200W & Tubulin binding. & $-1.8 \%$ \\
\hline PAC10 & YGR078C & Tubulin binding. & $-3.4 \%$ \\
\hline \multicolumn{4}{|c|}{ enzyme regulator activity } \\
\hline SPL2 & YHR136C & Enzyme inhibitor activity. & $-4.0 \%$ \\
\hline \multicolumn{4}{|c|}{ Others } \\
\hline MIC27 & YNL100W & $\begin{array}{l}\text { a mitochondrial inner membrane complex that } \\
\text { extends into the intermembrane space and has a role } \\
\text { in the maintenance of crista junctions, inner } \\
\text { membrane architecture, and formation of contact } \\
\text { sites to the outer membrane. }\end{array}$ & $-50.7 \%$ \\
\hline APQ12 & YIL040W & $\begin{array}{l}\text { Nuclear envelope/ER integral membrane protein; } \\
\text { interacts and functions with Brr6p and Brl1p in lipid } \\
\text { homeostasis }\end{array}$ & $-21.8 \%$ \\
\hline COX14 & YML129C & $\begin{array}{l}\text { Mitochondrial cytochrome c oxidase (complex IV) } \\
\text { assembly factor. }\end{array}$ & $-13.8 \%$ \\
\hline
\end{tabular}


Figure 13. The graph shows that $18 \%, 9 \%, 9 \%$ and $9 \%$ of the mutant strains most resistant to berberine are involved in tubulin binding, transmembrane transporter activity, transferase activity and ribosome structural constituent, respectively.

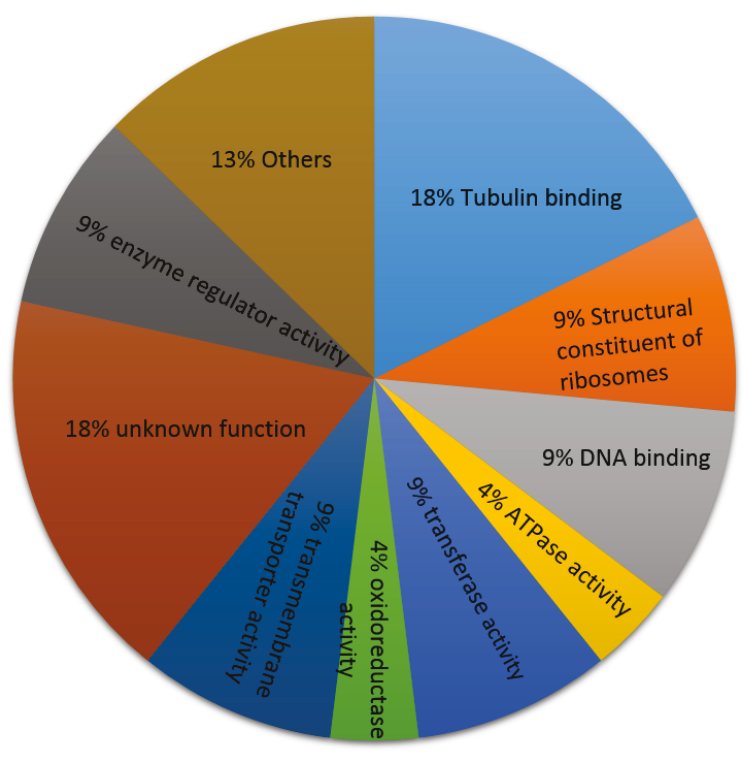

Figure 13. Functional distribution of 22 most resistant gene deletion strains to $0.13 \mathrm{mg} / \mathrm{ml}$ berberine. Deleted genes in resistant strains were classified by function using Go-terms. 


\section{Enrichment Analysis:}

For this analysis, I used the Gene Ontology Term Finder (http://www.yeastgenome.org/cgibin/GO/goTermFinder.pl\#default) to identify common features of the deleted genes in the most sensitive strains. Significant enrichments were observed for 'function' and 'process'. There was no significant enrichment observed for 'component' Indeed, 3 of 71 deletions were associated with process 'cellular polysaccharide catabolic/polysaccharide catabolic process' (Table4 A). Also, 4 of the 71 deletions were associated with function 'GTPase activator/ GTPase regulator activity/', and 2 of the deletions were associated with function 'four-way junction DNA binding' (Table 4 B).

Table (4 A). Shows the significant enrichment $(\mathrm{P} \leq 0.5)$ of process 'cellular polysaccharide catabolic' and 'polysaccharide catabolic' based on most sensitive mutants.

\begin{tabular}{|l|l|l|l|l|l|l|}
\hline \multicolumn{9}{|c|}{ Terms from the Process Ontology } \\
\hline $\begin{array}{l}\text { Gene Ontology } \\
\text { term }\end{array}$ & $\begin{array}{l}\text { Cluster } \\
\text { frequency }\end{array}$ & $\begin{array}{l}\text { Background } \\
\text { frequency }\end{array}$ & P-Value & FDR & $\begin{array}{l}\text { Expected } \\
\text { False } \\
\text { Positives }\end{array}$ & Genes annotated to the term \\
\hline $\begin{array}{l}\text { cellular } \\
\text { polysaccharide } \\
\text { catabolic } \\
\text { process |AmiGO }\end{array}$ & $\begin{array}{l}\text { 3 out of } \\
41 \text { genes, }\end{array}$ & $\begin{array}{l}10 \text { out of } \\
7165 \\
\text { background } \\
\text { genes, 0.1\% }\end{array}$ & 0.03812 & $0.10 \%$ & 0.10 & $\begin{array}{l}\text { SUC2/YIL162W, MSN1/YOL1 } \\
\text { 16W, GPH1/YPR160W }\end{array}$ \\
\hline $\begin{array}{l}\text { polysaccharide } \\
\text { catabolic } \\
\text { process |AmiGO }\end{array}$ & $\begin{array}{l}3 \text { out of } \\
71 \text { genes, } \\
4.2 \%\end{array}$ & $\begin{array}{l}11 \text { out of } \\
7165 \\
\text { background } \\
\text { genes, 0.2\% }\end{array}$ & 0.05204 & $0.07 \%$ & 0.14 & $\begin{array}{l}\text { SUC2/YIL162W, MSN1/YOL1 } \\
\text { 16W,GPH1/YPR160W }\end{array}$ \\
\hline
\end{tabular}


Table (4 B). Shows the significant enrichment $(\mathrm{P} \leq 0.5)$ of function 'GTPase activator activity' and 'DNA binding based on most sensitive mutants.

\begin{tabular}{|c|c|c|c|c|c|c|}
\hline \multicolumn{7}{|c|}{ Terms from the Function Ontology } \\
\hline $\begin{array}{l}\text { Gene Ontology } \\
\text { term }\end{array}$ & $\begin{array}{l}\text { Cluster } \\
\text { frequency }\end{array}$ & $\begin{array}{l}\text { Background } \\
\text { frequency }\end{array}$ & P-Value & FDR & $\begin{array}{l}\text { Expected } \\
\text { False } \\
\text { Positives }\end{array}$ & Genes annotated to the term \\
\hline $\begin{array}{l}\text { GTPase } \\
\text { activator } \\
\text { activity |AmiGo }\end{array}$ & $\begin{array}{l}4 \text { out of } \\
71 \text { genes, } \\
5.6 \%\end{array}$ & $\begin{array}{l}40 \text { out of } \\
7165 \\
\text { background } \\
\text { genes, } 0.6 \%\end{array}$ & 0.04080 & $0.34 \%$ & 0.34 & $\begin{array}{l}\text { GCS1/YDL226C, SAC7/YDR38 } \\
\text { 9W, NEL1/YHR035W,MSB4/Y } \\
\text { OL112W }\end{array}$ \\
\hline $\begin{array}{l}\text { GTPase } \\
\text { regulator } \\
\text { activity |AmiGo }\end{array}$ & $\begin{array}{l}4 \text { out of } \\
71 \text { genes, } \\
5.6 \%\end{array}$ & $\begin{array}{l}49 \text { out of } \\
7165 \\
\text { background } \\
\text { genes, } 0.7 \%\end{array}$ & 0.08845 & $0.31 \%$ & 0.62 & $\begin{array}{l}\text { GCS1/YDL226C, SAC7/YDR38 } \\
\text { 9W, NEL1/YHR035W,MSB4/Y } \\
\text { OL112W }\end{array}$ \\
\hline $\begin{array}{l}\text { four-way } \\
\text { junction DNA } \\
\text { binding |AmiGO }\end{array}$ & $\begin{array}{l}2 \text { out of } \\
71 \text { genes, } \\
2.8 \%\end{array}$ & $\begin{array}{l}6 \text { out of } 7165 \\
\text { background } \\
\text { genes, } 0.1 \%\end{array}$ & 0.09342 & $0.26 \%$ & 0.78 & $\begin{array}{l}\text { HMO1/YDR174W, MSH2/YO } \\
\text { L090W }\end{array}$ \\
\hline
\end{tabular}

Significant enrichment was observed among the most resistant strains for 'function', 'component' and 'process.' Indeed, 7 of 22 deletions were associated with process 'protein complex assembly/ protein complex subunit organization/protein complex biogenesis'; 6 of 22 most resistant strains were associted with 'cellular protein complex assembly', and 3 of the 22 were linked to 'tubulin complex assembly' process (Table5 A). Also, 4 of the 22 deletions were associated with function 'tubulin binding/cytoskeletal protein binding ' (Table5 B); and 3 of the deletions were associated with component 'prefoldin complex' (Table5 C). 
Table (5 A). Shows the significant enrichment $(\mathrm{P} \leq 0.5)$ of process 'tubulin complex assembly' and 'protein complex binding' based on most resistant mutants.

\begin{tabular}{|c|c|c|c|c|c|c|}
\hline \multicolumn{7}{|c|}{ Terms from the Process Ontology } \\
\hline $\begin{array}{l}\text { Gene Ontology } \\
\text { term }\end{array}$ & $\begin{array}{l}\text { Cluster } \\
\text { frequency }\end{array}$ & $\begin{array}{l}\text { Background } \\
\text { frequency }\end{array}$ & P-Value & FDR & $\begin{array}{l}\text { Expected } \\
\text { False } \\
\text { Positives }\end{array}$ & Genes annotated to the term \\
\hline $\begin{array}{l}\text { tubulin complex } \\
\text { assembly | AmiG } \\
\text { O }\end{array}$ & $\begin{array}{l}3 \text { out of } \\
22 \text { genes, } \\
13.6 \%\end{array}$ & $\begin{array}{l}10 \text { out of } \\
7165 \\
\text { background } \\
\text { genes, } 0.1 \%\end{array}$ & 0.00037 & $0.00 \%$ & 0.00 & $\begin{array}{l}\text { GIM4/YEL003W, PAC10/YGR } \\
\text { 078C, YKE2/YLR200W }\end{array}$ \\
\hline $\begin{array}{l}\text { protein complex } \\
\text { assembly | AmiG } \\
0\end{array}$ & $\begin{array}{l}7 \text { out of } \\
22 \text { genes, } \\
31.8 \%\end{array}$ & $\begin{array}{l}316 \text { out of } \\
7165 \\
\text { background } \\
\text { genes, } 4.4 \%\end{array}$ & 0.00368 & $0.00 \%$ & 0.00 & $\begin{array}{l}\text { DYN2/YDR424C, GIM4/YEL00 } \\
\text { 3W, BUD27/YFL023W, PAC1 } \\
\text { 0/YGR078C,YKE2/YLR200W, } \\
\text { COX14/YML129C, ASE1/YOR } \\
\text { 058C }\end{array}$ \\
\hline $\begin{array}{l}\text { protein complex } \\
\text { biogenesis |Ami } \\
\text { GO }\end{array}$ & $\begin{array}{l}7 \text { out of } \\
22 \text { genes, } \\
31.8 \%\end{array}$ & $\begin{array}{l}327 \text { out of } \\
7165 \\
\text { background } \\
\text { genes, } 4.6 \%\end{array}$ & 0.00459 & $0.00 \%$ & 0.00 & $\begin{array}{l}\text { DYN2/YDR424C, GIM4/YEL00 } \\
\text { 3W, BUD27/YFL023W, PAC1 } \\
\text { 0/YGR078C,YKE2/YLR200W, } \\
\text { COX14/YML129C, ASE1/YOR } \\
\text { 058C }\end{array}$ \\
\hline $\begin{array}{l}\text { cellular protein } \\
\text { complex } \\
\text { assembly | Ami } \\
\text { GO }\end{array}$ & $\begin{array}{l}6 \text { out of } \\
22 \text { genes, } \\
27.3 \%\end{array}$ & $\begin{array}{l}219 \text { out of } \\
7165 \\
\text { background } \\
\text { genes, 3.1\% }\end{array}$ & 0.00475 & $0.00 \%$ & 0.00 & $\begin{array}{l}\text { DYN2/YDR424C, GIM4/YEL00 } \\
\text { 3W, BUD27/YFL023W, PAC1 } \\
\text { 0/YGR078C,YKE2/YLR200W, } \\
\text { COX14/YML129C }\end{array}$ \\
\hline $\begin{array}{l}\text { protein complex } \\
\text { subunit } \\
\text { organization | A } \\
\text { miGO }\end{array}$ & $\begin{array}{l}7 \text { out of } \\
22 \text { genes, } \\
31.8 \%\end{array}$ & $\begin{array}{l}477 \text { out of } \\
7165 \\
\text { background } \\
\text { genes, } 6.7 \%\end{array}$ & 0.04948 & $0.08 \%$ & 0.38 & $\begin{array}{l}\text { DYN2/YDR424C, GIM4/YEL00 } \\
\text { 3W, BUD27/YFL023W, PAC1 } \\
\text { 0/YGR078C,YKE2/YLR200W, } \\
\text { COX14/YML129C, ASE1/YOR } \\
\text { 058C }\end{array}$ \\
\hline
\end{tabular}


Table (5 B). Shows the significant enrichment ( $\mathrm{P} \leq 0.5)$ of function 'tubulin binding' and 'cytoskeletal protein binding' based on most resistant mutants.

\begin{tabular}{|l|l|l|l|l|l|l|}
\hline \multicolumn{9}{|c|}{ Terms from the Function Ontology } \\
\hline $\begin{array}{l}\text { Gene Ontology } \\
\text { term }\end{array}$ & $\begin{array}{l}\text { Cluster } \\
\text { frequency }\end{array}$ & $\begin{array}{l}\text { Background } \\
\text { frequency }\end{array}$ & P-Value & FDR & $\begin{array}{l}\text { Expected } \\
\text { False } \\
\text { Positives }\end{array}$ & \multicolumn{1}{|c|}{$\begin{array}{l}\text { Genes annotated to the } \\
\text { term }\end{array}$} \\
\hline $\begin{array}{l}\text { tubulin } \\
\text { binding| AmiGO }\end{array}$ & $\begin{array}{l}4 \text { out of } \\
22 \text { genes, } \\
18.2 \%\end{array}$ & $\begin{array}{l}35 \text { out of } \\
7165 \\
\text { background } \\
\text { genes, 0.5\% }\end{array}$ & $4.26 e-05$ & $0.00 \%$ & 0.00 & $\begin{array}{l}\text { GIM4/YEL003W, PAC10/YG } \\
\text { R078C, YKE2/YLR200W,ASE } \\
1 / \text { YOR058C }\end{array}$ \\
\hline $\begin{array}{l}\text { cytoskeletal } \\
\text { protein } \\
\text { binding |AmiGO }\end{array}$ & $\begin{array}{l}4 \text { out of } \\
22 \text { genes, } \\
18.2 \%\end{array}$ & $\begin{array}{l}67 \text { out of } \\
7165 \\
\text { background } \\
\text { genes, 0.9\% }\end{array}$ & $\begin{array}{l}0.000590 . \\
08845\end{array}$ & $0.02 \%$ & 0.04 & $\begin{array}{l}\text { GIM4/YEL003W, PAC10/YG } \\
\text { R078C, YKE2/YLR200W,ASE } \\
1 / \text { YOR058C }\end{array}$ \\
\hline
\end{tabular}

Table (5 C). Shows the significant enrichment $(\mathrm{P} \leq 0.5)$ of component 'prefoldin complex' based on most resistant mutants.

\begin{tabular}{|l|l|l|l|l|l|l|}
\hline \multicolumn{9}{|c|}{ Terms from the Component Ontology } \\
\hline $\begin{array}{l}\text { Gene Ontology } \\
\text { term }\end{array}$ & $\begin{array}{l}\text { Cluster } \\
\text { frequency }\end{array}$ & $\begin{array}{l}\text { Background } \\
\text { frequency }\end{array}$ & P-Value & FDR & $\begin{array}{l}\text { Expected } \\
\text { False } \\
\text { Positives }\end{array}$ & $\begin{array}{l}\text { Genes annotated to the } \\
\text { term }\end{array}$ \\
\hline $\begin{array}{l}\text { prefoldin } \\
\text { complex |AmiGO }\end{array}$ & $\begin{array}{l}\text { 3 out of } \\
22 \text { genes, } \\
13.6 \%\end{array}$ & $\begin{array}{l}6 \text { out of } \\
7165 \\
\text { background } \\
\text { genes, 0.1\% }\end{array}$ & $2.74 \mathrm{e}-05$ & $0.00 \%$ & 0.00 & $\begin{array}{l}\text { GIM4/YEL003W, PAC10/YG } \\
\text { R078C,YKE2/YLR200W }\end{array}$ \\
\hline
\end{tabular}

\section{Membrane Leakage Assay:}

Berberine is reported to disrupt membrane function (Kang et al., 2015). However, deletions of genes involving membrane function were not prominent in the Go-Terms analysis results. Nevertheless, a membrane disruption assay was done to test the literature-based hypothesis.

The membrane-disrupting properties of berberine were investigated with a membrane leakage assay using LUVs. In this experiment the carrier control for berberine was YPD. From our 
assay, we did not observe a significant effect of berberine on the yeast-like lipid membrane (Figure 14).

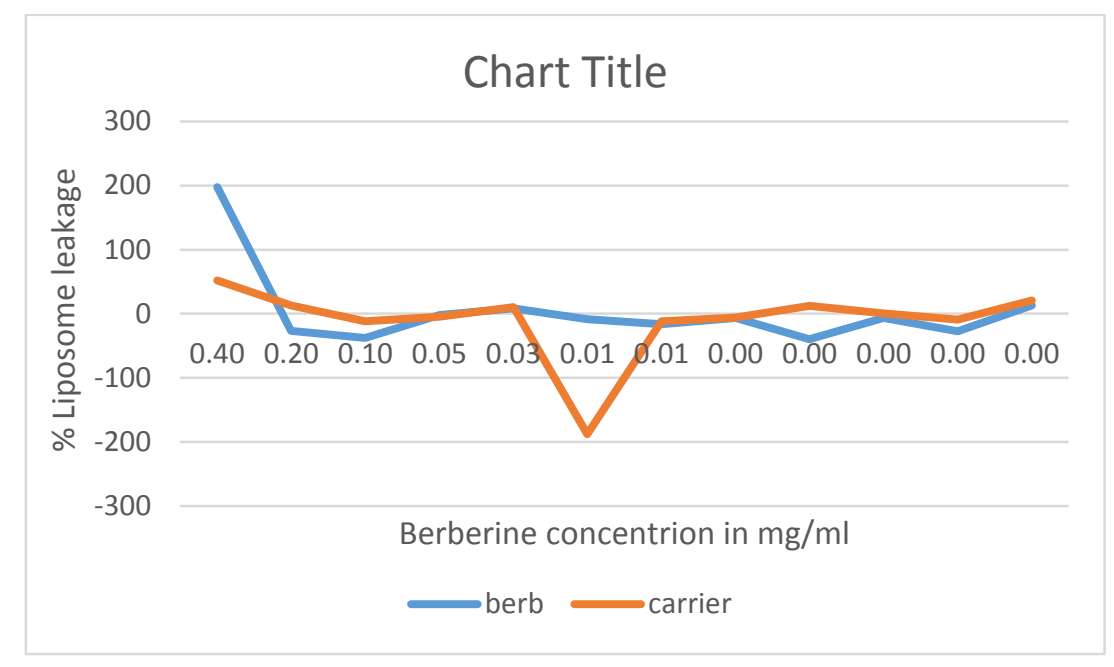

Figure 14. Shows that overall liposomes leakage \% is not significantly different from the carrier.

\section{'FLUORESCENCE MICROSCOPY'}

\section{Fluorescence microscopy.}

Preliminary viewing by fluorescence microscopy of berberine-stained ( $\geq 40 \mathrm{~min}$ ) yeast cells (sensitive, resistant and 'medium' sensitivity) indicated that there were three general staining patterns (Table 6): entire cell was stained moderately or very bright; very bright subcellular localization; moderate or very bright staining of the border of the cell. Aside from these different staining patterns, budding frequency was somewhat greater in 'berberineresistant' strains than in 'medium sensitivity' and berberine-sensitive strains. Cell size (longest axis) was not noticeably different between the three sensitivity groups. Based on the marked 
differences in staining pattern, we examined berberine staining patterns with confocal fluorescence microscopy.

Table 6. fluorescence microscopy of selected yeast strains treated with berberine for $\geq 40$.

\begin{tabular}{|c|c|c|c|c|}
\hline Strains & Brightness & Staining pattern & $\begin{array}{l}\text { Budding } \\
\text { frequency }\end{array}$ & $\begin{array}{l}\text { Average size } \\
(\mu \mathrm{m})\end{array}$ \\
\hline \multicolumn{5}{|c|}{ Medium sensitivity to berberine } \\
\hline S288C (wild type) & moderately bright & Entire cell & $56 \%$ & $5.2 \mu \mathrm{m}$ \\
\hline YGL086W & Very bright & Entire cell & $58 \%$ & $5.6 \mu \mathrm{m}$ \\
\hline YMR119W & Very bright & Subcellular localization & $54 \%$ & $5.3 \mu \mathrm{m}$ \\
\hline \multicolumn{5}{|c|}{ Sensitive to berberine } \\
\hline YML008C & very bright & Entire cell & $36 \%$ & $5.0 \mu \mathrm{m}$ \\
\hline YHR030C & very bright & Subcellular localization & $44 \%$ & $4.8 \mu \mathrm{m}$ \\
\hline YBL007C & very bright & Entire cell & $40 \%$ & $5.1 \mu \mathrm{m}$ \\
\hline YPR154W & moderately bright & Border of the cell & $49 \%$ & $5.0 \mu \mathrm{m}$ \\
\hline YGL173C & very bright & Entire cell & $41 \%$ & $4.8 \mu \mathrm{m}$ \\
\hline YDR388W & very bright & Border of the cell & $48 \%$ & $4.5 \mu \mathrm{m}$ \\
\hline YNL042W & moderately bright & Border of the cell & $41 \%$ & $6.4 \mu \mathrm{m}$ \\
\hline YOR153W & very bright & Entire cell & $55 \%$ & $6.0 \mu \mathrm{m}$ \\
\hline YMR110C & very bright & Entire cell & $52 \%$ & $4.2 \mu \mathrm{m}$ \\
\hline YDR378C & very bright & Entire cell & $57 \%$ & $6.1 \mu \mathrm{m}$ \\
\hline YDL226C & very bright & Subcellular localization & $48 \%$ & $6.4 \mu \mathrm{m}$ \\
\hline YDR289C & very bright & Entire cell & $59 \%$ & $5.6 \mu \mathrm{m}$ \\
\hline YIR002C & very bright & Entire cell & $55 \%$ & $5.6 \mu \mathrm{m}$ \\
\hline \multicolumn{5}{|c|}{ Resistant to berberine } \\
\hline YLR200W & very bright & Border of the cell & $65 \%$ & $5.0 \mu \mathrm{m}$ \\
\hline YGR078C & very bright & Entire cell & $69 \%$ & $4.6 \mu \mathrm{m}$ \\
\hline YOR058C & very bright & Entire cell & $72 \%$ & $6.0 \mu \mathrm{m}$ \\
\hline
\end{tabular}

\section{Confocal microscopy.}

Selected strains (berberine sensitive, resistant and medium sensitivity) were compared to the wild type strain (S288C) under the confocal microscope.

Several interesting berberine staining patterns were observed as illustrated in Figures 1517. As shown in Figure 15 some berberine enters YMR119W (medium sensitivity to berberine) and stains subcellular components (organelles) in the cell (small bright spots). Some cells stain 
brightly throughout the cell. These cells are likely dead or dying cells that are unable to exclude and/or pump out berberine. The non-staining structures (circles) evident in the yeast cells are the central vacuoles.

In figure 16, some berberine enters YDR388W cells (sensitive to berberine) and stains the border of the cell.

In Figure 17, some berberine enters YGR078C cells (resistant to berberine) and appears as bright spots within the cell. The bright spots likely represent a specific organelle (s). Occasionally, the entire cell is stained very brightly, representing dead or dying cells.

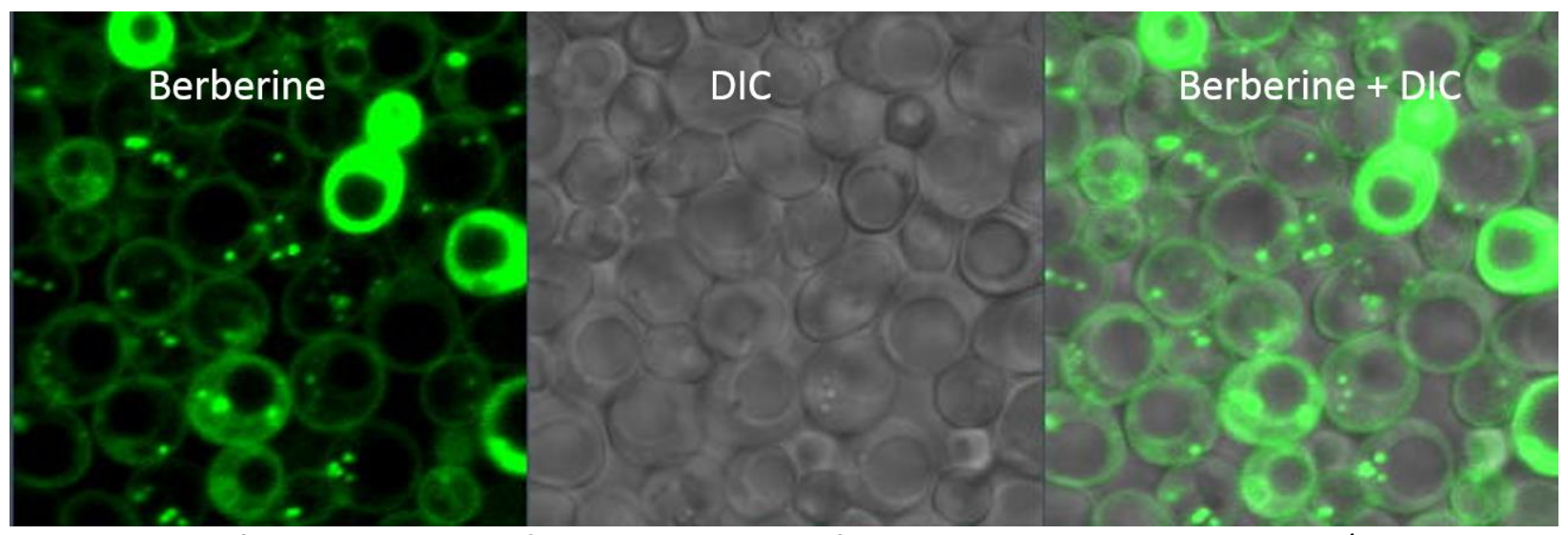

Figure 15. Confocal microscopy of YMR119W strain after $40 \mathrm{~min}$ exposure to $0.13 \mathrm{mg} / \mathrm{ml}$ berberine. Fluorescence micrograph is shown on the left, DIC (differential interference contrast) in the middle and both fluorescent-DIC overlay on the right. 

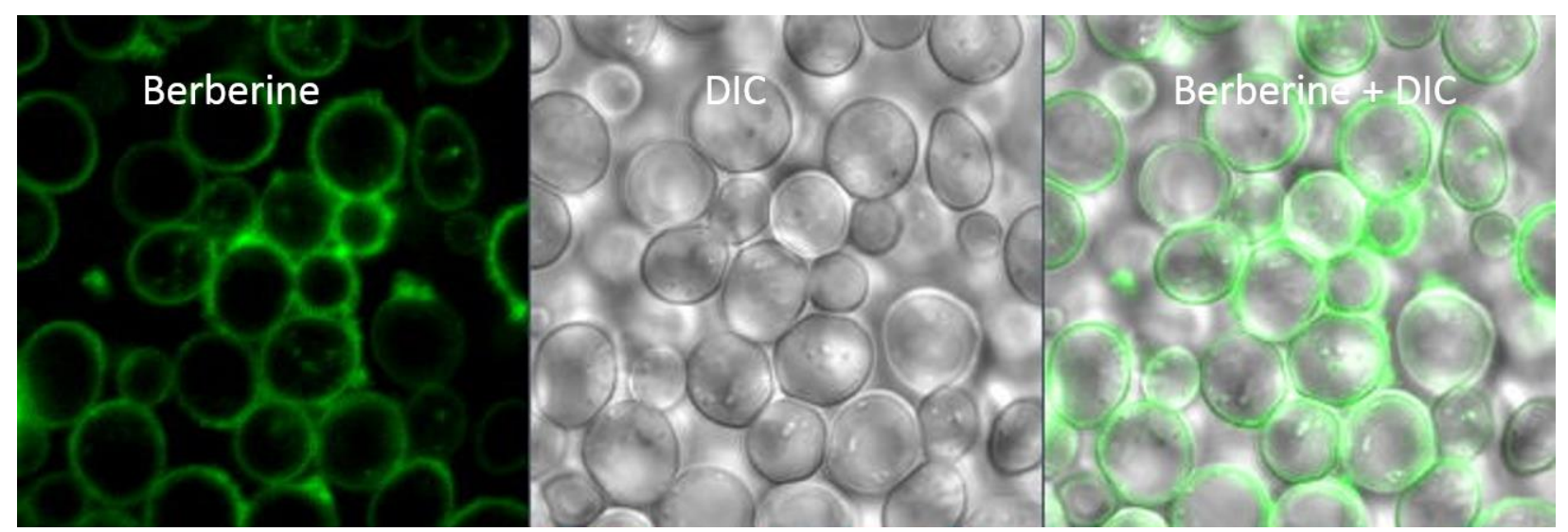

Figure 16. Confocal microscopy of YDR388W strain after 40 min exposure to $0.13 \mathrm{mg} / \mathrm{ml}$ berberine. Fluorescence micrograph is shown on the left, DIC (differential interference contrast) in the middle and both fluorescent-DIC overlay on the right.

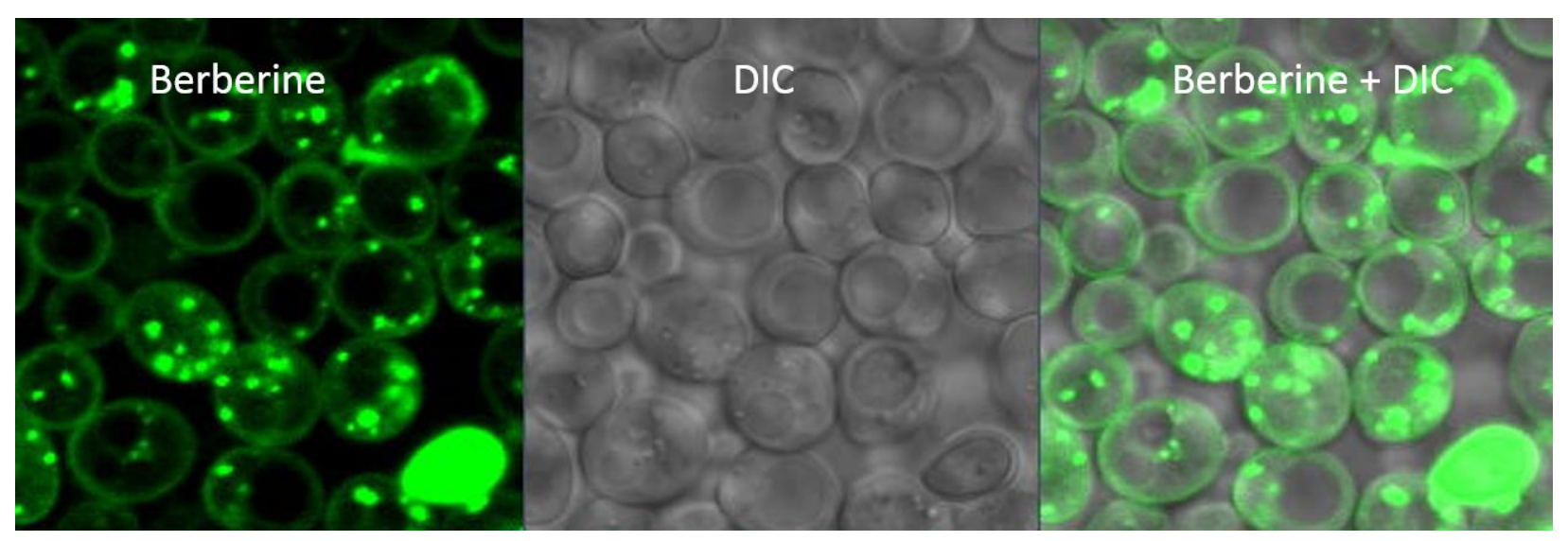

Figure 17. Confocal microscopy of YGR078C strain after $40 \mathrm{~min}$ exposure to $0.13 \mathrm{mg} / \mathrm{ml}$ berberine. Fluorescence micrograph is shown on the left, DIC (differential interference contrast) in the middle and both fluorescent-DIC overlay on the right.

To test whether the different berberine staining patterns correlated to berberine sensitivity, three resistant strains, two medium sensitivity strains, and three sensitive strains were examined. Following $\geq 40 \mathrm{~min}$ berberine exposure, confocal images were taken of 10 randomly-chosen fields of cells for each strain. The images were enumerated for 'diffuse' staining cells, bright subcellular organelles (spots) and bright (dead) staining cells (Table 7). The relative frequency of these cell staining patterns are presented in Figure 18. A one-way ANOVA with a 
post-hoc Tukey HSD test was used to determine significant differences in staining patterns among strains. As can be seen from this analysis (Figure 18), different staining patterns were not correlated to sensitivity class of the yeast strain. In general, resistant strains tended to have fewer cells with distinct 'spots' (subcellular organelle staining (Figure 18). Two resistant mutants (YGR078C and YOR058C) exibited a high frequency of cells with dim/ diffuse staining. Bright staining cell frequencies were clearly not distinctive in any of the sensitivity groups (Figure 18).

We were interested in the distinct organelle staining and did a second experiment using strains. YGL068W, YMR119W and YGR078C (Figure 19). In this experiment, the signficent difference in organelle staining patterns that were seen in the first experiment (Figure 18) were not evident; none of the three strains had distinct organelle staining patterns. This suggested that organelle staining pattern may be a dynamic process, that depends on the precise timing of berberine exposure. This, and identification of organelles that stain with berberine were investigated in the next section.

Table 7. Shows the three different staining pattern of berberine on some selected mutants.

\begin{tabular}{|l|l|l|l|l|}
\hline Strain & $\begin{array}{l}\text { Field\# (total } \\
\text { cells) }\end{array}$ & Dim/Diffuse & Spots & Bright \\
\hline \multirow{5}{*}{$\begin{array}{l}\text { YGR078C } \\
\text { (resistant) }\end{array}$} & $1(11)$ & 10 & 0 & 1 \\
\cline { 2 - 5 } & $2(12)$ & 7 & 3 & 2 \\
\cline { 2 - 5 } & $3(12)$ & 8 & 0 & 4 \\
\cline { 2 - 5 } & $4(15)$ & 11 & 1 & 3 \\
\cline { 2 - 5 } & $5(15)$ & 12 & 0 & 3 \\
\cline { 2 - 5 } & $6(11)$ & 7 & 4 & 0 \\
\cline { 2 - 5 } & $7(18)$ & 8 & 8 & 2 \\
\cline { 2 - 5 } & $8(9)$ & 7 & 0 & 2 \\
\cline { 2 - 5 } & $9(33)$ & 18 & 10 & 5 \\
\cline { 2 - 5 } & $10(34)$ & 18 & 7 & 9 \\
\hline \hline
\end{tabular}




\begin{tabular}{|c|c|c|c|c|}
\hline \multirow{10}{*}{$\begin{array}{l}\text { YOR058C } \\
\text { (resistant) }\end{array}$} & 1 (5) & 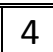 & 0 & 1 \\
\hline & $2(5)$ & 4 & 0 & 1 \\
\hline & $3(5)$ & 4 & 0 & 1 \\
\hline & $4(1)$ & 1 & 0 & 0 \\
\hline & $5(10)$ & 2 & 8 & 0 \\
\hline & $6(8)$ & 4 & 3 & 1 \\
\hline & $7(7)$ & 4 & 3 & 0 \\
\hline & $8(5)$ & 1 & 4 & 0 \\
\hline & $9(8)$ & 6 & 2 & 0 \\
\hline & $10(4)$ & 3 & 0 & 1 \\
\hline \multirow{10}{*}{$\begin{array}{l}\text { YLR200W } \\
\text { (resistant) }\end{array}$} & $11(4)$ & $\overline{\mid 1}$ & 3 & $\overline{0}$ \\
\hline & $2(5)$ & 4 & 1 & 0 \\
\hline & $3(6)$ & 4 & 2 & 0 \\
\hline & $4(6)$ & 4 & 2 & 0 \\
\hline & $5(7)$ & 2 & 5 & 0 \\
\hline & $6(7)$ & 1 & 6 & 0 \\
\hline & $7(4)$ & 2 & 2 & 0 \\
\hline & $8(4)$ & 1 & 3 & 0 \\
\hline & $9(4)$ & 2 & 2 & 0 \\
\hline & $10(7)$ & 3 & 4 & 0 \\
\hline \multirow{10}{*}{$\begin{array}{l}\text { YMR119W } \\
\text { (medium) }\end{array}$} & 1 (13) & 4 & 9 & $\overline{0}$ \\
\hline & $2(14)$ & 1 & 13 & 0 \\
\hline & $3(11)$ & 0 & 11 & 0 \\
\hline & $4(13)$ & 3 & 9 & 1 \\
\hline & $5(12)$ & 3 & 8 & 1 \\
\hline & $6(11)$ & 2 & 8 & 1 \\
\hline & $7(18)$ & 3 & 15 & 0 \\
\hline & $8(13)$ & 2 & 11 & 0 \\
\hline & $9(15)$ & 1 & 14 & 0 \\
\hline & \begin{tabular}{|l}
$10(11)$ \\
\end{tabular} & 1 & 10 & 0 \\
\hline \multirow{10}{*}{$\begin{array}{l}\text { YGL086W } \\
\text { (medium) }\end{array}$} & 1 (14) & 2 & 9 & 2 \\
\hline & $2(10)$ & 5 & 5 & 0 \\
\hline & $3(12)$ & 8 & 3 & 1 \\
\hline & $4(6)$ & 2 & 4 & 0 \\
\hline & $5(5)$ & 3 & 2 & 0 \\
\hline & $6(5)$ & 2 & 3 & 0 \\
\hline & $7(4)$ & 1 & 3 & 0 \\
\hline & $8(5)$ & 2 & 2 & 1 \\
\hline & $9(9)$ & 4 & 5 & 0 \\
\hline & $10(10)$ & 7 & 2 & 1 \\
\hline \multirow{6}{*}{$\begin{array}{l}\text { YHR030C } \\
\text { (sensitive) }\end{array}$} & $1(9)$ & 1 & 8 & 0 \\
\hline & $2(9)$ & 2 & 7 & 0 \\
\hline & $3(10)$ & 3 & 7 & 0 \\
\hline & $4(13)$ & 5 & 5 & 3 \\
\hline & $5(13)$ & 6 & 7 & 0 \\
\hline & 6 (14) & 4 & 10 & 0 \\
\hline
\end{tabular}




\begin{tabular}{|c|c|c|c|c|}
\hline & $7(10)$ & 7 & 3 & 0 \\
\hline & $8(7)$ & 4 & 0 & 3 \\
\hline & $9(10)$ & 6 & 4 & 0 \\
\hline & $10(10)$ & 5 & 5 & 0 \\
\hline \multirow{10}{*}{$\begin{array}{l}\text { YML008C } \\
\text { (sensitive) }\end{array}$} & 1 (5) & 1 & 2 & 2 \\
\hline & 2 (3) & 0 & 2 & 1 \\
\hline & $3(2)$ & 0 & 2 & 0 \\
\hline & $4(2)$ & 0 & 2 & 0 \\
\hline & $5(3)$ & 3 & 0 & 0 \\
\hline & $6(1)$ & 0 & 1 & 0 \\
\hline & $7(2)$ & 1 & 1 & 0 \\
\hline & $8(2)$ & 1 & 2 & 0 \\
\hline & $9(6)$ & 3 & 3 & 0 \\
\hline & $10(6)$ & 1 & 5 & 0 \\
\hline \multirow{10}{*}{$\begin{array}{l}\text { YPR154W } \\
\text { (sensitive) }\end{array}$} & 1 (11) & 2 & 6 & 3 \\
\hline & $2(12)$ & 4 & 6 & 2 \\
\hline & $3(14)$ & 4 & 8 & 2 \\
\hline & 4 (14) & 3 & 10 & 0 \\
\hline & $5(17)$ & 7 & 10 & 0 \\
\hline & $6(12)$ & 2 & 10 & 0 \\
\hline & 7 (9) & 3 & 6 & 0 \\
\hline & $8(10)$ & 3 & 4 & 3 \\
\hline & $9(15)$ & 4 & 9 & 2 \\
\hline & $10(16)$ & 2 & 14 & 0 \\
\hline
\end{tabular}




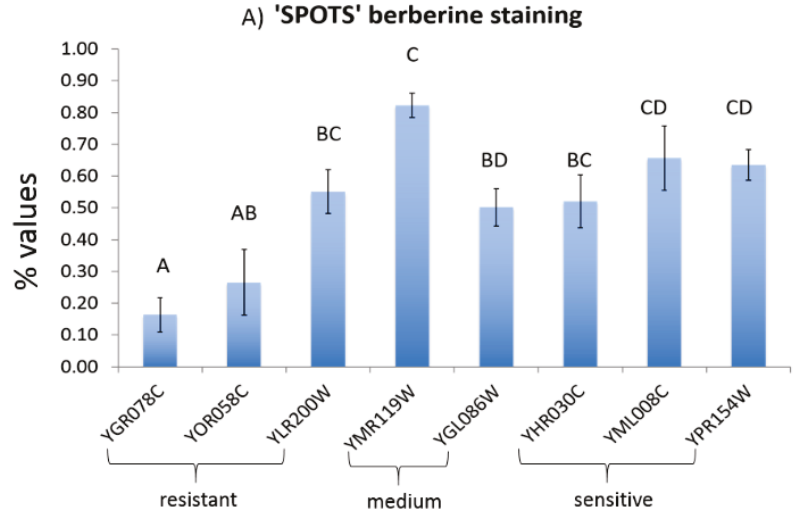

C) 'BRIGHT' berberine staining

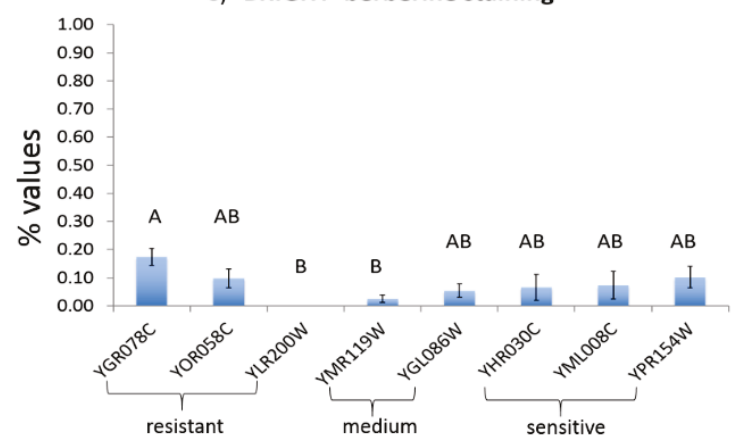

Figure 18. Berberine staining patterns from first experiment with selected resistant, medium sensitive and sensitive strains. Significant differences in berberine staining patterns based on Tukey's HSD test are indicated by different letters. 


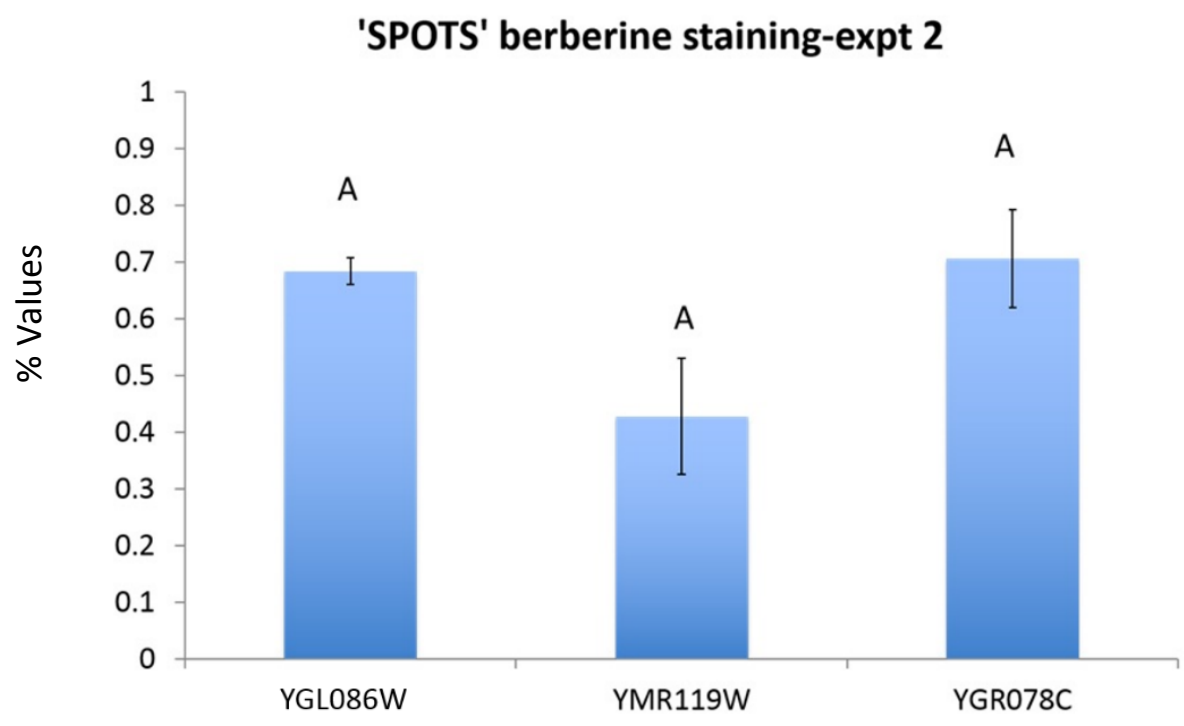

Figure 19. Results of second experiment assaying berberine staining patterns of strains that had distinct staining patterns in the first experiment. There were no significant differences in the frequencies of cells with berberine staining 'spots'.

\section{Analysis of berberine localization in yeast cells.}

\section{DAPI, Nile Red, Rhodamine 123 and berberine stains.}

We hypothesized that the bright berberine-staining spots evident in confocal micrographs could represent nuclei, mitochondria or oil droplets in the yeast cell. In order to determine where berberine is located, fluorescent stains DAPI (binds to DNA in mitochondria and nuclei), Nile Red (stains lipids) and Rhodamine 123 (stains mitochondria) were used alone and/or in combination with berberine staining (Figure 20). During short berberine exposure times ( $\leq 20$ minutes) berberine appears to localize to mitochondria (Figure 20C) that are located around the inside perimenter of the cell. During long berberine exposure times ( $\geq 40$ minutes) berberine appears to localize to oil droplets, based on. co-localization with Nile Red (Figure 20 A) and does not appear to co-localize with DAPI-staining structures (nucleus and mitochondria) (Figure $20 \mathrm{~B}$ ). As seen in 
Figure $20 \mathrm{~B}$, berberine is not colocalized with DAPI when exposed to berberine for $\geq 1$ hour (does not localize to mitochondria and nuclei).

A
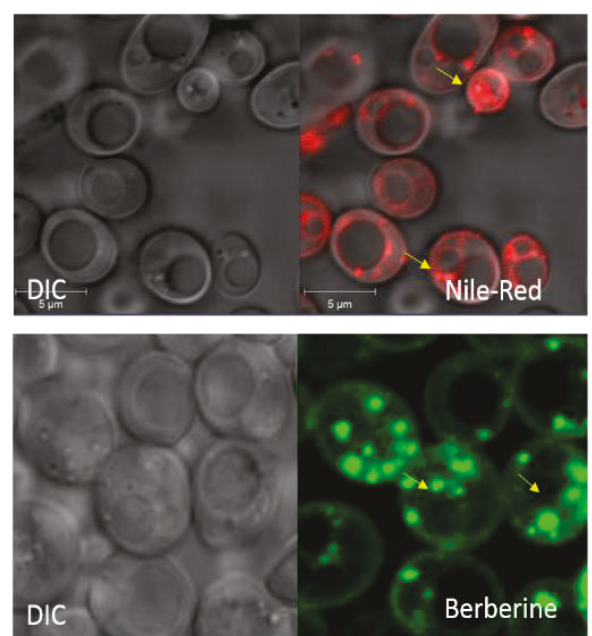

B
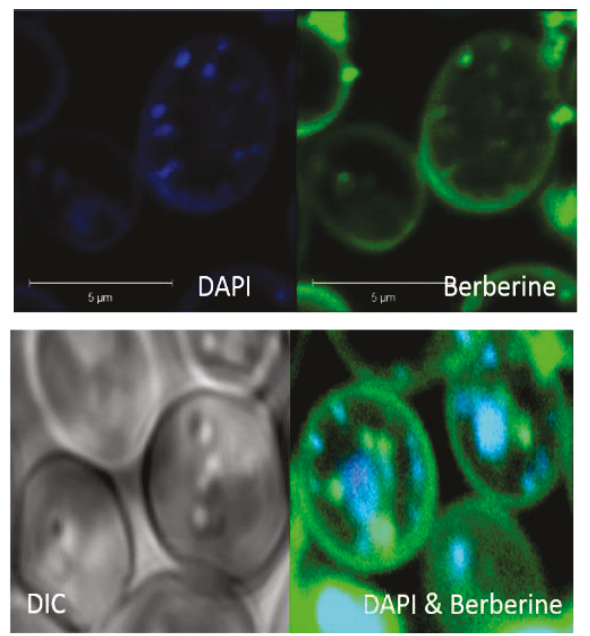

C
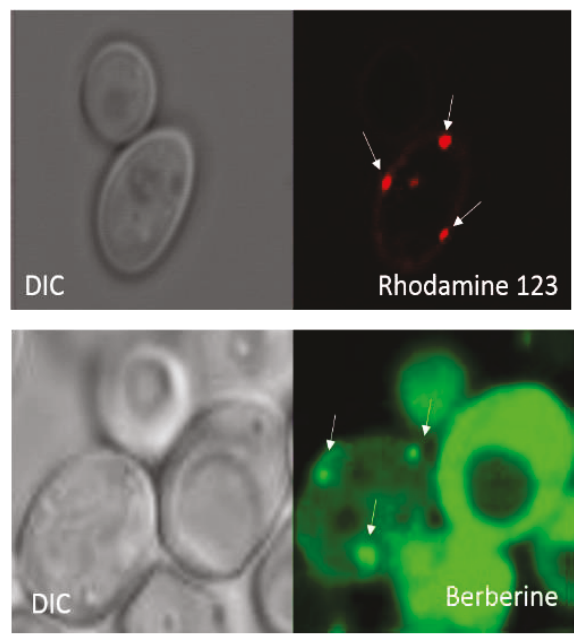

Figure 20. Live cell imaging of YPR154W, (A) long ( $\geq 40 \mathrm{~min}$ ) berberine exposure results in berberine localized to oil droplets. In the top right of the panel is shown Nile Red oil droplet staining (yellow arrows) that correspond to organelles that are stained with berberine (bottom right). (B) in long berberine exposures ( $\geq 40 \mathrm{~min}$ ) berberine and DAPI (nucleus and mitochondria) do not co-localized, On the top left of the panel nuclei and mitochondria are indicated in blue by DAPI staining. On the top right of the panel berberine staining is shown in green. In the bottom right of the panel berberine and DAPI staining are overlaid to show nuclei and mitochondrial DAPI signal in blue that is not localized with berberine (green). (C) With a short ( $\leq 20 \mathrm{~min}$ ) exposure to $0.13 \mathrm{mg} / \mathrm{ml}$ berberine, followed by $10 \mathrm{~min}$ exposure both rhodamine 123 stain (stains mitochondria) and berberine appear to localize to the mitochondria (white arrows) that are arranged along the inside perimeter of the cell. 


\section{Discussion:}

Due to the pathological nature of many fungal strains, their spreading in monoculture farming and their increasing resistance to previously used antifungal agents, there is an increasing need for the development of new antifungals. Berberine is an outstanding candidate in this regard (Manske et al., 1953). Berberine is a plant alkaloid found in several genera but mostly in the root bark of Berberis vulgaris (Etcu and Goina, 1970). Plants containing berberine have been used to treat cardiovascular, digestive and inflammatory diseases (Kulkarni and Dhir, 2010). The study by Ball et al., (2006) indicated that berberine is a stronger antibacterial agent when combined with 5-MHC (Ball et al., 2006). Berberine shows an inhibitory action against multiple strains of Malassezia and Candida fungi (Vollekova et al., 2001 and Park et al., 1999).

In the current study, we examined antifungal properties of berberine on the yeast Saccharamyces cerevisiae. S. cerevisiae was our model of choice because it is the most extensively studied eukaryotic model organism in molecular and cellular biology. Because the $S$. cerevisiae genome has been sequenced and a library of about 4700 mutant strains are available, we were able to test these mutants for their sensitivity to berberine and, in the process, gain some insights on berberine's antifungal mode of action.

\section{MIC and GDA:}

Many studies have suggested an antimicrobial and an antifungal activity of the plant alkaloid berberine. To investigate the potential of berberine as an antifungal agent, MIC assays were performed on S. cerevisiae. In this study, S. cerevisiae showed enough sensitivity to berberine, so that GDA assays could be performed with a concentration of $0.13 \mathrm{mg} / \mathrm{ml}$. 
A library of about 4700 S. cerevisiae mutant strains was tested through GDA assays to identify some possible modes of action of berberine on S. cerevisiae. Functional analysis of GDA results revealed the importance of certain cell processes in the yeast's sensitivity to berberine. Remarkably, a $70 \%-100 \%$ inhibition was observed in several mutant strains (YHR030C, YGR260W, YGL173C and YOR202W), suggesting that the deleted genes in these stains are involved in S.cerevisiae's sensitivity to berberine. Indeed, the genomic analysis suggests that these mutants' higher sensitivity to berberine is related to some defects in their actin filaments as Table 2 shows.

Previous studies have shown that berberine can accumulate in cell membranes and cause leaks. Therefore, mutations in genes involved in actin filament could make these yeast strains more sensitive to berberine as actin filaments have an important function in determining the shape and movement of the cell membrane. Another proposed mode of action of berberine is its intercalation in DNA, thereby interfering with processes such as transcription by RNA polymerase. As Table (2) shows, the YDR289C and YGL173C mutations play a role in gene transcription by RNA polymerase II. Such mutations could, therefore, make the RNA polymerase II even less able to perform gene transcription, making those mutants even more sensitive to berberine. These mutants would be interesting to investigate under Fluorescent microscopy. Finally, a third possible mode of action of berberine that I identified from my literature research and GDA assays is through reduction of 24-SMT activity. $24-\mathrm{SMT}$ is an enzyme that catalyzes a chemical reaction which is necessary for the biosynthesis of sterols and, therefore, essential for cell growth. These sterol molecules are located in both the lipid particles in the cell membrane and the mitochondrial outer membrane. As seen in Table (2), the YML008C mutation affects 24- 
SMT activity as well, possibly making the yeast even more sensitive to berberine. Park et al. (1999) indicated that with increasing berberine concentrations there were dramatic reductions in ergosterol accumulation in the yeast $C$. albicans. Because ergosterol is a crucial component of fungal cell membranes, this effect of berberine on $C$. albicans could support this antifungal mode of action of berberine. The YMLO08C mutant would, therefore, be a good candidate as well for an investigation under the microscope.

The study by Ball et al. (2006) hypothesized that berberine was removed through a MultiDrug Resistance (MDR) pumps. MICs were determined by theses authors for two purposes; to establish relevant concentrations of berberine for experimental purposes and to test the possibility of the importance of an S. aureus MDR pump (NorA) in berberine containing culture conditions (Ball et al., 2006). From these results, it is clear that INF55, a known MDR inhibitor, is an effective antimicrobial inhibitor that accumulate in bacteria. INF55 alone has no significant inhibitory effect on S. aureus growth at concentrations exceeding even $525 \mu \mathrm{M}$. MICs were established to be 40,325 and $>625 \mu \mathrm{M}$, respectively. This result alone is compelling evidence that the NorA MDR pump is important for S. aureus survival in the presence of berberine (Ball et al., 2006). This indicates a remarkable potentiation of berberine antimicrobial activity in the presence of $\mathrm{INF}_{55}$. Furthermore, when S.aureus was incubated with a chemically synthesized berberine-INF55 hybrid molecule, MICs were determined to be reduced by another $50 \%, 75 \%$ and 87.5\%, respectively (Ball et al., 2006). 
In this study, the Minimum Inhibition Concentration value of berberine was determined by using liquid broth protocol on S288C. Since the MIC test was carried out using discontinuous values, the exact MIC value could not be determined by just an MIC test (Canetti et al., 1969). Nevertheless, the MIC analysis provided some idea of an appropriate berberine concentration to use in GDA tests. Based on the test GDA runs, $0.13 \mathrm{mg} / \mathrm{ml}$ was determined as an appropriate concentration since the mutants on the plate exhibited 90-65\% inhibition.

The antifungal activity of berberine was examined using a chemical- genomic screen with about 4700 yeast strains, each with a different, non-essential gene deleted to $0.13 \mathrm{mg} / \mathrm{ml}$ of berberine. The GDA and SGAtools analysis in this study showed 71 strains most sensitive to berberine that had a colony size reduction $\geq 60 \%$. As showed in Table 2 , we distinguished 17 different categories out of the sensitive strains based on SGAtools. Out of the 17 categories, three main groups had mutations in genes that are directly involved in the cytoskeleton, cell membrane integrity or cell division: cytoskeletal protein binding (2.86\%), transferase activity (15.7\%) and RNA and mRNA binding (5.7\%). Also, 30\% of the 22 resistant deletion mutations identified from GDA analysis were identified as linked to tubulin binding. This group of mutants was enriched in tubulin associated genes ( $P$ value was $<0.10)$.

$60 \%-100 \%$ inhibition can be seen in mutants YHR030C, YGR260W, YGL173C, YML008C and YOR202W are the top most sensitive strains to berberine at the concentration of $0.13 \mathrm{mg} / \mathrm{ml}$. The YHR030C mutant (SLT2 gene) was $100 \%$ sensitive to berberine in the GDA assays which is interesting because the SLT2 gene codes for some domains on the MAP kinase. The YGR260W (TNA1 gene) was $78.5 \%$ sensitive to berberine. This gene codes for a transmembrane transporter in the plasma membrane and mitochondrial membrane. The HIS3 (YOR202W) gene codes for a 
histidine biosynthetic enzyme (Hope and Kevin, 1985). The KEM1 gene (YGL173C) is also known as XRN1 (Kenna et al., 1993). And is located on chromosome VII. It encodes the 5'-3' exoribonuclease enzyme which is involved in mRNA by turning the genes on and off, in this case, this enzyme is responsible for and actual degradation of the MRNA with positive regulation of transcription initiation and elongation via RNA Pol II interactions (Haimovich et al., 2013). The ERG6 gene, modified on the YML008C mutant, encodes for the 24- SMT enzyme involved in cell growth.

\section{GO Term Analysis:}

Based on_the GDA most sensitive mutants we used the GO Term tools to identify the genes involved in the yeast sensitivity to berberine. Among all the genes that were affected in the sensitive mutants, most of them were related to actin filaments, the cell membrane's integrity, lipids binding and cytokinesis which are interrelated because actin filaments are linked to the cell membrane and give it its shape and some of its functions (Table 2).

\section{Membrane Leakage Assay:}

Ball et al., 2006 and Kang et al., 2015 proposed that berberine disrupts membrane function but this was not supported by the GDA results (Table 2 and 3 ) or by the liposome leakage assay (Figure 14) in this study. However, because membranes are associated with other complexes, including cytoskeletal elements, berberine may affect membrane structure indirectly through other associations. 


\section{Drop-Out:}

As it is shown in Figure 9, the colony size and number were decreased in the presence of berberine at the concentration of $0.13 \mathrm{mg} / \mathrm{ml}$ in the experimental plates of the drop-out experiment compared to the control plates. Which suggests that the strains in figure 9 were identified as sensitive strains. This experiment shows a correlation between the GDA and Dropout experiments because the most sensitive mutants in GDA are almost the same most sensitive mutants in drop outs (Figure 8).

\section{Growth Curve:}

This experiment was an attempt to predict the growth behavior of yeast cells of $S$. cerevisiae treated with berberine. The growth rates differences can easily be seen in the proposed experiment (Figure 11). A sharp increase in the growth rate was observed in the resistant strains YLR200W and YGR078C in the presence of berberine compared to the wild type growth rate. On the other hand, the growth rate dropped down upon some of the most sensitive strains compared to the wild type growth rate, and the growth rate of the wild-type decreased in the presence of berberine much more than in the resistant ones.

\section{Fluorescence microscopy:}

A study by Stermitz et al., 2009 claims that berberine seems to bind strongly to DNA and the bound form of its DNA shows an increased fluorescence (Stermitz et al., 2009). As Table 6 shows, YML008C seemed to be a sensitive strain to berberine compared to the wild type with an 
MIC of $0.13 \mathrm{mg} / \mathrm{ml}$. As it was mentioned before, the ERG6 gene encodes for the 24- SMT enzyme necessary for cell growth.

The YHR030C mutant (SLT2 gene) also seemed to be strongly sensitive to berberine with an MIC $0.13 \mathrm{mg} / \mathrm{ml}$. Fluorescent Microscopy shows that subcellular localization of the cell was stained with berberine with $44 \%$ of budding frequency. The SL2 gene is involved in MAP kinase activity in the membrane and cytoplasm.

The YGL173C mutant (KEM1 gene) showed high sensitivity to berberine at the concertation of $0.13 \mathrm{mg} / \mathrm{ml}$. Under the microscope, the entire cell was stained with berberine. This was expected, considering that KEM1 gene's function is related to chromatin and mRNA.

Another sensitive strain was YDL226C (GCS1 gene). As seen under the fluorescence microscope, berberine was observed at the subcellular level. The function of the GCS1 gene which is related to the plasma membrane. The GCS1 gene encodes an ATR GTPase activator which is found on chromosome IV. During the cell cycle, this enzyme has the responsibility for the reorganization of actin filaments all the way between the ER and Golgi and between the Golgi and Plasma Membrane (Cherry et al., 2012; Blader et al., 1999; Poon et al., 1996; Poon et al., 1999).

Another sensitive strain was YDR378C mutant (LSM6 gene) showed 57\% of budding frequency in the presence of berberine, and the whole cell was stained. The LSM6 gene encodes for an RNA binding protein located on chromosome IV and is involved in the development of SSU- 
rRNA maturation and mRNA splicing via spliceosome (Cherry et al., 2012; Hernandez et al., 2004; Li et al., 2009; Stevens and Abelson, 1999).

Lastly, YDR388W mutant (RVS167 gene) showed 48\% of budding frequency in the presence of the antifungal berberine, and only the border of the cell was stained. The RVS167 gene encodes for an actin-associated protein which is involved in regulation of cytoskeleton, endocytosis and starvation and osmotic stress responses. The RVS167 gene, present on chromosome IV, encodes for a protein involved in the actin cortical patch localization and lipid tube assembly (Cherry et al., 2012).

\section{Confocal microscopy.}

A study on Candida albicans by Sanjiveeni Dhamgaye et al., 2014 claims that berberine treatment resulted in dysfunctional mitochondria and the cell wall integrity. In their study, they examined Candida albicans, which is a pathogenic yeast, by using an electronic microscope to view the internal organelles.

Recently, lipids droplets organelles in yeast have gained many researchers' attention (Maja Radulovic et al., 2013). S. cerevisiae is a useful model to know the pathways of natural lipids metabolism because of its natural lipids synthesis feature. The exact mechanism that involved in LD formation remains unknown, however, some researchers have established the biochemical pathways that linked to the formation of neutral lipids.

Saccharomyces cerevisiae's neutral lipids are mainly triacylglycerols (TGA) and steryl esters (STE). Also, they are part of intracellular structures called lipid droplets. Saccharomyces cerevisiae storage compounds are synthesized under stress conditions but not during exponential 
growth (Maja Radulovic et al., 2013). Indeed, lipid droplets are formed by a hydrophobic core of triacylglycerol (TGA) and steryl esters (STE) surrounded by a phospholipid monolayer with some particular proteins embedded (Jing Pu1,2 et al., 2011). Neutral lipids have the ability to be hydrolyzed into ergosterol, fatty acids, and diacylglycerol, that is used to build blocks for membrane synthesis and for producing energy (Maja Radulovic et al., 2013).

Previous studies showed that lipid droplets are highly energetic and able to make the interaction between other organelles (Martin and Parton, 2006; Goodman, 2008; Murphy et al., 2009; Zhang et al., 2010; Jing Pu1,2 et al., 2011). Specifically, lipids droplets usually have the ability to interact with endosome, mitochondria, ER and peroxisomes. However, the exact functions of these organelles remain unidentified.

A study by Jing Pu et al., 2011 claims that the interaction can occur between lipids droplets and mitochondria in S. cerevisiae due to some proteins exchange between the two organelles. In their study they examined 22 lipid droplets proteins and only 8 of them seemed to interact with prey proteins, Erg6 and Pet10 were identified as the most active ones in such interactions between lipids droplets and mitochondria in yeast cells.

In the current study, we used a confocal microscope to look at the way berberine was taken into the cell. In this experiment, we used different kinds of staining to confirm our suspicion of the stained organelles in the yeast cell. We stained the yeast cell with DAPI stain to see the nucleus and then the sample was stained with DAPI and berberine together. We found that the berberine didn't stain the nucleus. As it can be seen in Figure $20 \mathrm{~B}, \geq 40 \mathrm{~min}$ of bereberine exposure within the yeast cells followed by 2 hours exposure to DAPI stain before viewing by confocal microscopy; berberine and DAPI don't co-localized. Total time of berberine exposure (2 
hrs and $40 \mathrm{~min}$ ) the berberine is not localized to nuclei or mitochondria. Then we stained the sample with Nile blue and berberine together, and both seemed to stain the same organelles. Berberine co-localizes with Nile-Red (Figure 20 A). Another stain was used to confirm our prediction; we stained the cells with rhodamine 123 to stain the mitochondria and berberine to look at the stained organelles. Short berberine exposure ( $\leq 20$ minutes) stained the mitochondria with both stains in some cases, and after $\geq 40$ minutes of berberine exposure surprisingly we found that berberine was taken up in parts of other organelles which might be lipid droplets (Figure 20). Our prediction from this experiment is that berberine travels from the mitochondria to oil droplets. Future work is needed to confirm this theory. The conclusion from this experiment is that berberine seems to accumulate in lipid bodies.

Indeed, several of the mutants examined by confocal microscopy had berberine localized to the periphery of the cell (Table 6). These mutants should be examined again to see if the staining pattern is reproducible. In fact, these mutants maybe a valuable tool in looking at how berberine is taken up by living cells.

Figure 12. shows that almost $16 \%$ of the most sensitive mutants to berberine were associated with hydrolase activity which relates to lysosomes (Li and Kana, 2009). Lysosomes are organelles that have degradative enzymes and also have been found to fuse with the plasma membrane in the yeast cell (Li and Kana, 2009). These organelles are possible to see under the confocal microscope using lysosome fluorescence stains. The possibility that berberine localizes to the lysosome should be investigated. 


\section{One-Way ANOVA analysis:}

ANOVA analysis is a wise statistical method to test the difference in means between groups (Ding et al., 2014). In the present study, 1-way ANOVA analysis with a post-hoc Tukey Honestly Significant Difference (HSD) test with a significance level of $5 \%$ was performed for statistical differences in berberine staining patterns among selected yeast strains by using an online tool from SCISTAT for 1-way ANOVA (http://scistatcalc.blogspot.ca/2013/11/one-wayanova-test-calculator-in.html). The results of confocal microscopy analysis led to the identification of three uptake patterns groups (dim, spots and bright) as shown in Table 7.

The graphs in Figure 18 and 19 are granted from some yeast strains and show that the berberine staining is relatively consistent for a given time in a given experiment. This is evident from the relatively small standard errors in, for example, experiment 1 (Figure 18 A). However, when the time of berberine exposure differs, the staining pattern also differs, as can be seen by comparing results from experiment 1 and 2 (experiment 2 in Figure 19). This led us to investigate the pattern of staining following relatively short vs. long berberine exposure times.

In addition, we investigated the performance of berberine staining on eight yeast strains using confocal microscopy followed by feature selection technique test (ANOVA). The 'BRIGHT' cells were identified as dead cells, and that was not a great deal of variety. The 'SPOTS' cells showed that the berberine associated with oil droplets in the yeast cell. Indeed, there were no significant differences in the frequencies of cells with berberine staining 'spots' (Figure 19). 


\section{Conclusion:}

The aim of this study was to investigate berberine's potential as an antifungal agent against yeast, a well-documented model eukaryotic unicellular organism. In the process, we hoped to gain some insights on berberine's mode of action by using a comprehensive library of mutants. Previous research already showed some antifungal activity of berberine and indicated that berberine interferes with membrane function, DNA, actin filaments and some important enzymatic reactions. In order to carry out this research project, we chose first to use MIC with a view to quantifying the minimum concentration at which the wildtype S288C yeast is significantly inhibited. We could then carry on with testing the 4700 mutant strains for their sensitivity to berberine, using a GDA. Finally, once we had identified the most sensitive mutants through GDA, we searched, using GO TERMS, for the functions of the genes that were modified in the sensitive mutants and tested them with a drop-out, fluorescence microscopy, and confocal microscopy to see the cellular distribution of berberine in the strains of interest.

The most sensitive mutants identified through GDA were mostly related to actin filaments functions, the cell membrane's integrity and cytokinesis. This indicates that berberine is interfering with actin filaments and the cell membrane. Fluorescence microscopy confirmed this, as berberine was mostly concentrated on the plasma membrane of the yeast cells in most berberine sensitive strains. An additional evidence in regards to the interaction between the mitochondria and oil droplets in the presence of berberine is provided by the confocal microscopy results in the current study. DAPI, Nile Red and Rhodamine 123 stains were used as a double staining with berberine to confirm our hypothesis. The findings of 1-way ANOVA analysis 
and confocal microscopy investigation provide evidence that berberine can associate with lipids droplets in the yeast cell.

Researching the mode of action of berberine on S. cerevisiae was the main goal of our research project. Thanks to GDA, we could identify several mutants which showed a strong sensitivity to berberine. Further investigation with ANOVA analysis and confocal microscopy indicated a strong correlation between oil droplets functions and berberine's mode of action. Our results are therefore promising for finding berberine's mode of action on S. cerevisiae, and further research should aim at investigating how berberine interacts with lipids droplets. In order to do this, real time fluorescence microscopy could be used, using yeast cells that were transformed with fluorescent GFP-actin genes or other fluorescent actin gene fusions. This method would allow us to visualize and track in real-time the interactions of berberine with lipids droplets or/and actin filaments while yeast cells are being incubated in a berberine-containing medium. In order to investigate the possible binding of berberine to lipids droplets' proteins or actin-binding proteins, pull-down assays could be performed. Because berberine has been shown to inhibit 24-SMT, one of the few enzymes found in yeast lipid droplets (Athenstaedt $\mathrm{K}$ et al., 1999), enzymatic activity of $24-\mathrm{SMT}$ in the presence of berberine should be measured by enzymatic methyltransferase assays. Furthermore, because the synergistic association of berberine with synthetic antifungal agents such as fluconazole could reduce the concentration of drugs needed and thereby prevent the rise of drug-resistant fungi, we should also test berberine's synergistic action with other synthetic antifungal agents. 
Although these results are promising in order to gain an understanding of berberine's mode of action at the molecular and cellular level, we should bear in mind that the fungus kingdom is part of the eukaryotic domain and as such, berberine is likely to be toxic to humans and other members of the eukaryotic domain as well at higher concentrations. Indeed, research on berberine's neurotoxicity (Kysenius et al., 2014) has shown that berberine sensitizes neurons to glutamate and rotenone injury. 


\section{$\underline{\text { References: }}$}

Andrews, J. M. 2001. Determinination of minimum inhibitory concentrations. Journal of Antimicrobial chemotherapy. 48: 5 -16.

Athenstaedt, K., Zweytick, D., Jandrositz, A., Kohlwein, S. D., \& Daum, G. (1999). Identification and Characterization of Major Lipid Particle Proteins of the Yeast Saccharomyces cerevisiae. Journal of Bacteriology, 181(20), 6441-6448.

Ball, A. R., G. Casadei, S. Samosorn, J. B. Bremner, F. M. Ausubel, T. I. Moy, and K. Lewis. (2006) Conjugating Berberine to a Multidrug Resistance Pump Inhibitor Creates an Effective Antimicrobial. ACS Chemical Biology. 1(9): 594 - 587.

Babbar OP, Chhatwal VK, Ray IB, Mehra MK. (1982). Effect of berberine chloride eye drops on clinically positive trachoma patients. Indian J Med Res. 76:S83-S82.

Bouvier-Navé P, Husselstein T and Benveniste P. (1998). Two families of sterol methyltransferases are involved in the first and the second methylation steps of plant sterol biosynthesis. European Journal of Biochemistry. 256:88-96.

Cherry, J. M., Hong, E. L., Amundsen, C., Balakrishnan, R., Binkley, G., Chan, E. T., ... Wong, E. D. (2012). Saccharomyces Genome Database : the genomics resource of budding yeast, 40(November 2011), 700-705. http://doi.org/10.1093/nar/gkr1029.

Ding, H., Feng, P., Chen, W., \& Lin, H. (2014). Identification of bacteriophage virion proteins by the ANOVA feature selection and analysis. Molecular BioSystems, 10(8), 2229-2235.

Etcu P, Goina T. (1970). Neue Methoden zur Extrahierung der Alkaloide aus Berberis vulgaris. Planta medica. 18:372-75.

Freile. M., \& et al. (2006). Antifungal Activity of Aqueous Extracts and of Berberine Isolated from Berberis heterophylla. 83-8. 
Goodman, J.M. (2008). The gregarious lipid droplet. J Biol Chem 283, 28005-28009.

Hope, I. Struhl K. (1985). GCN4 protein, synthesize in vitro, binds HIS3 regulatory sequences: Implications for general control of amino acid biosynthetic genes in yeast. Cell, 177-188.

Iwazaki, R., Endo, E., Ueda-Nakamura, T., Nakamura, C., Garcia, L., \& Filho, B. (2009). In vitro antifungal activity of the berberine and its synergism with fluconazole. Antonie Van Leeuwenhoek, 201-205.

Irith Wiegand, Kai Hilpert, \& Robert E W Hancock. (2008). Agar and broth dilution methods to determine the minimal inhibitory concentration (MIC) of antimicrobial substances. Nature Protocols. 163-175.

Jaroslaw M. Boberek1, Jem Stach2, Liam Good. (2010). Genetic Evidence for Inhibition of Bacterial Division Protein FtsZ by Berberine. University of London, London. 1-7.

Jing Pu, Cheol Woong Ha, Shuyan Zhang, Jong Pil Jung, Won-Ki Huh,Pingsheng Liu. (2011). Interactomic study on interaction between lipid droplets and mitochondria. 151-747.

Kaneda Y, Torii M, Tanaka T, Aikawa M. (1991). In vitro effects of berberine sulfate on the growth and structure of Entamoeba histolytica, Giardia lamblia, and Trichomonas vaginalis. Ann Trop Med Parasitol. 85:417-425.

Kenna M, Stevens A, McCammon M, Douglas M. (1993). An Essential Yeast Gene with Homology to Exonuclease-Encoding XRN1/KEM1 Gene Also Encodes a protein with Exoribonuclease Activity. American Society for Microbiology. 341-350.

Kang, S., Li, Z., Yin, Z., Jia, R., Song, X., Li, L... Yin, I. (2015). The antibacterial mechanism of berberine against actinobacillus pleuropneumoniae. Natural Product Research, 29(23), 2203.

Kim Lewis \& Frederick M Ausubel. (2006). Prospects for plant-derived antibacterials. Nature Biotechnology.1504-1507. 
Kysenius K, Brunello CA \& Huttunen HJ. (2014). Mitochondria and NMDA Receptor-Dependent Toxicity of Berberine Sensitizes Neurons to Glutamate and Rotenone Injury. PLoS ONE 9(9): e107129. doi: 10.1371/journal.pone.0107129.

Lambert, R. J. W., P.N. Skandamis, P. J. Coote, and G.-J. E. Nychas. (2001). A study of the minimum inhibitory concentration and mode of action of oregano essential oil, thymol and carvacrol. Journal of Applied Microbiology. 91: 453 - 462.

Li, S. C., \& Kane, P. M. (2009). The yeast lysosome-like vacuole: Endpoint and crossroads. BBA Molecular Cell Research, 1793(4), 650-663.

Marin-Neto JA, Maciel BC, Secches AL, Gallo L. (1988). Cardiovascular effects of berberine in patients with severe congestive heart failure. ClinCardiol. 11:253-260.

Martin, S., and Parton, R.G. (2006). Lipid droplets: a unified view of a dynamic organelle. Nat Rev Mol Cell Biol 7, 373-378.

Manske, G.B., and Homes, H.L. (1953). The Alkaloids. VOI III. New York: Academic Press. 432.

Memarian, N., Jessulat, M., Alirezaie, J., Mir-Rashed, N., Jianhua Xu, J., Zareie, M., Smith, M. and Golshani, A. (2007). Colony size measurement of the yeast gene deletion strains for functional genomics. BMC Bioinformatics. 8:117.

Moore, D., and Frazer, L.N. (2002). Essential Fungal Genetics. Springer.

Murphy, S., Martin, S., and Parton, R.G. (2009). Lipid dropletorganelle interactions; sharing the fats. Biochim Biophys Acta 1791, 441-447.

Manastır, L., Ergon, M. C. and Yücesoy, M. (2011), Investigation of mutations in Erg11 gene of fluconazole resistant Candida albicans isolates from Turkish hospitals. Mycoses, 54: 99-104. 
C.M. Mann and J.L. Markham. (1998). A new method for determining the minimum inhibitory concentration of essential oils. Journal of Applied Microbiology. 538-544.

Nobukazu, S., Yazaki, K. (2007). Human MDR1 and MRP1 recognize berberine as their transport substracte. Biotechnol. Biochem. 71:242-245.

Oruc Hasan H. (2010). Fungicides and their effects on animals, Fungicides, Odile Carisse (Ed.), ISBN: 978-953-307-266-1, In Tech, Available from:

http://www.intechopen.com/books/fungicides/fungicides-and-their-effects-on-animals.

Park. K.-S., K.-C. Kang, J.-H. Kim, D. J. Adams, T.-N. Johng, and Y.-K. Paik. (1999). Differential inhibitory effects of protoberberines on sterol and chitin biosynthesis in Candida albicans. Journal of Antimicrobial Chemotherapy. 43: 667 - 674.

Quan H, Cao YY, Xu Z, Zhao JX, Gao PH, Qin XF, Jiang YY. (2006). Potent in vitro synergism of fluconazole and berberine chloride against clinical isolates of Candida albicans resistant to fluconazole. Journal Antimicrob Agents Chemother. 50(3):1096-9.

Radulovic, M., Knittelfelder, O., Cristobal-Sarramian, A., Kolb, D., Wolinski, H., \& Kohlwein, S. D. (2013). The emergence of lipid droplets in yeast: Current status and experimental approaches. Current Genetics, 59(4): 231-242.

Sanjiveeni Dhamgaye, Sanjiveeni Dhamgaye, Frédéric Devaux, Patrick Vandeputte, Nikesh Kumar Khandelwal, Dominique Sanglard, Gauranga Mukhopadhyay, Rajendra Prasad. (2014). Molecular Mechanisms of Action of Herbal Antifungal Alkaloid Berberine, in Candida albicans. PLOS ONE, 9(8), e104554.

Stermitz, F., Lorenz, P., Tawara, J., Zenewicz, L., \& Lewis, K. (2000). Synergy in a medicinal plant: Antimicrobial action of berberine potentiated by 5'-methoxyhydnocarpin, a multidrug pump inhibitor. Proceedings of the National Academy of Sciences, 1433-1437. 
Sunil Kumar Battu, Michael A. Repka, Sindhuri Maddineni, Amar G. Chittiboyina, Mitchell A. Ave ry, Soumyajit Majumda. (2010). Physicochemical Characterization of Berberine Chloride: A Perspective in the Development of a Solution Dosage Form for Oral Delivery. AAPS PharmSciTech. 1466-1475

http://sgatools.ccbr.utoronto.ca/.

http://scistatcalc.blogspot.ca/2013/11/one-way-anova-test-calculator-in.html

http://www.yeastgenome.org/cgi-bin/GO/goTermFinder.pl\#default.

Wang YX, Yao XJ, Tan YH. (1994) Effects of berberine on delayed afterdepolarizations in ventricular muscles in vitro and in vivo. J Cardiovasc Pharmacol. 23:716-722.

Zhang, S., Du, Y., Wang, Y., and Liu, P. (2010). Lipid Droplet - A Cellular Organelle for Lipid Metabolism. Acta Biophisica Sinica 26, 97-105.

Zhou. X., \& et al. (2009). A genome-wide screen in Saccharomyces cerevisiae reveals pathways affected by arsenic toxicity. Genomics, 294-307. 


\section{Appendix 1 GENE}

Ghyda's

Fatima's

Average Average

$\begin{array}{llll}\text { ORF plate row column } & x 3 & x 2\end{array}$

\begin{tabular}{|c|c|c|c|c|c|c|}
\hline HIS3 & YOR202W & 1 & 1 & 1 & -14.2308 & \\
\hline HIS3 & YOR202W & 1 & 1 & 2 & 14.67297 & \\
\hline HIS3 & YOR202W & 1 & 1 & 3 & 8.597301 & \\
\hline HIS3 & YOR202W & 1 & 1 & 4 & 8.070146 & \\
\hline HIS3 & YOR202W & 1 & 1 & 5 & 13.53352 & \\
\hline HIS3 & YOR202W & 1 & 1 & 6 & -8.86208 & \\
\hline HIS3 & YOR202W & 1 & 1 & 7 & -16.2149 & \\
\hline HIS3 & YOR202W & 1 & 1 & 8 & 0.515528 & \\
\hline HIS3 & YOR202W & 1 & 1 & 9 & -42.659 & \\
\hline HIS3 & YOR202W & 1 & 1 & 10 & 16.47829 & \\
\hline HIS3 & YOR202W & 1 & 1 & 11 & 5.570054 & \\
\hline HIS3 & YOR202W & 1 & 1 & 12 & 9.674323 & \\
\hline HIS3 & YOR202W & 1 & 1 & 13 & 14.09307 & \\
\hline HIS3 & YOR202W & 1 & 1 & 14 & 11.32904 & \\
\hline HIS3 & YOR202W & 1 & 1 & 15 & 10.07011 & \\
\hline HIS3 & YOR202W & 1 & 1 & 16 & -6.42092 & \\
\hline HIS3 & YOR202W & 1 & 1 & 17 & 7.472276 & \\
\hline HIS3 & YOR202W & 1 & 1 & 18 & 8.773368 & \\
\hline HIS3 & YOR202W & 1 & 1 & 19 & 10.25571 & \\
\hline HIS3 & YOR202W & 1 & 1 & 20 & -1.98611 & \\
\hline HIS3 & YOR202W & 1 & 1 & 21 & -10.4015 & \\
\hline HIS3 & YOR202W & 1 & 1 & 22 & 2.975541 & \\
\hline HIS3 & YOR202W & 1 & 1 & 23 & 17.74155 & \\
\hline HIS3 & YOR202W & 1 & 1 & 24 & 9.171372 & \\
\hline \multirow[t]{2}{*}{ HIS3 } & YOR202W & 1 & 2 & 1 & -3.84309 & \\
\hline & YBR030W & 1 & 2 & 2 & 43.04095 & 49.81447 \\
\hline \multirow[t]{5}{*}{ HDR1 } & YBR138C & 1 & 2 & 3 & 31.19816 & 21.7898 \\
\hline & YBR028C & 1 & 2 & 4 & 8.265776 & 35.96395 \\
\hline & YBR137W & 1 & 2 & 5 & 19.70116 & 42.63876 \\
\hline & YBR027C & 1 & 2 & 6 & 42.37332 & 33.83057 \\
\hline & YBR134W & 1 & 2 & 7 & 49.84178 & 46.45752 \\
\hline ETR1 & YBR026C & 1 & 2 & 8 & 48.34441 & 44.95729 \\
\hline \multirow[t]{2}{*}{ AGP2 } & YBR132C & 1 & 2 & 9 & 7.316037 & -9.81426 \\
\hline & YBR025C & 1 & 2 & 10 & \#DIV/0! & 48.94615 \\
\hline CCZ1 & YBR131W & 1 & 2 & 11 & 12.17321 & 39.05786 \\
\hline SCO2 & YBR024W & 1 & 2 & 12 & 43.77514 & 41.0427 \\
\hline SHE3 & YBR130C & 1 & 2 & 13 & 9.34691 & 32.01168 \\
\hline CHS3 & YBR023C & 1 & 2 & 14 & 63.5221 & 71.15447 \\
\hline OPY1 & YBR129C & 1 & 2 & 15 & 15.79232 & 35.03087 \\
\hline
\end{tabular}




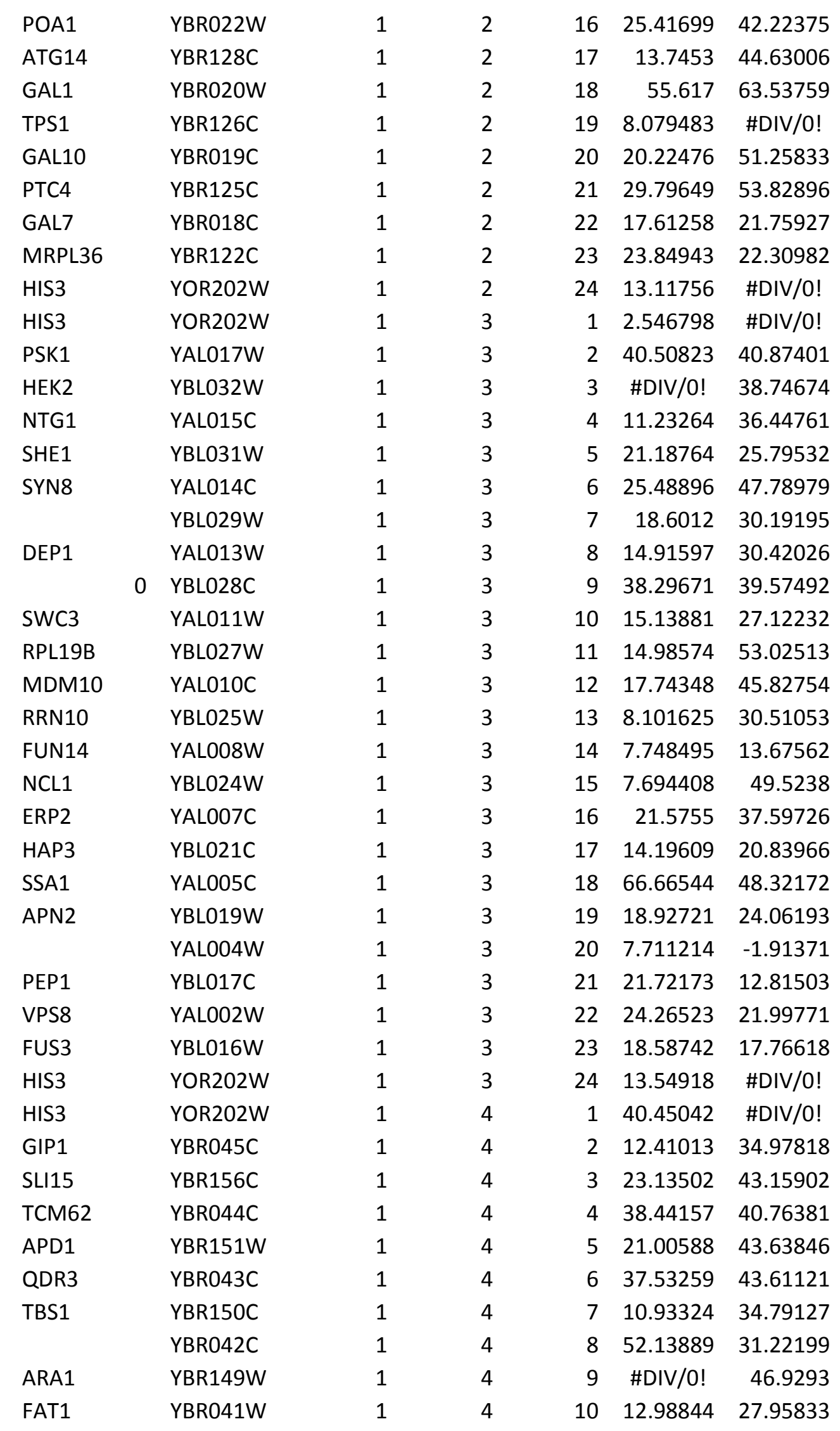




\begin{tabular}{|c|c|c|c|c|c|c|}
\hline YSW1 & YBR148W & 1 & 4 & 11 & 33.09511 & 35.25832 \\
\hline \multirow[t]{2}{*}{ FIG1 } & YBR040W & 1 & 4 & 12 & 22.95009 & 15.61638 \\
\hline & YBR147W & 1 & 4 & 13 & 15.68589 & 25.91725 \\
\hline CSG2 & YBR036C & 1 & 4 & 14 & 64.17575 & 71.13533 \\
\hline MRPS9 & YBR146W & 1 & 4 & 15 & 25.20914 & 24.18807 \\
\hline HMT1 & YBR034C & 1 & 4 & 16 & 19.14123 & 42.08514 \\
\hline ADH5 & YBR145W & 1 & 4 & 17 & 20.83562 & 47.70084 \\
\hline \multirow[t]{4}{*}{ EDS1 } & YBR033W & 1 & 4 & 18 & 53.55355 & 44.1145 \\
\hline & YBR144C & 1 & 4 & 19 & 44.43626 & 48.52256 \\
\hline & YBR032W & 1 & 4 & 20 & 19.23735 & 34.45318 \\
\hline & YBR141C & 1 & 4 & 21 & 29.10213 & 34.95783 \\
\hline \multirow[t]{2}{*}{ RPL4A } & YBR031W & 1 & 4 & 22 & 24.38362 & 1.733746 \\
\hline & YBR139W & 1 & 4 & 23 & -1.72709 & 47.12423 \\
\hline HIS3 & YOR202W & 1 & 4 & 24 & 4.906628 & \#DIV/0! \\
\hline HIS3 & YOR202W & 1 & 5 & 1 & -28.1202 & \#DIV/0! \\
\hline FUN21 & YAL031C & 1 & 5 & 2 & 14.33573 & 55.2091 \\
\hline SAS3 & YBL052C & 1 & 5 & 3 & 18.0169 & 50.58812 \\
\hline SNC1 & YAL030W & 1 & 5 & 4 & 19.43915 & 32.78424 \\
\hline PIN4 & YBL051C & 1 & 5 & 5 & 60.49303 & 68.19636 \\
\hline MYO4 & YAL029C & 1 & 5 & 6 & 23.57635 & 44.80369 \\
\hline $\mathrm{MOH} 1$ & YBL049W & 1 & 5 & 7 & 51.84055 & 57.52913 \\
\hline \multirow[t]{3}{*}{ FRT2 } & YAL028W & 1 & 5 & 8 & 26.27412 & 46.48414 \\
\hline & YBL048W & 1 & 5 & 9 & 10.20136 & 40.26323 \\
\hline & YAL027W & 1 & 5 & 10 & 19.0936 & 52.27239 \\
\hline EDE1 & YBL047C & 1 & 5 & 11 & 11.40138 & 20.89416 \\
\hline \multirow[t]{2}{*}{ DRS2 } & YAL026C & 1 & 5 & 12 & 3.144376 & 1.605761 \\
\hline & YBL046W & 1 & 5 & 13 & 16.60259 & 31.48875 \\
\hline PMT2 & YAL023C & 1 & 5 & 14 & 39.38882 & 32.0132 \\
\hline ECM13 & YBL043W & 1 & 5 & 15 & 17.58105 & 19.49217 \\
\hline FUN26 & YAL022C & 1 & 5 & 16 & -6.23647 & 35.19058 \\
\hline FUI1 & YBL042C & 1 & 5 & 17 & 3.022699 & 26.09944 \\
\hline ATS1 & YAL020C & 1 & 5 & 18 & 19.78251 & 48.87117 \\
\hline URA7 & YBL039C & 1 & 5 & 19 & 22.39959 & 22.11492 \\
\hline FUN30 & YAL019W & 1 & 5 & 20 & 13.96942 & 24.23332 \\
\hline \multirow[t]{3}{*}{ APL3 } & YBL037W & 1 & 5 & 21 & 46.91291 & 38.2944 \\
\hline & YAL018C & 1 & 5 & 22 & -5.70862 & 38.94163 \\
\hline & YBL036C & 1 & 5 & 23 & 14.15078 & 34.63124 \\
\hline HIS3 & YOR202W & 1 & 5 & 24 & 6.410862 & \#DIV/0! \\
\hline HIS3 & YOR202W & 1 & 6 & 1 & 15.58694 & \#DIV/0! \\
\hline UBP14 & YBR058C & 1 & 6 & 2 & 12.19436 & 41.40742 \\
\hline SSE2 & YBR169C & 1 & 6 & 3 & -0.61049 & 0.60389 \\
\hline MUM2 & YBR057C & 1 & 6 & 4 & -10.1148 & 31.58316 \\
\hline PEX32 & YBR168W & 1 & 6 & 5 & 7.639346 & 41.37826 \\
\hline
\end{tabular}




\begin{tabular}{|c|c|c|c|c|c|c|}
\hline & YBR056W & 1 & 6 & 6 & -17.2579 & 45.05267 \\
\hline TYR1 & YBR166C & 1 & 6 & 7 & 3.968564 & 40.35981 \\
\hline YRO2 & YBR054W & 1 & 6 & 8 & 26.82616 & 47.14612 \\
\hline \multirow[t]{2}{*}{ UBS1 } & YBR165W & 1 & 6 & 9 & -6.11366 & 14.41654 \\
\hline & YBR053C & 1 & 6 & 10 & 29.39988 & 57.53442 \\
\hline \multirow[t]{3}{*}{ ARL1 } & YBR164C & 1 & 6 & 11 & 21.80092 & 22.56824 \\
\hline & YBR052C & 1 & 6 & 12 & 37.68394 & 36.11719 \\
\hline & YBR162W- & & & & & \\
\hline \multirow[t]{2}{*}{ YSY6 } & $A$ & 1 & 6 & 13 & -5.97023 & 24.73325 \\
\hline & YBR051W & 1 & 6 & 14 & 25.35196 & 25.70231 \\
\hline TOS1 & YBR162C & 1 & 6 & 15 & 37.86547 & 31.78813 \\
\hline REG2 & YBR050C & 1 & 6 & 16 & 14.78411 & 44.84239 \\
\hline $\mathrm{CSH} 1$ & YBR161W & 1 & 6 & 17 & 11.35155 & 42.51641 \\
\hline \multirow[t]{2}{*}{ RPS11B } & YBR048W & 1 & 6 & 18 & -2.44582 & 39.22714 \\
\hline & YBR159W & 1 & 6 & 19 & -0.36704 & 36.97976 \\
\hline FMP23 & YBR047W & 1 & 6 & 20 & 24.70878 & 41.71011 \\
\hline AMN1 & YBR158W & 1 & 6 & 21 & 31.84421 & 47.83999 \\
\hline ZTA1 & YBR046C & 1 & 6 & 22 & 49.67872 & 39.91169 \\
\hline ICS2 & YBR157C & 1 & 6 & 23 & 19.35499 & 52.03154 \\
\hline HIS3 & YOR202W & 1 & 6 & 24 & -2.22016 & \#DIV/0! \\
\hline HIS3 & YOR202W & 1 & 7 & 1 & -6.529 & \#DIV/0! \\
\hline ACS1 & YAL054C & 1 & 7 & 2 & 12.80465 & 42.45327 \\
\hline \multirow[t]{2}{*}{ PRX1 } & YBL064C & 1 & 7 & 3 & 13.60354 & 31.36482 \\
\hline & YAL053W & 1 & 7 & 4 & 55.94984 & 36.3293 \\
\hline KIP1 & YBL063W & 1 & 7 & 5 & 12.05134 & 26.27383 \\
\hline \multirow[t]{3}{*}{ OAF1 } & YAL051W & 1 & 7 & 6 & 44.89791 & 33.064 \\
\hline & YBL062W & 1 & 7 & 7 & 42.73494 & 57.47576 \\
\hline & YAL049C & 1 & 7 & 8 & 12.50389 & 36.29772 \\
\hline \multirow[t]{5}{*}{ SKT5 } & YBL061C & 1 & 7 & 9 & 22.99517 & 33.72785 \\
\hline & YAL045C & 1 & 7 & 10 & 31.78411 & 44.84885 \\
\hline & YBL060W & 1 & 7 & 11 & 16.82321 & 47.77667 \\
\hline & YAL043C-A & 1 & 7 & 12 & 46.19639 & 33.56205 \\
\hline & YBL059W & 1 & 7 & 13 & 14.09693 & 29.28146 \\
\hline ERV46 & YAL042W & 1 & 7 & 14 & 15.76087 & 48.35096 \\
\hline PTH2 & YBL057C & 1 & 7 & 15 & 13.51532 & 34.29793 \\
\hline CLN3 & YAL040C & 1 & 7 & 16 & 27.96972 & 51.88719 \\
\hline \multirow[t]{3}{*}{ PTC3 } & YBL056W & 1 & 7 & 17 & 32.48074 & 13.91206 \\
\hline & YAL037W & 1 & 7 & 18 & 19.52329 & 54.50662 \\
\hline & YBL055C & 1 & 7 & 19 & 9.301877 & 52.69591 \\
\hline \multirow[t]{2}{*}{ RBG1 } & YAL036C & 1 & 7 & 20 & 15.84776 & 45.90629 \\
\hline & YBL054W & 1 & 7 & 21 & 24.42267 & 15.69849 \\
\hline \multirow[t]{2}{*}{ FUN19 } & YAL034C & 1 & 7 & 22 & 2.802363 & 43.62612 \\
\hline & YBL053W & 1 & 7 & 23 & 23.06308 & 37.50065 \\
\hline
\end{tabular}




\begin{tabular}{|c|c|c|c|c|c|c|}
\hline HIS3 & YOR202W & 1 & 7 & 24 & -4.26538 & \#DIV/0! \\
\hline \multirow[t]{2}{*}{ HIS3 } & YOR202W & 1 & 8 & 1 & 9.747801 & \#DIV/0! \\
\hline & YBR071W & 1 & 8 & 2 & 13.94803 & 47.67391 \\
\hline SMP1 & YBR182C & 1 & 8 & 3 & 10.98999 & 44.62207 \\
\hline TAT1 & YBR069C & 1 & 8 & 4 & 1.512456 & 15.38485 \\
\hline RPS6B & YBR181C & 1 & 8 & 5 & 8.524925 & 24.1389 \\
\hline BAP2 & YBR068C & 1 & 8 & 6 & -9.80563 & 37.84818 \\
\hline DTR1 & YBR180W & 1 & 8 & 7 & 14.47332 & 45.91993 \\
\hline \multirow[t]{2}{*}{ TIP1 } & YBR067C & 1 & 8 & 8 & 48.62183 & 35.23213 \\
\hline & YBR178W & 1 & 8 & 9 & 17.91925 & 37.10189 \\
\hline NRG2 & YBR066C & 1 & 8 & 10 & 16.97764 & 45.88465 \\
\hline EHT1 & YBR177C & 1 & 8 & 11 & 15.2882 & 50.67294 \\
\hline ECM2 & YBR065C & 1 & 8 & 12 & 9.346162 & 43.80011 \\
\hline \multirow[t]{2}{*}{ ECM31 } & YBR176W & 1 & 8 & 13 & 12.8758 & 32.68434 \\
\hline & YBR064W & 1 & 8 & 14 & 19.78251 & 48.79559 \\
\hline \multirow[t]{4}{*}{ SWD3 } & YBR175W & 1 & 8 & 15 & 14.98111 & 6.497777 \\
\hline & YBR063C & 1 & 8 & 16 & 31.97084 & 40.13003 \\
\hline & YBR174C & 1 & 8 & 17 & 21.17402 & 1.030137 \\
\hline & YBR062C & 1 & 8 & 18 & 24.59764 & 22.44624 \\
\hline SMY2 & YBR172C & 1 & 8 & 19 & 26.04847 & 45.87122 \\
\hline TRM7 & YBR061C & 1 & 8 & 20 & 27.64541 & 47.27797 \\
\hline SEC66 & YBR171W & 1 & 8 & 21 & 13.46785 & 19.67038 \\
\hline AKL1 & YBR059C & 1 & 8 & 22 & 15.49141 & 37.25011 \\
\hline NPL4 & YBR170C & 1 & 8 & 23 & -2.85492 & 27.67434 \\
\hline HIS3 & YOR202W & 1 & 8 & 24 & -17.8041 & \#DIV/0! \\
\hline \multirow[t]{2}{*}{ HIS3 } & YOR202W & 1 & 9 & 1 & -23.6664 & \#DIV/0! \\
\hline & YAL066W & 1 & 9 & 2 & 17.39135 & 40.16782 \\
\hline \multirow[t]{2}{*}{ NUP170 } & YBL079W & 1 & 9 & 3 & -0.81018 & 44.03432 \\
\hline & YAL065C & 1 & 9 & 4 & 3.825752 & 31.54517 \\
\hline \multirow[t]{2}{*}{ ATG8 } & YBL078C & 1 & 9 & 5 & 61.70397 & 54.32237 \\
\hline & YAL064C-A & 1 & 9 & 6 & 18.53881 & 36.09136 \\
\hline SSA3 & YBL075C & 1 & 9 & 7 & 32.97021 & 45.07801 \\
\hline GDH3 & YAL062W & 1 & 9 & 8 & 21.26147 & 23.47316 \\
\hline \multirow[t]{3}{*}{ RPS8A } & YBL072C & 1 & 9 & 9 & 6.172854 & 13.57802 \\
\hline & YAL061W & 1 & 9 & 10 & 9.525706 & 50.83646 \\
\hline & YBL071C & 1 & 9 & 11 & 10.86418 & 39.96568 \\
\hline \multirow[t]{2}{*}{$\mathrm{BDH} 1$} & YAL060W & 1 & 9 & 12 & 16.8598 & 40.10747 \\
\hline & YBL070C & 1 & 9 & 13 & 28.19301 & 49.48362 \\
\hline ECM1 & YAL059W & 1 & 9 & 14 & 19.00575 & \#DIV/0! \\
\hline AST1 & YBL069W & 1 & 9 & 15 & 0.924124 & \#DIV/0! \\
\hline CNE1 & YAL058W & 1 & 9 & 16 & -2.86618 & \#DIV/0! \\
\hline \multirow[t]{2}{*}{ PRS4 } & YBL068W & 1 & 9 & 17 & 42.39178 & 58.89055 \\
\hline & YAL058C-A & 1 & 9 & 18 & 27.78871 & 50.38313 \\
\hline
\end{tabular}




\begin{tabular}{|c|c|c|c|c|c|c|}
\hline UBP13 & YBL067C & 1 & 9 & 19 & 23.12039 & 50.83707 \\
\hline GPB2 & YAL056W & 1 & 9 & 20 & 21.40369 & 46.74451 \\
\hline SEF1 & YBL066C & 1 & 9 & 21 & 31.12945 & 44.23039 \\
\hline \multirow[t]{2}{*}{ PEX22 } & YAL055W & 1 & 9 & 22 & 5.00431 & 27.16322 \\
\hline & YBL065W & 1 & 9 & 23 & \#DIV/0! & 31.86207 \\
\hline HIS3 & YOR202W & 1 & 9 & 24 & -15.9064 & \#DIV/0! \\
\hline \multirow[t]{2}{*}{ HIS3 } & YOR202W & 1 & 10 & 1 & -3.23111 & \#DIV/0! \\
\hline & YBR090C & 1 & 10 & 2 & 38.24872 & 48.12082 \\
\hline KTR4 & YBR199W & 1 & 10 & 3 & 4.797959 & 29.23201 \\
\hline \multirow[t]{2}{*}{ MIS1 } & YBR084W & 1 & 10 & 4 & -0.72731 & 19.47169 \\
\hline & YBR197C & 1 & 10 & 5 & -6.45753 & 28.72797 \\
\hline RPL19A & YBR084C-A & 1 & 10 & 6 & 19.02823 & 50.15852 \\
\hline MSI1 & YBR195C & 1 & 10 & 7 & 13.42262 & 25.27821 \\
\hline TEC1 & YBR083W & 1 & 10 & 8 & -21.2521 & 37.87053 \\
\hline SOY1 & YBR194W & 1 & 10 & 9 & 17.05427 & 47.75521 \\
\hline UBC4 & YBR082C & 1 & 10 & 10 & 20.90432 & 43.37693 \\
\hline RPS9B & YBR189W & 1 & 10 & 11 & 38.36467 & 44.88291 \\
\hline SLM4 & YBR077C & 1 & 10 & 12 & \#DIV/0! & 29.46306 \\
\hline NTC20 & YBR188C & 1 & 10 & 13 & 40.09965 & 51.13953 \\
\hline \multirow[t]{3}{*}{ ECM8 } & YBR076W & 1 & 10 & 14 & 8.584098 & \#DIV/O! \\
\hline & YBR187W & 1 & 10 & 15 & 30.62229 & \#DIV/0! \\
\hline & YBR075W & 1 & 10 & 16 & 58.65138 & \#DIV/0! \\
\hline \multirow[t]{2}{*}{$\mathrm{PCH} 2$} & YBR186W & 1 & 10 & 17 & 33.31948 & 53.86576 \\
\hline & YBR074W & 1 & 10 & 18 & 10.37947 & 37.67122 \\
\hline MBA1 & YBR185C & 1 & 10 & 19 & 23.84445 & 44.7371 \\
\hline \multirow[t]{2}{*}{$\mathrm{RDH} 54$} & YBR073W & 1 & 10 & 20 & 11.85165 & 29.43462 \\
\hline & YBR184W & 1 & 10 & 21 & 17.37403 & 52.45274 \\
\hline HSP26 & YBR072W & 1 & 10 & 22 & 49.11487 & 59.76986 \\
\hline YPC1 & YBR183W & 1 & 10 & 23 & 36.05024 & 50.44771 \\
\hline HIS3 & YOR202W & 1 & 10 & 24 & 22.00294 & \#DIV/0! \\
\hline HIS3 & YOR202W & 1 & 11 & 1 & 2.545615 & \#DIV/0! \\
\hline \multirow[t]{3}{*}{ UIP3 } & YAR027W & 1 & 11 & 2 & 20.74006 & 40.97874 \\
\hline & YBL094C & 1 & 11 & 3 & 62.78998 & 62.156 \\
\hline & YAR023C & 1 & 11 & 4 & 10.33667 & 37.15923 \\
\hline SCS22 & YBL091C-A & 1 & 11 & 5 & 3.132937 & 31.00592 \\
\hline PAU7 & YAR020C & 1 & 11 & 6 & -1.02976 & 19.88413 \\
\hline MAP2 & YBL091C & 1 & 11 & 7 & 23.71743 & 43.95053 \\
\hline KIN3 & YAR018C & 1 & 11 & 8 & 13.15324 & 30.38225 \\
\hline AVT5 & YBL089W & 1 & 11 & 9 & 16.39789 & 44.70744 \\
\hline ADE1 & YAR015W & 1 & 11 & 10 & 22.41737 & \#DIV/0! \\
\hline TEL1 & YBL088C & 1 & 11 & 11 & 17.4015 & 31.40378 \\
\hline BUD14 & YAR014C & 1 & 11 & 12 & 21.40783 & 28.04814 \\
\hline RPL23A & YBL087C & 1 & 11 & 13 & 11.83469 & 21.66421 \\
\hline
\end{tabular}




\begin{tabular}{|c|c|c|c|c|c|c|}
\hline \multirow[t]{2}{*}{ SWD1 } & YAR003W & 1 & 11 & 14 & 19.51961 & 25.49691 \\
\hline & YBL086C & 1 & 11 & 15 & 24.17233 & 44.85876 \\
\hline NUP60 & YAR002W & 1 & 11 & 16 & 28.70147 & 27.95677 \\
\hline BOI1 & YBL085W & 1 & 11 & 17 & 2.51561 & 51.7413 \\
\hline \multirow[t]{3}{*}{ ERP1 } & YAR002C-A & 1 & 11 & 18 & 7.176993 & 42.477 \\
\hline & YBL083C & 1 & 11 & 19 & 18.42295 & 32.68245 \\
\hline & YAL068C & 1 & 11 & 20 & 9.789055 & 46.73335 \\
\hline RHK1 & YBL082C & 1 & 11 & 21 & -1.1495 & 32.38532 \\
\hline \multirow[t]{2}{*}{ SEO1 } & YAL067C & 1 & 11 & 22 & 48.8567 & 23.92708 \\
\hline & YBL081W & 1 & 11 & 23 & 21.45724 & 50.39905 \\
\hline HIS3 & YOR202W & 1 & 11 & 24 & 15.45855 & \#DIV/0! \\
\hline HIS3 & YOR202W & 1 & 12 & 1 & -9.60089 & \#DIV/0! \\
\hline YMC2 & YBR104W & 1 & 12 & 2 & 7.106782 & 43.59025 \\
\hline NGR1 & YBR212W & 1 & 12 & 3 & -8.04217 & 35.01048 \\
\hline SIF2 & YBR103W & 1 & 12 & 4 & \#DIV/0! & 33.04424 \\
\hline ERV15 & YBR210W & 1 & 12 & 5 & 5.073727 & 27.23709 \\
\hline \multirow[t]{3}{*}{ FES1 } & YBR101C & 1 & 12 & 6 & 0.353322 & 51.17505 \\
\hline & YBR209W & 1 & 12 & 7 & 11.20799 & 26.395 \\
\hline & YBR100W & 1 & 12 & 8 & 35.81348 & 48.1 \\
\hline \multirow[t]{2}{*}{ DUR1,2 } & YBR208C & 1 & 12 & 9 & \#DIV/0! & 45.65015 \\
\hline & YBR099C & 1 & 12 & 10 & 15.61465 & 47.75 \\
\hline FTH1 & YBR207W & 1 & 12 & 11 & 17.72523 & 42.85536 \\
\hline \multirow[t]{2}{*}{ MMS4 } & YBR098W & 1 & 12 & 12 & 25.88227 & 37.37882 \\
\hline & YBR206W & 1 & 12 & 13 & 20.14854 & 54.51831 \\
\hline RXT2 & YBR095C & 1 & 12 & 14 & 27.15864 & 28.31986 \\
\hline \multirow[t]{3}{*}{ KTR3 } & YBR205W & 1 & 12 & 15 & 10.08012 & 49.87289 \\
\hline & YBR094W & 1 & 12 & 16 & \#DIV/0! & 42.32339 \\
\hline & YBR204C & 1 & 12 & 17 & 7.714464 & 42.9328 \\
\hline PHO5 & YBR093C & 1 & 12 & 18 & -8.04097 & 34.9759 \\
\hline $\cos 111$ & YBR203W & 1 & 12 & 19 & 5.41316 & 46.19796 \\
\hline PHO3 & YBR092C & 1 & 12 & 20 & 39.37427 & 57.45291 \\
\hline \multirow[t]{2}{*}{ DER1 } & YBR201W & 1 & 12 & 21 & 34.42956 & 49.78331 \\
\hline & YBR090C-A & 1 & 12 & 22 & 8.948376 & 47.47931 \\
\hline BEM1 & YBR200W & 1 & 12 & 23 & 12.00924 & 21.9844 \\
\hline HIS3 & YOR202W & 1 & 12 & 24 & 18.03214 & \#DIV/0! \\
\hline \multirow[t]{2}{*}{ HIS3 } & YOR202W & 1 & 13 & 1 & 9.234345 & \#DIV/0! \\
\hline & YAR047C & 1 & 13 & 2 & -5.9658 & 30.48417 \\
\hline NTH2 & YBR001C & 1 & 13 & 3 & 1.72782 & 41.1063 \\
\hline \multirow[t]{3}{*}{ OSH1 } & YAR044W & 1 & 13 & 4 & -0.84182 & 46.68489 \\
\hline & YBL107C & 1 & 13 & 5 & \#DIV/O! & 44.07464 \\
\hline & YAR043C & 1 & 13 & 6 & 18.67683 & 36.97685 \\
\hline SRO77 & YBL106C & 1 & 13 & 7 & \#DIV/0! & 48.66437 \\
\hline SWH1 & YAR042W & 1 & 13 & 8 & 20.59303 & 51.02876 \\
\hline
\end{tabular}




\begin{tabular}{|c|c|c|c|c|c|c|}
\hline & YBL104C & 1 & 13 & 9 & 17.06596 & 38.59321 \\
\hline & YAR040C & 1 & 13 & 10 & 15.59577 & 43.51871 \\
\hline \multirow[t]{2}{*}{ RTG3 } & YBL103C & 1 & 13 & 11 & 15.33704 & 19.45455 \\
\hline & YAR037W & 1 & 13 & 12 & 15.24088 & 23.02449 \\
\hline SFT2 & YBL102W & 1 & 13 & 13 & 5.995412 & 41.39472 \\
\hline YAT1 & YAR035W & 1 & 13 & 14 & 21.08294 & 22.56661 \\
\hline ECM21 & YBL101C & 1 & 13 & 15 & 34.05883 & 41.94115 \\
\hline \multirow[t]{3}{*}{ PRM9 } & YAR031W & 1 & 13 & 16 & \#DIV/0! & 51.07804 \\
\hline & YBL100C & 1 & 13 & 17 & \#DIV/0! & 38.5716 \\
\hline & YAR030C & 1 & 13 & 18 & 18.30806 & 47.24017 \\
\hline \multirow[t]{5}{*}{ BNA4 } & YBL098W & 1 & 13 & 19 & 11.61919 & 52.35325 \\
\hline & YAR029W & 1 & 13 & 20 & 17.2655 & 46.50227 \\
\hline & YBL096C & 1 & 13 & 21 & 21.49198 & 53.37714 \\
\hline & YAR028W & 1 & 13 & 22 & 16.74757 & 45.1443 \\
\hline & YBL095W & 1 & 13 & 23 & 20.99781 & 43.78964 \\
\hline HIS3 & YOR202W & 1 & 13 & 24 & 5.103767 & \#DIV/O! \\
\hline HIS3 & YOR202W & 1 & 14 & 1 & 33.62272 & \#DIV/0! \\
\hline GRS1 & YBR121C & 1 & 14 & 2 & -0.21244 & 23.96414 \\
\hline TDP1 & YBR223C & 1 & 14 & 3 & 35.66962 & 41.01239 \\
\hline MUD1 & YBR119W & 1 & 14 & 4 & 31.02962 & 34.12149 \\
\hline \multirow[t]{2}{*}{ PCS60 } & YBR222C & 1 & 14 & 5 & 12.58208 & 25.04367 \\
\hline & YBR116C & 1 & 14 & 6 & 32.2141 & 39.9037 \\
\hline PDB1 & YBR221C & 1 & 14 & 7 & 29.92838 & 53.73983 \\
\hline \multirow[t]{2}{*}{ LYS2 } & YBR115C & 1 & 14 & 8 & 20.8141 & 35.90924 \\
\hline & YBR220C & 1 & 14 & 9 & 4.014024 & 39.22125 \\
\hline \multirow[t]{3}{*}{ RAD16 } & YBR114W & 1 & 14 & 10 & 14.96979 & 47.58635 \\
\hline & YBR219C & 1 & 14 & 11 & 10.15824 & 38.00982 \\
\hline & YBR113W & 1 & 14 & 12 & -2.65293 & 19.6293 \\
\hline PYC2 & YBR218C & 1 & 14 & 13 & 19.72733 & 42.18247 \\
\hline YSA1 & YBR111C & 1 & 14 & 14 & 16.43147 & 44.56894 \\
\hline \multirow[t]{2}{*}{ ATG12 } & YBR217W & 1 & 14 & 15 & 12.37093 & 37.32802 \\
\hline & YBR108W & 1 & 14 & 16 & \#DIV/0! & 47.92103 \\
\hline YBP1 & YBR216C & 1 & 14 & 17 & \#DIV/0! & 47.77915 \\
\hline IML3 & YBR107C & 1 & 14 & 18 & 23.99197 & 14.26191 \\
\hline HPC2 & YBR215W & 1 & 14 & 19 & 16.09829 & 47.17768 \\
\hline PHO88 & YBR106W & 1 & 14 & 20 & 14.77767 & 25.0788 \\
\hline SDS24 & YBR214W & 1 & 14 & 21 & 14.33603 & 50.56419 \\
\hline VID24 & YBR105C & 1 & 14 & 22 & -0.53921 & 37.033 \\
\hline MET8 & YBR213W & 1 & 14 & 23 & 42.05011 & 57.04445 \\
\hline HIS3 & YOR202W & 1 & 14 & 24 & -5.66844 & \#DIV/0! \\
\hline HIS3 & YOR202W & 1 & 15 & 1 & 3.845827 & \#DIV/0! \\
\hline \multirow[t]{2}{*}{$\mathrm{ACH} 1$} & YBL015W & 1 & 15 & 2 & 16.61236 & 38.93417 \\
\hline & YBR016W & 1 & 15 & 3 & 23.9481 & 46.34659 \\
\hline
\end{tabular}




\begin{tabular}{|c|c|c|c|c|c|c|}
\hline FMT1 & YBL013W & 1 & 15 & 4 & 3.0223 & 12.24314 \\
\hline MNN2 & YBR015C & 1 & 15 & 5 & \#DIV/0! & 43.59529 \\
\hline \multirow[t]{6}{*}{ SCT1 } & YBL011W & 1 & 15 & 6 & 8.328853 & \#DIV/0! \\
\hline & YBR014C & 1 & 15 & 7 & 26.74358 & \#DIV/0! \\
\hline & YBL010C & 1 & 15 & 8 & 17.77941 & 50.26636 \\
\hline & YBR013C & 1 & 15 & 9 & 12.34257 & 33.61807 \\
\hline & YBL009W & 1 & 15 & 10 & 31.74401 & 42.12058 \\
\hline & YBR012C & 1 & 15 & 11 & 16.70642 & 41.31622 \\
\hline HIR1 & YBL008W & 1 & 15 & 12 & 16.08351 & 50.30949 \\
\hline НHТ1 & YBR010W & 1 & 15 & 13 & 40.02875 & 38.97687 \\
\hline SLA1 & YBL007C & 1 & 15 & 14 & 88.50865 & 72.27051 \\
\hline HHF1 & YBR009C & 1 & 15 & 15 & -7.15078 & 34.7872 \\
\hline PDR3 & YBL005W & 1 & 15 & 16 & 11.92729 & 30.13005 \\
\hline FLR1 & YBR008C & 1 & 15 & 17 & 23.17911 & 35.16163 \\
\hline HTA2 & YBL003C & 1 & 15 & 18 & 13.2202 & 41.79188 \\
\hline DSF2 & YBR007C & 1 & 15 & 19 & 49.97662 & 54.67359 \\
\hline ECM15 & YBL001C & 1 & 15 & 20 & 35.01874 & 52.70864 \\
\hline UGA2 & YBR006W & 1 & 15 & 21 & 31.27211 & 47.46629 \\
\hline FLO1 & YAR050W & 1 & 15 & 22 & 10.58375 & 50.64246 \\
\hline RCR1 & YBR005W & 1 & 15 & 23 & 14.02771 & 42.54578 \\
\hline HIS3 & YOR202W & 1 & 15 & 24 & -2.74729 & \#DIV/0! \\
\hline HIS3 & YOR202W & 1 & 16 & 1 & 17.67762 & \#DIV/0! \\
\hline HIS3 & YOR202W & 1 & 16 & 2 & 20.59839 & \#DIV/0! \\
\hline HIS3 & YOR202W & 1 & 16 & 3 & 14.25478 & \#DIV/O! \\
\hline HIS3 & YOR202W & 1 & 16 & 4 & -14.1763 & \#DIV/0! \\
\hline HIS3 & YOR202W & 1 & 16 & 5 & \#DIV/0! & \#DIV/0! \\
\hline HIS3 & YOR202W & 1 & 16 & 6 & 7.049678 & \#DIV/0! \\
\hline HIS3 & YOR202W & 1 & 16 & 7 & -5.3782 & \#DIV/0! \\
\hline HIS3 & YOR202W & 1 & 16 & 8 & 12.94464 & \#DIV/0! \\
\hline HIS3 & YOR202W & 1 & 16 & 9 & 26.13047 & \#DIV/0! \\
\hline HIS3 & YOR202W & 1 & 16 & 10 & 30.06855 & \#DIV/0! \\
\hline HIS3 & YOR202W & 1 & 16 & 11 & 9.357009 & \#DIV/O! \\
\hline HIS3 & YOR202W & 1 & 16 & 12 & 12.4787 & \#DIV/0! \\
\hline HIS3 & YOR202W & 1 & 16 & 13 & 15.59897 & \#DIV/0! \\
\hline HIS3 & YOR202W & 1 & 16 & 14 & 31.56846 & \#DIV/O! \\
\hline HIS3 & YOR202W & 1 & 16 & 15 & \#DIV/0! & \#DIV/0! \\
\hline HIS3 & YOR202W & 1 & 16 & 16 & 19.98234 & \#DIV/0! \\
\hline HIS3 & YOR202W & 1 & 16 & 17 & 20.21798 & \#DIV/0! \\
\hline HIS3 & YOR202W & 1 & 16 & 18 & 15.08847 & \#DIV/O! \\
\hline HIS3 & YOR202W & 1 & 16 & 19 & 12.289 & \#DIV/0! \\
\hline HIS3 & YOR202W & 1 & 16 & 20 & -2.21998 & \#DIV/0! \\
\hline HIS3 & YOR202W & 1 & 16 & 21 & 3.245053 & \#DIV/0! \\
\hline HIS3 & YOR202W & 1 & 16 & 22 & 4.614257 & \#DIV/O! \\
\hline
\end{tabular}




\begin{tabular}{|c|c|c|c|c|c|c|}
\hline HIS3 & YOR202W & 1 & 16 & 23 & -9.2944 & \#DIV/0! \\
\hline HIS3 & YOR202W & 1 & 16 & 24 & 20.36035 & \#DIV/0! \\
\hline HIS3 & YOR202W & 3 & 1 & 1 & 62.59128 & \#DIV/0! \\
\hline HIS3 & YOR202W & 3 & 1 & 2 & 3.084673 & \#DIV/0! \\
\hline HIS3 & YOR202W & 3 & 1 & 3 & 11.80692 & \#DIV/0! \\
\hline HIS3 & YOR202W & 3 & 1 & 4 & 7.26562 & \#DIV/0! \\
\hline HIS3 & YOR202W & 3 & 1 & 5 & 2.211391 & \#DIV/0! \\
\hline HIS3 & YOR202W & 3 & 1 & 6 & -7.483 & \#DIV/O! \\
\hline HIS3 & YOR202W & 3 & 1 & 7 & 18.14248 & \#DIV/0! \\
\hline HIS3 & YOR202W & 3 & 1 & 8 & 12.95304 & \#DIV/0! \\
\hline HIS3 & YOR202W & 3 & 1 & 9 & 19.04429 & \#DIV/0! \\
\hline HIS3 & YOR202W & 3 & 1 & 10 & 12.97181 & \#DIV/0! \\
\hline HIS3 & YOR202W & 3 & 1 & 11 & 21.35916 & \#DIV/0! \\
\hline HIS3 & YOR202W & 3 & 1 & 12 & 28.55879 & \#DIV/O! \\
\hline HIS3 & YOR202W & 3 & 1 & 13 & 2.951219 & \#DIV/0! \\
\hline HIS3 & YOR202W & 3 & 1 & 14 & 23.71495 & \#DIV/0! \\
\hline HIS3 & YOR202W & 3 & 1 & 15 & 10.55482 & \#DIV/0! \\
\hline HIS3 & YOR202W & 3 & 1 & 16 & 34.32457 & \#DIV/0! \\
\hline HIS3 & YOR202W & 3 & 1 & 17 & 6.256505 & \#DIV/0! \\
\hline HIS3 & YOR202W & 3 & 1 & 18 & 7.079899 & \#DIV/O! \\
\hline HIS3 & YOR202W & 3 & 1 & 19 & 11.44934 & \#DIV/0! \\
\hline HIS3 & YOR202W & 3 & 1 & 20 & 4.222856 & \#DIV/0! \\
\hline HIS3 & YOR202W & 3 & 1 & 21 & 4.019112 & \#DIV/0! \\
\hline HIS3 & YOR202W & 3 & 1 & 22 & -2.089 & \#DIV/O! \\
\hline HIS3 & YOR202W & 3 & 1 & 23 & -6.17371 & \#DIV/0! \\
\hline HIS3 & YOR202W & 3 & 1 & 24 & -17.9897 & \#DIV/0! \\
\hline \multirow[t]{2}{*}{ HIS3 } & YOR202W & 3 & 2 & 1 & 37.10456 & \#DIV/0! \\
\hline & YDR199W & 3 & 2 & 2 & 22.85261 & 30.69547 \\
\hline \multirow[t]{2}{*}{ OMS1 } & YDR316W & 3 & 2 & 3 & 13.38031 & 35.75428 \\
\hline & YDR198C & 3 & 2 & 4 & 20.9195 & 40.01653 \\
\hline \multirow[t]{3}{*}{ IPK1 } & YDR315C & 3 & 2 & 5 & 30.39003 & 34.78934 \\
\hline & YDR193W & 3 & 2 & 6 & 23.76251 & 20.50271 \\
\hline & YDR314C & 3 & 2 & 7 & 28.95709 & 50.09361 \\
\hline NUP42 & YDR192C & 3 & 2 & 8 & 25.62311 & 29.15713 \\
\hline PIB1 & YDR313C & 3 & 2 & 9 & 60.44509 & 26.57986 \\
\hline HST4 & YDR191W & 3 & 2 & 10 & 32.43823 & 11.84673 \\
\hline \multirow[t]{2}{*}{ SSF2 } & YDR312W & 3 & 2 & 11 & 48.26358 & 42.79744 \\
\hline & YDR186C & 3 & 2 & 12 & 16.71318 & 51.99524 \\
\hline \multirow[t]{2}{*}{ SUM1 } & YDR310C & 3 & 2 & 13 & 29.21517 & 43.10226 \\
\hline & YDR185C & 3 & 2 & 14 & 11.84354 & 49.43692 \\
\hline GIC2 & YDR309C & 3 & 2 & 15 & 9.598124 & 47.19575 \\
\hline \multirow[t]{2}{*}{ ATC1 } & YDR184C & 3 & 2 & 16 & 23.80024 & 48.78625 \\
\hline & YDR307W & 3 & 2 & 17 & 7.097609 & 60.54249 \\
\hline
\end{tabular}




\begin{tabular}{|c|c|c|c|c|c|c|}
\hline \multirow[t]{2}{*}{ PLP1 } & YDR183W & 3 & 2 & 18 & 31.8117 & 49.87057 \\
\hline & YDR306C & 3 & 2 & 19 & 11.53547 & 49.15004 \\
\hline SAS4 & YDR181C & 3 & 2 & 20 & 33.85531 & 50.38985 \\
\hline \multirow[t]{3}{*}{ HNT2 } & YDR305C & 3 & 2 & 21 & 22.12061 & 31.32452 \\
\hline & YDR179W- & & & & & \\
\hline & $A$ & 3 & 2 & 22 & 25.99453 & 30.7884 \\
\hline CPR5 & YDR304C & 3 & 2 & 23 & 88.13559 & 58.16797 \\
\hline HIS3 & YOR202W & 3 & 2 & 24 & \#DIV/0! & \#DIV/0! \\
\hline \multirow[t]{3}{*}{ HIS3 } & YOR202W & 3 & 3 & 1 & 32.115 & \#DIV/0! \\
\hline & YDL199C & 3 & 3 & 2 & 45.10559 & 53.28751 \\
\hline & YDR090C & 3 & 3 & 3 & 18.94684 & 19.41452 \\
\hline \multirow[t]{2}{*}{ ASF2 } & YDL197C & 3 & 3 & 4 & 37.3363 & 50.14655 \\
\hline & YDR089W & 3 & 3 & 5 & 19.2417 & 36.37502 \\
\hline SNF3 & YDL194W & 3 & 3 & 6 & 32.72019 & 60.15176 \\
\hline AFR1 & YDR085C & 3 & 3 & 7 & 50.48491 & 27.45962 \\
\hline ARF1 & YDL192W & 3 & 3 & 8 & 12.03508 & 41.72932 \\
\hline TVP23 & YDR084C & 3 & 3 & 9 & 16.85002 & 39.57521 \\
\hline RPL35A & YDL191W & 3 & 3 & 10 & 23.55473 & 61.92526 \\
\hline RRP8 & YDR083W & 3 & 3 & 11 & 21.89648 & 55.75702 \\
\hline UFD2 & YDL190C & 3 & 3 & 12 & 16.0042 & 53.38863 \\
\hline VPS41 & YDR080W & 3 & 3 & 13 & 33.98429 & 67.65571 \\
\hline RBS1 & YDL189W & 3 & 3 & 14 & 13.60757 & 11.4716 \\
\hline SED1 & YDR077W & 3 & 3 & 15 & 15.786 & 60.95513 \\
\hline PPH22 & YDL188C & 3 & 3 & 16 & 24.15854 & 61.91308 \\
\hline \multirow[t]{2}{*}{ RAD55 } & YDR076W & 3 & 3 & 17 & 61.90502 & 58.79025 \\
\hline & YDL187C & 3 & 3 & 18 & 21.51364 & 64.91604 \\
\hline \multirow[t]{2}{*}{ PPH3 } & YDR075W & 3 & 3 & 19 & 15.83369 & 55.10702 \\
\hline & YDL186W & 3 & 3 & 20 & 19.90558 & 60.53031 \\
\hline TPS2 & YDR074W & 3 & 3 & 21 & \#DIV/O! & 45.45492 \\
\hline TFP1 & YDL185W & 3 & 3 & 22 & 24.06044 & 55.18745 \\
\hline SNF11 & YDR073W & 3 & 3 & 23 & 8.660164 & 41.49773 \\
\hline HIS3 & YOR202W & 3 & 3 & 24 & -9.26073 & \#DIV/0! \\
\hline HIS3 & YOR202W & 3 & 4 & 1 & 43.76751 & \#DIV/O! \\
\hline ADR1 & YDR216W & 3 & 4 & 2 & 21.92495 & 22.08503 \\
\hline \multirow[t]{2}{*}{ MSN5 } & YDR335W & 3 & 4 & 3 & 78.4881 & 66.46933 \\
\hline & YDR215C & 3 & 4 & 4 & 32.11992 & 31.69425 \\
\hline SWR1 & YDR334W & 3 & 4 & 5 & 17.04068 & 47.469 \\
\hline \multirow[t]{2}{*}{ AHA1 } & YDR214W & 3 & 4 & 6 & 11.87147 & 25.75834 \\
\hline & YDR333C & 3 & 4 & 7 & 41.11907 & 30.48272 \\
\hline \multirow[t]{3}{*}{ UPC2 } & YDR213W & 3 & 4 & 8 & 15.95218 & 51.38671 \\
\hline & YDR332W & 3 & 4 & 9 & 11.19563 & 36.36685 \\
\hline & YDR210W & 3 & 4 & 10 & 53.76282 & 31.9691 \\
\hline UBX5 & YDR330W & 3 & 4 & 11 & 32.25114 & 54.40772 \\
\hline
\end{tabular}




\begin{tabular}{|c|c|c|c|c|c|c|}
\hline & YDR209C & 3 & 4 & 12 & 22.44391 & 57.87755 \\
\hline PEX3 & YDR329C & 3 & 4 & 13 & 6.992011 & 42.07841 \\
\hline UME6 & YDR207C & 3 & 4 & 14 & 40.12917 & 44.69466 \\
\hline ASP1 & YDR321W & 3 & 4 & 15 & 53.18383 & 60.40874 \\
\hline EBS1 & YDR206W & 3 & 4 & 16 & 55.74098 & 63.9272 \\
\hline SWA2 & YDR320C & 3 & 4 & 17 & 26.7277 & 56.25499 \\
\hline \multirow[t]{3}{*}{ MSC2 } & YDR205W & 3 & 4 & 18 & 46.38874 & 62.02884 \\
\hline & YDR319C & 3 & 4 & 19 & 22.71912 & 42.59032 \\
\hline & YDR203W & 3 & 4 & 20 & 14.11822 & 45.01419 \\
\hline MCM21 & YDR318W & 3 & 4 & 21 & 15.78399 & 54.49936 \\
\hline \multirow[t]{2}{*}{ RAV2 } & YDR202C & 3 & 4 & 22 & 25.21191 & 48.39028 \\
\hline & YDR317W & 3 & 4 & 23 & 62.22349 & 60.39059 \\
\hline HIS3 & YOR202W & 3 & 4 & 24 & \#DIV/0! & \#DIV/O! \\
\hline HIS3 & YOR202W & 3 & 5 & 1 & 28.64989 & \#DIV/0! \\
\hline \multirow[t]{2}{*}{ RRI1 } & YDL216C & 3 & 5 & 2 & 22.66316 & 50.33794 \\
\hline & YDR102C & 3 & 5 & 3 & 23.088 & 44.87101 \\
\hline GDH2 & YDL215C & 3 & 5 & 4 & 20.10311 & 24.91685 \\
\hline ARX1 & YDR101C & 3 & 5 & 5 & 16.54219 & 31.52896 \\
\hline PRR2 & YDL214C & 3 & 5 & 6 & 26.2862 & 35.21229 \\
\hline TVP15 & YDR100W & 3 & 5 & 7 & 15.34787 & 51.49365 \\
\hline NOP6 & YDL213C & 3 & 5 & 8 & 19.63536 & 42.91776 \\
\hline \multirow[t]{2}{*}{ BMH2 } & YDR099W & 3 & 5 & 9 & 37.361 & 31.38281 \\
\hline & YDL211C & 3 & 5 & 10 & 31.97351 & 68.86433 \\
\hline GRX3 & YDR098C & 3 & 5 & 11 & 18.75 & 40.14024 \\
\hline UGA4 & YDL210W & 3 & 5 & 12 & 9.181527 & 56.22473 \\
\hline \multirow[t]{2}{*}{ MSH6 } & YDR097C & 3 & 5 & 13 & 23.50601 & 33.22011 \\
\hline & YDL206W & 3 & 5 & 14 & 18.56841 & 69.64451 \\
\hline GIS1 & YDR096W & 3 & 5 & 15 & 14.86268 & 13.43769 \\
\hline \multirow[t]{4}{*}{ RTN2 } & YDL204W & 3 & 5 & 16 & 13.11419 & 62.33266 \\
\hline & YDR095C & 3 & 5 & 17 & 31.48545 & 58.20077 \\
\hline & YDL203C & 3 & 5 & 18 & 24.78972 & 42.33964 \\
\hline & YDR094W & 3 & 5 & 19 & 28.50849 & 66.13817 \\
\hline TRM8 & YDL201W & 3 & 5 & 20 & 16.86633 & 56.39381 \\
\hline DNF2 & YDR093W & 3 & 5 & 21 & 13.89465 & 39.23435 \\
\hline MGT1 & YDL200C & 3 & 5 & 22 & 60.83935 & 61.82308 \\
\hline UBC13 & YDR092W & 3 & 5 & 23 & 43.85849 & 60.89474 \\
\hline HIS3 & YOR202W & 3 & 5 & 24 & 7.992485 & \#DIV/0! \\
\hline HIS3 & YOR202W & 3 & 6 & 1 & 19.95646 & \#DIV/0! \\
\hline RTN1 & YDR233C & 3 & 6 & 2 & 34.25896 & 44.07066 \\
\hline TRP4 & YDR354W & 3 & 6 & 3 & 47.73227 & 26.86887 \\
\hline \multirow[t]{2}{*}{ IVY1 } & YDR229W & 3 & 6 & 4 & 41.97109 & 45.3783 \\
\hline & YDR352W & 3 & 6 & 5 & 38.81054 & 45.45775 \\
\hline SIR4 & YDR227W & 3 & 6 & 6 & 35.72056 & 56.17133 \\
\hline
\end{tabular}




\begin{tabular}{|c|c|c|c|c|c|c|}
\hline SBE2 & YDR351W & 3 & 6 & 7 & 39.46438 & 56.50129 \\
\hline HTA1 & YDR225W & 3 & 6 & 8 & 13.80454 & 31.8986 \\
\hline YPS7 & YDR349C & 3 & 6 & 9 & 12.27713 & 38.46711 \\
\hline \multirow[t]{3}{*}{ CRF1 } & YDR223W & 3 & 6 & 10 & 12.36197 & 37.84848 \\
\hline & YDR348C & 3 & 6 & 11 & 22.55576 & 57.85635 \\
\hline & YDR222W & 3 & 6 & 12 & 33.096 & 58.88009 \\
\hline \multirow[t]{2}{*}{ SVF1 } & YDR346C & 3 & 6 & 13 & 15.46036 & 46.51773 \\
\hline & YDR221W & 3 & 6 & 14 & 33.60774 & 68.85277 \\
\hline \multirow[t]{5}{*}{ HXT3 } & YDR345C & 3 & 6 & 15 & 27.26217 & 55.04742 \\
\hline & YDR220C & 3 & 6 & 16 & 15.14538 & 67.68572 \\
\hline & YDR344C & 3 & 6 & 17 & 83.05831 & 45.32327 \\
\hline & YDR219C & 3 & 6 & 18 & 18.47375 & 23.31813 \\
\hline & YDR340W & 3 & 6 & 19 & 15.17619 & 51.71359 \\
\hline \multirow[t]{2}{*}{ SPR28 } & YDR218C & 3 & 6 & 20 & 14.51314 & 33.62685 \\
\hline & YDR338C & 3 & 6 & 21 & 19.32206 & 54.41752 \\
\hline \multirow[t]{2}{*}{ RAD9 } & YDR217C & 3 & 6 & 22 & 29.80897 & 25.66198 \\
\hline & YDR336W & 3 & 6 & 23 & 32.45228 & 19.6795 \\
\hline HIS3 & YOR202W & 3 & 6 & 24 & 20.46442 & \#DIV/0! \\
\hline HIS3 & YOR202W & 3 & 7 & 1 & 23.40384 & \#DIV/0! \\
\hline OST4 & YDL232W & 3 & 7 & 2 & 41.39422 & -3.56201 \\
\hline RBF64 & YDR117C & 3 & 7 & 3 & 19.96075 & 56.55521 \\
\hline BRE4 & YDL231C & 3 & 7 & 4 & 29.36612 & 29.59299 \\
\hline MRPL1 & YDR116C & 3 & 7 & 5 & 37.90266 & 48.30797 \\
\hline \multirow[t]{2}{*}{ PTP1 } & YDL230W & 3 & 7 & 6 & 21.98909 & 43.17433 \\
\hline & YDR112W & 3 & 7 & 7 & 24.14955 & 60.73219 \\
\hline SSB1 & YDL229W & 3 & 7 & 8 & 29.37227 & 33.33234 \\
\hline ALT2 & YDR111C & 3 & 7 & 9 & 35.22032 & 47.43898 \\
\hline $\mathrm{HO}$ & YDL227C & 3 & 7 & 10 & 37.9998 & 55.32622 \\
\hline FOB1 & YDR110W & 3 & 7 & 11 & 5.192641 & 48.07159 \\
\hline GCS1 & YDL226C & 3 & 7 & 12 & 82.19847 & 89.25583 \\
\hline STE5 & YDR103W & 3 & 7 & 13 & 20.71745 & 69.62351 \\
\hline \multirow[t]{2}{*}{ WHI4 } & YDL224C & 3 & 7 & 14 & 17.45446 & 60.27318 \\
\hline & YDR109C & 3 & 7 & 15 & 13.69657 & 66.25113 \\
\hline HBT1 & YDL223C & 3 & 7 & 16 & 35.19497 & 75.17536 \\
\hline GSG1 & YDR108W & 3 & 7 & 17 & 9.905566 & 59.14178 \\
\hline \multirow[t]{2}{*}{ FMP45 } & YDL222C & 3 & 7 & 18 & 25.40323 & 60.57434 \\
\hline & YDR107C & 3 & 7 & 19 & -3.00216 & 57.80536 \\
\hline DTD1 & YDL219W & 3 & 7 & 20 & 26.09371 & 55.21034 \\
\hline \multirow[t]{2}{*}{ TMS1 } & YDR105C & 3 & 7 & 21 & 11.89632 & 60.54784 \\
\hline & YDL218W & 3 & 7 & 22 & 12.10015 & 62.82533 \\
\hline SPO71 & YDR104C & 3 & 7 & 23 & 30.74965 & 8.402971 \\
\hline HIS3 & YOR202W & 3 & 7 & 24 & 34.49274 & \#DIV/0! \\
\hline HIS3 & YOR202W & 3 & 8 & 1 & 28.46632 & \#DIV/0! \\
\hline
\end{tabular}




\begin{tabular}{|c|c|c|c|c|c|c|}
\hline BTT1 & YDR252W & 3 & 8 & 2 & 40.38154 & 47.53969 \\
\hline VPS74 & YDR372C & 3 & 8 & 3 & 41.67209 & 53.92004 \\
\hline PAM1 & YDR251W & 3 & 8 & 4 & 30.67209 & 42.68788 \\
\hline \multirow[t]{4}{*}{ CTS2 } & YDR371W & 3 & 8 & 5 & 20.49269 & \#DIV/0! \\
\hline & YDR250C & 3 & 8 & 6 & 28.66761 & \#DIV/0! \\
\hline & YDR370C & 3 & 8 & 7 & 38.14608 & 45.931 \\
\hline & YDR249C & 3 & 8 & 8 & 44.5957 & 60.00976 \\
\hline \multirow[t]{2}{*}{ XRS2 } & YDR369C & 3 & 8 & 9 & 21.98543 & 49.14661 \\
\hline & YDR248C & 3 & 8 & 10 & 21.88597 & 50.6236 \\
\hline YPR1 & YDR368W & 3 & 8 & 11 & 34.30717 & 44.61521 \\
\hline \multirow[t]{2}{*}{ VHS1 } & YDR247W & 3 & 8 & 12 & 55.84672 & 49.51755 \\
\hline & YDR363W- & & & & & \\
\hline SEM1 & $A$ & 3 & 8 & 13 & 61.96709 & -7.3304 \\
\hline MNN10 & YDR245W & 3 & 8 & 14 & 42.62309 & 60.53972 \\
\hline ESC2 & YDR363W & 3 & 8 & 15 & 67.20645 & 67.89652 \\
\hline \multirow[t]{2}{*}{ PEX5 } & YDR244W & 3 & 8 & 16 & 35.08231 & 67.73436 \\
\hline & YDR360W & 3 & 8 & 17 & 13.16597 & 59.8904 \\
\hline BUD26 & YDR241W & 3 & 8 & 18 & 15.38036 & 51.15623 \\
\hline \multirow[t]{2}{*}{ VID21 } & YDR359C & 3 & 8 & 19 & 86.03116 & 43.49648 \\
\hline & YDR239C & 3 & 8 & 20 & 30.56341 & 38.95089 \\
\hline GGA1 & YDR358W & 3 & 8 & 21 & 21.97963 & 33.12749 \\
\hline \multirow[t]{2}{*}{ LYS4 } & YDR234W & 3 & 8 & 22 & 7.822341 & 32.61036 \\
\hline & YDR357C & 3 & 8 & 23 & 25.70333 & 42.51662 \\
\hline HIS3 & YOR202W & 3 & 8 & 24 & 15.64483 & \#DIV/0! \\
\hline HIS3 & YOR202W & 3 & 9 & 1 & 22.38103 & \#DIV/0! \\
\hline NTH1 & YDR001C & 3 & 9 & 2 & 33.87709 & 53.19736 \\
\hline FIN1 & YDR130C & 3 & 9 & 3 & 20.59028 & 28.37531 \\
\hline \multirow[t]{3}{*}{ AAD4 } & YDL243C & 3 & 9 & 4 & 30.96323 & 53.95016 \\
\hline & YDR128W & 3 & 9 & 5 & 35.81638 & 52.3088 \\
\hline & YDL242W & 3 & 9 & 6 & 31.31738 & 51.05396 \\
\hline \multirow[t]{2}{*}{ ARO1 } & YDR127W & 3 & 9 & 7 & 61.7155 & 50.9294 \\
\hline & YDL241W & 3 & 9 & 8 & 40.49679 & 47.47541 \\
\hline SWF1 & YDR126W & 3 & 9 & 9 & \#DIV/0! & 49.96709 \\
\hline LRG1 & YDL240W & 3 & 9 & 10 & 15.68237 & 34.45309 \\
\hline ECM18 & YDR125C & 3 & 9 & 11 & 29.14514 & 38.77601 \\
\hline \multirow[t]{2}{*}{ ADY3 } & YDL239C & 3 & 9 & 12 & 17.83809 & 36.66303 \\
\hline & YDR124W & 3 & 9 & 13 & -3.23934 & 65.91323 \\
\hline GUD1 & YDL238C & 3 & 9 & 14 & 7.038854 & 45.33443 \\
\hline \multirow[t]{2}{*}{ INO2 } & YDR123C & 3 & 9 & 15 & 44.14504 & 43.75477 \\
\hline & YDL237W & 3 & 9 & 16 & 71.76192 & 44.52147 \\
\hline KIN1 & YDR122W & 3 & 9 & 17 & 24.71606 & 46.59664 \\
\hline PHO13 & YDL236W & 3 & 9 & 18 & 69.26109 & 66.19127 \\
\hline DPB4 & YDR121W & 3 & 9 & 19 & 27.72634 & 63.89731 \\
\hline
\end{tabular}




\begin{tabular}{|c|c|c|c|c|c|c|}
\hline GYP7 & YDL234C & 3 & 9 & 20 & 27.38971 & 65.24803 \\
\hline \multirow[t]{3}{*}{ TRM1 } & YDR120C & 3 & 9 & 21 & 15.01682 & 49.92822 \\
\hline & YDL233W & 3 & 9 & 22 & 8.027933 & 52.5285 \\
\hline & YDR119W & 3 & 9 & 23 & 30.31339 & 36.80206 \\
\hline HIS3 & YOR202W & 3 & 9 & 24 & 13.50496 & \#DIV/0! \\
\hline HIS3 & YOR202W & 3 & 10 & 1 & 3.542973 & \#DIV/0! \\
\hline \multirow[t]{3}{*}{ DIN7 } & YDR263C & 3 & 10 & 2 & 24.77834 & 51.66734 \\
\hline & YDR387C & 3 & 10 & 3 & 21.5024 & 60.06822 \\
\hline & YDR262W & 3 & 10 & 4 & 39.91445 & 51.27846 \\
\hline MUS81 & YDR386W & 3 & 10 & 5 & 31.2968 & 61.55749 \\
\hline EXG2 & YDR261C & 3 & 10 & 6 & 63.62918 & 48.33578 \\
\hline EFT2 & YDR385W & 3 & 10 & 7 & 10.43699 & 43.1677 \\
\hline SWM1 & YDR260C & 3 & 10 & 8 & 33.86588 & 52.52711 \\
\hline АTO3 & YDR384C & 3 & 10 & 9 & 8.016421 & 58.41571 \\
\hline YAP6 & YDR259C & 3 & 10 & 10 & 20.47526 & 40.57477 \\
\hline NKP1 & YDR383C & 3 & 10 & 11 & 43.61307 & 60.5914 \\
\hline HSP78 & YDR258C & 3 & 10 & 12 & 43.96857 & 57.78874 \\
\hline RPP2B & YDR382W & 3 & 10 & 13 & 38.33384 & 65.69937 \\
\hline SET7 & YDR257C & 3 & 10 & 14 & 24.50098 & 55.48248 \\
\hline AR010 & YDR380W & 3 & 10 & 15 & 26.01809 & 45.35058 \\
\hline CTA1 & YDR256C & 3 & 10 & 16 & 3.722149 & 62.84159 \\
\hline RGA2 & YDR379W & 3 & 10 & 17 & 16.5098 & 52.32028 \\
\hline RMD5 & YDR255C & 3 & 10 & 18 & \#DIV/0! & 44.44848 \\
\hline LSM6 & YDR378C & 3 & 10 & 19 & 82.76534 & 83.03797 \\
\hline $\mathrm{CHL} 4$ & YDR254W & 3 & 10 & 20 & 26.53183 & 62.52408 \\
\hline BCS1 & YDR375C & 3 & 10 & 21 & \#DIV/0! & 36.33882 \\
\hline \multirow[t]{2}{*}{ MET32 } & YDR253C & 3 & 10 & 22 & 23.82007 & 63.85464 \\
\hline & YDR374C & 3 & 10 & 23 & 31.02606 & 25.1706 \\
\hline HIS3 & YOR202W & 3 & 10 & 24 & 9.539923 & \#DIV/0! \\
\hline \multirow[t]{2}{*}{ HIS3 } & YOR202W & 3 & 11 & 1 & 10.81366 & \#DIV/0! \\
\hline & YDR015C & 3 & 11 & 2 & 40.74143 & 42.03404 \\
\hline EKI1 & YDR147W & 3 & 11 & 3 & 47.82024 & 50.26738 \\
\hline RAD61 & YDR014W & 3 & 11 & 4 & 16.93756 & 52.25766 \\
\hline SWI5 & YDR146C & 3 & 11 & 5 & 15.6541 & 41.00834 \\
\hline SNQ2 & YDR011W & 3 & 11 & 6 & 22.18699 & 52.26987 \\
\hline \multirow[t]{2}{*}{ MKC7 } & YDR144C & 3 & 11 & 7 & 41.21736 & 60.96221 \\
\hline & YDR010C & 3 & 11 & 8 & 73.62313 & 74.48038 \\
\hline SAN1 & YDR143C & 3 & 11 & 9 & 23.31505 & 45.33738 \\
\hline GAL3 & YDR009W & 3 & 11 & 10 & 18.10477 & 40.56334 \\
\hline \multirow[t]{2}{*}{ PEX7 } & YDR142C & 3 & 11 & 11 & 0.77856 & 54.79971 \\
\hline & YDR008C & 3 & 11 & 12 & 11.12943 & 37.94375 \\
\hline RUB1 & YDR139C & 3 & 11 & 13 & 25.4871 & 62.38774 \\
\hline TRP1 & YDR007W & 3 & 11 & 14 & 24.84341 & 60.42563 \\
\hline
\end{tabular}




\begin{tabular}{|c|c|c|c|c|c|c|}
\hline YCF1 & YDR135C & 3 & 11 & 15 & 25.6055 & 30.41028 \\
\hline \multirow[t]{2}{*}{ SOK1 } & YDR006C & 3 & 11 & 16 & 61.97476 & 60.47612 \\
\hline & YDR134C & 3 & 11 & 17 & 11.62956 & 47.73324 \\
\hline \multirow[t]{2}{*}{ MAF1 } & YDR005C & 3 & 11 & 18 & 24.28284 & 55.3068 \\
\hline & YDR133C & 3 & 11 & 19 & 9.426679 & -16.4948 \\
\hline \multirow[t]{2}{*}{ RAD57 } & YDR004W & 3 & 11 & 20 & 39.50667 & 60.44283 \\
\hline & YDR132C & 3 & 11 & 21 & 23.67569 & 61.07637 \\
\hline \multirow[t]{2}{*}{ RCR2 } & YDR003W & 3 & 11 & 22 & 17.88228 & 61.80492 \\
\hline & YDR131C & 3 & 11 & 23 & 61.73303 & 41.46238 \\
\hline HIS3 & YOR202W & 3 & 11 & 24 & 50.22415 & \#DIV/0! \\
\hline HIS3 & YOR202W & 3 & 12 & 1 & 5.042267 & \#DIV/0! \\
\hline RNH2O2 & YDR279W & 3 & 12 & 2 & 41.89186 & 40.95026 \\
\hline \multirow[t]{2}{*}{ DIT1 } & YDR403W & 3 & 12 & 3 & 45.71934 & 48.50231 \\
\hline & YDR278C & 3 & 12 & 4 & 21.97566 & 26.19048 \\
\hline DIT2 & YDR402C & 3 & 12 & 5 & 41.62049 & 42.84753 \\
\hline \multirow[t]{2}{*}{ MTH1 } & YDR277C & 3 & 12 & 6 & 12.47581 & 57.21838 \\
\hline & YDR401W & 3 & 12 & 7 & 27.32191 & 57.87476 \\
\hline PMP3 & YDR276C & 3 & 12 & 8 & 23.52626 & 51.73225 \\
\hline URH1 & YDR400W & 3 & 12 & 9 & 12.43781 & 59.99561 \\
\hline BSC2 & YDR275W & 3 & 12 & 10 & 4.871824 & 64.55635 \\
\hline \multirow[t]{2}{*}{ HPT1 } & YDR399W & 3 & 12 & 11 & 26.59633 & 50.49878 \\
\hline & YDR274C & 3 & 12 & 12 & 94.82759 & 54.86533 \\
\hline SXM1 & YDR395W & 3 & 12 & 13 & 19.17554 & 44.04389 \\
\hline DON1 & YDR273W & 3 & 12 & 14 & 16.89534 & 59.6131 \\
\hline SHE9 & YDR393W & 3 & 12 & 15 & 23.86908 & 58.82707 \\
\hline GLO2 & YDR272W & 3 & 12 & 16 & 13.91917 & 56.75559 \\
\hline SPT3 & YDR392W & 3 & 12 & 17 & 26.97017 & 34.96886 \\
\hline \multirow[t]{3}{*}{ CCC2 } & YDR270W & 3 & 12 & 18 & 44.63412 & 51.48148 \\
\hline & YDR391C & 3 & 12 & 19 & 20.46042 & 58.78261 \\
\hline & YDR266C & 3 & 12 & 20 & 68.91361 & 53.49806 \\
\hline SAC7 & YDR389W & 3 & 12 & 21 & 62.8511 & 74.30804 \\
\hline PEX10 & YDR265W & 3 & 12 & 22 & 25.77547 & 35.1709 \\
\hline RVS167 & YDR388W & 3 & 12 & 23 & 87.4883 & 87.80285 \\
\hline HIS3 & YOR202W & 3 & 12 & 24 & -14.2129 & \#DIV/0! \\
\hline \multirow[t]{2}{*}{ HIS3 } & YOR202W & 3 & 13 & 1 & 8.111555 & \#DIV/0! \\
\hline & YDR056C & 3 & 13 & 2 & 12.54408 & 55.01831 \\
\hline HOM2 & YDR158W & 3 & 13 & 3 & 47.44952 & 47.93911 \\
\hline \multirow[t]{3}{*}{ PST1 } & YDR055W & 3 & 13 & 4 & 14.99744 & 47.53904 \\
\hline & YDR157W & 3 & 13 & 5 & 16.36508 & 61.52711 \\
\hline & YDR051C & 3 & 13 & 6 & 64.79809 & 58.5696 \\
\hline \multirow[t]{2}{*}{ RPA14 } & YDR156W & 3 & 13 & 7 & 64.38951 & 59.93964 \\
\hline & YDR049W & 3 & 13 & 8 & 69.42035 & 75.05184 \\
\hline CPR1 & YDR155C & 3 & 13 & 9 & 10.1873 & 40.22905 \\
\hline
\end{tabular}




\begin{tabular}{|c|c|c|c|c|c|c|}
\hline & YDR048C & 3 & 13 & 10 & 14.9545 & 61.59713 \\
\hline & YDR154C & 3 & 13 & 11 & 12.50088 & 68.16124 \\
\hline RPS11A & YDR025W & 3 & 13 & 12 & 66.13534 & 73.05511 \\
\hline ENT5 & YDR153C & 3 & 13 & 13 & 22.77674 & 69.32912 \\
\hline FYV1 & YDR024W & 3 & 13 & 14 & 23.19325 & 59.30485 \\
\hline GIR2 & YDR152W & 3 & 13 & 15 & 15.01849 & 63.02979 \\
\hline CIS1 & YDR022C & 3 & 13 & 16 & 18.74589 & 46.55619 \\
\hline \multirow[t]{2}{*}{ CTH1 } & YDR151C & 3 & 13 & 17 & 20.47756 & 66.96762 \\
\hline & YDR020C & 3 & 13 & 18 & 13.37332 & 31.91545 \\
\hline NUM1 & YDR150W & 3 & 13 & 19 & 46.79751 & 55.15311 \\
\hline \multirow[t]{3}{*}{ GCV1 } & YDR019C & 3 & 13 & 20 & 48.17554 & 73.57228 \\
\hline & YDR149C & 3 & 13 & 21 & 63.3572 & 73.60735 \\
\hline & YDR018C & 3 & 13 & 22 & 19.52796 & 58.54768 \\
\hline KGD2 & YDR148C & 3 & 13 & 23 & 29.79481 & 43.92628 \\
\hline HIS3 & YOR202W & 3 & 13 & 24 & 32.70699 & \#DIV/0! \\
\hline HIS3 & YOR202W & 3 & 14 & 1 & 1.426333 & \#DIV/0! \\
\hline SUR2 & YDR297W & 3 & 14 & 2 & 51.69177 & 37.7286 \\
\hline SIP1 & YDR422C & 3 & 14 & 3 & 31.10041 & 45.75483 \\
\hline DPL1 & YDR294C & 3 & 14 & 4 & 23.71585 & 48.69587 \\
\hline AR080 & YDR421W & 3 & 14 & 5 & 21.8067 & 25.60958 \\
\hline SSD1 & YDR293C & 3 & 14 & 6 & 36.20755 & 64.00744 \\
\hline \multirow[t]{2}{*}{ HKR1 } & YDR420W & 3 & 14 & 7 & 21.28528 & 50.18576 \\
\hline & YDR291W & 3 & 14 & 8 & 61.37172 & 68.32257 \\
\hline RAD30 & YDR419W & 3 & 14 & 9 & 61.10185 & 54.97303 \\
\hline \multirow[t]{3}{*}{ RTT103 } & YDR289C & 3 & 14 & 10 & 88.69965 & 79.95029 \\
\hline & YDR415C & 3 & 14 & 11 & 62.56278 & 63.26389 \\
\hline & YDR287W & 3 & 14 & 12 & 46.69506 & 68.35408 \\
\hline \multirow[t]{2}{*}{ ERD1 } & YDR414C & 3 & 14 & 13 & 25.43062 & 33.5311 \\
\hline & YDR286C & 3 & 14 & 14 & 60.82399 & 62.51505 \\
\hline DFM1 & YDR411C & 3 & 14 & 15 & 24.5398 & 57.3082 \\
\hline ZIP1 & YDR285W & 3 & 14 & 16 & 21.57855 & 56.22519 \\
\hline STE14 & YDR410C & 3 & 14 & 17 & 61.39284 & 78.1405 \\
\hline DPP1 & YDR284C & 3 & 14 & 18 & 18.66243 & 59.15456 \\
\hline \multirow[t]{2}{*}{ SIZ1 } & YDR409W & 3 & 14 & 19 & 28.60431 & 57.00218 \\
\hline & YDR282C & 3 & 14 & 20 & 25.98596 & 47.9177 \\
\hline ADE8 & YDR408C & 3 & 14 & 21 & 93.49845 & 73.32237 \\
\hline PHM6 & YDR281C & 3 & 14 & 22 & 23.24783 & 65.69248 \\
\hline PDR15 & YDR406W & 3 & 14 & 23 & 3.914169 & 49.56 \\
\hline HIS3 & YOR202W & 3 & 14 & 24 & 17.73762 & \#DIV/0! \\
\hline HIS3 & YOR202W & 3 & 15 & 1 & 37.83594 & \#DIV/0! \\
\hline IPT1 & YDR072C & 3 & 15 & 2 & 34.44669 & 31.77171 \\
\hline CSN9 & YDR179C & 3 & 15 & 3 & 11.96189 & 47.14132 \\
\hline PAA1 & YDR071C & 3 & 15 & 4 & 61.0168 & 52.86219 \\
\hline
\end{tabular}




\begin{tabular}{|c|c|c|c|c|c|c|}
\hline SDH4 & YDR178W & 3 & 15 & 5 & 16.5046 & 41.0199 \\
\hline FMP16 & YDR070C & 3 & 15 & 6 & 38.40583 & 49.06776 \\
\hline HMO1 & YDR174W & 3 & 15 & 7 & 77.38357 & 55.91312 \\
\hline DOS2 & YDR068W & 3 & 15 & 8 & 26.04243 & 61.46056 \\
\hline \multirow[t]{2}{*}{ ARG82 } & YDR173C & 3 & 15 & 9 & 49.63229 & 37.34648 \\
\hline & YDR067C & 3 & 15 & 10 & 12.12848 & 52.20762 \\
\hline \multirow[t]{2}{*}{ HSP42 } & YDR171W & 3 & 15 & 11 & 11.98983 & 48.0426 \\
\hline & YDR066C & 3 & 15 & 12 & 23.82258 & 60.05493 \\
\hline \multirow[t]{2}{*}{ STB3 } & YDR169C & 3 & 15 & 13 & 32.99331 & 62.96812 \\
\hline & YDR063W & 3 & 15 & 14 & 65.81048 & 71.40279 \\
\hline \multirow[t]{2}{*}{ TRM82 } & YDR165W & 3 & 15 & 15 & 46.47736 & 63.80161 \\
\hline & YDR061W & 3 & 15 & 16 & 62.49316 & 66.15842 \\
\hline CWC15 & YDR163W & 3 & 15 & 17 & 28.94607 & 44.29178 \\
\hline UBC5 & YDR059C & 3 & 15 & 18 & 30.18338 & 65.12579 \\
\hline NBP2 & YDR162C & 3 & 15 & 19 & 44.67517 & 66.7472 \\
\hline TGL2 & YDR058C & 3 & 15 & 20 & 43.47329 & 77.40789 \\
\hline TCl1 & YDR161W & 3 & 15 & 21 & 67.24482 & 56.69761 \\
\hline YOS9 & YDR057W & 3 & 15 & 22 & 20.21401 & 62.65046 \\
\hline SAC3 & YDR159W & 3 & 15 & 23 & 92.75362 & 30.39333 \\
\hline HIS3 & YOR202W & 3 & 15 & 24 & 29.33136 & \#DIV/0! \\
\hline HIS3 & YOR202W & 3 & 16 & 1 & 17.32597 & \#DIV/0! \\
\hline HIS3 & YOR202W & 3 & 16 & 2 & -18.3273 & \#DIV/0! \\
\hline HIS3 & YOR202W & 3 & 16 & 3 & 35.24306 & \#DIV/0! \\
\hline HIS3 & YOR202W & 3 & 16 & 4 & 45.62677 & \#DIV/0! \\
\hline HIS3 & YOR202W & 3 & 16 & 5 & 23.57691 & \#DIV/0! \\
\hline HIS3 & YOR202W & 3 & 16 & 6 & -1.34064 & \#DIV/0! \\
\hline HIS3 & YOR202W & 3 & 16 & 7 & -12.3456 & \#DIV/0! \\
\hline HIS3 & YOR202W & 3 & 16 & 8 & 9.679629 & \#DIV/0! \\
\hline HIS3 & YOR202W & 3 & 16 & 9 & 35.47287 & \#DIV/0! \\
\hline HIS3 & YOR202W & 3 & 16 & 10 & 10.1704 & \#DIV/0! \\
\hline HIS3 & YOR202W & 3 & 16 & 11 & 31.41082 & \#DIV/0! \\
\hline HIS3 & YOR202W & 3 & 16 & 12 & 31.98518 & \#DIV/0! \\
\hline HIS3 & YOR202W & 3 & 16 & 13 & 44.76845 & \#DIV/0! \\
\hline HIS3 & YOR202W & 3 & 16 & 14 & 33.16972 & \#DIV/0! \\
\hline HIS3 & YOR202W & 3 & 16 & 15 & 58.43176 & \#DIV/0! \\
\hline HIS3 & YOR202W & 3 & 16 & 16 & 18.23752 & \#DIV/0! \\
\hline HIS3 & YOR202W & 3 & 16 & 17 & -6.31583 & \#DIV/0! \\
\hline HIS3 & YOR202W & 3 & 16 & 18 & 80.60995 & \#DIV/0! \\
\hline HIS3 & YOR202W & 3 & 16 & 19 & 13.04756 & \#DIV/0! \\
\hline HIS3 & YOR202W & 3 & 16 & 20 & -2.45313 & \#DIV/0! \\
\hline HIS3 & YOR202W & 3 & 16 & 21 & 35.43347 & \#DIV/0! \\
\hline HIS3 & YOR202W & 3 & 16 & 22 & -24.8295 & \#DIV/0! \\
\hline HIS3 & YOR202W & 3 & 16 & 23 & 38.61555 & \#DIV/0! \\
\hline
\end{tabular}




\begin{tabular}{|c|c|c|c|c|c|c|}
\hline HIS3 & YOR202W & 3 & 16 & 24 & 21.74736 & \#DIV/0! \\
\hline HIS3 & YOR202W & 4 & 1 & 1 & 26.71394 & \#DIV/0! \\
\hline HIS3 & YOR202W & 4 & 1 & 2 & 38.05029 & \#DIV/0! \\
\hline HIS3 & YOR202W & 4 & 1 & 3 & 21.69743 & \#DIV/0! \\
\hline HIS3 & YOR202W & 4 & 1 & 4 & 27.79557 & \#DIV/0! \\
\hline HIS3 & YOR202W & 4 & 1 & 5 & 35.449 & \#DIV/0! \\
\hline HIS3 & YOR202W & 4 & 1 & 6 & 30.2189 & \#DIV/0! \\
\hline HIS3 & YOR202W & 4 & 1 & 7 & 20.04142 & \#DIV/0! \\
\hline HIS3 & YOR202W & 4 & 1 & 8 & 19.46996 & \#DIV/O! \\
\hline HIS3 & YOR202W & 4 & 1 & 9 & 38.9989 & \#DIV/0! \\
\hline HIS3 & YOR202W & 4 & 1 & 10 & 8.234969 & \#DIV/O! \\
\hline HIS3 & YOR202W & 4 & 1 & 11 & 31.80811 & \#DIV/O! \\
\hline HIS3 & YOR202W & 4 & 1 & 12 & 17.46763 & \#DIV/0! \\
\hline HIS3 & YOR202W & 4 & 1 & 13 & 28.32314 & \#DIV/0! \\
\hline HIS3 & YOR202W & 4 & 1 & 14 & 33.8902 & \#DIV/0! \\
\hline HIS3 & YOR202W & 4 & 1 & 15 & 31.06232 & \#DIV/O! \\
\hline HIS3 & YOR202W & 4 & 1 & 16 & 30.57897 & \#DIV/0! \\
\hline HIS3 & YOR202W & 4 & 1 & 17 & 11.80598 & \#DIV/0! \\
\hline HIS3 & YOR202W & 4 & 1 & 18 & 24.18537 & \#DIV/0! \\
\hline HIS3 & YOR202W & 4 & 1 & 19 & 34.81422 & \#DIV/O! \\
\hline HIS3 & YOR202W & 4 & 1 & 20 & 11.43403 & \#DIV/0! \\
\hline HIS3 & YOR202W & 4 & 1 & 21 & 36.6913 & \#DIV/0! \\
\hline HIS3 & YOR202W & 4 & 1 & 22 & 24.63748 & \#DIV/0! \\
\hline HIS3 & YOR202W & 4 & 1 & 23 & 27.16595 & \#DIV/O! \\
\hline HIS3 & YOR202W & 4 & 1 & 24 & 61.88322 & \#DIV/0! \\
\hline HIS3 & YOR202W & 4 & 2 & 1 & 48.02466 & \#DIV/0! \\
\hline \multirow[t]{2}{*}{ RPL34A } & YER056C-A & 4 & 2 & 2 & 57.92346 & 36.72517 \\
\hline & YER158C & 4 & 2 & 3 & 65.13643 & 46.08094 \\
\hline \multirow[t]{2}{*}{$\mathrm{FCY} 2$} & YER056C & 4 & 2 & 4 & 36.87703 & 13.89466 \\
\hline & YER156C & 4 & 2 & 5 & 33.6919 & 34.98097 \\
\hline HIS1 & YER055C & 4 & 2 & 6 & 23.20418 & 17.69944 \\
\hline BEM2 & YER155C & 4 & 2 & 7 & 33.38881 & 35.459 \\
\hline GIP2 & YER054C & 4 & 2 & 8 & 37.18053 & 40.72799 \\
\hline PET122 & YER153C & 4 & 2 & 9 & 50.52556 & 13.82617 \\
\hline \multirow[t]{2}{*}{ PIC2 } & YER053C & 4 & 2 & 10 & 15.28273 & -0.64863 \\
\hline & YER152C & 4 & 2 & 11 & 11.55852 & 43.27879 \\
\hline HOM3 & YER052C & 4 & 2 & 12 & 40.23016 & 17.78372 \\
\hline \multirow[t]{2}{*}{ UBP3 } & YER151C & 4 & 2 & 13 & 30.61427 & 33.19192 \\
\hline & YER051W & 4 & 2 & 14 & 34.6556 & 35.8881 \\
\hline \multirow[t]{2}{*}{ SPI1 } & YER150W & 4 & 2 & 15 & 34.53783 & 17.68054 \\
\hline & YER049W & 4 & 2 & 16 & 21.28552 & 21.20626 \\
\hline PEA2 & YER149C & 4 & 2 & 17 & 18.82474 & 4.334318 \\
\hline CAJ1 & YER048C & 4 & 2 & 18 & 48.42395 & 46.60348 \\
\hline
\end{tabular}




\begin{tabular}{|c|c|c|c|c|c|c|}
\hline FTR1 & YER145C & 4 & 2 & 19 & 43.81593 & 4.561395 \\
\hline SAP1 & YER047C & 4 & 2 & 20 & 39.61657 & 17.1524 \\
\hline \multirow[t]{3}{*}{ UBP5 } & YER144C & 4 & 2 & 21 & -3.75278 & 31.51027 \\
\hline & YER046W- & & & & & \\
\hline & $A$ & 4 & 2 & 22 & 19.88784 & 27.83366 \\
\hline DDI1 & YER143W & 4 & 2 & 23 & -0.32504 & 22.57942 \\
\hline HIS3 & YOR202W & 4 & 2 & 24 & 50.27736 & \#DIV/0! \\
\hline HIS3 & YOR202W & 4 & 3 & 1 & 53.71743 & \#DIV/0! \\
\hline LRS4 & YDR439W & 4 & 3 & 2 & 52.70896 & 35.73329 \\
\hline \multirow[t]{2}{*}{ VAC8 } & YEL013W & 4 & 3 & 3 & 52.70736 & 33.97069 \\
\hline & YDR438W & 4 & 3 & 4 & 14.79923 & 28.5122 \\
\hline UBC8 & YEL012W & 4 & 3 & 5 & 12.82155 & 11.23525 \\
\hline PPZ2 & YDR436W & 4 & 3 & 6 & 39.74352 & 14.23968 \\
\hline GLC3 & YEL011W & 4 & 3 & 7 & \#DIV/0! & 32.14607 \\
\hline \multirow[t]{4}{*}{ PPM1 } & YDR435C & 4 & 3 & 8 & 37.51352 & 36.16354 \\
\hline & YEL010W & 4 & 3 & 9 & 17.5982 & 46.11995 \\
\hline & YDR431W & 4 & 3 & 10 & 17.66219 & 30.68218 \\
\hline & YEL008W & 4 & 3 & 11 & 4.4324 & 35.59565 \\
\hline CYM1 & YDR430C & 4 & 3 & 12 & 11.90422 & 34.2393 \\
\hline \multirow[t]{4}{*}{ TOS9 } & YEL007W & 4 & 3 & 13 & 20.01198 & 28.28532 \\
\hline & YDR428C & 4 & 3 & 14 & 14.11485 & 32.72446 \\
\hline & YEL006W & 4 & 3 & 15 & 31.4815 & 36.5114 \\
\hline & YDR426C & 4 & 3 & 16 & 46.58333 & 39.81286 \\
\hline VAB2 & YEL005C & 4 & 3 & 17 & 45.73824 & 13.35043 \\
\hline SNX41 & YDR425W & 4 & 3 & 18 & 31.58866 & 27.05667 \\
\hline YEA4 & YEL004W & 4 & 3 & 19 & -12.068 & -7.58727 \\
\hline DYN2 & YDR424C & 4 & 3 & 20 & -8.93784 & -15.1625 \\
\hline GIM4 & YEL003W & 4 & 3 & 21 & -8.24408 & -37.7994 \\
\hline \multirow[t]{2}{*}{ CAD1 } & YDR423C & 4 & 3 & 22 & 41.71461 & 44.19246 \\
\hline & YEL001C & 4 & 3 & 23 & 41.18659 & 21.23585 \\
\hline HIS3 & YOR202W & 4 & 3 & 24 & 59.13716 & \#DIV/0! \\
\hline \multirow[t]{2}{*}{ HIS3 } & YOR202W & 4 & 4 & 1 & 55.92652 & \#DIV/0! \\
\hline & YER066W & 4 & 4 & 2 & 36.83123 & 32.29358 \\
\hline \multirow[t]{2}{*}{ ECM32 } & YER176W & 4 & 4 & 3 & 43.67436 & 61.88408 \\
\hline & YER066C-A & 4 & 4 & 4 & 30.89789 & 33.43444 \\
\hline TMT1 & YER175C & 4 & 4 & 5 & 42.88391 & 35.95639 \\
\hline ICL1 & YER065C & 4 & 4 & 6 & 14.22685 & 41.4658 \\
\hline \multirow[t]{2}{*}{ GRX4 } & YER174C & 4 & 4 & 7 & 44.25536 & 45.64714 \\
\hline & YER064C & 4 & 4 & 8 & 15.89871 & 26.43169 \\
\hline RAD24 & YER173W & 4 & 4 & 9 & 18.85699 & 36.73347 \\
\hline THO1 & YER063W & 4 & 4 & 10 & 41.77527 & 30.45956 \\
\hline ADK2 & YER170W & 4 & 4 & 11 & 32.28955 & 29.81699 \\
\hline HOR2 & YER062C & 4 & 4 & 12 & 10.73089 & 43.74032 \\
\hline
\end{tabular}




\begin{tabular}{|c|c|c|c|c|c|c|}
\hline BCK2 & YER167W & 4 & 4 & 13 & 16.38059 & 29.35287 \\
\hline CEM1 & YER061C & 4 & 4 & 14 & 1.439414 & 26.92815 \\
\hline \multirow[t]{2}{*}{ DNF1 } & YER166W & 4 & 4 & 15 & 46.465 & 51.71771 \\
\hline & YER060W- & & & & & \\
\hline FCY22 & A & 4 & 4 & 16 & 49.77578 & 34.15376 \\
\hline CHD1 & YER164W & 4 & 4 & 17 & 10.75281 & 35.91553 \\
\hline \multirow[t]{2}{*}{ FCY21 } & YER060W & 4 & 4 & 18 & 17.91282 & -3.21645 \\
\hline & YER163C & 4 & 4 & 19 & 30.63902 & 39.34958 \\
\hline PCL6 & YER059W & 4 & 4 & 20 & 34.4131 & 37.70618 \\
\hline RAD4 & YER162C & 4 & 4 & 21 & 35.56396 & 30.6103 \\
\hline HMF1 & YER057C & 4 & 4 & 22 & 18.36274 & 25.09394 \\
\hline SPT2 & YER161C & 4 & 4 & 23 & 21.05052 & 24.04097 \\
\hline HIS3 & YOR202W & 4 & 4 & 24 & 35.67369 & \#DIV/O! \\
\hline \multirow[t]{2}{*}{ HIS3 } & YOR202W & 4 & 5 & 1 & 79.45923 & \#DIV/0! \\
\hline & YDR459C & 4 & 5 & 2 & 52.06016 & 16.95972 \\
\hline \multirow[t]{2}{*}{ SPF1 } & YEL031W & 4 & 5 & 3 & 2.210735 & 42.48462 \\
\hline & YDR458C & 4 & 5 & 4 & 23.72795 & 37.57549 \\
\hline \multirow[t]{3}{*}{ ECM10 } & YEL030W & 4 & 5 & 5 & 29.62103 & 49.0986 \\
\hline & YDR455C & 4 & 5 & 6 & 18.59272 & 38.5701 \\
\hline & YEL028W & 4 & 5 & 7 & 47.64266 & 45.47786 \\
\hline TSA2 & YDR453C & 4 & 5 & 8 & 27.39426 & 22.10551 \\
\hline SRI1 & YEL025C & 4 & 5 & 9 & 55.85138 & 16.18514 \\
\hline \multirow[t]{2}{*}{ PPN1 } & YDR452W & 4 & 5 & 10 & 7.970071 & 31.27575 \\
\hline & YEL023C & 4 & 5 & 11 & -0.25765 & 27.72812 \\
\hline \multirow[t]{2}{*}{ YHP1 } & YDR451C & 4 & 5 & 12 & 17.72585 & 43.49632 \\
\hline & YEL020C & 4 & 5 & 13 & 18.33739 & 19.09829 \\
\hline RPS17B & YDR447C & 4 & 5 & 14 & 29.56758 & 38.17245 \\
\hline GTT3 & YEL017W & 4 & 5 & 15 & 23.93381 & 47.66443 \\
\hline ECM11 & YDR446W & 4 & 5 & 16 & 28.48034 & \#DIV/0! \\
\hline \multirow[t]{2}{*}{ PMP2 } & YEL017C-A & 4 & 5 & 17 & 29.99434 & 40.68575 \\
\hline & YDR445C & 4 & 5 & 18 & 11.27469 & 31.73145 \\
\hline NPP2 & YEL016C & 4 & 5 & 19 & 18.63024 & 43.59053 \\
\hline APT2 & YDR441C & 4 & 5 & 20 & 45.99576 & 46.37671 \\
\hline EDC3 & YEL015W & 4 & 5 & 21 & 39.35344 & 42.12801 \\
\hline \multirow[t]{2}{*}{ DOT1 } & YDR440W & 4 & 5 & 22 & 26.51847 & \#DIV/0! \\
\hline & YEL014C & 4 & 5 & 23 & 32.34597 & \#DIV/0! \\
\hline HIS3 & YOR202W & 4 & 5 & 24 & 26.35788 & \#DIV/0! \\
\hline \multirow[t]{6}{*}{ HIS3 } & YOR202W & 4 & 6 & 1 & 45.01116 & \#DIV/O! \\
\hline & YER079W & 4 & 6 & 2 & 10.27018 & 34.71368 \\
\hline & YER187W & 4 & 6 & 3 & 1.842414 & 21.43401 \\
\hline & YER078C & 4 & 6 & 4 & -2.80544 & 51.07189 \\
\hline & YER186C & 4 & 6 & 5 & 12.21965 & 20.25724 \\
\hline & YER077C & 4 & 6 & 6 & 18.14489 & -5.18892 \\
\hline
\end{tabular}




\begin{tabular}{|c|c|c|c|c|c|c|}
\hline & YER185W & 4 & 6 & 7 & 3.444033 & 47.1882 \\
\hline \multirow[t]{2}{*}{ PTP3 } & YER075C & 4 & 6 & 8 & -0.56187 & 36.54151 \\
\hline & YER184C & 4 & 6 & 9 & 22.16152 & 30.65127 \\
\hline RPS24A & YER074W & 4 & 6 & 10 & 34.66303 & 31.8277 \\
\hline FAU1 & YER183C & 4 & 6 & 11 & 18.31689 & 38.21654 \\
\hline ALD5 & YER073W & 4 & 6 & 12 & 24.36832 & 41.66977 \\
\hline FMP10 & YER182W & 4 & 6 & 13 & 18.51777 & 40.60577 \\
\hline \multirow[t]{3}{*}{ VTC1 } & YER072W & 4 & 6 & 14 & 43.10749 & 49.01149 \\
\hline & YER181C & 4 & 6 & 15 & 23.04296 & 46.7135 \\
\hline & YER071C & 4 & 6 & 16 & 14.51207 & 52.19064 \\
\hline ISC10 & YER180C & 4 & 6 & 17 & 11.20707 & 32.83722 \\
\hline ARG5,6 & YER069W & 4 & 6 & 18 & 25.35138 & 38.36802 \\
\hline \multirow[t]{2}{*}{ DMC1 } & YER179W & 4 & 6 & 19 & 33.41768 & 20.27268 \\
\hline & YER067W & 4 & 6 & 20 & 28.68217 & -17.5055 \\
\hline \multirow[t]{2}{*}{ PDA1 } & YER178W & 4 & 6 & 21 & 23.18628 & 34.51454 \\
\hline & YER067C-A & 4 & 6 & 22 & 35.48378 & 41.43936 \\
\hline $\mathrm{BMH} 1$ & YER177W & 4 & 6 & 23 & 35.65036 & 44.31135 \\
\hline HIS3 & YOR202W & 4 & 6 & 24 & 27.86883 & \#DIV/O! \\
\hline HIS3 & YOR202W & 4 & 7 & 1 & 51.24111 & \#DIV/0! \\
\hline DIG2 & YDR480W & 4 & 7 & 2 & 28.3928 & 17.07463 \\
\hline PAU2 & YEL049W & 4 & 7 & 3 & 22.18209 & 37.35887 \\
\hline \multirow[t]{4}{*}{ PEX29 } & YDR479C & 4 & 7 & 4 & 8.506846 & 37.75768 \\
\hline & YEL048C & 4 & 7 & 5 & 26.27757 & 33.79565 \\
\hline & YDR476C & 4 & 7 & 6 & 33.16622 & 36.82387 \\
\hline & YEL047C & 4 & 7 & 7 & 27.84571 & 36.27628 \\
\hline \multirow[t]{3}{*}{ JIP4 } & YDR475C & 4 & 7 & 8 & 42.21879 & 52.60183 \\
\hline & YEL043W & 4 & 7 & 9 & 24.86401 & 45.61349 \\
\hline & YDR474C & 4 & 7 & 10 & 27.78193 & 48.86349 \\
\hline GDA1 & YEL042W & 4 & 7 & 11 & 81.01777 & 48.60865 \\
\hline \multirow[t]{2}{*}{ RPL27B } & YDR471W & 4 & 7 & 12 & \#DIV/0! & 36.07277 \\
\hline & YEL041W & 4 & 7 & 13 & -0.37173 & 41.30796 \\
\hline SDC1 & YDR469W & 4 & 7 & 14 & -2.49834 & 8.675214 \\
\hline \multirow[t]{2}{*}{ UTR2 } & YEL040W & 4 & 7 & 15 & 23.48663 & 45.43253 \\
\hline & YDR467C & 4 & 7 & 16 & 26.654 & 45.13116 \\
\hline CYC7 & YEL039C & 4 & 7 & 17 & 31.05418 & 40.52471 \\
\hline PKH3 & YDR466W & 4 & 7 & 18 & 10.41611 & 57.48374 \\
\hline UTR4 & YEL038W & 4 & 7 & 19 & 32.63726 & 42.10894 \\
\hline RMT2 & YDR465C & 4 & 7 & 20 & 19.73545 & 34.75867 \\
\hline RAD23 & YEL037C & 4 & 7 & 21 & 17.58916 & 38.51816 \\
\hline \multirow[t]{2}{*}{ STP1 } & YDR463W & 4 & 7 & 22 & 28.88493 & 36.85169 \\
\hline & YEL033W & 4 & 7 & 23 & 27.56575 & 28.28742 \\
\hline HIS3 & YOR202W & 4 & 7 & 24 & 39.60656 & \#DIV/0! \\
\hline HIS3 & YOR202W & 4 & 8 & 1 & 50.10285 & \#DIV/0! \\
\hline
\end{tabular}




\begin{tabular}{|c|c|c|c|c|c|c|}
\hline IES5 & YER092W & 4 & 8 & 2 & 41.20353 & 36.59021 \\
\hline \multirow[t]{2}{*}{ BUD27 } & YFL023W & 4 & 8 & 3 & -47.6829 & -10.8722 \\
\hline & YER091C-A & 4 & 8 & 4 & 28.67782 & 31.11039 \\
\hline GAT1 & YFL021W & 4 & 8 & 5 & 26.31884 & 37.91836 \\
\hline MET6 & YER091C & 4 & 8 & 6 & 16.39615 & 28.78962 \\
\hline PAU5 & YFL020C & 4 & 8 & 7 & 3.660274 & 42.31933 \\
\hline \multirow[t]{2}{*}{ TRP2 } & YER090W & 4 & 8 & 8 & 12.77538 & 29.18746 \\
\hline & YFL019C & 4 & 8 & 9 & 0.784584 & 25.55456 \\
\hline DOT6 & YER088C & 4 & 8 & 10 & 34.55682 & 30.75927 \\
\hline \multirow[t]{4}{*}{ LPD1 } & YFL018C & 4 & 8 & 11 & 34.64446 & 32.78986 \\
\hline & YER087C-A & 4 & 8 & 12 & 49.27309 & 47.34092 \\
\hline & YFL015C & 4 & 8 & 13 & 18.31898 & 43.39065 \\
\hline & YER085C & 4 & 8 & 14 & 15.24487 & 44.15674 \\
\hline \multirow[t]{4}{*}{ HSP12 } & YFL014W & 4 & 8 & 15 & 23.03177 & 27.70504 \\
\hline & YER084W & 4 & 8 & 16 & 6.566926 & 32.72535 \\
\hline & YFL013W- & & & & & \\
\hline & $A$ & 4 & 8 & 17 & 19.80392 & 41.52779 \\
\hline RMD7 & YER083C & 4 & 8 & 18 & 20.32185 & 19.23327 \\
\hline HXT10 & YFL011W & 4 & 8 & 19 & 37.39116 & 24.8835 \\
\hline \multirow[t]{2}{*}{ SER3 } & YER081W & 4 & 8 & 20 & 14.70056 & 42.51599 \\
\hline & YFL006W & 4 & 8 & 21 & 5.147078 & 26.23852 \\
\hline \multirow[t]{2}{*}{ FMP29 } & YER080W & 4 & 8 & 22 & 36.96825 & 11.54891 \\
\hline & YER188W & 4 & 8 & 23 & 27.08474 & 39.41058 \\
\hline HIS3 & YOR202W & 4 & 8 & 24 & 34.0385 & \#DIV/0! \\
\hline HIS3 & YOR202W & 4 & 9 & 1 & 48.7552 & \#DIV/0! \\
\hline PUF6 & YDR496C & 4 & 9 & 2 & 34.74513 & 26.22688 \\
\hline SIT1 & YEL065W & 4 & 9 & 3 & 24.34157 & 45.39333 \\
\hline RSM28 & YDR494W & 4 & 9 & 4 & 48.73796 & 45.08553 \\
\hline AVT2 & YEL064C & 4 & 9 & 5 & 6.332361 & 41.52039 \\
\hline IZH1 & YDR492W & 4 & 9 & 6 & 5.947618 & 28.83462 \\
\hline \multirow[t]{2}{*}{ CAN1 } & YEL063C & 4 & 9 & 7 & 21.41996 & 39.28568 \\
\hline & YDR491C & 4 & 9 & 8 & 37.08382 & 23.0098 \\
\hline NPR2 & YEL062W & 4 & 9 & 9 & 2.804127 & 47.01176 \\
\hline PKH1 & YDR490C & 4 & 9 & 10 & 40.15333 & 42.95113 \\
\hline CIN8 & YEL061C & 4 & 9 & 11 & 31.72495 & 69.32193 \\
\hline PAC11 & YDR488C & 4 & 9 & 12 & 25.37783 & 29.59734 \\
\hline PRB1 & YEL060C & 4 & 9 & 13 & 13.4757 & 45.42592 \\
\hline \multirow[t]{2}{*}{ VPS60 } & YDR486C & 4 & 9 & 14 & 38.55369 & 24.14404 \\
\hline & YEL059W & 4 & 9 & 15 & 5.061494 & 27.12556 \\
\hline \multirow[t]{2}{*}{ VPS72 } & YDR485C & 4 & 9 & 16 & 31.95953 & 40.70652 \\
\hline & YEL057C & 4 & 9 & 17 & 43.43017 & 48.51014 \\
\hline KRE2 & YDR483W & 4 & 9 & 18 & 15.9376 & 35.94007 \\
\hline HAT2 & YEL056W & 4 & 9 & 19 & -7.17565 & 40.32491 \\
\hline
\end{tabular}




\begin{tabular}{|c|c|c|c|c|c|c|}
\hline CWC21 & YDR482C & 4 & 9 & 20 & 18.54619 & 34.49626 \\
\hline MAK10 & YEL053C & 4 & 9 & 21 & 26.1426 & 9.692411 \\
\hline $\mathrm{PHO} 8$ & YDR481C & 4 & 9 & 22 & 14.70318 & 22.62771 \\
\hline AFG1 & YEL052W & 4 & 9 & 23 & 22.09269 & 53.00756 \\
\hline HIS3 & YOR202W & 4 & 9 & 24 & 37.75155 & \#DIV/O! \\
\hline \multirow[t]{2}{*}{ HIS3 } & YOR202W & 4 & 10 & 1 & 51.52634 & \#DIV/0! \\
\hline & YER113C & 4 & 10 & 2 & 21.44073 & 40.85416 \\
\hline RPO41 & YFL036W & 4 & 10 & 3 & 56.03378 & 33.44121 \\
\hline \multirow[t]{2}{*}{ SWI4 } & YER111C & 4 & 10 & 4 & 20.15728 & 32.49466 \\
\hline & YFL035C-B & 4 & 10 & 5 & 39.51039 & 28.20513 \\
\hline \multirow[t]{3}{*}{ FLO8 } & YER109C & 4 & 10 & 6 & 16.38865 & 37.04345 \\
\hline & YFL034W & 4 & 10 & 7 & 13.58501 & 35.01975 \\
\hline & YER108C & 4 & 10 & 8 & 17.51303 & 34.94546 \\
\hline RPL22B & YFL034C-A & 4 & 10 & 9 & 3.523138 & 52.33422 \\
\hline \multirow[t]{2}{*}{ MAM1 } & YER106W & 4 & 10 & 10 & 12.53252 & 37.64971 \\
\hline & YFL032W & 4 & 10 & 11 & 17.71886 & 34.1683 \\
\hline AST2 & YER101C & 4 & 10 & 12 & 56.5828 & 36.31607 \\
\hline HAC1 & YFL031W & 4 & 10 & 13 & 60.35412 & 41.69271 \\
\hline UBP9 & YER098W & 4 & 10 & 14 & 19.44077 & 38.67725 \\
\hline \multirow[t]{2}{*}{ AGX1 } & YFL030W & 4 & 10 & 15 & 19.889 & 32.84557 \\
\hline & YER097W & 4 & 10 & 16 & 18.10634 & 44.26634 \\
\hline CAF16 & YFL028C & 4 & 10 & 17 & 13.58629 & 37.55162 \\
\hline SHC1 & YER096W & 4 & 10 & 18 & 45.4073 & 41.35473 \\
\hline GYP8 & YFL027C & 4 & 10 & 19 & 50.1175 & 47.64333 \\
\hline RAD51 & YER095W & 4 & 10 & 20 & 52.55475 & 46.25328 \\
\hline \multirow[t]{2}{*}{ STE2 } & YFL026W & 4 & 10 & 21 & 25.49475 & 34.85762 \\
\hline & YER093C-A & 4 & 10 & 22 & 30.87242 & 22.23499 \\
\hline BST1 & YFL025C & 4 & 10 & 23 & 30.91638 & 33.7601 \\
\hline HIS3 & YOR202W & 4 & 10 & 24 & 25.38388 & \#DIV/0! \\
\hline HIS3 & YOR202W & 4 & 11 & 1 & 39.32684 & \#DIV/O! \\
\hline \multirow[t]{2}{*}{ EMI1 } & YDR512C & 4 & 11 & 2 & 58.20043 & 25.60967 \\
\hline & YER010C & 4 & 11 & 3 & 25.88462 & 47.76924 \\
\hline ACN9 & YDR511W & 4 & 11 & 4 & -3.42866 & 31.88018 \\
\hline \multirow[t]{2}{*}{ PAC2 } & YER007W & 4 & 11 & 5 & 32.54716 & 37.56187 \\
\hline & YDR509W & 4 & 11 & 6 & 46.0711 & 31.67429 \\
\hline RBF20 & YER007C-A & 4 & 11 & 7 & 39.41045 & 42.70148 \\
\hline GNP1 & YDR508C & 4 & 11 & 8 & 10.109 & 42.29936 \\
\hline \multirow[t]{2}{*}{ YND1 } & YER005W & 4 & 11 & 9 & 17.23344 & 29.58484 \\
\hline & YDR506C & 4 & 11 & 10 & 18.85778 & 35.80706 \\
\hline FMP52 & YER004W & 4 & 11 & 11 & 1.477348 & 39.00938 \\
\hline PSP1 & YDR505C & 4 & 11 & 12 & 22.06486 & 50.5481 \\
\hline NOP16 & YER002W & 4 & 11 & 13 & 34.0407 & 25.24782 \\
\hline SPG3 & YDR504C & 4 & 11 & 14 & 13.53682 & 35.95308 \\
\hline
\end{tabular}




\begin{tabular}{|c|c|c|c|c|c|c|}
\hline MNN1 & YER001W & 4 & 11 & 15 & 2.879572 & 51.4343 \\
\hline LPP1 & YDR503C & 4 & 11 & 16 & 20.15953 & 38.80423 \\
\hline DLD3 & YEL071W & 4 & 11 & 17 & 7.532888 & 21.2224 \\
\hline \multirow[t]{2}{*}{ PLM2 } & YDR501W & 4 & 11 & 18 & -8.58531 & 32.80727 \\
\hline & YEL068C & 4 & 11 & 19 & 21.88146 & 28.06009 \\
\hline \multirow[t]{2}{*}{ RPL37B } & YDR500C & 4 & 11 & 20 & 46.0724 & 25.57866 \\
\hline & YEL067C & 4 & 11 & 21 & 49.08145 & 27.44923 \\
\hline ITR1 & YDR497C & 4 & 11 & 22 & 45.22216 & 40.37749 \\
\hline HPA3 & YEL066W & 4 & 11 & 23 & 23.66906 & 25.99357 \\
\hline HIS3 & YOR202W & 4 & 11 & 24 & 29.76106 & \#DIV/0! \\
\hline HIS3 & YOR202W & 4 & 12 & 1 & 46.62803 & \#DIV/0! \\
\hline \multirow[t]{2}{*}{ DSE1 } & YER124C & 4 & 12 & 2 & 54.53444 & 30.38095 \\
\hline & YFL051C & 4 & 12 & 3 & 34.00965 & 36.71032 \\
\hline YCK3 & YER123W & 4 & 12 & 4 & 50.16016 & 17.19253 \\
\hline \multirow[t]{3}{*}{ ALR2 } & YFL050C & 4 & 12 & 5 & -2.63966 & \#DIV/0! \\
\hline & YER121W & 4 & 12 & 6 & 18.0501 & 24.80131 \\
\hline & YFL049W & 4 & 12 & 7 & -9.98671 & 40.59426 \\
\hline SCS2 & YER120W & 4 & 12 & 8 & 1.956473 & 32.4229 \\
\hline \multirow[t]{2}{*}{ EMP47 } & YFL048C & 4 & 12 & 9 & 20.18963 & 44.14568 \\
\hline & YER119C-A & 4 & 12 & 10 & 4.956872 & 36.00618 \\
\hline RGD2 & YFL047W & 4 & 12 & 11 & 18.98252 & 34.24417 \\
\hline AVT6 & YER119C & 4 & 12 & 12 & 3.161688 & 37.03252 \\
\hline FMP32 & YFL046W & 4 & 12 & 13 & 19.71639 & 31.21471 \\
\hline SHO1 & YER118C & 4 & 12 & 14 & 21.0673 & 36.78481 \\
\hline YOD1 & YFL044C & 4 & 12 & 15 & 21.90253 & 36.77783 \\
\hline \multirow[t]{2}{*}{ RPL23B } & YER117W & 4 & 12 & 16 & 22.54378 & 34.22318 \\
\hline & YFL043C & 4 & 12 & 17 & 26.964 & 42.87438 \\
\hline \multirow[t]{2}{*}{ SLX8 } & YER116C & 4 & 12 & 18 & 8.54379 & 48.36677 \\
\hline & YFL042C & 4 & 12 & 19 & 18.21683 & 38.21753 \\
\hline SPR6 & YER115C & 4 & 12 & 20 & 15.18329 & 34.32825 \\
\hline FET5 & YFL041W & 4 & 12 & 21 & 31.78065 & 38.5455 \\
\hline \multirow[t]{2}{*}{ BOI2 } & YER114C & 4 & 12 & 22 & 18.53142 & 29.3143 \\
\hline & YFL040W & 4 & 12 & 23 & 26.56309 & 26.24855 \\
\hline HIS3 & YOR202W & 4 & 12 & 24 & 16.3727 & \#DIV/0! \\
\hline HIS3 & YOR202W & 4 & 13 & 1 & 40.22051 & \#DIV/0! \\
\hline HLR1 & YDR528W & 4 & 13 & 2 & 27.68492 & 34.21677 \\
\hline FIR1 & $\begin{array}{l}\text { YER032W } \\
\text { YDR525W- }\end{array}$ & 4 & 13 & 3 & 40.83548 & 38.25187 \\
\hline SNA2 & $A$ & 4 & 13 & 4 & 15.23809 & 41.40943 \\
\hline YРT31 & YER031C & 4 & 13 & 5 & 58.92794 & 52.79473 \\
\hline \multirow[t]{2}{*}{ API2 } & YDR525W & 4 & 13 & 6 & 5.114075 & 29.03909 \\
\hline & YER030W & 4 & 13 & 7 & 40.54197 & 40.93129 \\
\hline AGE1 & YDR524C & 4 & 13 & 8 & 53.53343 & 54.95371 \\
\hline
\end{tabular}




\begin{tabular}{|c|c|c|c|c|c|c|}
\hline MIG3 & YER028C & 4 & 13 & 9 & 20.61048 & 35.12214 \\
\hline SPS2 & YDR522C & 4 & 13 & 10 & 43.81029 & 46.8308 \\
\hline \multirow[t]{2}{*}{ GAL83 } & YER027C & 4 & 13 & 11 & 39.43287 & 41.40204 \\
\hline & YDR520C & 4 & 13 & 12 & 24.07946 & 54.36376 \\
\hline YAT2 & YER024W & 4 & 13 & 13 & 30.59243 & 22.74709 \\
\hline FPR2 & YDR519W & 4 & 13 & 14 & 13.20859 & 50.91899 \\
\hline GPA2 & YER020W & 4 & 13 & 15 & 35.76468 & 31.9676 \\
\hline GRH1 & YDR517W & 4 & 13 & 16 & 50.54381 & 42.57092 \\
\hline ISC1 & YER019W & 4 & 13 & 17 & 61.07476 & 40.94302 \\
\hline EMI2 & YDR516C & 4 & 13 & 18 & 23.98201 & 39.88631 \\
\hline \multirow[t]{2}{*}{$\mathrm{SBH} 2$} & YER019C-A & 4 & 13 & 19 & 14.11355 & 19.36151 \\
\hline & YDR514C & 4 & 13 & 20 & 28.18608 & 43.95933 \\
\hline BIM1 & YER016W & 4 & 13 & 21 & 35.25096 & 49.1388 \\
\hline TTR1 & YDR513W & 4 & 13 & 22 & 23.28769 & 53.15175 \\
\hline TIR1 & YER011W & 4 & 13 & 23 & 27.64999 & 40.67222 \\
\hline HIS3 & YOR202W & 4 & 13 & 24 & 30.71963 & \#DIV/0! \\
\hline HIS3 & YOR202W & 4 & 14 & 1 & 30.30968 & \#DIV/0! \\
\hline \multirow[t]{3}{*}{ MAG1 } & YER142C & 4 & 14 & 2 & 50.25653 & 32.3186 \\
\hline & YFR011C & 4 & 14 & 3 & 56.26093 & 49.52826 \\
\hline & YER140W & 4 & 14 & 4 & 40.5653 & 31.1138 \\
\hline \multirow[t]{2}{*}{ UBP6 } & YFR010W & 4 & 14 & 5 & 84.33053 & 69.65307 \\
\hline & YER139C & 4 & 14 & 6 & 12.95625 & 28.64502 \\
\hline \multirow[t]{2}{*}{ GCN20 } & YFR009W & 4 & 14 & 7 & 30.88558 & 49.32655 \\
\hline & YER137C & 4 & 14 & 8 & 40.59755 & 49.81554 \\
\hline \multirow[t]{5}{*}{ FAR7 } & YFR008W & 4 & 14 & 9 & 53.26105 & 37.05289 \\
\hline & YER135C & 4 & 14 & 10 & 67.90142 & 71.85142 \\
\hline & YFR007W & 4 & 14 & 11 & -1.40206 & 37.82571 \\
\hline & YER134C & 4 & 14 & 12 & 30.75611 & 40.39168 \\
\hline & YFR006W & 4 & 14 & 13 & 39.30355 & 42.1875 \\
\hline PMD1 & YER132C & 4 & 14 & 14 & 23.09191 & 44.88391 \\
\hline AAD6 & YFL056C & 4 & 14 & 15 & 29.57909 & 40.63152 \\
\hline RPS26B & YER131W & 4 & 14 & 16 & 36.73114 & 57.56286 \\
\hline \multirow[t]{3}{*}{ AGP3 } & YFL055W & 4 & 14 & 17 & 33.05792 & 44.81036 \\
\hline & YER130C & 4 & 14 & 18 & 21.16596 & 29.10007 \\
\hline & YFL054C & 4 & 14 & 19 & 37.36984 & 56.41848 \\
\hline PAK1 & YER129W & 4 & 14 & 20 & 30.08624 & 33.13124 \\
\hline \multirow[t]{3}{*}{ DAK2 } & YFL053W & 4 & 14 & 21 & 5.336589 & 51.08707 \\
\hline & YER128W & 4 & 14 & 22 & 47.59548 & 31.81704 \\
\hline & YFL052W & 4 & 14 & 23 & 33.68224 & 43.99944 \\
\hline HIS3 & YOR202W & 4 & 14 & 24 & -2.86428 & \#DIV/0! \\
\hline \multirow[t]{2}{*}{ HIS3 } & YOR202W & 4 & 15 & 1 & 42.64548 & \#DIV/0! \\
\hline & YDR541C & 4 & 15 & 2 & 54.33592 & 43.72921 \\
\hline SPO73 & YER046W & 4 & 15 & 3 & 54.47679 & 52.04877 \\
\hline
\end{tabular}




\begin{tabular}{|c|c|c|c|c|c|c|}
\hline & YDR540C & 4 & 15 & 4 & 38.1191 & 46.83808 \\
\hline \multirow[t]{2}{*}{ ACA1 } & YER045C & 4 & 15 & 5 & 36.75878 & 40.07665 \\
\hline & YDR539W & 4 & 15 & 6 & 38.69495 & 33.19095 \\
\hline MEI4 & YER044C-A & 4 & 15 & 7 & 48.4437 & 41.75692 \\
\hline PAD1 & YDR538W & 4 & 15 & 8 & 61.27497 & 51.61017 \\
\hline \multirow[t]{3}{*}{ MXR1 } & YER042W & 4 & 15 & 9 & 12.54643 & 50.65372 \\
\hline & YDR537C & 4 & 15 & 10 & 22.11742 & 40.55002 \\
\hline & YER039C-A & 4 & 15 & 11 & 43.36615 & 36.21269 \\
\hline STL1 & YDR536W & 4 & 15 & 12 & 54.14377 & 66.35045 \\
\hline \multirow[t]{2}{*}{ YEN1 } & YER041W & 4 & 15 & 13 & 6.372687 & 51.18263 \\
\hline & YDR535C & 4 & 15 & 14 & 16.43194 & 54.68052 \\
\hline HVG1 & YER039C & 4 & 15 & 15 & 61.48248 & 60.88331 \\
\hline FIT1 & $\begin{array}{l}\text { YDR534C } \\
\text { YER038W- }\end{array}$ & 4 & 15 & 16 & 51.71144 & 30.49376 \\
\hline FMP49 & $A$ & 4 & 15 & 17 & 34.56795 & 44.45636 \\
\hline HSP31 & YDR533C & 4 & 15 & 18 & 6.107344 & 54.01281 \\
\hline \multirow[t]{3}{*}{ EDC2 } & YER035W & 4 & 15 & 19 & 41.06713 & 48.23245 \\
\hline & YDR532C & 4 & 15 & 20 & 44.79211 & 40.00143 \\
\hline & YER034W & 4 & 15 & 21 & 62.51711 & 41.26662 \\
\hline APA2 & YDR530C & 4 & 15 & 22 & 74.14536 & 36.3772 \\
\hline ZRG8 & YER033C & 4 & 15 & 23 & 26.69419 & 44.74581 \\
\hline HIS3 & YOR202W & 4 & 15 & 24 & 23.35197 & \#DIV/O! \\
\hline HIS3 & YOR202W & 4 & 16 & 1 & 36.94443 & \#DIV/0! \\
\hline HIS3 & YOR202W & 4 & 16 & 2 & 56.46612 & \#DIV/0! \\
\hline HIS3 & YOR202W & 4 & 16 & 3 & 62.62329 & \#DIV/0! \\
\hline HIS3 & YOR202W & 4 & 16 & 4 & 57.03788 & \#DIV/0! \\
\hline HIS3 & YOR202W & 4 & 16 & 5 & 52.29925 & \#DIV/O! \\
\hline HIS3 & YOR202W & 4 & 16 & 6 & 35.75316 & \#DIV/O! \\
\hline HIS3 & YOR202W & 4 & 16 & 7 & 16.37577 & \#DIV/O! \\
\hline HIS3 & YOR202W & 4 & 16 & 8 & 9.902601 & \#DIV/0! \\
\hline HIS3 & YOR202W & 4 & 16 & 9 & 16.39585 & \#DIV/0! \\
\hline HIS3 & YOR202W & 4 & 16 & 10 & 22.12437 & \#DIV/O! \\
\hline HIS3 & YOR202W & 4 & 16 & 11 & 32.84963 & \#DIV/0! \\
\hline HIS3 & YOR202W & 4 & 16 & 12 & -0.39265 & \#DIV/O! \\
\hline HIS3 & YOR202W & 4 & 16 & 13 & 24.71307 & \#DIV/O! \\
\hline HIS3 & YOR202W & 4 & 16 & 14 & 25.34782 & \#DIV/0! \\
\hline HIS3 & YOR202W & 4 & 16 & 15 & 27.01296 & \#DIV/0! \\
\hline HIS3 & YOR202W & 4 & 16 & 16 & 22.12932 & \#DIV/0! \\
\hline HIS3 & YOR202W & 4 & 16 & 17 & 41.94099 & \#DIV/O! \\
\hline HIS3 & YOR202W & 4 & 16 & 18 & 48.58485 & \#DIV/0! \\
\hline HIS3 & YOR202W & 4 & 16 & 19 & 10.64602 & \#DIV/0! \\
\hline HIS3 & YOR202W & 4 & 16 & 20 & 18.67441 & \#DIV/0! \\
\hline HIS3 & YOR202W & 4 & 16 & 21 & 22.50149 & \#DIV/O! \\
\hline
\end{tabular}




\begin{tabular}{|c|c|c|c|c|c|c|}
\hline HIS3 & YOR202W & 4 & 16 & 22 & 30.89583 & \#DIV/0! \\
\hline HIS3 & YOR202W & 4 & 16 & 23 & 44.38233 & \#DIV/0! \\
\hline HIS3 & YOR202W & 4 & 16 & 24 & 32.25595 & \#DIV/0! \\
\hline HIS3 & YOR202W & 5 & 1 & 1 & 38.7242 & \#DIV/0! \\
\hline HIS3 & YOR202W & 5 & 1 & 2 & 31.07417 & \#DIV/0! \\
\hline HIS3 & YOR202W & 5 & 1 & 3 & 53.63393 & \#DIV/0! \\
\hline HIS3 & YOR202W & 5 & 1 & 4 & 7.694015 & \#DIV/O! \\
\hline HIS3 & YOR202W & 5 & 1 & 5 & 29.04501 & \#DIV/0! \\
\hline HIS3 & YOR202W & 5 & 1 & 6 & 18.49333 & \#DIV/0! \\
\hline HIS3 & YOR202W & 5 & 1 & 7 & 37.26281 & \#DIV/0! \\
\hline HIS3 & YOR202W & 5 & 1 & 8 & 23.27115 & \#DIV/0! \\
\hline HIS3 & YOR202W & 5 & 1 & 9 & 37.29075 & \#DIV/0! \\
\hline HIS3 & YOR202W & 5 & 1 & 10 & 21.8441 & \#DIV/O! \\
\hline HIS3 & YOR202W & 5 & 1 & 11 & 36.9186 & \#DIV/0! \\
\hline HIS3 & YOR202W & 5 & 1 & 12 & -13.8029 & \#DIV/0! \\
\hline HIS3 & YOR202W & 5 & 1 & 13 & 32.97631 & \#DIV/0! \\
\hline HIS3 & YOR202W & 5 & 1 & 14 & 29.44346 & \#DIV/0! \\
\hline HIS3 & YOR202W & 5 & 1 & 15 & 37.73508 & \#DIV/0! \\
\hline HIS3 & YOR202W & 5 & 1 & 16 & 31.14878 & \#DIV/0! \\
\hline HIS3 & YOR202W & 5 & 1 & 17 & 38.0503 & \#DIV/0! \\
\hline HIS3 & YOR202W & 5 & 1 & 18 & 32.96437 & \#DIV/0! \\
\hline HIS3 & YOR202W & 5 & 1 & 19 & 15.6704 & \#DIV/0! \\
\hline HIS3 & YOR202W & 5 & 1 & 20 & 13.04967 & \#DIV/0! \\
\hline HIS3 & YOR202W & 5 & 1 & 21 & 32.32855 & \#DIV/0! \\
\hline HIS3 & YOR202W & 5 & 1 & 22 & 33.26853 & \#DIV/0! \\
\hline HIS3 & YOR202W & 5 & 1 & 23 & 34.17546 & \#DIV/0! \\
\hline HIS3 & YOR202W & 5 & 1 & 24 & 19.67817 & \#DIV/0! \\
\hline HIS3 & YOR202W & 5 & 2 & 1 & 46.88271 & \#DIV/0! \\
\hline \multirow[t]{4}{*}{ CLG1 } & YGL215W & 5 & 2 & 2 & 20.81323 & 51.6293 \\
\hline & YGR051C & 5 & 2 & 3 & 48.87479 & 40.02507 \\
\hline & YGL214W & 5 & 2 & 4 & 13.73137 & 51.76467 \\
\hline & YGR050C & 5 & 2 & 5 & 27.00449 & 53.16308 \\
\hline SKI8 & YGL213C & 5 & 2 & 6 & 37.57812 & 39.91028 \\
\hline SCM4 & YGR049W & 5 & 2 & 7 & 40.26559 & 39.18162 \\
\hline \multirow[t]{2}{*}{ VAM7 } & YGL212W & 5 & 2 & 8 & 24.0368 & 55.59801 \\
\hline & YGR045C & 5 & 2 & 9 & 10.56278 & 51.91257 \\
\hline NCS6 & YGL211W & 5 & 2 & 10 & 23.63995 & 47.91524 \\
\hline RME1 & YGR044C & 5 & 2 & 11 & 19.82679 & 51.21624 \\
\hline \multirow[t]{2}{*}{ YPT32 } & YGL210W & 5 & 2 & 12 & 32.73398 & 36.81975 \\
\hline & YGR043C & 5 & 2 & 13 & -19.4399 & 51.58158 \\
\hline \multirow[t]{2}{*}{ MIG2 } & YGL209W & 5 & 2 & 14 & 4.633267 & 45.87108 \\
\hline & YGR042W & 5 & 2 & 15 & 34.01866 & 34.0813 \\
\hline SIP2 & YGL208W & 5 & 2 & 16 & 15.15057 & 47.06885 \\
\hline
\end{tabular}




\begin{tabular}{|c|c|c|c|c|c|c|}
\hline BUD9 & YGR041W & 5 & 2 & 17 & 33.60125 & 58.70131 \\
\hline POX1 & YGL205W & 5 & 2 & 18 & 11.25598 & 32.45295 \\
\hline KSS1 & YGR040W & 5 & 2 & 19 & 4.606378 & 40.01313 \\
\hline \multirow[t]{2}{*}{ KEX1 } & YGL203C & 5 & 2 & 20 & 32.1499 & 21.2946 \\
\hline & YGR039W & 5 & 2 & 21 & 46.91149 & 20.56829 \\
\hline ARO8 & YGL202W & 5 & 2 & 22 & 44.94495 & 32.93766 \\
\hline ORM1 & YGR038W & 5 & 2 & 23 & 20.12554 & 45.14459 \\
\hline HIS3 & YOR202W & 5 & 2 & 24 & 18.02168 & \#DIV/0! \\
\hline \multirow[t]{2}{*}{ HIS3 } & YOR202W & 5 & 3 & 1 & 25.18894 & \#DIV/0! \\
\hline & YFR022W & 5 & 3 & 2 & 6.651156 & 49.66424 \\
\hline SCY1 & YGL083W & 5 & 3 & 3 & 35.57859 & 38.36928 \\
\hline \multirow[t]{4}{*}{ ATG18 } & YFR021W & 5 & 3 & 4 & 3.774326 & 43.93215 \\
\hline & YGL082W & 5 & 3 & 5 & 18.89395 & 51.46377 \\
\hline & YFR020W & 5 & 3 & 6 & 22.17815 & 54.75245 \\
\hline & YGL081W & 5 & 3 & 7 & 18.96032 & 31.81649 \\
\hline FAB1 & YFR019W & 5 & 3 & 8 & 62.28744 & 47.36256 \\
\hline \multirow[t]{4}{*}{ FMP37 } & YGL080W & 5 & 3 & 9 & 6.234111 & 48.64319 \\
\hline & YFR018C & 5 & 3 & 10 & 17.02243 & 40.84492 \\
\hline & YGL079W & 5 & 3 & 11 & 21.48863 & 38.74768 \\
\hline & YFR017C & 5 & 3 & 12 & 14.07141 & 41.72608 \\
\hline \multirow[t]{2}{*}{ DBP3 } & YGL078C & 5 & 3 & 13 & -4.89446 & 40.81007 \\
\hline & YFR016C & 5 & 3 & 14 & -7.55955 & 36.83503 \\
\hline HNM1 & YGL077C & 5 & 3 & 15 & -14.3367 & 37.62901 \\
\hline GSY1 & YFR015C & 5 & 3 & 16 & 12.8001 & 41.55556 \\
\hline NPY1 & YGL067W & 5 & 3 & 17 & 17.08311 & 51.95626 \\
\hline CMK1 & YFR014C & 5 & 3 & 18 & 24.46471 & 34.75117 \\
\hline SGF73 & YGL066W & 5 & 3 & 19 & 40.40307 & 26.25578 \\
\hline IOC3 & YFR013W & 5 & 3 & 20 & 43.43531 & 38.60336 \\
\hline \multirow[t]{2}{*}{ PYC1 } & YGL062W & 5 & 3 & 21 & 14.28705 & 21.31671 \\
\hline & YFR012W & 5 & 3 & 22 & 25.18089 & 37.41827 \\
\hline YBP2 & YGL060W & 5 & 3 & 23 & 24.40436 & 37.32616 \\
\hline HIS3 & YOR202W & 5 & 3 & 24 & 1.431999 & \#DIV/0! \\
\hline HIS3 & YOR202W & 5 & 4 & 1 & 10.51147 & \#DIV/0! \\
\hline SHE10 & YGL228W & 5 & 4 & 2 & 24.78609 & 67.49462 \\
\hline PEX8 & YGR077C & 5 & 4 & 3 & -1.36527 & 54.63082 \\
\hline VID30 & YGL227W & 5 & 4 & 4 & -1.56811 & 31.5483 \\
\hline \multirow[t]{3}{*}{ UPF3 } & YGR072W & 5 & 4 & 5 & 55.83078 & 48.91468 \\
\hline & YGL226W & 5 & 4 & 6 & 33.08207 & 52.25386 \\
\hline & YGR071C & 5 & 4 & 7 & 43.14798 & 55.03419 \\
\hline OST5 & YGL226C-A & 5 & 4 & 8 & 11.55094 & 49.05524 \\
\hline ROM1 & YGR070W & 5 & 4 & 9 & 10.93506 & 45.70369 \\
\hline \multirow[t]{2}{*}{ SDT1 } & YGL224C & 5 & 4 & 10 & 49.85035 & 35.32033 \\
\hline & YGR069W & 5 & 4 & 11 & 36.42683 & 36.7136 \\
\hline
\end{tabular}




\begin{tabular}{|c|c|c|c|c|c|c|}
\hline \multirow[t]{2}{*}{ EDC1 } & YGL222C & 5 & 4 & 12 & 34.90539 & 35.85105 \\
\hline & YGR066C & 5 & 4 & 13 & 41.16303 & 38.58911 \\
\hline NIF3 & YGL221C & 5 & 4 & 14 & 4.101544 & 49.22663 \\
\hline SPR3 & YGR059W & 5 & 4 & 15 & 8.830716 & 47.56991 \\
\hline \multirow[t]{6}{*}{ MDM34 } & YGL219C & 5 & 4 & 16 & 34.46662 & 19.7886 \\
\hline & YGR058W & 5 & 4 & 17 & 30.48979 & 28.47922 \\
\hline & YGL218W & 5 & 4 & 18 & 65.05832 & -13.2913 \\
\hline & YGR054W & 5 & 4 & 19 & \#DIV/0! & 42.99804 \\
\hline & YGL217C & 5 & 4 & 20 & 25.95482 & 15.85419 \\
\hline & YGR053C & 5 & 4 & 21 & 20.14116 & 38.65795 \\
\hline KIP3 & YGL216W & 5 & 4 & 22 & 4.26419 & 31.76644 \\
\hline FMP48 & YGR052W & 5 & 4 & 23 & 7.23056 & 34.0778 \\
\hline HIS3 & YOR202W & 5 & 4 & 24 & 20.04713 & \#DIV/0! \\
\hline HIS3 & YOR202W & 5 & 5 & 1 & 16.82403 & \#DIV/0! \\
\hline \multirow[t]{2}{*}{ PHO4 } & YFR034C & 5 & 5 & 2 & 9.014936 & 44.30091 \\
\hline & YGL109W & 5 & 5 & 3 & -9.77632 & 50.05198 \\
\hline \multirow[t]{2}{*}{ QCR6 } & YFR033C & 5 & 5 & 4 & 18.34746 & 39.50019 \\
\hline & YGL108C & 5 & 5 & 5 & 37.39799 & 45.76262 \\
\hline RPL29 & YFR032C-A & 5 & 5 & 6 & 25.40063 & 23.52749 \\
\hline \multirow[t]{2}{*}{ ARC1 } & YGL105W & 5 & 5 & 7 & 35.47864 & 58.23782 \\
\hline & YFR032C & 5 & 5 & 8 & 23.1643 & 44.30673 \\
\hline VPS73 & YGL104C & 5 & 5 & 9 & 9.608858 & 40.05724 \\
\hline \multirow[t]{2}{*}{ RPL2A } & YFR031C-A & 5 & 5 & 10 & 11.80105 & 46.02356 \\
\hline & YGL101W & 5 & 5 & 11 & 36.65742 & 41.70841 \\
\hline MET10 & YFR030W & 5 & 5 & 12 & 13.86982 & 45.8027 \\
\hline \multirow[t]{2}{*}{ LIF1 } & YGL090W & 5 & 5 & 13 & \#DIV/0! & 52.15758 \\
\hline & YFR026C & 5 & 5 & 14 & 4.833925 & 41.20551 \\
\hline $\operatorname{MF}(\mathrm{ALPHA}) 2$ & YGL089C & 5 & 5 & 15 & 18.86157 & 40.32173 \\
\hline HIS2 & YFR025C & 5 & 5 & 16 & 2.563943 & 52.56175 \\
\hline MMS2 & YGL087C & 5 & 5 & 17 & 36.31819 & 43.89358 \\
\hline LSB3 & YFR024C-A & 5 & 5 & 18 & 33.51815 & 28.88149 \\
\hline \multirow[t]{3}{*}{ MAD1 } & YGL086W & 5 & 5 & 19 & 47.49379 & 41.57163 \\
\hline & YFR024C & 5 & 5 & 20 & 52.24918 & 40.13566 \\
\hline & YGL085W & 5 & 5 & 21 & -11.7685 & 30.3377 \\
\hline PES4 & YFR023W & 5 & 5 & 22 & 8.016787 & 36.83046 \\
\hline GUP1 & YGL084C & 5 & 5 & 23 & 37.54069 & 45.41704 \\
\hline HIS3 & YOR202W & 5 & 5 & 24 & 28.45838 & \#DIV/0! \\
\hline HIS3 & YOR202W & 5 & 6 & 1 & 24.44682 & \#DIV/0! \\
\hline TAD1 & YGL243W & 5 & 6 & 2 & -0.27536 & 51.68819 \\
\hline \multirow[t]{2}{*}{ DBF2 } & YGR092W & 5 & 6 & 3 & 44.03851 & 42.5903 \\
\hline & YGL242C & 5 & 6 & 4 & 39.50775 & 67.57987 \\
\hline NNF2 & YGR089W & 5 & 6 & 5 & 49.04915 & 46.34531 \\
\hline KAP114 & YGL241W & 5 & 6 & 6 & 9.249709 & 46.90658 \\
\hline
\end{tabular}




\begin{tabular}{|c|c|c|c|c|c|c|}
\hline СТT1 & YGR088W & 5 & 6 & 7 & 7.26041 & 40.66396 \\
\hline HAP2 & YGL237C & 5 & 6 & 8 & 44.9857 & 38.7478 \\
\hline PDC6 & YGR087C & 5 & 6 & 9 & 21.99692 & 48.95878 \\
\hline MTO1 & YGL236C & 5 & 6 & 10 & 60.98048 & 28.85244 \\
\hline \multirow[t]{2}{*}{ PIL1 } & YGR086C & 5 & 6 & 11 & 31.65963 & 48.76825 \\
\hline & YGL235W & 5 & 6 & 12 & 56.3267 & 31.42661 \\
\hline RPL11B & YGR085C & 5 & 6 & 13 & 39.39967 & 39.85605 \\
\hline ADE5,7 & YGL234W & 5 & 6 & 14 & 68.32791 & 58.43816 \\
\hline MRP13 & YGR084C & 5 & 6 & 15 & 38.1766 & 16.76215 \\
\hline TAN1 & YGL232W & 5 & 6 & 16 & 20.45183 & 27.37138 \\
\hline \multirow[t]{2}{*}{ SLX9 } & YGR081C & 5 & 6 & 17 & 36.58072 & 17.69174 \\
\hline & YGL231C & 5 & 6 & 18 & 13.91326 & 39.06977 \\
\hline \multirow[t]{3}{*}{ TWF1 } & YGR080W & 5 & 6 & 19 & 40.22585 & 29.76147 \\
\hline & YGL230C & 5 & 6 & 20 & 19.45017 & 38.80326 \\
\hline & YGR079W & 5 & 6 & 21 & 23.26675 & 40.98804 \\
\hline SAP4 & YGL229C & 5 & 6 & 22 & 33.82221 & 28.00293 \\
\hline PAC10 & YGR078C & 5 & 6 & 23 & -3.46076 & 25.61174 \\
\hline HIS3 & YOR202W & 5 & 6 & 24 & -6.16019 & \#DIV/0! \\
\hline HIS3 & YOR202W & 5 & 7 & 1 & 34.04321 & \#DIV/0! \\
\hline RMD8 & YFR048W & 5 & 7 & 2 & -10.1556 & 47.61252 \\
\hline SNT2 & YGL131C & 5 & 7 & 3 & 21.45428 & 49.01068 \\
\hline BNA6 & YFR047C & 5 & 7 & 4 & 11.55953 & 52.24658 \\
\hline SOH1 & YGL127C & 5 & 7 & 5 & 49.29621 & 25.36889 \\
\hline CNN1 & YFR046C & 5 & 7 & 6 & 25.55539 & 50.53846 \\
\hline \multirow[t]{2}{*}{ SCS3 } & YGL126W & 5 & 7 & 7 & -7.42916 & 39.58979 \\
\hline & YFR045W & 5 & 7 & 8 & 19.07123 & 48.854 \\
\hline \multirow[t]{2}{*}{ MET13 } & YGL125W & 5 & 7 & 9 & 32.81173 & 43.16734 \\
\hline & YFR044C & 5 & 7 & 10 & -11.6319 & 40.3152 \\
\hline \multirow[t]{2}{*}{ MON1 } & YGL124C & 5 & 7 & 11 & 40.1798 & 11.34273 \\
\hline & YFR043C & 5 & 7 & 12 & 26.57264 & 48.5217 \\
\hline GPG1 & YGL121C & 5 & 7 & 13 & -0.24478 & 49.13528 \\
\hline \multirow[t]{2}{*}{ ERJ5 } & YFR041C & 5 & 7 & 14 & 19.18937 & 68.87598 \\
\hline & YGL118C & 5 & 7 & 15 & 7.559597 & 44.7584 \\
\hline \multirow[t]{3}{*}{ SAP155 } & YFR040W & 5 & 7 & 16 & 26.62449 & 42.76555 \\
\hline & YGL117W & 5 & 7 & 17 & 31.32502 & 40.39826 \\
\hline & YFR039C & 5 & 7 & 18 & 24.13451 & 48.60051 \\
\hline \multirow[t]{4}{*}{ SNF4 } & YGL115W & 5 & 7 & 19 & 53.77795 & 17.77857 \\
\hline & YFR038W & 5 & 7 & 20 & 29.32051 & 42.46338 \\
\hline & YGL114W & 5 & 7 & 21 & 50.33494 & 25.35634 \\
\hline & YFR035C & 5 & 7 & 22 & 37.86979 & 35.979 \\
\hline CUE3 & YGL110C & 5 & 7 & 23 & 11.89394 & 47.54487 \\
\hline HIS3 & YOR202W & 5 & 7 & 24 & 31.76075 & \#DIV/0! \\
\hline HIS3 & YOR202W & 5 & 8 & 1 & 40.3497 & \#DIV/0! \\
\hline
\end{tabular}




\begin{tabular}{|c|c|c|c|c|c|c|}
\hline \multirow[t]{2}{*}{ MNT2 } & YGL257C & 5 & 8 & 2 & 36.44233 & 59.63895 \\
\hline & YGR110W & 5 & 8 & 3 & 17.24206 & 59.96801 \\
\hline $\mathrm{ADH} 4$ & YGL256W & 5 & 8 & 4 & 9.224723 & 63.33475 \\
\hline CLB6 & YGR109C & 5 & 8 & 5 & 70.54465 & 51.01465 \\
\hline ZRT1 & YGL255W & 5 & 8 & 6 & 34.9435 & 54.99481 \\
\hline CLB1 & YGR108W & 5 & 8 & 7 & 30.75596 & 57.69148 \\
\hline \multirow[t]{2}{*}{ FZF1 } & YGL254W & 5 & 8 & 8 & 13.35593 & 52.68921 \\
\hline & YGR107W & 5 & 8 & 9 & 3.298955 & 56.59561 \\
\hline \multirow[t]{2}{*}{ HXK2 } & YGL253W & 5 & 8 & 10 & 9.211843 & 45.2618 \\
\hline & YGR106C & 5 & 8 & 11 & 19.15047 & 57.88176 \\
\hline RTG2 & YGL252C & 5 & 8 & 12 & 30.01369 & 42.78575 \\
\hline VMA21 & YGR105W & 5 & 8 & 13 & \#DIV/0! & 36.2608 \\
\hline HFM1 & YGL251C & 5 & 8 & 14 & -3.68424 & 55.14255 \\
\hline \multirow[t]{2}{*}{ PCP1 } & YGR101W & 5 & 8 & 15 & \#DIV/0! & 49.70399 \\
\hline & YGL250W & 5 & 8 & 16 & 46.71986 & 49.44512 \\
\hline MDR1 & YGR100W & 5 & 8 & 17 & 30.33162 & 56.713 \\
\hline ZIP2 & YGL249W & 5 & 8 & 18 & 36.68491 & 36.10003 \\
\hline ASK10 & YGR097W & 5 & 8 & 19 & 41.96953 & 64.62403 \\
\hline PDE1 & YGL248W & 5 & 8 & 20 & 45.43249 & 34.52565 \\
\hline TPC1 & YGR096W & 5 & 8 & 21 & 1.523938 & 40.24844 \\
\hline \multirow[t]{2}{*}{ RTF1 } & YGL244W & 5 & 8 & 22 & 28.54289 & 3.996555 \\
\hline & YGR093W & 5 & 8 & 23 & 29.35205 & 40.79286 \\
\hline HIS3 & YOR202W & 5 & 8 & 24 & 22.94946 & \#DIV/0! \\
\hline \multirow[t]{2}{*}{ HIS3 } & YOR202W & 5 & 9 & 1 & 59.65734 & \#DIV/0! \\
\hline & YGL007W & 5 & 9 & 2 & 50.64212 & 34.98713 \\
\hline ARO2 & YGL148W & 5 & 9 & 3 & 43.50715 & 59.61124 \\
\hline PMC1 & YGL006W & 5 & 9 & 4 & 45.59756 & 49.41851 \\
\hline RPL9A & YGL147C & 5 & 9 & 5 & 37.15267 & 33.47117 \\
\hline \multirow[t]{2}{*}{ COG7 } & YGL005C & 5 & 9 & 6 & 27.81867 & 48.72951 \\
\hline & YGL146C & 5 & 9 & 7 & 26.02711 & 52.46429 \\
\hline RPN14 & YGL004C & 5 & 9 & 8 & -1.1104 & 40.1271 \\
\hline ROG1 & YGL144C & 5 & 9 & 9 & 21.26261 & 41.91592 \\
\hline ERP6 & YGL002W & 5 & 9 & 10 & 45.04695 & 41.34009 \\
\hline \multirow[t]{8}{*}{ HUL5 } & YGL141W & 5 & 9 & 11 & 25.25001 & 52.38663 \\
\hline & YFR057W & 5 & 9 & 12 & 30.57476 & 42.80182 \\
\hline & YGL140C & 5 & 9 & 13 & 45.95875 & 46.67549 \\
\hline & YFR056C & 5 & 9 & 14 & 9.285527 & 50.17541 \\
\hline & YGL139W & 5 & 9 & 15 & 33.41252 & 32.09511 \\
\hline & YFR055W & 5 & 9 & 16 & 32.05671 & 50.54241 \\
\hline & YGL138C & 5 & 9 & 17 & 37.67831 & 41.04405 \\
\hline & YFR054C & 5 & 9 & 18 & -0.18818 & 35.79418 \\
\hline MRM2 & YGL136C & 5 & 9 & 19 & 17.23816 & 41.25967 \\
\hline HXK1 & YFR053C & 5 & 9 & 20 & 15.68049 & 45.84332 \\
\hline
\end{tabular}




\begin{tabular}{|c|c|c|c|c|c|c|}
\hline ITC1 & YGL133W & 5 & 9 & 21 & 42.20611 & 49.1976 \\
\hline \multirow[t]{2}{*}{ YMR31 } & YFR049W & 5 & 9 & 22 & 38.34615 & 26.04361 \\
\hline & YGL132W & 5 & 9 & 23 & 43.40811 & 40.74744 \\
\hline HIS3 & YOR202W & 5 & 9 & 24 & 40.48503 & \#DIV/0! \\
\hline HIS3 & YOR202W & 5 & 10 & 1 & 42.18125 & \#DIV/0! \\
\hline \multirow[t]{2}{*}{ STF2 } & YGR008C & 5 & 10 & 2 & 35.81112 & 36.57777 \\
\hline & YGR130C & 5 & 10 & 3 & 41.01938 & 23.54381 \\
\hline MUQ1 & YGR007W & 5 & 10 & 4 & 20.64533 & 58.69689 \\
\hline SYF2 & YGR129W & 5 & 10 & 5 & 42.37855 & 46.36585 \\
\hline \multirow[t]{2}{*}{ PEX31 } & YGR004W & 5 & 10 & 6 & 16.54416 & 54.57442 \\
\hline & YGR127W & 5 & 10 & 7 & 22.25023 & 55.62733 \\
\hline \multirow[t]{4}{*}{ CUL3 } & YGR003W & 5 & 10 & 8 & 41.56154 & 56.77115 \\
\hline & YGR126W & 5 & 10 & 9 & 26.3824 & 39.69996 \\
\hline & YGR001C & 5 & 10 & 10 & 20.80361 & 41.06684 \\
\hline & YGR125W & 5 & 10 & 11 & 17.16395 & 41.71358 \\
\hline $\cos 12$ & YGL263W & 5 & 10 & 12 & 26.26455 & 31.45695 \\
\hline \multirow[t]{2}{*}{ ASN2 } & YGR124W & 5 & 10 & 13 & 28.59173 & 31.43706 \\
\hline & YGL262W & 5 & 10 & 14 & 62.63813 & 76.53481 \\
\hline \multirow[t]{2}{*}{ PPT1 } & YGR123C & 5 & 10 & 15 & 16.16338 & 49.12468 \\
\hline & YGL261C & 5 & 10 & 16 & 40.48697 & 47.2797 \\
\hline \multirow[t]{2}{*}{ MEP1 } & YGR121C & 5 & 10 & 17 & \#DIV/0! & 40.16194 \\
\hline & YGL260W & 5 & 10 & 18 & 23.84223 & 32.10322 \\
\hline RPS23A & YGR118W & 5 & 10 & 19 & \#DIV/0! & 47.53959 \\
\hline \multirow[t]{2}{*}{ YPS5 } & YGL259W & 5 & 10 & 20 & 21.359 & 35.7439 \\
\hline & YGR117C & 5 & 10 & 21 & 16.98881 & 56.25683 \\
\hline \multirow[t]{2}{*}{ VEL1 } & YGL258W & 5 & 10 & 22 & 9.368101 & 28.101 \\
\hline & YGR111W & 5 & 10 & 23 & 13.32634 & 53.02986 \\
\hline HIS3 & YOR202W & 5 & 10 & 24 & 31.69167 & \#DIV/0! \\
\hline HIS3 & YOR202W & 5 & 11 & 1 & 29.42712 & \#DIV/0! \\
\hline PIB2 & YGL023C & 5 & 11 & 2 & 51.38958 & 55.44075 \\
\hline YIP5 & YGL161C & 5 & 11 & 3 & 48.37193 & 51.88847 \\
\hline \multirow[t]{2}{*}{ ALK1 } & YGL021W & 5 & 11 & 4 & 42.93855 & 45.11814 \\
\hline & YGL160W & 5 & 11 & 5 & 50.06017 & 33.18525 \\
\hline \multirow[t]{2}{*}{ MDM39 } & YGL020C & 5 & 11 & 6 & 24.34202 & 31.08822 \\
\hline & YGL159W & 5 & 11 & 7 & -0.07514 & 31.09525 \\
\hline CKB1 & YGL019W & 5 & 11 & 8 & 23.97843 & 62.52214 \\
\hline RCK1 & YGL158W & 5 & 11 & 9 & 18.55562 & 44.04701 \\
\hline \multirow[t]{2}{*}{ ATE1 } & YGL017W & 5 & 11 & 10 & 35.03785 & 37.60333 \\
\hline & YGL157W & 5 & 11 & 11 & 48.08284 & 65.77471 \\
\hline KAP122 & YGL016W & 5 & 11 & 12 & 70.96038 & 41.46669 \\
\hline \multirow[t]{2}{*}{ AMS1 } & YGL156W & 5 & 11 & 13 & 9.729354 & 48.46625 \\
\hline & YGL015C & 5 & 11 & 14 & 18.07569 & 49.54029 \\
\hline LYS5 & YGL154C & 5 & 11 & 15 & 21.05505 & 36.67463 \\
\hline
\end{tabular}




\begin{tabular}{|c|c|c|c|c|c|c|}
\hline PUF4 & YGL014W & 5 & 11 & 16 & 27.97822 & 28.83269 \\
\hline PEX14 & YGL153W & 5 & 11 & 17 & 26.41696 & 36.94136 \\
\hline \multirow[t]{3}{*}{ PDR1 } & YGL013C & 5 & 11 & 18 & 60.69326 & 56.88967 \\
\hline & YGL152C & 5 & 11 & 19 & 14.56558 & 42.85002 \\
\hline & YGL010W & 5 & 11 & 20 & 23.80071 & 17.21622 \\
\hline NUT1 & YGL151W & 5 & 11 & 21 & 65.98859 & 50.93342 \\
\hline \multirow[t]{2}{*}{ LEU1 } & YGL009C & 5 & 11 & 22 & 26.28132 & 39.00463 \\
\hline & YGL149W & 5 & 11 & 23 & 35.20848 & 56.02981 \\
\hline HIS3 & YOR202W & 5 & 11 & 24 & 37.32039 & \#DIV/0! \\
\hline \multirow[t]{2}{*}{ HIS3 } & YOR202W & 5 & 12 & 1 & 13.41719 & \#DIV/0! \\
\hline & YGR022C & 5 & 12 & 2 & 19.93755 & 41.98435 \\
\hline \multirow[t]{2}{*}{ BTN2 } & YGR142W & 5 & 12 & 3 & 46.09586 & 53.38681 \\
\hline & YGR021W & 5 & 12 & 4 & 30.09553 & 19.53655 \\
\hline VPS62 & YGR141W & 5 & 12 & 5 & 66.38116 & 48.973 \\
\hline \multirow[t]{3}{*}{ UGA1 } & YGR019W & 5 & 12 & 6 & 27.23968 & 54.54446 \\
\hline & YGR139W & 5 & 12 & 7 & 0.406378 & 56.96999 \\
\hline & YGR018C & 5 & 12 & 8 & 33.15949 & 47.66957 \\
\hline \multirow[t]{4}{*}{ TPO2 } & YGR138C & 5 & 12 & 9 & 22.12748 & 33.45519 \\
\hline & YGR017W & 5 & 12 & 10 & 35.65586 & 49.22754 \\
\hline & YGR137W & 5 & 12 & 11 & 41.12107 & 48.40241 \\
\hline & YGR016W & 5 & 12 & 12 & 15.76573 & 51.52479 \\
\hline \multirow[t]{2}{*}{ LSB1 } & YGR136W & 5 & 12 & 13 & 30.57796 & 54.18209 \\
\hline & YGR015C & 5 & 12 & 14 & 15.55593 & 49.9283 \\
\hline PRE9 & YGR135W & 5 & 12 & 15 & 25.56424 & 30.0895 \\
\hline MSB2 & YGR014W & 5 & 12 & 16 & 9.633324 & 44.18993 \\
\hline \multirow[t]{2}{*}{ CAF130 } & YGR134W & 5 & 12 & 17 & 44.88369 & 57.90344 \\
\hline & YGR012W & 5 & 12 & 18 & 23.99419 & 38.70412 \\
\hline \multirow[t]{2}{*}{ PEX4 } & YGR133W & 5 & 12 & 19 & 4.072959 & 54.14942 \\
\hline & YGR011W & 5 & 12 & 20 & 31.45248 & 48.66923 \\
\hline PHB1 & YGR132C & 5 & 12 & 21 & 22.2651 & 36.20644 \\
\hline \multirow[t]{2}{*}{ NMA2 } & YGR010W & 5 & 12 & 22 & 37.2945 & 42.87202 \\
\hline & YGR131W & 5 & 12 & 23 & 24.6833 & 53.84169 \\
\hline HIS3 & YOR202W & 5 & 12 & 24 & 37.06482 & \#DIV/0! \\
\hline \multirow[t]{2}{*}{ HIS3 } & YOR202W & 5 & 13 & 1 & 2.855737 & \#DIV/0! \\
\hline & YGL041C & 5 & 13 & 2 & 27.63797 & 37.81096 \\
\hline \multirow[t]{2}{*}{ SAE2 } & YGL175C & 5 & 13 & 3 & 36.23059 & 41.53858 \\
\hline & YGL039W & 5 & 13 & 4 & 28.824 & 52.36343 \\
\hline \multirow[t]{2}{*}{ BUD13 } & YGL174W & 5 & 13 & 5 & 7.327883 & 24.84531 \\
\hline & YGL036W & 5 & 13 & 6 & 55.96879 & 45.3536 \\
\hline KEM1 & YGL173C & 5 & 13 & 7 & 90.93231 & 82.93234 \\
\hline MIG1 & YGL035C & 5 & 13 & 8 & 60.97222 & 66.38851 \\
\hline \multirow[t]{2}{*}{ SPO74 } & YGL170C & 5 & 13 & 9 & 21.19815 & 49.41396 \\
\hline & YGL034C & 5 & 13 & 10 & 37.47898 & 39.95467 \\
\hline
\end{tabular}




\begin{tabular}{|c|c|c|c|c|c|c|}
\hline HUR1 & YGL168W & 5 & 13 & 11 & 34.86246 & -1.91933 \\
\hline AGA2 & YGL032C & 5 & 13 & 12 & 49.68201 & 65.55195 \\
\hline PMR1 & YGL167C & 5 & 13 & 13 & 51.45259 & 16.73868 \\
\hline RPL24A & YGL031C & 5 & 13 & 14 & 43.05286 & 32.18977 \\
\hline CUP2 & YGL166W & 5 & 13 & 15 & 26.02628 & 38.64701 \\
\hline \multirow[t]{2}{*}{ SCW11 } & YGL028C & 5 & 13 & 16 & 19.31242 & 34.81853 \\
\hline & YGL165C & 5 & 13 & 17 & 37.07985 & 49.54145 \\
\hline CWH41 & YGL027C & 5 & 13 & 18 & 33.1946 & 41.04168 \\
\hline YRB30 & YGL164C & 5 & 13 & 19 & 26.25939 & 29.57825 \\
\hline TRP5 & YGL026C & 5 & 13 & 20 & 41.30613 & 72.41394 \\
\hline \multirow[t]{2}{*}{ RAD54 } & YGL163C & 5 & 13 & 21 & \#DIV/0! & 46.91052 \\
\hline & YGL024W & 5 & 13 & 22 & 88.97161 & 71.03039 \\
\hline SUT1 & YGL162W & 5 & 13 & 23 & 43.8145 & 51.147 \\
\hline HIS3 & YOR202W & 5 & 13 & 24 & 33.85362 & \#DIV/0! \\
\hline HIS3 & YOR202W & 5 & 14 & 1 & 7.400167 & \#DIV/0! \\
\hline ACB1 & YGR037C & 5 & 14 & 2 & 13.35239 & 44.90217 \\
\hline \multirow[t]{2}{*}{ RTS3 } & YGR161C & 5 & 14 & 3 & 23.16224 & 51.31695 \\
\hline & YGR035C & 5 & 14 & 4 & 41.32242 & 53.44766 \\
\hline $\mathrm{CHO} 2$ & YGR157W & 5 & 14 & 5 & 72.61742 & 45.13724 \\
\hline \multirow[t]{2}{*}{ RPL26B } & YGR034W & 5 & 14 & 6 & 48.40928 & 58.1908 \\
\hline & YGR154C & 5 & 14 & 7 & 62.14598 & 25.90909 \\
\hline FMP17 & YGR033C & 5 & 14 & 8 & 46.77966 & 58.1606 \\
\hline TOS10 & YGR153W & 5 & 14 & 9 & 52.93708 & 59.1804 \\
\hline GSC2 & YGR032W & 5 & 14 & 10 & 48.27302 & 67.36949 \\
\hline \multirow[t]{3}{*}{ RSR1 } & YGR152C & 5 & 14 & 11 & 36.44667 & 53.48892 \\
\hline & YGR031W & 5 & 14 & 12 & 45.30311 & 46.71845 \\
\hline & YGR151C & 5 & 14 & 13 & 21.26239 & 41.48229 \\
\hline \multirow[t]{2}{*}{ MSP1 } & YGR028W & 5 & 14 & 14 & 28.07551 & 53.60531 \\
\hline & YGR149W & 5 & 14 & 15 & 29.67173 & 48.80595 \\
\hline RPS25A & YGR027C & 5 & 14 & 16 & 63.17844 & 73.59317 \\
\hline \multirow[t]{4}{*}{ RPL24B } & YGR148C & 5 & 14 & 17 & 58.56273 & 43.46178 \\
\hline & YGR026W & 5 & 14 & 18 & 51.50711 & 48.21942 \\
\hline & YGR146C & 5 & 14 & 19 & 59.59547 & 46.40141 \\
\hline & YGR025W & 5 & 14 & 20 & 29.98778 & 33.12875 \\
\hline THI4 & YGR144W & 5 & 14 & 21 & 18.29138 & 47.49086 \\
\hline MTL1 & YGR023W & 5 & 14 & 22 & 10.12913 & 46.9907 \\
\hline SKN1 & YGR143W & 5 & 14 & 23 & 6.970411 & 32.7551 \\
\hline HIS3 & YOR202W & 5 & 14 & 24 & 5.952991 & \#DIV/0! \\
\hline \multirow[t]{3}{*}{ HIS3 } & YOR202W & 5 & 15 & 1 & 11.51248 & \#DIV/O! \\
\hline & YGL059W & 5 & 15 & 2 & 33.87478 & \#DIV/0! \\
\hline & YGL199C & 5 & 15 & 3 & 59.78737 & \#DIV/0! \\
\hline RAD6 & YGL058W & 5 & 15 & 4 & 32.23311 & \#DIV/0! \\
\hline YIP4 & YGL198W & 5 & 15 & 5 & 47.91209 & \#DIV/0! \\
\hline
\end{tabular}




\begin{tabular}{|c|c|c|c|c|c|c|}
\hline & YGL057C & 5 & 15 & 6 & 35.54447 & \#DIV/0! \\
\hline MDS3 & YGL197W & 5 & 15 & 7 & 50.17678 & \#DIV/0! \\
\hline \multirow[t]{2}{*}{ SDS23 } & YGL056C & 5 & 15 & 8 & 39.64406 & \#DIV/0! \\
\hline & YGL196W & 5 & 15 & 9 & 48.95521 & \#DIV/0! \\
\hline ERV14 & YGL054C & 5 & 15 & 10 & 58.18701 & \#DIV/0! \\
\hline GCN1 & YGL195W & 5 & 15 & 11 & 44.24714 & \#DIV/0! \\
\hline PRM8 & YGL053W & 5 & 15 & 12 & 65.70195 & \#DIV/0! \\
\hline HOS2 & YGL194C & 5 & 15 & 13 & 20.42526 & \#DIV/0! \\
\hline MST27 & YGL051W & 5 & 15 & 14 & 45.7339 & \#DIV/0! \\
\hline \multirow[t]{2}{*}{ GTS1 } & YGL181W & 5 & 15 & 15 & 64.21906 & \#DIV/0! \\
\hline & YGL050W & 5 & 15 & 16 & 40.50079 & \#DIV/0! \\
\hline \multirow[t]{2}{*}{ ATG1 } & YGL180W & 5 & 15 & 17 & 22.24194 & \#DIV/0! \\
\hline & YGL046W & 5 & 15 & 18 & 63.08408 & \#DIV/0! \\
\hline TOS3 & YGL179C & 5 & 15 & 19 & 21.83325 & \#DIV/0! \\
\hline \multirow[t]{2}{*}{ RIM8 } & YGL045W & 5 & 15 & 20 & 73.76697 & \#DIV/0! \\
\hline & YGL177W & 5 & 15 & 21 & 65.76399 & \#DIV/0! \\
\hline \multirow[t]{2}{*}{ DST1 } & YGL043W & 5 & 15 & 22 & 65.63447 & \#DIV/0! \\
\hline & YGL176C & 5 & 15 & 23 & 15.24525 & \#DIV/0! \\
\hline HIS3 & YOR202W & 5 & 15 & 24 & 7.060082 & \#DIV/0! \\
\hline HIS3 & YOR202W & 5 & 16 & 1 & 31.74008 & \#DIV/0! \\
\hline HIS3 & YOR202W & 5 & 16 & 2 & 37.55392 & \#DIV/0! \\
\hline HIS3 & YOR202W & 5 & 16 & 3 & 31.6662 & \#DIV/0! \\
\hline HIS3 & YOR202W & 5 & 16 & 4 & 19.206 & \#DIV/0! \\
\hline HIS3 & YOR202W & 5 & 16 & 5 & 9.082818 & \#DIV/0! \\
\hline HIS3 & YOR202W & 5 & 16 & 6 & 37.29934 & \#DIV/0! \\
\hline HIS3 & YOR202W & 5 & 16 & 7 & 12.3627 & \#DIV/0! \\
\hline HIS3 & YOR202W & 5 & 16 & 8 & 22.64632 & \#DIV/0! \\
\hline HIS3 & YOR202W & 5 & 16 & 9 & 44.25205 & \#DIV/0! \\
\hline HIS3 & YOR202W & 5 & 16 & 10 & 20.50153 & \#DIV/0! \\
\hline HIS3 & YOR202W & 5 & 16 & 11 & 41.20891 & \#DIV/0! \\
\hline HIS3 & YOR202W & 5 & 16 & 12 & 25.87216 & \#DIV/0! \\
\hline HIS3 & YOR202W & 5 & 16 & 13 & 54.23153 & \#DIV/0! \\
\hline HIS3 & YOR202W & 5 & 16 & 14 & 50.94189 & \#DIV/0! \\
\hline HIS3 & YOR202W & 5 & 16 & 15 & 22.17765 & \#DIV/0! \\
\hline HIS3 & YOR202W & 5 & 16 & 16 & 33.78083 & \#DIV/0! \\
\hline HIS3 & YOR202W & 5 & 16 & 17 & 51.97084 & \#DIV/0! \\
\hline HIS3 & YOR202W & 5 & 16 & 18 & 39.06077 & \#DIV/0! \\
\hline HIS3 & YOR202W & 5 & 16 & 19 & 51.23042 & \#DIV/0! \\
\hline HIS3 & YOR202W & 5 & 16 & 20 & 46.6015 & \#DIV/0! \\
\hline HIS3 & YOR202W & 5 & 16 & 21 & 57.63553 & \#DIV/0! \\
\hline HIS3 & YOR202W & 5 & 16 & 22 & 38.69375 & \#DIV/0! \\
\hline HIS3 & YOR202W & 5 & 16 & 23 & 31.99408 & \#DIV/0! \\
\hline HIS3 & YOR202W & 5 & 16 & 24 & 8.416836 & \#DIV/0! \\
\hline
\end{tabular}




\begin{tabular}{|c|c|c|c|c|c|c|}
\hline HIS3 & YOR202W & 6 & 1 & 1 & 73.3949 & \#DIV/0! \\
\hline HIS3 & YOR202W & 6 & 1 & 2 & 19.83211 & \#DIV/0! \\
\hline HIS3 & YOR202W & 6 & 1 & 3 & \#DIV/0! & \#DIV/0! \\
\hline HIS3 & YOR202W & 6 & 1 & 4 & 30.25847 & \#DIV/0! \\
\hline HIS3 & YOR202W & 6 & 1 & 5 & 10.73291 & \#DIV/0! \\
\hline HIS3 & YOR202W & 6 & 1 & 6 & 19.03256 & \#DIV/0! \\
\hline HIS3 & YOR202W & 6 & 1 & 7 & 20.4219 & \#DIV/0! \\
\hline HIS3 & YOR202W & 6 & 1 & 8 & 17.42204 & \#DIV/0! \\
\hline HIS3 & YOR202W & 6 & 1 & 9 & 46.38518 & \#DIV/0! \\
\hline HIS3 & YOR202W & 6 & 1 & 10 & 5.379879 & \#DIV/0! \\
\hline HIS3 & YOR202W & 6 & 1 & 11 & 58.52087 & \#DIV/0! \\
\hline HIS3 & YOR202W & 6 & 1 & 12 & 10.19726 & \#DIV/0! \\
\hline HIS3 & YOR202W & 6 & 1 & 13 & 2.77953 & \#DIV/0! \\
\hline HIS3 & YOR202W & 6 & 1 & 14 & -6.262 & \#DIV/0! \\
\hline HIS3 & YOR202W & 6 & 1 & 15 & -8.10935 & \#DIV/0! \\
\hline HIS3 & YOR202W & 6 & 1 & 16 & 26.8343 & \#DIV/0! \\
\hline HIS3 & YOR202W & 6 & 1 & 17 & 21.19987 & \#DIV/0! \\
\hline HIS3 & YOR202W & 6 & 1 & 18 & 25.14743 & \#DIV/0! \\
\hline HIS3 & YOR202W & 6 & 1 & 19 & 8.465489 & \#DIV/0! \\
\hline HIS3 & YOR202W & 6 & 1 & 20 & 40.66212 & \#DIV/0! \\
\hline HIS3 & YOR202W & 6 & 1 & 21 & 22.98051 & \#DIV/0! \\
\hline HIS3 & YOR202W & 6 & 1 & 22 & 30.26043 & \#DIV/0! \\
\hline HIS3 & YOR202W & 6 & 1 & 23 & 13.50734 & \#DIV/0! \\
\hline HIS3 & YOR202W & 6 & 1 & 24 & 5.532187 & \#DIV/0! \\
\hline HIS3 & YOR202W & 6 & 2 & 1 & 54.07631 & \#DIV/0! \\
\hline SSF1 & YHR066W & 6 & 2 & 2 & 36.74109 & -1.83794 \\
\hline SVP26 & YHR181W & 6 & 2 & 3 & \#DIV/0! & 21.47876 \\
\hline \multirow[t]{2}{*}{ GIC1 } & YHR061C & 6 & 2 & 4 & \#DIV/0! & 24.26471 \\
\hline & YHR180W & 6 & 2 & 5 & 26.80505 & 54.4228 \\
\hline FYV4 & YHR059W & 6 & 2 & 6 & 26.28295 & 15.33833 \\
\hline OYE2 & YHR179W & 6 & 2 & 7 & 32.56714 & 34.08114 \\
\hline CPR2 & YHR057C & 6 & 2 & 8 & 12.30024 & 46.5276 \\
\hline STB5 & YHR178W & 6 & 2 & 9 & \#DIV/0! & 39.61868 \\
\hline SMF2 & YHR050W & 6 & 2 & 10 & 39.40031 & 41.26905 \\
\hline FMO1 & YHR176W & 6 & 2 & 11 & \#DIV/0! & 31.14899 \\
\hline FSH1 & YHR049W & 6 & 2 & 12 & -2.67765 & 43.3691 \\
\hline \multirow[t]{2}{*}{ ATG7 } & YHR171W & 6 & 2 & 13 & 26.20935 & 39.376 \\
\hline & YHR049C-A & 6 & 2 & 14 & 16.31251 & -2.33844 \\
\hline \multirow[t]{2}{*}{ THP2 } & YHR167W & 6 & 2 & 15 & 13.70792 & 33.945 \\
\hline & YHR048W & 6 & 2 & 16 & 46.80811 & 38.85257 \\
\hline SOL3 & YHR163W & 6 & 2 & 17 & 36.53648 & 39.46488 \\
\hline \multirow[t]{2}{*}{ AAP1' } & YHR047C & 6 & 2 & 18 & 38.04386 & 40.55347 \\
\hline & YHR162W & 6 & 2 & 19 & 22.78439 & 50.78123 \\
\hline
\end{tabular}




\begin{tabular}{|c|c|c|c|c|c|c|}
\hline INM1 & YHR046C & 6 & 2 & 20 & 48.76383 & 30.35756 \\
\hline \multirow[t]{2}{*}{ YAP1801 } & YHR161C & 6 & 2 & 21 & 34.30404 & 44.02599 \\
\hline & YHR045W & 6 & 2 & 22 & 19.28223 & 42.48931 \\
\hline PEX18 & YHR160C & 6 & 2 & 23 & -5.53848 & 38.29496 \\
\hline HIS3 & YOR202W & 6 & 2 & 24 & 8.163675 & \#DIV/O! \\
\hline HIS3 & YOR202W & 6 & 3 & 1 & -4.13213 & \#DIV/0! \\
\hline PBP1 & YGR178C & 6 & 3 & 2 & 20.80026 & 33.31514 \\
\hline SHU1 & YHL006C & 6 & 3 & 3 & 90.42765 & 35.97089 \\
\hline \multirow[t]{3}{*}{ ATF2 } & YGR177C & 6 & 3 & 4 & \#DIV/0! & 23.9622 \\
\hline & YHL005C & 6 & 3 & 5 & -2.13663 & -25.1761 \\
\hline & YGR176W & 6 & 3 & 6 & 10.85933 & 44.31092 \\
\hline LAG1 & YHL003C & 6 & 3 & 7 & 38.7211 & 39.44844 \\
\hline CBP4 & YGR174C & 6 & 3 & 8 & 19.04434 & 20.28977 \\
\hline HSE1 & YHL002W & 6 & 3 & 9 & \#DIV/0! & 21.73023 \\
\hline RBG2 & YGR173W & 6 & 3 & 10 & 56.25964 & 32.2435 \\
\hline $\cos 6$ & YGR295C & 6 & 3 & 11 & -6.21435 & 45.19996 \\
\hline \multirow[t]{2}{*}{ PSD2 } & YGR170W & 6 & 3 & 12 & -10.5031 & 28.92077 \\
\hline & YGR290W & 6 & 3 & 13 & 9.041263 & 37.39796 \\
\hline PUS6 & YGR169C & 6 & 3 & 14 & 8.145165 & 27.75733 \\
\hline \multirow[t]{3}{*}{ MAL13 } & YGR288W & 6 & 3 & 15 & 34.26153 & 36.66249 \\
\hline & YGR168C & 6 & 3 & 16 & 47.52214 & 28.90426 \\
\hline & YGR287C & 6 & 3 & 17 & 42.90298 & 38.59504 \\
\hline KRE11 & YGR166W & 6 & 3 & 18 & 52.86029 & 32.38961 \\
\hline \multirow[t]{2}{*}{$\mathrm{BIO} 2$} & YGR286C & 6 & 3 & 19 & 50.91913 & 33.64561 \\
\hline & YGR164W & 6 & 3 & 20 & 5.432713 & 43.25105 \\
\hline ERV29 & YGR284C & 6 & 3 & 21 & 2.692638 & 33.8384 \\
\hline \multirow[t]{2}{*}{ GTR2 } & YGR163W & 6 & 3 & 22 & 19.02815 & 15.31825 \\
\hline & YGR283C & 6 & 3 & 23 & 13.32971 & 33.97036 \\
\hline HIS3 & YOR202W & 6 & 3 & 24 & 7.124697 & \#DIV/0! \\
\hline HIS3 & YOR202W & 6 & 4 & 1 & -2.60551 & \#DIV/0! \\
\hline KSP1 & YHR082C & 6 & 4 & 2 & 29.79625 & 11.55258 \\
\hline RPN10 & YHR200W & 6 & 4 & 3 & 16.99004 & 36.93192 \\
\hline LRP1 & YHR081W & 6 & 4 & 4 & \#DIV/0! & 32.06637 \\
\hline \multirow[t]{2}{*}{ FMP34 } & YHR199C & 6 & 4 & 5 & \#DIV/0! & 32.18207 \\
\hline & YHR080C & 6 & 4 & 6 & 8.579356 & 47.90373 \\
\hline \multirow[t]{2}{*}{ FMP22 } & YHR198C & 6 & 4 & 7 & 63.9822 & 29.85755 \\
\hline & YHR079C-B & 6 & 4 & 8 & 10.76444 & 46.93955 \\
\hline NVJ1 & YHR195W & 6 & 4 & 9 & \#DIV/0! & 19.05309 \\
\hline IRE1 & YHR079C & 6 & 4 & 10 & 20.68609 & 33.74112 \\
\hline \multirow[t]{2}{*}{ MDM31 } & YHR194W & 6 & 4 & 11 & 14.14983 & 40.37862 \\
\hline & YHR078W & 6 & 4 & 12 & 19.90628 & 45.1289 \\
\hline EGD2 & YHR193C & 6 & 4 & 13 & 8.5453 & 34.47505 \\
\hline NMD2 & YHR077C & 6 & 4 & 14 & 40.85651 & 57.85338 \\
\hline
\end{tabular}




\begin{tabular}{|c|c|c|c|c|c|c|}
\hline CTF8 & YHR191C & 6 & 4 & 15 & 26.04096 & 55.01425 \\
\hline PTC7 & YHR076W & 6 & 4 & 16 & \#DIV/0! & 32.89942 \\
\hline PTH1 & YHR189W & 6 & 4 & 17 & \#DIV/0! & 55.31896 \\
\hline PPE1 & YHR075C & 6 & 4 & 18 & 57.51411 & 53.54483 \\
\hline PFS1 & YHR185C & 6 & 4 & 19 & 35.57787 & 53.1929 \\
\hline OSH3 & YHR073W & 6 & 4 & 20 & 35.45995 & 31.48553 \\
\hline SSP1 & YHR184W & 6 & 4 & 21 & 21.13599 & 37.84258 \\
\hline \multirow[t]{2}{*}{ HTD2 } & YHR067W & 6 & 4 & 22 & 48.23111 & 68.13785 \\
\hline & YHR182W & 6 & 4 & 23 & 23.62088 & 54.75124 \\
\hline HIS3 & YOR202W & 6 & 4 & 24 & -0.36075 & \#DIV/0! \\
\hline HIS3 & YOR202W & 6 & 5 & 1 & -4.02915 & \#DIV/0! \\
\hline FYV8 & YGR196C & 6 & 5 & 2 & 6.797389 & 13.50148 \\
\hline OPI1 & YHL020C & 6 & 5 & 3 & 64.26779 & 44.92131 \\
\hline XKS1 & YGR194C & 6 & 5 & 4 & 31.23337 & 48.63946 \\
\hline APM2 & YHL019C & 6 & 5 & 5 & -8.0247 & 38.34408 \\
\hline \multirow[t]{2}{*}{ PDX1 } & YGR193C & 6 & 5 & 6 & 48.38136 & 54.50639 \\
\hline & YHL017W & 6 & 5 & 7 & -15.4426 & 49.87786 \\
\hline TDH3 & YGR192C & 6 & 5 & 8 & 23.66955 & 39.7105 \\
\hline DUR3 & YHL016C & 6 & 5 & 9 & 18.60103 & 30.23206 \\
\hline $\mathrm{CRH} 1$ & YGR189C & 6 & 5 & 10 & 0.253246 & 29.2793 \\
\hline YLF2 & YHL014C & 6 & 5 & 11 & -3.99561 & 49.64768 \\
\hline \multirow[t]{2}{*}{ BUB1 } & YGR188C & 6 & 5 & 12 & 39.66909 & 55.11214 \\
\hline & YHL013C & 6 & 5 & 13 & 9.237949 & 53.78433 \\
\hline \multirow[t]{2}{*}{ HGH1 } & YGR187C & 6 & 5 & 14 & 78.20812 & 33.16997 \\
\hline & YHL012W & 6 & 5 & 15 & 5.2944 & 39.14626 \\
\hline \multirow[t]{2}{*}{ UBR1 } & YGR184C & 6 & 5 & 16 & -12.5323 & 61.28926 \\
\hline & YHL010C & 6 & 5 & 17 & -1.31212 & 59.88016 \\
\hline QCR9 & YGR183C & 6 & 5 & 18 & 1.35159 & 44.57095 \\
\hline \multirow[t]{3}{*}{ YAP3 } & YHL009C & 6 & 5 & 19 & 81.98368 & 58.78782 \\
\hline & YGR182C & 6 & 5 & 20 & 56.80174 & 40.63415 \\
\hline & YHL008C & 6 & 5 & 21 & 18.00724 & 58.51234 \\
\hline TIM13 & YGR181W & 6 & 5 & 22 & -6.73409 & 50.35579 \\
\hline STE20 & YHL007C & 6 & 5 & 23 & 35.12376 & 56.97918 \\
\hline HIS3 & YOR202W & 6 & 5 & 24 & -1.40157 & \#DIV/0! \\
\hline HIS3 & YOR202W & 6 & 6 & 1 & 46.48882 & \#DIV/0! \\
\hline \multirow[t]{2}{*}{ YPT35 } & YHR105W & 6 & 6 & 2 & 19.09476 & 38.65755 \\
\hline & YIL006W & 6 & 6 & 3 & 13.15401 & 38.97505 \\
\hline GRE3 & YHR104W & 6 & 6 & 4 & 37.81525 & 51.16817 \\
\hline EPS1 & YIL005W & 6 & 6 & 5 & 48.69756 & 50.89635 \\
\hline SBE22 & YHR103W & 6 & 6 & 6 & 8.57391 & 58.73651 \\
\hline \multirow[t]{3}{*}{ INP51 } & YIL002C & 6 & 6 & 7 & 46.52677 & 57.37947 \\
\hline & YHR097C & 6 & 6 & 8 & 4.403783 & 45.72938 \\
\hline & YIL001W & 6 & 6 & 9 & -0.47462 & 30.60479 \\
\hline
\end{tabular}




\begin{tabular}{|c|c|c|c|c|c|c|}
\hline \multirow[t]{4}{*}{ HXT5 } & YHR096C & 6 & 6 & 10 & 21.69105 & 59.54507 \\
\hline & YHR210C & 6 & 6 & 11 & 4.198754 & 52.46112 \\
\hline & YHR095W & 6 & 6 & 12 & 46.0061 & 46.82004 \\
\hline & YHR209W & 6 & 6 & 13 & 4.039219 & 50.2193 \\
\hline HXT1 & YHR094C & 6 & 6 & 14 & 30.96157 & 48.09384 \\
\hline SET5 & YHR207C & 6 & 6 & 15 & 7.337022 & 49.93622 \\
\hline AHT1 & YHR093W & 6 & 6 & 16 & 28.39347 & 42.84283 \\
\hline SKN7 & YHR206W & 6 & 6 & 17 & 23.16288 & 45.39877 \\
\hline HXT4 & YHR092C & 6 & 6 & 18 & -7.06559 & 42.44792 \\
\hline \multirow[t]{2}{*}{ MNL1 } & YHR204W & 6 & 6 & 19 & 25.91754 & 36.67193 \\
\hline & YHR087W & 6 & 6 & 20 & 16.2382 & 53.53263 \\
\hline RPS4B & YHR203C & 6 & 6 & 21 & 35.32061 & 19.17096 \\
\hline \multirow[t]{2}{*}{ NAM8 } & YHR086W & 6 & 6 & 22 & -16.0443 & 30.79198 \\
\hline & YHR202W & 6 & 6 & 23 & 7.038061 & 52.72382 \\
\hline HIS3 & YOR202W & 6 & 6 & 24 & 3.793589 & \#DIV/0! \\
\hline HIS3 & YOR202W & 6 & 7 & 1 & 36.52869 & \#DIV/0! \\
\hline TRX2 & YGR209C & 6 & 7 & 2 & 8.846314 & 33.7424 \\
\hline GOS1 & YHL031C & 6 & 7 & 3 & 39.75921 & 34.44437 \\
\hline SER2 & YGR208W & 6 & 7 & 4 & 21.8844 & 46.90931 \\
\hline \multirow[t]{4}{*}{ ECM29 } & YHL030W & 6 & 7 & 5 & -0.65264 & 47.43616 \\
\hline & YGR207C & 6 & 7 & 6 & 13.2498 & 56.72458 \\
\hline & YHL029C & 6 & 7 & 7 & 21.42274 & 52.86765 \\
\hline & YGR206W & 6 & 7 & 8 & 7.011898 & 41.84002 \\
\hline \multirow[t]{2}{*}{ WSC4 } & YHL028W & 6 & 7 & 9 & 42.22763 & 42.88131 \\
\hline & YGR205W & 6 & 7 & 10 & 29.84207 & 47.94338 \\
\hline \multirow[t]{3}{*}{ RIM101 } & YHL027W & 6 & 7 & 11 & 21.48387 & 43.71369 \\
\hline & YGR203W & 6 & 7 & 12 & 20.54645 & 43.17148 \\
\hline & YHL026C & 6 & 7 & 13 & -1.91241 & 46.94733 \\
\hline РCT1 & YGR202C & 6 & 7 & 14 & 5.882906 & 31.38804 \\
\hline \multirow[t]{2}{*}{ SNF6 } & YHL025W & 6 & 7 & 15 & -3.76227 & 62.96745 \\
\hline & YGR201C & 6 & 7 & 16 & -16.3758 & 42.23144 \\
\hline RIM4 & YHL024W & 6 & 7 & 17 & 6.621069 & 38.19606 \\
\hline ELP2 & YGR200C & 6 & 7 & 18 & 23.01523 & 22.88381 \\
\hline RMD11 & YHL023C & 6 & 7 & 19 & 22.12737 & 23.4551 \\
\hline PMT6 & YGR199W & 6 & 7 & 20 & -6.7367 & 54.06651 \\
\hline SPO11 & YHL022C & 6 & 7 & 21 & -10.3326 & 55.46735 \\
\hline SNG1 & YGR197C & 6 & 7 & 22 & -4.41436 & 43.68343 \\
\hline FMP12 & YHL021C & 6 & 7 & 23 & 2.341754 & 57.04357 \\
\hline HIS3 & YOR202W & 6 & 7 & 24 & 26.77381 & \#DIV/0! \\
\hline HIS3 & YOR202W & 6 & 8 & 1 & 40.45605 & \#DIV/0! \\
\hline TOM71 & YHR117W & 6 & 8 & 2 & 10.4002 & 38.56085 \\
\hline SNL1 & YIL016W & 6 & 8 & 3 & 67.80894 & 44.35638 \\
\hline $\operatorname{cox} 23$ & YHR116W & 6 & 8 & 4 & 61.26612 & 3.9413 \\
\hline
\end{tabular}




\begin{tabular}{|c|c|c|c|c|c|c|}
\hline BAR1 & YIL015W & 6 & 8 & 5 & 10.79429 & 50.58869 \\
\hline \multirow[t]{2}{*}{ DMA1 } & YHR115C & 6 & 8 & 6 & 15.92164 & 49.05735 \\
\hline & YIL015C-A & 6 & 8 & 7 & 26.42114 & 55.51675 \\
\hline BZZ1 & YHR114W & 6 & 8 & 8 & 4.562776 & 54.258 \\
\hline \multirow[t]{2}{*}{ MNT3 } & YIL014W & 6 & 8 & 9 & 22.62647 & 41.81424 \\
\hline & YHR113W & 6 & 8 & 10 & 17.5121 & 47.52992 \\
\hline \multirow[t]{3}{*}{ PDR11 } & YIL013C & 6 & 8 & 11 & 16.54904 & 61.04257 \\
\hline & YHR112C & 6 & 8 & 12 & 56.36624 & 67.58309 \\
\hline & YIL012W & 6 & 8 & 13 & 38.24241 & 20.60984 \\
\hline UBA4 & YHR111W & 6 & 8 & 14 & 12.04256 & 51.45807 \\
\hline TIR3 & YIL011W & 6 & 8 & 15 & 2.818638 & 54.98996 \\
\hline ERP5 & YHR110W & 6 & 8 & 16 & 9.759205 & 40.0812 \\
\hline DOT5 & YIL010W & 6 & 8 & 17 & 19.16891 & 44.93235 \\
\hline CTM1 & YHR109W & 6 & 8 & 18 & 19.82253 & 46.05038 \\
\hline EST3 & YIL009C-A & 6 & 8 & 19 & \#DIV/0! & 64.19758 \\
\hline GGA2 & YHR108W & 6 & 8 & 20 & 13.71357 & 42.48851 \\
\hline URM1 & YIL008W & 6 & 8 & 21 & \#DIV/O! & 16.60341 \\
\hline TRR2 & YHR106W & 6 & 8 & 22 & 64.49547 & 57.55936 \\
\hline NAS2 & YIL007C & 6 & 8 & 23 & 8.699321 & 54.69772 \\
\hline HIS3 & YOR202W & 6 & 8 & 24 & 42.36979 & \#DIV/O! \\
\hline HIS3 & YOR202W & 6 & 9 & 1 & -10.1664 & \#DIV/0! \\
\hline DIE2 & YGR227W & 6 & 9 & 2 & 18.74346 & 31.04561 \\
\hline \multirow[t]{3}{*}{ ECM34 } & YHL043W & 6 & 9 & 3 & 54.42374 & 49.61464 \\
\hline & YGR226C & 6 & 9 & 4 & 2.764336 & 43.28515 \\
\hline & YHL042W & 6 & 9 & 5 & -1.12989 & 43.15011 \\
\hline \multirow[t]{2}{*}{ AMA1 } & YGR225W & 6 & 9 & 6 & 47.13246 & 51.27082 \\
\hline & YHL041W & 6 & 9 & 7 & 23.51528 & 56.71468 \\
\hline AZR1 & YGR224W & 6 & 9 & 8 & 28.39399 & 47.52564 \\
\hline ARN1 & YHL040C & 6 & 9 & 9 & 33.04112 & 56.80455 \\
\hline \multirow[t]{2}{*}{ HSV2 } & YGR223C & 6 & 9 & 10 & 5.920883 & 54.29531 \\
\hline & YHL039W & 6 & 9 & 11 & 62.36288 & 44.73034 \\
\hline \multirow[t]{2}{*}{ TOS2 } & YGR221C & 6 & 9 & 12 & 23.63205 & 55.39656 \\
\hline & YHL037C & 6 & 9 & 13 & 22.43704 & 55.24008 \\
\hline $\mathrm{CCH} 1$ & YGR217W & 6 & 9 & 14 & 19.00864 & 39.45109 \\
\hline MUP3 & YHL036W & 6 & 9 & 15 & 36.85848 & 31.37255 \\
\hline RPSOA & YGR214W & 6 & 9 & 16 & 3.109768 & 60.37559 \\
\hline VMR1 & YHL035C & 6 & 9 & 17 & 34.96325 & 34.66077 \\
\hline RTA1 & YGR213C & 6 & 9 & 18 & 43.44341 & 50.60417 \\
\hline SBP1 & YHL034C & 6 & 9 & 19 & 10.5967 & 37.0255 \\
\hline SLI1 & YGR212W & 6 & 9 & 20 & -4.61158 & 53.82306 \\
\hline \multirow[t]{2}{*}{ RPL8A } & YHL033C & 6 & 9 & 21 & 65.6283 & 53.25847 \\
\hline & YGR210C & 6 & 9 & 22 & 32.33825 & 51.23551 \\
\hline GUT1 & YHL032C & 6 & 9 & 23 & 16.3625 & 57.46834 \\
\hline
\end{tabular}




\begin{tabular}{|c|c|c|c|c|c|c|}
\hline HIS3 & YOR202W & 6 & 9 & 24 & 5.646233 & \#DIV/0! \\
\hline \multirow[t]{3}{*}{ HIS3 } & YOR202W & 6 & 10 & 1 & 5.322136 & \#DIV/0! \\
\hline & YHR132W- & & & & & \\
\hline & $A$ & 6 & 10 & 2 & 23.73629 & 39.08763 \\
\hline CAP2 & YIL034C & 6 & 10 & 3 & 16.19184 & 47.92753 \\
\hline \multirow[t]{3}{*}{ ECM14 } & YHR132C & 6 & 10 & 4 & 17.38248 & 46.71543 \\
\hline & YIL032C & 6 & 10 & 5 & -1.2708 & 38.59025 \\
\hline & YHR131C & 6 & 10 & 6 & 2.008186 & 44.96676 \\
\hline \multirow[t]{3}{*}{ SSM4 } & YIL030C & 6 & 10 & 7 & 35.65949 & 42.41009 \\
\hline & YHR130C & 6 & 10 & 8 & -4.61972 & 50.45091 \\
\hline & YIL029C & 6 & 10 & 9 & 38.51812 & 43.19576 \\
\hline \multirow[t]{3}{*}{ ARP1 } & YHR129C & 6 & 10 & 10 & 0.822865 & 54.99818 \\
\hline & YIL028W & 6 & 10 & 11 & 61.25366 & 41.46689 \\
\hline & YHR127W & 6 & 10 & 12 & \#DIV/0! & 54.56729 \\
\hline \multirow[t]{5}{*}{ KRE27 } & YIL027C & 6 & 10 & 13 & 48.48402 & 35.51155 \\
\hline & YHR126C & 6 & 10 & 14 & \#DIV/0! & 40.87691 \\
\hline & YIL025C & 6 & 10 & 15 & 41.57779 & 43.65176 \\
\hline & YHR125W & 6 & 10 & 16 & 22.96377 & 47.06297 \\
\hline & YIL024C & 6 & 10 & 17 & -0.86689 & 48.52351 \\
\hline \multirow[t]{2}{*}{ NDT80 } & YHR124W & 6 & 10 & 18 & -14.9148 & 48.56527 \\
\hline & YIL023C & 6 & 10 & 19 & 22.24251 & 57.40136 \\
\hline EPT1 & YHR123W & 6 & 10 & 20 & 26.64352 & 40.67155 \\
\hline HIS6 & YIL020C & 6 & 10 & 21 & 12.66771 & 33.13131 \\
\hline LSM12 & YHR121W & 6 & 10 & 22 & 53.00535 & 47.41722 \\
\hline VID28 & YIL017C & 6 & 10 & 23 & 68.12073 & 55.71807 \\
\hline HIS3 & YOR202W & 6 & 10 & 24 & -15.4298 & \#DIV/0! \\
\hline HIS3 & YOR202W & 6 & 11 & 1 & -15.2167 & \#DIV/0! \\
\hline KEL2 & YGR238C & 6 & 11 & 2 & 52.54375 & 56.53123 \\
\hline \multirow[t]{3}{*}{ VPS29 } & YHR012W & 6 & 11 & 3 & -3.77084 & 45.18687 \\
\hline & YGR237C & 6 & 11 & 4 & 21.60891 & 63.01971 \\
\hline & YHR009C & 6 & 11 & 5 & 11.51512 & 35.90379 \\
\hline SPG1 & YGR236C & 6 & 11 & 6 & 40.73465 & 35.49579 \\
\hline \multirow[t]{2}{*}{ STP2 } & YHR006W & 6 & 11 & 7 & 7.304613 & 30.68797 \\
\hline & YGR235C & 6 & 11 & 8 & 14.23355 & 49.22652 \\
\hline GPA1 & YHR005C & 6 & 11 & 9 & 54.65892 & 51.47884 \\
\hline YHB1 & YGR234W & 6 & 11 & 10 & -12.4054 & 52.10505 \\
\hline NEM1 & YHR004C & 6 & 11 & 11 & -7.15758 & 39.64041 \\
\hline \multirow[t]{2}{*}{ PHO81 } & YGR233C & 6 & 11 & 12 & \#DIV/0! & -5.57269 \\
\hline & YHR003C & 6 & 11 & 13 & 1.424723 & 28.01088 \\
\hline \multirow[t]{2}{*}{ PHB2 } & YGR231C & 6 & 11 & 14 & 23.06235 & 59.6919 \\
\hline & YHR001W- & & & & & \\
\hline QCR10 & $A$ & 6 & 11 & 15 & 38.21183 & 41.14345 \\
\hline NAS6 & YGR232W & 6 & 11 & 16 & 3.005224 & 53.11335 \\
\hline
\end{tabular}




\begin{tabular}{|c|c|c|c|c|c|c|}
\hline ARN2 & YHL047C & 6 & 11 & 17 & -2.76036 & 59.95523 \\
\hline \multirow[t]{2}{*}{ BNS1 } & YGR230W & 6 & 11 & 18 & 34.67763 & 44.36996 \\
\hline & YHL046C & 6 & 11 & 19 & 13.11824 & 47.78273 \\
\hline \multirow[t]{4}{*}{ SMI1 } & YGR229C & 6 & 11 & 20 & 15.70328 & 33.80319 \\
\hline & YHL045W & 6 & 11 & 21 & 9.260947 & 37.50736 \\
\hline & YGR228W & 6 & 11 & 22 & 33.3697 & 41.76368 \\
\hline & YHL044W & 6 & 11 & 23 & 13.11395 & 59.74744 \\
\hline HIS3 & YOR202W & 6 & 11 & 24 & 22.95399 & \#DIV/0! \\
\hline HIS3 & YOR202W & 6 & 12 & 1 & 31.62364 & \#DIV/O! \\
\hline DSE2 & YHR143W & 6 & 12 & 2 & 46.44512 & 47.96233 \\
\hline PIG2 & YIL045W & 6 & 12 & 3 & 4.994499 & 37.75652 \\
\hline CHS7 & YHR142W & 6 & 12 & 4 & 15.73843 & 69.77551 \\
\hline \multirow[t]{2}{*}{ AGE2 } & YIL044C & 6 & 12 & 5 & \#DIV/0! & 51.48903 \\
\hline & YHR140W & 6 & 12 & 6 & 8.681371 & 36.86084 \\
\hline \multirow[t]{3}{*}{ CBR1 } & YIL043C & 6 & 12 & 7 & 19.94704 & 52.26389 \\
\hline & YHR139C-A & 6 & 12 & 8 & 12.35022 & 41.99726 \\
\hline & YIL042C & 6 & 12 & 9 & -13.491 & 63.90173 \\
\hline SPS100 & YHR139C & 6 & 12 & 10 & 50.15969 & 34.32944 \\
\hline \multirow[t]{2}{*}{ GVP36 } & YIL041W & 6 & 12 & 11 & 50.29896 & 28.83516 \\
\hline & YHR138C & 6 & 12 & 12 & 9.173267 & 57.61614 \\
\hline APQ12 & YIL040W & 6 & 12 & 13 & -21.8477 & -11.1769 \\
\hline \multirow[t]{2}{*}{ ARO9 } & YHR137W & 6 & 12 & 14 & 70.83291 & 59.90954 \\
\hline & YIL039W & 6 & 12 & 15 & 61.70658 & 57.73223 \\
\hline SPL2 & YHR136C & 6 & 12 & 16 & -4.0555 & 23.79759 \\
\hline NOT3 & YIL038C & 6 & 12 & 17 & 4.302396 & 52.03735 \\
\hline YCK1 & YHR135C & 6 & 12 & 18 & 3.773629 & 37.78349 \\
\hline PRM2 & YIL037C & 6 & 12 & 19 & 3.587086 & 34.61691 \\
\hline WSS1 & YHR134W & 6 & 12 & 20 & 36.08261 & 48.42109 \\
\hline CST6 & YIL036W & 6 & 12 & 21 & 26.37013 & 32.80719 \\
\hline NSG1 & YHR133C & 6 & 12 & 22 & 15.07025 & 35.79856 \\
\hline CKA1 & YIL035C & 6 & 12 & 23 & 34.26541 & 24.75432 \\
\hline HIS3 & YOR202W & 6 & 12 & 24 & -18.9281 & \#DIV/0! \\
\hline \multirow[t]{3}{*}{ HIS3 } & YOR202W & 6 & 13 & 1 & -1.40988 & \#DIV/0! \\
\hline & YGR259C & 6 & 13 & 2 & 3.379462 & 53.3612 \\
\hline & YHR029C & 6 & 13 & 3 & 0.179923 & 65.51054 \\
\hline GND2 & YGR256W & 6 & 13 & 4 & -27.8969 & 48.40461 \\
\hline \multirow[t]{2}{*}{ DAP2 } & YHR028C & 6 & 13 & 5 & \#DIV/0! & 49.69817 \\
\hline & YGR250C & 6 & 13 & 6 & \#DIV/0! & 39.95297 \\
\hline THR1 & YHR025W & 6 & 13 & 7 & 55.72831 & 44.5761 \\
\hline \multirow[t]{2}{*}{ MGA1 } & YGR249W & 6 & 13 & 8 & 45.62198 & 46.71933 \\
\hline & YHR022C & 6 & 13 & 9 & 30.16787 & 59.45271 \\
\hline SOL4 & YGR248W & 6 & 13 & 10 & 8.955304 & 61.01545 \\
\hline
\end{tabular}


YHR021W-

\begin{tabular}{|c|c|c|c|c|c|c|}
\hline ECM12 & $A$ & 6 & 13 & 11 & -0.73427 & 54.19453 \\
\hline CPD1 & YGR247W & 6 & 13 & 12 & 30.59993 & 45.8033 \\
\hline RPS27B & YHR021C & 6 & 13 & 13 & 38.28588 & 60.88117 \\
\hline LSC2 & YGR244C & 6 & 13 & 14 & 16.61137 & 38.19518 \\
\hline ARG4 & YHR018C & 6 & 13 & 15 & 58.96735 & 46.42194 \\
\hline FMP43 & YGR243W & 6 & 13 & 16 & \#DIV/0! & 44.74488 \\
\hline \multirow[t]{2}{*}{ YSC83 } & YHR017W & 6 & 13 & 17 & 42.60289 & 29.02997 \\
\hline & YGR242W & 6 & 13 & 18 & 13.62754 & 48.75867 \\
\hline YSC84 & YHR016C & 6 & 13 & 19 & 34.73791 & 48.43812 \\
\hline YAP1802 & YGR241C & 6 & 13 & 20 & 55.44365 & 30.05486 \\
\hline MIP6 & YHR015W & 6 & 13 & 21 & 9.917365 & 46.67404 \\
\hline PEX21 & YGR239C & 6 & 13 & 22 & 19.63842 & 52.22834 \\
\hline SPO13 & YHR014W & 6 & 13 & 23 & 44.86853 & 29.16522 \\
\hline HIS3 & YOR202W & 6 & 13 & 24 & 18.6096 & \#DIV/0! \\
\hline \multirow[t]{3}{*}{ HIS3 } & YOR202W & 6 & 14 & 1 & 31.90227 & \#DIV/0! \\
\hline & YHR159W & 6 & 14 & 2 & 10.38785 & 54.39167 \\
\hline & YIL059C & 6 & 14 & 3 & \#DIV/0! & 47.50563 \\
\hline \multirow[t]{2}{*}{ KEL1 } & YHR158C & 6 & 14 & 4 & 62.09386 & 48.89304 \\
\hline & YIL058W & 6 & 14 & 5 & 4.631028 & 55.03773 \\
\hline \multirow[t]{2}{*}{ REC104 } & YHR157W & 6 & 14 & 6 & 54.7245 & 54.77653 \\
\hline & YIL057C & 6 & 14 & 7 & 14.77932 & 33.50371 \\
\hline \multirow[t]{2}{*}{ LIN1 } & YHR156C & 6 & 14 & 8 & \#DIV/0! & 41.05929 \\
\hline & YIL056W & 6 & 14 & 9 & 69.78774 & 23.43813 \\
\hline \multirow[t]{2}{*}{ YSP1 } & YHR155W & 6 & 14 & 10 & 63.27751 & 61.11583 \\
\hline & YIL055C & 6 & 14 & 11 & 11.26467 & 41.37605 \\
\hline \multirow[t]{2}{*}{ RTT107 } & YHR154W & 6 & 14 & 12 & 21.33549 & 46.79824 \\
\hline & YIL054W & 6 & 14 & 13 & 45.36452 & 57.19279 \\
\hline SPO16 & YHR153C & 6 & 14 & 14 & \#DIV/0! & 38.72151 \\
\hline RHR2 & YIL053W & 6 & 14 & 15 & 64.91228 & 72.8951 \\
\hline SPO12 & YHR152W & 6 & 14 & 16 & -6.33256 & 18.80677 \\
\hline \multirow[t]{2}{*}{ RPL34B } & YIL052C & 6 & 14 & 17 & 53.01256 & 56.73715 \\
\hline & YHR151C & 6 & 14 & 18 & 1.531271 & 41.61234 \\
\hline PCL7 & YIL050W & 6 & 14 & 19 & 22.14533 & 57.56959 \\
\hline PEX28 & YHR150W & 6 & 14 & 20 & -2.72875 & 59.61589 \\
\hline DFG10 & YIL049W & 6 & 14 & 21 & 35.30317 & 45.42446 \\
\hline CRP1 & YHR146W & 6 & 14 & 22 & \#DIV/0! & 53.83931 \\
\hline SYG1 & YIL047C & 6 & 14 & 23 & \#DIV/0! & 63.18884 \\
\hline HIS3 & YOR202W & 6 & 14 & 24 & 17.68689 & \#DIV/0! \\
\hline HIS3 & YOR202W & 6 & 15 & 1 & 17.54641 & \#DIV/0! \\
\hline BGL2 & YGR282C & 6 & 15 & 2 & 25.39247 & \#DIV/0! \\
\hline DOG1 & YHR044C & 6 & 15 & 3 & 76.1745 & 59.4648 \\
\hline YOR1 & YGR281W & 6 & 15 & 4 & 66.89152 & 42.33403 \\
\hline
\end{tabular}




\begin{tabular}{|c|c|c|c|c|c|c|}
\hline DOG2 & YHR043C & 6 & 15 & 5 & 53.43661 & 43.17436 \\
\hline SCW4 & YGR279C & 6 & 15 & 6 & 52.12102 & 56.22542 \\
\hline SRB2 & YHR041C & 6 & 15 & 7 & 60.11349 & 65.55224 \\
\hline RTT102 & YGR275W & 6 & 15 & 8 & \#DIV/0! & 50.66195 \\
\hline MSC7 & YHR039C & 6 & 15 & 9 & 23.50293 & 46.56423 \\
\hline YTA7 & YGR270W & 6 & 15 & 10 & 24.22931 & 44.74644 \\
\hline \multirow[t]{3}{*}{ PUT2 } & YHR037W & 6 & 15 & 11 & 54.41916 & 57.06293 \\
\hline & YGR269W & 6 & 15 & 12 & 57.24564 & 57.9335 \\
\hline & YHR035W & 6 & 15 & 13 & 70.04043 & 46.63168 \\
\hline HUA1 & YGR268C & 6 & 15 & 14 & \#DIV/0! & 54.60571 \\
\hline \multirow[t]{5}{*}{ PIH1 } & YHR034C & 6 & 15 & 15 & \#DIV/0! & 60.22199 \\
\hline & YGR266W & 6 & 15 & 16 & 50.8638 & 49.28571 \\
\hline & YHR033W & 6 & 15 & 17 & 9.691595 & 51.93094 \\
\hline & YGR263C & 6 & 15 & 18 & 18.71104 & 47.74111 \\
\hline & YHR032W & 6 & 15 & 19 & 44.74774 & 65.01536 \\
\hline APL6 & YGR261C & 6 & 15 & 20 & 95.96774 & 53.08199 \\
\hline RRM3 & YHR031C & 6 & 15 & 21 & 13.72717 & 59.48985 \\
\hline TNA1 & YGR260W & 6 & 15 & 22 & 78.5 & 53.84576 \\
\hline SLT2 & YHR030C & 6 & 15 & 23 & 100 & 94.5223 \\
\hline HIS3 & YOR202W & 6 & 15 & 24 & 29.39022 & \#DIV/0! \\
\hline HIS3 & YOR202W & 6 & 16 & 1 & 19.86513 & \#DIV/0! \\
\hline HIS3 & YOR202W & 6 & 16 & 2 & -23.369 & \#DIV/0! \\
\hline HIS3 & YOR202W & 6 & 16 & 3 & 44.38641 & \#DIV/0! \\
\hline HIS3 & YOR202W & 6 & 16 & 4 & \#DIV/0! & \#DIV/O! \\
\hline HIS3 & YOR202W & 6 & 16 & 5 & 78.94737 & \#DIV/0! \\
\hline HIS3 & YOR202W & 6 & 16 & 6 & -5.94711 & \#DIV/0! \\
\hline HIS3 & YOR202W & 6 & 16 & 7 & 16.3406 & \#DIV/0! \\
\hline HIS3 & YOR202W & 6 & 16 & 8 & -10.0312 & \#DIV/0! \\
\hline HIS3 & YOR202W & 6 & 16 & 9 & -20.6579 & \#DIV/0! \\
\hline HIS3 & YOR202W & 6 & 16 & 10 & 19.9324 & \#DIV/0! \\
\hline HIS3 & YOR202W & 6 & 16 & 11 & 41.62154 & \#DIV/0! \\
\hline HIS3 & YOR202W & 6 & 16 & 12 & -19.7093 & \#DIV/0! \\
\hline HIS3 & YOR202W & 6 & 16 & 13 & 35.85822 & \#DIV/0! \\
\hline HIS3 & YOR202W & 6 & 16 & 14 & 48.59316 & \#DIV/0! \\
\hline HIS3 & YOR202W & 6 & 16 & 15 & 2.507672 & \#DIV/0! \\
\hline HIS3 & YOR202W & 6 & 16 & 16 & -27.0098 & \#DIV/0! \\
\hline HIS3 & YOR202W & 6 & 16 & 17 & -2.05263 & \#DIV/0! \\
\hline HIS3 & YOR202W & 6 & 16 & 18 & -10.0082 & \#DIV/0! \\
\hline HIS3 & YOR202W & 6 & 16 & 19 & -12.1956 & \#DIV/0! \\
\hline HIS3 & YOR202W & 6 & 16 & 20 & -16.1339 & \#DIV/0! \\
\hline HIS3 & YOR202W & 6 & 16 & 21 & -2.82043 & \#DIV/0! \\
\hline HIS3 & YOR202W & 6 & 16 & 22 & 38.77759 & \#DIV/0! \\
\hline HIS3 & YOR202W & 6 & 16 & 23 & 50.5007 & \#DIV/O! \\
\hline
\end{tabular}




\begin{tabular}{|c|c|c|c|c|c|c|}
\hline HIS3 & YOR202W & 6 & 16 & 24 & -2.69978 & \#DIV/0! \\
\hline HIS3 & YOR202W & 7 & 1 & 1 & 47.7708 & \#DIV/0! \\
\hline HIS3 & YOR202W & 7 & 1 & 2 & 40.38395 & \#DIV/0! \\
\hline HIS3 & YOR202W & 7 & 1 & 3 & 13.66037 & \#DIV/0! \\
\hline HIS3 & YOR202W & 7 & 1 & 4 & 22.4272 & \#DIV/0! \\
\hline HIS3 & YOR202W & 7 & 1 & 5 & 49.88736 & \#DIV/0! \\
\hline HIS3 & YOR202W & 7 & 1 & 6 & -0.41928 & \#DIV/O! \\
\hline HIS3 & YOR202W & 7 & 1 & 7 & 21.46954 & \#DIV/0! \\
\hline HIS3 & YOR202W & 7 & 1 & 8 & -9.09607 & \#DIV/0! \\
\hline HIS3 & YOR202W & 7 & 1 & 9 & 20.80531 & \#DIV/0! \\
\hline HIS3 & YOR202W & 7 & 1 & 10 & 36.72352 & \#DIV/0! \\
\hline HIS3 & YOR202W & 7 & 1 & 11 & -8.35666 & \#DIV/0! \\
\hline HIS3 & YOR202W & 7 & 1 & 12 & 10.98883 & \#DIV/O! \\
\hline HIS3 & YOR202W & 7 & 1 & 13 & -0.95144 & \#DIV/0! \\
\hline HIS3 & YOR202W & 7 & 1 & 14 & 14.50915 & \#DIV/0! \\
\hline HIS3 & YOR202W & 7 & 1 & 15 & 45.39158 & \#DIV/0! \\
\hline HIS3 & YOR202W & 7 & 1 & 16 & 15.10454 & \#DIV/0! \\
\hline HIS3 & YOR202W & 7 & 1 & 17 & 5.7703 & \#DIV/0! \\
\hline HIS3 & YOR202W & 7 & 1 & 18 & 3.998793 & \#DIV/0! \\
\hline HIS3 & YOR202W & 7 & 1 & 19 & 0.519678 & \#DIV/0! \\
\hline HIS3 & YOR202W & 7 & 1 & 20 & 3.590053 & \#DIV/0! \\
\hline HIS3 & YOR202W & 7 & 1 & 21 & 28.59914 & \#DIV/0! \\
\hline HIS3 & YOR202W & 7 & 1 & 22 & 14.12521 & \#DIV/0! \\
\hline HIS3 & YOR202W & 7 & 1 & 23 & 48.54083 & \#DIV/0! \\
\hline HIS3 & YOR202W & 7 & 1 & 24 & 37.97724 & \#DIV/0! \\
\hline HIS3 & YOR202W & 7 & 2 & 1 & 74.82021 & \#DIV/0! \\
\hline \multirow[t]{2}{*}{ GZF3 } & YJL110C & 7 & 2 & 2 & 34.25023 & 47.60832 \\
\hline & YJL215C & 7 & 2 & 3 & 35.18566 & 39.43638 \\
\hline PRM10 & YJL108C & 7 & 2 & 4 & 29.99452 & 28.28782 \\
\hline HXT8 & YJL214W & 7 & 2 & 5 & 33.11709 & 53.70342 \\
\hline \multirow[t]{2}{*}{ SET4 } & YJL105W & 7 & 2 & 6 & 15.97757 & 39.60415 \\
\hline & YJL213W & 7 & 2 & 7 & 24.66565 & -14.1195 \\
\hline GSH1 & YJL101C & 7 & 2 & 8 & 48.65296 & 33.98934 \\
\hline OPT1 & YJL212C & 7 & 2 & 9 & 36.35001 & 9.671502 \\
\hline \multirow[t]{2}{*}{ LSB6 } & YJL100W & 7 & 2 & 10 & 42.31195 & 14.57908 \\
\hline & YJL211C & 7 & 2 & 11 & 42.74516 & 31.88341 \\
\hline CHS6 & YJL099W & 7 & 2 & 12 & 38.98858 & 57.93083 \\
\hline PEX2 & YJL210W & 7 & 2 & 13 & 6.038144 & 42.575 \\
\hline SAP185 & YJL098W & 7 & 2 & 14 & \#DIV/0! & 10.04969 \\
\hline NUC1 & YJL208C & 7 & 2 & 15 & 13.8681 & 24.12715 \\
\hline \multirow[t]{2}{*}{ BCK1 } & YJL095W & 7 & 2 & 16 & 1.267879 & 47.19278 \\
\hline & YJL207C & 7 & 2 & 17 & -0.699 & 29.77346 \\
\hline KHA1 & YJL094C & 7 & 2 & 18 & \#DIV/0! & 30.54083 \\
\hline
\end{tabular}




\begin{tabular}{|c|c|c|c|c|c|c|}
\hline & YJL206C-A & 7 & 2 & 19 & 11.0038 & 21.63324 \\
\hline \multirow[t]{2}{*}{ TOK1 } & YJL093C & 7 & 2 & 20 & 23.02256 & 29.91992 \\
\hline & YJL206C & 7 & 2 & 21 & 43.56508 & 22.76062 \\
\hline SIP4 & YJL089W & 7 & 2 & 22 & 4.118804 & 43.64704 \\
\hline RCY1 & YJL204C & 7 & 2 & 23 & 3.32599 & 14.24682 \\
\hline HIS3 & YOR202W & 7 & 2 & 24 & -9.92165 & \#DIV/0! \\
\hline HIS3 & YOR202W & 7 & 3 & 1 & \#DIV/0! & \#DIV/0! \\
\hline \multirow[t]{2}{*}{ SEC28 } & YIL076W & 7 & 3 & 2 & 36.0773 & 30.67695 \\
\hline & YIR020C & 7 & 3 & 3 & 57.73154 & 15.77167 \\
\hline SER33 & YIL074C & 7 & 3 & 4 & -0.67286 & 28.01865 \\
\hline MUC1 & YIR019C & 7 & 3 & 5 & 25.5661 & 15.1139 \\
\hline SPO22 & YIL073C & 7 & 3 & 6 & 19.55394 & 45.1816 \\
\hline YAP5 & YIR018W & 7 & 3 & 7 & 12.42822 & 27.11357 \\
\hline HOP1 & YIL072W & 7 & 3 & 8 & 21.22667 & 34.25635 \\
\hline MET28 & YIR017C & 7 & 3 & 9 & 11.70689 & 31.1778 \\
\hline \multirow[t]{2}{*}{$\mathrm{PCl} 8$} & YIL071C & 7 & 3 & 10 & 36.86233 & 37.92357 \\
\hline & YIR016W & 7 & 3 & 11 & 0.03742 & 26.32539 \\
\hline \multirow[t]{3}{*}{ MAM33 } & YIL070C & 7 & 3 & 12 & 27.78934 & 34.67822 \\
\hline & YIR014W & 7 & 3 & 13 & 11.1672 & 45.27692 \\
\hline & YIL067C & 7 & 3 & 14 & 56.06061 & 18.9896 \\
\hline GAT4 & YIR013C & 7 & 3 & 15 & 2.037701 & 29.06442 \\
\hline RNR3 & YIL066C & 7 & 3 & 16 & 15.21541 & 22.42597 \\
\hline MSL1 & YIR009W & 7 & 3 & 17 & 6.390397 & 41.34706 \\
\hline \multirow[t]{3}{*}{ FIS1 } & YIL065C & 7 & 3 & 18 & -1.4606 & 29.76842 \\
\hline & YIR007W & 7 & 3 & 19 & 48.43445 & 54.67993 \\
\hline & YIL064W & 7 & 3 & 20 & 34.529 & 25.26404 \\
\hline \multirow[t]{3}{*}{ IST3 } & YIR005W & 7 & 3 & 21 & 21.83383 & 16.93578 \\
\hline & YIL060W & 7 & 3 & 22 & 37.03603 & 40.11271 \\
\hline & YIR003W & 7 & 3 & 23 & 5.675394 & 38.178 \\
\hline HIS3 & YOR202W & 7 & 3 & 24 & 3.925965 & \#DIV/0! \\
\hline \multirow[t]{3}{*}{ HIS3 } & YOR202W & 7 & 4 & 1 & 68.1054 & \#DIV/0! \\
\hline & YJL131C & 7 & 4 & 2 & 40.3791 & 43.38887 \\
\hline & YJR011C & 7 & 4 & 3 & 52.75608 & 58.26585 \\
\hline URA2 & YJL130C & 7 & 4 & 4 & 14.92056 & 17.80874 \\
\hline MET3 & YJR010W & 7 & 4 & 5 & 31.5081 & 34.88083 \\
\hline PBS2 & YJL128C & 7 & 4 & 6 & 21.40439 & 26.06538 \\
\hline SPC1 & YJR010C-A & 7 & 4 & 7 & 29.2618 & 41.95291 \\
\hline NIT2 & YJL126W & 7 & 4 & 8 & 10.48045 & 18.67954 \\
\hline $\mathrm{TDH} 2$ & YJR009C & 7 & 4 & 9 & 16.25967 & 22.19947 \\
\hline \multirow[t]{3}{*}{ LSM1 } & YJL124C & 7 & 4 & 10 & 28.4877 & 54.69176 \\
\hline & YJR008W & 7 & 4 & 11 & 13.84545 & 42.0345 \\
\hline & YJL123C & 7 & 4 & 12 & 14.33814 & 25.64111 \\
\hline APL1 & YJR005W & 7 & 4 & 13 & 13.95024 & 29.59685 \\
\hline
\end{tabular}




\begin{tabular}{|c|c|c|c|c|c|c|}
\hline & YJL122W & 7 & 4 & 14 & 36.62504 & 39.79278 \\
\hline & YJR003C & 7 & 4 & 15 & 62.01693 & 59.42503 \\
\hline RPE1 & YJL121C & 7 & 4 & 16 & 18.4159 & 7.655784 \\
\hline AVT1 & YJR001W & 7 & 4 & 17 & 18.30617 & 30.76948 \\
\hline \multirow[t]{2}{*}{ NCA3 } & YJL116C & 7 & 4 & 18 & 61.8551 & 57.7802 \\
\hline & YJL218W & 7 & 4 & 19 & 34.04194 & 30.59075 \\
\hline \multirow[t]{2}{*}{ ASF1 } & YJL115W & 7 & 4 & 20 & 72.1756 & 35.30948 \\
\hline & YJL217W & 7 & 4 & 21 & 21.70957 & 24.90913 \\
\hline \multirow[t]{2}{*}{ MDV1 } & YJL112W & 7 & 4 & 22 & 14.58181 & 38.08756 \\
\hline & YJL216C & 7 & 4 & 23 & 41.20267 & 49.71867 \\
\hline HIS3 & YOR202W & 7 & 4 & 24 & 11.9288 & \#DIV/0! \\
\hline HIS3 & YOR202W & 7 & 5 & 1 & 77.26236 & \#DIV/0! \\
\hline PRK1 & YIL095W & 7 & 5 & 2 & 43.15202 & 1.376147 \\
\hline MGA2 & YIR033W & 7 & 5 & 3 & 30.32771 & 30.9719 \\
\hline RSM25 & YIL093C & 7 & 5 & 4 & 43.7838 & 44.68183 \\
\hline \multirow[t]{2}{*}{ DAL3 } & YIR032C & 7 & 5 & 5 & 39.21682 & 26.09274 \\
\hline & YIL092W & 7 & 5 & 6 & 41.22262 & 18.63813 \\
\hline DAL7 & YIR031C & 7 & 5 & 7 & 38.56436 & 43.47191 \\
\hline ICE2 & YIL090W & 7 & 5 & 8 & 17.60007 & 58.74185 \\
\hline \multirow[t]{2}{*}{ DCG1 } & YIR030C & 7 & 5 & 9 & 19.74513 & 25.28784 \\
\hline & YIL089W & 7 & 5 & 10 & 55.25866 & 57.01375 \\
\hline DAL2 & YIR029W & 7 & 5 & 11 & 11.1147 & 32.10751 \\
\hline AVT7 & YIL088C & 7 & 5 & 12 & 50.00596 & 57.68641 \\
\hline \multirow[t]{2}{*}{ DAL4 } & YIR028W & 7 & 5 & 13 & 23.69568 & 42.20516 \\
\hline & YIL087C & 7 & 5 & 14 & 7.600052 & 46.59544 \\
\hline \multirow[t]{2}{*}{ DAL1 } & YIR027C & 7 & 5 & 15 & 6.122696 & 29.0172 \\
\hline & YIL086C & 7 & 5 & 16 & 14.21783 & 33.35867 \\
\hline MND2 & YIR025W & 7 & 5 & 17 & 10.14981 & 47.67732 \\
\hline SDS3 & YIL084C & 7 & 5 & 18 & 55.66513 & 37.94379 \\
\hline GIF1 & YIR024C & 7 & 5 & 19 & 37.10336 & 11.02279 \\
\hline AIR1 & YIL079C & 7 & 5 & 20 & 11.51909 & 43.18105 \\
\hline \multirow[t]{3}{*}{ DAL81 } & YIR023W & 7 & 5 & 21 & 13.64936 & 23.82281 \\
\hline & YIL077C & 7 & 5 & 22 & 29.92361 & 52.6917 \\
\hline & YIR020W-B & 7 & 5 & 23 & \#DIV/0! & 25.78851 \\
\hline HIS3 & YOR202W & 7 & 5 & 24 & 32.22976 & \#DIV/0! \\
\hline \multirow[t]{2}{*}{ HIS3 } & YOR202W & 7 & 6 & 1 & 46.63869 & \#DIV/0! \\
\hline & YJL144W & 7 & 6 & 2 & 38.76463 & 34.32879 \\
\hline \multirow[t]{2}{*}{ CPR7 } & YJR032W & 7 & 6 & 3 & 39.23194 & 37.92028 \\
\hline & YJL142C & 7 & 6 & 4 & 11.23682 & 47.02553 \\
\hline GEA1 & YJR031C & 7 & 6 & 5 & 12.03122 & 47.02277 \\
\hline \multirow[t]{2}{*}{ YAK1 } & YJL141C & 7 & 6 & 6 & 37.78894 & 45.8675 \\
\hline & YJR030C & 7 & 6 & 7 & 12.23057 & 39.22956 \\
\hline YUR1 & YJL139C & 7 & 6 & 8 & 7.653637 & 39.76197 \\
\hline
\end{tabular}




\begin{tabular}{|c|c|c|c|c|c|c|}
\hline & YJR026W & 7 & 6 & 9 & 16.954 & 26.63061 \\
\hline TIF2 & YJL138C & 7 & 6 & 10 & 80.14676 & 18.4862 \\
\hline BNA1 & YJR025C & 7 & 6 & 11 & 2.025525 & 33.0235 \\
\hline \multirow[t]{2}{*}{ GLG2 } & YJL137C & 7 & 6 & 12 & 16.16224 & 44.19198 \\
\hline & YJR024C & 7 & 6 & 13 & 11.10164 & 38.84404 \\
\hline RPS21B & YJL136C & 7 & 6 & 14 & 16.40041 & 48.41618 \\
\hline \multirow[t]{3}{*}{ REC107 } & YJR021C & 7 & 6 & 15 & 4.527059 & 40.44028 \\
\hline & YJL135W & 7 & 6 & 16 & 31.62676 & 37.09283 \\
\hline & YJR020W & 7 & 6 & 17 & 17.4143 & 33.74566 \\
\hline LCB3 & YJL134W & 7 & 6 & 18 & 6.708104 & 17.16694 \\
\hline TES1 & YJR019C & 7 & 6 & 19 & 11.7803 & 36.24025 \\
\hline \multirow[t]{3}{*}{ MRS3 } & YJL133W & 7 & 6 & 20 & 36.19718 & 27.04331 \\
\hline & YJR015W & 7 & 6 & 21 & -1.34615 & 31.13314 \\
\hline & YJL132W & 7 & 6 & 22 & 40.80967 & 26.17335 \\
\hline RBF22 & YJR014W & 7 & 6 & 23 & 12.69657 & 29.36011 \\
\hline HIS3 & YOR202W & 7 & 6 & 24 & 6.159689 & \#DIV/O! \\
\hline \multirow[t]{4}{*}{ HIS3 } & YOR202W & 7 & 7 & 1 & 85.19313 & \#DIV/0! \\
\hline & YIL110W & 7 & 7 & 2 & 66.34363 & 50.97267 \\
\hline & YJL007C & 7 & 7 & 3 & 50.29534 & 47.48114 \\
\hline & YIL108W & 7 & 7 & 4 & 38.27625 & 36.69446 \\
\hline SYS1 & YJL004C & 7 & 7 & 5 & 34.54136 & 23.01113 \\
\hline \multirow[t]{2}{*}{ PFK26 } & YIL107C & 7 & 7 & 6 & 26.41688 & 43.7593 \\
\hline & YIR044C & 7 & 7 & 7 & 14.5165 & 34.50808 \\
\hline \multirow[t]{2}{*}{ LIT2 } & YIL105C & 7 & 7 & 8 & 2.12414 & 57.01159 \\
\hline & YIR043C & 7 & 7 & 9 & 8.725911 & 26.4186 \\
\hline \multirow[t]{2}{*}{ DPH1 } & YIL103W & 7 & 7 & 10 & 24.85665 & 27.1053 \\
\hline & YIR042C & 7 & 7 & 11 & 38.9013 & 23.29571 \\
\hline XBP1 & YIL101C & 7 & 7 & 12 & 48.84476 & 35.69654 \\
\hline \multirow[t]{2}{*}{ YPS6 } & YIR039C & 7 & 7 & 13 & 24.18227 & 49.09601 \\
\hline & YIL100W & 7 & 7 & 14 & 25.19417 & 36.29777 \\
\hline GTT1 & YIR038C & 7 & 7 & 15 & 30.59072 & 32.33227 \\
\hline SGA1 & YIL099W & 7 & 7 & 16 & 50.96106 & 31.66408 \\
\hline HYR1 & YIR037W & 7 & 7 & 17 & 32.65788 & 36.58001 \\
\hline \multirow[t]{2}{*}{ FMC1 } & YIL098C & 7 & 7 & 18 & 33.70114 & 46.15978 \\
\hline & YIR036C & 7 & 7 & 19 & 56.92452 & 54.66792 \\
\hline \multirow[t]{3}{*}{ FYV10 } & YIL097W & 7 & 7 & 20 & 29.93874 & 49.28528 \\
\hline & YIR035C & 7 & 7 & 21 & 24.02002 & 42.26515 \\
\hline & YIL096C & 7 & 7 & 22 & 36.82871 & 41.18804 \\
\hline LYS1 & YIR034C & 7 & 7 & 23 & 29.56513 & 40.96211 \\
\hline HIS3 & YOR202W & 7 & 7 & 24 & 0.780206 & \#DIV/0! \\
\hline HIS3 & YOR202W & 7 & 8 & 1 & 28.96996 & \#DIV/0! \\
\hline FBP26 & YJL155C & 7 & 8 & 2 & \#DIV/0! & 32.21652 \\
\hline ISY1 & YJR050W & 7 & 8 & 3 & 27.65884 & 32.80727 \\
\hline
\end{tabular}




\begin{tabular}{|c|c|c|c|c|c|c|}
\hline VPS35 & YJL154C & 7 & 8 & 4 & 44.02971 & 42.30014 \\
\hline UTR1 & YJR049C & 7 & 8 & 5 & 23.22889 & 54.6845 \\
\hline INO1 & YJL153C & 7 & 8 & 6 & 16.39696 & 34.74576 \\
\hline \multirow[t]{2}{*}{ CYC1 } & YJR048W & 7 & 8 & 7 & 19.02407 & 22.00413 \\
\hline & YJL152W & 7 & 8 & 8 & 25.71535 & 46.69144 \\
\hline ANB1 & YJR047C & 7 & 8 & 9 & 11.99567 & 23.02577 \\
\hline SNA3 & YJL151C & 7 & 8 & 10 & 29 & 55.0322 \\
\hline \multirow[t]{2}{*}{ POL32 } & YJR043C & 7 & 8 & 11 & 33.85598 & 32.65704 \\
\hline & YJL150W & 7 & 8 & 12 & 25.99093 & 36.10518 \\
\hline \multirow[t]{3}{*}{ GEF1 } & YJR040W & 7 & 8 & 13 & 21.39213 & 35.92995 \\
\hline & YJL149W & 7 & 8 & 14 & 15.55399 & 54.16346 \\
\hline & YJR038C & 7 & 8 & 15 & 8.352542 & 24.98136 \\
\hline \multirow[t]{3}{*}{ RPA34 } & YJL148W & 7 & 8 & 16 & 15.92619 & 49.16349 \\
\hline & YJR037W & 7 & 8 & 17 & 4.436392 & 31.15211 \\
\hline & YJL147C & 7 & 8 & 18 & 12.44308 & 34.53055 \\
\hline HUL4 & YJR036C & 7 & 8 & 19 & 9.87231 & 48.21364 \\
\hline IDS2 & YJL146W & 7 & 8 & 20 & 41.46973 & 31.52032 \\
\hline RAD26 & YJR035W & 7 & 8 & 21 & 31.473 & 26.51947 \\
\hline SFH5 & YJL145W & 7 & 8 & 22 & 19.61102 & 47.58028 \\
\hline RAV1 & YJR033C & 7 & 8 & 23 & 6.202271 & 22.70741 \\
\hline HIS3 & YOR202W & 7 & 8 & 24 & 22.31725 & \#DIV/0! \\
\hline HIS3 & YOR202W & 7 & 9 & 1 & 21.86533 & \#DIV/0! \\
\hline \multirow[t]{2}{*}{ GIN1 } & YIL130W & 7 & 9 & 2 & 17.54745 & 41.84466 \\
\hline & YJL028W & 7 & 9 & 3 & 18.52088 & 51.4514 \\
\hline \multirow[t]{2}{*}{ MET18 } & YIL128W & 7 & 9 & 4 & 18.98921 & 24.11546 \\
\hline & YJL027C & 7 & 9 & 5 & 33.76887 & 23.46795 \\
\hline AYR1 & YIL124W & 7 & 9 & 6 & 8.290944 & 27.10476 \\
\hline APS3 & YJL024C & 7 & 9 & 7 & 6.3126 & 21.78292 \\
\hline SIM1 & YIL123W & 7 & 9 & 8 & 17.66222 & 22.93743 \\
\hline PET130 & YJL023C & 7 & 9 & 9 & \#DIV/0! & 56.41429 \\
\hline \multirow[t]{2}{*}{ QDR1 } & YIL120W & 7 & 9 & 10 & 7.320674 & 51.41733 \\
\hline & YJL022W & 7 & 9 & 11 & 26.24604 & 32.22489 \\
\hline \multirow[t]{2}{*}{ RPI1 } & YIL119C & 7 & 9 & 12 & 1.643552 & 45.72692 \\
\hline & YJL021C & 7 & 9 & 13 & 2.354111 & 25.63522 \\
\hline PRM5 & YIL117C & 7 & 9 & 14 & 24.77286 & 47.1285 \\
\hline $\mathrm{BBC1}$ & YJL020C & 7 & 9 & 15 & 20.58915 & 28.83333 \\
\hline \multirow[t]{2}{*}{ HIS5 } & YIL116W & 7 & 9 & 16 & -2.49869 & 49.7853 \\
\hline & YJL017W & 7 & 9 & 17 & 9.245123 & 42.72347 \\
\hline \multirow[t]{2}{*}{ POR2 } & YIL114C & 7 & 9 & 18 & 18.55456 & 28.03994 \\
\hline & YJL016W & 7 & 9 & 19 & 20.99171 & 37.69828 \\
\hline SDP1 & YIL113W & 7 & 9 & 20 & 23.76166 & 55.53545 \\
\hline MAD3 & YJL013C & 7 & 9 & 21 & 8.62589 & 43.41564 \\
\hline HOS4 & YIL112W & 7 & 9 & 22 & 33.56159 & 53.60689 \\
\hline
\end{tabular}




\begin{tabular}{|c|c|c|c|c|c|c|}
\hline VTC4 & YJL012C & 7 & 9 & 23 & 10.38978 & 28.2717 \\
\hline HIS3 & YOR202W & 7 & 9 & 24 & 12.72539 & \#DIV/0! \\
\hline \multirow[t]{2}{*}{ HIS3 } & YOR202W & 7 & 10 & 1 & 9.502709 & \#DIV/0! \\
\hline & YJL169W & 7 & 10 & 2 & 37.5363 & 46.4637 \\
\hline LIA1 & YJR070C & 7 & 10 & 3 & 46.30792 & 26.68464 \\
\hline SET2 & YJL168C & 7 & 10 & 4 & 24.8652 & 43.6217 \\
\hline HAM1 & YJR069C & 7 & 10 & 5 & 15.96246 & 46.11323 \\
\hline HAL5 & YJL165C & 7 & 10 & 6 & 10.42696 & 55.02449 \\
\hline NTA1 & YJR062C & 7 & 10 & 7 & 25.86247 & 28.37183 \\
\hline \multirow[t]{3}{*}{ TPK1 } & YJL164C & 7 & 10 & 8 & 34.39236 & 37.00919 \\
\hline & YJR061W & 7 & 10 & 9 & 30.01328 & 43.32437 \\
\hline & YJL163C & 7 & 10 & 10 & 29.78569 & 43.60755 \\
\hline CBF1 & YJR060W & 7 & 10 & 11 & 25.24277 & 27.99504 \\
\hline JJJ 2 & YJL162C & 7 & 10 & 12 & 18.55666 & 39.3183 \\
\hline PTK2 & YJR059W & 7 & 10 & 13 & 13.67394 & 32.61599 \\
\hline FMP33 & YJL161W & 7 & 10 & 14 & 16.46137 & 35.13065 \\
\hline \multirow[t]{3}{*}{ APS2 } & YJR058C & 7 & 10 & 15 & 9.627057 & 47.77466 \\
\hline & YJL160C & 7 & 10 & 16 & 8.27646 & 60.89241 \\
\hline & YJR054W & 7 & 10 & 17 & 11.75761 & 39.9122 \\
\hline HSP150 & YJL159W & 7 & 10 & 18 & 4.89723 & 51.99213 \\
\hline BFA1 & YJR053W & 7 & 10 & 19 & 18.87314 & 52.08657 \\
\hline CIS3 & YJL158C & 7 & 10 & 20 & 26.86813 & 45.81961 \\
\hline RAD7 & YJR052W & 7 & 10 & 21 & 2.710864 & 49.71958 \\
\hline FAR1 & YJL157C & 7 & 10 & 22 & 11.69155 & 48.90309 \\
\hline OSM1 & YJR051W & 7 & 10 & 23 & 62.41906 & 31.82731 \\
\hline HIS3 & YOR202W & 7 & 10 & 24 & 29.84778 & \#DIV/0! \\
\hline HIS3 & YOR202W & 7 & 11 & 1 & 29.41265 & \#DIV/0! \\
\hline ECM37 & YIL146C & 7 & 11 & 2 & 37.53181 & 54.28964 \\
\hline UBX6 & YJL048C & 7 & 11 & 3 & 14.68951 & 42.4453 \\
\hline PAN6 & YIL145C & 7 & 11 & 4 & -18.2228 & 41.49546 \\
\hline \multirow[t]{3}{*}{ RTT101 } & YJL047C & 7 & 11 & 5 & 14.23043 & 38.74957 \\
\hline & YIL141W & 7 & 11 & 6 & 2.776484 & 41.83087 \\
\hline & YJL046W & 7 & 11 & 7 & 29.218 & 34.31214 \\
\hline \multirow[t]{2}{*}{ AXL2 } & YIL140W & 7 & 11 & 8 & 8.926363 & 38.67091 \\
\hline & YJL045W & 7 & 11 & 9 & 28.20234 & 44.35154 \\
\hline REV7 & YIL139C & 7 & 11 & 10 & 20.96499 & 48.40502 \\
\hline GYP6 & YJL044C & 7 & 11 & 11 & 13.89994 & 38.33352 \\
\hline \multirow[t]{2}{*}{ TPM2 } & YIL138C & 7 & 11 & 12 & 15.20215 & 46.01585 \\
\hline & YJL043W & 7 & 11 & 13 & 19.19093 & 40.29257 \\
\hline RBF108 & YIL137C & 7 & 11 & 14 & 30.27561 & 34.1401 \\
\hline MHP1 & YJL042W & 7 & 11 & 15 & 7.741018 & 41.70651 \\
\hline \multirow[t]{2}{*}{ VHS2 } & YIL135C & 7 & 11 & 16 & 31.52226 & 48.83854 \\
\hline & YJL038C & 7 & 11 & 17 & 17.53967 & \#DIV/0! \\
\hline
\end{tabular}




\begin{tabular}{|c|c|c|c|c|c|c|}
\hline \multirow[t]{2}{*}{ FLX1 } & YIL134W & 7 & 11 & 18 & 26.6143 & \#DIV/0! \\
\hline & YJL037W & 7 & 11 & 19 & 27.44518 & 45.17339 \\
\hline RPL16A & YIL133C & 7 & 11 & 20 & 44.5295 & 42.1796 \\
\hline SNX4 & YJL036W & 7 & 11 & 21 & 13.77244 & 50.43154 \\
\hline CSM2 & YIL132C & 7 & 11 & 22 & 5.549091 & 57.39135 \\
\hline MAD2 & YJL030W & 7 & 11 & 23 & 24.50176 & 45.39838 \\
\hline HIS3 & YOR202W & 7 & 11 & 24 & 33.93101 & \#DIV/0! \\
\hline HIS3 & YOR202W & 7 & 12 & 1 & 17.4348 & \#DIV/O! \\
\hline \multirow[t]{3}{*}{ MNN5 } & YJL186W & 7 & 12 & 2 & 35.02767 & 44.83443 \\
\hline & YJR087W & 7 & 12 & 3 & 23.56348 & 38.06507 \\
\hline & YJL185C & 7 & 12 & 4 & 2.084556 & 39.16968 \\
\hline CSN12 & YJR084W & 7 & 12 & 5 & 46.37163 & 42.48767 \\
\hline MNN11 & YJL183W & 7 & 12 & 6 & 21.01784 & 22.09951 \\
\hline \multirow[t]{2}{*}{ ACF4 } & YJR083C & 7 & 12 & 7 & 17.02389 & 44.94752 \\
\hline & YJL182C & 7 & 12 & 8 & 7.222796 & 50.65747 \\
\hline \multirow[t]{2}{*}{ EAF6 } & YJR082C & 7 & 12 & 9 & 47.00193 & 22.0017 \\
\hline & YJL181W & 7 & 12 & 10 & 26.10835 & 29.87116 \\
\hline FMP26 & YJR080C & 7 & 12 & 11 & 28.22178 & 38.01618 \\
\hline \multirow[t]{2}{*}{ ATG27 } & YJL178C & 7 & 12 & 12 & 0.980593 & 55.87654 \\
\hline & YJR079W & 7 & 12 & 13 & 16.63608 & 23.78276 \\
\hline SWI3 & YJL176C & 7 & 12 & 14 & 5.903944 & 49.98052 \\
\hline \multirow[t]{2}{*}{ BNA2 } & YJR078W & 7 & 12 & 15 & 6.95806 & 46.88494 \\
\hline & YJL175W & 7 & 12 & 16 & 20.21645 & 55.21114 \\
\hline MIR1 & YJR077C & 7 & 12 & 17 & 13.14708 & \#DIV/O! \\
\hline CPS1 & YJL172W & 7 & 12 & 18 & 8.407078 & \#DIV/0! \\
\hline \multirow[t]{2}{*}{ HOC1 } & YJR075W & 7 & 12 & 19 & 17.04339 & 27.99978 \\
\hline & YJL171C & 7 & 12 & 20 & 5.812764 & 60.81204 \\
\hline MOG1 & YJR074W & 7 & 12 & 21 & 59.32203 & 40.88969 \\
\hline ASG7 & YJL170C & 7 & 12 & 22 & 18.36038 & 48.60526 \\
\hline OPI3 & YJR073C & 7 & 12 & 23 & 19.86079 & 48.71881 \\
\hline HIS3 & YOR202W & 7 & 12 & 24 & 23.26724 & \#DIV/0! \\
\hline \multirow[t]{2}{*}{ HIS3 } & YOR202W & 7 & 13 & 1 & 25.82758 & \#DIV/0! \\
\hline & YIL161W & 7 & 13 & 2 & 20.95964 & 52.39275 \\
\hline DLS1 & YJL065C & 7 & 13 & 3 & \#DIV/0! & 51.75917 \\
\hline \multirow[t]{2}{*}{ POT1 } & YIL160C & 7 & 13 & 4 & 38.39544 & 38.60646 \\
\hline & YJL064W & 7 & 13 & 5 & 16.73449 & 46.42154 \\
\hline BNR1 & YIL159W & 7 & 13 & 6 & 3.492189 & 48.00277 \\
\hline LAS21 & YJL062W & 7 & 13 & 7 & 17.86973 & 33.20954 \\
\hline FMP35 & YIL157C & 7 & 13 & 8 & 23.85519 & 43.12603 \\
\hline BNA3 & YJL060W & 7 & 13 & 9 & 2.971384 & 31.09009 \\
\hline UBP7 & YIL156W & 7 & 13 & 10 & 12.99898 & 34.49572 \\
\hline YHC3 & YJL059W & 7 & 13 & 11 & 0.046589 & 40.78596 \\
\hline GUT2 & YIL155C & 7 & 13 & 12 & 14.6658 & 47.0035 \\
\hline
\end{tabular}




\begin{tabular}{|c|c|c|c|c|c|c|}
\hline BIT61 & YJL058C & 7 & 13 & 13 & 9.135552 & 35.18152 \\
\hline IMP2' & YIL154C & 7 & 13 & 14 & 21.90313 & 52.28834 \\
\hline IKS1 & YJL057C & 7 & 13 & 15 & 22.38092 & 50.69174 \\
\hline \multirow[t]{3}{*}{ RRD1 } & YIL153W & 7 & 13 & 16 & 12.71064 & 58.04691 \\
\hline & YJL055W & 7 & 13 & 17 & 28.07245 & 60.63377 \\
\hline & YIL152W & 7 & 13 & 18 & 5.651009 & 52.39651 \\
\hline PEP8 & YJL053W & 7 & 13 & 19 & 14.89431 & 42.11125 \\
\hline \multirow[t]{2}{*}{ MLP2 } & YIL149C & 7 & 13 & 20 & 10.25048 & 57.8226 \\
\hline & YJL051W & 7 & 13 & 21 & 8.112987 & 12.05836 \\
\hline \multirow[t]{2}{*}{ RPL40A } & YIL148W & 7 & 13 & 22 & 37.29663 & 61.12102 \\
\hline & YJL049W & 7 & 13 & 23 & 39.67426 & 46.60176 \\
\hline HIS3 & YOR202W & 7 & 13 & 24 & 31.49991 & \#DIV/0! \\
\hline HIS3 & YOR202W & 7 & 14 & 1 & 60.67103 & \#DIV/0! \\
\hline ECM25 & YJL201W & 7 & 14 & 2 & 59.35757 & \#DIV/0! \\
\hline YUH1 & YJR099W & 7 & 14 & 3 & 66.80873 & 63.20703 \\
\hline \multirow[t]{2}{*}{ MBB1 } & YJL199C & 7 & 14 & 4 & 34.41558 & 42.42251 \\
\hline & YJR098C & 7 & 14 & 5 & 33.14241 & 45.20132 \\
\hline PHO90 & YJL198W & 7 & 14 & 6 & 31.42791 & 43.43719 \\
\hline JJJ3 & YJR097W & 7 & 14 & 7 & 39.54175 & 50.37772 \\
\hline \multirow[t]{2}{*}{ UBP12 } & YJL197W & 7 & 14 & 8 & 31.99531 & 44.87288 \\
\hline & YJR096W & 7 & 14 & 9 & 33.38163 & 25.79785 \\
\hline ELO1 & YJL196C & 7 & 14 & 10 & 27.85523 & 54.34493 \\
\hline \multirow[t]{2}{*}{ SFC1 } & YJR095W & 7 & 14 & 11 & 37.63941 & 55.68714 \\
\hline & $\begin{array}{l}\text { YJL193W } \\
\text { YJR094W- }\end{array}$ & 7 & 14 & 12 & 36.9697 & 57.32902 \\
\hline RPL43B & $A$ & 7 & 14 & 13 & 20.84681 & 49.24494 \\
\hline SOP4 & YJL192C & 7 & 14 & 14 & 24.0307 & 53.03948 \\
\hline IME1 & YJR094C & 7 & 14 & 15 & 0.50942 & 42.48374 \\
\hline RPS14B & YJL191W & 7 & 14 & 16 & 19.90386 & 43.82435 \\
\hline BUD4 & YJR092W & 7 & 14 & 17 & 19.1874 & 21.41441 \\
\hline RPS22A & YJL190C & 7 & 14 & 18 & 39.58363 & 39.62799 \\
\hline JSN1 & YJR091C & 7 & 14 & 19 & 6.075602 & 60.27102 \\
\hline BUD19 & YJL188C & 7 & 14 & 20 & 36.78122 & 61.94472 \\
\hline GRR1 & YJR090C & 7 & 14 & 21 & 10.00576 & \#DIV/0! \\
\hline \multirow[t]{2}{*}{ SWE1 } & YJL187C & 7 & 14 & 22 & 22.62172 & 50.38937 \\
\hline & YJR088C & 7 & 14 & 23 & 22.65304 & 41.21521 \\
\hline HIS3 & YOR202W & 7 & 14 & 24 & 34.00512 & \#DIV/O! \\
\hline HIS3 & YOR202W & 7 & 15 & 1 & \#DIV/0! & \#DIV/0! \\
\hline MPH1 & YIR002C & 7 & 15 & 2 & 89.90634 & 77.28423 \\
\hline ARG3 & YJL088W & 7 & 15 & 3 & 49.67532 & 21.67089 \\
\hline \multirow[t]{2}{*}{ SGN1 } & YIR001C & 7 & 15 & 4 & 42.75723 & 67.50825 \\
\hline & YJL084C & 7 & 15 & 5 & 8.298104 & 61.15546 \\
\hline VTH1 & YIL173W & 7 & 15 & 6 & 27.17975 & 35.98238 \\
\hline
\end{tabular}




\begin{tabular}{|c|c|c|c|c|c|c|}
\hline TAX4 & YJL083W & 7 & 15 & 7 & 57.22222 & 37.96676 \\
\hline HXT12 & YIL170W & 7 & 15 & 8 & 21.13489 & 47.81903 \\
\hline PRY3 & YJL078C & 7 & 15 & 9 & 26.42962 & 50.33725 \\
\hline SDL1 & YIL168W & 7 & 15 & 10 & 14.84725 & 56.01235 \\
\hline ICS3 & YJL077C & 7 & 15 & 11 & \#DIV/0! & 45.26854 \\
\hline SDL1 & YIL167W & 7 & 15 & 12 & 43.38302 & 40.99666 \\
\hline \multirow[t]{2}{*}{ JEM1 } & YJL073W & 7 & 15 & 13 & 37.00402 & 56.44613 \\
\hline & YIL166C & 7 & 15 & 14 & 18.50204 & 40.93938 \\
\hline \multirow[t]{3}{*}{ ARG2 } & YJL071W & 7 & 15 & 15 & 33.64304 & 49.41623 \\
\hline & YIL165C & 7 & 15 & 16 & 19.09885 & 38.3078 \\
\hline & YJL070C & 7 & 15 & 17 & \#DIV/0! & 55.49415 \\
\hline \multirow[t]{4}{*}{ NIT1 } & YIL164C & 7 & 15 & 18 & 30.04423 & 43.32638 \\
\hline & YJL068C & 7 & 15 & 19 & 20.61348 & 44.52592 \\
\hline & YIL163C & 7 & 15 & 20 & 21.26286 & 45.27166 \\
\hline & YJL067W & 7 & 15 & 21 & 47.9892 & 51.15364 \\
\hline SUC2 & YIL162W & 7 & 15 & 22 & 60.24169 & 63.83548 \\
\hline MPM1 & YJL066C & 7 & 15 & 23 & 76.19647 & 38.39804 \\
\hline HIS3 & YOR202W & 7 & 15 & 24 & 23.05273 & \#DIV/0! \\
\hline HIS3 & YOR202W & 7 & 16 & 1 & 62.18504 & \#DIV/0! \\
\hline HIS3 & YOR202W & 7 & 16 & 2 & 52.76863 & \#DIV/0! \\
\hline HIS3 & YOR202W & 7 & 16 & 3 & 58.32751 & \#DIV/0! \\
\hline HIS3 & YOR202W & 7 & 16 & 4 & 54.15006 & \#DIV/0! \\
\hline HIS3 & YOR202W & 7 & 16 & 5 & 39.00292 & \#DIV/0! \\
\hline HIS3 & YOR202W & 7 & 16 & 6 & 58.07623 & \#DIV/0! \\
\hline HIS3 & YOR202W & 7 & 16 & 7 & 29.51861 & \#DIV/0! \\
\hline HIS3 & YOR202W & 7 & 16 & 8 & 15.16534 & \#DIV/0! \\
\hline HIS3 & YOR202W & 7 & 16 & 9 & 29.68826 & \#DIV/0! \\
\hline HIS3 & YOR202W & 7 & 16 & 10 & 16.34339 & \#DIV/0! \\
\hline HIS3 & YOR202W & 7 & 16 & 11 & 9.368952 & \#DIV/0! \\
\hline HIS3 & YOR202W & 7 & 16 & 12 & 24.54545 & \#DIV/0! \\
\hline HIS3 & YOR202W & 7 & 16 & 13 & 11.53687 & \#DIV/0! \\
\hline HIS3 & YOR202W & 7 & 16 & 14 & 20.82576 & \#DIV/0! \\
\hline HIS3 & YOR202W & 7 & 16 & 15 & 16.19249 & \#DIV/0! \\
\hline HIS3 & YOR202W & 7 & 16 & 16 & 15.44883 & \#DIV/0! \\
\hline HIS3 & YOR202W & 7 & 16 & 17 & 10.37394 & \#DIV/0! \\
\hline HIS3 & YOR202W & 7 & 16 & 18 & 13.14793 & \#DIV/0! \\
\hline HIS3 & YOR202W & 7 & 16 & 19 & 8.999413 & \#DIV/0! \\
\hline HIS3 & YOR202W & 7 & 16 & 20 & 14.67754 & \#DIV/0! \\
\hline HIS3 & YOR202W & 7 & 16 & 21 & 29.07879 & \#DIV/0! \\
\hline HIS3 & YOR202W & 7 & 16 & 22 & 25.99124 & \#DIV/0! \\
\hline HIS3 & YOR202W & 7 & 16 & 23 & 17.14514 & \#DIV/0! \\
\hline HIS3 & YOR202W & 7 & 16 & 24 & 35.68742 & \#DIV/0! \\
\hline HIS3 & YOR202W & 8 & 1 & 1 & 17.5493 & \#DIV/0! \\
\hline
\end{tabular}




\begin{tabular}{|c|c|c|c|c|c|c|}
\hline HIS3 & YOR202W & 8 & 1 & 2 & 9.803929 & \#DIV/0! \\
\hline HIS3 & YOR202W & 8 & 1 & 3 & 12.21997 & \#DIV/0! \\
\hline HIS3 & YOR202W & 8 & 1 & 4 & 36.18506 & \#DIV/0! \\
\hline HIS3 & YOR202W & 8 & 1 & 5 & 13.22311 & \#DIV/0! \\
\hline HIS3 & YOR202W & 8 & 1 & 6 & 27.81013 & \#DIV/0! \\
\hline HIS3 & YOR202W & 8 & 1 & 7 & 31.17164 & \#DIV/0! \\
\hline HIS3 & YOR202W & 8 & 1 & 8 & 42.71052 & \#DIV/0! \\
\hline HIS3 & YOR202W & 8 & 1 & 9 & 45.46983 & \#DIV/0! \\
\hline HIS3 & YOR202W & 8 & 1 & 10 & 40.72495 & \#DIV/0! \\
\hline HIS3 & YOR202W & 8 & 1 & 11 & 39.43731 & \#DIV/0! \\
\hline HIS3 & YOR202W & 8 & 1 & 12 & 26.11342 & \#DIV/0! \\
\hline HIS3 & YOR202W & 8 & 1 & 13 & 14.70019 & \#DIV/0! \\
\hline HIS3 & YOR202W & 8 & 1 & 14 & 24.98425 & \#DIV/0! \\
\hline HIS3 & YOR202W & 8 & 1 & 15 & 43.90235 & \#DIV/0! \\
\hline HIS3 & YOR202W & 8 & 1 & 16 & 24.28837 & \#DIV/0! \\
\hline HIS3 & YOR202W & 8 & 1 & 17 & 12.23695 & \#DIV/0! \\
\hline HIS3 & YOR202W & 8 & 1 & 18 & 38.93114 & \#DIV/0! \\
\hline HIS3 & YOR202W & 8 & 1 & 19 & 42.50731 & \#DIV/0! \\
\hline HIS3 & YOR202W & 8 & 1 & 20 & 43.25217 & \#DIV/0! \\
\hline HIS3 & YOR202W & 8 & 1 & 21 & 35.01287 & \#DIV/0! \\
\hline HIS3 & YOR202W & 8 & 1 & 22 & 27.93066 & \#DIV/0! \\
\hline HIS3 & YOR202W & 8 & 1 & 23 & 24.46448 & \#DIV/0! \\
\hline HIS3 & YOR202W & 8 & 1 & 24 & 34.39505 & \#DIV/0! \\
\hline \multirow[t]{2}{*}{ HIS3 } & YOR202W & 8 & 2 & 1 & 27.06069 & \#DIV/0! \\
\hline & YKL187C & 8 & 2 & 2 & 44.80023 & 38.69048 \\
\hline TIF1 & YKR059W & 8 & 2 & 3 & 33.39682 & 51.934 \\
\hline ASH1 & YKL185W & 8 & 2 & 4 & 37.33207 & 50.45499 \\
\hline GLG1 & YKR058W & 8 & 2 & 5 & 63.54551 & 57.54729 \\
\hline SPE1 & YKL184W & 8 & 2 & 6 & 45.65552 & 44.55966 \\
\hline RPS21A & YKR057W & 8 & 2 & 7 & 12.01287 & 18.07478 \\
\hline LOT5 & YKL183W & 8 & 2 & 8 & 6.139764 & 18.80839 \\
\hline TRM2 & YKR056W & 8 & 2 & 9 & 9.576642 & 39.58645 \\
\hline COY1 & YKL179C & 8 & 2 & 10 & -3.15785 & 10.32914 \\
\hline RHO4 & YKR055W & 8 & 2 & 11 & 46.49162 & 7.998338 \\
\hline STE3 & YKL178C & 8 & 2 & 12 & 4.659141 & 37.17765 \\
\hline \multirow[t]{2}{*}{ DYN1 } & YKR054C & 8 & 2 & 13 & -18.3893 & 44.33395 \\
\hline & YKL177W & 8 & 2 & 14 & 23.31082 & 38.38782 \\
\hline YSR3 & YKR053C & 8 & 2 & 15 & 43.50702 & 13.37874 \\
\hline LST4 & YKL176C & 8 & 2 & 16 & 4.678892 & 32.9692 \\
\hline MRS4 & YKR052C & 8 & 2 & 17 & 1.917864 & -9.99037 \\
\hline \multirow[t]{2}{*}{ ZRT3 } & YKL175W & 8 & 2 & 18 & 4.606115 & 32.9258 \\
\hline & YKR051W & 8 & 2 & 19 & 32.48312 & 31.09134 \\
\hline TPO5 & YKL174C & 8 & 2 & 20 & -12.1163 & 35.69929 \\
\hline
\end{tabular}




\begin{tabular}{|c|c|c|c|c|c|c|}
\hline \multirow[t]{2}{*}{ TRK2 } & YKR050W & 8 & 2 & 21 & 0.504922 & 43.02794 \\
\hline & YKL171W & 8 & 2 & 22 & 32.90274 & 9.903128 \\
\hline FMP46 & YKR049C & 8 & 2 & 23 & 11.19938 & 41.70292 \\
\hline HIS3 & YOR202W & 8 & 2 & 24 & 15.47371 & \#DIV/0! \\
\hline HIS3 & YOR202W & 8 & 3 & 1 & 58.48262 & \#DIV/O! \\
\hline STE24 & YJR117W & 8 & 3 & 2 & 35.35501 & 39.97353 \\
\hline \multirow[t]{2}{*}{ LHS1 } & YKL073W & 8 & 3 & 3 & 31.02885 & 56.19504 \\
\hline & YJR116W & 8 & 3 & 4 & 41.50739 & 53.99621 \\
\hline \multirow[t]{5}{*}{ STB6 } & YKL072W & 8 & 3 & 5 & 7.271707 & 51.96637 \\
\hline & YJR115W & 8 & 3 & 6 & 41.1448 & 38.40165 \\
\hline & YKL071W & 8 & 3 & 7 & 9.711443 & 39.76707 \\
\hline & YJR111C & 8 & 3 & 8 & 14.90876 & 27.57891 \\
\hline & YKL070W & 8 & 3 & 9 & 34.23816 & 42.87751 \\
\hline \multirow[t]{2}{*}{ YMR1 } & YJR110W & 8 & 3 & 10 & 28.80283 & 38.07746 \\
\hline & YKL069W & 8 & 3 & 11 & 62.41039 & 40.98535 \\
\hline CPA2 & YJR109C & 8 & 3 & 12 & 36.00004 & 46.23017 \\
\hline NUP100 & YKL068W & 8 & 3 & 13 & 10.73049 & 43.83945 \\
\hline ABM1 & YJR108W & 8 & 3 & 14 & -2.41567 & 28.97809 \\
\hline \multirow[t]{3}{*}{ YNK1 } & YKL067W & 8 & 3 & 15 & 24.15593 & 33.16869 \\
\hline & YJR107W & 8 & 3 & 16 & 29.05552 & 42.82063 \\
\hline & YKL066W & 8 & 3 & 17 & 9.451519 & 24.00061 \\
\hline ECM27 & YJR106W & 8 & 3 & 18 & 40.83926 & 21.61373 \\
\hline YET1 & YKL065C & 8 & 3 & 19 & 6.681418 & 42.58634 \\
\hline URA8 & YJR103W & 8 & 3 & 20 & 19.33631 & 29.3286 \\
\hline \multirow[t]{3}{*}{ MNR2 } & YKL064W & 8 & 3 & 21 & 50.15137 & 42.71533 \\
\hline & YJR100C & 8 & 3 & 22 & 43.80194 & 4.510785 \\
\hline & YKL063C & 8 & 3 & 23 & 20.916 & 16.52798 \\
\hline HIS3 & YOR202W & 8 & 3 & 24 & 15.82525 & \#DIV/O! \\
\hline HIS3 & YOR202W & 8 & 4 & 1 & 58.01545 & \#DIV/0! \\
\hline LOS1 & YKL205W & 8 & 4 & 2 & 15.18435 & 54.3963 \\
\hline ECM4 & YKR076W & 8 & 4 & 3 & 42.03848 & 37.47797 \\
\hline \multirow[t]{3}{*}{ EAP1 } & YKL204W & 8 & 4 & 4 & 65.04054 & 56.01439 \\
\hline & YKR074W & 8 & 4 & 5 & 3.48798 & 30.11642 \\
\hline & YKL202W & 8 & 4 & 6 & 52.65638 & 52.58892 \\
\hline SIS2 & YKR072C & 8 & 4 & 7 & 32.79182 & 35.74238 \\
\hline \multirow[t]{3}{*}{ MNN4 } & YKL201C & 8 & 4 & 8 & 25.33541 & 47.42525 \\
\hline & YKR070W & 8 & 4 & 9 & 48.5029 & 35.20184 \\
\hline & YKL200C & 8 & 4 & 10 & 7.296817 & 33.87391 \\
\hline MET1 & YKR069W & 8 & 4 & 11 & -28.7171 & 46.52458 \\
\hline YKT9 & YKL199C & 8 & 4 & 12 & 0.715698 & 31.74885 \\
\hline GPT2 & YKR067W & 8 & 4 & 13 & 9.764923 & 53.90509 \\
\hline PTK1 & YKL198C & 8 & 4 & 14 & -26.7023 & 47.28452 \\
\hline CCP1 & YKR066C & 8 & 4 & 15 & 10.69788 & 31.43167 \\
\hline
\end{tabular}




\begin{tabular}{|c|c|c|c|c|c|c|}
\hline PEX1 & YKL197C & 8 & 4 & 16 & 21.51412 & 48.75085 \\
\hline FMP18 & YKR065C & 8 & 4 & 17 & -7.41158 & 40.08432 \\
\hline \multirow[t]{2}{*}{$\mathrm{DPH} 2$} & YKL191W & 8 & 4 & 18 & -2.03693 & 53.45069 \\
\hline & YKR064W & 8 & 4 & 19 & 39.51101 & 38.8486 \\
\hline CNB1 & YKL190W & 8 & 4 & 20 & 41.64262 & 43.4917 \\
\hline KTR2 & YKR061W & 8 & 4 & 21 & 20.73224 & 41.10056 \\
\hline PXA2 & YKL188C & 8 & 4 & 22 & 28.71193 & 46.64995 \\
\hline UTP30 & YKR060W & 8 & 4 & 23 & 36.89816 & 29.62069 \\
\hline HIS3 & YOR202W & 8 & 4 & 24 & 12.28645 & \#DIV/0! \\
\hline HIS3 & YOR202W & 8 & 5 & 1 & 41.36022 & \#DIV/0! \\
\hline \multirow[t]{3}{*}{ STR2 } & YJR130C & 8 & 5 & 2 & 20.98488 & 44.64619 \\
\hline & YKL091C & 8 & 5 & 3 & 40.57831 & 59.93678 \\
\hline & YJR129C & 8 & 5 & 4 & 23.16351 & 54.95599 \\
\hline \multirow[t]{2}{*}{ CUE2 } & YKL090W & 8 & 5 & 5 & 74.06774 & 59.09029 \\
\hline & YJR128W & 8 & 5 & 6 & 10.5056 & 53.447 \\
\hline SRX1 & YKL086W & 8 & 5 & 7 & 25.31822 & 52.11163 \\
\hline ZMS1 & YJR127C & 8 & 5 & 8 & 20.41505 & 61.12939 \\
\hline MDH1 & YKL085W & 8 & 5 & 9 & 14.97506 & 38.85922 \\
\hline VPS70 & YJR126C & 8 & 5 & 10 & 37.07156 & 55.0996 \\
\hline HOT13 & YKL084W & 8 & 5 & 11 & 12.28254 & 53.24021 \\
\hline ENT3 & YJR125C & 8 & 5 & 12 & 13.16175 & 47.88165 \\
\hline \multirow[t]{2}{*}{ TEF4 } & YKL081W & 8 & 5 & 13 & 16.61617 & 19.22714 \\
\hline & YJR124C & 8 & 5 & 14 & 15.25984 & 53.37704 \\
\hline SMY1 & YKL079W & 8 & 5 & 15 & 11.99866 & 34.65316 \\
\hline \multirow[t]{3}{*}{ ATP2 } & YJR121W & 8 & 5 & 16 & 32.31739 & 49.29059 \\
\hline & YKL077W & 8 & 5 & 17 & 55.48079 & 33.7728 \\
\hline & YJR120W & 8 & 5 & 18 & 22.52277 & 56.17085 \\
\hline \multirow[t]{3}{*}{ PSY1 } & YKL076C & 8 & 5 & 19 & 60.60439 & 48.23541 \\
\hline & YJR119C & 8 & 5 & 20 & 49.97794 & 32.54083 \\
\hline & YKL075C & 8 & 5 & 21 & 15.8361 & 26.67297 \\
\hline ILM1 & YJR118C & 8 & 5 & 22 & 29.99436 & 27.01267 \\
\hline MUD2 & YKL074C & 8 & 5 & 23 & 23.82721 & 28.80818 \\
\hline HIS3 & YOR202W & 8 & 5 & 24 & 17.45605 & \#DIV/0! \\
\hline HIS3 & YOR202W & 8 & 6 & 1 & 12.21838 & \#DIV/0! \\
\hline SRY1 & YKL218C & 8 & 6 & 2 & 58.13532 & 72.2091 \\
\hline SRP40 & YKR092C & 8 & 6 & 3 & 35.2186 & 43.19257 \\
\hline JEN1 & YKL217W & 8 & 6 & 4 & 37.35901 & 56.98801 \\
\hline SRL3 & YKR091W & 8 & 6 & 5 & 21.39981 & 33.75375 \\
\hline URA1 & YKL216W & 8 & 6 & 6 & 27.94189 & 43.16077 \\
\hline \multirow[t]{2}{*}{ PXL1 } & YKR090W & 8 & 6 & 7 & 66.44437 & 61.75961 \\
\hline & YKL215C & 8 & 6 & 8 & 13.42103 & 46.2015 \\
\hline STC1 & YKR089C & 8 & 6 & 9 & 17.29462 & 60.74014 \\
\hline YRA2 & YKL214C & 8 & 6 & 10 & 36.27207 & 41.95893 \\
\hline
\end{tabular}




\begin{tabular}{|c|c|c|c|c|c|c|}
\hline TVP38 & YKR088C & 8 & 6 & 11 & 26.45964 & 55.24143 \\
\hline DOA1 & YKL213C & 8 & 6 & 12 & 16.07143 & 59.3898 \\
\hline OMA1 & YKR087C & 8 & 6 & 13 & 43.17286 & 61.41623 \\
\hline SAC1 & YKL212W & 8 & 6 & 14 & 67.47411 & 45.71429 \\
\hline HBS1 & YKR084C & 8 & 6 & 15 & 16.85541 & 25.76425 \\
\hline TRP3 & YKL211C & 8 & 6 & 16 & 17.75136 & 56.52971 \\
\hline NUP133 & YKR082W & 8 & 6 & 17 & 11.54264 & 41.9055 \\
\hline CBT1 & YKL208W & 8 & 6 & 18 & 37.40048 & 45.28856 \\
\hline \multirow[t]{5}{*}{ MTD1 } & YKR080W & 8 & 6 & 19 & 32.64533 & 47.6044 \\
\hline & YKL207W & 8 & 6 & 20 & 46.54925 & 26.1859 \\
\hline & YKR078W & 8 & 6 & 21 & 36.47015 & 9.472425 \\
\hline & YKL206C & 8 & 6 & 22 & 20.86386 & 40.00565 \\
\hline & YKR077W & 8 & 6 & 23 & 24.91326 & 50.68749 \\
\hline HIS3 & YOR202W & 8 & 6 & 24 & 11.17041 & \#DIV/0! \\
\hline HIS3 & YOR202W & 8 & 7 & 1 & 54.5192 & \#DIV/0! \\
\hline HMS2 & YJR147W & 8 & 7 & 2 & 35.06179 & 36.41045 \\
\hline \multirow[t]{3}{*}{ LAP4 } & YKL103C & 8 & 7 & 3 & 58.09913 & 73.01172 \\
\hline & YJR146W & 8 & 7 & 4 & 64.40313 & 57.13359 \\
\hline & YKL102C & 8 & 7 & 5 & 27.51153 & 62.88606 \\
\hline RPS4A & YJR145C & 8 & 7 & 6 & 34.10402 & 56.64145 \\
\hline \multirow[t]{3}{*}{ HSL1 } & YKL101W & 8 & 7 & 7 & 65.704 & 70.67797 \\
\hline & YJR142W & 8 & 7 & 8 & 40.07888 & 56.7529 \\
\hline & YKL100C & 8 & 7 & 9 & 47.61051 & 62.46586 \\
\hline \multirow[t]{2}{*}{ HIR3 } & YJR140C & 8 & 7 & 10 & 32.41395 & 39.42748 \\
\hline & YKL098W & 8 & 7 & 11 & 39.09967 & 43.37058 \\
\hline \multirow[t]{2}{*}{ HOM6 } & YJR139C & 8 & 7 & 12 & 48.39371 & 71.59785 \\
\hline & YKL097C & 8 & 7 & 13 & 39.16982 & 48.75506 \\
\hline \multirow[t]{2}{*}{ ECM17 } & YJR137C & 8 & 7 & 14 & 23.20416 & 60.24929 \\
\hline & YKL096W- & & & & & \\
\hline CWP2 & $A$ & 8 & 7 & 15 & 60.36627 & 67.22024 \\
\hline MCM22 & YJR135C & 8 & 7 & 16 & 21.54718 & 53.82009 \\
\hline CWP1 & YKL096W & 8 & 7 & 17 & 19.59799 & -14.7805 \\
\hline SGM1 & YJR134C & 8 & 7 & 18 & 20.86781 & 39.40045 \\
\hline YJU3 & YKL094W & 8 & 7 & 19 & 41.04393 & 56.95951 \\
\hline XPT1 & YJR133W & 8 & 7 & 20 & 21.44805 & 52.9986 \\
\hline MBR1 & YKL093W & 8 & 7 & 21 & 80.23075 & 70.88892 \\
\hline MNS1 & YJR131W & 8 & 7 & 22 & 29.43809 & 18.22202 \\
\hline BUD2 & YKL092C & 8 & 7 & 23 & 46.69888 & 52.71841 \\
\hline HIS3 & YOR202W & 8 & 7 & 24 & 12.22443 & \#DIV/0! \\
\hline \multirow[t]{2}{*}{ HIS3 } & YOR202W & 8 & 8 & 1 & 35.98409 & \#DIV/0! \\
\hline & YKR012C & 8 & 8 & 2 & 23.35218 & 46.02715 \\
\hline NFT1 & YKR103W & 8 & 8 & 3 & 73.69357 & 68.30346 \\
\hline TOS5 & YKR011C & 8 & 8 & 4 & 66.60051 & 32.04064 \\
\hline
\end{tabular}




\begin{tabular}{|c|c|c|c|c|c|c|}
\hline FLO10 & YKR102W & 8 & 8 & 5 & 47.45934 & 48.70441 \\
\hline TOF2 & YKR010C & 8 & 8 & 6 & 20.36871 & 35.11021 \\
\hline SIR1 & YKR101W & 8 & 8 & 7 & 59.42515 & 53.21583 \\
\hline FOX2 & YKR009C & 8 & 8 & 8 & 38.8044 & 54.71902 \\
\hline SKG1 & YKR100C & 8 & 8 & 9 & 62.84941 & 39.78874 \\
\hline MEH1 & YKR007W & 8 & 8 & 10 & 19.50225 & 51.86561 \\
\hline \multirow[t]{2}{*}{ BAS1 } & YKR099W & 8 & 8 & 11 & 64.03763 & 45.79787 \\
\hline & YKR005C & 8 & 8 & 12 & 38.49562 & 48.56412 \\
\hline UBP11 & YKR098C & 8 & 8 & 13 & 66.27927 & 26.45737 \\
\hline OSH6 & YKR003W & 8 & 8 & 14 & 17.22734 & 48.60406 \\
\hline PCK1 & YKR097W & 8 & 8 & 15 & 39.54293 & 57.1345 \\
\hline \multirow[t]{3}{*}{ VPS1 } & YKR001C & 8 & 8 & 16 & 44.6304 & 41.30762 \\
\hline & YKR096W & 8 & 8 & 17 & 19.48571 & 40.68432 \\
\hline & YKL222C & 8 & 8 & 18 & 32.04278 & 56.41414 \\
\hline MLP1 & YKR095W & 8 & 8 & 19 & 35.49235 & 57.34337 \\
\hline $\mathrm{MCH} 2$ & YKL221W & 8 & 8 & 20 & 59.30265 & 55.31987 \\
\hline RPL40B & YKR094C & 8 & 8 & 21 & 27.51609 & 66.27959 \\
\hline FRE2 & YKL220C & 8 & 8 & 22 & 33.4927 & 58.43673 \\
\hline PTR2 & YKR093W & 8 & 8 & 23 & 34.67556 & 60.11245 \\
\hline HIS3 & YOR202W & 8 & 8 & 24 & 21.46271 & \#DIV/0! \\
\hline HIS3 & YOR202W & 8 & 9 & 1 & 41.5443 & 54.9901 \\
\hline LAC1 & YKL008C & 8 & 9 & 2 & 46.01186 & 48.15051 \\
\hline OAC1 & YKL120W & 8 & 9 & 3 & 63.3558 & 39.07814 \\
\hline CAP1 & YKL007W & 8 & 9 & 4 & 49.91356 & 56.195 \\
\hline SBA1 & YKL117W & 8 & 9 & 5 & 44.21425 & 51.53387 \\
\hline RPL14A & YKL006W & 8 & 9 & 6 & 39.91347 & 62.53666 \\
\hline PRR1 & YKL116C & 8 & 9 & 7 & 39.31096 & 34.77366 \\
\hline \multirow[t]{2}{*}{ BYE1 } & YKL005C & 8 & 9 & 8 & \#DIV/0! & 68.33565 \\
\hline & YKL115C & 8 & 9 & 9 & 61.51946 & 53.97577 \\
\hline MET14 & YKL001C & 8 & 9 & 10 & 30.24593 & 64.6638 \\
\hline \multirow[t]{2}{*}{ APN1 } & YKL114C & 8 & 9 & 11 & 41.5931 & 59.67548 \\
\hline & YJR154W & 8 & 9 & 12 & 24.02748 & 46.38395 \\
\hline RAD27 & YKL113C & 8 & 9 & 13 & \#DIV/0! & 58.07524 \\
\hline PGU1 & YJR153W & 8 & 9 & 14 & 65.0093 & 15.62495 \\
\hline KTI12 & YKL110C & 8 & 9 & 15 & 33.15034 & 30.13872 \\
\hline DAL5 & YJR152W & 8 & 9 & 16 & 42.02773 & 56.18517 \\
\hline HAP4 & YKL109W & 8 & 9 & 17 & 21.49884 & 50.35819 \\
\hline \multirow[t]{3}{*}{ DAN1 } & YJR150C & 8 & 9 & 18 & \#DIV/0! & 60.0053 \\
\hline & YKL107W & 8 & 9 & 19 & 50.98345 & 54.91484 \\
\hline & YJR149W & 8 & 9 & 20 & 60.68319 & 20.3179 \\
\hline AAT1 & YKL106W & 8 & 9 & 21 & 52.28165 & 56.95631 \\
\hline \multirow[t]{2}{*}{ BAT2 } & YJR148W & 8 & 9 & 22 & 65.22506 & 23.57222 \\
\hline & YKL105C & 8 & 9 & 23 & 23.12127 & \#DIV/0! \\
\hline
\end{tabular}




\begin{tabular}{|c|c|c|c|c|c|c|}
\hline HIS3 & YOR202W & 8 & 9 & 24 & 7.677169 & \#DIV/0! \\
\hline HIS3 & YOR202W & 8 & 10 & 1 & 73.2202 & \#DIV/0! \\
\hline DBP7 & YKR024C & 8 & 10 & 2 & 9.910684 & 59.07529 \\
\hline \multirow[t]{2}{*}{ PUF3 } & YLL013C & 8 & 10 & 3 & 28.8582 & 45.66679 \\
\hline & YKR023W & 8 & 10 & 4 & 19.81218 & 55.30978 \\
\hline \multirow[t]{2}{*}{ YEH1 } & YLL012W & 8 & 10 & 5 & 64.76177 & 64.0438 \\
\hline & YKR021W & 8 & 10 & 6 & 18.81271 & 56.7264 \\
\hline PSR1 & YLL010C & 8 & 10 & 7 & 38.01149 & 52.02639 \\
\hline VPS51 & YKR020W & 8 & 10 & 8 & 20.00765 & 19.4084 \\
\hline cox17 & YLL009C & 8 & 10 & 9 & 41.73968 & 36.12639 \\
\hline \multirow[t]{3}{*}{ IRS4 } & YKR019C & 8 & 10 & 10 & 36.06076 & 12.23058 \\
\hline & YLL007C & 8 & 10 & 11 & 12.57232 & 21.42147 \\
\hline & YKR018C & 8 & 10 & 12 & 14.77223 & 54.60317 \\
\hline \multirow[t]{2}{*}{ MMM1 } & YLL006W & 8 & 10 & 13 & \#DIV/0! & 62.68581 \\
\hline & YKR017C & 8 & 10 & 14 & 46.72824 & 48.10719 \\
\hline SPO75 & YLL005C & 8 & 10 & 15 & 36.31111 & 23.49786 \\
\hline FMP13 & YKR016W & 8 & 10 & 16 & 13.05049 & 46.68803 \\
\hline \multirow[t]{2}{*}{ RTT109 } & YLL002W & 8 & 10 & 17 & 34.27547 & 36.8503 \\
\hline & YKR015C & 8 & 10 & 18 & 51.6051 & 42.72044 \\
\hline DNM1 & YLL001W & 8 & 10 & 19 & 31.46234 & 28.67321 \\
\hline \multirow[t]{2}{*}{ YPT52 } & YKR014C & 8 & 10 & 20 & 14.58661 & 55.14783 \\
\hline & YKR106W & 8 & 10 & 21 & 23.14219 & 37.35538 \\
\hline \multirow[t]{2}{*}{ PRY2 } & YKR013W & 8 & 10 & 22 & 41.58936 & 56.21086 \\
\hline & YKR105C & 8 & 10 & 23 & 62.26073 & 68.30252 \\
\hline HIS3 & YOR202W & 8 & 10 & 24 & 30.75332 & \#DIV/0! \\
\hline \multirow[t]{3}{*}{ HIS3 } & YOR202W & 8 & 11 & 1 & 46.99414 & \#DIV/0! \\
\hline & YKL030W & 8 & 11 & 2 & 57.29328 & 54.25414 \\
\hline & YKL136W & 8 & 11 & 3 & 40.41114 & 57.84147 \\
\hline \multirow[t]{3}{*}{ MAE1 } & YKL029C & 8 & 11 & 4 & 5.064693 & 29.25861 \\
\hline & YKL133C & 8 & 11 & 5 & 52.09608 & 37.70979 \\
\hline & YKL027W & 8 & 11 & 6 & 28.88158 & 19.84729 \\
\hline RMA1 & YKL132C & 8 & 11 & 7 & 41.8919 & 47.70255 \\
\hline \multirow[t]{2}{*}{ GPX1 } & YKL026C & 8 & 11 & 8 & 28.59168 & 48.65477 \\
\hline & YKL131W & 8 & 11 & 9 & 30.76367 & 40.70146 \\
\hline PAN3 & YKL025C & 8 & 11 & 10 & 15.69961 & 52.46324 \\
\hline \multirow[t]{2}{*}{ SHE2 } & YKL130C & 8 & 11 & 11 & 33.75352 & 22.2933 \\
\hline & YKL023W & 8 & 11 & 12 & 43.21663 & 49.41376 \\
\hline MYO3 & YKL129C & 8 & 11 & 13 & 27.88387 & 40.77814 \\
\hline SPT23 & YKL020C & 8 & 11 & 14 & 33.1521 & 22.52339 \\
\hline PMU1 & YKL128C & 8 & 11 & 15 & 43.07635 & 18.4172 \\
\hline HCS1 & YKL017C & 8 & 11 & 16 & 27.94916 & 37.74467 \\
\hline PGM1 & YKL127W & 8 & 11 & 17 & 30.81339 & 16.55403 \\
\hline PUT3 & YKL015W & 8 & 11 & 18 & 43.79838 & 21.53202 \\
\hline
\end{tabular}




\begin{tabular}{|c|c|c|c|c|c|c|}
\hline $\mathrm{SSH} 4$ & YKL124W & 8 & 11 & 19 & 28.77006 & 28.78379 \\
\hline \multirow[t]{2}{*}{ UFD4 } & YKL010C & 8 & 11 & 20 & 25.81183 & 38.45213 \\
\hline & YKL123W & 8 & 11 & 21 & 43.82545 & 31.96903 \\
\hline \multirow[t]{2}{*}{ MRT4 } & YKL009W & 8 & 11 & 22 & 59.18743 & 46.70957 \\
\hline & YKL121W & 8 & 11 & 23 & 43.4547 & 51.27376 \\
\hline HIS3 & YOR202W & 8 & 11 & 24 & 17.34153 & \#DIV/0! \\
\hline HIS3 & $\begin{array}{l}\text { YOR202W } \\
\text { YKR035W- }\end{array}$ & 8 & 12 & 1 & 38.39916 & \#DIV/0! \\
\hline DID2 & A & 8 & 12 & 2 & 48.41211 & 42.41811 \\
\hline \multirow[t]{3}{*}{ HSP104 } & YLL026W & 8 & 12 & 3 & 41.19829 & 57.96139 \\
\hline & YKR035C & 8 & 12 & 4 & 38.94203 & 50.99928 \\
\hline & YLL025W & 8 & 12 & 5 & 3.860317 & 21.47085 \\
\hline DAL80 & YKR034W & 8 & 12 & 6 & 20.06368 & 58.12416 \\
\hline \multirow[t]{4}{*}{ SSA2 } & YLL024C & 8 & 12 & 7 & 22.61878 & 59.8349 \\
\hline & YKR033C & 8 & 12 & 8 & 22.07382 & 55.15113 \\
\hline & YLL023C & 8 & 12 & 9 & 28.00314 & 55.75789 \\
\hline & YKR032W & 8 & 12 & 10 & 24.12621 & 49.2093 \\
\hline SPA2 & YLL021W & 8 & 12 & 11 & 67.33897 & 56.43288 \\
\hline \multirow[t]{2}{*}{ SPO14 } & YKR031C & 8 & 12 & 12 & 5.556103 & 45.46263 \\
\hline & YLL020C & 8 & 12 & 13 & 23.59085 & 41.62564 \\
\hline GMH1 & YKR030W & 8 & 12 & 14 & 39.07987 & 26.09246 \\
\hline KNS1 & YLL019C & 8 & 12 & 15 & 38.46416 & 42.48682 \\
\hline SET3 & YKR029C & 8 & 12 & 16 & \#DIV/0! & 54.44558 \\
\hline SDC25 & YLL017W & 8 & 12 & 17 & 3.456468 & 48.62357 \\
\hline SAP190 & YKR028W & 8 & 12 & 18 & 7.083826 & 47.52937 \\
\hline SDC25 & YLL016W & 8 & 12 & 19 & 29.44858 & 14.84717 \\
\hline FMP50 & YKR027W & 8 & 12 & 20 & \#DIV/0! & 33.19088 \\
\hline BPT1 & YLL015W & 8 & 12 & 21 & 37.48063 & 44.8668 \\
\hline \multirow[t]{2}{*}{ GCN3 } & YKR026C & 8 & 12 & 22 & 54.43805 & 29.59848 \\
\hline & YLL014W & 8 & 12 & 23 & 13.80155 & 46.65173 \\
\hline HIS3 & YOR202W & 8 & 12 & 24 & 58.31521 & \#DIV/0! \\
\hline \multirow[t]{2}{*}{ HIS3 } & YOR202W & 8 & 13 & 1 & 42.26383 & \#DIV/0! \\
\hline & YKL044W & 8 & 13 & 2 & 26.92172 & 36.42916 \\
\hline RPS27A & YKL156W & 8 & 13 & 3 & 34.84557 & 34.90997 \\
\hline \multirow[t]{2}{*}{ PHD1 } & YKL043W & 8 & 13 & 4 & 40.67396 & 43.31621 \\
\hline & YKL151C & 8 & 13 & 5 & 34.81576 & 37.90362 \\
\hline VPS24 & YKL041W & 8 & 13 & 6 & 32.61428 & 32.6492 \\
\hline MCR1 & YKL150W & 8 & 13 & 7 & 3.575527 & 36.06847 \\
\hline NFU1 & YKL040C & 8 & 13 & 8 & 14.26766 & 40.21615 \\
\hline DBR1 & YKL149C & 8 & 13 & 9 & 14.56414 & 47.42828 \\
\hline PTM1 & YKL039W & 8 & 13 & 10 & 15.03643 & -8.00171 \\
\hline SDH1 & YKL148C & 8 & 13 & 11 & 28.46404 & 24.46757 \\
\hline RGT1 & YKL038W & 8 & 13 & 12 & 36.59942 & 60.80059 \\
\hline
\end{tabular}




\begin{tabular}{|c|c|c|c|c|c|c|}
\hline & YKL147C & 8 & 13 & 13 & 24.23858 & 40.11222 \\
\hline & YKL037W & 8 & 13 & 14 & 36.94679 & 27.06254 \\
\hline AVT3 & YKL146W & 8 & 13 & 15 & 20.90624 & 49.26272 \\
\hline TUL1 & YKL034W & 8 & 13 & 16 & 32.9775 & 25.22465 \\
\hline \multirow[t]{3}{*}{ MRP8 } & YKL142W & 8 & 13 & 17 & 27.25544 & 48.58503 \\
\hline & YKL033W- & & & & & \\
\hline & $A$ & 8 & 13 & 18 & 37.32703 & \#DIV/O! \\
\hline TGL1 & YKL140W & 8 & 13 & 19 & 43.96667 & \#DIV/0! \\
\hline IXR1 & YKL032C & 8 & 13 & 20 & 46.60468 & 25.18026 \\
\hline \multirow[t]{3}{*}{ CTK1 } & YKL139W & 8 & 13 & 21 & 49.24731 & 28.20669 \\
\hline & YKL031W & 8 & 13 & 22 & 27.91369 & 62.48098 \\
\hline & YKL137W & 8 & 13 & 23 & 20.62597 & 43.80408 \\
\hline HIS3 & YOR202W & 8 & 13 & 24 & 27.86619 & \#DIV/0! \\
\hline HIS3 & YOR202W & 8 & 14 & 1 & 47.85042 & \#DIV/0! \\
\hline NAP1 & YKR048C & 8 & 14 & 2 & 47.7485 & 34.10388 \\
\hline \multirow[t]{3}{*}{ RPL8B } & YLL045C & 8 & 14 & 3 & 28.3541 & 38.87661 \\
\hline & YKR047W & 8 & 14 & 4 & 19.2577 & 61.76193 \\
\hline & YLL044W & 8 & 14 & 5 & 24.65326 & 52.35482 \\
\hline PET10 & YKR046C & 8 & 14 & 6 & 18.18412 & 46.54318 \\
\hline \multirow[t]{2}{*}{ FPS1 } & YLL043W & 8 & 14 & 7 & 62.69796 & 71.08414 \\
\hline & YKR045C & 8 & 14 & 8 & 17.45162 & 36.63431 \\
\hline ATG10 & YLL042C & 8 & 14 & 9 & 21.5744 & 33.38676 \\
\hline UIP5 & YKR044W & 8 & 14 & 10 & 20.23734 & 39.63379 \\
\hline \multirow[t]{2}{*}{$\mathrm{SDH} 2$} & YLL041C & 8 & 14 & 11 & 13.80405 & 18.53318 \\
\hline & YKR043C & 8 & 14 & 12 & 24.39807 & 53.13296 \\
\hline VPS13 & YLL040C & 8 & 14 & 13 & 27.21735 & 36.65472 \\
\hline UTH1 & YKR042W & 8 & 14 & 14 & 21.64672 & 16.1207 \\
\hline \multirow[t]{2}{*}{ UBI4 } & YLL039C & 8 & 14 & 15 & 36.34193 & 50.43306 \\
\hline & YKR041W & 8 & 14 & 16 & 16.24093 & 42.08978 \\
\hline \multirow[t]{3}{*}{ ENT4 } & YLL038C & 8 & 14 & 17 & 41.95834 & 47.17393 \\
\hline & YKR040C & 8 & 14 & 18 & 36.58554 & \#DIV/0! \\
\hline & YLL032C & 8 & 14 & 19 & 42.03059 & \#DIV/0! \\
\hline \multirow[t]{2}{*}{ GAP1 } & YKR039W & 8 & 14 & 20 & 17.50182 & 58.49519 \\
\hline & YLL029W & 8 & 14 & 21 & 11.20878 & 32.77494 \\
\hline CAF4 & YKR036C & 8 & 14 & 22 & 52.16997 & 40.72601 \\
\hline TPO1 & YLL028W & 8 & 14 & 23 & 32.67132 & 35.29989 \\
\hline HIS3 & YOR202W & 8 & 14 & 24 & 21.57009 & \#DIV/0! \\
\hline HIS3 & YOR202W & 8 & 15 & 1 & 55.98778 & \#DIV/0! \\
\hline MSN4 & YKL062W & 8 & 15 & 2 & 23.94979 & 46.56181 \\
\hline \multirow[t]{2}{*}{ KKQ8 } & YKL168C & 8 & 15 & 3 & 44.88888 & 45.12513 \\
\hline & YKL061W & 8 & 15 & 4 & 39.21001 & 38.85036 \\
\hline MRP49 & YKL167C & 8 & 15 & 5 & 33.98683 & 30.26709 \\
\hline RBF18 & YKL056C & 8 & 15 & 6 & 41.82703 & 59.40918 \\
\hline
\end{tabular}




\begin{tabular}{|c|c|c|c|c|c|c|}
\hline TPK3 & YKL166C & 8 & 15 & 7 & 18.98129 & 34.04248 \\
\hline OAR1 & YKL055C & 8 & 15 & 8 & 3.294128 & 18.87325 \\
\hline \multirow[t]{2}{*}{ PIR1 } & YKL164C & 8 & 15 & 9 & 26.01334 & 34.24642 \\
\hline & YKL053W & 8 & 15 & 10 & 13.8443 & 47.78543 \\
\hline PIR3 & YKL163W & 8 & 15 & 11 & 42.09463 & 34.1986 \\
\hline \multirow[t]{2}{*}{ MDM35 } & YKL053C-A & 8 & 15 & 12 & 49.74792 & 35.6445 \\
\hline & YKL162C & 8 & 15 & 13 & 26.43762 & 23.99141 \\
\hline \multirow[t]{3}{*}{ SFK1 } & YKL051W & 8 & 15 & 14 & 20.31627 & 46.20336 \\
\hline & YKL161C & 8 & 15 & 15 & 16.71843 & 56.29259 \\
\hline & YKL050C & 8 & 15 & 16 & 24.98655 & 24.63151 \\
\hline ELF1 & YKL160W & 8 & 15 & 17 & 48.38003 & 36.74044 \\
\hline ELM1 & YKL048C & 8 & 15 & 18 & 21.2063 & \#DIV/0! \\
\hline \multirow[t]{3}{*}{ RCN1 } & YKL159C & 8 & 15 & 19 & 44.3412 & 53.93779 \\
\hline & YKL047W & 8 & 15 & 20 & 23.82539 & 49.05465 \\
\hline & YKL158W & 8 & 15 & 21 & 43.10778 & 51.81369 \\
\hline DCW1 & YKL046C & 8 & 15 & 22 & 37.18591 & 60.84743 \\
\hline APE2 & YKL157W & 8 & 15 & 23 & 62.21741 & 41.9978 \\
\hline HIS3 & YOR202W & 8 & 15 & 24 & 25.36618 & \#DIV/O! \\
\hline HIS3 & YOR202W & 8 & 16 & 1 & 61.02496 & \#DIV/0! \\
\hline HIS3 & YOR202W & 8 & 16 & 2 & 32.29209 & \#DIV/0! \\
\hline HIS3 & YOR202W & 8 & 16 & 3 & 31.76842 & \#DIV/0! \\
\hline HIS3 & YOR202W & 8 & 16 & 4 & 10.58327 & \#DIV/O! \\
\hline HIS3 & YOR202W & 8 & 16 & 5 & 10.86124 & \#DIV/0! \\
\hline HIS3 & YOR202W & 8 & 16 & 6 & 5.943188 & \#DIV/0! \\
\hline HIS3 & YOR202W & 8 & 16 & 7 & 42.7996 & \#DIV/0! \\
\hline HIS3 & YOR202W & 8 & 16 & 8 & -2.53638 & \#DIV/O! \\
\hline HIS3 & YOR202W & 8 & 16 & 9 & 16.99783 & \#DIV/0! \\
\hline HIS3 & YOR202W & 8 & 16 & 10 & 4.720066 & \#DIV/O! \\
\hline HIS3 & YOR202W & 8 & 16 & 11 & -16.7845 & \#DIV/0! \\
\hline HIS3 & YOR202W & 8 & 16 & 12 & -9.42851 & \#DIV/0! \\
\hline HIS3 & YOR202W & 8 & 16 & 13 & 2.598335 & \#DIV/0! \\
\hline HIS3 & YOR202W & 8 & 16 & 14 & -16.1566 & \#DIV/0! \\
\hline HIS3 & YOR202W & 8 & 16 & 15 & 11.2146 & \#DIV/0! \\
\hline HIS3 & YOR202W & 8 & 16 & 16 & 19.53796 & \#DIV/0! \\
\hline HIS3 & YOR202W & 8 & 16 & 17 & 30.04114 & \#DIV/0! \\
\hline HIS3 & YOR202W & 8 & 16 & 18 & -4.15292 & \#DIV/0! \\
\hline HIS3 & YOR202W & 8 & 16 & 19 & -2.01225 & \#DIV/0! \\
\hline HIS3 & YOR202W & 8 & 16 & 20 & 12.22334 & \#DIV/0! \\
\hline HIS3 & YOR202W & 8 & 16 & 21 & 18.54077 & \#DIV/0! \\
\hline HIS3 & YOR202W & 8 & 16 & 22 & 22.16803 & \#DIV/0! \\
\hline HIS3 & YOR202W & 8 & 16 & 23 & 0.405442 & \#DIV/0! \\
\hline HIS3 & YOR202W & 8 & 16 & 24 & 40.98711 & \#DIV/0! \\
\hline HIS3 & YOR202W & 9 & 1 & 1 & 57.4588 & \#DIV/0! \\
\hline
\end{tabular}




\begin{tabular}{|c|c|c|c|c|c|c|}
\hline HIS3 & YOR202W & 9 & 1 & 2 & 21.06133 & \#DIV/0! \\
\hline HIS3 & YOR202W & 9 & 1 & 3 & 33.87916 & \#DIV/0! \\
\hline HIS3 & YOR202W & 9 & 1 & 4 & 40.19131 & \#DIV/0! \\
\hline HIS3 & YOR202W & 9 & 1 & 5 & 18.80476 & \#DIV/0! \\
\hline HIS3 & YOR202W & 9 & 1 & 6 & 23.13825 & \#DIV/0! \\
\hline HIS3 & YOR202W & 9 & 1 & 7 & 15.48585 & \#DIV/0! \\
\hline HIS3 & YOR202W & 9 & 1 & 8 & 28.55271 & \#DIV/0! \\
\hline HIS3 & YOR202W & 9 & 1 & 9 & 32.55455 & \#DIV/0! \\
\hline HIS3 & YOR202W & 9 & 1 & 10 & 29.44078 & \#DIV/0! \\
\hline HIS3 & YOR202W & 9 & 1 & 11 & 21.76699 & \#DIV/0! \\
\hline HIS3 & YOR202W & 9 & 1 & 12 & 30.49095 & \#DIV/0! \\
\hline HIS3 & YOR202W & 9 & 1 & 13 & 32.86522 & \#DIV/0! \\
\hline HIS3 & YOR202W & 9 & 1 & 14 & 35.23409 & \#DIV/0! \\
\hline HIS3 & YOR202W & 9 & 1 & 15 & 24.0837 & \#DIV/0! \\
\hline HIS3 & YOR202W & 9 & 1 & 16 & 38.49592 & \#DIV/0! \\
\hline HIS3 & YOR202W & 9 & 1 & 17 & 17.4438 & \#DIV/0! \\
\hline HIS3 & YOR202W & 9 & 1 & 18 & 34.73702 & \#DIV/0! \\
\hline HIS3 & YOR202W & 9 & 1 & 19 & 26.0232 & \#DIV/0! \\
\hline HIS3 & YOR202W & 9 & 1 & 20 & 29.82263 & \#DIV/0! \\
\hline HIS3 & YOR202W & 9 & 1 & 21 & 23.76549 & \#DIV/0! \\
\hline HIS3 & YOR202W & 9 & 1 & 22 & 17.05332 & \#DIV/0! \\
\hline HIS3 & YOR202W & 9 & 1 & 23 & -6.06186 & \#DIV/0! \\
\hline HIS3 & YOR202W & 9 & 1 & 24 & 6.857719 & \#DIV/0! \\
\hline \multirow[t]{2}{*}{ HIS3 } & YOR202W & 9 & 2 & 1 & 51.62173 & \#DIV/0! \\
\hline & YLR217W & 9 & 2 & 2 & 24.28484 & 54.20317 \\
\hline CHS5 & YLR330W & 9 & 2 & 3 & 18.2842 & 35.06072 \\
\hline CPR6 & YLR216C & 9 & 2 & 4 & -0.50371 & 15.27709 \\
\hline REC102 & YLR329W & 9 & 2 & 5 & 18.02571 & 33.4174 \\
\hline FRE1 & YLR214W & 9 & 2 & 6 & 31.58928 & 22.03959 \\
\hline NMA1 & YLR328W & 9 & 2 & 7 & 52.41103 & 22.00498 \\
\hline CRR1 & YLR213C & 9 & 2 & 8 & 34.5981 & 6.705307 \\
\hline \multirow[t]{3}{*}{ RBF9 } & YLR327C & 9 & 2 & 9 & 28.67189 & 23.59225 \\
\hline & YLR211C & 9 & 2 & 10 & 39.24281 & 42.51842 \\
\hline & YLR326W & 9 & 2 & 11 & 31.84812 & 13.33761 \\
\hline CLB4 & YLR210W & 9 & 2 & 12 & 56.72155 & 54.18872 \\
\hline RPL38 & YLR325C & 9 & 2 & 13 & 12.00068 & 20.51268 \\
\hline PNP1 & YLR209C & 9 & 2 & 14 & 24.4374 & 36.77583 \\
\hline PEX30 & YLR324W & 9 & 2 & 15 & 14.15205 & 25.82338 \\
\hline HRD3 & YLR207W & 9 & 2 & 16 & 25.79995 & -10.8181 \\
\hline MMS22 & YLR320W & 9 & 2 & 17 & 38.7537 & 15.88251 \\
\hline ENT2 & YLR206W & 9 & 2 & 18 & 32.92692 & 33.77412 \\
\hline BUD6 & YLR319C & 9 & 2 & 19 & 13.31177 & -3.95628 \\
\hline HMX1 & YLR205C & 9 & 2 & 20 & 15.51728 & 36.73333 \\
\hline
\end{tabular}




\begin{tabular}{|c|c|c|c|c|c|c|}
\hline EST2 & YLR318W & 9 & 2 & 21 & 23.64611 & 9.353516 \\
\hline YKE2 & YLR200W & 9 & 2 & 22 & -1.83324 & 47.17525 \\
\hline NKP2 & YLR315W & 9 & 2 & 23 & 34.35208 & 41.96695 \\
\hline HIS3 & YOR202W & 9 & 2 & 24 & 7.021468 & \#DIV/0! \\
\hline HIS3 & YOR202W & 9 & 3 & 1 & 36.47171 & \#DIV/O! \\
\hline JLP1 & YLL057C & 9 & 3 & 2 & 29.00624 & 34.77406 \\
\hline \multirow[t]{2}{*}{ CHA4 } & YLR098C & 9 & 3 & 3 & 25.38852 & 29.90927 \\
\hline & YLL056C & 9 & 3 & 4 & 26.57889 & 43.72922 \\
\hline \multirow[t]{2}{*}{ HRT3 } & YLR097C & 9 & 3 & 5 & 27.41958 & 52.66243 \\
\hline & YLL055W & 9 & 3 & 6 & 19.07015 & 17.18608 \\
\hline \multirow[t]{2}{*}{ KIN2 } & YLR096W & 9 & 3 & 7 & 19.99838 & 42.84284 \\
\hline & YLL054C & 9 & 3 & 8 & \#DIV/0! & 24.91564 \\
\hline \multirow[t]{2}{*}{ IOC2 } & YLR095C & 9 & 3 & 9 & 13.71259 & 16.88203 \\
\hline & YLL053C & 9 & 3 & 10 & 8.21482 & 36.36188 \\
\hline GIS3 & YLR094C & 9 & 3 & 11 & 13.75749 & 44.28904 \\
\hline AQY2 & YLL052C & 9 & 3 & 12 & 20.38563 & 16.04714 \\
\hline NYV1 & YLR093C & 9 & 3 & 13 & 9.221996 & 23.06297 \\
\hline FRE6 & YLL051C & 9 & 3 & 14 & 14.13995 & 25.71519 \\
\hline \multirow[t]{3}{*}{ SUL2 } & YLR092W & 9 & 3 & 15 & 34.77322 & 35.0658 \\
\hline & YLL049W & 9 & 3 & 16 & 11.25555 & 53.52057 \\
\hline & YLR091W & 9 & 3 & 17 & 36.78284 & 33.11492 \\
\hline YBT1 & YLL048C & 9 & 3 & 18 & 34.68371 & 30.18485 \\
\hline \multirow[t]{2}{*}{ XDJ1 } & YLR090W & 9 & 3 & 19 & 18.96451 & 32.75741 \\
\hline & YLL047W & 9 & 3 & 20 & 27.38438 & 45.52847 \\
\hline ALT1 & YLR089C & 9 & 3 & 21 & 23.16048 & 41.13529 \\
\hline RNP1 & YLL046C & 9 & 3 & 22 & 24.50838 & 47.24927 \\
\hline CSF1 & YLR087C & 9 & 3 & 23 & 27.98698 & 43.73451 \\
\hline HIS3 & YOR202W & 9 & 3 & 24 & 13.14721 & \#DIV/0! \\
\hline HIS3 & YOR202W & 9 & 4 & 1 & 26.21897 & \#DIV/0! \\
\hline \multirow[t]{2}{*}{ TOP3 } & YLR234W & 9 & 4 & 2 & 7.949497 & 36.35972 \\
\hline & YLR345W & 9 & 4 & 3 & 16.46123 & 35.12865 \\
\hline EST1 & YLR233C & 9 & 4 & 4 & 18.32517 & 7.326188 \\
\hline \multirow[t]{2}{*}{ RPL26A } & YLR344W & 9 & 4 & 5 & 10.26347 & 22.94047 \\
\hline & YLR232W & 9 & 4 & 6 & 27.96343 & 26.8306 \\
\hline GAS2 & YLR343W & 9 & 4 & 7 & 31.7194 & 40.90475 \\
\hline BNA5 & YLR231C & 9 & 4 & 8 & 12.87282 & 14.57353 \\
\hline FKS1 & YLR342W & 9 & 4 & 9 & 7.962236 & 27.31577 \\
\hline ECM22 & YLR228C & 9 & 4 & 10 & 17.88911 & 36.23011 \\
\hline \multirow[t]{4}{*}{ SPO77 } & YLR341W & 9 & 4 & 11 & 16.35939 & 29.41391 \\
\hline & YLR225C & 9 & 4 & 12 & 7.472478 & 28.04748 \\
\hline & YLR338W & 9 & 4 & 13 & 21.19974 & 20.53617 \\
\hline & YLR224W & 9 & 4 & 14 & 12.43481 & 42.44284 \\
\hline VRP1 & YLR337C & 9 & 4 & 15 & 59.19639 & 72.01804 \\
\hline
\end{tabular}




\begin{tabular}{|c|c|c|c|c|c|c|}
\hline RSA3 & YLR221C & 9 & 4 & 16 & 12.26297 & 37.42757 \\
\hline NUP2 & YLR335W & 9 & 4 & 17 & 9.203509 & 35.13287 \\
\hline \multirow[t]{2}{*}{$\mathrm{CCC} 1$} & YLR220W & 9 & 4 & 18 & 14.30382 & 47.10008 \\
\hline & YLR334C & 9 & 4 & 19 & 41.36733 & 42.68577 \\
\hline MSC3 & YLR219W & 9 & 4 & 20 & 28.40173 & 43.04072 \\
\hline \multirow[t]{2}{*}{ RPS25B } & YLR333C & 9 & 4 & 21 & 31.95723 & 43.10231 \\
\hline & YLR218C & 9 & 4 & 22 & 35.67863 & 9.905605 \\
\hline MID2 & YLR332W & 9 & 4 & 23 & 34.2881 & 49.71042 \\
\hline HIS3 & YOR202W & 9 & 4 & 24 & 12.15772 & \#DIV/0! \\
\hline HIS3 & YOR202W & 9 & 5 & 1 & 27.19864 & \#DIV/0! \\
\hline \multirow[t]{2}{*}{ LOT6 } & YLR011W & 9 & 5 & 2 & 38.69685 & 50.8072 \\
\hline & YLR114C & 9 & 5 & 3 & \#DIV/0! & 27.37295 \\
\hline SSK1 & YLR006C & 9 & 5 & 4 & \#DIV/0! & 38.79042 \\
\hline \multirow[t]{6}{*}{ HOG1 } & YLR113W & 9 & 5 & 5 & 45.8051 & \#DIV/0! \\
\hline & YLR004C & 9 & 5 & 6 & 21.73942 & 33.43599 \\
\hline & YLR112W & 9 & 5 & 7 & 14.09368 & 24.36192 \\
\hline & YLR003C & 9 & 5 & 8 & 15.7457 & 50.20118 \\
\hline & YLR111W & 9 & 5 & 9 & 15.76018 & 38.06829 \\
\hline & YLR001C & 9 & 5 & 10 & 10.2667 & 37.44029 \\
\hline CCW12 & YLR110C & 9 & 5 & 11 & 18.23464 & 13.06241 \\
\hline AYT1 & YLL063C & 9 & 5 & 12 & 29.72729 & 49.96663 \\
\hline AHP1 & YLR109W & 9 & 5 & 13 & 1.215876 & 24.81419 \\
\hline \multirow[t]{2}{*}{ MHT1 } & YLL062C & 9 & 5 & 14 & 21.16645 & 42.11461 \\
\hline & YLR108C & 9 & 5 & 15 & 9.3398 & 12.56679 \\
\hline MMP1 & YLL061W & 9 & 5 & 16 & 17.70637 & 30.02664 \\
\hline REX3 & YLR107W & 9 & 5 & 17 & 25.58412 & 29.6839 \\
\hline \multirow[t]{3}{*}{ GTT2 } & YLL060C & 9 & 5 & 18 & 19.47093 & 40.06686 \\
\hline & YLR104W & 9 & 5 & 19 & 12.99436 & 26.06878 \\
\hline & YLL059C & 9 & 5 & 20 & 28.92454 & 30.33433 \\
\hline \multirow[t]{2}{*}{ APC9 } & YLR102C & 9 & 5 & 21 & 22.45823 & 41.16164 \\
\hline & YLL058W & 9 & 5 & 22 & 47.75315 & 24.19618 \\
\hline ICT1 & YLR099C & 9 & 5 & 23 & 22.33825 & 50.80652 \\
\hline HIS3 & YOR202W & 9 & 5 & 24 & 6.467423 & \#DIV/0! \\
\hline HIS3 & YOR202W & 9 & 6 & 1 & 19.9237 & \#DIV/0! \\
\hline SYM1 & YLR251W & 9 & 6 & 2 & 60.58592 & 51.64909 \\
\hline VPS38 & YLR360W & 9 & 6 & 3 & 14.53434 & 2.620284 \\
\hline SSP120 & YLR250W & 9 & 6 & 4 & 9.27124 & 37.65674 \\
\hline $\mathrm{RSC2}$ & YLR357W & 9 & 6 & 5 & 26.18326 & \#DIV/0! \\
\hline \multirow[t]{3}{*}{ RCK2 } & YLR248W & 9 & 6 & 6 & 6.413949 & 42.04345 \\
\hline & YLR356W & 9 & 6 & 7 & 19.38979 & 32.07385 \\
\hline & YLR247C & 9 & 6 & 8 & 12.20736 & 48.69158 \\
\hline TAL1 & YLR354C & 9 & 6 & 9 & 12.54291 & 47.37232 \\
\hline ERF2 & YLR246W & 9 & 6 & 10 & 8.159586 & 36.4503 \\
\hline
\end{tabular}




\begin{tabular}{|c|c|c|c|c|c|c|}
\hline BUD8 & YLR353W & 9 & 6 & 11 & 8.570019 & 40.10042 \\
\hline \multirow[t]{3}{*}{ ARV1 } & YLR242C & 9 & 6 & 12 & 16.05537 & 16.45228 \\
\hline & YLR352W & 9 & 6 & 13 & 15.07086 & 32.22614 \\
\hline & YLR241W & 9 & 6 & 14 & 2.965428 & 54.60254 \\
\hline NIT3 & YLR351C & 9 & 6 & 15 & 12.27571 & 28.35251 \\
\hline LIP2 & YLR239C & 9 & 6 & 16 & 23.99866 & 23.85063 \\
\hline ORM2 & YLR350W & 9 & 6 & 17 & 10.3707 & 46.54145 \\
\hline \multirow[t]{3}{*}{ THI7 } & YLR237W & 9 & 6 & 18 & 46.61102 & 52.83831 \\
\hline & YLR349W & 9 & 6 & 19 & 20.40517 & 50.4138 \\
\hline & YLR236C & 9 & 6 & 20 & 14.06977 & 40.50663 \\
\hline \multirow[t]{3}{*}{ DIC1 } & YLR348C & 9 & 6 & 21 & 12.87067 & 34.91547 \\
\hline & YLR235C & 9 & 6 & 22 & 23.91159 & 40.13095 \\
\hline & YLR346C & 9 & 6 & 23 & 34.39745 & 57.74224 \\
\hline HIS3 & YOR202W & 9 & 6 & 24 & 17.94784 & \#DIV/0! \\
\hline HIS3 & YOR202W & 9 & 7 & 1 & 15.54706 & \#DIV/0! \\
\hline IZH3 & YLR023C & 9 & 7 & 2 & 22.27016 & 29.06268 \\
\hline \multirow[t]{2}{*}{ ZRT2 } & YLR130C & 9 & 7 & 3 & 21.25171 & 54.7544 \\
\hline & YLR021W & 9 & 7 & 4 & 28.41189 & 63.62986 \\
\hline DCN1 & YLR128W & 9 & 7 & 5 & 15.19132 & \#DIV/0! \\
\hline \multirow[t]{2}{*}{ YEH3 } & YLR020C & 9 & 7 & 6 & 12.23012 & 57.37616 \\
\hline & YLR126C & 9 & 7 & 7 & 16.71186 & 42.58199 \\
\hline \multirow[t]{2}{*}{ PSR2 } & YLR019W & 9 & 7 & 8 & 11.2401 & 38.04569 \\
\hline & YLR125W & 9 & 7 & 9 & 21.40033 & 42.68643 \\
\hline \multirow[t]{2}{*}{ РOM34 } & YLR018C & 9 & 7 & 10 & 18.04235 & 56.34894 \\
\hline & YLR124W & 9 & 7 & 11 & 12.74638 & 54.22192 \\
\hline \multirow[t]{4}{*}{ MEU1 } & YLR017W & 9 & 7 & 12 & 38.46457 & 46.47184 \\
\hline & YLR123C & 9 & 7 & 13 & 18.13253 & 49.43749 \\
\hline & YLR016C & 9 & 7 & 14 & 10.95548 & 23.44119 \\
\hline & YLR122C & 9 & 7 & 15 & 7.48111 & 28.36842 \\
\hline BRE2 & YLR015W & 9 & 7 & 16 & 24.42669 & 26.99895 \\
\hline YPS3 & YLR121C & 9 & 7 & 17 & 9.739441 & 31.36483 \\
\hline PPR1 & YLR014C & 9 & 7 & 18 & 13.52716 & 45.01079 \\
\hline YPS1 & YLR120C & 9 & 7 & 19 & 12.52271 & 33.67799 \\
\hline GAT3 & YLR013W & 9 & 7 & 20 & 26.81403 & 34.16857 \\
\hline \multirow[t]{3}{*}{ SRN2 } & YLR119W & 9 & 7 & 21 & 22.94622 & 46.03929 \\
\hline & YLR012C & 9 & 7 & 22 & -3.36095 & 27.97738 \\
\hline & YLR118C & 9 & 7 & 23 & 8.184407 & 27.40578 \\
\hline HIS3 & YOR202W & 9 & 7 & 24 & 18.42223 & \#DIV/0! \\
\hline HIS3 & YOR202W & 9 & 8 & 1 & 8.38169 & \#DIV/0! \\
\hline RPS28B & YLR264W & 9 & 8 & 2 & 21.15684 & 43.01855 \\
\hline SUR4 & YLR372W & 9 & 8 & 3 & 23.47273 & 45.32339 \\
\hline RED1 & YLR263W & 9 & 8 & 4 & 25.64473 & 38.42601 \\
\hline ROM2 & YLR371W & 9 & 8 & 5 & 21.67489 & 61.86293 \\
\hline
\end{tabular}




\begin{tabular}{|c|c|c|c|c|c|c|}
\hline RBF7 & YLR262C-A & 9 & 8 & 6 & 25.08173 & \#DIV/0! \\
\hline ARC18 & YLR370C & 9 & 8 & 7 & 13.40159 & \#DIV/0! \\
\hline YPT6 & YLR262C & 9 & 8 & 8 & 15.88997 & 42.44434 \\
\hline \multirow[t]{2}{*}{ MDM30 } & YLR368W & 9 & 8 & 9 & 10.3173 & 49.26315 \\
\hline & YLR261C & 9 & 8 & 10 & 12.03637 & 53.61925 \\
\hline RPS22B & YLR367W & 9 & 8 & 11 & 10.27913 & 33.92102 \\
\hline \multirow[t]{6}{*}{ GSY2 } & YLR258W & 9 & 8 & 12 & 20.21205 & 48.00037 \\
\hline & YLR366W & 9 & 8 & 13 & 7.226004 & 29.28614 \\
\hline & YLR257W & 9 & 8 & 14 & 5.62164 & 49.78001 \\
\hline & YLR365W & 9 & 8 & 15 & -1.16177 & 48.16728 \\
\hline & YLR255C & 9 & 8 & 16 & 26.66269 & 42.27462 \\
\hline & YLR364W & 9 & 8 & 17 & 18.17265 & 36.85024 \\
\hline NDL1 & YLR254C & 9 & 8 & 18 & -4.64598 & 48.97688 \\
\hline \multirow[t]{2}{*}{ NMD4 } & YLR363C & 9 & 8 & 19 & 29.05548 & 47.62897 \\
\hline & YLR253W & 9 & 8 & 20 & 14.78281 & -4.94441 \\
\hline \multirow[t]{2}{*}{ STE11 } & YLR362W & 9 & 8 & 21 & 32.05241 & 27.82112 \\
\hline & YLR252W & 9 & 8 & 22 & 21.89143 & 33.98296 \\
\hline DCR2 & YLR361C & 9 & 8 & 23 & 38.6787 & 47.86629 \\
\hline HIS3 & YOR202W & 9 & 8 & 24 & 13.25918 & \#DIV/0! \\
\hline HIS3 & YOR202W & 9 & 9 & 1 & 11.15296 & \#DIV/O! \\
\hline RIC1 & YLR039C & 9 & 9 & 2 & 21.70528 & 46.81246 \\
\hline SPE4 & YLR146C & 9 & 9 & 3 & 26.19962 & 50.28193 \\
\hline cox12 & YLR038C & 9 & 9 & 4 & 23.84503 & 7.526125 \\
\hline ACF2 & YLR144C & 9 & 9 & 5 & 12.71778 & 48.28594 \\
\hline \multirow[t]{3}{*}{ DAN2 } & YLR037C & 9 & 9 & 6 & 10.18551 & \#DIV/0! \\
\hline & YLR143W & 9 & 9 & 7 & 6.211059 & \#DIV/0! \\
\hline & YLR036C & 9 & 9 & 8 & -0.91494 & 44.72643 \\
\hline PUT1 & YLR142W & 9 & 9 & 9 & 20.17635 & 71.67942 \\
\hline MLH2 & YLR035C & 9 & 9 & 10 & 12.94873 & 46.1349 \\
\hline NHA1 & YLR138W & 9 & 9 & 11 & 26.43572 & 28.25912 \\
\hline \multirow[t]{2}{*}{ SMF3 } & YLR034C & 9 & 9 & 12 & 12.89044 & 58.38577 \\
\hline & YLR137W & 9 & 9 & 13 & 7.330812 & 28.59092 \\
\hline RAD5 & YLR032W & 9 & 9 & 14 & 7.763307 & \#DIV/0! \\
\hline \multirow[t]{2}{*}{ TIS11 } & YLR136C & 9 & 9 & 15 & 13.17275 & \#DIV/0! \\
\hline & YLR031W & 9 & 9 & 16 & 3.514322 & \#DIV/0! \\
\hline \multirow[t]{2}{*}{ SLX4 } & YLR135W & 9 & 9 & 17 & 15.33313 & 41.33783 \\
\hline & YLR030W & 9 & 9 & 18 & 5.75938 & 50.03109 \\
\hline PDC5 & YLR134W & 9 & 9 & 19 & 9.659147 & 40.14805 \\
\hline ADE16 & YLR028C & 9 & 9 & 20 & 10.67221 & 27.50777 \\
\hline CKI1 & YLR133W & 9 & 9 & 21 & 40.99735 & 17.37017 \\
\hline UBR2 & YLR024C & 9 & 9 & 22 & 13.84925 & 33.43983 \\
\hline ACE2 & YLR131C & 9 & 9 & 23 & 24.98582 & 24.45374 \\
\hline HIS3 & YOR202W & 9 & 9 & 24 & 22.56284 & \#DIV/0! \\
\hline
\end{tabular}




\begin{tabular}{|c|c|c|c|c|c|c|}
\hline \multirow[t]{2}{*}{ HIS3 } & YOR202W & 9 & 10 & 1 & 13.59225 & \#DIV/0! \\
\hline & YLR281C & 9 & 10 & 2 & 19.90757 & 43.87301 \\
\hline \multirow[t]{2}{*}{ REH1 } & YLR387C & 9 & 10 & 3 & 23.14027 & 48.00304 \\
\hline & YLR280C & 9 & 10 & 4 & 21.29994 & 49.61606 \\
\hline \multirow[t]{2}{*}{ VAC14 } & YLR386W & 9 & 10 & 5 & 18.07355 & 38.56775 \\
\hline & YLR279W & 9 & 10 & 6 & 12.20376 & 42.35523 \\
\hline \multirow[t]{2}{*}{ SWC7 } & YLR385C & 9 & 10 & 7 & 8.033941 & 40.57359 \\
\hline & YLR278C & 9 & 10 & 8 & 21.14383 & 54.41672 \\
\hline IKI3 & YLR384C & 9 & 10 & 9 & \#DIV/0! & 35.91002 \\
\hline PIG1 & YLR273C & 9 & 10 & 10 & 13.47932 & 49.8996 \\
\hline \multirow[t]{2}{*}{ CTF3 } & YLR381W & 9 & 10 & 11 & 11.43755 & 39.60574 \\
\hline & YLR271W & 9 & 10 & 12 & 19.21764 & 49.62946 \\
\hline \multirow[t]{2}{*}{ CSR1 } & YLR380W & 9 & 10 & 13 & 9.164564 & 43.50813 \\
\hline & YLR269C & 9 & 10 & 14 & 40.06456 & \#DIV/0! \\
\hline FBP1 & YLR377C & 9 & 10 & 15 & 19.3288 & \#DIV/0! \\
\hline SEC22 & YLR268W & 9 & 10 & 16 & 5.97097 & \#DIV/0! \\
\hline PSY3 & YLR376C & 9 & 10 & 17 & 15.20983 & 26.53471 \\
\hline BOP2 & YLR267W & 9 & 10 & 18 & 16.3522 & 40.6674 \\
\hline STP3 & YLR375W & 9 & 10 & 19 & 13.7163 & 36.87447 \\
\hline \multirow[t]{2}{*}{ PDR8 } & YLR266C & 9 & 10 & 20 & 16.26903 & 55.00475 \\
\hline & YLR374C & 9 & 10 & 21 & 25.14126 & 29.32174 \\
\hline NEJ1 & YLR265C & 9 & 10 & 22 & 20.93821 & 38.18296 \\
\hline VID22 & YLR373C & 9 & 10 & 23 & 25.64683 & 18.62684 \\
\hline HIS3 & YOR202W & 9 & 10 & 24 & 25.1131 & \#DIV/O! \\
\hline \multirow[t]{4}{*}{ HIS3 } & YOR202W & 9 & 11 & 1 & 17.68468 & \#DIV/0! \\
\hline & YLR053C & 9 & 11 & 2 & 13.16469 & 54.67352 \\
\hline & YLR171W & 9 & 11 & 3 & 16.50726 & 42.11311 \\
\hline & YLR050C & 9 & 11 & 4 & 24.48356 & 56.16099 \\
\hline \multirow[t]{3}{*}{ APS1 } & YLR170C & 9 & 11 & 5 & 21.95846 & 62.74399 \\
\hline & YLR049C & 9 & 11 & 6 & 1.425076 & 51.6429 \\
\hline & YLR169W & 9 & 11 & 7 & 15.20021 & 40.96086 \\
\hline \multirow[t]{3}{*}{ RPSOB } & YLR048W & 9 & 11 & 8 & 8.502399 & 48.2714 \\
\hline & YLR168C & 9 & 11 & 9 & 28.68295 & 37.93193 \\
\hline & YLR047C & 9 & 11 & 10 & 18.98984 & 67.23941 \\
\hline \multirow[t]{3}{*}{ PUS5 } & YLR165C & 9 & 11 & 11 & 37.58324 & 43.5699 \\
\hline & YLR046C & 9 & 11 & 12 & 15.1544 & 50.95636 \\
\hline & YLR164W & 9 & 11 & 13 & 18.6229 & 18.90828 \\
\hline PDC1 & YLR044C & 9 & 11 & 14 & 13.23168 & 21.63024 \\
\hline RNH2O3 & YLR154C & 9 & 11 & 15 & 7.319227 & 52.68026 \\
\hline \multirow[t]{3}{*}{ TRX1 } & YLR043C & 9 & 11 & 16 & 32.56274 & 43.20506 \\
\hline & YLR152C & 9 & 11 & 17 & 9.647659 & 49.79271 \\
\hline & YLR042C & 9 & 11 & 18 & 23.07854 & 22.7258 \\
\hline PCD1 & YLR151C & 9 & 11 & 19 & 7.459535 & 52.35656 \\
\hline
\end{tabular}




\begin{tabular}{|c|c|c|c|c|c|c|}
\hline & YLR041W & 9 & 11 & 20 & 29.95394 & 25.82522 \\
\hline \multirow[t]{3}{*}{ STM1 } & YLR150W & 9 & 11 & 21 & 50.79612 & 45.72334 \\
\hline & YLR040C & 9 & 11 & 22 & 21.3698 & 27.34247 \\
\hline & YLR149C & 9 & 11 & 23 & \#DIV/0! & 32.30488 \\
\hline HIS3 & YOR202W & 9 & 11 & 24 & 27.49363 & \#DIV/0! \\
\hline \multirow[t]{3}{*}{ HIS3 } & YOR202W & 9 & 12 & 1 & 28.9271 & \#DIV/0! \\
\hline & YLR294C & 9 & 12 & 2 & 19.67454 & 33.12894 \\
\hline & YLR400W & 9 & 12 & 3 & 11.17699 & 60.97124 \\
\hline SEC72 & YLR292C & 9 & 12 & 4 & 39.08692 & 19.57194 \\
\hline \multirow[t]{2}{*}{ SKI2 } & YLR398C & 9 & 12 & 5 & 25.93874 & 64.57228 \\
\hline & YLR290C & 9 & 12 & 6 & 20.3525 & 45.80337 \\
\hline cox8 & YLR395C & 9 & 12 & 7 & 12.74347 & 34.02099 \\
\hline GUF1 & YLR289W & 9 & 12 & 8 & 13.93784 & 59.44568 \\
\hline CST9 & YLR394W & 9 & 12 & 9 & 34.95569 & 66.30208 \\
\hline RPS30A & YLR287C-A & 9 & 12 & 10 & 14.31204 & 51.92666 \\
\hline \multirow[t]{3}{*}{ ATP10 } & YLR393W & 9 & 12 & 11 & 25.25122 & 53.7375 \\
\hline & YLR287C & 9 & 12 & 12 & 13.59991 & 49.62238 \\
\hline & YLR392C & 9 & 12 & 13 & 3.39831 & 44.65204 \\
\hline \multirow[t]{2}{*}{ CTS1 } & YLR286C & 9 & 12 & 14 & 12.74237 & 36.43116 \\
\hline & YLR391W & 9 & 12 & 15 & 18.75309 & 53.21799 \\
\hline NNT1 & $\begin{array}{l}\text { YLR285W } \\
\text { YLR390W- }\end{array}$ & 9 & 12 & 16 & 5.171418 & 37.80426 \\
\hline CCW14 & $A$ & 9 & 12 & 17 & 26.63438 & 41.95779 \\
\hline $\mathrm{ECl} 1$ & YLR284C & 9 & 12 & 18 & 11.12631 & 24.46235 \\
\hline \multirow[t]{2}{*}{ ECM19 } & YLR390W & 9 & 12 & 19 & 13.18487 & 31.03507 \\
\hline & YLR283W & 9 & 12 & 20 & 15.73644 & 49.79479 \\
\hline \multirow[t]{2}{*}{ STE23 } & YLR389C & 9 & 12 & 21 & 12.27309 & 57.53841 \\
\hline & YLR282C & 9 & 12 & 22 & 33.86503 & 25.9182 \\
\hline RPS29A & YLR388W & 9 & 12 & 23 & 34.15602 & 14.01361 \\
\hline HIS3 & YOR202W & 9 & 12 & 24 & 28.93935 & \#DIV/0! \\
\hline \multirow[t]{2}{*}{ HIS3 } & YOR202W & 9 & 13 & 1 & 13.73954 & \#DIV/0! \\
\hline & YLR065C & 9 & 13 & 2 & 26.4261 & 36.17528 \\
\hline \multirow[t]{2}{*}{ TOS4 } & YLR183C & 9 & 13 & 3 & 29.11363 & 56.99825 \\
\hline & YLR064W & 9 & 13 & 4 & 14.3893 & 46.55285 \\
\hline \multirow[t]{2}{*}{ SWI6 } & YLR182W & 9 & 13 & 5 & 11.14101 & 44.56581 \\
\hline & YLR063W & 9 & 13 & 6 & 27.47341 & 57.19301 \\
\hline VTA1 & YLR181C & 9 & 13 & 7 & 33.79293 & 43.19844 \\
\hline BUD28 & YLR062C & 9 & 13 & 8 & 12.34874 & 33.77452 \\
\hline SAM1 & YLR180W & 9 & 13 & 9 & 7.074368 & 38.78251 \\
\hline \multirow[t]{2}{*}{ RPL22A } & YLR061W & 9 & 13 & 10 & 66.61653 & 45.75298 \\
\hline & YLR179C & 9 & 13 & 11 & 8.939119 & 43.23798 \\
\hline REX2 & YLR059C & 9 & 13 & 12 & 27.20798 & 53.42429 \\
\hline TFS1 & YLR178C & 9 & 13 & 13 & 12.64207 & 63.2085 \\
\hline
\end{tabular}




\begin{tabular}{|c|c|c|c|c|c|c|}
\hline \multirow[t]{3}{*}{ SHM2 } & YLR058C & 9 & 13 & 14 & 38.68336 & 41.5388 \\
\hline & YLR177W & 9 & 13 & 15 & 24.18414 & 3.728792 \\
\hline & YLR057W & 9 & 13 & 16 & 15.9283 & 46.71022 \\
\hline RFX1 & YLR176C & 9 & 13 & 17 & 19.86232 & 40.80696 \\
\hline ERG3 & YLR056W & 9 & 13 & 18 & 52.85093 & 34.44502 \\
\hline IDP2 & YLR174W & 9 & 13 & 19 & 42.09533 & 31.9445 \\
\hline \multirow[t]{2}{*}{ SPT8 } & YLR055C & 9 & 13 & 20 & 24.97102 & 15.21089 \\
\hline & YLR173W & 9 & 13 & 21 & 22.39431 & 9.665599 \\
\hline OsW2 & YLR054C & 9 & 13 & 22 & 29.7062 & 47.9119 \\
\hline DPH5 & YLR172C & 9 & 13 & 23 & 10.83048 & 52.54937 \\
\hline HIS3 & YOR202W & 9 & 13 & 24 & 37.20939 & \#DIV/O! \\
\hline HIS3 & YOR202W & 9 & 14 & 1 & 20.35963 & \#DIV/O! \\
\hline \multirow[t]{6}{*}{ SPH1 } & YLR313C & 9 & 14 & 2 & 20.09138 & 43.92666 \\
\hline & YLR414C & 9 & 14 & 3 & 40.21754 & 45.93644 \\
\hline & YLR312C & 9 & 14 & 4 & 6.148006 & 44.19748 \\
\hline & YLR413W & 9 & 14 & 5 & 10.0715 & 41.02044 \\
\hline & YLR311C & 9 & 14 & 6 & 10.5258 & 50.26737 \\
\hline & YLR412W & 9 & 14 & 7 & 10.02368 & 34.40593 \\
\hline IMH1 & YLR309C & 9 & 14 & 8 & 19.10201 & 40.45108 \\
\hline VIP1 & YLR410W & 9 & 14 & 9 & 19.17144 & 65.77981 \\
\hline \multirow[t]{2}{*}{ CDA1 } & YLR307W & 9 & 14 & 10 & 10.54871 & 49.70736 \\
\hline & YLR408C & 9 & 14 & 11 & 12.90867 & 40.872 \\
\hline \multirow[t]{2}{*}{ UBC12 } & YLR306W & 9 & 14 & 12 & 14.34426 & 47.09116 \\
\hline & YLR407W & 9 & 14 & 13 & 16.90226 & 31.76445 \\
\hline MET17 & YLR303W & 9 & 14 & 14 & 21.01435 & 50.32109 \\
\hline RPL31B & YLR406C & 9 & 14 & 15 & 22.857 & 47.22158 \\
\hline EXG1 & YLR300W & 9 & 14 & 16 & 27.36286 & 59.29023 \\
\hline DUS4 & YLR405W & 9 & 14 & 17 & 10.72571 & 52.36443 \\
\hline \multirow[t]{5}{*}{ ECM38 } & YLR299W & 9 & 14 & 18 & 15.88126 & 41.31315 \\
\hline & YLR404W & 9 & 14 & 19 & 12.64175 & 48.3288 \\
\hline & YLR297W & 9 & 14 & 20 & 42.2164 & 44.5928 \\
\hline & YLR402W & 9 & 14 & 21 & 42.82446 & 51.00729 \\
\hline & YLR296W & 9 & 14 & 22 & 22.10605 & 46.95362 \\
\hline DUS3 & YLR401C & 9 & 14 & 23 & 21.65621 & 56.86983 \\
\hline HIS3 & YOR202W & 9 & 14 & 24 & 27.15776 & \#DIV/0! \\
\hline HIS3 & YOR202W & 9 & 15 & 1 & 8.824707 & \#DIV/0! \\
\hline \multirow[t]{2}{*}{ ARP6 } & YLR085C & 9 & 15 & 2 & 32.74709 & \#DIV/0! \\
\hline & YLR199C & 9 & 15 & 3 & 27.90944 & \#DIV/0! \\
\hline \multirow[t]{2}{*}{ RAX2 } & YLR084C & 9 & 15 & 4 & 26.5277 & \#DIV/0! \\
\hline & YLR194C & 9 & 15 & 5 & 23.84319 & \#DIV/0! \\
\hline \multirow[t]{2}{*}{ EMP70 } & YLR083C & 9 & 15 & 6 & 5.546369 & \#DIV/0! \\
\hline & YLR193C & 9 & 15 & 7 & 28.55225 & \#DIV/0! \\
\hline SRL2 & YLR082C & 9 & 15 & 8 & 34.7526 & \#DIV/O! \\
\hline
\end{tabular}




\begin{tabular}{|c|c|c|c|c|c|c|}
\hline HCR1 & YLR192C & 9 & 15 & 9 & 43.69923 & \#DIV/0! \\
\hline GAL2 & YLR081W & 9 & 15 & 10 & 57.50428 & \#DIV/0! \\
\hline PEX13 & YLR191W & 9 & 15 & 11 & 39.2223 & \#DIV/0! \\
\hline EMP46 & YLR080W & 9 & 15 & 12 & 36.74596 & \#DIV/0! \\
\hline MMR1 & YLR190W & 9 & 15 & 13 & 26.05289 & \#DIV/0! \\
\hline SIC1 & YLR079W & 9 & 15 & 14 & 71.79241 & \#DIV/0! \\
\hline ATG26 & YLR189C & 9 & 15 & 15 & 13.39387 & \#DIV/0! \\
\hline FMP25 & YLR077W & 9 & 15 & 16 & 24.618 & \#DIV/0! \\
\hline \multirow[t]{2}{*}{ MDL1 } & YLR188W & 9 & 15 & 17 & 12.3767 & \#DIV/O! \\
\hline & YLR073C & 9 & 15 & 18 & 4.481861 & \#DIV/0! \\
\hline \multirow[t]{2}{*}{ SKG3 } & YLR187W & 9 & 15 & 19 & 14.64193 & \#DIV/0! \\
\hline & YLR072W & 9 & 15 & 20 & 6.208911 & \#DIV/0! \\
\hline RPL37A & YLR185W & 9 & 15 & 21 & 32.17185 & \#DIV/0! \\
\hline \multirow[t]{2}{*}{ XYL2 } & YLR070C & 9 & 15 & 22 & 27.64432 & \#DIV/0! \\
\hline & YLR184W & 9 & 15 & 23 & 23.809 & \#DIV/0! \\
\hline HIS3 & YOR202W & 9 & 15 & 24 & 17.71322 & \#DIV/0! \\
\hline HIS3 & YOR202W & 9 & 16 & 1 & 12.88204 & \#DIV/0! \\
\hline HIS3 & YOR202W & 9 & 16 & 2 & 34.58404 & \#DIV/0! \\
\hline HIS3 & YOR202W & 9 & 16 & 3 & 26.57541 & \#DIV/0! \\
\hline HIS3 & YOR202W & 9 & 16 & 4 & 25.39819 & \#DIV/0! \\
\hline HIS3 & YOR202W & 9 & 16 & 5 & 24.12751 & \#DIV/0! \\
\hline HIS3 & YOR202W & 9 & 16 & 6 & 13.01909 & \#DIV/0! \\
\hline HIS3 & YOR202W & 9 & 16 & 7 & 40.00141 & \#DIV/O! \\
\hline HIS3 & YOR202W & 9 & 16 & 8 & 21.38016 & \#DIV/0! \\
\hline HIS3 & YOR202W & 9 & 16 & 9 & 21.11744 & \#DIV/0! \\
\hline HIS3 & YOR202W & 9 & 16 & 10 & 31.44188 & \#DIV/0! \\
\hline HIS3 & YOR202W & 9 & 16 & 11 & 8.762099 & \#DIV/0! \\
\hline HIS3 & YOR202W & 9 & 16 & 12 & 23.1112 & \#DIV/0! \\
\hline HIS3 & YOR202W & 9 & 16 & 13 & 39.77691 & \#DIV/0! \\
\hline HIS3 & YOR202W & 9 & 16 & 14 & 53.52176 & \#DIV/0! \\
\hline HIS3 & YOR202W & 9 & 16 & 15 & 9.92095 & \#DIV/0! \\
\hline HIS3 & YOR202W & 9 & 16 & 16 & 4.993088 & \#DIV/0! \\
\hline HIS3 & YOR202W & 9 & 16 & 17 & 15.61876 & \#DIV/0! \\
\hline HIS3 & YOR202W & 9 & 16 & 18 & 23.86705 & \#DIV/0! \\
\hline HIS3 & YOR202W & 9 & 16 & 19 & 11.97678 & \#DIV/0! \\
\hline HIS3 & YOR202W & 9 & 16 & 20 & 14.98133 & \#DIV/0! \\
\hline HIS3 & YOR202W & 9 & 16 & 21 & 22.67893 & \#DIV/O! \\
\hline HIS3 & YOR202W & 9 & 16 & 22 & 29.58635 & \#DIV/0! \\
\hline HIS3 & YOR202W & 9 & 16 & 23 & 35.90419 & \#DIV/0! \\
\hline HIS3 & YOR202W & 9 & 16 & 24 & 28.7844 & \#DIV/0! \\
\hline HIS3 & YOR202W & 10 & 1 & 1 & 42.83692 & \#DIV/0! \\
\hline HIS3 & YOR202W & 10 & 1 & 2 & 47.37918 & \#DIV/0! \\
\hline HIS3 & YOR202W & 10 & 1 & 3 & 44.84734 & \#DIV/0! \\
\hline
\end{tabular}




\begin{tabular}{|c|c|c|c|c|c|c|}
\hline HIS3 & YOR202W & 10 & 1 & 4 & 59.67131 & \#DIV/0! \\
\hline HIS3 & YOR202W & 10 & 1 & 5 & 22.94658 & \#DIV/0! \\
\hline HIS3 & YOR202W & 10 & 1 & 6 & 15.40138 & \#DIV/0! \\
\hline HIS3 & YOR202W & 10 & 1 & 7 & 42.37552 & \#DIV/0! \\
\hline HIS3 & YOR202W & 10 & 1 & 8 & 27.68248 & \#DIV/0! \\
\hline HIS3 & YOR202W & 10 & 1 & 9 & 35.10265 & \#DIV/0! \\
\hline HIS3 & YOR202W & 10 & 1 & 10 & 31.37277 & \#DIV/0! \\
\hline HIS3 & YOR202W & 10 & 1 & 11 & 32.89158 & \#DIV/0! \\
\hline HIS3 & YOR202W & 10 & 1 & 12 & 39.87012 & \#DIV/0! \\
\hline HIS3 & YOR202W & 10 & 1 & 13 & 42.50867 & \#DIV/0! \\
\hline HIS3 & YOR202W & 10 & 1 & 14 & 41.861 & \#DIV/O! \\
\hline HIS3 & YOR202W & 10 & 1 & 15 & 31.29113 & \#DIV/O! \\
\hline HIS3 & YOR202W & 10 & 1 & 16 & 28.92011 & \#DIV/0! \\
\hline HIS3 & YOR202W & 10 & 1 & 17 & 43.53138 & \#DIV/0! \\
\hline HIS3 & YOR202W & 10 & 1 & 18 & 40.54111 & \#DIV/0! \\
\hline HIS3 & YOR202W & 10 & 1 & 19 & 29.30738 & \#DIV/O! \\
\hline HIS3 & YOR202W & 10 & 1 & 20 & 39.80368 & \#DIV/0! \\
\hline HIS3 & YOR202W & 10 & 1 & 21 & 36.51184 & \#DIV/0! \\
\hline HIS3 & YOR202W & 10 & 1 & 22 & 36.86686 & \#DIV/0! \\
\hline HIS3 & YOR202W & 10 & 1 & 23 & 30.95992 & \#DIV/O! \\
\hline HIS3 & YOR202W & 10 & 1 & 24 & 67.3542 & \#DIV/0! \\
\hline HIS3 & YOR202W & 10 & 2 & 1 & 51.85391 & \#DIV/0! \\
\hline \multirow[t]{3}{*}{ IOC4 } & YMR044W & 10 & 2 & 2 & 19.62205 & \#DIV/0! \\
\hline & YMR158C- & & & & & \\
\hline & B & 10 & 2 & 3 & 33.76683 & 25.87769 \\
\hline ARG80 & YMR042W & 10 & 2 & 4 & 4.461939 & 43.37793 \\
\hline \multirow[t]{2}{*}{ FMP39 } & YMR157C & 10 & 2 & 5 & 35.7757 & 23.18062 \\
\hline & YMR041C & 10 & 2 & 6 & 18.81145 & 23.22011 \\
\hline TPP1 & YMR156C & 10 & 2 & 7 & 10.3221 & 29.72243 \\
\hline \multirow[t]{2}{*}{ YET2 } & YMR040W & 10 & 2 & 8 & 37.52813 & 32.57914 \\
\hline & YMR155W & 10 & 2 & 9 & 32.04908 & 36.31248 \\
\hline SUB1 & YMR039C & 10 & 2 & 10 & 46.6141 & 53.34728 \\
\hline RIM13 & YMR154C & 10 & 2 & 11 & 61.4488 & 36.21783 \\
\hline LYS7 & YMR038C & 10 & 2 & 12 & 45.78316 & 43.60375 \\
\hline NUP53 & YMR153W & 10 & 2 & 13 & 64.37822 & 25.19609 \\
\hline \multirow[t]{3}{*}{ MSN2 } & YMR037C & 10 & 2 & 14 & 46.286 & 33.57876 \\
\hline & YMR153C- & & & & & \\
\hline & A & 10 & 2 & 15 & 50.65327 & 8.282622 \\
\hline MIH1 & YMR036C & 10 & 2 & 16 & 51.94236 & 35.36471 \\
\hline YIM1 & YMR152W & 10 & 2 & 17 & 31.37148 & 42.07084 \\
\hline \multirow[t]{3}{*}{ IMP2 } & YMR035W & 10 & 2 & 18 & 35.98238 & 32.77185 \\
\hline & YMR148W & 10 & 2 & 19 & 37.30093 & -5.45509 \\
\hline & YMR034C & 10 & 2 & 20 & 25.99778 & 2.619433 \\
\hline
\end{tabular}


$\begin{array}{llllll}\text { YMR147W } & 10 & 2 & 21 & 31.71453 & 44.29612\end{array}$

YMR031W-

\begin{tabular}{|c|c|c|c|c|c|c|}
\hline & & & & & & \\
\hline NDE1 & YMR145C & 10 & 2 & 23 & 44.02175 & 34.10759 \\
\hline HIS3 & YOR202W & 10 & 2 & 24 & 37.16792 & \#DIV/0! \\
\hline HIS3 & YOR202W & 10 & 3 & 1 & 22.47596 & \#DIV/0! \\
\hline & YLR428C & 10 & 3 & 2 & 19.89408 & 41.45776 \\
\hline MFT1 & YML062C & 10 & 3 & 3 & 14.35653 & 32.92234 \\
\hline MAG2 & YLR427W & 10 & 3 & 4 & 45.90081 & 23.78161 \\
\hline OGG1 & YML060W & 10 & 3 & 5 & 8.500963 & 27.75762 \\
\hline & YLR426W & 10 & 3 & 6 & 34.8185 & 28.86728 \\
\hline NTE1 & YML059C & 10 & 3 & 7 & 45.88462 & 34.04762 \\
\hline TUS1 & YLR425W & 10 & 3 & 8 & 47.96236 & 51.85508 \\
\hline SML1 & YML058W & 10 & 3 & 9 & 34.47516 & 31.86247 \\
\hline ATG17 & YLR423C & 10 & 3 & 10 & 44.27646 & 36.69104 \\
\hline & YML058C- & & & & & \\
\hline & A & 10 & 3 & 11 & 44.56181 & 45.86919 \\
\hline & YLR422W & 10 & 3 & 12 & 49.44849 & 23.78121 \\
\hline CMP2 & YML057W & 10 & 3 & 13 & 33.96131 & 46.77024 \\
\hline RPN13 & YLR421C & 10 & 3 & 14 & 54.74088 & 15.23856 \\
\hline IMD4 & YML056C & 10 & 3 & 15 & 52.1605 & 32.84677 \\
\hline URA4 & YLR420W & 10 & 3 & 16 & 44.56451 & 29.59977 \\
\hline SPC2 & YML055W & 10 & 3 & 17 & 40.37445 & 40.43695 \\
\hline CDC73 & YLR418C & 10 & 3 & 18 & 25.69524 & 38.14866 \\
\hline CYB2 & YML054C & 10 & 3 & 19 & 40.17466 & 40.09504 \\
\hline & YLR416C & 10 & 3 & 20 & 62.64544 & 38.5469 \\
\hline & YML053C & 10 & 3 & 21 & 42.86295 & 34.52165 \\
\hline & YLR415C & 10 & 3 & 22 & 43.0511 & 23.08201 \\
\hline SUR7 & YML052W & 10 & 3 & 23 & 35.40427 & 36.27961 \\
\hline HIS3 & YOR202W & 10 & 3 & 24 & 37.48315 & \#DIV/0! \\
\hline HIS3 & YOR202W & 10 & 4 & 1 & 14.53572 & \#DIV/0! \\
\hline RIM9 & YMR063W & 10 & 4 & 2 & 17.22911 & 37.46069 \\
\hline ALD2 & YMR170C & 10 & 4 & 3 & 32.92344 & 46.08745 \\
\hline TOM37 & YMR060C & 10 & 4 & 4 & 39.91537 & -0.30311 \\
\hline ALD3 & YMR169c & 10 & 4 & 5 & 43.58917 & 37.86968 \\
\hline FET3 & YMR058W & 10 & 4 & 6 & 18.1209 & 27.55076 \\
\hline MLH1 & YMR167W & 10 & 4 & 7 & 42.95986 & 61.68934 \\
\hline & YMR057C & 10 & 4 & 8 & 23.67593 & 43.30435 \\
\hline & YMR166C & 10 & 4 & 9 & 34.58905 & 32.94301 \\
\hline AAC1 & YMR056C & 10 & 4 & 10 & 38.72093 & \#DIV/0! \\
\hline MSS11 & YMR164C & 10 & 4 & 11 & 7.77248 & \#DIV/0! \\
\hline BUB2 & YMR055C & 10 & 4 & 12 & 31.21082 & \#DIV/0! \\
\hline & YMR163C & 10 & 4 & 13 & 19.22055 & 46.49972 \\
\hline
\end{tabular}




\begin{tabular}{|c|c|c|c|c|c|c|}
\hline STV1 & YMR054W & 10 & 4 & 14 & 63.66297 & 8.744118 \\
\hline DNF3 & YMR162C & 10 & 4 & 15 & 32.62389 & 38.03604 \\
\hline STB2 & YMR053C & 10 & 4 & 16 & 40.25194 & 32.89143 \\
\hline HLJ1 & YMR161W & 10 & 4 & 17 & 1.734034 & 43.42847 \\
\hline \multirow[t]{3}{*}{ FAR3 } & YMR052W & 10 & 4 & 18 & 55.14408 & 34.86046 \\
\hline & $\begin{array}{l}\text { YMR160W } \\
\text { YMR052C- }\end{array}$ & 10 & 4 & 19 & 39.69482 & 51.53752 \\
\hline & $A$ & 10 & 4 & 20 & 43.09807 & 40.0014 \\
\hline ATG16 & YMR159C & 10 & 4 & 21 & 18.83383 & 29.38159 \\
\hline \multirow[t]{2}{*}{ CSM3 } & $\begin{array}{l}\text { YMR048W } \\
\text { YMR158W- }\end{array}$ & 10 & 4 & 22 & 36.25886 & 45.14026 \\
\hline & $A$ & 10 & 4 & 23 & 44.55246 & 46.06105 \\
\hline HIS3 & YOR202W & 10 & 4 & 24 & 28.43698 & \#DIV/0! \\
\hline HIS3 & YOR202W & 10 & 5 & 1 & 41.17391 & \#DIV/O! \\
\hline SIR3 & $\begin{array}{l}\text { YLR442C } \\
\text { YML081C- }\end{array}$ & 10 & 5 & 2 & 41.0349 & 49.12301 \\
\hline ATP18 & $A$ & 10 & 5 & 3 & 33.15152 & 43.48984 \\
\hline RPS1A & YLR441C & 10 & 5 & 4 & 29.68867 & 52.58135 \\
\hline WAR1 & YML076C & 10 & 5 & 5 & 43.77797 & 42.93686 \\
\hline CAR2 & YLR438W & 10 & 5 & 6 & 45.17389 & 39.05721 \\
\hline \multirow[t]{2}{*}{ HMG1 } & YML075C & 10 & 5 & 7 & 42.70892 & 34.24315 \\
\hline & YLR437C & 10 & 5 & 8 & 42.10912 & 44.25698 \\
\hline FPR3 & YML074C & 10 & 5 & 9 & 20.3926 & 8.394899 \\
\hline ECM30 & YLR436C & 10 & 5 & 10 & 44.00875 & 32.0284 \\
\hline TCB3 & YML072C & 10 & 5 & 11 & 39.92547 & \#DIV/0! \\
\hline TSR2 & YLR435W & 10 & 5 & 12 & 61.90498 & \#DIV/0! \\
\hline \multirow[t]{2}{*}{ COG8 } & YML071C & 10 & 5 & 13 & 44.09919 & 37.04697 \\
\hline & YLR434C & 10 & 5 & 14 & 35.13685 & 29.23896 \\
\hline DAK1 & YML070W & 10 & 5 & 15 & 52.639 & 37.37604 \\
\hline CNA1 & YLR433C & 10 & 5 & 16 & 48.20085 & 37.20052 \\
\hline ITT1 & YML068W & 10 & 5 & 17 & 50.59747 & 37.18823 \\
\hline IMD3 & YLR432W & 10 & 5 & 18 & 34.23555 & 49.60204 \\
\hline ERV41 & YML067C & 10 & 5 & 19 & 48.0802 & -16.617 \\
\hline ATG23 & YLR431C & 10 & 5 & 20 & 25.18312 & 39.74376 \\
\hline SMA2 & YML066C & 10 & 5 & 21 & 65.91018 & 40.25173 \\
\hline CRN1 & YLR429W & 10 & 5 & 22 & 29.45322 & 41.6476 \\
\hline RPS1B & YML063W & 10 & 5 & 23 & 22.02794 & 43.5854 \\
\hline HIS3 & YOR202W & 10 & 5 & 24 & 37.5919 & \#DIV/0! \\
\hline \multirow[t]{3}{*}{ HIS3 } & YOR202W & 10 & 6 & 1 & 62.4695 & \#DIV/0! \\
\hline & YMR086C- & & & & & \\
\hline & $A$ & 10 & 6 & 2 & 33.31046 & 38.38625 \\
\hline \multirow[t]{3}{*}{ SPT21 } & YMR179W & 10 & 6 & 3 & 40.87323 & 42.55197 \\
\hline & YMR085W & 10 & 6 & 4 & 29.91071 & 48.9622 \\
\hline & YMR178W & 10 & 6 & 5 & 48.75165 & 27.2942 \\
\hline
\end{tabular}




\begin{tabular}{|c|c|c|c|c|c|c|}
\hline NAM7 & YMR080C & 10 & 6 & 6 & 33.07646 & 34.16703 \\
\hline MMT1 & YMR177W & 10 & 6 & 7 & 24.87124 & 28.96175 \\
\hline CTF18 & YMR078C & 10 & 6 & 8 & 16.079 & 41.08631 \\
\hline ECM5 & YMR176W & 10 & 6 & 9 & 39.96824 & 40.18369 \\
\hline RCO1 & YMR075W & 10 & 6 & 10 & 23.27044 & 32.40886 \\
\hline \multirow[t]{3}{*}{ SIP18 } & YMR175w & 10 & 6 & 11 & 47.30776 & 32.88135 \\
\hline & YMR075C- & & & & & \\
\hline & A & 10 & 6 & 12 & 29.17759 & 23.86399 \\
\hline \multirow[t]{4}{*}{ PAI3 } & YMR174C & 10 & 6 & 13 & 29.39376 & 50.05007 \\
\hline & YMR073C & 10 & 6 & 14 & 51.30639 & 31.20654 \\
\hline & YMR173W- & & & & & \\
\hline & $A$ & 10 & 6 & 15 & 47.91861 & 38.30462 \\
\hline МOT3 & YMR070W & 10 & 6 & 16 & 28.85407 & 31.15184 \\
\hline DDR48 & YMR173W & 10 & 6 & 17 & 21.79731 & 50.21438 \\
\hline AVO2 & YMR068W & 10 & 6 & 18 & 30.30263 & 48.5452 \\
\hline НОТ1 & YMR172W & 10 & 6 & 19 & 16.38225 & 43.15949 \\
\hline \multirow[t]{3}{*}{ UBX4 } & YMR067C & 10 & 6 & 20 & 50.25854 & 36.02125 \\
\hline & YMR172C- & & & & & \\
\hline & $A$ & 10 & 6 & 21 & 47.39698 & 43.19516 \\
\hline \multirow[t]{2}{*}{ KAR5 } & YMR065W & 10 & 6 & 22 & 54.56715 & 46.91869 \\
\hline & YMR171C & 10 & 6 & 23 & 53.10995 & 44.40972 \\
\hline HIS3 & YOR202W & 10 & 6 & 24 & 23.28118 & \#DIV/0! \\
\hline HIS3 & YOR202W & 10 & 7 & 1 & 49.86355 & \#DIV/0! \\
\hline FMP27 & YLR454W & 10 & 7 & 2 & 33.64727 & 50.86202 \\
\hline CAC2 & YML102W & 10 & 7 & 3 & 28.74535 & 8.195734 \\
\hline \multirow[t]{3}{*}{ RIF2 } & YLR453C & 10 & 7 & 4 & 37.94774 & 39.98193 \\
\hline & YML012C- & & & & & \\
\hline & $A$ & 10 & 7 & 5 & 20.11507 & 39.21296 \\
\hline SST2 & YLR452C & 10 & 7 & 6 & 41.819 & 37.61189 \\
\hline CUE4 & YML101C & 10 & 7 & 7 & 49.59298 & 47.58055 \\
\hline \multirow[t]{3}{*}{ LEU3 } & YLR451W & 10 & 7 & 8 & 37.54734 & 52.18735 \\
\hline & YML100W- & & & & & \\
\hline & $A$ & 10 & 7 & 9 & 45.71486 & 52.83488 \\
\hline HMG2 & YLR450W & 10 & 7 & 10 & 52.49184 & 54.73915 \\
\hline TSL1 & YML100W & 10 & 7 & 11 & 49.56582 & 51.27559 \\
\hline FPR4 & YLR449W & 10 & 7 & 12 & 17.91861 & 44.94001 \\
\hline ARG81 & YML099C & 10 & 7 & 13 & 19.70803 & -0.19184 \\
\hline RPL6B & YLR448W & 10 & 7 & 14 & 56.30563 & 71.19333 \\
\hline \multirow[t]{4}{*}{ VPS9 } & YML097C & 10 & 7 & 15 & \#DIV/0! & 41.45493 \\
\hline & YLR446W & 10 & 7 & 16 & 39.7285 & 46.71795 \\
\hline & YML096W & 10 & 7 & 17 & 33.49805 & 43.56084 \\
\hline & YLR445W & 10 & 7 & 18 & 42.39467 & 47.92783 \\
\hline \multirow[t]{2}{*}{ RAD10 } & YML095C & 10 & 7 & 19 & 26.95007 & 40.08259 \\
\hline & YLR444C & 10 & 7 & 20 & 55.165 & 42.10937 \\
\hline
\end{tabular}




\begin{tabular}{|c|c|c|c|c|c|c|}
\hline GIM5 & YML094W & 10 & 7 & 21 & 42.94996 & 18.84007 \\
\hline \multirow[t]{2}{*}{ ECM7 } & YLR443W & 10 & 7 & 22 & 32.92887 & 23.4274 \\
\hline & YML090W & 10 & 7 & 23 & 45.08734 & 51.81971 \\
\hline HIS3 & YOR202W & 10 & 7 & 24 & 39.06123 & \#DIV/0! \\
\hline \multirow[t]{2}{*}{ HIS3 } & YOR202W & 10 & 8 & 1 & 43.08294 & \#DIV/0! \\
\hline & YMR103C & 10 & 8 & 2 & 39.83626 & 44.0917 \\
\hline \multirow[t]{2}{*}{ APP2 } & YMR192W & 10 & 8 & 3 & 18.91997 & 54.35747 \\
\hline & YMR102C & 10 & 8 & 4 & 35.00096 & 34.03046 \\
\hline SPG5 & YMR191W & 10 & 8 & 5 & 39.81429 & 42.69927 \\
\hline SRT1 & YMR101C & 10 & 8 & 6 & 59.30717 & 44.46869 \\
\hline SGS1 & YMR190C & 10 & 8 & 7 & 36.71046 & 45.8671 \\
\hline MUB1 & YMR100W & 10 & 8 & 8 & 37.36545 & 32.48612 \\
\hline \multirow[t]{2}{*}{ GCV2 } & YMR189W & 10 & 8 & 9 & 30.69909 & 47.34901 \\
\hline & YMR099C & 10 & 8 & 10 & 41.00529 & 50.93952 \\
\hline MRPS17 & YMR188C & 10 & 8 & 11 & 46.77837 & 47.41754 \\
\hline \multirow[t]{2}{*}{ SNZ1 } & YMR096W & 10 & 8 & 12 & 39.37673 & 40.55242 \\
\hline & YMR187C & 10 & 8 & 13 & 23.46428 & 45.20043 \\
\hline SNO1 & YMR095C & 10 & 8 & 14 & 38.95093 & 48.48277 \\
\hline HSC82 & YMR186W & 10 & 8 & 15 & 25.30971 & 51.33275 \\
\hline AIP1 & YMR092C & 10 & 8 & 16 & 37.67272 & 35.09223 \\
\hline $\mathrm{SSO} 2$ & YMR183C & 10 & 8 & 17 & 28.79886 & 53.45902 \\
\hline VBA1 & YMR088C & 10 & 8 & 18 & 43.06068 & 18.45389 \\
\hline \multirow[t]{4}{*}{ RGM1 } & YMR182C & 10 & 8 & 19 & 51.96875 & 59.74972 \\
\hline & YMR087W & 10 & 8 & 20 & 26.43127 & 46.03719 \\
\hline & YMR181C & 10 & 8 & 21 & 44.39211 & 43.66667 \\
\hline & YMR086W & 10 & 8 & 22 & 1.585109 & 47.64351 \\
\hline CTL1 & YMR180C & 10 & 8 & 23 & 23.13925 & 66.66353 \\
\hline HIS3 & YOR202W & 10 & 8 & 24 & 35.21756 & \#DIV/0! \\
\hline HIS3 & YOR202W & 10 & 9 & 1 & 50.13336 & \#DIV/0! \\
\hline YAP1 & YML007W & 10 & 9 & 2 & 44.69664 & 45.48843 \\
\hline NGL3 & YML118W & 10 & 9 & 3 & 11.32398 & 22.58416 \\
\hline \multirow[t]{3}{*}{ GIS4 } & YML006C & 10 & 9 & 4 & 49.55349 & 51.99593 \\
\hline & YML117W- & & & & & \\
\hline & $A$ & 10 & 9 & 5 & 43.08082 & 44.43005 \\
\hline TRM12 & YML005W & 10 & 9 & 6 & 45.98129 & 42.29207 \\
\hline NAB6 & YML117W & 10 & 9 & 7 & 19.6445 & 47.58761 \\
\hline GLO1 & YML004C & 10 & 9 & 8 & 15.28488 & 36.71902 \\
\hline \multirow[t]{2}{*}{ ATR1 } & YML116W & 10 & 9 & 9 & 46.99253 & 46.11695 \\
\hline & YML003W & 10 & 9 & 10 & 47.49211 & 50.96916 \\
\hline \multirow[t]{2}{*}{ DAT1 } & YML113W & 10 & 9 & 11 & 46.02012 & 53.55188 \\
\hline & YML002W & 10 & 9 & 12 & 62.05433 & 51.63136 \\
\hline ZDS2 & YML109W & 10 & 9 & 13 & 43.15182 & 46.15799 \\
\hline YPT7 & YML001W & 10 & 9 & 14 & 33.03031 & 40.6731 \\
\hline
\end{tabular}




\begin{tabular}{|c|c|c|c|c|c|c|}
\hline & YML108W & 10 & 9 & 15 & 42.10616 & 18.13827 \\
\hline \multirow[t]{3}{*}{ PAU4 } & YLR461W & 10 & 9 & 16 & 41.76278 & 52.62261 \\
\hline & YML107C & 10 & 9 & 17 & 28.7263 & 30.23131 \\
\hline & YLR460C & 10 & 9 & 18 & 36.45194 & 51.00495 \\
\hline \multirow[t]{2}{*}{ URA5 } & YML106W & 10 & 9 & 19 & 29.14571 & 12.89778 \\
\hline & YLR456W & 10 & 9 & 20 & 40.70325 & 61.76637 \\
\hline \multirow[t]{2}{*}{ MDM1 } & YML104C & 10 & 9 & 21 & 24.0675 & 53.44529 \\
\hline & YLR455W & 10 & 9 & 22 & 50.31233 & 56.66897 \\
\hline NUP188 & YML103C & 10 & 9 & 23 & 44.67255 & 48.46815 \\
\hline HIS3 & YOR202W & 10 & 9 & 24 & 31.69569 & \#DIV/0! \\
\hline HIS3 & YOR202W & 10 & 10 & 1 & 72.82102 & \#DIV/O! \\
\hline \multirow[t]{2}{*}{ ASI1 } & YMR119W & 10 & 10 & 2 & 60.05813 & 49.57577 \\
\hline & YMR206W & 10 & 10 & 3 & 38.36739 & 44.31629 \\
\hline ASC1 & YMR116C & 10 & 10 & 4 & 55.94638 & 60.16973 \\
\hline PFK2 & YMR205C & 10 & 10 & 5 & 44.43329 & 55.44016 \\
\hline \multirow[t]{3}{*}{ FMP24 } & YMR115W & 10 & 10 & 6 & 36.23991 & 43.30834 \\
\hline & YMR204C & 10 & 10 & 7 & 17.25624 & 45.37436 \\
\hline & YMR114C & 10 & 10 & 8 & 35.11714 & 51.67294 \\
\hline \multirow[t]{2}{*}{ ERG2 } & YMR202W & 10 & 10 & 9 & 34.58207 & 50.25723 \\
\hline & YMR111C & 10 & 10 & 10 & 42.40131 & 49.62356 \\
\hline \multirow[t]{2}{*}{ RAD14 } & YMR201C & 10 & 10 & 11 & 48.33168 & 39.91632 \\
\hline & YMR110C & 10 & 10 & 12 & 63.3272 & 82.90472 \\
\hline CLN1 & YMR199W & 10 & 10 & 13 & 41.00594 & 28.18727 \\
\hline MYO5 & YMR109W & 10 & 10 & 14 & 32.13448 & 31.57326 \\
\hline CIK1 & YMR198W & 10 & 10 & 15 & 30.11586 & 27.43779 \\
\hline \multirow[t]{2}{*}{ SPG4 } & YMR107W & 10 & 10 & 16 & 39.4967 & 57.27494 \\
\hline & YMR196W & 10 & 10 & 17 & 31.23518 & 49.9035 \\
\hline YKU80 & YMR106C & 10 & 10 & 18 & 21.60926 & 47.87205 \\
\hline ICY1 & YMR195W & 10 & 10 & 19 & 26.60571 & 51.2178 \\
\hline PGM2 & YMR105C & 10 & 10 & 20 & 38.54733 & 62.39635 \\
\hline RPL36A & YMR194W & 10 & 10 & 21 & 28.69618 & 14.58726 \\
\hline \multirow[t]{3}{*}{ YPK2 } & YMR104C & 10 & 10 & 22 & 37.94158 & 56.14931 \\
\hline & YMR193C- & & & & & \\
\hline & $A$ & 10 & 10 & 23 & 49.15547 & 52.52572 \\
\hline HIS3 & YOR202W & 10 & 10 & 24 & 47.9408 & \#DIV/0! \\
\hline HIS3 & YOR202W & 10 & 11 & 1 & 46.71083 & \#DIV/0! \\
\hline OST6 & YML019W & 10 & 11 & 2 & 52.20222 & 49.32054 \\
\hline \multirow[t]{3}{*}{ MVP1 } & YMR004W & 10 & 11 & 3 & 36.92672 & 26.09414 \\
\hline & YML018C & 10 & 11 & 4 & 47.38899 & 46.55529 \\
\hline & YMR003W & 10 & 11 & 5 & 34.61502 & 49.84607 \\
\hline \multirow[t]{2}{*}{ PSP2 } & YML017W & 10 & 11 & 6 & 17.72905 & 42.30721 \\
\hline & YMR002W & 10 & 11 & 7 & 36.18506 & 51.97445 \\
\hline PPZ1 & YML016C & 10 & 11 & 8 & 37.42941 & 34.26025 \\
\hline
\end{tabular}




\begin{tabular}{|c|c|c|c|c|c|c|}
\hline & YML131W & 10 & 11 & 9 & 25.98265 & 40.90061 \\
\hline SEL1 & YML013W & 10 & 11 & 10 & 30.97271 & 28.07683 \\
\hline \multirow[t]{3}{*}{ MSC1 } & YML128C & 10 & 11 & 11 & 29.83011 & 46.9141 \\
\hline & YML013C- & & & & & \\
\hline & $A$ & 10 & 11 & 12 & 62.00125 & 71.19694 \\
\hline TUB3 & YML124C & 10 & 11 & 13 & 37.40553 & 32.05409 \\
\hline ERV25 & YML012W & 10 & 11 & 14 & 16.39793 & 53.5316 \\
\hline \multirow[t]{5}{*}{ PHO84 } & YML123C & 10 & 11 & 15 & 31.32531 & 48.42337 \\
\hline & YML011C & 10 & 11 & 16 & 25.10534 & 23.12948 \\
\hline & YML122C & 10 & 11 & 17 & 19.47918 & 58.84891 \\
\hline & YML010C- & & & & & \\
\hline & B & 10 & 11 & 18 & 13.16588 & 38.25317 \\
\hline GTR1 & YML121W & 10 & 11 & 19 & 27.17506 & 62.28457 \\
\hline MRPL39 & YML009c & 10 & 11 & 20 & 32.07289 & 44.10299 \\
\hline NDI1 & YML120C & 10 & 11 & 21 & 61.44918 & 65.41203 \\
\hline \multirow[t]{2}{*}{ ERG6 } & YML008C & 10 & 11 & 22 & 71.13887 & 78.36417 \\
\hline & YML119W & 10 & 11 & 23 & 38.57475 & 60.97833 \\
\hline HIS3 & YOR202W & 10 & 11 & 24 & 21.38989 & \#DIV/0! \\
\hline HIS3 & YOR202W & 10 & 12 & 1 & 48.84739 & \#DIV/O! \\
\hline JLP2 & YMR132C & 10 & 12 & 2 & 55.36284 & 45.33644 \\
\hline \multirow[t]{2}{*}{ MRE11 } & YMR224C & 10 & 12 & 3 & 56.99218 & 55.06446 \\
\hline & YMR130W & 10 & 12 & 4 & 52.21438 & 27.13201 \\
\hline UBP8 & YMR223W & 10 & 12 & 5 & 45.96679 & 21.45267 \\
\hline POM152 & YMR129W & 10 & 12 & 6 & 44.52728 & 70.08557 \\
\hline $\mathrm{FSH} 2$ & YMR222C & 10 & 12 & 7 & 30.29218 & 36.82635 \\
\hline SAS2 & YMR127C & 10 & 12 & 8 & 60.63106 & 58.85974 \\
\hline FMP42 & YMR221C & 10 & 12 & 9 & 25.88106 & 49.59077 \\
\hline DLT1 & YMR126C & 10 & 12 & 10 & 26.79884 & 41.88615 \\
\hline \multirow[t]{2}{*}{ ESC1 } & YMR219W & 10 & 12 & 11 & 24.10217 & 50.50505 \\
\hline & YMR124W & 10 & 12 & 12 & 32.42428 & 50.28394 \\
\hline SKY1 & YMR216C & 10 & 12 & 13 & 18.62292 & 13.52525 \\
\hline PKR1 & YMR123W & 10 & 12 & 14 & 25.46609 & 41.73691 \\
\hline \multirow[t]{2}{*}{ GAS3 } & YMR215W & 10 & 12 & 15 & 16.84488 & 31.15804 \\
\hline & YMR122C & 10 & 12 & 16 & 19.39603 & 30.94717 \\
\hline SCJ1 & YMR214W & 10 & 12 & 17 & 15.05493 & 45.08635 \\
\hline \multirow[t]{2}{*}{ RPL15B } & YMR121C & 10 & 12 & 18 & 21.94175 & 21.29433 \\
\hline & YMR210W & 10 & 12 & 19 & 14.16009 & 45.29084 \\
\hline \multirow[t]{4}{*}{ ADE17 } & YMR120C & 10 & 12 & 20 & 7.125982 & 21.44969 \\
\hline & YMR209C & 10 & 12 & 21 & 19.82431 & 58.48706 \\
\hline & YMR119W- & & & & & \\
\hline & A & 10 & 12 & 22 & 38.69477 & 41.61339 \\
\hline HFA1 & YMR207C & 10 & 12 & 23 & 61.45638 & 41.77326 \\
\hline HIS3 & YOR202W & 10 & 12 & 24 & 38.74459 & \#DIV/0! \\
\hline
\end{tabular}




\begin{tabular}{|c|c|c|c|c|c|c|}
\hline HIS3 & YOR202W & 10 & 13 & 1 & 51.61618 & \#DIV/0! \\
\hline \multirow[t]{3}{*}{ SRC1 } & YML034W & 10 & 13 & 2 & 53.04516 & 45.00496 \\
\hline & YMR018W & 10 & 13 & 3 & 51.18015 & 52.05848 \\
\hline & YML033W & 10 & 13 & 4 & 47.96325 & 45.39874 \\
\hline SPO20 & YMR017W & 10 & 13 & 5 & 39.84983 & 43.13776 \\
\hline RAD52 & YML032C & 10 & 13 & 6 & 43.87832 & 56.85873 \\
\hline \multirow[t]{2}{*}{ SOK2 } & YMR016C & 10 & 13 & 7 & 44.04365 & 43.5048 \\
\hline & YML030W & 10 & 13 & 8 & 15.21545 & 43.97872 \\
\hline ERG5 & YMR015C & 10 & 13 & 9 & 25.2159 & 53.88258 \\
\hline USA1 & YML029W & 10 & 13 & 10 & 33.20137 & 23.83178 \\
\hline CLU1 & YMR012W & 10 & 13 & 11 & 26.59264 & 54.25746 \\
\hline TSA1 & YML028W & 10 & 13 & 12 & 25.9331 & 56.37859 \\
\hline HXT2 & YMR011W & 10 & 13 & 13 & 28.25489 & 44.11168 \\
\hline \multirow[t]{2}{*}{ YOX1 } & YML027W & 10 & 13 & 14 & 23.74187 & 58.40084 \\
\hline & YMR010W & 10 & 13 & 15 & 55.03556 & 58.56277 \\
\hline \multirow[t]{2}{*}{ RPS18B } & YML026C & 10 & 13 & 16 & 23.24748 & 47.24338 \\
\hline & YMR009W & 10 & 13 & 17 & 40.72833 & 49.5274 \\
\hline APT1 & YML022W & 10 & 13 & 18 & 74.99206 & 56.38013 \\
\hline PLB1 & YMR008C & 10 & 13 & 19 & 20.77399 & 45.77874 \\
\hline \multirow[t]{3}{*}{ UNG1 } & YML021C & 10 & 13 & 20 & 56.66352 & 64.5017 \\
\hline & YMR007W & 10 & 13 & 21 & 29.00392 & 34.14148 \\
\hline & YML020W & 10 & 13 & 22 & 34.93444 & 55.13476 \\
\hline PLB2 & YMR006C & 10 & 13 & 23 & 36.91767 & 68.65396 \\
\hline HIS3 & YOR202W & 10 & 13 & 24 & 57.92475 & \#DIV/0! \\
\hline \multirow[t]{3}{*}{ HIS3 } & YOR202W & 10 & 14 & 1 & 45.54301 & \#DIV/0! \\
\hline & YMR144W & 10 & 14 & 2 & 33.13478 & 41.6434 \\
\hline & $\begin{array}{l}\text { YMR244C- } \\
\text { A }\end{array}$ & 10 & 14 & 3 & 57.83555 & 29.38205 \\
\hline RPS16A & YMR143W & 10 & 14 & 4 & 54.69684 & 43.05833 \\
\hline \multirow[t]{2}{*}{ ZRC1 } & YMR243C & 10 & 14 & 5 & 44.32454 & 44.88808 \\
\hline & YMR141C & 10 & 14 & 6 & 49.15282 & 59.84376 \\
\hline YHM2 & YMR241W & 10 & 14 & 7 & 50.8438 & 49.33867 \\
\hline SIP5 & YMR140W & 10 & 14 & 8 & 24.46352 & \#DIV/0! \\
\hline DFG5 & YMR238W & 10 & 14 & 9 & 16.91844 & 36.14006 \\
\hline RIM11 & YMR139W & 10 & 14 & 10 & 20.15475 & 51.96982 \\
\hline $\mathrm{BCH} 1$ & YMR237W & 10 & 14 & 11 & 15.17903 & 51.95479 \\
\hline CIN4 & YMR138W & 10 & 14 & 12 & 16.39222 & 65.53207 \\
\hline RNH1 & YMR234W & 10 & 14 & 13 & 28.97362 & 34.26048 \\
\hline PSO2 & YMR137C & 10 & 14 & 14 & 21.19568 & 55.10079 \\
\hline TRI1 & YMR233W & 10 & 14 & 15 & 31.09353 & 49.92905 \\
\hline GAT2 & YMR136W & 10 & 14 & 16 & 28.67078 & 55.37523 \\
\hline FUS2 & YMR232W & 10 & 14 & 17 & 41.94933 & 59.01515 \\
\hline
\end{tabular}


YMR135W-

\begin{tabular}{|c|c|c|c|c|c|c|}
\hline & $A$ & 10 & 14 & 18 & 29.36135 & 52.79024 \\
\hline RPS10B & YMR230W & 10 & 14 & 19 & 39.10826 & 51.94784 \\
\hline \multirow[t]{2}{*}{ GID8 } & YMR135C & 10 & 14 & 20 & 12.27728 & 40.61005 \\
\hline & YMR226C & 10 & 14 & 21 & 36.19107 & 62.69036 \\
\hline REC114 & YMR133W & 10 & 14 & 22 & 36.80576 & 3.891695 \\
\hline MRPL44 & YMR225C & 10 & 14 & 23 & -13.3826 & 23.87129 \\
\hline HIS3 & YOR202W & 10 & 14 & 24 & 37.62052 & \#DIV/0! \\
\hline HIS3 & YOR202W & 10 & 15 & 1 & 35.61098 & \#DIV/O! \\
\hline \multirow[t]{3}{*}{ GAL80 } & YML051W & 10 & 15 & 2 & 30.80971 & \#DIV/0! \\
\hline & $\begin{array}{l}\text { YMR031C } \\
\text { YML048W- }\end{array}$ & 10 & 15 & 3 & 52.84744 & \#DIV/0! \\
\hline & $A$ & 10 & 15 & 4 & 56.85786 & \#DIV/0! \\
\hline RSF1 & YMR030W & 10 & 15 & 5 & 66.69633 & \#DIV/0! \\
\hline GSF2 & YML048W & 10 & 15 & 6 & 55.76128 & \#DIV/0! \\
\hline FAR8 & YMR029C & 10 & 15 & 7 & 48.21185 & \#DIV/0! \\
\hline PRM6 & YML047C & 10 & 15 & 8 & 25.37687 & \#DIV/0! \\
\hline HRT2 & YMR027W & 10 & 15 & 9 & 37.10681 & \#DIV/O! \\
\hline CAT2 & YML042W & 10 & 15 & 10 & 39.32599 & \#DIV/0! \\
\hline PEX12 & YMR026C & 10 & 15 & 11 & 17.64948 & \#DIV/0! \\
\hline VPS71 & YML041C & 10 & 15 & 12 & 8.211322 & \#DIV/0! \\
\hline CSI1 & YMR025W & 10 & 15 & 13 & 29.3661 & \#DIV/O! \\
\hline YMD8 & YML038C & 10 & 15 & 14 & 67.73658 & \#DIV/0! \\
\hline \multirow[t]{2}{*}{ MSS1 } & YMR023C & 10 & 15 & 15 & 29.16556 & \#DIV/0! \\
\hline & YML037C & 10 & 15 & 16 & 35.46512 & \#DIV/O! \\
\hline \multirow[t]{2}{*}{ QRI8 } & YMR022W & 10 & 15 & 17 & 35.84895 & \#DIV/O! \\
\hline & YML036W & 10 & 15 & 18 & 34.13301 & \#DIV/0! \\
\hline \multirow[t]{3}{*}{ MAC1 } & YMR021C & 10 & 15 & 19 & 17.60826 & \#DIV/O! \\
\hline & YML035C- & & & & & \\
\hline & $A$ & 10 & 15 & 20 & 16.89893 & \#DIV/0! \\
\hline FMS1 & YMR020W & 10 & 15 & 21 & 10.91939 & \#DIV/0! \\
\hline AMD1 & YML035C & 10 & 15 & 22 & \#DIV/0! & \#DIV/0! \\
\hline STB4 & YMR019W & 10 & 15 & 23 & -43.7811 & \#DIV/0! \\
\hline HIS3 & YOR202W & 10 & 15 & 24 & 47.76503 & \#DIV/0! \\
\hline HIS3 & YOR202W & 10 & 16 & 1 & 16.49406 & \#DIV/0! \\
\hline HIS3 & YOR202W & 10 & 16 & 2 & 19.86982 & \#DIV/0! \\
\hline HIS3 & YOR202W & 10 & 16 & 3 & 16.92307 & \#DIV/0! \\
\hline HIS3 & YOR202W & 10 & 16 & 4 & 34.11274 & \#DIV/0! \\
\hline HIS3 & YOR202W & 10 & 16 & 5 & 24.04141 & \#DIV/O! \\
\hline HIS3 & YOR202W & 10 & 16 & 6 & 32.83653 & \#DIV/O! \\
\hline HIS3 & YOR202W & 10 & 16 & 7 & 20.83658 & \#DIV/0! \\
\hline HIS3 & YOR202W & 10 & 16 & 8 & 35.77074 & \#DIV/O! \\
\hline HIS3 & YOR202W & 10 & 16 & 9 & 19.718 & \#DIV/0! \\
\hline
\end{tabular}




\begin{tabular}{|c|c|c|c|c|c|c|}
\hline HIS3 & YOR202W & 10 & 16 & 10 & 27.46834 & \#DIV/0! \\
\hline HIS3 & YOR202W & 10 & 16 & 11 & 24.10146 & \#DIV/0! \\
\hline HIS3 & YOR202W & 10 & 16 & 12 & 15.10989 & \#DIV/0! \\
\hline HIS3 & YOR202W & 10 & 16 & 13 & 18.9169 & \#DIV/0! \\
\hline HIS3 & YOR202W & 10 & 16 & 14 & 12.4369 & \#DIV/0! \\
\hline HIS3 & YOR202W & 10 & 16 & 15 & 8.960068 & \#DIV/0! \\
\hline HIS3 & YOR202W & 10 & 16 & 16 & 15.37525 & \#DIV/0! \\
\hline HIS3 & YOR202W & 10 & 16 & 17 & 41.26015 & \#DIV/0! \\
\hline HIS3 & YOR202W & 10 & 16 & 18 & 18.18132 & \#DIV/0! \\
\hline HIS3 & YOR202W & 10 & 16 & 19 & 21.51746 & \#DIV/0! \\
\hline HIS3 & YOR202W & 10 & 16 & 20 & 31.44673 & \#DIV/0! \\
\hline HIS3 & YOR202W & 10 & 16 & 21 & 18.54005 & \#DIV/0! \\
\hline HIS3 & YOR202W & 10 & 16 & 22 & 47.87815 & \#DIV/0! \\
\hline HIS3 & YOR202W & 10 & 16 & 23 & 41.57034 & \#DIV/0! \\
\hline HIS3 & YOR202W & 10 & 16 & 24 & 53.31812 & \#DIV/0! \\
\hline HIS3 & YOR202W & 11 & 1 & 1 & 47.05002 & \#DIV/O! \\
\hline HIS3 & YOR202W & 11 & 1 & 2 & 50.61361 & \#DIV/0! \\
\hline HIS3 & YOR202W & 11 & 1 & 3 & 38.82689 & \#DIV/0! \\
\hline HIS3 & YOR202W & 11 & 1 & 4 & 24.09088 & \#DIV/0! \\
\hline HIS3 & YOR202W & 11 & 1 & 5 & 31.90076 & \#DIV/0! \\
\hline HIS3 & YOR202W & 11 & 1 & 6 & 40.23765 & \#DIV/0! \\
\hline HIS3 & YOR202W & 11 & 1 & 7 & 35.34005 & \#DIV/0! \\
\hline HIS3 & YOR202W & 11 & 1 & 8 & 31.26728 & \#DIV/O! \\
\hline HIS3 & YOR202W & 11 & 1 & 9 & 41.50851 & \#DIV/0! \\
\hline HIS3 & YOR202W & 11 & 1 & 10 & 37.8965 & \#DIV/0! \\
\hline HIS3 & YOR202W & 11 & 1 & 11 & 40.80782 & \#DIV/0! \\
\hline HIS3 & YOR202W & 11 & 1 & 12 & 39.2816 & \#DIV/0! \\
\hline HIS3 & YOR202W & 11 & 1 & 13 & 37.12522 & \#DIV/0! \\
\hline HIS3 & YOR202W & 11 & 1 & 14 & 57.80357 & \#DIV/0! \\
\hline HIS3 & YOR202W & 11 & 1 & 15 & 49.52545 & \#DIV/0! \\
\hline HIS3 & YOR202W & 11 & 1 & 16 & 39.89658 & \#DIV/0! \\
\hline HIS3 & YOR202W & 11 & 1 & 17 & 49.59167 & \#DIV/0! \\
\hline HIS3 & YOR202W & 11 & 1 & 18 & 33.70418 & \#DIV/O! \\
\hline HIS3 & YOR202W & 11 & 1 & 19 & 48.36607 & \#DIV/0! \\
\hline HIS3 & YOR202W & 11 & 1 & 20 & 36.6659 & \#DIV/0! \\
\hline HIS3 & YOR202W & 11 & 1 & 21 & 35.40305 & \#DIV/0! \\
\hline HIS3 & YOR202W & 11 & 1 & 22 & 52.29425 & \#DIV/0! \\
\hline HIS3 & YOR202W & 11 & 1 & 23 & 40.31789 & \#DIV/0! \\
\hline HIS3 & YOR202W & 11 & 1 & 24 & 16.59295 & \#DIV/0! \\
\hline \multirow[t]{3}{*}{ HIS3 } & YOR202W & 11 & 2 & 1 & 19.86898 & \#DIV/O! \\
\hline & YNL146W & 11 & 2 & 2 & 22.77202 & \#DIV/0! \\
\hline & YNL274C & 11 & 2 & 3 & 12.86557 & 41.05757 \\
\hline MFA2 & YNL145W & 11 & 2 & 4 & 32.51365 & 31.80745 \\
\hline
\end{tabular}




\begin{tabular}{|c|c|c|c|c|c|c|}
\hline \multirow[t]{2}{*}{ TOF1 } & YNL273W & 11 & 2 & 5 & 18.99374 & 25.65067 \\
\hline & YNL144C & 11 & 2 & 6 & 34.81898 & 22.8549 \\
\hline \multirow[t]{2}{*}{ BNI1 } & YNL271C & 11 & 2 & 7 & 70.37637 & 51.76872 \\
\hline & YNL143C & 11 & 2 & 8 & 32.84621 & 34.54444 \\
\hline ALP1 & YNL270C & 11 & 2 & 9 & 38.96485 & 32.54836 \\
\hline MEP2 & YNL142W & 11 & 2 & 10 & 29.71909 & 33.94034 \\
\hline LYP1 & YNL268W & 11 & 2 & 11 & 41.63662 & 31.75512 \\
\hline \multirow[t]{3}{*}{$\mathrm{AAH} 1$} & YNL141W & 11 & 2 & 12 & 32.9243 & 51.01424 \\
\hline & YNL266W & 11 & 2 & 13 & 15.56051 & 19.06756 \\
\hline & YNL140C & 11 & 2 & 14 & 32.13826 & 9.242201 \\
\hline IST1 & YNL265C & 11 & 2 & 15 & 11.04636 & 48.42385 \\
\hline EAF7 & YNL136W & 11 & 2 & 16 & 8.628366 & 13.66343 \\
\hline ATX1 & YNL259C & 11 & 2 & 17 & 55.17258 & 40.14859 \\
\hline FPR1 & YNL135C & 11 & 2 & 18 & 1.448238 & -8.71387 \\
\hline \multirow[t]{2}{*}{ SIP3 } & YNL257C & 11 & 2 & 19 & 23.9862 & 36.15718 \\
\hline & YNL134C & 11 & 2 & 20 & 14.61596 & 37.44923 \\
\hline GIS2 & YNL255C & 11 & 2 & 21 & 4.719154 & 14.35482 \\
\hline \multirow[t]{2}{*}{ CPT1 } & YNL130C & 11 & 2 & 22 & 59.64443 & 24.39308 \\
\hline & YNL254C & 11 & 2 & 23 & 41.16029 & 30.88205 \\
\hline HIS3 & YOR202W & 11 & 2 & 24 & 35.43427 & \#DIV/O! \\
\hline HIS3 & YOR202W & 11 & 3 & 1 & 15.74138 & \#DIV/0! \\
\hline \multirow[t]{5}{*}{ GFD1 } & YMR255W & 11 & 3 & 2 & 32.33758 & 42.59079 \\
\hline & YNL035C & 11 & 3 & 3 & 41.38834 & 35.08918 \\
\hline & YMR254C & 11 & 3 & 4 & 30.60926 & 47.78897 \\
\hline & YNL034W & 11 & 3 & 5 & 55.28451 & 35.4187 \\
\hline & YMR253C & 11 & 3 & 6 & 35.61367 & 31.24256 \\
\hline \multirow[t]{2}{*}{ SIW14 } & YNL032W & 11 & 3 & 7 & 18.28923 & 40.61866 \\
\hline & YMR252C & 11 & 3 & 8 & 22.30482 & 31.78609 \\
\hline НHT2 & $\begin{array}{l}\text { YNL031C } \\
\text { YMR251W- }\end{array}$ & 11 & 3 & 9 & 13.62992 & 7.012749 \\
\hline HOR7 & $A$ & 11 & 3 & 10 & 50.48938 & 50.05315 \\
\hline \multirow[t]{2}{*}{ HHF2 } & YNL030W & 11 & 3 & 11 & 34.99024 & 31.53969 \\
\hline & YMR251W & 11 & 3 & 12 & 44.5634 & 27.49591 \\
\hline KTR5 & YNL029C & 11 & 3 & 13 & \#DIV/0! & 38.0148 \\
\hline \multirow[t]{3}{*}{ GAD1 } & YMR250W & 11 & 3 & 14 & 45.51224 & 24.61509 \\
\hline & YNL028W & 11 & 3 & 15 & 28.36485 & 38.74555 \\
\hline & YMR247C & 11 & 3 & 16 & 37.88382 & 57.7939 \\
\hline CRZ1 & YNL027W & 11 & 3 & 17 & 43.4517 & 24.70737 \\
\hline \multirow[t]{3}{*}{ FAA4 } & YMR246W & 11 & 3 & 18 & 28.6715 & -0.25532 \\
\hline & YNL024C & 11 & 3 & 19 & 25.3947 & 24.92048 \\
\hline & YMR245W & 11 & 3 & 20 & 26.44046 & -0.26441 \\
\hline \multirow[t]{2}{*}{ FAP1 } & YNL023C & 11 & 3 & 21 & 17.70223 & 52.84654 \\
\hline & YMR244W & 11 & 3 & 22 & 28.83058 & 24.45031 \\
\hline
\end{tabular}




\begin{tabular}{|c|c|c|c|c|c|c|}
\hline & YNL022C & 11 & 3 & 23 & 45.17065 & -28.6228 \\
\hline HIS3 & YOR202W & 11 & 3 & 24 & -7.26855 & \#DIV/0! \\
\hline HIS3 & YOR202W & 11 & 4 & 1 & 42.66225 & \#DIV/0! \\
\hline SKO1 & YNL167C & 11 & 4 & 2 & 35.68476 & 39.59622 \\
\hline PCL1 & YNL289W & 11 & 4 & 3 & 36.80518 & 42.13213 \\
\hline BNI5 & YNL166C & 11 & 4 & 4 & 50.07369 & 36.0176 \\
\hline \multirow[t]{2}{*}{ CAF40 } & YNL288W & 11 & 4 & 5 & 46.00534 & 52.29337 \\
\hline & YNL165W & 11 & 4 & 6 & 14.98734 & 19.18844 \\
\hline CUS2 & YNL286W & 11 & 4 & 7 & 46.58645 & 43.07277 \\
\hline \multirow[t]{2}{*}{ IBD2 } & YNL164C & 11 & 4 & 8 & 44.7373 & 5.600804 \\
\hline & YNL285W & 11 & 4 & 9 & 36.27148 & 31.41418 \\
\hline RPL42A & YNL162W & 11 & 4 & 10 & -17.8609 & -13.5947 \\
\hline WSC2 & YNL283C & 11 & 4 & 11 & 38.26279 & 34.4033 \\
\hline ASI2 & YNL159C & 11 & 4 & 12 & 38.32415 & 26.29054 \\
\hline \multirow[t]{2}{*}{$\mathrm{HCH} 1$} & YNL281W & 11 & 4 & 13 & 37.36512 & 28.01063 \\
\hline & YNL157W & 11 & 4 & 14 & 51.11166 & -11.4979 \\
\hline ERG24 & YNL280C & 11 & 4 & 15 & 26.02196 & 34.06181 \\
\hline NSG2 & YNL156C & 11 & 4 & 16 & 15.35081 & 14.71327 \\
\hline \multirow[t]{2}{*}{ PRM1 } & YNL279W & 11 & 4 & 17 & -0.38379 & 31.34716 \\
\hline & YNL155W & 11 & 4 & 18 & 32.96605 & 37.26981 \\
\hline CAF120 & YNL278W & 11 & 4 & 19 & 18.31616 & 27.01286 \\
\hline YCK2 & YNL154C & 11 & 4 & 20 & 32.01308 & 44.89681 \\
\hline MET2 & YNL277W & 11 & 4 & 21 & 26.2037 & 29.16896 \\
\hline GIM3 & YNL153C & 11 & 4 & 22 & -14.0281 & 26.83718 \\
\hline BOR1 & YNL275W & 11 & 4 & 23 & 6.393079 & 48.95873 \\
\hline HIS3 & YOR202W & 11 & 4 & 24 & 7.098064 & \#DIV/0! \\
\hline HIS3 & YOR202W & 11 & 5 & 1 & 45.33801 & \#DIV/0! \\
\hline URA10 & YMR271C & 11 & 5 & 2 & 41.31021 & 35.69065 \\
\hline \multirow[t]{2}{*}{ COX5A } & YNL052W & 11 & 5 & 3 & 13.72513 & -8.15312 \\
\hline & YMR269W & 11 & 5 & 4 & 50.07586 & 3.180257 \\
\hline COG5 & YNL051W & 11 & 5 & 5 & 51.37831 & -3.32965 \\
\hline \multirow[t]{3}{*}{ RSN1 } & YMR266W & 11 & 5 & 6 & 47.38616 & 36.66436 \\
\hline & YNL050C & 11 & 5 & 7 & 32.91287 & 22.81826 \\
\hline & YMR265C & 11 & 5 & 8 & 16.09077 & 21.57347 \\
\hline SFB2 & YNL049C & 11 & 5 & 9 & 44.99685 & 29.97835 \\
\hline \multirow[t]{2}{*}{ CUE1 } & YMR264W & 11 & 5 & 10 & 34.79694 & 21.33467 \\
\hline & YNL046W & 11 & 5 & 11 & 60.43616 & 30.55558 \\
\hline \multirow[t]{3}{*}{ SAP30 } & YMR263W & 11 & 5 & 12 & 35.29995 & 18.54587 \\
\hline & YNL045W & 11 & 5 & 13 & 49.35731 & 20.20691 \\
\hline & YMR262W & 11 & 5 & 14 & 35.97618 & 34.64912 \\
\hline YIP3 & YNL044W & 11 & 5 & 15 & 53.20912 & 8.769673 \\
\hline \multirow[t]{2}{*}{ TPS3 } & YMR261C & 11 & 5 & 16 & 32.66436 & 33.08824 \\
\hline & YNL043C & 11 & 5 & 17 & 34.61152 & 28.19632 \\
\hline
\end{tabular}




\begin{tabular}{|c|c|c|c|c|c|c|}
\hline & YMR259C & 11 & 5 & 18 & 41.45047 & 28.83995 \\
\hline \multirow[t]{3}{*}{ COG6 } & YNL041C & 11 & 5 & 19 & 35.9968 & 19.7705 \\
\hline & YMR258C & 11 & 5 & 20 & 55.68243 & 42.89617 \\
\hline & YNL040W & 11 & 5 & 21 & 64.54696 & 37.79575 \\
\hline $\operatorname{cox} 7$ & YMR256C & 11 & 5 & 22 & 22.20705 & -24.156 \\
\hline IDH1 & YNL037C & 11 & 5 & 23 & -6.91257 & 4.545455 \\
\hline HIS3 & YOR202W & 11 & 5 & 24 & 19.40125 & \#DIV/0! \\
\hline \multirow[t]{2}{*}{ HIS3 } & YOR202W & 11 & 6 & 1 & 35.29024 & \#DIV/0! \\
\hline & YNL190W & 11 & 6 & 2 & 36.05828 & 48.20049 \\
\hline \multirow[t]{2}{*}{ RPL18B } & YNL301C & 11 & 6 & 3 & 10.46819 & 23.6057 \\
\hline & YNL187W & 11 & 6 & 4 & 21.28792 & 24.32651 \\
\hline TOS6 & YNL300W & 11 & 6 & 5 & 23.64327 & 11.71546 \\
\hline NPR1 & YNL183C & 11 & 6 & 6 & 48.47898 & 20.5127 \\
\hline TRF5 & YNL299W & 11 & 6 & 7 & 45.72095 & 50.74511 \\
\hline SRF6 & YNL179C & 11 & 6 & 8 & 55.20473 & 20.7479 \\
\hline \multirow[t]{2}{*}{ CLA4 } & YNL298W & 11 & 6 & 9 & 34.58032 & 48.80952 \\
\hline & YNL176C & 11 & 6 & 10 & 38.97107 & 40.26918 \\
\hline MON2 & YNL297C & 11 & 6 & 11 & 40.89677 & 25.80071 \\
\hline \multirow[t]{2}{*}{ NOP13 } & YNL175C & 11 & 6 & 12 & 48.55905 & -10.3131 \\
\hline & YNL296W & 11 & 6 & 13 & 20.10255 & 4.540522 \\
\hline \multirow[t]{3}{*}{ MDG1 } & YNL173C & 11 & 6 & 14 & 30.62251 & 32.02819 \\
\hline & YNL295W & 11 & 6 & 15 & 17.09324 & 5.520276 \\
\hline & YNL171C & 11 & 6 & 16 & 18.94726 & 29.91858 \\
\hline \multirow[t]{2}{*}{ RIM21 } & YNL294C & 11 & 6 & 17 & 22.43935 & 11.78186 \\
\hline & YNL170W & 11 & 6 & 18 & 46.14255 & 7.6765 \\
\hline MSB3 & YNL293W & 11 & 6 & 19 & 38.49225 & 14.13664 \\
\hline PSD1 & YNL169C & 11 & 6 & 20 & 32.1948 & 16.23724 \\
\hline PUS4 & YNL292W & 11 & 6 & 21 & 25.05523 & 37.01669 \\
\hline FMP41 & YNL168C & 11 & 6 & 22 & -5.76435 & 8.139432 \\
\hline MID1 & YNL291C & 11 & 6 & 23 & 44.30572 & 54.48926 \\
\hline HIS3 & YOR202W & 11 & 6 & 24 & 26.54081 & \#DIV/0! \\
\hline HIS3 & YOR202W & 11 & 7 & 1 & 61.67211 & \#DIV/0! \\
\hline YKU70 & YMR284W & 11 & 7 & 2 & 39.2096 & 43.26395 \\
\hline $\mathrm{FKH} 2$ & YNL068C & 11 & 7 & 3 & 32.82811 & 31.82268 \\
\hline RIT1 & YMR283C & 11 & 7 & 4 & 42.45662 & 38.21608 \\
\hline RPL9B & YNL067W & 11 & 7 & 5 & 53.88762 & 52.1602 \\
\hline AEP2 & YMR282C & 11 & 7 & 6 & 39.90056 & 25.41633 \\
\hline SUN4 & YNL066W & 11 & 7 & 7 & 52.80967 & 32.39136 \\
\hline CAT8 & YMR280C & 11 & 7 & 8 & 41.11553 & 13.03943 \\
\hline \multirow[t]{2}{*}{ AQR1 } & YNL065W & 11 & 7 & 9 & 53.33813 & 46.0363 \\
\hline & YMR279C & 11 & 7 & 10 & 36.39972 & 30.17738 \\
\hline \multirow[t]{2}{*}{ CPA2 } & YJR109C & 11 & 7 & 11 & 44.4764 & 37.78027 \\
\hline & YMR278W & 11 & 7 & 12 & 31.5912 & 41.49348 \\
\hline
\end{tabular}




\begin{tabular}{|c|c|c|c|c|c|c|}
\hline YDJ1 & YNL064C & 11 & 7 & 13 & 49.48386 & 21.91702 \\
\hline DSK2 & YMR276W & 11 & 7 & 14 & 54.97322 & 10.60437 \\
\hline MTQ1 & YNL063W & 11 & 7 & 15 & 33.94396 & 22.6696 \\
\hline \multirow[t]{2}{*}{ BUL1 } & YMR275C & 11 & 7 & 16 & 48.50412 & 41.38537 \\
\hline & YNL058C & 11 & 7 & 17 & 27.55593 & 30.8302 \\
\hline \multirow[t]{2}{*}{ RCE1 } & YMR274C & 11 & 7 & 18 & 55.05199 & 40.70226 \\
\hline & YNL057W & 11 & 7 & 19 & 27.3175 & 2.544348 \\
\hline \multirow[t]{2}{*}{ ZDS1 } & YMR273C & 11 & 7 & 20 & 18.47485 & 43.52533 \\
\hline & YNL056W & 11 & 7 & 21 & 22.70562 & 22.05429 \\
\hline SCS7 & YMR272C & 11 & 7 & 22 & 2.672379 & 28.48883 \\
\hline VAC7 & YNL054W & 11 & 7 & 23 & 39.31963 & 25.02949 \\
\hline HIS3 & YOR202W & 11 & 7 & 24 & 29.9473 & \#DIV/0! \\
\hline HIS3 & YOR202W & 11 & 8 & 1 & 33.9766 & \#DIV/0! \\
\hline PSY2 & YNL201C & 11 & 8 & 2 & 51.50773 & 57.77526 \\
\hline \multirow[t]{2}{*}{ HXT14 } & YNL318C & 11 & 8 & 3 & 41.50541 & 44.2029 \\
\hline & YNL200C & 11 & 8 & 4 & 20.4817 & 35.91278 \\
\hline PHA2 & YNL316C & 11 & 8 & 5 & 41.89949 & 18.33815 \\
\hline GCR2 & YNL199C & 11 & 8 & 6 & 45.35318 & 2.586246 \\
\hline \multirow[t]{2}{*}{ ATP11 } & YNL315C & 11 & 8 & 7 & 56.08646 & 31.38145 \\
\hline & YNL198C & 11 & 8 & 8 & 44.83457 & 42.86922 \\
\hline DAL82 & YNL314W & 11 & 8 & 9 & 49.94704 & -1.69645 \\
\hline \multirow[t]{2}{*}{ WHI3 } & YNL197C & 11 & 8 & 10 & 30.01074 & 46.8702 \\
\hline & YNL311C & 11 & 8 & 11 & 38.96084 & 24.31098 \\
\hline SLZ1 & YNL196C & 11 & 8 & 12 & 21.12869 & 21.18499 \\
\hline \multirow[t]{2}{*}{ STB1 } & YNL309W & 11 & 8 & 13 & 27.15001 & 20.89732 \\
\hline & YNL195C & 11 & 8 & 14 & 35.78059 & 39.73262 \\
\hline \multirow[t]{4}{*}{ MCK1 } & YNL307C & 11 & 8 & 15 & 54.02928 & 40.92607 \\
\hline & YNL194C & 11 & 8 & 16 & 60.09012 & 24.94064 \\
\hline & YNL305C & 11 & 8 & 17 & 49.23865 & 13.54532 \\
\hline & YNL193W & 11 & 8 & 18 & 56.52429 & 29.4053 \\
\hline YPT11 & YNL304W & 11 & 8 & 19 & 52.50595 & 12.77101 \\
\hline \multirow[t]{3}{*}{ CHS1 } & YNL192W & 11 & 8 & 20 & 55.18534 & 40.86474 \\
\hline & YNL303W & 11 & 8 & 21 & 19.11871 & 24.54331 \\
\hline & YNL191W & 11 & 8 & 22 & 43.64679 & 32.02666 \\
\hline RPS19B & YNL302C & 11 & 8 & 23 & 43.16856 & 6.613085 \\
\hline HIS3 & YOR202W & 11 & 8 & 24 & 38.69966 & \#DIV/0! \\
\hline HIS3 & YOR202W & 11 & 9 & 1 & 28.26382 & \#DIV/0! \\
\hline PRP12 & YMR302C & 11 & 9 & 2 & 29.80202 & 61.81536 \\
\hline SWS2 & YNL081C & 11 & 9 & 3 & -8.61981 & -51.2021 \\
\hline \multirow[t]{2}{*}{ ADE4 } & YMR300C & 11 & 9 & 4 & 42.99534 & 38.6669 \\
\hline & YNL080C & 11 & 9 & 5 & 47.53295 & 19.80184 \\
\hline DYN3 & YMR299C & 11 & 9 & 6 & 26.31941 & 35.05552 \\
\hline TPM1 & YNL079C & 11 & 9 & 7 & \#DIV/0! & 15.64317 \\
\hline
\end{tabular}




\begin{tabular}{|c|c|c|c|c|c|c|}
\hline PRC1 & YMR297W & 11 & 9 & 8 & 47.58926 & 40.49066 \\
\hline \multirow[t]{2}{*}{ NIS1 } & YNL078W & 11 & 9 & 9 & \#DIV/0! & 25.17973 \\
\hline & YMR295C & 11 & 9 & 10 & 8.418171 & 30.2504 \\
\hline \multirow[t]{2}{*}{ APJ1 } & YNL077W & 11 & 9 & 11 & 34.75359 & 45.78863 \\
\hline & $\begin{array}{l}\text { YMR294W- } \\
\text { A }\end{array}$ & 11 & 9 & 12 & 36.74219 & 43.76747 \\
\hline MKS1 & YNL076W & 11 & 9 & 13 & 35.28502 & 13.15452 \\
\hline JNM1 & YMR294W & 11 & 9 & 14 & 28.43116 & 4.981266 \\
\hline MLF3 & YNL074C & 11 & 9 & 15 & 38.35654 & 36.59127 \\
\hline GOT1 & YMR292W & 11 & 9 & 16 & 37.93696 & 31.02633 \\
\hline \multirow[t]{2}{*}{ RNH201 } & YNL072W & 11 & 9 & 17 & 26.7073 & 30.52747 \\
\hline & YMR291W & 11 & 9 & 18 & 60.84444 & 35.53111 \\
\hline LAT1 & YNL071W & 11 & 9 & 19 & 52.22069 & 41.20081 \\
\hline ABZ2 & YMR289W & 11 & 9 & 20 & 47.32203 & 14.30584 \\
\hline TOM7 & YNL070W & 11 & 9 & 21 & 32.01339 & 33.54452 \\
\hline NGL2 & YMR285C & 11 & 9 & 22 & -7.37524 & 32.24432 \\
\hline RPL16B & YNL069C & 11 & 9 & 23 & 27.91664 & 58.92983 \\
\hline HIS3 & YOR202W & 11 & 9 & 24 & 57.65116 & \#DIV/0! \\
\hline \multirow[t]{2}{*}{ HIS3 } & YOR202W & 11 & 10 & 1 & 3.51597 & \#DIV/0! \\
\hline & YNL217W & 11 & 10 & 2 & 25.69728 & 43.95874 \\
\hline PEX6 & YNL329C & 11 & 10 & 3 & 31.36578 & 41.88479 \\
\hline IES2 & YNL215W & 11 & 10 & 4 & 31.72154 & 38.01748 \\
\hline MDJ2 & YNL328C & 11 & 10 & 5 & 42.88339 & 37.29869 \\
\hline PEX17 & YNL214W & 11 & 10 & 6 & 43.74246 & 19.25229 \\
\hline EGT2 & YNL327W & 11 & 10 & 7 & 45.69738 & 6.271998 \\
\hline VID27 & YNL212W & 11 & 10 & 8 & 58.51749 & 27.23999 \\
\hline \multirow[t]{2}{*}{ PFA3 } & YNL326C & 11 & 10 & 9 & 34.19378 & 28.65021 \\
\hline & YNL211C & 11 & 10 & 10 & 41.93323 & 28.27891 \\
\hline \multirow[t]{3}{*}{ FIG4 } & YNL325C & 11 & 10 & 11 & 37.18384 & 35.16536 \\
\hline & YNL208W & 11 & 10 & 12 & 41.88646 & 15.40818 \\
\hline & YNL324W & 11 & 10 & 13 & 52.76545 & 48.04543 \\
\hline RTT106 & YNL206C & 11 & 10 & 14 & 12.87434 & 19.204 \\
\hline \multirow[t]{2}{*}{ LEM3 } & YNL323W & 11 & 10 & 15 & 40.8984 & -4.77871 \\
\hline & YNL205C & 11 & 10 & 16 & 37.93291 & 34.91985 \\
\hline KRE1 & YNL322C & 11 & 10 & 17 & 51.58964 & 27.45596 \\
\hline \multirow[t]{4}{*}{ SPS18 } & YNL204C & 11 & 10 & 18 & 32.21396 & 37.76139 \\
\hline & YNL321W & 11 & 10 & 19 & 41.02368 & 21.1019 \\
\hline & YNL203C & 11 & 10 & 20 & -9.08032 & -18.492 \\
\hline & YNL320W & 11 & 10 & 21 & 42.9721 & 40.58571 \\
\hline \multirow[t]{2}{*}{ SPS19 } & YNL202W & 11 & 10 & 22 & 25.49391 & 35.29693 \\
\hline & YNL319W & 11 & 10 & 23 & 28.76224 & 41.06266 \\
\hline HIS3 & YOR202W & 11 & 10 & 24 & 14.34759 & \#DIV/0! \\
\hline HIS3 & YOR202W & 11 & 11 & 1 & 5.864888 & \#DIV/0! \\
\hline
\end{tabular}




\begin{tabular}{|c|c|c|c|c|c|c|}
\hline \multirow[t]{2}{*}{ TGL3 } & YMR313C & 11 & 11 & 2 & 52.12183 & 39.84053 \\
\hline & YNL095C & 11 & 11 & 3 & 36.20045 & 35.85986 \\
\hline ELP6 & YMR312W & 11 & 11 & 4 & 31.48184 & 40.42717 \\
\hline APP1 & YNL094W & 11 & 11 & 5 & 32.3237 & 39.78097 \\
\hline GLC8 & YMR311C & 11 & 11 & 6 & 36.8115 & 33.71686 \\
\hline \multirow[t]{3}{*}{ YPT53 } & YNL093W & 11 & 11 & 7 & 54.34151 & 26.80556 \\
\hline & YMR310C & 11 & 11 & 8 & 19.54888 & 23.79806 \\
\hline & YNL092W & 11 & 11 & 9 & 30.05856 & -2.85532 \\
\hline GAS1 & YMR307W & 11 & 11 & 10 & -0.63937 & -33.1065 \\
\hline NST1 & YNL091W & 11 & 11 & 11 & 52.13016 & -3.27717 \\
\hline FKS3 & YMR306W & 11 & 11 & 12 & 49.67428 & 18.15191 \\
\hline \multirow[t]{3}{*}{$\mathrm{RHO} 2$} & $\begin{array}{l}\text { YNL090W } \\
\text { YMR306C- }\end{array}$ & 11 & 11 & 13 & \#DIV/0! & 12.55656 \\
\hline & $A$ & 11 & 11 & 14 & 32.78392 & 33.14229 \\
\hline & YNL089C & 11 & 11 & 15 & 52.88165 & 33.12693 \\
\hline SCW10 & YMR305C & 11 & 11 & 16 & 55.60281 & 11.54276 \\
\hline TCB2 & YNL087W & 11 & 11 & 17 & 49.31341 & 11.07424 \\
\hline UBP15 & YMR304W & 11 & 11 & 18 & 21.30071 & 13.3072 \\
\hline \multirow[t]{2}{*}{ MKT1 } & $\begin{array}{l}\text { YNL085W } \\
\text { YMR304C- }\end{array}$ & 11 & 11 & 19 & 62.33775 & 55.22989 \\
\hline & $A$ & 11 & 11 & 20 & 52.75314 & 14.17663 \\
\hline SAL1 & YNL083W & 11 & 11 & 21 & 34.84422 & 29.54623 \\
\hline $\mathrm{ADH} 2$ & YMR303C & 11 & 11 & 22 & 1.4531 & 5.414776 \\
\hline PMS1 & YNL082W & 11 & 11 & 23 & 34.01342 & 45.91533 \\
\hline HIS3 & YOR202W & 11 & 11 & 24 & 44.16498 & \#DIV/0! \\
\hline HIS3 & YOR202W & 11 & 12 & 1 & 15.41704 & \#DIV/0! \\
\hline \multirow[t]{2}{*}{ BNI4 } & YNL233W & 11 & 12 & 2 & 47.14462 & 39.25252 \\
\hline & YNR004W & 11 & 12 & 3 & 17.43005 & 36.23091 \\
\hline PDR16 & YNL231C & 11 & 12 & 4 & 29.83534 & 26.77586 \\
\hline FUN34 & YNR002C & 11 & 12 & 5 & 52.82657 & 36.29045 \\
\hline ELA1 & YNL230C & 11 & 12 & 6 & 47.44133 & 4.970881 \\
\hline CIT1 & YNR001C & 11 & 12 & 7 & 34.18935 & 32.08533 \\
\hline URE2 & YNL229C & 11 & 12 & 8 & 58.33464 & 24.6534 \\
\hline \multirow[t]{3}{*}{ DOM34 } & YNL001W & 11 & 12 & 9 & 55.32864 & 60.15394 \\
\hline & YNL228W & 11 & 12 & 10 & 54.66491 & 21.59519 \\
\hline & YNL338W & 11 & 12 & 11 & 37.33138 & 39.83123 \\
\hline JJJ1 & YNL227C & 11 & 12 & 12 & 25.99126 & 40.38005 \\
\hline \multirow[t]{4}{*}{$\cos 1$} & YNL336W & 11 & 12 & 13 & 2.825836 & 47.61325 \\
\hline & YNL226W & 11 & 12 & 14 & 32.74206 & 37.15806 \\
\hline & YNL335W & 11 & 12 & 15 & 45.92822 & 38.11497 \\
\hline & YNL224C & 11 & 12 & 16 & 51.13873 & 37.31284 \\
\hline SNO2 & YNL334C & 11 & 12 & 17 & 35.04156 & 22.63575 \\
\hline ATG4 & YNL223W & 11 & 12 & 18 & 24.36752 & 28.74894 \\
\hline
\end{tabular}




\begin{tabular}{|c|c|c|c|c|c|c|}
\hline SNZ2 & YNL333W & 11 & 12 & 19 & 29.51346 & 33.60588 \\
\hline ALG9 & YNL219C & 11 & 12 & 20 & 36.81706 & 47.96176 \\
\hline THI12 & YNL332W & 11 & 12 & 21 & 2.173852 & 33.19421 \\
\hline MGS1 & YNL218W & 11 & 12 & 22 & 21.28492 & 48.83924 \\
\hline RPD3 & YNL330C & 11 & 12 & 23 & 13.56397 & 44.0002 \\
\hline HIS3 & YOR202W & 11 & 12 & 24 & 34.25592 & \#DIV/0! \\
\hline HIS3 & YOR202W & 11 & 13 & 1 & 16.24325 & \#DIV/0! \\
\hline \multirow[t]{4}{*}{ YRF1-6 } & YNL339C & 11 & 13 & 2 & 36.849 & 28.8783 \\
\hline & YNL115C & 11 & 13 & 3 & 38.30806 & 36.73298 \\
\hline & YMR326C & 11 & 13 & 4 & 45.27586 & 34.89034 \\
\hline & YNL108C & 11 & 13 & 5 & 33.70421 & 34.33211 \\
\hline SNO4 & YMR322C & 11 & 13 & 6 & 31.42478 & 35.95795 \\
\hline \multirow[t]{2}{*}{ YAF9 } & YNL107W & 11 & 13 & 7 & 22.18617 & 51.06317 \\
\hline & YMR320W & 11 & 13 & 8 & 41.1778 & 24.02331 \\
\hline INP52 & YNL106C & 11 & 13 & 9 & 35.75317 & 7.711807 \\
\hline \multirow[t]{2}{*}{ FET4 } & YMR319C & 11 & 13 & 10 & 3.966112 & 43.41802 \\
\hline & YNL105W & 11 & 13 & 11 & 20.14064 & 44.132 \\
\hline ADH6 & YMR318C & 11 & 13 & 12 & 2.153335 & 13.5398 \\
\hline \multirow[t]{2}{*}{ LEU4 } & YNL104C & 11 & 13 & 13 & \#DIV/0! & 34.30332 \\
\hline & YMR317W & 11 & 13 & 14 & -0.1549 & 36.31417 \\
\hline AVT4 & YNL101W & 11 & 13 & 15 & 3.582122 & 33.0767 \\
\hline \multirow[t]{3}{*}{ DIA1 } & YMR316W & 11 & 13 & 16 & 8.365394 & 46.87645 \\
\hline & $\begin{array}{l}\text { YNL100W } \\
\text { YMR316C- }\end{array}$ & 11 & 13 & 17 & -50.7086 & 41.44628 \\
\hline & B & 11 & 13 & 18 & 1.536586 & -2.08313 \\
\hline \multirow[t]{3}{*}{ OCA1 } & YNL099C & 11 & 13 & 19 & 10.64327 & 31.7158 \\
\hline & YMR316C- & & & & & \\
\hline & $A$ & 11 & 13 & 20 & 42.65323 & 25.24242 \\
\hline \multirow[t]{2}{*}{ RAS2 } & YNL098C & 11 & 13 & 21 & 0.974713 & 29.90602 \\
\hline & YMR315W & 11 & 13 & 22 & 71.11069 & 48.74031 \\
\hline PHO23 & YNL097C & 11 & 13 & 23 & 23.41954 & 23.22181 \\
\hline HIS3 & YOR202W & 11 & 13 & 24 & 42.08586 & \#DIV/0! \\
\hline HIS3 & YOR202W & 11 & 14 & 1 & 53.59091 & \#DIV/0! \\
\hline \multirow[t]{2}{*}{ TEX1 } & YNL253W & 11 & 14 & 2 & 40.40294 & 29.96186 \\
\hline & YNR018W & 11 & 14 & 3 & 74.69588 & 8.722441 \\
\hline MPA43 & YNL249C & 11 & 14 & 4 & 33.53246 & 40.63349 \\
\hline SMM1 & YNR015W & 11 & 14 & 5 & 33.33968 & 30.76196 \\
\hline \multirow[t]{2}{*}{ VPS75 } & YNL246W & 11 & 14 & 6 & 7.183186 & 16.39592 \\
\hline & YNR014W & 11 & 14 & 7 & 7.098796 & 21.98738 \\
\hline ATG2 & YNL242W & 11 & 14 & 8 & 11.85528 & 39.55428 \\
\hline PHO91 & YNR013C & 11 & 14 & 9 & 7.656081 & 34.80948 \\
\hline ZWF1 & YNL241C & 11 & 14 & 10 & -3.17204 & 47.76625 \\
\hline URK1 & YNR012W & 11 & 14 & 11 & 4.400397 & 26.50181 \\
\hline
\end{tabular}




\begin{tabular}{|c|c|c|c|c|c|c|}
\hline LAP3 & YNL239W & 11 & 14 & 12 & 11.2575 & 34.17242 \\
\hline CSE2 & YNR010W & 11 & 14 & 13 & 30.04309 & 36.39344 \\
\hline \multirow[t]{2}{*}{ KEX2 } & YNL238W & 11 & 14 & 14 & \#DIV/0! & -12.5906 \\
\hline & YNR009W & 11 & 14 & 15 & 24.21393 & 29.8203 \\
\hline YTP1 & YNL237W & 11 & 14 & 16 & 10.74394 & 42.26702 \\
\hline LRO1 & YNR008W & 11 & 14 & 17 & \#DIV/0! & 47.52066 \\
\hline SIN4 & YNL236W & 11 & 14 & 18 & 24.89849 & -115.292 \\
\hline \multirow[t]{2}{*}{ ATG3 } & YNR007C & 11 & 14 & 19 & 21.05815 & 39.80279 \\
\hline & YNL235C & 11 & 14 & 20 & 44.16084 & 29.30238 \\
\hline \multirow[t]{3}{*}{ VPS27 } & YNR006W & 11 & 14 & 21 & \#DIV/0! & -73.7665 \\
\hline & YNL234W & 11 & 14 & 22 & 56.38045 & 31.8248 \\
\hline & YNR005C & 11 & 14 & 23 & 24.90444 & 44.7221 \\
\hline HIS3 & YOR202W & 11 & 14 & 24 & 41.76653 & \#DIV/0! \\
\hline HIS3 & YOR202W & 11 & 15 & 1 & 48.52391 & \#DIV/0! \\
\hline HDA1 & YNL021W & 11 & 15 & 2 & 34.35247 & \#DIV/0! \\
\hline NRK1 & YNL129W & 11 & 15 & 3 & 30.4297 & \#DIV/0! \\
\hline ARK1 & YNL020C & 11 & 15 & 4 & 36.92154 & \#DIV/0! \\
\hline TEP1 & YNL128W & 11 & 15 & 5 & 22.34507 & \#DIV/0! \\
\hline PUB1 & YNL016W & 11 & 15 & 6 & -2.8824 & \#DIV/0! \\
\hline FAR11 & YNL127W & 11 & 15 & 7 & 34.05664 & \#DIV/O! \\
\hline PBI2 & YNL015W & 11 & 15 & 8 & 41.4451 & \#DIV/0! \\
\hline \multirow[t]{3}{*}{ ESBP6 } & YNL125C & 11 & 15 & 9 & 31.91567 & \#DIV/0! \\
\hline & YNL013C & 11 & 15 & 10 & 68.52332 & \#DIV/0! \\
\hline & YNL123W & 11 & 15 & 11 & 11.02966 & \#DIV/0! \\
\hline \multirow[t]{3}{*}{ SPO1 } & YNL012W & 11 & 15 & 12 & 18.50788 & \#DIV/0! \\
\hline & YNL122C & 11 & 15 & 13 & 15.94729 & \#DIV/0! \\
\hline & YNL010W & 11 & 15 & 14 & 7.325814 & \#DIV/0! \\
\hline TOM70 & YNL121C & 11 & 15 & 15 & 33.48038 & \#DIV/O! \\
\hline \multirow[t]{2}{*}{ IDP3 } & YNL009W & 11 & 15 & 16 & 23.00396 & \#DIV/0! \\
\hline & YNL120C & 11 & 15 & 17 & \#DIV/0! & \#DIV/O! \\
\hline ASI3 & YNL008C & 11 & 15 & 18 & 6.895028 & \#DIV/0! \\
\hline NCS2 & YNL119W & 11 & 15 & 19 & 19.17785 & \#DIV/O! \\
\hline HRB1 & YNL004W & 11 & 15 & 20 & 26.36385 & \#DIV/0! \\
\hline MLS1 & YNL117W & 11 & 15 & 21 & 13.42689 & \#DIV/0! \\
\hline PET8 & YNL003C & 11 & 15 & 22 & 19.04632 & \#DIV/0! \\
\hline DMA2 & YNL116W & 11 & 15 & 23 & 50.26272 & \#DIV/0! \\
\hline HIS3 & YOR202W & 11 & 15 & 24 & 43.74358 & \#DIV/0! \\
\hline HIS3 & YOR202W & 11 & 16 & 1 & 43.56546 & \#DIV/0! \\
\hline HIS3 & YOR202W & 11 & 16 & 2 & 37.99158 & \#DIV/0! \\
\hline HIS3 & YOR202W & 11 & 16 & 3 & 61.87696 & \#DIV/0! \\
\hline HIS3 & YOR202W & 11 & 16 & 4 & 50.25403 & \#DIV/0! \\
\hline HIS3 & YOR202W & 11 & 16 & 5 & 35.5956 & \#DIV/0! \\
\hline HIS3 & YOR202W & 11 & 16 & 6 & 54.19124 & \#DIV/O! \\
\hline
\end{tabular}




\begin{tabular}{|c|c|c|c|c|c|c|}
\hline HIS3 & YOR202W & 11 & 16 & 7 & 51.45738 & \#DIV/O! \\
\hline HIS3 & YOR202W & 11 & 16 & 8 & 46.57367 & \#DIV/0! \\
\hline HIS3 & YOR202W & 11 & 16 & 9 & 24.59967 & \#DIV/O! \\
\hline HIS3 & YOR202W & 11 & 16 & 10 & 49.04934 & \#DIV/0! \\
\hline HIS3 & YOR202W & 11 & 16 & 11 & 52.64747 & \#DIV/0! \\
\hline HIS3 & YOR202W & 11 & 16 & 12 & 41.74596 & \#DIV/0! \\
\hline HIS3 & YOR202W & 11 & 16 & 13 & 54.15284 & \#DIV/0! \\
\hline HIS3 & YOR202W & 11 & 16 & 14 & 16.90088 & \#DIV/0! \\
\hline HIS3 & YOR202W & 11 & 16 & 15 & 34.78684 & \#DIV/O! \\
\hline HIS3 & YOR202W & 11 & 16 & 16 & 35.81055 & \#DIV/0! \\
\hline HIS3 & YOR202W & 11 & 16 & 17 & 39.76732 & \#DIV/0! \\
\hline HIS3 & YOR202W & 11 & 16 & 18 & 35.36041 & \#DIV/0! \\
\hline HIS3 & YOR202W & 11 & 16 & 19 & 46.66222 & \#DIV/0! \\
\hline HIS3 & YOR202W & 11 & 16 & 20 & 39.77161 & \#DIV/0! \\
\hline HIS3 & YOR202W & 11 & 16 & 21 & 32.78236 & \#DIV/0! \\
\hline HIS3 & YOR202W & 11 & 16 & 22 & 21.06155 & \#DIV/0! \\
\hline HIS3 & YOR202W & 11 & 16 & 23 & 10.15666 & \#DIV/0! \\
\hline HIS3 & YOR202W & 11 & 16 & 24 & 30.96307 & \#DIV/0! \\
\hline HIS3 & YOR202W & 12 & 1 & 1 & 59.05506 & \#DIV/0! \\
\hline HIS3 & YOR202W & 12 & 1 & 2 & 73.8777 & \#DIV/0! \\
\hline HIS3 & YOR202W & 12 & 1 & 3 & \#DIV/0! & \#DIV/0! \\
\hline HIS3 & YOR202W & 12 & 1 & 4 & 66.72356 & \#DIV/0! \\
\hline HIS3 & YOR202W & 12 & 1 & 5 & 59.37463 & \#DIV/0! \\
\hline HIS3 & YOR202W & 12 & 1 & 6 & 42.96792 & \#DIV/0! \\
\hline HIS3 & YOR202W & 12 & 1 & 7 & -630.555 & \#DIV/0! \\
\hline HIS3 & YOR202W & 12 & 1 & 8 & 10.08454 & \#DIV/0! \\
\hline HIS3 & YOR202W & 12 & 1 & 9 & 35.15212 & \#DIV/0! \\
\hline HIS3 & YOR202W & 12 & 1 & 10 & 45.96248 & \#DIV/0! \\
\hline HIS3 & YOR202W & 12 & 1 & 11 & 56.00762 & \#DIV/0! \\
\hline HIS3 & YOR202W & 12 & 1 & 12 & 37.84614 & \#DIV/0! \\
\hline HIS3 & YOR202W & 12 & 1 & 13 & 35.89856 & \#DIV/0! \\
\hline HIS3 & YOR202W & 12 & 1 & 14 & 20.29281 & \#DIV/O! \\
\hline HIS3 & YOR202W & 12 & 1 & 15 & 5.24626 & \#DIV/0! \\
\hline HIS3 & YOR202W & 12 & 1 & 16 & 45.05218 & \#DIV/O! \\
\hline HIS3 & YOR202W & 12 & 1 & 17 & 18.93795 & \#DIV/0! \\
\hline HIS3 & YOR202W & 12 & 1 & 18 & 62.53694 & \#DIV/0! \\
\hline HIS3 & YOR202W & 12 & 1 & 19 & 66.1896 & \#DIV/0! \\
\hline HIS3 & YOR202W & 12 & 1 & 20 & 64.89771 & \#DIV/0! \\
\hline HIS3 & YOR202W & 12 & 1 & 21 & 27.52129 & \#DIV/0! \\
\hline HIS3 & YOR202W & 12 & 1 & 22 & 21.08344 & \#DIV/0! \\
\hline HIS3 & YOR202W & 12 & 1 & 23 & 21.20578 & \#DIV/0! \\
\hline HIS3 & YOR202W & 12 & 1 & 24 & 30.08974 & \#DIV/0! \\
\hline HIS3 & YOR202W & 12 & 2 & 1 & 27.19689 & \#DIV/O! \\
\hline
\end{tabular}




\begin{tabular}{|c|c|c|c|c|c|c|}
\hline & YOR006C & 12 & 2 & 2 & \#DIV/0! & 45.30245 \\
\hline & YOR097C & 12 & 2 & 3 & 28.83863 & 45.68673 \\
\hline DNL4 & YOR005C & 12 & 2 & 4 & 47.45427 & 31.45697 \\
\hline ARF3 & YOR094W & 12 & 2 & 5 & 71.76614 & 53.91496 \\
\hline \multirow[t]{2}{*}{ YSP3 } & YOR003W & 12 & 2 & 6 & 49.16645 & 14.95244 \\
\hline & YOR093C & 12 & 2 & 7 & 52.05772 & 29.65953 \\
\hline ALG6 & YOR002W & 12 & 2 & 8 & 24.7419 & 42.86287 \\
\hline ECM3 & YOR092W & 12 & 2 & 9 & 33.1057 & 40.23949 \\
\hline RRP6 & YOR001W & 12 & 2 & 10 & 18.6868 & 35.73128 \\
\hline \multirow[t]{2}{*}{ RBF46 } & YOR091W & 12 & 2 & 11 & -5.89055 & 48.38473 \\
\hline & YOL163W & 12 & 2 & 12 & 10.15931 & 53.13296 \\
\hline \multirow[t]{2}{*}{ PTC5 } & YOR090C & 12 & 2 & 13 & 18.30564 & 23.34393 \\
\hline & YOL162W & 12 & 2 & 14 & 21.3553 & 47.15779 \\
\hline \multirow[t]{4}{*}{ VPS21 } & YOR089C & 12 & 2 & 15 & 43.98181 & 6.495862 \\
\hline & YOL160W & 12 & 2 & 16 & 2.147012 & 36.90284 \\
\hline & YOR088W & 12 & 2 & 17 & 14.29592 & 39.90367 \\
\hline & YOL159C & 12 & 2 & 18 & 76.08216 & 37.85006 \\
\hline YVC1 & YOR087W & 12 & 2 & 19 & 46.40752 & 20.13863 \\
\hline ENB1 & YOL158C & 12 & 2 & 20 & 27.43497 & 50.11864 \\
\hline \multirow[t]{2}{*}{ TCB1 } & YOR086C & 12 & 2 & 21 & 19.55999 & 47.35352 \\
\hline & YOL155C & 12 & 2 & 22 & 32.87246 & 49.80738 \\
\hline OST3 & YOR085W & 12 & 2 & 23 & 25.12704 & 43.30412 \\
\hline HIS3 & YOR202W & 12 & 2 & 24 & 52.45116 & \#DIV/0! \\
\hline HIS3 & YOR202W & 12 & 3 & 1 & 48.10789 & \#DIV/0! \\
\hline SSK2 & YNR031C & 12 & 3 & 2 & 53.6241 & 31.30436 \\
\hline THI20 & YOL055C & 12 & 3 & 3 & 11.08454 & 46.46183 \\
\hline ECM39 & YNR030W & 12 & 3 & 4 & 79.15971 & 26.43378 \\
\hline \multirow[t]{3}{*}{ PSH1 } & YOL054W & 12 & 3 & 5 & 52.16477 & 39.61024 \\
\hline & YNR029C & 12 & 3 & 6 & 73.45123 & 43.56432 \\
\hline & YOL053W & 12 & 3 & 7 & 53.15629 & 33.72891 \\
\hline \multirow[t]{2}{*}{ CPR8 } & YNR028W & 12 & 3 & 8 & 18.74201 & 40.98567 \\
\hline & YOL053C-A & 12 & 3 & 9 & 5.400658 & 66.3469 \\
\hline BUD17 & YNR027W & 12 & 3 & 10 & -3.47935 & 39.4306 \\
\hline \multirow[t]{4}{*}{ SPE2 } & YOL052C & 12 & 3 & 11 & 7.148223 & 31.44672 \\
\hline & YNR025C & 12 & 3 & 12 & 42.00884 & 47.44809 \\
\hline & YOL050C & 12 & 3 & 13 & 38.56741 & 47.19102 \\
\hline & YNR024W & 12 & 3 & 14 & 14.44825 & 44.45213 \\
\hline GSH2 & YOL049W & 12 & 3 & 15 & -4.87361 & 45.58239 \\
\hline \multirow[t]{5}{*}{ MRPL50 } & YNR022C & 12 & 3 & 16 & 69.62641 & 51.85673 \\
\hline & YOL048C & 12 & 3 & 17 & 30.30467 & 30.11696 \\
\hline & YNR021W & 12 & 3 & 18 & 60.64757 & 51.84155 \\
\hline & YOL047C & 12 & 3 & 19 & 27.50339 & 36.64155 \\
\hline & YNR020C & 12 & 3 & 20 & 27.32383 & 30.43237 \\
\hline
\end{tabular}




\begin{tabular}{|c|c|c|c|c|c|c|}
\hline & YOL046C & 12 & 3 & 21 & 30.12501 & 43.64227 \\
\hline ARE2 & YNR019W & 12 & 3 & 22 & 41.28821 & 57.32938 \\
\hline PSK2 & YOL045W & 12 & 3 & 23 & -6.73865 & 39.78197 \\
\hline HIS3 & YOR202W & 12 & 3 & 24 & 5.747023 & \#DIV/O! \\
\hline HIS3 & YOR202W & 12 & 4 & 1 & 37.33495 & \#DIV/0! \\
\hline \multirow[t]{4}{*}{ ERP4 } & YOR016C & 12 & 4 & 2 & 32.64618 & 50.38318 \\
\hline & YOR112W & 12 & 4 & 3 & 10.83705 & 37.75412 \\
\hline & YOR015W & 12 & 4 & 4 & 19.23282 & 47.87356 \\
\hline & YOR111W & 12 & 4 & 5 & 36.60453 & 46.69252 \\
\hline RTS1 & YOR014W & 12 & 4 & 6 & 44.35618 & 36.00604 \\
\hline \multirow[t]{2}{*}{ INP53 } & YOR109W & 12 & 4 & 7 & \#DIV/0! & 53.63486 \\
\hline & YOR013W & 12 & 4 & 8 & 6.652516 & 46.57848 \\
\hline \multirow[t]{2}{*}{ LEU9 } & YOR108W & 12 & 4 & 9 & 12.07387 & 51.86263 \\
\hline & YOR012W & 12 & 4 & 10 & -25.4154 & 41.59675 \\
\hline RGS2 & YOR107W & 12 & 4 & 11 & 3.125698 & 33.18773 \\
\hline AUS1 & YOR011W & 12 & 4 & 12 & 20.94453 & 40.47655 \\
\hline VAM3 & YOR106W & 12 & 4 & 13 & 37.7524 & 57.10414 \\
\hline \multirow[t]{2}{*}{ TIR2 } & YOR010C & 12 & 4 & 14 & 41.34811 & 29.18712 \\
\hline & YOR105W & 12 & 4 & 15 & 39.10108 & 54.26694 \\
\hline TIR4 & YOR009W & 12 & 4 & 16 & 25.49241 & 52.30538 \\
\hline \multirow[t]{3}{*}{ PIN2 } & YOR104W & 12 & 4 & 17 & 46.60947 & 51.12734 \\
\hline & YOR008C- & & & & & \\
\hline & $A$ & 12 & 4 & 18 & 55.00119 & 47.30772 \\
\hline RAS1 & YOR101W & 12 & 4 & 19 & 17.02136 & 46.36101 \\
\hline SLG1 & YOR008C & 12 & 4 & 20 & 35.61463 & 49.39412 \\
\hline CRC1 & YOR100C & 12 & 4 & 21 & 30.28609 & 58.68362 \\
\hline SGT2 & YOR007C & 12 & 4 & 22 & 20.95397 & 52.3775 \\
\hline KTR1 & YOR099W & 12 & 4 & 23 & 34.02672 & 48.0434 \\
\hline HIS3 & YOR202W & 12 & 4 & 24 & 11.52013 & \#DIV/0! \\
\hline HIS3 & YOR202W & 12 & 5 & 1 & 34.84626 & \#DIV/0! \\
\hline LYS9 & YNR050C & 12 & 5 & 2 & 44.35163 & 39.87005 \\
\hline RTG1 & YOL067C & 12 & 5 & 3 & 25.00334 & \#DIV/0! \\
\hline MSO1 & YNR049C & 12 & 5 & 4 & 22.40021 & 51.46745 \\
\hline \multirow[t]{2}{*}{ INP54 } & YOL065C & 12 & 5 & 5 & 15.93211 & 47.21833 \\
\hline & YNR048W & 12 & 5 & 6 & 51.36123 & 40.10506 \\
\hline \multirow[t]{2}{*}{ MET22 } & YOL064C & 12 & 5 & 7 & -8.85155 & 31.658 \\
\hline & YNR047W & 12 & 5 & 8 & 75.62477 & 28.90851 \\
\hline MOR1 & YOL063C & 12 & 5 & 9 & 6.95732 & 40.5793 \\
\hline PET494 & YNR045W & 12 & 5 & 10 & 15.72001 & 39.29487 \\
\hline \multirow[t]{2}{*}{ APM4 } & YOL062C & 12 & 5 & 11 & 25.65131 & 54.45282 \\
\hline & YNR042W & 12 & 5 & 12 & -19.4244 & 53.7155 \\
\hline \multirow[t]{2}{*}{ PRS5 } & YOL061W & 12 & 5 & 13 & 19.85529 & 46.53653 \\
\hline & YNR040W & 12 & 5 & 14 & 6.04726 & 49.98711 \\
\hline
\end{tabular}




\begin{tabular}{|c|c|c|c|c|c|c|}
\hline MAM3 & YOL060C & 12 & 5 & 15 & 4.854753 & 43.58196 \\
\hline ZRG17 & YNR039C & 12 & 5 & 16 & 23.55812 & 58.1794 \\
\hline GPD2 & YOL059W & 12 & 5 & 17 & 36.815 & 60.6192 \\
\hline SOL1 & YNR034W & 12 & 5 & 18 & 38.38569 & 51.89076 \\
\hline ARG1 & YOL058W & 12 & 5 & 19 & 32.97086 & 45.36397 \\
\hline \multirow[t]{3}{*}{ PPG1 } & YNR032W & 12 & 5 & 20 & 26.4079 & 38.80765 \\
\hline & YOL057W & 12 & 5 & 21 & 9.728931 & 59.39254 \\
\hline & YNR032C- & & & & & \\
\hline HUB1 & $A$ & 12 & 5 & 22 & 44.69804 & 34.70767 \\
\hline GPM3 & YOL056W & 12 & 5 & 23 & 39.44863 & 58.30064 \\
\hline HIS3 & YOR202W & 12 & 5 & 24 & 31.19525 & \#DIV/0! \\
\hline HIS3 & YOR202W & 12 & 6 & 1 & 31.7783 & \#DIV/0! \\
\hline \multirow[t]{2}{*}{ CIN5 } & YOR028C & 12 & 6 & 2 & 26.0455 & 49.4844 \\
\hline & YOR129C & 12 & 6 & 3 & 3.686846 & 49.07653 \\
\hline STI1 & YOR027W & 12 & 6 & 4 & 24.28425 & 43.39146 \\
\hline RGA1 & YOR127W & 12 & 6 & 5 & 52.51077 & 33.23561 \\
\hline BUB3 & YOR026W & 12 & 6 & 6 & -1.77093 & 33.42119 \\
\hline $\mathrm{IAH} 1$ & YOR126C & 12 & 6 & 7 & -1.73885 & 50.34705 \\
\hline HST3 & YOR025W & 12 & 6 & 8 & -6.79658 & 34.11372 \\
\hline \multirow[t]{2}{*}{ UBP2 } & YOR124C & 12 & 6 & 9 & 39.16574 & 38.18236 \\
\hline & YOR024W & 12 & 6 & 10 & -0.57676 & 49.12236 \\
\hline LEO1 & YOR123C & 12 & 6 & 11 & 43.87783 & \#DIV/0! \\
\hline \multirow[t]{3}{*}{$\mathrm{AHC1}$} & YOR023C & 12 & 6 & 12 & -24.404 & 58.33448 \\
\hline & YOR121C & 12 & 6 & 13 & 24.90914 & 50.4969 \\
\hline & YOR022C & 12 & 6 & 14 & 8.425616 & 41.5006 \\
\hline \multirow[t]{4}{*}{ GCY1 } & YOR120W & 12 & 6 & 15 & 59.80719 & 63.69215 \\
\hline & YOR021C & 12 & 6 & 16 & 44.59856 & 36.80404 \\
\hline & YOR118W & 12 & 6 & 17 & -11.3735 & 50.0445 \\
\hline & YOR019W & 12 & 6 & 18 & 24.56291 & 37.57308 \\
\hline TRS33 & YOR115C & 12 & 6 & 19 & 55.67802 & 43.34535 \\
\hline \multirow[t]{2}{*}{ ROD1 } & YOR018W & 12 & 6 & 20 & 10.77435 & 54.97793 \\
\hline & YOR114W & 12 & 6 & 21 & 31.79202 & 51.31432 \\
\hline PET127 & YOR017W & 12 & 6 & 22 & 10.10244 & 35.19115 \\
\hline AZF1 & YOR113W & 12 & 6 & 23 & 49.51126 & 39.0534 \\
\hline HIS3 & YOR202W & 12 & 6 & 24 & 11.14311 & \#DIV/0! \\
\hline \multirow[t]{4}{*}{ HIS3 } & YOR202W & 12 & 7 & 1 & 27.79019 & \#DIV/0! \\
\hline & YNR064C & 12 & 7 & 2 & 18.0952 & 47.66089 \\
\hline & YOL085C & 12 & 7 & 3 & 16.98329 & 35.58706 \\
\hline & YNR063W & 12 & 7 & 4 & 11.74034 & 43.17334 \\
\hline \multirow[t]{4}{*}{ PHM7 } & YOL084W & 12 & 7 & 5 & 67.79686 & 43.21577 \\
\hline & YNR062C & 12 & 7 & 6 & 26.22882 & 42.03878 \\
\hline & YOL083W & 12 & 7 & 7 & 3.562095 & 28.44439 \\
\hline & YNR061C & 12 & 7 & 8 & 6.69805 & 51.92144 \\
\hline
\end{tabular}




\begin{tabular}{|c|c|c|c|c|c|c|}
\hline ATG19 & YOL082W & 12 & 7 & 9 & 7.968603 & 29.18872 \\
\hline FRE4 & YNR060W & 12 & 7 & 10 & -3.13358 & 61.17711 \\
\hline IRA2 & YOL081W & 12 & 7 & 11 & 86.74397 & 36.49578 \\
\hline MNT4 & YNR059W & 12 & 7 & 12 & 6.43366 & 52.90072 \\
\hline REX4 & YOL080C & 12 & 7 & 13 & 18.04609 & 64.14892 \\
\hline \multirow[t]{2}{*}{$\mathrm{BIO3}$} & YNR058W & 12 & 7 & 14 & 15.28059 & 36.5895 \\
\hline & YOL079W & 12 & 7 & 15 & 38.40802 & 59.97025 \\
\hline \multirow[t]{2}{*}{$\mathrm{BIO4}$} & YNR057C & 12 & 7 & 16 & 29.4363 & 52.19399 \\
\hline & YOL075C & 12 & 7 & 17 & 32.95621 & 61.28985 \\
\hline BIO5 & YNR056C & 12 & 7 & 18 & -5.09623 & 50.8378 \\
\hline EMI5 & YOL071W & 12 & 7 & 19 & 34.77414 & 35.55215 \\
\hline \multirow[t]{2}{*}{ HOL1 } & YNR055C & 12 & 7 & 20 & 48.21362 & 58.22729 \\
\hline & YOL070C & 12 & 7 & 21 & 26.51386 & 48.83792 \\
\hline BRE5 & YNR051C & 12 & 7 & 22 & 40.91359 & 54.58322 \\
\hline HST1 & YOL068C & 12 & 7 & 23 & 29.22751 & 57.89098 \\
\hline HIS3 & YOR202W & 12 & 7 & 24 & 6.083993 & \#DIV/0! \\
\hline HIS3 & YOR202W & 12 & 8 & 1 & 9.63796 & \#DIV/0! \\
\hline GLO4 & YOR040W & 12 & 8 & 2 & 16.79038 & 45.34088 \\
\hline ARP8 & YOR141C & 12 & 8 & 3 & 13.89616 & 14.7444 \\
\hline CKB2 & YOR039W & 12 & 8 & 4 & 37.94614 & 31.80394 \\
\hline SFL1 & YOR140W & 12 & 8 & 5 & 4.332155 & 42.19401 \\
\hline \multirow[t]{2}{*}{ HIR2 } & YOR038C & 12 & 8 & 6 & -8.07808 & 38.92201 \\
\hline & YOR139C & 12 & 8 & 7 & 46.86243 & 61.68946 \\
\hline CYC2 & YOR037W & 12 & 8 & 8 & -3.38074 & 33.2785 \\
\hline RUP1 & YOR138C & 12 & 8 & 9 & 15.77622 & 53.49122 \\
\hline SHE4 & YOR035C & 12 & 8 & 10 & 44.18073 & 29.26997 \\
\hline SIA1 & YOR137C & 12 & 8 & 11 & 37.94773 & 47.48077 \\
\hline AKR2 & YOR034C & 12 & 8 & 12 & 18.76902 & 48.79475 \\
\hline IDH2 & YOR136W & 12 & 8 & 13 & 16.43912 & 52.10876 \\
\hline \multirow[t]{2}{*}{ EXO1 } & YOR033C & 12 & 8 & 14 & \#DIV/0! & 45.0224 \\
\hline & YOR135C & 12 & 8 & 15 & \#DIV/0! & 30.86152 \\
\hline HMS1 & YOR032C & 12 & 8 & 16 & 28.87404 & 63.59373 \\
\hline BAG7 & YOR134W & 12 & 8 & 17 & 33.07355 & 48.40224 \\
\hline CRS5 & YOR031W & 12 & 8 & 18 & 6.519734 & 50.15573 \\
\hline EFT1 & YOR133W & 12 & 8 & 19 & 36.05131 & 57.07172 \\
\hline DFG16 & YOR030W & 12 & 8 & 20 & 25.95354 & 53.89394 \\
\hline \multirow[t]{3}{*}{ VPS17 } & YOR132W & 12 & 8 & 21 & 0.214961 & 50.84403 \\
\hline & YOR029W & 12 & 8 & 22 & 52.48624 & 48.70971 \\
\hline & YOR131C & 12 & 8 & 23 & \#DIV/0! & 62.70625 \\
\hline HIS3 & YOR202W & 12 & 8 & 24 & -12.6848 & \#DIV/0! \\
\hline HIS3 & YOR202W & 12 & 9 & 1 & 28.83223 & \#DIV/0! \\
\hline IZH2 & YOL002C & 12 & 9 & 2 & 19.00737 & 39.10256 \\
\hline IZH4 & YOL101C & 12 & 9 & 3 & -8.84542 & 31.18715 \\
\hline
\end{tabular}




\begin{tabular}{|c|c|c|c|c|c|c|}
\hline \multirow[t]{2}{*}{ PHO80 } & YOL001W & 12 & 9 & 4 & 28.41792 & 27.24701 \\
\hline & YOL099C & 12 & 9 & 5 & 1.714299 & 23.99409 \\
\hline \multirow[t]{2}{*}{$\cos 10$} & YNR075W & 12 & 9 & 6 & 14.50792 & 43.48354 \\
\hline & YOL098C & 12 & 9 & 7 & 20.7067 & 27.05056 \\
\hline AlF1 & YNR074C & 12 & 9 & 8 & 12.81338 & 38.57994 \\
\hline \multirow[t]{2}{*}{ HMI1 } & YOL095C & 12 & 9 & 9 & 49.55823 & 35.09033 \\
\hline & YNR073C & 12 & 9 & 10 & \#DIV/0! & 25.87519 \\
\hline TRM10 & YOL093W & 12 & 9 & 11 & 30.52344 & 48.09287 \\
\hline \multirow[t]{3}{*}{ HXT17 } & YNR072W & 12 & 9 & 12 & 15.93087 & 47.81503 \\
\hline & YOL092W & 12 & 9 & 13 & 48.08711 & 52.98055 \\
\hline & YNR071C & 12 & 9 & 14 & -12.4249 & 50.8068 \\
\hline SPO21 & YOL091W & 12 & 9 & 15 & \#DIV/0! & 54.75201 \\
\hline BSC5 & YNR069C & 12 & 9 & 16 & 43.65913 & 61.05716 \\
\hline MSH2 & YOL090W & 12 & 9 & 17 & 72.19658 & 74.03499 \\
\hline DSE4 & YNR067C & 12 & 9 & 18 & 57.66449 & 51.07058 \\
\hline \multirow[t]{2}{*}{ HAL9 } & YOL089C & 12 & 9 & 19 & 10.77279 & 44.66505 \\
\hline & YNR066C & 12 & 9 & 20 & 62.81694 & 56.99024 \\
\hline MPD2 & YOL088C & 12 & 9 & 21 & 65.20108 & 58.13544 \\
\hline \multirow[t]{2}{*}{ YSN1 } & YNR065C & 12 & 9 & 22 & 18.75797 & 59.77144 \\
\hline & YOL087C & 12 & 9 & 23 & 44.23981 & 55.67125 \\
\hline HIS3 & YOR202W & 12 & 9 & 24 & 11.08721 & \#DIV/0! \\
\hline \multirow[t]{2}{*}{ HIS3 } & YOR202W & 12 & 10 & 1 & 13.40805 & \#DIV/0! \\
\hline & YOR053W & 12 & 10 & 2 & 3.494515 & 46.16802 \\
\hline \multirow[t]{4}{*}{ SEY1 } & YOR165W & 12 & 10 & 3 & 23.24063 & 38.7562 \\
\hline & YOR052C & 12 & 10 & 4 & 50.24452 & 18.67487 \\
\hline & YOR164C & 12 & 10 & 5 & 54.26328 & 21.50439 \\
\hline & YOR051C & 12 & 10 & 6 & 14.93555 & 41.34478 \\
\hline \multirow[t]{2}{*}{ DDP1 } & YOR163W & 12 & 10 & 7 & 25.67749 & 50.35738 \\
\hline & YOR050C & 12 & 10 & 8 & -8.77734 & 46.13582 \\
\hline YRR1 & YOR162C & 12 & 10 & 9 & 18.63003 & 51.32484 \\
\hline RSB1 & YOR049C & 12 & 10 & 10 & 55.39414 & 39.56626 \\
\hline PNS1 & YOR161C & 12 & 10 & 11 & 0.464133 & 48.35714 \\
\hline STD1 & YOR047C & 12 & 10 & 12 & 26.73917 & 40.70618 \\
\hline NFI1 & YOR156C & 12 & 10 & 13 & 40.8437 & 39.36121 \\
\hline \multirow[t]{3}{*}{ TOM6 } & YOR045W & 12 & 10 & 14 & 33.59042 & 56.38352 \\
\hline & YOR154W & 12 & 10 & 15 & 68.2435 & 54.664 \\
\hline & YOR044W & 12 & 10 & 16 & -17.2947 & 51.47447 \\
\hline PDR5 & YOR153W & 12 & 10 & 17 & 84.15179 & 80.97113 \\
\hline \multirow[t]{2}{*}{ WHI2 } & YOR043W & 12 & 10 & 18 & 13.13846 & 49.51164 \\
\hline & YOR152C & 12 & 10 & 19 & -1.37837 & 41.84184 \\
\hline CUE5 & YOR042W & 12 & 10 & 20 & 17.4806 & 42.57691 \\
\hline ELG1 & YOR144C & 12 & 10 & 21 & 16.63032 & 40.69861 \\
\hline SRF5 & YOR041C & 12 & 10 & 22 & 30.62079 & 55.59227 \\
\hline
\end{tabular}




\begin{tabular}{|c|c|c|c|c|c|c|}
\hline LSC1 & YOR142W & 12 & 10 & 23 & \#DIV/0! & 50.73571 \\
\hline HIS3 & YOR202W & 12 & 10 & 24 & \#DIV/0! & \#DIV/0! \\
\hline \multirow[t]{2}{*}{ HIS3 } & YOR202W & 12 & 11 & 1 & 17.28151 & \#DIV/0! \\
\hline & YOL014W & 12 & 11 & 2 & -0.6305 & 40.17094 \\
\hline \multirow[t]{2}{*}{ SKM1 } & YOL113W & 12 & 11 & 3 & 41.17515 & 28.97602 \\
\hline & $\begin{array}{l}\text { YOL013W- } \\
\text { A }\end{array}$ & 12 & 11 & 4 & 35.59361 & \\
\hline MSB4 & YOL112W & 12 & 11 & 5 & 74.73786 & 65.51127 \\
\hline \multirow[t]{2}{*}{ HRD1 } & YOL013C & 12 & 11 & 6 & 10.89824 & -7.6817 \\
\hline & YOL111C & 12 & 11 & 7 & 20.75372 & 34.97746 \\
\hline HTZ1 & YOL012C & 12 & 11 & 8 & 15.53715 & 19.23048 \\
\hline SHR5 & YOL110W & 12 & 11 & 9 & 30.15825 & 53.32537 \\
\hline PLB3 & YOL011W & 12 & 11 & 10 & 5.690752 & 58.25724 \\
\hline ZEO1 & YOL109W & 12 & 11 & 11 & 73.00362 & 44.8346 \\
\hline MDM12 & YOL009C & 12 & 11 & 12 & 17.31953 & 30.70224 \\
\hline \multirow[t]{5}{*}{ INO4 } & YOL108C & 12 & 11 & 13 & 61.79355 & 52.45919 \\
\hline & YOL008W & 12 & 11 & 14 & 7.580758 & 36.5165 \\
\hline & YOL107W & 12 & 11 & 15 & 1.701754 & 50.55631 \\
\hline & YOL007C & 12 & 11 & 16 & -6.3741 & 42.64835 \\
\hline & YOL106W & 12 & 11 & 17 & 18.98849 & 40.12194 \\
\hline TOP1 & YOL006C & 12 & 11 & 18 & 85.17077 & 18.49187 \\
\hline WSC3 & YOL105C & 12 & 11 & 19 & \#DIV/0! & 51.86027 \\
\hline SIN3 & YOL004W & 12 & 11 & 20 & 74.68676 & 29.30042 \\
\hline \multirow[t]{2}{*}{ NDJ1 } & YOL104C & 12 & 11 & 21 & 42.08959 & 39.98203 \\
\hline & YOL003C & 12 & 11 & 22 & 66.54929 & 72.92097 \\
\hline ITR2 & YOL103W & 12 & 11 & 23 & 28.2056 & 53.11376 \\
\hline HIS3 & YOR202W & 12 & 11 & 24 & 23.12288 & \#DIV/0! \\
\hline HIS3 & YOR202W & 12 & 12 & 1 & 47.93419 & \#DIV/0! \\
\hline VPS5 & YOR069W & 12 & 12 & 2 & -7.4738 & -0.22918 \\
\hline FYV12 & YOR183W & 12 & 12 & 3 & -3.99645 & 22.34631 \\
\hline VAM10 & YOR068C & 12 & 12 & 4 & 37.4131 & 15.26104 \\
\hline RPS30B & YOR182C & 12 & 12 & 5 & 41.53892 & 29.73458 \\
\hline ALG8 & YOR067C & 12 & 12 & 6 & 15.03258 & 32.58599 \\
\hline \multirow[t]{2}{*}{ GAC1 } & YOR178C & 12 & 12 & 7 & 28.92907 & 40.66628 \\
\hline & YOR066W & 12 & 12 & 8 & 15.74584 & 33.43437 \\
\hline MPC54 & YOR177C & 12 & 12 & 9 & 3.910849 & 39.40359 \\
\hline \multirow[t]{2}{*}{ CYT1 } & YOR065W & 12 & 12 & 10 & 82.82353 & 46.71472 \\
\hline & YOR175C & 12 & 12 & 11 & \#DIV/0! & 42.1328 \\
\hline YNG1 & YOR064C & 12 & 12 & 12 & -13.871 & 57.76414 \\
\hline \multirow[t]{2}{*}{ DCS2 } & YOR173W & 12 & 12 & 13 & 18.71677 & 46.51987 \\
\hline & YOR062C & 12 & 12 & 14 & 11.74016 & 49.27545 \\
\hline YRM1 & YOR172W & 12 & 12 & 15 & 19.39001 & 44.05622 \\
\hline CKA2 & YOR061W & 12 & 12 & 16 & 34.47744 & 50.38983 \\
\hline
\end{tabular}




\begin{tabular}{|c|c|c|c|c|c|c|}
\hline \multirow[t]{3}{*}{ LCB4 } & YOR171C & 12 & 12 & 17 & 33.80887 & 52.1311 \\
\hline & YOR059C & 12 & 12 & 18 & -14.43 & 35.71462 \\
\hline & YOR170W & 12 & 12 & 19 & 34.1074 & 49.44762 \\
\hline ASE1 & YOR058C & 12 & 12 & 20 & -5.41453 & 51.99043 \\
\hline \multirow[t]{3}{*}{ RPS28A } & YOR167C & 12 & 12 & 21 & 25.18235 & 47.89479 \\
\hline & YOR055W & 12 & 12 & 22 & 11.42567 & 29.98557 \\
\hline & YOR166C & 12 & 12 & 23 & 29.08707 & 43.09608 \\
\hline HIS3 & YOR202W & 12 & 12 & 24 & 10.08184 & \#DIV/O! \\
\hline \multirow[t]{2}{*}{ HIS3 } & YOR202W & 12 & 13 & 1 & 19.49311 & \#DIV/O! \\
\hline & YOL029C & 12 & 13 & 2 & 71.99381 & 35.37308 \\
\hline YGK3 & YOL128C & 12 & 13 & 3 & 29.54809 & 27.89417 \\
\hline YAP7 & YOL028C & 12 & 13 & 4 & -1.91625 & 16.89632 \\
\hline $\mathrm{MDH} 2$ & YOL126C & 12 & 13 & 5 & 25.88394 & 35.6698 \\
\hline \multirow[t]{2}{*}{ MDM38 } & YOL027C & 12 & 13 & 6 & 41.34758 & 13.74963 \\
\hline & YOL124C & 12 & 13 & 7 & 46.37332 & 36.39592 \\
\hline LAG2 & YOL025W & 12 & 13 & 8 & 31.72969 & 47.30089 \\
\hline \multirow[t]{2}{*}{ SMF1 } & YOL122C & 12 & 13 & 9 & 8.434252 & 39.78737 \\
\hline & YOL024W & 12 & 13 & 10 & 28.00135 & 34.76471 \\
\hline RPS19A & YOL121C & 12 & 13 & 11 & -9.24024 & 46.86314 \\
\hline TAT2 & YOL020W & 12 & 13 & 12 & 39.75433 & 50.69408 \\
\hline $\mathrm{MCH} 4$ & YOL119C & 12 & 13 & 13 & 50.47549 & 56.5142 \\
\hline \multirow[t]{2}{*}{ TOS7 } & YOL019W & 12 & 13 & 14 & 35.86178 & 49.17203 \\
\hline & YOL118C & 12 & 13 & 15 & 59.74044 & 44.82456 \\
\hline TLG2 & YOL018C & 12 & 13 & 16 & 63.35441 & 18.18585 \\
\hline $\mathrm{RR} / 2$ & YOL117W & 12 & 13 & 17 & 59.20508 & 57.49999 \\
\hline ESC8 & YOL017W & 12 & 13 & 18 & 8.869514 & 47.7859 \\
\hline MSN1 & YOL116W & 12 & 13 & 19 & 67.82326 & 43.31011 \\
\hline CMK2 & YOL016C & 12 & 13 & 20 & \#DIV/0! & 34.82749 \\
\hline \multirow[t]{3}{*}{ TRF4 } & YOL115W & 12 & 13 & 21 & 32.94251 & 37.69211 \\
\hline & YOL015W & 12 & 13 & 22 & 51.84287 & 33.68144 \\
\hline & YOL114C & 12 & 13 & 23 & 73.77634 & 45.34664 \\
\hline HIS3 & YOR202W & 12 & 13 & 24 & 50.16304 & \#DIV/O! \\
\hline \multirow[t]{2}{*}{ HIS3 } & YOR202W & 12 & 14 & 1 & 51.47034 & \#DIV/0! \\
\hline & YOR084W & 12 & 14 & 2 & -0.65438 & 19.1463 \\
\hline LIP5 & YOR196C & 12 & 14 & 3 & 24.556 & 16.5288 \\
\hline WHI5 & YOR083W & 12 & 14 & 4 & 34.54242 & 29.49829 \\
\hline \multirow[t]{2}{*}{ SLK19 } & YOR195W & 12 & 14 & 5 & -8.94693 & 35.32147 \\
\hline & YOR082C & 12 & 14 & 6 & 22.43611 & 30.53356 \\
\hline PEX27 & YOR193W & 12 & 14 & 7 & 19.55065 & 29.85572 \\
\hline \multirow[t]{2}{*}{ STC2 } & YOR081C & 12 & 14 & 8 & 19.79907 & 33.58435 \\
\hline & YOR192C & 12 & 14 & 9 & 38.73476 & 36.01512 \\
\hline DIA2 & YOR080W & 12 & 14 & 10 & 37.06303 & 7.215736 \\
\hline RIS1 & YOR191W & 12 & 14 & 11 & 47.04891 & 34.03433 \\
\hline
\end{tabular}




\begin{tabular}{|c|c|c|c|c|c|c|}
\hline ATX2 & YOR079C & 12 & 14 & 12 & 27.26062 & 43.98316 \\
\hline SPR1 & YOR190W & 12 & 14 & 13 & 25.46476 & 40.16963 \\
\hline BUD21 & YOR078W & 12 & 14 & 14 & 53.86314 & 25.55587 \\
\hline IES4 & YOR189W & 12 & 14 & 15 & 26.14977 & 37.76234 \\
\hline SKI7 & YOR076C & 12 & 14 & 16 & 19.28126 & 59.38672 \\
\hline \multirow[t]{4}{*}{ MSB1 } & YOR188W & 12 & 14 & 17 & 63.36141 & 58.73553 \\
\hline & YOR072W & 12 & 14 & 18 & 52.92247 & 44.43561 \\
\hline & YOR186W & 12 & 14 & 19 & 60.42398 & 45.91152 \\
\hline & YOR071C & 12 & 14 & 20 & 16.81216 & 52.01767 \\
\hline GSP2 & YOR185C & 12 & 14 & 21 & \#DIV/0! & 48.94606 \\
\hline GYP1 & YOR070C & 12 & 14 & 22 & 44.29226 & 40.03741 \\
\hline SER1 & YOR184W & 12 & 14 & 23 & 55.5212 & 48.63587 \\
\hline HIS3 & YOR202W & 12 & 14 & 24 & 24.47955 & \#DIV/0! \\
\hline HIS3 & YOR202W & 12 & 15 & 1 & 43.73148 & \#DIV/0! \\
\hline PEX15 & YOL044W & 12 & 15 & 2 & \#DIV/0! & \#DIV/0! \\
\hline FRE7 & YOL152W & 12 & 15 & 3 & 48.28455 & \#DIV/0! \\
\hline NTG2 & YOL043C & 12 & 15 & 4 & 12.64696 & \#DIV/O! \\
\hline GRE2 & YOL151W & 12 & 15 & 5 & 26.57182 & \#DIV/0! \\
\hline \multirow[t]{2}{*}{ NGL1 } & YOL042W & 12 & 15 & 6 & 2.038401 & \#DIV/0! \\
\hline & YOL150C & 12 & 15 & 7 & 5.978108 & \#DIV/O! \\
\hline NOP12 & YOL041C & 12 & 15 & 8 & 24.67559 & \#DIV/O! \\
\hline PEX11 & YOL147C & 12 & 15 & 9 & 71.01944 & \#DIV/0! \\
\hline RPP2A & YOL039W & 12 & 15 & 10 & 51.42985 & \#DIV/0! \\
\hline \multirow[t]{4}{*}{ PPM2 } & YOL141W & 12 & 15 & 11 & 2.985544 & \#DIV/0! \\
\hline & YOL037C & 12 & 15 & 12 & 24.84656 & \#DIV/0! \\
\hline & YOL138C & 12 & 15 & 13 & 56.09297 & \#DIV/0! \\
\hline & YOL036W & 12 & 15 & 14 & 45.23761 & \#DIV/0! \\
\hline \multirow[t]{2}{*}{ BSC6 } & YOL137W & 12 & 15 & 15 & 87.65182 & \#DIV/0! \\
\hline & YOL035C & 12 & 15 & 16 & 8.569473 & \#DIV/0! \\
\hline \multirow[t]{2}{*}{ PFK27 } & YOL136C & 12 & 15 & 17 & 52.08888 & \#DIV/0! \\
\hline & YOL032W & 12 & 15 & 18 & 22.64267 & \#DIV/0! \\
\hline GAS4 & YOL132W & 12 & 15 & 19 & 74.08064 & \#DIV/0! \\
\hline \multirow[t]{2}{*}{ SIL1 } & YOL031C & 12 & 15 & 20 & 57.21357 & \#DIV/0! \\
\hline & YOL131W & 12 & 15 & 21 & 20.49665 & \#DIV/0! \\
\hline GAS5 & YOL030W & 12 & 15 & 22 & 30.09148 & \#DIV/0! \\
\hline VPS68 & YOL129W & 12 & 15 & 23 & 16.01597 & \#DIV/0! \\
\hline HIS3 & YOR202W & 12 & 15 & 24 & 36.11111 & \#DIV/0! \\
\hline HIS3 & YOR202W & 12 & 16 & 1 & 12.72851 & \#DIV/0! \\
\hline HIS3 & YOR202W & 12 & 16 & 2 & 5.776963 & \#DIV/0! \\
\hline HIS3 & YOR202W & 12 & 16 & 3 & 20.53695 & \#DIV/0! \\
\hline HIS3 & YOR202W & 12 & 16 & 4 & 3.302157 & \#DIV/0! \\
\hline HIS3 & YOR202W & 12 & 16 & 5 & 19.98847 & \#DIV/0! \\
\hline HIS3 & YOR202W & 12 & 16 & 6 & 22.36989 & \#DIV/O! \\
\hline
\end{tabular}




\begin{tabular}{|c|c|c|c|c|c|c|}
\hline HIS3 & YOR202W & 12 & 16 & 7 & 22.07676 & \#DIV/0! \\
\hline HIS3 & YOR202W & 12 & 16 & 8 & 66.37569 & \#DIV/0! \\
\hline HIS3 & YOR202W & 12 & 16 & 9 & 31.88491 & \#DIV/0! \\
\hline HIS3 & YOR202W & 12 & 16 & 10 & 22.78739 & \#DIV/0! \\
\hline HIS3 & YOR202W & 12 & 16 & 11 & 10.1768 & \#DIV/0! \\
\hline HIS3 & YOR202W & 12 & 16 & 12 & 39.65703 & \#DIV/0! \\
\hline HIS3 & YOR202W & 12 & 16 & 13 & 21.92362 & \#DIV/0! \\
\hline HIS3 & YOR202W & 12 & 16 & 14 & 53.51541 & \#DIV/0! \\
\hline HIS3 & YOR202W & 12 & 16 & 15 & 15.79857 & \#DIV/O! \\
\hline HIS3 & YOR202W & 12 & 16 & 16 & 63.42269 & \#DIV/0! \\
\hline HIS3 & YOR202W & 12 & 16 & 17 & 33.24134 & \#DIV/0! \\
\hline HIS3 & YOR202W & 12 & 16 & 18 & -2.06823 & \#DIV/0! \\
\hline HIS3 & YOR202W & 12 & 16 & 19 & -5.27042 & \#DIV/0! \\
\hline HIS3 & YOR202W & 12 & 16 & 20 & -7.6381 & \#DIV/0! \\
\hline HIS3 & YOR202W & 12 & 16 & 21 & 33.60059 & \#DIV/0! \\
\hline HIS3 & YOR202W & 12 & 16 & 22 & 28.2771 & \#DIV/0! \\
\hline HIS3 & YOR202W & 12 & 16 & 23 & 33.31545 & \#DIV/0! \\
\hline HIS3 & YOR202W & 12 & 16 & 24 & 52.09193 & \#DIV/0! \\
\hline HIS3 & YOR202W & 13 & 1 & 1 & 26.48651 & \#DIV/0! \\
\hline HIS3 & YOR202W & 13 & 1 & 2 & 7.567002 & \#DIV/0! \\
\hline HIS3 & YOR202W & 13 & 1 & 3 & 34.79204 & \#DIV/0! \\
\hline HIS3 & YOR202W & 13 & 1 & 4 & 36.46083 & \#DIV/0! \\
\hline HIS3 & YOR202W & 13 & 1 & 5 & 24.30101 & \#DIV/0! \\
\hline HIS3 & YOR202W & 13 & 1 & 6 & 24.41042 & \#DIV/0! \\
\hline HIS3 & YOR202W & 13 & 1 & 7 & -15.7761 & \#DIV/0! \\
\hline HIS3 & YOR202W & 13 & 1 & 8 & 12.01751 & \#DIV/0! \\
\hline HIS3 & YOR202W & 13 & 1 & 9 & 3.738786 & \#DIV/0! \\
\hline HIS3 & YOR202W & 13 & 1 & 10 & 6.597691 & \#DIV/0! \\
\hline HIS3 & YOR202W & 13 & 1 & 11 & -12.4023 & \#DIV/0! \\
\hline HIS3 & YOR202W & 13 & 1 & 12 & 3.549697 & \#DIV/0! \\
\hline HIS3 & YOR202W & 13 & 1 & 13 & -6.07998 & \#DIV/0! \\
\hline HIS3 & YOR202W & 13 & 1 & 14 & 12.62234 & \#DIV/0! \\
\hline HIS3 & YOR202W & 13 & 1 & 15 & 17.48829 & \#DIV/0! \\
\hline HIS3 & YOR202W & 13 & 1 & 16 & 13.1487 & \#DIV/0! \\
\hline HIS3 & YOR202W & 13 & 1 & 17 & 8.627123 & \#DIV/0! \\
\hline HIS3 & YOR202W & 13 & 1 & 18 & 25.32106 & \#DIV/0! \\
\hline HIS3 & YOR202W & 13 & 1 & 19 & 6.214244 & \#DIV/0! \\
\hline HIS3 & YOR202W & 13 & 1 & 20 & 1.732775 & \#DIV/0! \\
\hline HIS3 & YOR202W & 13 & 1 & 21 & -8.4059 & \#DIV/0! \\
\hline HIS3 & YOR202W & 13 & 1 & 22 & 5.731819 & \#DIV/0! \\
\hline HIS3 & YOR202W & 13 & 1 & 23 & 8.363402 & \#DIV/0! \\
\hline HIS3 & YOR202W & 13 & 1 & 24 & 3.477516 & \#DIV/0! \\
\hline HIS3 & YOR202W & 13 & 2 & 1 & 44.93664 & \#DIV/0! \\
\hline
\end{tabular}




\begin{tabular}{|c|c|c|c|c|c|c|}
\hline LPE10 & YPL060W & 13 & 2 & 2 & 43.27845 & 53.87349 \\
\hline DAP1 & YPL170W & 13 & 2 & 3 & 42.82676 & 41.89538 \\
\hline \multirow[t]{2}{*}{ PDR12 } & YPL058C & 13 & 2 & 4 & 35.31309 & 45.2065 \\
\hline & YPL168W & 13 & 2 & 5 & 22.77019 & 32.68096 \\
\hline SUR1 & YPL057C & 13 & 2 & 6 & 9.18222 & 30.23397 \\
\hline \multirow[t]{3}{*}{ REV3 } & YPL167C & 13 & 2 & 7 & 13.2605 & 18.75104 \\
\hline & YPL056C & 13 & 2 & 8 & 26.71441 & 58.01756 \\
\hline & YPL166W & 13 & 2 & 9 & 26.30217 & 48.12111 \\
\hline LGE1 & YPL055C & 13 & 2 & 10 & 27.44745 & 37.69977 \\
\hline SET6 & YPL165C & 13 & 2 & 11 & 32.97721 & 36.6692 \\
\hline LEE1 & YPL054W & 13 & 2 & 12 & 20.85593 & 61.91951 \\
\hline MLH3 & YPL164C & 13 & 2 & 13 & 21.48191 & 41.48757 \\
\hline KTR6 & YPL053C & 13 & 2 & 14 & 21.39473 & 32.73076 \\
\hline SVS1 & YPL163C & 13 & 2 & 15 & 14.72583 & 9.610195 \\
\hline \multirow[t]{2}{*}{ OAZ1 } & YPL052W & 13 & 2 & 16 & 13.26925 & 21.01441 \\
\hline & YPL162C & 13 & 2 & 17 & 26.58671 & 29.13288 \\
\hline ARL3 & YPL051W & 13 & 2 & 18 & 10.37126 & 17.52546 \\
\hline BEM4 & YPL161C & 13 & 2 & 19 & 16.52705 & 37.25219 \\
\hline CAM1 & YPL048W & 13 & 2 & 20 & 17.39374 & 35.50439 \\
\hline PET20 & YPL159C & 13 & 2 & 21 & 7.31714 & 32.71341 \\
\hline SGF11 & YPL047W & 13 & 2 & 22 & 10.39173 & 50.27529 \\
\hline TGS1 & YPL157W & 13 & 2 & 23 & 0.623043 & 24.80338 \\
\hline HIS3 & YOR202W & 13 & 2 & 24 & 8.488304 & \#DIV/O! \\
\hline HIS3 & YOR202W & 13 & 3 & 1 & 26.21684 & \#DIV/0! \\
\hline MCT1 & YOR221C & 13 & 3 & 2 & 18.18963 & 34.76712 \\
\hline SNC2 & YOR327C & 13 & 3 & 3 & 15.9037 & 32.71838 \\
\hline STE13 & YOR219C & 13 & 3 & 4 & 8.320822 & 34.07279 \\
\hline FRT1 & YOR324C & 13 & 3 & 5 & 23.73864 & 33.24328 \\
\hline \multirow[t]{3}{*}{ RUD3 } & YOR216C & 13 & 3 & 6 & 28.2275 & 34.72099 \\
\hline & YOR322C & 13 & 3 & 7 & 7.793734 & 33.85678 \\
\hline & YOR215C & 13 & 3 & 8 & 18.73797 & 33.19606 \\
\hline \multirow[t]{2}{*}{ РMT3 } & YOR321W & 13 & 3 & 9 & 17.58031 & 42.04063 \\
\hline & YOR214C & 13 & 3 & 10 & 23.18174 & 47.49283 \\
\hline GNT1 & YOR320C & 13 & 3 & 11 & 16.1179 & 54.59783 \\
\hline \multirow[t]{2}{*}{ SAS5 } & YOR213C & 13 & 3 & 12 & 33.64988 & 55.24479 \\
\hline & YOR318C & 13 & 3 & 13 & 17.03782 & 20.74511 \\
\hline STE4 & YOR212W & 13 & 3 & 14 & 11.32604 & 36.76742 \\
\hline FAA1 & YOR317W & 13 & 3 & 15 & 12.9403 & 20.08849 \\
\hline NPT1 & YOR209C & 13 & 3 & 16 & 18.07 & 28.39161 \\
\hline COT1 & YOR316C & 13 & 3 & 17 & 12.63064 & 27.95944 \\
\hline \multirow[t]{2}{*}{ PTP2 } & YOR208W & 13 & 3 & 18 & 11.97105 & 17.36736 \\
\hline & YOR315W & 13 & 3 & 19 & 5.145808 & 20.89372 \\
\hline HIS3 & YOR202W & 13 & 3 & 20 & 9.669208 & 27.14927 \\
\hline
\end{tabular}




\begin{tabular}{|c|c|c|c|c|c|c|}
\hline & YOR314W & 13 & 3 & 21 & 11.19972 & 38.94093 \\
\hline MCA1 & YOR197W & 13 & 3 & 22 & 4.626047 & 40.00946 \\
\hline SPS4 & YOR313C & 13 & 3 & 23 & 8.359761 & 42.10775 \\
\hline HIS3 & YOR202W & 13 & 3 & 24 & 4.790994 & 27.41379 \\
\hline HIS3 & YOR202W & 13 & 4 & 1 & \#DIV/0! & \#DIV/0! \\
\hline \multirow[t]{5}{*}{ YTA6 } & YPL074W & 13 & 4 & 2 & -3.02105 & 57.37533 \\
\hline & YPL183C & 13 & 4 & 3 & 35.50573 & 22.56809 \\
\hline & YPL073C & 13 & 4 & 4 & 20.27575 & 56.13431 \\
\hline & YPL182C & 13 & 4 & 5 & 1.802536 & 40.30345 \\
\hline & YPL071C & 13 & 4 & 6 & 11.33996 & 46.04347 \\
\hline CTI6 & YPL181W & 13 & 4 & 7 & 17.11745 & 48.1721 \\
\hline MUK1 & YPL070W & 13 & 4 & 8 & 10.43905 & 39.41947 \\
\hline TCO89 & YPL180W & 13 & 4 & 9 & 4.826412 & 16.71923 \\
\hline BTS1 & YPL069C & 13 & 4 & 10 & -7.52238 & 47.03172 \\
\hline \multirow[t]{2}{*}{ PPQ1 } & YPL179W & 13 & 4 & 11 & 20.36488 & 28.60503 \\
\hline & YPL068C & 13 & 4 & 12 & 18.91949 & 27.66985 \\
\hline \multirow[t]{2}{*}{ CBC2 } & YPL178W & 13 & 4 & 13 & 55.55947 & 58.39973 \\
\hline & YPL067C & 13 & 4 & 14 & 19.23304 & 31.12305 \\
\hline \multirow[t]{3}{*}{ CUP9 } & YPL177C & 13 & 4 & 15 & 11.59303 & 39 \\
\hline & YPL066W & 13 & 4 & 16 & 8.344433 & 45.39515 \\
\hline & YPL176C & 13 & 4 & 17 & 5.489418 & 43.02742 \\
\hline CWC27 & YPL064C & 13 & 4 & 18 & 15.83611 & 56.51344 \\
\hline \multirow[t]{2}{*}{ NIP100 } & YPL174C & 13 & 4 & 19 & 18.84409 & 65.37755 \\
\hline & YPL062W & 13 & 4 & 20 & 27.14442 & 33.04572 \\
\hline cox10 & YPL172C & 13 & 4 & 21 & 41.07143 & -14.7929 \\
\hline ALD6 & YPL061W & 13 & 4 & 22 & 8.322948 & 17.30424 \\
\hline OYE3 & YPL171C & 13 & 4 & 23 & 13.3108 & 26.30644 \\
\hline HIS3 & YOR202W & 13 & 4 & 24 & 12.87879 & \#DIV/0! \\
\hline HIS3 & YOR202W & 13 & 5 & 1 & 29.38362 & \#DIV/0! \\
\hline RPL33B & YOR234C & 13 & 5 & 2 & 13.30796 & 49.38771 \\
\hline PUT4 & YOR348C & 13 & 5 & 3 & 9.967207 & 25.92284 \\
\hline KIN4 & YOR233W & 13 & 5 & 4 & 13.20861 & 41.26393 \\
\hline PYK2 & YOR347C & 13 & 5 & 5 & 21.76719 & 56.18849 \\
\hline MKK1 & YOR231W & 13 & 5 & 6 & 24.65145 & 65.52137 \\
\hline REV1 & YOR346W & 13 & 5 & 7 & 3.245663 & 56.52287 \\
\hline WTM1 & YOR230W & 13 & 5 & 8 & 4.26899 & 25.90327 \\
\hline TYE7 & YOR344C & 13 & 5 & 9 & 1.898007 & 27.07127 \\
\hline \multirow[t]{5}{*}{ WTM2 } & YOR229W & 13 & 5 & 10 & 48.43217 & 48.22134 \\
\hline & YOR343C & 13 & 5 & 11 & -1.77633 & 46.08629 \\
\hline & YOR228C & 13 & 5 & 12 & 0.866271 & 31.9452 \\
\hline & YOR342C & 13 & 5 & 13 & 7.968097 & 28.86676 \\
\hline & YOR227W & 13 & 5 & 14 & 16.65342 & 53.43349 \\
\hline UBC11 & YOR339C & 13 & 5 & 15 & 11.97739 & 15.70816 \\
\hline
\end{tabular}




\begin{tabular}{|c|c|c|c|c|c|c|}
\hline \multirow[t]{3}{*}{ ISU2 } & YOR226C & 13 & 5 & 16 & 1.254022 & 62.58065 \\
\hline & YOR338W & 13 & 5 & 17 & 22.50189 & 46.2488 \\
\hline & YOR225W & 13 & 5 & 18 & 17.21509 & 61.06718 \\
\hline \multirow[t]{2}{*}{ TEA1 } & YOR337W & 13 & 5 & 19 & 10.77169 & 23.15802 \\
\hline & YOR223W & 13 & 5 & 20 & 22.23592 & 33.50209 \\
\hline MRS2 & YOR334W & 13 & 5 & 21 & 7.816414 & 6.475407 \\
\hline ODC2 & YOR222W & 13 & 5 & 22 & 13.49906 & 18.19142 \\
\hline PDR10 & YOR328W & 13 & 5 & 23 & 13.34007 & 56.20201 \\
\hline HIS3 & YOR202W & 13 & 5 & 24 & 9.147731 & \#DIV/0! \\
\hline \multirow[t]{2}{*}{ HIS3 } & YOR202W & 13 & 6 & 1 & 18.55294 & \#DIV/0! \\
\hline & YPL095C & 13 & 6 & 2 & 17.24041 & 54.83548 \\
\hline OXR1 & YPL196W & 13 & 6 & 3 & -17.4303 & 47.84894 \\
\hline SSU1 & YPL092W & 13 & 6 & 4 & 13.5437 & 44.28544 \\
\hline APL5 & YPL195W & 13 & 6 & 5 & -4.4933 & 37.91982 \\
\hline GLR1 & YPL091W & 13 & 6 & 6 & 6.839922 & 63.03966 \\
\hline DDC1 & YPL194W & 13 & 6 & 7 & 7.256897 & 55.20084 \\
\hline RPS6A & YPL090C & 13 & 6 & 8 & 17.57009 & 56.19703 \\
\hline PRM3 & YPL192C & 13 & 6 & 9 & 1.118788 & 39.64249 \\
\hline \multirow[t]{3}{*}{ RLM1 } & YPL089C & 13 & 6 & 10 & 14.42437 & 37.87591 \\
\hline & YPL191C & 13 & 6 & 11 & 1.195055 & 45.21355 \\
\hline & YPL088W & 13 & 6 & 12 & 13.58901 & 42.13589 \\
\hline GUP2 & YPL189W & 13 & 6 & 13 & 22.38931 & 52.57818 \\
\hline YDC1 & YPL087W & 13 & 6 & 14 & 63.38503 & 57.3288 \\
\hline MF(ALPHA)1 & YPL187W & 13 & 6 & 15 & 17.19954 & 38.72827 \\
\hline ELP3 & YPL086C & 13 & 6 & 16 & 13.28702 & 13.34145 \\
\hline UIP4 & YPL186C & 13 & 6 & 17 & 10.33092 & 35.82134 \\
\hline \multirow[t]{4}{*}{ RPS9A } & YPL081W & 13 & 6 & 18 & 33.97188 & 41.4941 \\
\hline & YPL185W & 13 & 6 & 19 & 12.63113 & 30.6464 \\
\hline & YPL080C & 13 & 6 & 20 & 5.600541 & 63.60517 \\
\hline & YPL184C & 13 & 6 & 21 & 12.91742 & 32.34472 \\
\hline RPL21B & $\begin{array}{l}\text { YPL079W } \\
\text { YPL183W- }\end{array}$ & 13 & 6 & 22 & 5.876098 & 43.47777 \\
\hline GON5 & $A$ & 13 & 6 & 23 & 4.133176 & 20.17318 \\
\hline HIS3 & YOR202W & 13 & 6 & 24 & 10.07687 & \#DIV/0! \\
\hline HIS3 & YOR202W & 13 & 7 & 1 & 1.327511 & \#DIV/0! \\
\hline RBF17 & YOR252W & 13 & 7 & 2 & 11.33511 & 54.21369 \\
\hline \multirow[t]{2}{*}{ PDE2 } & YOR360C & 13 & 7 & 3 & 10.15553 & 44.04339 \\
\hline & YOR251C & 13 & 7 & 4 & 9.892244 & 35.66798 \\
\hline VTS1 & YOR359W & 13 & 7 & 5 & 3.151633 & 19.13434 \\
\hline SRL1 & YOR247W & 13 & 7 & 6 & 4.33125 & 19.00794 \\
\hline \multirow[t]{2}{*}{ HAP5 } & YOR358W & 13 & 7 & 7 & -10.52 & 43.72692 \\
\hline & YOR246C & 13 & 7 & 8 & 4.838647 & 45.49047 \\
\hline GRD19 & YOR357C & 13 & 7 & 9 & 12.06619 & 44.8512 \\
\hline
\end{tabular}




\begin{tabular}{|c|c|c|c|c|c|c|}
\hline \multirow[t]{2}{*}{ DGA1 } & YOR245C & 13 & 7 & 10 & 6.875811 & 40.54853 \\
\hline & YOR356W & 13 & 7 & 11 & -6.80748 & 60.17722 \\
\hline PUS7 & YOR243C & 13 & 7 & 12 & 61.35222 & 71.84002 \\
\hline GDS1 & YOR355W & 13 & 7 & 13 & 57.01281 & 52.63913 \\
\hline SSP2 & YOR242C & 13 & 7 & 14 & 4.239038 & 43.26962 \\
\hline MSC6 & YOR354C & 13 & 7 & 15 & 10.66802 & 22.28484 \\
\hline \multirow[t]{3}{*}{ ABP140 } & YOR239W & 13 & 7 & 16 & 6.929402 & 29.04958 \\
\hline & YOR352W & 13 & 7 & 17 & 2.841112 & 37.12747 \\
\hline & YOR238W & 13 & 7 & 18 & 11.35318 & 35.58422 \\
\hline MEK1 & YOR351C & 13 & 7 & 19 & 13.86913 & 66.58175 \\
\hline HES1 & YOR237W & 13 & 7 & 20 & 19.04404 & 59.21221 \\
\hline \multirow[t]{2}{*}{ MNE1 } & YOR350C & 13 & 7 & 21 & -0.18005 & 38.32795 \\
\hline & YOR235W & 13 & 7 & 22 & 33.64018 & 37.3442 \\
\hline CIN1 & YOR349W & 13 & 7 & 23 & 5.241885 & 47.04394 \\
\hline HIS3 & YOR202W & 13 & 7 & 24 & 20.26504 & \#DIV/0! \\
\hline \multirow[t]{5}{*}{ HIS3 } & YOR202W & 13 & 8 & 1 & 27.74774 & \#DIV/0! \\
\hline & YPL108W & 13 & 8 & 2 & -10.8593 & \#DIV/0! \\
\hline & YPL208W & 13 & 8 & 3 & 10.08959 & 48.38399 \\
\hline & YPL107W & 13 & 8 & 4 & 6.9498 & 28.62738 \\
\hline & YPL207W & 13 & 8 & 5 & 6.224401 & 24.53613 \\
\hline \multirow[t]{4}{*}{ SSE1 } & YPL106C & 13 & 8 & 6 & -15.1986 & 44.14459 \\
\hline & YPL206C & 13 & 8 & 7 & 10.60793 & 40.95935 \\
\hline & YPL105C & 13 & 8 & 8 & 3.3558 & 25.57114 \\
\hline & YPL205C & 13 & 8 & 9 & -1.58779 & 35.27833 \\
\hline FMP30 & YPL103C & 13 & 8 & 10 & -6.21546 & 39.58741 \\
\hline \multirow[t]{2}{*}{ TPK2 } & YPL203W & 13 & 8 & 11 & 4.765294 & 44.07395 \\
\hline & YPL102C & 13 & 8 & 12 & \#DIV/0! & 56.04698 \\
\hline AFT2 & YPL202C & 13 & 8 & 13 & 3.921506 & 39.4206 \\
\hline \multirow[t]{2}{*}{ ELP4 } & YPL101W & 13 & 8 & 14 & \#DIV/0! & 58.87411 \\
\hline & YPL201C & 13 & 8 & 15 & 11.42558 & 31.33877 \\
\hline ATG21 & YPL100W & 13 & 8 & 16 & 17.17072 & 38.13038 \\
\hline CSM4 & YPL200W & 13 & 8 & 17 & 1.96283 & 18.51859 \\
\hline \multirow[t]{3}{*}{ FMP14 } & YPL099C & 13 & 8 & 18 & 12.04619 & 48.01733 \\
\hline & YPL199C & 13 & 8 & 19 & 12.29067 & 22.68216 \\
\hline & YPL098C & 13 & 8 & 20 & -11.5475 & 56.90008 \\
\hline RPL7B & YPL198W & 13 & 8 & 21 & 22.2517 & 48.19471 \\
\hline \multirow[t]{2}{*}{ PNG1 } & YPL096W & 13 & 8 & 22 & 3.963599 & 58.68603 \\
\hline & YPL197C & 13 & 8 & 23 & 15.33766 & 50.30943 \\
\hline HIS3 & YOR202W & 13 & 8 & 24 & 5.939799 & 29.13711 \\
\hline \multirow[t]{3}{*}{ HIS3 } & YOR202W & 13 & 9 & 1 & -8.59339 & \#DIV/0! \\
\hline & YOR271C & 13 & 9 & 2 & -3.01685 & 63.41628 \\
\hline & YOR378W & 13 & 9 & 3 & -3.37342 & 52.90315 \\
\hline VPH1 & YOR270C & 13 & 9 & 4 & -18.5948 & 39.90392 \\
\hline
\end{tabular}




\begin{tabular}{|c|c|c|c|c|c|c|}
\hline ATF1 & YOR377W & 13 & 9 & 5 & -3.21496 & 33.57883 \\
\hline \multirow[t]{3}{*}{ PAC1 } & YOR269W & 13 & 9 & 6 & 24.94948 & 41.42137 \\
\hline & YOR376W & 13 & 9 & 7 & -13.7963 & 43.55956 \\
\hline & YOR268C & 13 & 9 & 8 & -3.76435 & 31.5356 \\
\hline GDH1 & YOR375C & 13 & 9 & 9 & 4.352764 & 22.34591 \\
\hline HRK1 & YOR267C & 13 & 9 & 10 & 16.55416 & 48.58092 \\
\hline ALD4 & YOR374W & 13 & 9 & 11 & 7.467144 & 30.77183 \\
\hline PNT1 & YOR266W & 13 & 9 & 12 & 25.57935 & 62.18334 \\
\hline GPB1 & YOR371C & 13 & 9 & 13 & 17.34563 & 20.84698 \\
\hline RBL2 & YOR265W & 13 & 9 & 14 & 12.17045 & 19.72205 \\
\hline RAD17 & YOR368W & 13 & 9 & 15 & 12.91476 & 42.23974 \\
\hline DSE3 & YOR264W & 13 & 9 & 16 & 26.28447 & 59.42569 \\
\hline \multirow[t]{3}{*}{ SCP1 } & YOR367W & 13 & 9 & 17 & 50.97749 & 60.98282 \\
\hline & YOR263C & 13 & 9 & 18 & 9.125758 & 53.71068 \\
\hline & YOR365C & 13 & 9 & 19 & -4.30871 & 22.15446 \\
\hline \multirow[t]{2}{*}{ OSW1 } & YOR255W & 13 & 9 & 20 & 14.50907 & 45.10174 \\
\hline & YOR364W & 13 & 9 & 21 & 20.23792 & 58.87541 \\
\hline NAT5 & YOR253W & 13 & 9 & 22 & 10.99406 & 44.75705 \\
\hline PIP2 & YOR363C & 13 & 9 & 23 & 5.46627 & 2.482355 \\
\hline HIS3 & YOR202W & 13 & 9 & 24 & 12.80642 & \#DIV/0! \\
\hline HIS3 & YOR202W & 13 & 10 & 1 & 3.799552 & \#DIV/0! \\
\hline \multirow[t]{2}{*}{ MEI5 } & YPL121C & 13 & 10 & 2 & 25.09452 & 35.47414 \\
\hline & YPL225W & 13 & 10 & 3 & -3.37591 & 53.2037 \\
\hline VPS30 & YPL120W & 13 & 10 & 4 & 5.645617 & 15.36178 \\
\hline MMT2 & YPL224C & 13 & 10 & 5 & 25.48254 & 34.41037 \\
\hline DBP1 & YPL119C & 13 & 10 & 6 & 9.734077 & 63.84199 \\
\hline GRE1 & YPL223C & 13 & 10 & 7 & 9.456266 & 19.47616 \\
\hline HOS3 & YPL116W & 13 & 10 & 8 & 37.34871 & 64.90368 \\
\hline FMP40 & YPL222W & 13 & 10 & 9 & 6.934025 & 34.4864 \\
\hline BEM3 & YPL115C & 13 & 10 & 10 & 2.680672 & 38.89486 \\
\hline \multirow[t]{2}{*}{ BOP1 } & YPL221W & 13 & 10 & 11 & 0.789962 & 41.1953 \\
\hline & YPL114W & 13 & 10 & 12 & 18.62356 & 56.46593 \\
\hline \multirow[t]{2}{*}{ RPL1A } & YPL220W & 13 & 10 & 13 & -2.46612 & 52.20307 \\
\hline & YPL113C & 13 & 10 & 14 & 22.78101 & 65.41427 \\
\hline PCL8 & YPL219W & 13 & 10 & 15 & 1.253743 & 22.78673 \\
\hline \multirow[t]{2}{*}{ PEX25 } & YPL112C & 13 & 10 & 16 & 6.940848 & 43.68543 \\
\hline & YPL216W & 13 & 10 & 17 & 6.433192 & 58.7486 \\
\hline CAR1 & YPL111W & 13 & 10 & 18 & 26.29519 & 51.74399 \\
\hline \multirow[t]{2}{*}{ THI6 } & YPL214C & 13 & 10 & 19 & -1.8903 & 55.33706 \\
\hline & YPL110C & 13 & 10 & 20 & 51.11274 & 11.92484 \\
\hline \multirow[t]{2}{*}{ LEA1 } & YPL213W & 13 & 10 & 21 & 36.31885 & 41.99392 \\
\hline & YPL109C & 13 & 10 & 22 & 11.78253 & 63.21638 \\
\hline PUS1 & YPL212C & 13 & 10 & 23 & 9.436722 & 35.97154 \\
\hline
\end{tabular}




\begin{tabular}{|c|c|c|c|c|c|c|}
\hline HIS3 & YOR202W & 13 & 10 & 24 & 13.79025 & \#DIV/0! \\
\hline HIS3 & YOR202W & 13 & 11 & 1 & 51.72291 & \#DIV/0! \\
\hline FMP31 & YOR286W & 13 & 11 & 2 & -3.22699 & 44.52213 \\
\hline \multirow[t]{2}{*}{ CHL1 } & YPL008W & 13 & 11 & 3 & 3.434461 & 45.76439 \\
\hline & YOR285W & 13 & 11 & 4 & 12.78429 & 57.63085 \\
\hline LSP1 & YPL004C & 13 & 11 & 5 & -0.72016 & 44.27935 \\
\hline HUA2 & YOR284W & 13 & 11 & 6 & -1.88992 & 50.08333 \\
\hline \multirow[t]{2}{*}{ ULA1 } & YPL003W & 13 & 11 & 7 & 3.281849 & 32.35495 \\
\hline & YOR283W & 13 & 11 & 8 & 13.37351 & 51.55985 \\
\hline HAT1 & YPL001W & 13 & 11 & 9 & 11.31575 & 47.76029 \\
\hline FSH3 & YOR280C & 13 & 11 & 10 & -6.95818 & 39.73215 \\
\hline PHR1 & YOR386W & 13 & 11 & 11 & 8.619864 & 63.26452 \\
\hline \multirow[t]{3}{*}{ RFM1 } & YOR279C & 13 & 11 & 12 & 25.65914 & 30.95032 \\
\hline & YOR385W & 13 & 11 & 13 & 1.09375 & 56.95044 \\
\hline & YOR277C & 13 & 11 & 14 & 16.98902 & 46.59463 \\
\hline FRE5 & YOR384W & 13 & 11 & 15 & 14.60088 & 59.53161 \\
\hline CAF20 & YOR276W & 13 & 11 & 16 & 12.43078 & 55.59503 \\
\hline FIT3 & YOR383C & 13 & 11 & 17 & -6.96966 & 51.23374 \\
\hline RIM20 & YOR275C & 13 & 11 & 18 & 15.83133 & 45.99336 \\
\hline FIT2 & YOR382W & 13 & 11 & 19 & -8.74171 & 60.28813 \\
\hline MOD5 & YOR274W & 13 & 11 & 20 & -2.7036 & 28.93279 \\
\hline FRE3 & YOR381W & 13 & 11 & 21 & 5.456357 & 48.35225 \\
\hline TPO4 & YOR273C & 13 & 11 & 22 & 9.982008 & 58.76219 \\
\hline RDR1 & YOR380W & 13 & 11 & 23 & 11.372 & 51.63814 \\
\hline HIS3 & YOR202W & 13 & 11 & 24 & 14.75215 & \#DIV/0! \\
\hline HIS3 & YOR202W & 13 & 12 & 1 & 3.172624 & \#DIV/O! \\
\hline \multirow[t]{2}{*}{ UME1 } & YPL139C & 13 & 12 & 2 & 5.633361 & 42.12283 \\
\hline & YPL245W & 13 & 12 & 3 & 7.92239 & 51.01848 \\
\hline SPP1 & YPL138C & 13 & 12 & 4 & -7.70211 & 24.98963 \\
\hline \multirow[t]{2}{*}{ HUT1 } & YPL244C & 13 & 12 & 5 & 3.320543 & 43.91975 \\
\hline & YPL137C & 13 & 12 & 6 & 27.0903 & 16.04938 \\
\hline \multirow[t]{2}{*}{ CIN2 } & YPL241C & 13 & 12 & 7 & -0.21111 & 26.26431 \\
\hline & YPL136W & 13 & 12 & 8 & 4.342906 & 29.24835 \\
\hline HSP82 & YPL240C & 13 & 12 & 9 & 9.075937 & 64.29329 \\
\hline ISU1 & YPL135W & 13 & 12 & 10 & 9.420622 & 47.62927 \\
\hline YAR1 & YPL239W & 13 & 12 & 11 & 26.65507 & 25.21934 \\
\hline \multirow[t]{2}{*}{ ODC1 } & YPL134C & 13 & 12 & 12 & -6.65365 & 56.04052 \\
\hline & YPL236C & 13 & 12 & 13 & -10.718 & 49.09387 \\
\hline RDS2 & YPL133C & 13 & 12 & 14 & 6.304185 & 15.20035 \\
\hline SSO1 & YPL232W & 13 & 12 & 15 & -4.9225 & 62.45755 \\
\hline \multirow[t]{2}{*}{ SPO19 } & YPL130W & 13 & 12 & 16 & -1.3206 & 26.74555 \\
\hline & YPL230W & 13 & 12 & 17 & 18.97553 & 50.94427 \\
\hline HHO1 & YPL127C & 13 & 12 & 18 & 14.41688 & 63.366 \\
\hline
\end{tabular}




\begin{tabular}{|c|c|c|c|c|c|c|}
\hline & YPL229W & 13 & 12 & 19 & 10.26759 & 60.86922 \\
\hline KAP120 & YPL125W & 13 & 12 & 20 & \#DIV/0! & 44.66234 \\
\hline ALG5 & YPL227C & 13 & 12 & 21 & \#DIV/0! & 32.68851 \\
\hline RNY1 & YPL123C & 13 & 12 & 22 & 18.92084 & 56.50819 \\
\hline NEW1 & YPL226W & 13 & 12 & 23 & 11.42989 & 49.25031 \\
\hline HIS3 & YOR202W & 13 & 12 & 24 & 11.64213 & \#DIV/0! \\
\hline \multirow[t]{2}{*}{ HIS3 } & YOR202W & 13 & 13 & 1 & 2.786197 & \#DIV/0! \\
\hline & YOR300W & 13 & 13 & 2 & 7.214014 & 15.39855 \\
\hline SKS1 & YPL026C & 13 & 13 & 3 & 14.88008 & 42.61963 \\
\hline \multirow[t]{2}{*}{ BUD7 } & YOR299W & 13 & 13 & 4 & -1.19467 & 59.67418 \\
\hline & YPL025C & 13 & 13 & 5 & 2.536612 & 53.22075 \\
\hline MUM3 & YOR298W & 13 & 13 & 6 & 40.03629 & 47.58018 \\
\hline NCE4 & $\begin{array}{l}\text { YPL024W } \\
\text { YOR298C- }\end{array}$ & 13 & 13 & 7 & -4.54115 & 58.4352 \\
\hline MBF1 & $A$ & 13 & 13 & 8 & 0.821239 & 59.5917 \\
\hline MET12 & YPL023C & 13 & 13 & 9 & 2.011628 & 44.55692 \\
\hline TIM18 & YOR297C & 13 & 13 & 10 & 10.19585 & 26.32541 \\
\hline \multirow[t]{2}{*}{ RAD1 } & YPL022W & 13 & 13 & 11 & 61.39616 & 58.78807 \\
\hline & YOR296W & 13 & 13 & 12 & 17.98068 & 61.80411 \\
\hline ECM23 & YPL021W & 13 & 13 & 13 & 13.43386 & 66.13863 \\
\hline RPS10A & YOR293W & 13 & 13 & 14 & -4.12163 & -4.0265 \\
\hline \multirow[t]{2}{*}{ VTC3 } & YPL019C & 13 & 13 & 15 & -5.3031 & 67.05953 \\
\hline & YOR292C & 13 & 13 & 16 & -7.3898 & 47.02764 \\
\hline \multirow[t]{5}{*}{ CTF19 } & YPL018W & 13 & 13 & 17 & 4.785806 & 48.18655 \\
\hline & YOR291W & 13 & 13 & 18 & -3.65449 & 44.40559 \\
\hline & YPL017C & 13 & 13 & 19 & 8.58608 & 62.0926 \\
\hline & YOR289W & 13 & 13 & 20 & \#DIV/0! & 49.76574 \\
\hline & YPL014W & 13 & 13 & 21 & \#DIV/0! & 57.83046 \\
\hline \multirow[t]{2}{*}{ MPD1 } & YOR288C & 13 & 13 & 22 & 16.65285 & 68.94425 \\
\hline & YPL009C & 13 & 13 & 23 & 12.95891 & 65.92749 \\
\hline HIS3 & YOR202W & 13 & 13 & 24 & 21.21653 & \#DIV/0! \\
\hline HIS3 & YOR202W & 13 & 14 & 1 & 8.495273 & \#DIV/0! \\
\hline \multirow[t]{2}{*}{ PRM4 } & YPL156C & 13 & 14 & 2 & -8.3403 & 37.85346 \\
\hline & YPL260W & 13 & 14 & 3 & 36.18473 & 32.64944 \\
\hline KIP2 & YPL155C & 13 & 14 & 4 & 2.349984 & 47.84316 \\
\hline APM1 & YPL259C & 13 & 14 & 5 & 5.704885 & 31.51613 \\
\hline PEP4 & YPL154C & 13 & 14 & 6 & 39.56565 & 24.22827 \\
\hline THI21 & YPL258C & 13 & 14 & 7 & -16.2078 & 28.77167 \\
\hline \multirow[t]{3}{*}{ RRD2 } & YPL152W & 13 & 14 & 8 & -7.03701 & 63.41857 \\
\hline & YPL257W & 13 & 14 & 9 & -5.27623 & 36.42442 \\
\hline & YPL150W & 13 & 14 & 10 & 34.87955 & 49.09274 \\
\hline CLN2 & YPL256C & 13 & 14 & 11 & -5.94133 & 52.53962 \\
\hline ATG5 & YPL149W & 13 & 14 & 12 & 36.37576 & 3.915663 \\
\hline
\end{tabular}




\begin{tabular}{|c|c|c|c|c|c|c|}
\hline VIK1 & YPL253C & 13 & 14 & 13 & -15.1911 & 49.65946 \\
\hline PXA1 & YPL147W & 13 & 14 & 14 & -2.53806 & 68.93301 \\
\hline ICY2 & YPL250C & 13 & 14 & 15 & -0.35697 & 57.57706 \\
\hline KES1 & YPL145C & 13 & 14 & 16 & 20.12471 & 73.93441 \\
\hline \multirow[t]{2}{*}{ GYP5 } & YPL249C & 13 & 14 & 17 & -16.2598 & 12.8499 \\
\hline & YPL144W & 13 & 14 & 18 & -18.7159 & 38.65712 \\
\hline \multirow[t]{3}{*}{ GAL4 } & YPL248C & 13 & 14 & 19 & -4.72488 & 31.85515 \\
\hline & YPL141C & 13 & 14 & 20 & 7.744162 & 39.61789 \\
\hline & YPL247C & 13 & 14 & 21 & 1.899713 & 53.54656 \\
\hline MKK2 & YPL140C & 13 & 14 & 22 & -2.39174 & 19.46063 \\
\hline RBD2 & YPL246C & 13 & 14 & 23 & 22.32735 & 42.19589 \\
\hline HIS3 & YOR202W & 13 & 14 & 24 & 26.66952 & \#DIV/0! \\
\hline HIS3 & YOR202W & 13 & 15 & 1 & 7.667055 & \#DIV/0! \\
\hline RPL20B & YOR312C & 13 & 15 & 2 & 3.054414 & \#DIV/O! \\
\hline ELC1 & YPL046C & 13 & 15 & 3 & 4.677939 & \#DIV/0! \\
\hline \multirow[t]{4}{*}{ HSD1 } & YOR311C & 13 & 15 & 4 & 14.30203 & \#DIV/0! \\
\hline & YPL041C & 13 & 15 & 5 & 4.692303 & \#DIV/0! \\
\hline & YOR309C & 13 & 15 & 6 & 30.7537 & \#DIV/0! \\
\hline & YPL039W & 13 & 15 & 7 & -5.9285 & \#DIV/0! \\
\hline SNU66 & YOR308C & 13 & 15 & 8 & -6.33686 & \#DIV/0! \\
\hline MET31 & YPL038W & 13 & 15 & 9 & -1.90283 & \#DIV/0! \\
\hline SLY41 & YOR307C & 13 & 15 & 10 & 32.43384 & \#DIV/0! \\
\hline EGD1 & YPL037C & 13 & 15 & 11 & 48.09497 & \#DIV/0! \\
\hline MCH5 & YOR306C & 13 & 15 & 12 & -2.02842 & \#DIV/0! \\
\hline PMA2 & YPL036W & 13 & 15 & 13 & 2.423817 & \#DIV/0! \\
\hline \multirow[t]{2}{*}{ CPA1 } & YOR303W & 13 & 15 & 14 & 5.458608 & \#DIV/0! \\
\hline & YPL034W & 13 & 15 & 15 & -1.90285 & \#DIV/0! \\
\hline \multirow[t]{4}{*}{ ISW2 } & YOR304W & 13 & 15 & 16 & 32.0483 & \#DIV/0! \\
\hline & YPL033C & 13 & 15 & 17 & 8.277786 & \#DIV/0! \\
\hline & YOR304C- & & & & & \\
\hline & $A$ & 13 & 15 & 18 & 16.41092 & \#DIV/0! \\
\hline \multirow[t]{3}{*}{ SVL3 } & YPL032C & 13 & 15 & 19 & 23.61824 & \#DIV/0! \\
\hline & YOR302W & 13 & 15 & 20 & 8.803137 & \#DIV/0! \\
\hline & YPL030W & 13 & 15 & 21 & 15.8532 & \#DIV/0! \\
\hline RAX1 & YOR301W & 13 & 15 & 22 & 19.06449 & \#DIV/0! \\
\hline SMA1 & YPL027W & 13 & 15 & 23 & 3.392494 & \#DIV/0! \\
\hline HIS3 & YOR202W & 13 & 15 & 24 & 20.1597 & \#DIV/0! \\
\hline HIS3 & YOR202W & 13 & 16 & 1 & 7.482381 & \#DIV/0! \\
\hline HIS3 & YOR202W & 13 & 16 & 2 & 43.42992 & \#DIV/0! \\
\hline HIS3 & YOR202W & 13 & 16 & 3 & 19.15464 & \#DIV/0! \\
\hline HIS3 & YOR202W & 13 & 16 & 4 & 5.386399 & \#DIV/0! \\
\hline HIS3 & YOR202W & 13 & 16 & 5 & -2.82388 & \#DIV/0! \\
\hline HIS3 & YOR202W & 13 & 16 & 6 & 2.458364 & \#DIV/0! \\
\hline
\end{tabular}




\begin{tabular}{|c|c|c|c|c|c|c|}
\hline HIS3 & YOR202W & 13 & 16 & 7 & 15.13037 & \#DIV/O! \\
\hline HIS3 & YOR202W & 13 & 16 & 8 & 42.46498 & \#DIV/0! \\
\hline HIS3 & YOR202W & 13 & 16 & 9 & 45.98928 & \#DIV/O! \\
\hline HIS3 & YOR202W & 13 & 16 & 10 & 36.99565 & \#DIV/0! \\
\hline HIS3 & YOR202W & 13 & 16 & 11 & 24.84398 & \#DIV/0! \\
\hline HIS3 & YOR202W & 13 & 16 & 12 & 12.95646 & \#DIV/0! \\
\hline HIS3 & YOR202W & 13 & 16 & 13 & 29.69318 & \#DIV/0! \\
\hline HIS3 & YOR202W & 13 & 16 & 14 & 14.25848 & \#DIV/0! \\
\hline HIS3 & YOR202W & 13 & 16 & 15 & 6.626262 & \#DIV/O! \\
\hline HIS3 & YOR202W & 13 & 16 & 16 & 16.60429 & \#DIV/0! \\
\hline HIS3 & YOR202W & 13 & 16 & 17 & 30.5991 & \#DIV/0! \\
\hline HIS3 & YOR202W & 13 & 16 & 18 & 7.704671 & \#DIV/0! \\
\hline HIS3 & YOR202W & 13 & 16 & 19 & 25.14203 & \#DIV/0! \\
\hline HIS3 & YOR202W & 13 & 16 & 20 & 37.3418 & \#DIV/0! \\
\hline HIS3 & YOR202W & 13 & 16 & 21 & 0.960095 & \#DIV/0! \\
\hline HIS3 & YOR202W & 13 & 16 & 22 & 16.01894 & \#DIV/0! \\
\hline HIS3 & YOR202W & 13 & 16 & 23 & 22.17879 & \#DIV/0! \\
\hline HIS3 & YOR202W & 13 & 16 & 24 & 12.45932 & \#DIV/0! \\
\hline HIS3 & YOR202W & 14 & 1 & 1 & 36.9123 & \#DIV/0! \\
\hline HIS3 & YOR202W & 14 & 1 & 2 & 30.17381 & \#DIV/0! \\
\hline HIS3 & YOR202W & 14 & 1 & 3 & 51.31128 & \#DIV/0! \\
\hline HIS3 & YOR202W & 14 & 1 & 4 & 47.64855 & \#DIV/0! \\
\hline HIS3 & YOR202W & 14 & 1 & 5 & 38.87168 & \#DIV/0! \\
\hline HIS3 & YOR202W & 14 & 1 & 6 & 20.26431 & \#DIV/0! \\
\hline HIS3 & YOR202W & 14 & 1 & 7 & 28.17501 & \#DIV/0! \\
\hline HIS3 & YOR202W & 14 & 1 & 8 & 35.13335 & \#DIV/0! \\
\hline HIS3 & YOR202W & 14 & 1 & 9 & 36.55488 & \#DIV/0! \\
\hline HIS3 & YOR202W & 14 & 1 & 10 & 23.13213 & \#DIV/0! \\
\hline HIS3 & YOR202W & 14 & 1 & 11 & 9.987056 & \#DIV/0! \\
\hline HIS3 & YOR202W & 14 & 1 & 12 & 48.78536 & \#DIV/0! \\
\hline HIS3 & YOR202W & 14 & 1 & 13 & 40.33163 & \#DIV/0! \\
\hline HIS3 & YOR202W & 14 & 1 & 14 & 18.44553 & \#DIV/O! \\
\hline HIS3 & YOR202W & 14 & 1 & 15 & 20.06743 & \#DIV/0! \\
\hline HIS3 & YOR202W & 14 & 1 & 16 & 21.80289 & \#DIV/0! \\
\hline HIS3 & YOR202W & 14 & 1 & 17 & 36.82287 & \#DIV/0! \\
\hline HIS3 & YOR202W & 14 & 1 & 18 & 41.62371 & \#DIV/0! \\
\hline HIS3 & YOR202W & 14 & 1 & 19 & 56.83251 & \#DIV/0! \\
\hline HIS3 & YOR202W & 14 & 1 & 20 & 24.21582 & \#DIV/0! \\
\hline HIS3 & YOR202W & 14 & 1 & 21 & 27.88606 & \#DIV/0! \\
\hline HIS3 & YOR202W & 14 & 1 & 22 & 37.72962 & \#DIV/0! \\
\hline HIS3 & YOR202W & 14 & 1 & 23 & 50.99197 & \#DIV/0! \\
\hline HIS3 & YOR202W & 14 & 1 & 24 & 10.30617 & \#DIV/0! \\
\hline HIS3 & YOR202W & 14 & 2 & 1 & -0.24582 & \#DIV/0! \\
\hline
\end{tabular}




\begin{tabular}{|c|c|c|c|c|c|c|}
\hline TIF4632 & YGL049C & 14 & 2 & 2 & 9.175269 & 47.1482 \\
\hline \multirow[t]{3}{*}{ IES1 } & YFL013C & 14 & 2 & 3 & 51.29803 & 35.25106 \\
\hline & YGL042C & 14 & 2 & 4 & 52.63001 & 59.35638 \\
\hline & YFL012W & 14 & 2 & 5 & 36.98917 & 36.93473 \\
\hline \multirow[t]{2}{*}{ ECM33 } & YBR078W & 14 & 2 & 6 & 37.16767 & 19.58333 \\
\hline & YFL010W- & & & & & \\
\hline AUA1 & $A$ & 14 & 2 & 7 & 30.21452 & 58.23759 \\
\hline RPL17B & YJL177W & 14 & 2 & 8 & 17.89634 & 47.8923 \\
\hline \multirow[t]{2}{*}{ WWM1 } & YFL010C & 14 & 2 & 9 & 38.7262 & 37.54123 \\
\hline & YGR122W & 14 & 2 & 10 & 33.57033 & 63.78099 \\
\hline \multirow[t]{2}{*}{ BLM3 } & YFL007W & 14 & 2 & 11 & 27.52645 & 49.6306 \\
\hline & YGR068C & 14 & 2 & 12 & 31.14079 & 48.64191 \\
\hline \multirow[t]{2}{*}{ VTC2 } & YFL004W & 14 & 2 & 13 & 11.47903 & 52.61385 \\
\hline & YGR067C & 14 & 2 & 14 & 30.83006 & 54.23209 \\
\hline MSH4 & YFL003C & 14 & 2 & 15 & 18.27466 & 23.37584 \\
\hline ADE6 & YGR061C & 14 & 2 & 16 & 54.17608 & 44.97899 \\
\hline DEG1 & YFL001W & 14 & 2 & 17 & 20.5572 & 33.59734 \\
\hline \multirow[t]{2}{*}{ MUP1 } & YGR055W & 14 & 2 & 18 & -28.2415 & 34.47668 \\
\hline & YGR255C & 14 & 2 & 19 & 40.26664 & 26.77398 \\
\hline RCS1 & YGL071W & 14 & 2 & 20 & 31.68991 & 50.70086 \\
\hline ENO1 & YGR254W & 14 & 2 & 21 & 24.66568 & 57.28808 \\
\hline \multirow[t]{2}{*}{ PUS2 } & YGL063W & 14 & 2 & 22 & 42.66607 & 57.18485 \\
\hline & YGR122C-A & 14 & 2 & 23 & 16.82134 & 54.00141 \\
\hline HIS3 & YOR202W & 14 & 2 & 24 & 33.67302 & \#DIV/0! \\
\hline HIS3 & YOR202W & 14 & 3 & 1 & 14.75154 & \#DIV/0! \\
\hline SAM3 & YPL274W & 14 & 3 & 2 & 26.65243 & 43.3881 \\
\hline CLB5 & YPR120C & 14 & 3 & 3 & 11.36125 & 43.91619 \\
\hline SAM4 & YPL273W & 14 & 3 & 4 & 62.48474 & 45.88346 \\
\hline \multirow[t]{3}{*}{ CLB2 } & YPR119W & 14 & 3 & 5 & 9.551946 & 35.89797 \\
\hline & YPL272C & 14 & 3 & 6 & 32.29511 & 40.24122 \\
\hline & YPR117W & 14 & 3 & 7 & 31.02245 & 32.19985 \\
\hline \multirow[t]{2}{*}{ MDL2 } & YPL270W & 14 & 3 & 8 & 20.14971 & 42.6864 \\
\hline & YPR115W & 14 & 3 & 9 & 20.03621 & 65.01865 \\
\hline \multirow[t]{2}{*}{ KAR9 } & YPL269W & 14 & 3 & 10 & 25.66154 & 46.74567 \\
\hline & YPR114W & 14 & 3 & 11 & 41.48374 & 65.14593 \\
\hline ACM1 & YPL267W & 14 & 3 & 12 & 43.58078 & 52.39362 \\
\hline DBF20 & YPR111W & 14 & 3 & 13 & 44.68415 & 30.19796 \\
\hline \multirow[t]{3}{*}{ DIP5 } & YPL265W & 14 & 3 & 14 & 24.63126 & 41.30724 \\
\hline & YPR109W & 14 & 3 & 15 & 21.76947 & 53.03326 \\
\hline & YPL264C & 14 & 3 & 16 & 25.65969 & 62.54313 \\
\hline ISR1 & YPR106W & 14 & 3 & 17 & 23.39891 & 31.13715 \\
\hline \multirow[t]{2}{*}{ KEL3 } & YPL263C & 14 & 3 & 18 & 33.01156 & 34.23488 \\
\hline & YPR098C & 14 & 3 & 19 & 25.25678 & 45.62034 \\
\hline
\end{tabular}




\begin{tabular}{|c|c|c|c|c|c|c|}
\hline \multirow[t]{4}{*}{ FUM1 } & YPL262W & 14 & 3 & 20 & 25.15115 & 36.11414 \\
\hline & YPR097W & 14 & 3 & 21 & 22.54006 & 41.89159 \\
\hline & YPL261C & 14 & 3 & 22 & 22.71806 & 59.49301 \\
\hline & YPR096C & 14 & 3 & 23 & 58.72555 & 45.0293 \\
\hline HIS3 & YOR202W & 14 & 3 & 24 & 4.364608 & \#DIV/0! \\
\hline HIS3 & YOR202W & 14 & 4 & 1 & 19.95259 & \#DIV/0! \\
\hline ISN1 & YOR155C & 14 & 4 & 2 & 24.36795 & 56.48372 \\
\hline TOS8 & YGL096W & 14 & 4 & 3 & 66.72396 & 42.65192 \\
\hline VHS3 & YOR054C & 14 & 4 & 4 & 33.74682 & 64.94036 \\
\hline PAN2 & YGL094C & 14 & 4 & 5 & 34.61835 & 58.22077 \\
\hline RHO5 & YNL180C & 14 & 4 & 6 & 22.02672 & 37.92157 \\
\hline PNC1 & YGL037C & 14 & 4 & 7 & 1.106934 & 50.27628 \\
\hline BOP3 & YNL042W & 14 & 4 & 8 & 62.38898 & 75.74514 \\
\hline CGR1 & YGL029W & 14 & 4 & 9 & 45.80465 & 64.65836 \\
\hline CYT2 & YKL087C & 14 & 4 & 10 & 37.96628 & 35.26776 \\
\hline MAL12 & YGR292W & 14 & 4 & 11 & 24.16069 & 64.92447 \\
\hline \multirow[t]{2}{*}{ KGD1 } & YIL125W & 14 & 4 & 12 & 24.32599 & 57.17621 \\
\hline & YGR291C & 14 & 4 & 13 & 13.78617 & 62.90754 \\
\hline GEM1 & YAL048C & 14 & 4 & 14 & 67.08061 & 25.64838 \\
\hline \multirow[t]{2}{*}{ MAL11 } & YGR289C & 14 & 4 & 15 & 47.88408 & 63.68337 \\
\hline & YOR248W & 14 & 4 & 16 & 47.28661 & 61.04137 \\
\hline \multirow[t]{3}{*}{ RNH70 } & YGR276C & 14 & 4 & 17 & 29.72851 & 35.8495 \\
\hline & YOR220W & 14 & 4 & 18 & 43.18683 & 55.40045 \\
\hline & YGR273C & 14 & 4 & 19 & 31.84794 & 32.99826 \\
\hline \multirow[t]{2}{*}{ DCl1 } & YOR180C & 14 & 4 & 20 & -1.57994 & 56.86802 \\
\hline & YGR272C & 14 & 4 & 21 & 31.57331 & 32.34949 \\
\hline SYC1 & YOR179C & 14 & 4 & 22 & 38.73419 & 41.90193 \\
\hline SLH1 & YGR271W & 14 & 4 & 23 & 50.08236 & 57.46694 \\
\hline HIS3 & YOR202W & 14 & 4 & 24 & -0.10098 & \#DIV/0! \\
\hline \multirow[t]{2}{*}{ HIS3 } & YOR202W & 14 & 5 & 1 & -25.0985 & \#DIV/0! \\
\hline & YPR013C & 14 & 5 & 2 & 30.23701 & 43.40659 \\
\hline \multirow[t]{2}{*}{ MSS18 } & YPR134W & 14 & 5 & 3 & 30.36805 & 33.82374 \\
\hline & YPR012W & 14 & 5 & 4 & 6.052784 & 51.94795 \\
\hline RPS23B & YPR132W & 14 & 5 & 5 & 45.06805 & 42.57849 \\
\hline \multirow[t]{2}{*}{ SUT2 } & YPR009W & 14 & 5 & 6 & 17.53349 & 36.02797 \\
\hline & YPR130C & 14 & 5 & 7 & 21.65121 & 65.19562 \\
\hline HAA1 & YPR008W & 14 & 5 & 8 & 26.15146 & 60.13794 \\
\hline SCD6 & YPR129W & 14 & 5 & 9 & 41.25945 & 58.17348 \\
\hline REC8 & YPR007C & 14 & 5 & 10 & 35.83653 & 66.25 \\
\hline ANT1 & YPR128C & 14 & 5 & 11 & 20.0211 & 42.87109 \\
\hline \multirow[t]{2}{*}{ ICL2 } & YPR006C & 14 & 5 & 12 & 22.03011 & 58.69804 \\
\hline & YPR127W & 14 & 5 & 13 & 32.65711 & 42.38019 \\
\hline HAL1 & YPR005C & 14 & 5 & 14 & 46.68607 & 60.31869 \\
\hline
\end{tabular}




\begin{tabular}{|c|c|c|c|c|c|c|}
\hline & YPR126C & 14 & 5 & 15 & 34.09957 & 42.18577 \\
\hline & YPR004C & 14 & 5 & 16 & 26.60249 & 46.90194 \\
\hline & YPR125W & 14 & 5 & 17 & 30.38805 & 24.03065 \\
\hline & YPR003C & 14 & 5 & 18 & 31.44864 & 59.35849 \\
\hline & YPR123C & 14 & 5 & 19 & 20.66703 & 24.88191 \\
\hline PDH1 & YPR002W & 14 & 5 & 20 & 23.71422 & 60.60449 \\
\hline AXL1 & YPR122W & 14 & 5 & 21 & 25.12225 & 45.6655 \\
\hline CIT3 & YPR001W & 14 & 5 & 22 & 12.29696 & 44.34647 \\
\hline THI22 & YPR121W & 14 & 5 & 23 & 74.14697 & 57.15607 \\
\hline HIS3 & YOR202W & 14 & 5 & 24 & 34.83442 & \#DIV/0! \\
\hline HIS3 & YOR202W & 14 & 6 & 1 & 38.43516 & \#DIV/0! \\
\hline PET123 & YOR158W & 14 & 6 & 2 & 31.27475 & 45.44268 \\
\hline DJP1 & YIR004W & 14 & 6 & 3 & 33.03172 & 54.30876 \\
\hline \multirow[t]{2}{*}{ MRH1 } & YDR033W & 14 & 6 & 4 & 38.38665 & 52.91588 \\
\hline & YIL158W & 14 & 6 & 5 & 10.48999 & 45.86525 \\
\hline \multirow[t]{3}{*}{ PST2 } & YDR032C & 14 & 6 & 6 & 32.79789 & 59.35487 \\
\hline & YIL151C & 14 & 6 & 7 & 36.71371 & 51.37636 \\
\hline & YDR031W & 14 & 6 & 8 & 34.74043 & 47.49331 \\
\hline OM45 & YIL136W & 14 & 6 & 9 & 30.27547 & 35.90337 \\
\hline RAD28 & YDR030C & 14 & 6 & 10 & 32.73048 & 59.94224 \\
\hline \multirow[t]{2}{*}{ FKH1 } & YIL131C & 14 & 6 & 11 & 22.87317 & 43.87018 \\
\hline & YDR029W & 14 & 6 & 12 & 19.99708 & 42.79532 \\
\hline \multirow[t]{2}{*}{ POG1 } & YIL122W & 14 & 6 & 13 & 31.29263 & 53.12658 \\
\hline & YDR026C & 14 & 6 & 14 & 36.71973 & 39.03289 \\
\hline \multirow[t]{3}{*}{ COX5B } & YIL111W & 14 & 6 & 15 & 22.2235 & 48.46723 \\
\hline & YAL046C & 14 & 6 & 16 & 33.03802 & 44.47241 \\
\hline & YIL102C & 14 & 6 & 17 & 44.88971 & 38.62872 \\
\hline ADY4 & YLR227C & 14 & 6 & 18 & 20.87361 & 44.71597 \\
\hline \multirow[t]{2}{*}{ LYS12 } & YIL094C & 14 & 6 & 19 & 21.02152 & 46.81013 \\
\hline & YKR104W & 14 & 6 & 20 & 46.96468 & 53.35331 \\
\hline KTR7 & YIL085C & 14 & 6 & 21 & 35.34197 & 31.39796 \\
\hline \multirow[t]{2}{*}{ BAP3 } & YDR046C & 14 & 6 & 22 & 25.53804 & 41.1511 \\
\hline & YMR074C & 14 & 6 & 23 & 32.45595 & 37.56219 \\
\hline HIS3 & YOR202W & 14 & 6 & 24 & 22.07312 & \#DIV/0! \\
\hline HIS3 & YOR202W & 14 & 7 & 1 & 21.78612 & \#DIV/0! \\
\hline \multirow[t]{4}{*}{ YOP1 } & YPR028W & 14 & 7 & 2 & 17.3147 & 55.82864 \\
\hline & YPR152C & 14 & 7 & 3 & 27.59945 & 46.68271 \\
\hline & YPR027C & 14 & 7 & 4 & 19.77778 & 62.00788 \\
\hline & YPR150W & 14 & 7 & 5 & 63.28498 & 30.78527 \\
\hline ATH1 & YPR026W & 14 & 7 & 6 & 20.72234 & 37.23369 \\
\hline NCE102 & YPR149W & 14 & 7 & 7 & 25.5564 & 53.70207 \\
\hline \multirow[t]{2}{*}{ YME1 } & YPR024W & 14 & 7 & 8 & 26.44156 & 35.96407 \\
\hline & YPR148C & 14 & 7 & 9 & 23.45209 & 55.19923 \\
\hline
\end{tabular}




\begin{tabular}{|c|c|c|c|c|c|c|}
\hline \multirow[t]{4}{*}{ EAF3 } & YPR023C & 14 & 7 & 10 & 58.09895 & 56.33217 \\
\hline & YPR147C & 14 & 7 & 11 & 32.87236 & 42.82588 \\
\hline & YPR022C & 14 & 7 & 12 & 34.99849 & 51.40907 \\
\hline & YPR146C & 14 & 7 & 13 & 32.22457 & 36.59591 \\
\hline ATP20 & YPR020W & 14 & 7 & 14 & 38.10846 & 46.7931 \\
\hline ASN1 & YPR145W & 14 & 7 & 15 & 43.17968 & 55.19337 \\
\hline RLF2 & YPR018W & 14 & 7 & 16 & 25.57815 & -4.19197 \\
\hline KAR3 & YPR141C & 14 & 7 & 17 & 34.76452 & 39.08668 \\
\hline DSS4 & YPR017C & 14 & 7 & 18 & 42.67345 & 32.55741 \\
\hline \multirow[t]{2}{*}{ TAZ1 } & YPR140W & 14 & 7 & 19 & 32.99187 & 34.05517 \\
\hline & YPR015C & 14 & 7 & 20 & 75.14317 & 67.10334 \\
\hline \multirow[t]{2}{*}{ MEP3 } & YPR138C & 14 & 7 & 21 & 48.15978 & 64.41692 \\
\hline & YPR014C & 14 & 7 & 22 & 35.17831 & 48.45927 \\
\hline CTF4 & YPR135W & 14 & 7 & 23 & 64.53407 & 65.09615 \\
\hline HIS3 & YOR202W & 14 & 7 & 24 & 39.8533 & \#DIV/0! \\
\hline HIS3 & YOR202W & 14 & 8 & 1 & 34.7847 & \#DIV/0! \\
\hline \multirow[t]{3}{*}{ NRG1 } & YDR043C & 14 & 8 & 2 & 41.48491 & 35.25078 \\
\hline & YLR455W & 14 & 8 & 3 & 25.14191 & 44.77473 \\
\hline & YDR042C & 14 & 8 & 4 & 40.33586 & 54.87964 \\
\hline TOR1 & YJR066W & 14 & 8 & 5 & 34.67845 & 59.71698 \\
\hline EHD3 & YDR036C & 14 & 8 & 6 & 67.5549 & 46.65374 \\
\hline VPS55 & YJR044C & 14 & 8 & 7 & 58.17531 & 27.86623 \\
\hline \multirow[t]{2}{*}{ ARO3 } & YDR035W & 14 & 8 & 8 & 34.51484 & 42.8537 \\
\hline & YJR039W & 14 & 8 & 9 & 44.77662 & 46.22969 \\
\hline \multirow[t]{3}{*}{ LYS14 } & YDR034C & 14 & 8 & 10 & 37.15414 & 48.29126 \\
\hline & YJL103C & 14 & 8 & 11 & 40.78657 & 49.25414 \\
\hline & YPR039W & 14 & 8 & 12 & 41.38988 & 61.44297 \\
\hline VPS53 & YJL029C & 14 & 8 & 13 & 50.87142 & 0.360247 \\
\hline \multirow[t]{3}{*}{ UBP16 } & YPL072W & 14 & 8 & 14 & 21.53251 & 44.0338 \\
\hline & YNL086W & 14 & 8 & 15 & 27.74299 & 51.02879 \\
\hline & YPL035C & 14 & 8 & 16 & 26.7222 & 22.97723 \\
\hline MSG5 & YNL053W & 14 & 8 & 17 & 43.63031 & 55.65954 \\
\hline HST2 & YPL015C & 14 & 8 & 18 & 34.23316 & 56.0049 \\
\hline \multirow[t]{2}{*}{ SLM2 } & YNL047C & 14 & 8 & 19 & 37.87131 & 50.62743 \\
\hline & YOR240W & 14 & 8 & 20 & 35.06235 & 36.43839 \\
\hline \multirow[t]{4}{*}{ HEF3 } & YNL014W & 14 & 8 & 21 & 26.74271 & 44.69616 \\
\hline & YMR194C- & & & & & \\
\hline & $A$ & 14 & 8 & 22 & 39.37916 & 33.21293 \\
\hline & YNL011C & 14 & 8 & 23 & 27.45827 & 62.17892 \\
\hline HIS3 & YOR202W & 14 & 8 & 24 & 26.38023 & \#DIV/0! \\
\hline \multirow[t]{2}{*}{ HIS3 } & YOR202W & 14 & 9 & 1 & 23.2681 & \#DIV/0! \\
\hline & YPR045C & 14 & 9 & 2 & 28.2889 & 46.44829 \\
\hline BSP1 & YPR171W & 14 & 9 & 3 & 17.79319 & 46.89837 \\
\hline
\end{tabular}




\begin{tabular}{|c|c|c|c|c|c|c|}
\hline & YPR044C & 14 & 9 & 4 & 48.01421 & 52.11733 \\
\hline & YPR170C & 14 & 9 & 5 & 22.26347 & 52.23784 \\
\hline RPL43A & YPR043W & 14 & 9 & 6 & \#DIV/0! & 14.33291 \\
\hline MET16 & YPR167C & 14 & 9 & 7 & 32.30415 & 58.97182 \\
\hline PUF2 & YPR042C & 14 & 9 & 8 & 27.76225 & 50.51724 \\
\hline MMS1 & YPR164W & 14 & 9 & 9 & 40.00805 & 54.23815 \\
\hline TIP41 & YPR040W & 14 & 9 & 10 & 51.39483 & 48.54258 \\
\hline \multirow[t]{3}{*}{ GPH1 } & YPR160W & 14 & 9 & 11 & 79.20228 & 47.30421 \\
\hline & YPR038W & 14 & 9 & 12 & 38.38622 & 57.12455 \\
\hline & YPR158W & 14 & 9 & 13 & -24.3016 & 55.64792 \\
\hline \multirow[t]{2}{*}{ ERV2 } & YPR037C & 14 & 9 & 14 & 49.33835 & 62.30311 \\
\hline & YPR157W & 14 & 9 & 15 & 27.29459 & 58.31235 \\
\hline SRO7 & YPR032W & 14 & 9 & 16 & 35.30963 & 47.10817 \\
\hline TPO3 & YPR156C & 14 & 9 & 17 & 26.39376 & 34.67613 \\
\hline NTO1 & YPR031W & 14 & 9 & 18 & 22.95624 & 46.29037 \\
\hline NCA2 & YPR155C & 14 & 9 & 19 & 26.55688 & 55.92887 \\
\hline CSR2 & YPR030W & 14 & 9 & 20 & 49.17672 & 51.77203 \\
\hline PIN3 & YPR154W & 14 & 9 & 21 & 70.31432 & 80.93666 \\
\hline \multirow[t]{2}{*}{ APL4 } & YPR029C & 14 & 9 & 22 & 24.56038 & 69.33031 \\
\hline & YPR153W & 14 & 9 & 23 & 37.22873 & 39.95045 \\
\hline HIS3 & YOR202W & 14 & 9 & 24 & -0.63452 & \#DIV/O! \\
\hline HIS3 & YOR202W & 14 & 10 & 1 & -15.8962 & \#DIV/0! \\
\hline cox14 & YML129C & 14 & 10 & 2 & -13.8966 & 43.19189 \\
\hline SMP2 & YMR165C & 14 & 10 & 3 & -7.60684 & 35.59829 \\
\hline CTK3 & YML112W & 14 & 10 & 4 & -23.5928 & 25.52721 \\
\hline ASI1 & YMR119W & 14 & 10 & 5 & 17.47908 & 52.60641 \\
\hline \multirow[t]{2}{*}{ BUL2 } & YML111W & 14 & 10 & 6 & 46.98543 & 57.90986 \\
\hline & YMR118C & 14 & 10 & 7 & 27.848 & 38.84515 \\
\hline FAR10 & YLR238W & 14 & 10 & 8 & 48.02423 & 49.28893 \\
\hline CAT5 & YOR125C & 14 & 10 & 9 & -5.90278 & -1.54412 \\
\hline \multirow[t]{2}{*}{ RXT2 } & YBR095C & 14 & 10 & 10 & 31.34378 & 47.64674 \\
\hline & YOL153C & 14 & 10 & 11 & 43.2906 & 49.23729 \\
\hline \multirow[t]{2}{*}{ IST2 } & YBR086C & 14 & 10 & 12 & 39.62199 & 48.90412 \\
\hline & YOL073C & 14 & 10 & 13 & 42.43317 & 36.88024 \\
\hline AAC3 & YBR085W & 14 & 10 & 14 & 47.70183 & 54.48066 \\
\hline AGA1 & YNR044W & 14 & 10 & 15 & 48.60125 & 50.60496 \\
\hline UBC4 & YBR082C & 14 & 10 & 16 & 34.78079 & 40.64386 \\
\hline \multirow[t]{2}{*}{ ABZ1 } & YNR033W & 14 & 10 & 17 & 39.04537 & 47.04075 \\
\hline & YBR075W & 14 & 10 & 18 & 25.28513 & 57.15515 \\
\hline MRPL10 & YNL284C & 14 & 10 & 19 & 16.66629 & 43.61827 \\
\hline TRM7 & YBR061C & 14 & 10 & 20 & 38.28754 & 50.82697 \\
\hline \multirow[t]{2}{*}{ LSM7 } & YNL147W & 14 & 10 & 21 & 35.47679 & 17.67361 \\
\hline & YBR020W & 14 & 10 & 22 & 6.597607 & 51.10853 \\
\hline
\end{tabular}




\begin{tabular}{|c|c|c|c|c|c|c|}
\hline CYB5 & YNL111C & 14 & 10 & 23 & 16.92972 & 44.88087 \\
\hline HIS3 & YOR202W & 14 & 10 & 24 & 32.57082 & \#DIV/0! \\
\hline HIS3 & YOR202W & 14 & 11 & 1 & 30.94469 & \#DIV/0! \\
\hline JID1 & YPR061C & 14 & 11 & 2 & 33.40403 & 20.20732 \\
\hline HPA2 & YPR193C & 14 & 11 & 3 & 7.674817 & 54.28111 \\
\hline ARO7 & YPR060C & 14 & 11 & 4 & 19.3729 & 50.20162 \\
\hline \multirow[t]{2}{*}{ AQY1 } & YPR192W & 14 & 11 & 5 & 4.533001 & 41.65709 \\
\hline & YPR059C & 14 & 11 & 6 & 36.62577 & 49.96794 \\
\hline QCR2 & YPR191W & 14 & 11 & 7 & 33.87627 & 10.82911 \\
\hline YMC1 & YPR058W & 14 & 11 & 8 & 27.01964 & 41.81911 \\
\hline SKI3 & YPR189W & 14 & 11 & 9 & 18.39825 & 61.20176 \\
\hline BRR1 & YPR057W & 14 & 11 & 10 & 8.354038 & 40.56433 \\
\hline MLC2 & YPR188C & 14 & 11 & 11 & 27.14054 & 67.036 \\
\hline SMK1 & YPR054W & 14 & 11 & 12 & 39.37352 & 46.21621 \\
\hline \multirow[t]{2}{*}{ ATG13 } & YPR185W & 14 & 11 & 13 & 41.93104 & 43.41288 \\
\hline & YPR053C & 14 & 11 & 14 & 41.12037 & 25.07576 \\
\hline GDB1 & YPR184W & 14 & 11 & 15 & 49.8882 & 46.52589 \\
\hline NHP6A & YPR052C & 14 & 11 & 16 & -4.99718 & 56.1146 \\
\hline HDA3 & YPR179C & 14 & 11 & 17 & 30.0469 & 39.42996 \\
\hline \multirow[t]{3}{*}{ MAK3 } & YPR051W & 14 & 11 & 18 & 24.14708 & 26.83727 \\
\hline & YPR174C & 14 & 11 & 19 & 18.39542 & 54.81973 \\
\hline & YPR050C & 14 & 11 & 20 & 39.01818 & 54.38486 \\
\hline VPS4 & YPR173C & 14 & 11 & 21 & 59.98335 & 6.775261 \\
\hline \multirow[t]{2}{*}{ MCM16 } & YPR046W & 14 & 11 & 22 & 30.01575 & 26.84883 \\
\hline & YPR172W & 14 & 11 & 23 & 37.42431 & 66.7794 \\
\hline HIS3 & YOR202W & 14 & 11 & 24 & 13.91879 & \#DIV/0! \\
\hline HIS3 & YOR202W & 14 & 12 & 1 & 36.4248 & \#DIV/0! \\
\hline \multirow[t]{2}{*}{ RTG3 } & YBL103C & 14 & 12 & 2 & 27.67589 & 60.20508 \\
\hline & YNL109W & 14 & 12 & 3 & 27.11051 & 45.10841 \\
\hline LTE1 & YAL024C & 14 & 12 & 4 & 12.19901 & 55.67812 \\
\hline \multirow[t]{5}{*}{ RPS7B } & YNL096C & 14 & 12 & 5 & 8.925583 & 50.97693 \\
\hline & YDR271C & 14 & 12 & 6 & -34.5787 & 43.3314 \\
\hline & YOR379C & 14 & 12 & 7 & 37.33399 & 58.22556 \\
\hline & YDR269C & 14 & 12 & 8 & 28.41993 & 54.3213 \\
\hline & YOR366W & 14 & 12 & 9 & 22.62218 & 50.07873 \\
\hline \multirow[t]{2}{*}{ AMD2 } & YDR242W & 14 & 12 & 10 & 29.00369 & 54.19055 \\
\hline & YOR345C & 14 & 12 & 11 & 3.984635 & 48.79554 \\
\hline \multirow[t]{2}{*}{ BRE1 } & YDL074C & 14 & 12 & 12 & 50.85971 & 0.766912 \\
\hline & YOR333C & 14 & 12 & 13 & 44.10885 & 55.63587 \\
\hline \multirow[t]{2}{*}{ AAD3 } & YCR107W & 14 & 12 & 14 & 30.31278 & 57.85478 \\
\hline & YOR325W & 14 & 12 & 15 & 48.43762 & 57.08831 \\
\hline \multirow[t]{2}{*}{ MAL32 } & YBR299W & 14 & 12 & 16 & \#DIV/0! & 27.82051 \\
\hline & YOR309C & 14 & 12 & 17 & 56.91358 & 47.7607 \\
\hline
\end{tabular}




\begin{tabular}{|c|c|c|c|c|c|c|}
\hline UBS1 & YBR165W & 14 & 12 & 18 & 33.33491 & 53.89066 \\
\hline MCH5 & YOR306C & 14 & 12 & 19 & 28.97901 & 46.2361 \\
\hline \multirow[t]{2}{*}{ TEF2 } & YBR118W & 14 & 12 & 20 & \#DIV/0! & 50.04773 \\
\hline & YOR300W & 14 & 12 & 21 & 5.145261 & 42.16263 \\
\hline LYS2 & YBR115C & 14 & 12 & 22 & 36.47682 & 60.79117 \\
\hline ADE2 & YOR128C & 14 & 12 & 23 & \#DIV/0! & 63.79496 \\
\hline HIS3 & YOR202W & 14 & 12 & 24 & 23.01606 & \#DIV/O! \\
\hline HIS3 & YOR202W & 14 & 13 & 1 & 35.80267 & \#DIV/0! \\
\hline TKL1 & YPR074C & 14 & 13 & 2 & 1.884189 & 69.57948 \\
\hline IML2 & YJL082W & 14 & 13 & 3 & 31.10358 & 54.35896 \\
\hline LTP1 & YPR073C & 14 & 13 & 4 & 16.78388 & 59.08782 \\
\hline \multirow[t]{2}{*}{ PRY1 } & YJL079C & 14 & 13 & 5 & 2.155161 & 52.16648 \\
\hline & YPR071W & 14 & 13 & 6 & 18.72289 & 50.40832 \\
\hline SOD2 & YHR008C & 14 & 13 & 7 & 28.84296 & 51.17503 \\
\hline MED1 & & 14 & 13 & 8 & 27.67522 & 36.08147 \\
\hline ARR3 & YPR201W & 14 & 13 & 9 & 20.09814 & 54.64771 \\
\hline SPE3 & YPR069C & 14 & 13 & 10 & 28.56871 & 54.84337 \\
\hline ARR2 & YPR200C & 14 & 13 & 11 & 4.661511 & 51.41336 \\
\hline HOS1 & YPR068C & 14 & 13 & 12 & 40.96512 & 68.64962 \\
\hline ARR1 & YPR199C & 14 & 13 & 13 & 40.27588 & 51.66543 \\
\hline UBA3 & YPR066W & 14 & 13 & 14 & 55.89217 & 27.30935 \\
\hline SGE1 & YPR198W & 14 & 13 & 15 & 40.01937 & 66.01481 \\
\hline \multirow[t]{6}{*}{ ROX1 } & YPR065W & 14 & 13 & 16 & 57.11147 & 64.48811 \\
\hline & YPR197C & 14 & 13 & 17 & 48.49779 & 55.85687 \\
\hline & YPR064W & 14 & 13 & 18 & 18.41102 & 59.90669 \\
\hline & YPR196W & 14 & 13 & 19 & 38.86124 & 46.62309 \\
\hline & YPR063C & 14 & 13 & 20 & 36.35333 & 61.71418 \\
\hline & YPR195C & 14 & 13 & 21 & 55.91398 & 53.71382 \\
\hline FCY1 & YPR062W & 14 & 13 & 22 & 33.47104 & 64.31953 \\
\hline OPT2 & YPR194C & 14 & 13 & 23 & 58.14428 & 60.87059 \\
\hline HIS3 & YOR202W & 14 & 13 & 24 & 4.328984 & \#DIV/0! \\
\hline HIS3 & YOR202W & 14 & 14 & 1 & 37.53197 & \#DIV/0! \\
\hline PTC2 & YER089C & 14 & 14 & 2 & -4.29774 & 19.43301 \\
\hline SUE1 & YPR151C & 14 & 14 & 3 & \#DIV/0! & 30.88046 \\
\hline \multirow[t]{2}{*}{ SLF1 } & YDR515W & 14 & 14 & 4 & 12.62117 & 46.20061 \\
\hline & YPR118W & 14 & 14 & 5 & 44.79179 & 55.03647 \\
\hline \multirow[t]{2}{*}{ SAM2 } & YDR502C & 14 & 14 & 6 & 20.08167 & 51.45536 \\
\hline & YPR091C & 14 & 14 & 7 & 52.15675 & 29.58153 \\
\hline FMP36 & YDR493W & 14 & 14 & 8 & 43.97126 & 51.14857 \\
\hline MDM36 & YPR083W & 14 & 14 & 9 & 17.6392 & 55.42928 \\
\hline KRE2 & YDR483W & 14 & 14 & 10 & 20.79443 & 51.33903 \\
\hline AGC1 & YPR021C & 14 & 14 & 11 & 13.60032 & 50.15837 \\
\hline SNF1 & YDR477W & 14 & 14 & 12 & 27.84063 & 19.963 \\
\hline
\end{tabular}




\begin{tabular}{|c|c|c|c|c|c|c|}
\hline & YPR011C & 14 & 14 & 13 & 51.59299 & 51.39028 \\
\hline & YDR444W & 14 & 14 & 14 & 59.23022 & 48.529 \\
\hline & YPL158C & 14 & 14 & 15 & 32.12267 & 51.79539 \\
\hline & YDR326C & 14 & 14 & 16 & 39.72869 & 49.23142 \\
\hline & YML050W & 14 & 14 & 17 & 34.86551 & 67.55763 \\
\hline & YDR290W & 14 & 14 & 18 & 57.38112 & 41.97144 \\
\hline \multirow[t]{2}{*}{ CDA2 } & YLR308W & 14 & 14 & 19 & 32.74689 & 52.82208 \\
\hline & YFL063W & 14 & 14 & 20 & 16.02492 & 14.47832 \\
\hline $\mathrm{ADH} 1$ & YOL086C & 14 & 14 & 21 & 61.6466 & 49.5082 \\
\hline RIM15 & YFL033C & 14 & 14 & 22 & \#DIV/0! & 37.55266 \\
\hline MFA1 & YDR461W & 14 & 14 & 23 & 63.92335 & 68.09504 \\
\hline HIS3 & YOR202W & 14 & 14 & 24 & 21.76186 & \\
\hline HIS3 & YOR202W & 14 & 15 & 1 & 37.19737 & \\
\hline SYT1 & YPR095C & 14 & 15 & 2 & 46.98365 & \\
\hline HIS3 & YOR202W & 14 & 15 & 3 & 35.81453 & \\
\hline ASR1 & YPR093C & 14 & 15 & 4 & 29.72524 & \\
\hline \multirow[t]{2}{*}{ HIS3 } & YOR202W & 14 & 15 & 5 & 63.22978 & \\
\hline & YPR092W & 14 & 15 & 6 & 33.71853 & \\
\hline \multirow[t]{2}{*}{ HIS3 } & YOR202W & 14 & 15 & 7 & 25.79166 & \\
\hline & YPR090W & 14 & 15 & 8 & 47.92528 & \\
\hline \multirow[t]{2}{*}{ COQ2 } & YNR041C & 14 & 15 & 9 & \#DIV/0! & \\
\hline & YPR089W & 14 & 15 & 10 & 30.19352 & \\
\hline \multirow[t]{3}{*}{ PET191 } & YJR034W & 14 & 15 & 11 & 19.60908 & \\
\hline & YPR084W & 14 & 15 & 12 & 30.11371 & \\
\hline & YJL120W & 14 & 15 & 13 & 90.25615 & \\
\hline \multirow[t]{3}{*}{ MRL1 } & YPR079W & 14 & 15 & 14 & 28.26513 & \\
\hline & YJL119C & 14 & 15 & 15 & 43.15724 & \\
\hline & YPR078C & 14 & 15 & 16 & 71.74778 & \\
\hline \multirow[t]{4}{*}{ PHO86 } & YJL117W & 14 & 15 & 17 & 46.51102 & \\
\hline & YPR077C & 14 & 15 & 18 & 36.80181 & \\
\hline & YJL107C & 14 & 15 & 19 & 37.52155 & \\
\hline & YPR076W & 14 & 15 & 20 & 32.22154 & \\
\hline IME2 & YJL106W & 14 & 15 & 21 & 57.22116 & \\
\hline OPY2 & YPR075C & 14 & 15 & 22 & 63.6919 & \\
\hline HPR5 & YJL092W & 14 & 15 & 23 & -3.30731 & \\
\hline HIS3 & YOR202W & 14 & 15 & 24 & 27.65349 & \\
\hline HIS3 & YOR202W & 14 & 16 & 1 & 39.78851 & \\
\hline HIS3 & YOR202W & 14 & 16 & 2 & 27.25099 & \\
\hline HIS3 & YOR202W & 14 & 16 & 3 & 13.90402 & \\
\hline HIS3 & YOR202W & 14 & 16 & 4 & -0.73595 & \\
\hline HIS3 & YOR202W & 14 & 16 & 5 & -8.25802 & \\
\hline HIS3 & YOR202W & 14 & 16 & 6 & 13.57024 & \\
\hline HIS3 & YOR202W & 14 & 16 & 7 & 34.08325 & \\
\hline
\end{tabular}




$\begin{array}{lcrrrc}\text { HIS3 } & \text { YOR202W } & 14 & 16 & 8 & 39.91194 \\ \text { HIS3 } & \text { YOR202W } & 14 & 16 & 9 & 41.11782 \\ \text { HIS3 } & \text { YOR202W } & 14 & 16 & 10 & 52.64714 \\ \text { HIS3 } & \text { YOR202W } & 14 & 16 & 11 & 37.24949 \\ \text { HIS3 } & \text { YOR202W } & 14 & 16 & 12 & 66.00481 \\ \text { HIS3 } & \text { YOR202W } & 14 & 16 & 13 & 56.14281 \\ \text { HIS3 } & \text { YOR202W } & 14 & 16 & 14 & \# D I V / 0 ! \\ \text { HIS3 } & \text { YOR202W } & 14 & 16 & 15 & 45.71553 \\ \text { HIS3 } & \text { YOR202W } & 14 & 16 & 16 & \# D I V / 0 ! \\ \text { HIS3 } & \text { YOR202W } & 14 & 16 & 17 & \# D I V / 0 ! \\ \text { HIS3 } & \text { YOR202W } & 14 & 16 & 18 & \# D I V / 0 ! \\ \text { HIS3 } & \text { YOR202W } & 14 & 16 & 19 & \# D I V / 0 ! \\ \text { HIS3 } & \text { YOR202W } & 14 & 16 & 20 & 36.09822 \\ \text { HIS3 } & \text { YOR202W } & 14 & 16 & 21 & 8.739212 \\ \text { HIS3 } & \text { YOR202W } & 14 & 16 & 22 & 22.82663 \\ \text { HIS3 } & \text { YOR202W } & 14 & 16 & 23 & 17.22006 \\ \text { HIS3 } & \text { YOR202W } & 14 & 16 & 24 & 35.54909\end{array}$


Appendix 2

HIS3

HIS3

HIS3

HIS3

HIS3

HIS3

HIS3

HIS3

HIS3

HIS3

HIS3

HIS3

HIS3

HIS3

HIS3

HIS3

HIS3

HIS3

HIS3

HIS3

HIS3

HIS3

HIS3

HIS3

HIS3

HDR1

ETR1

AGP2

CCZ1

$\mathrm{SCO} 2$

SHE3

CHS3

OPY1

POA1

ATG14

plate

row

column

GHYDA $\times 3$ times

Fatimax 2 times

YOR202W

YOR202W

YOR202W

YOR202W

YOR202W

YOR202W

YOR202W

YOR202W

YOR202W

YOR202W

YOR202W

YOR202W

YOR202W

YOR202W

YOR202W

YOR202W

YOR202W

YOR202W

YOR202W

YOR202W

YOR202W

YOR202W

YOR202W

YOR202W

YOR202W

YBR030W

YBR138C

YBR028C

YBR137W

YBR027C

YBR134W

YBR026C

YBR132C

YBR025C

YBR131W

YBR024W

YBR130C

YBR023C

YBR129C

YBR022W

YBR128C
1

\#2

\#1

0

$-42.9812$

0.28885

$\begin{array}{lllll}2 & 50.35247 & 21.83099 & -28.1646\end{array}$

$\begin{array}{lllll}3 & 64.36782 & -31.411 & -7.16487\end{array}$

$\begin{array}{llll}4 & 32.75017 & -5.69358 & -2.84615\end{array}$

$\begin{array}{lllll}5 & 28.31784 & 24.38356 & -12.1008\end{array}$

$\begin{array}{lllll}6 & 29.48473 & -27.1516 & -28.9193\end{array}$

$\begin{array}{llll}7 & 28.35145 & -38.255 & -38.7413\end{array}$

$\begin{array}{llll}8 & -7.1618 & 19.61326 & -10.9049\end{array}$

$\begin{array}{lllll}9 & -10.1833 & 30.44164 & -148.235\end{array}$

$\begin{array}{lllll}10 & 32.98805 & 17.60228 & -1.15546\end{array}$

$\begin{array}{lllll}11 & 40.57725 & -6.74646 & -17.1206\end{array}$

$\begin{array}{lllll}12 & 40.59322 & -3.01075 & -8.5595\end{array}$

$\begin{array}{lllll}13 & 32.66376 & 12.35839 & -2.74295\end{array}$

$\begin{array}{lllll}14 & 43.05924 & 8.006536 & -17.0787\end{array}$

$\begin{array}{lllll}15 & 34.43548 & 23.79936 & -28.0245\end{array}$

$\begin{array}{llll}16 & -6.54912 & -3.16456 & -9.54907\end{array}$

$\begin{array}{lllll}17 & 49.53001 & -16.9913 & -10.1218\end{array}$

$\begin{array}{lllll}18 & 42.89963 & -3.56394 & -13.0156\end{array}$

$\begin{array}{lllll}19 & 30.97913 & 23.86207 & -24.0741\end{array}$

$\begin{array}{lllll}20 & 29.02939 & 12.45937 & -47.4471\end{array}$

$\begin{array}{lllll}21 & -6.54545 & 26.20791 & -50.8671\end{array}$

$\begin{array}{lllll}22 & 29.27461 & -12.1116 & -8.23643\end{array}$

$\begin{array}{lllll}23 & 36.96275 & -33.5714 & 49.83333\end{array}$

$\begin{array}{lllll}24 & 37.93353 & 5.042436 & -15.4618\end{array}$

$\begin{array}{lllll}1 & 9.566517 & 4.477612 & -25.5734\end{array}$

$\begin{array}{lllll}2 & 64.26914 & 51.34021 & 13.51351\end{array}$

$\begin{array}{lllll}3 & 54.7817 & 15.98174 & 22.83105\end{array}$

$\begin{array}{llll}4 & 23.63014 & 1.980198 & -0.81301\end{array}$

$\begin{array}{lllll}5 & 15.68895 & 23.62924 & 19.78528\end{array}$

$\begin{array}{lllll}6 & 36.69634 & 25.28147 & 65.14215\end{array}$

$\begin{array}{lllll}7 & 57.96371 & 44.53782 & 47.02381\end{array}$

$\begin{array}{lllll}8 & 34.43354 & 31.67982 & 78.91986\end{array}$

$\begin{array}{lllll}9 & 26.77858 & -8.50394 & 3.673469\end{array}$

$10 \quad 12.35955$

100

$11 \quad 21.0806$

$2.418208 \quad 13.02083$

$\begin{array}{ll}12 & 25.14507\end{array}$

$\begin{array}{ll}38.45127 \quad 67.72908 \\ 11.57742 & -11.8721\end{array}$

$\begin{array}{lllll}13 & 28.33545 & 11.57742 & -11.8721\end{array}$

$14 \quad 52.80326$

$74.34312 \quad 63.41991$

$\begin{array}{ll}15 & 26.92308 \\ 16 & 37.59162\end{array}$

0.896057

19.55782

$\begin{array}{llll}16 & 37.59162 & 14.94253 & 23.71681\end{array}$

$\begin{array}{llll}17 & 50.25316 & 1.260504 & -10.2778\end{array}$
63.26531

19.6

44.60695

47.64595

32.17726

31.28713

50.23847

$-0.21459$

55.07246

42.88538

52.589

41.59593

74.49568

33.07544

49.67532

53.149
36.36364

23.97959

27.32095

37.63158

35.48387

61.62791

39.67611

$-19.4139$

42.81984

35.23035

29.4964

22.42744

67.81327

36.9863

34.77218

36.11111 


\begin{tabular}{|c|c|c|c|c|c|c|c|c|c|c|}
\hline GAL1 & & YBR020W & 1 & 2 & 18 & 55.52194 & 45.60669 & 65.72238 & $\begin{array}{l}66.95122 \\
\text { missing }\end{array}$ & 60.12397 \\
\hline TPS1 & & YBR126C & 1 & 2 & 19 & 22.01258 & 3.165468 & -0.9396 & colony & missing colony \\
\hline GAL10 & & YBR019C & 1 & 2 & 20 & -7.80347 & 21.49362 & 46.98413 & 57.75281 & 44.76386 \\
\hline PTC4 & & YBR125C & 1 & 2 & 21 & 37.67535 & 20.57292 & 31.1412 & 68.79536 & 38.86256 \\
\hline GAL7 & & YBR018C & 1 & 2 & 22 & 17.53555 & 37.98077 & -2.67857 & 18.34532 & 25.17321 \\
\hline MRPL36 & & YBR122C & 1 & 2 & 23 & 10.31308 & 7.663782 & 53.57143 & 17.01783 & 27.60181 \\
\hline HIS3 & & YOR202W & 1 & 2 & 24 & 36.72966 & 4.829545 & -2.20653 & & \\
\hline HIS3 & & YOR202W & 1 & 3 & 1 & 59.43285 & -25 & -26.7925 & & \\
\hline PSK1 & & YAL017W & 1 & 3 & 2 & 58.32549 & 38.94325 & 24.25595 & 39.56386 & 42.18415 \\
\hline HEK2 & & YBL032W & 1 & 3 & 3 & 41.56545 & 56.08035 & \#DIV/0! & 46.79803 & 30.69544 \\
\hline NTG1 & & YAL015C & 1 & 3 & 4 & 26.24878 & -21.1509 & 28.6 & 46.29948 & 26.59574 \\
\hline SHE1 & & YBL031W & 1 & 3 & 5 & 10.55901 & -3.07918 & 56.08309 & 11.18143 & 40.40921 \\
\hline \multirow[t]{2}{*}{ SYN8 } & & YAL014C & 1 & 3 & 6 & 25.6182 & 24.19006 & 26.65862 & 53.47432 & 42.10526 \\
\hline & & YBL029W & 1 & 3 & 7 & 17.48879 & 29.5 & 8.814815 & 33.39161 & 26.99229 \\
\hline \multirow[t]{2}{*}{ DEP1 } & & YAL013W & 1 & 3 & 8 & 28.867 & 21.99662 & -6.1157 & 46.69261 & 14.14791 \\
\hline & 0 & YBL028C & 1 & 3 & 9 & 26.08696 & 60.73298 & 28.07018 & 47.28171 & 31.86813 \\
\hline SWC3 & & YAL011W & 1 & 3 & 10 & 36.5353 & -10.6545 & 19.53563 & 58.29528 & -4.05063 \\
\hline RPL19B & & YBL027W & 1 & 3 & 11 & -3.89294 & 23.41463 & 25.43554 & 62.80702 & 43.24324 \\
\hline MDM10 & & YAL010C & 1 & 3 & 12 & 34.01786 & 5.426357 & 13.78621 & 56.88351 & 34.77157 \\
\hline RRN10 & & YBL025W & 1 & 3 & 13 & 30.28624 & 14.14286 & -20.1242 & 30.45872 & 30.56235 \\
\hline FUN14 & & YAL008W & 1 & 3 & 14 & 34.19958 & 15.5096 & -26.4637 & 3.361345 & 23.9899 \\
\hline NCL1 & & YBL024W & 1 & 3 & 15 & 30.5102 & 13.9738 & -21.4008 & 64.09861 & 34.94898 \\
\hline ERP2 & & YAL007C & 1 & 3 & 16 & 43.21839 & 29.26491 & -7.75681 & 27.31092 & 47.8836 \\
\hline HAP3 & & YBL021C & 1 & 3 & 17 & 30.38982 & 4.447268 & 7.751196 & 34.91461 & 6.764706 \\
\hline SSA1 & & YAL005C & 1 & 3 & 18 & 37.86008 & 95.82043 & 66.31579 & 40.34091 & 56.30252 \\
\hline \multirow[t]{2}{*}{ APN2 } & & YBL019W & 1 & 3 & 19 & 45.38922 & 19.55882 & -8.16641 & 24.08293 & 24.04092 \\
\hline & & YAL004W & 1 & 3 & 20 & 46.42857 & -25.7143 & 2.419355 & -34.3008 & 30.47337 \\
\hline PEP1 & & YBL017C & 1 & 3 & 21 & 43.72367 & 1.775148 & 19.66637 & -10.8642 & 36.49425 \\
\hline VPS8 & & YAL002W & 1 & 3 & 22 & 8.660131 & -1.77353 & 65.90909 & 33.85049 & 10.14493 \\
\hline FUS3 & & YBL016W & 1 & 3 & 23 & 14.19753 & 6.985294 & 34.57944 & -1.52905 & 37.0614 \\
\hline HIS3 & & YOR202W & 1 & 3 & 24 & 33.7386 & -2.29226 & 9.201213 & & \\
\hline HIS3 & & YOR202W & 1 & 4 & 1 & 66.83969 & 0.969163 & 53.54239 & & \\
\hline GIP1 & & YBR045C & 1 & 4 & 2 & 13.89925 & 6.559264 & 16.77187 & 34.22371 & 35.73265 \\
\hline SLI15 & & YBR156C & 1 & 4 & 3 & 28.77698 & 11.32404 & 29.30403 & 47.50403 & 38.81402 \\
\hline TCM62 & & YBR044C & 1 & 4 & 4 & 5.910165 & 90.87523 & 18.53933 & 39.70944 & 41.81818 \\
\hline APD1 & & YBR151W & 1 & 4 & 5 & 27.99642 & 22.26667 & 12.75456 & 48.75 & 38.52691 \\
\hline QDR3 & & YBR043C & 1 & 4 & 6 & 40.03466 & 43.27177 & 29.29134 & 48.25784 & 38.96458 \\
\hline \multirow[t]{2}{*}{ TBS1 } & & YBR150C & 1 & 4 & 7 & 21.15732 & 8.710801 & 2.931596 & 29.47559 & 40.10695 \\
\hline & & YBR042C & 1 & 4 & 8 & 44.04762 & 38.32061 & 74.04844 & 26.56827 & 35.87571 \\
\hline ARA1 & & YBR149W & 1 & 4 & 9 & 27.35043 & 100 & \#DIV/0! & 12.73063 & 81.12798 \\
\hline FAT1 & & YBR041W & 1 & 4 & 10 & 23.05581 & 8.025682 & 7.883817 & 30.22222 & 25.69444 \\
\hline YSW1 & & YBR148W & 1 & 4 & 11 & 50.68664 & -4.06404 & 52.66272 & 41.28167 & 29.23497 \\
\hline
\end{tabular}




\begin{tabular}{|c|c|c|c|c|c|c|c|c|c|}
\hline \multirow[t]{2}{*}{ FIG1 } & YBR040W & 1 & 4 & 12 & 38.79159 & 53.20057 & -23.1419 & 3.232759 & 28 \\
\hline & YBR147W & 1 & 4 & 13 & 31.31407 & 12.66667 & 3.076923 & 34.80663 & 17.02786 \\
\hline CSG2 & YBR036C & 1 & 4 & 14 & 58.36431 & 68.34016 & 65.82278 & 64.89362 & 77.37705 \\
\hline MRPS9 & YBR146W & 1 & 4 & 15 & 18.85776 & 28.44037 & 28.3293 & 36.48425 & 11.89189 \\
\hline HMT1 & YBR034C & 1 & 4 & 16 & 21.53316 & 19.07152 & 16.81901 & 49.1018 & 35.06849 \\
\hline ADH5 & YBR145W & 1 & 4 & 17 & 24.8366 & 26.71429 & 10.95596 & 40.45872 & 54.94297 \\
\hline \multirow[t]{4}{*}{ EDS1 } & YBR033W & 1 & 4 & 18 & 68.19013 & 32.73026 & 59.74026 & 62.21889 & 26.0101 \\
\hline & YBR144C & 1 & 4 & 19 & 48.78049 & 24.5283 & 60 & 47.16007 & 49.88506 \\
\hline & YBR032W & 1 & 4 & 20 & 34.07672 & 17.98365 & 5.651672 & 44.58204 & 24.32432 \\
\hline & YBR141C & 1 & 4 & 21 & 43.92523 & 27.52881 & 15.85233 & 40.70513 & 29.21053 \\
\hline \multirow[t]{2}{*}{ RPL4A } & YBR031W & 1 & 4 & 22 & 39.8892 & 47.93233 & -14.6707 & -1.17647 & 4.643963 \\
\hline & YBR139W & 1 & 4 & 23 & -16.0194 & 7.52551 & 3.312629 & 51.73745 & 42.51101 \\
\hline HIS3 & YOR202W & 1 & 4 & 24 & 30.1826 & -4.37117 & -11.0915 & & \\
\hline HIS3 & YOR202W & 1 & 5 & 1 & 45.15195 & -2.56223 & -126.95 & & \\
\hline FUN21 & YAL031C & 1 & 5 & 2 & 22.07547 & -22.4234 & 43.35512 & 63.54319 & 46.875 \\
\hline SAS3 & YBL052C & 1 & 5 & 3 & 50.83426 & -10.6349 & 13.85135 & 62.22597 & 38.95028 \\
\hline SNC1 & YAL030W & 1 & 5 & 4 & 29.89691 & -0.96618 & 29.38671 & 27.77778 & 37.7907 \\
\hline PIN4 & YBL051C & 1 & 5 & 5 & 59.71944 & 59.00305 & 62.7566 & 75.04303 & 61.34969 \\
\hline MYO4 & YAL029C & 1 & 5 & 6 & 39.73384 & 17.58794 & 13.40726 & 45.66372 & 43.94366 \\
\hline $\mathrm{MOH} 1$ & YBL049W & 1 & 5 & 7 & 38.41962 & 61.97836 & 55.12367 & 52.75459 & 62.30366 \\
\hline \multirow[t]{3}{*}{ FRT2 } & YAL028W & 1 & 5 & 8 & 33.63095 & 29.57958 & 15.61181 & 46.92308 & 46.0452 \\
\hline & YBL048W & 1 & 5 & 9 & 25.19347 & -13.0368 & 18.44741 & 47.28546 & 33.241 \\
\hline & YAL027W & 1 & 5 & 10 & 18.94439 & 24.97149 & 13.36493 & 57.31523 & 47.22955 \\
\hline EDE1 & YBL047C & 1 & 5 & 11 & 15.60748 & 10.15038 & 8.446298 & 2.843602 & 38.94472 \\
\hline \multirow[t]{2}{*}{ DRS2 } & YAL026C & 1 & 5 & 12 & 17.22054 & 6.312769 & -14.1002 & 43.27087 & -40.0593 \\
\hline & YBL046W & 1 & 5 & 13 & 20.65574 & 19.41176 & 9.74026 & 39.37008 & 23.60743 \\
\hline РMT2 & YAL023C & 1 & 5 & 14 & 26.90058 & 6.758833 & 84.50704 & 33.33333 & 30.69307 \\
\hline ECM13 & YBL043W & 1 & 5 & 15 & 31.87441 & 17.78976 & 3.078983 & -1.47368 & 40.45802 \\
\hline FUN26 & YAL022C & 1 & 5 & 16 & -38.4211 & 11.3307 & 8.380952 & 29.71619 & 40.66496 \\
\hline FUI1 & YBL042C & 1 & 5 & 17 & -22.9947 & 19.279 & 12.78375 & 38.61004 & 13.58885 \\
\hline ATS1 & YAL020C & 1 & 5 & 18 & 23.24723 & 20.02725 & 16.07306 & 58.13559 & 39.60674 \\
\hline URA7 & YBL039C & 1 & 5 & 19 & 26.33552 & 12.53561 & 28.32765 & 15.78947 & 28.44037 \\
\hline FUN30 & YAL019W & 1 & 5 & 20 & 30.33135 & 13.13131 & -1.5544 & 27.98054 & 20.48611 \\
\hline \multirow[t]{3}{*}{ APL3 } & YBL037W & 1 & 5 & 21 & 35.44776 & 55.92388 & 49.36709 & 55.38462 & 21.20419 \\
\hline & YAL018C & 1 & 5 & 22 & -27.6316 & 11.75573 & -1.25 & 52.88326 & 25 \\
\hline & YBL036C & 1 & 5 & 23 & 32.71318 & 9.27673 & 0.462428 & 50.46729 & 18.79518 \\
\hline HIS3 & YOR202W & 1 & 5 & 24 & 26.32479 & -8.22622 & 1.134021 & & \\
\hline HIS3 & YOR202W & 1 & 6 & 1 & 31.07235 & -11.3614 & 27.04987 & & \\
\hline UBP14 & YBR058C & 1 & 6 & 2 & 2.505695 & -16.6856 & 50.76297 & 44.40735 & 38.40749 \\
\hline SSE2 & YBR169C & 1 & 6 & 3 & 24.18699 & -31.1667 & 5.148206 & -26.555 & 27.7628 \\
\hline MUM2 & YBR057C & 1 & 6 & 4 & 18.09623 & -43.0493 & -5.3913 & 46.26087 & 16.90544 \\
\hline \multirow[t]{2}{*}{ PEX32 } & YBR168W & 1 & 6 & 5 & 11.69355 & 0 & 11.22449 & 51.98728 & 30.76923 \\
\hline & YBR056W & 1 & 6 & 6 & 32.85714 & -23.5197 & -61.1111 & 51.93966 & 38.16568 \\
\hline
\end{tabular}




\begin{tabular}{|c|c|c|c|c|c|c|c|c|c|}
\hline TYR1 & YBR166C & 1 & 6 & 7 & 9.89011 & -19.5122 & 21.52778 & 43.76947 & 36.95015 \\
\hline YRO2 & YBR054W & 1 & 6 & 8 & 28.22142 & 13.63636 & 38.62069 & 60.9589 & 33.33333 \\
\hline \multirow[t]{2}{*}{ UBS1 } & YBR165W & 1 & 6 & 9 & 21.35562 & -33.7625 & -5.93407 & 7.053942 & 21.77914 \\
\hline & YBR053C & 1 & 6 & 10 & 59.76139 & 16.32928 & 12.10898 & 62.12766 & 52.94118 \\
\hline \multirow[t]{2}{*}{ ARL1 } & YBR164C & 1 & 6 & 11 & 33.0131 & 12.56983 & 19.81982 & 25.4833 & 19.65318 \\
\hline & $\begin{array}{l}\text { YBR052C } \\
\text { YBR162W- }\end{array}$ & 1 & 6 & 12 & 36.50794 & 47.91386 & 28.63002 & 49.78541 & 22.44898 \\
\hline \multirow[t]{2}{*}{ YSY6 } & A & 1 & 6 & 13 & 24.52999 & -46.003 & 3.562341 & 21.74629 & 27.72021 \\
\hline & YBR051W & 1 & 6 & 14 & 23.16765 & 35.92552 & 16.9627 & 30.5835 & 20.82111 \\
\hline TOS1 & YBR162C & 1 & 6 & 15 & 18.06358 & 12.85956 & 82.67327 & 28.4264 & 35.14986 \\
\hline REG2 & YBR050C & 1 & 6 & 16 & -1.00376 & 29.17251 & 16.18357 & 51.34281 & 38.34197 \\
\hline CSH1 & YBR161W & 1 & 6 & 17 & 37.11968 & 1.981707 & -5.04673 & 50.37936 & 34.65347 \\
\hline \multirow[t]{2}{*}{ RPS11B } & YBR048W & 1 & 6 & 18 & -30.2108 & -2.69058 & 25.56391 & 43.78378 & 34.67049 \\
\hline & YBR159W & 1 & 6 & 19 & -17.6556 & 19.48925 & -2.93478 & 46.09495 & 27.86458 \\
\hline FMP23 & YBR047W & 1 & 6 & 20 & 16.27907 & 33.24324 & 24.60401 & 52.31481 & 31.1054 \\
\hline AMN1 & YBR158W & 1 & 6 & 21 & 39.13956 & 46.22642 & 10.16667 & 56.19946 & 39.48052 \\
\hline ZTA1 & YBR046C & 1 & 6 & 22 & 32.57677 & 65.52306 & 50.93633 & 39.68254 & 40.14085 \\
\hline ICS2 & YBR157C & 1 & 6 & 23 & 38.1295 & 23.35845 & -3.42298 & 57.95918 & 46.1039 \\
\hline HIS3 & YOR202W & 1 & 6 & 24 & -2.71941 & -17.9598 & 14.01869 & & \\
\hline HIS3 & YOR202W & 1 & 7 & 1 & 7.854251 & -37.9167 & 10.47542 & & \\
\hline ACS1 & YAL054C & 1 & 7 & 2 & 17.6525 & 35.97884 & -15.2174 & 49.20914 & 35.6974 \\
\hline \multirow[t]{2}{*}{ PRX1 } & YBL064C & 1 & 7 & 3 & 24.71452 & 10.94148 & 5.154639 & 32.69962 & 30.03003 \\
\hline & YAL053W & 1 & 7 & 4 & 44.57364 & 100 & 23.27586 & 55.7947 & 16.86391 \\
\hline KIP1 & YBL063W & 1 & 7 & 5 & 36.82771 & -21.8134 & 21.13971 & 16.09907 & 36.4486 \\
\hline \multirow[t]{3}{*}{ OAF1 } & YAL051W & 1 & 7 & 6 & 40.39548 & 31.79825 & 62.5 & 37.76978 & 28.35821 \\
\hline & YBL062W & 1 & 7 & 7 & 36.97674 & 40 & 51.22807 & 65.23888 & 49.71264 \\
\hline & YALO49C & 1 & 7 & 8 & 34.58236 & -4.51436 & 7.443683 & 47.08819 & 25.50725 \\
\hline \multirow[t]{5}{*}{ SKT5 } & YBL061C & 1 & 7 & 9 & 13.39387 & 45.77114 & 9.820486 & 38.63216 & 28.82353 \\
\hline & YAL045C & 1 & 7 & 10 & 14.92281 & 32.24299 & 48.18653 & 56.54854 & 33.14917 \\
\hline & YBL060W & 1 & 7 & 11 & 34.65909 & 11.94581 & 3.864734 & 57.02875 & 38.52459 \\
\hline & YAL043C-A & 1 & 7 & 12 & 35.64728 & 66.57825 & 36.36364 & 48.57571 & 18.54839 \\
\hline & YBL059W & 1 & 7 & 13 & 27.23005 & 17.50973 & -2.44898 & 25.39964 & 33.16327 \\
\hline ERV46 & YAL042W & 1 & 7 & 14 & 24.58159 & 28.98936 & -6.28834 & 54.84728 & 41.85464 \\
\hline PTH2 & YBL057C & 1 & 7 & 15 & 11.69772 & 17.88512 & 10.96311 & 36.89482 & 31.70103 \\
\hline CLN3 & YALO4OC & 1 & 7 & 16 & 35.72744 & 24.91389 & 23.26784 & 66.93227 & 36.84211 \\
\hline \multirow[t]{3}{*}{ PTC3 } & YBL056W & 1 & 7 & 17 & 8.294931 & 48.83721 & 40.31008 & 16.80261 & 11.02151 \\
\hline & YAL037W & 1 & 7 & 18 & 28.29132 & 21.26514 & 9.013398 & 62.94278 & 46.07046 \\
\hline & YBL055C & 1 & 7 & 19 & -4.02299 & 20.53973 & 11.38889 & 60.13865 & 45.25316 \\
\hline \multirow[t]{2}{*}{ RBG1 } & YAL036C & 1 & 7 & 20 & -9.46502 & 26.47482 & 30.53348 & 45.93023 & 45.88235 \\
\hline & YBL054W & 1 & 7 & 21 & 32.41973 & 28.60539 & 12.2429 & 13.88286 & 17.51412 \\
\hline \multirow[t]{2}{*}{ FUN19 } & YAL034C & 1 & 7 & 22 & 20.08368 & 24.02023 & -35.6968 & 55.02717 & 32.22506 \\
\hline & YBL053W & 1 & 7 & 23 & 7.972028 & 14.05564 & 47.16157 & 46.89826 & 28.10304 \\
\hline HIS3 & YOR202W & 1 & 7 & 24 & -30.8908 & 15.8701 & 2.224576 & & \\
\hline
\end{tabular}




\begin{tabular}{|c|c|c|c|c|c|c|c|c|c|}
\hline \multirow[t]{2}{*}{ HIS3 } & YOR202W & 1 & 8 & 1 & -13.6364 & -21.709 & 64.58877 & & \\
\hline & YBR071W & 1 & 8 & 2 & 11.11111 & 16.47635 & 14.25662 & 50.62305 & 44.72477 \\
\hline SMP1 & YBR182C & 1 & 8 & 3 & -1.7982 & 13.93939 & 20.82879 & 50.07564 & 39.16849 \\
\hline TAT1 & YBR069C & 1 & 8 & 4 & 18.07349 & -23.5632 & 10.0271 & 31.67331 & -0.90361 \\
\hline RPS6B & YBR181C & 1 & 8 & 5 & 28.61416 & -20.623 & 17.5836 & 23.60248 & 24.67532 \\
\hline BAP2 & YBR068C & 1 & 8 & 6 & 26.85714 & -2.86494 & -53.4091 & 46.96707 & 28.72928 \\
\hline DTR1 & YBR180W & 1 & 8 & 7 & 38.60511 & 12.53298 & -7.71812 & 57.32899 & 34.51087 \\
\hline \multirow[t]{2}{*}{ TIP1 } & YBR067C & 1 & 8 & 8 & 28.01932 & 58.16876 & 59.67742 & 46.74457 & 23.71968 \\
\hline & YBR178W & 1 & 8 & 9 & 27.62022 & -0.96774 & 27.10526 & 30.65539 & 43.54839 \\
\hline NRG2 & YBR066C & 1 & 8 & 10 & 17.28516 & 11.0424 & 22.60536 & 52.61438 & 39.15493 \\
\hline EHT1 & YBR177C & 1 & 8 & 11 & 29.55975 & 8.15822 & 8.14664 & 56.31825 & 45.02762 \\
\hline ECM2 & YBR065C & 1 & 8 & 12 & 34.19741 & 9.184994 & -15.3439 & 55.65217 & 31.94805 \\
\hline \multirow[t]{2}{*}{ ECM31 } & YBR176W & 1 & 8 & 13 & 17.92015 & 2.255639 & 18.45161 & 20.49689 & 44.87179 \\
\hline & YBR064W & 1 & 8 & 14 & 23.34884 & 14.2487 & 21.75 & 62.51656 & 35.07463 \\
\hline \multirow[t]{4}{*}{ SWD3 } & YBR175W & 1 & 8 & 15 & 20.88608 & 2.42915 & 21.62809 & -3.73832 & 16.73387 \\
\hline & YBR063C & 1 & 8 & 16 & 18.73479 & 34.4358 & 42.74194 & 36.1204 & 44.13965 \\
\hline & YBR174C & 1 & 8 & 17 & 16.64671 & 29.30556 & 17.56979 & 4.8 & -2.73973 \\
\hline & YBR062C & 1 & 8 & 18 & 40.90909 & 18.04511 & 14.83871 & 0 & 44.89247 \\
\hline SMY2 & YBR172C & 1 & 8 & 19 & 38.23916 & 15.65657 & 24.2497 & 57.3913 & 34.35115 \\
\hline TRM7 & YBR061C & 1 & 8 & 20 & 33.45622 & 20.54422 & 28.9358 & 54.33855 & 40.21739 \\
\hline SEC66 & YBR171W & 1 & 8 & 21 & 22.85068 & 47.51678 & -29.9639 & 24.84076 & 14.5 \\
\hline AKL1 & YBR059C & 1 & 8 & 22 & 35.15249 & -3.40219 & 14.72393 & 52.82392 & 21.6763 \\
\hline NPL4 & YBR170C & 1 & 8 & 23 & -1.64609 & 89.95633 & -96.875 & 34.57944 & 20.76923 \\
\hline HIS3 & YOR202W & 1 & 8 & 24 & -80.9612 & 7.613636 & 19.93534 & & \\
\hline \multirow[t]{2}{*}{ HIS3 } & YOR202W & 1 & 9 & 1 & 1.693405 & -61.4137 & -11.279 & & \\
\hline & YAL066W & 1 & 9 & 2 & 29.84389 & 38.87588 & -16.5457 & 36.74589 & 43.58974 \\
\hline \multirow[t]{2}{*}{ NUP170 } & YBL079W & 1 & 9 & 3 & 6.607495 & -13.6667 & 4.628633 & 54.08805 & 33.98058 \\
\hline & YAL065C & 1 & 9 & 4 & 20.23692 & -24.7706 & 16.01098 & 43.14815 & 19.9422 \\
\hline \multirow[t]{2}{*}{ ATG8 } & YBL078C & 1 & 9 & 5 & 61.40684 & 67.78976 & 55.91532 & 47.7327 & 60.91205 \\
\hline & YAL064C-A & 1 & 9 & 6 & 39.12232 & 41.36546 & -24.8714 & 41.7931 & 30.38961 \\
\hline SSA3 & YBL075C & 1 & 9 & 7 & 29.86301 & 100 & -30.9524 & 53.14534 & 37.01068 \\
\hline GDH3 & YAL062W & 1 & 9 & 8 & 28.57143 & 29.47067 & 5.742297 & 14.70588 & 32.24044 \\
\hline \multirow[t]{3}{*}{ RPS8A } & YBL072C & 1 & 9 & 9 & 41.02823 & 21.5625 & -44.0722 & 19.14498 & 8.01105 \\
\hline & YAL061W & 1 & 9 & 10 & 24.97897 & -21.179 & 24.77718 & 55.38721 & 46.28571 \\
\hline & YBL071C & 1 & 9 & 11 & 26.21277 & 26.66667 & -20.2869 & 35.65891 & 44.27245 \\
\hline \multirow[t]{2}{*}{$\mathrm{BDH} 1$} & YAL060W & 1 & 9 & 12 & 43.11558 & 13.29715 & -5.83333 & 43.65904 & 36.55589 \\
\hline & YBL070C & 1 & 9 & 13 & 19.02801 & 47.86151 & 17.68953 & $\begin{array}{l}60.375 \\
\text { missing }\end{array}$ & 38.59223 \\
\hline ECM1 & YAL059W & 1 & 9 & 14 & 15.02183 & 22.96748 & 19.02792 & $\begin{array}{l}\text { colony } \\
\text { missing }\end{array}$ & missing colony \\
\hline AST1 & YBL069W & 1 & 9 & 15 & 12.95681 & 6.690562 & -16.875 & $\begin{array}{l}\text { colony } \\
\text { missing }\end{array}$ & missing colony \\
\hline CNE1 & YAL058W & 1 & 9 & 16 & 25.07599 & 41.95046 & -75.625 & colony & missing colony \\
\hline
\end{tabular}




\begin{tabular}{|c|c|c|c|c|c|c|c|c|c|}
\hline \multirow[t]{2}{*}{ PRS4 } & YBL068W & 1 & 9 & 17 & 31.94313 & 40.11494 & 55.11727 & 65.4321 & 52.34899 \\
\hline & YAL058C-A & 1 & 9 & 18 & 28.38542 & 38.46928 & 16.51143 & 56.02941 & 44.73684 \\
\hline UBP13 & YBL067C & 1 & 9 & 19 & 43.74121 & 48.63582 & -23.0159 & 56.19469 & 45.47945 \\
\hline GPB2 & YAL056W & 1 & 9 & 20 & 54.89978 & 27.43628 & -18.125 & 63.65031 & 29.83871 \\
\hline SEF1 & YBL066C & 1 & 9 & 21 & 13.80671 & 36.81241 & 42.76923 & 30.56604 & 57.89474 \\
\hline \multirow[t]{2}{*}{ PEX22 } & YAL055W & 1 & 9 & 22 & 11.34632 & 13.10345 & -9.43683 & 39.26471 & 15.06173 \\
\hline & YBL065W & 1 & 9 & 23 & \#DIV/0! & 100 & 31.52174 & 43.72414 & 20 \\
\hline HIS3 & YOR202W & 1 & 9 & 24 & -37.8824 & -26.1421 & 16.30537 & & \\
\hline \multirow[t]{2}{*}{ HIS3 } & YOR202W & 1 & 10 & 1 & 7.85381 & 10.7565 & -28.3037 & & \\
\hline & YBR090C & 1 & 10 & 2 & 31.0219 & 42.72623 & 40.99804 & 56.19835 & 40.04329 \\
\hline KTR4 & YBR199W & 1 & 10 & 3 & 19.05679 & 10.43478 & -15.0977 & 29.81818 & 28.64583 \\
\hline \multirow[t]{2}{*}{ MIS1 } & YBR084W & 1 & 10 & 4 & 5.382436 & -20.3412 & 12.77683 & 41.63569 & -2.69231 \\
\hline & YBR197C & 1 & 10 & 5 & -10.9215 & -2.01005 & -6.44105 & 35.56405 & 21.89189 \\
\hline RPL19A & YBR084C-A & 1 & 10 & 6 & 22.33251 & 19.73568 & 15.0165 & 52.13523 & 48.18182 \\
\hline MSI1 & YBR195C & 1 & 10 & 7 & 26.20632 & 18.01075 & -3.94922 & 24.70785 & 25.84856 \\
\hline TEC1 & YBR083W & 1 & 10 & 8 & 25.31447 & 49.89035 & -138.961 & 37.80069 & 37.94038 \\
\hline SOY1 & YBR194W & 1 & 10 & 9 & 20.76649 & 21.95846 & 8.437856 & 49.02724 & 46.48318 \\
\hline UBC4 & YBR082C & 1 & 10 & 10 & 22.73902 & 26.00806 & 13.96588 & 45.81818 & 40.93567 \\
\hline RPS9B & YBR189W & 1 & 10 & 11 & 34.13304 & 31.32328 & 49.63768 & 52.02073 & 37.7451 \\
\hline SLM4 & YBR077C & 1 & 10 & 12 & 33.85417 & 71.8232 & \#DIV/O! & 29.72259 & 29.20354 \\
\hline NTC20 & YBR188C & 1 & 10 & 13 & 41.10813 & 38.08256 & 41.10825 & $\begin{array}{l}61.5894 \\
\text { missing }\end{array}$ & 40.68966 \\
\hline \multirow[t]{3}{*}{ ECM8 } & YBR076W & 1 & 10 & 14 & 19.31429 & 30.76233 & -24.3243 & $\begin{array}{l}\text { colony } \\
\text { missing }\end{array}$ & missing colony \\
\hline & YBR187W & 1 & 10 & 15 & 22.40829 & 44.05772 & 25.40084 & $\begin{array}{l}\text { colony } \\
\text { missing }\end{array}$ & missing colony \\
\hline & YBR075W & 1 & 10 & 16 & 13.68421 & 100 & 62.26994 & colony & missing colony \\
\hline \multirow[t]{2}{*}{$\mathrm{PCH} 2$} & YBR186W & 1 & 10 & 17 & 16.93345 & 52.72727 & 30.29772 & 65.90909 & 41.82243 \\
\hline & YBR074W & 1 & 10 & 18 & -29.303 & 21.38518 & 39.05622 & 35.01094 & 40.33149 \\
\hline MBA1 & YBR185C & 1 & 10 & 19 & 21.841 & 28.33969 & 21.35266 & 56.69643 & 32.77778 \\
\hline \multirow[t]{2}{*}{ RDH54 } & YBR073W & 1 & 10 & 20 & 27.35962 & 18.54015 & -10.3448 & 23.44689 & 35.42234 \\
\hline & YBR184W & 1 & 10 & 21 & 17.54967 & 41.12512 & -6.55271 & 65.23605 & 39.66942 \\
\hline HSP26 & YBR072W & 1 & 10 & 22 & 41.76829 & 61.05691 & 44.51939 & 75.7716 & 43.76812 \\
\hline YPC1 & YBR183W & 1 & 10 & 23 & 63.24222 & 14.85232 & 30.05618 & 61.02757 & 39.86784 \\
\hline HIS3 & YOR202W & 1 & 10 & 24 & 9.16442 & 11.02941 & 45.81498 & & \\
\hline HIS3 & YOR202W & 1 & 11 & 1 & 7.439311 & 14.11458 & -13.9171 & & \\
\hline \multirow[t]{3}{*}{ UIP3 } & YAR027W & 1 & 11 & 2 & 24.24242 & 14.71861 & 23.25915 & 36.15917 & 45.79832 \\
\hline & YBL094C & 1 & 11 & 3 & 58.29876 & 74.44988 & 55.6213 & 58.03982 & 66.27219 \\
\hline & YAR023C & 1 & 11 & 4 & 11.56602 & -4.37756 & 23.82155 & 44.79718 & 29.52128 \\
\hline SCS22 & YBL091C-A & 1 & 11 & 5 & 1.350135 & -8.43373 & 16.48241 & 25.41966 & 36.59218 \\
\hline PAU7 & YAR020C & 1 & 11 & 6 & 19.35194 & 23.08892 & -45.5301 & 16.96607 & 22.8022 \\
\hline MAP2 & YBL091C & 1 & 11 & 7 & 51.29151 & 17.74436 & 2.116402 & 51.35135 & 36.54971 \\
\hline KIN3 & YAR018C & 1 & 11 & 8 & 18.01386 & 5.982906 & 15.46296 & 32.76451 & 28 \\
\hline
\end{tabular}




\begin{tabular}{|c|c|c|c|c|c|c|c|c|c|}
\hline AVT5 & YBL089W & 1 & 11 & 9 & 27.64561 & 0.956938 & 20.59113 & $\begin{array}{l}42.18456 \\
\text { missing }\end{array}$ & 47.23032 \\
\hline ADE1 & YAR015W & 1 & 11 & 10 & 36.10063 & 20.57026 & 10.58122 & colony & missing colony \\
\hline TEL1 & YBL088C & 1 & 11 & 11 & 22.31183 & 19.03553 & 10.85714 & 22.35529 & 40.45226 \\
\hline BUD14 & YAR014C & 1 & 11 & 12 & 10.25641 & 15.44172 & 38.52535 & 31.41762 & 24.67866 \\
\hline RPL23A & YBL087C & 1 & 11 & 13 & 20.92338 & 3.419973 & 11.16071 & 16.39344 & 26.93498 \\
\hline \multirow[t]{2}{*}{ SWD1 } & YAR003W & 1 & 11 & 14 & 32.29614 & 40.47323 & -14.2105 & 27.90015 & 23.09368 \\
\hline & YBL086C & 1 & 11 & 15 & 30.81264 & 28.47854 & 13.22581 & 53.47299 & 36.24454 \\
\hline NUP60 & YAR002W & 1 & 11 & 16 & 23.04385 & 65.87537 & -2.81481 & 26.50177 & 29.41176 \\
\hline BOI1 & YBL085W & 1 & 11 & 17 & -33.9073 & 44.82339 & -3.36927 & 60.37483 & 43.10777 \\
\hline \multirow[t]{3}{*}{ ERP1 } & YAR002C-A & 1 & 11 & 18 & -26.2248 & 20.28136 & 27.4744 & 44.75043 & 40.20356 \\
\hline & YBL083C & 1 & 11 & 19 & 15.67982 & 34.55621 & 5.032823 & 32.85124 & 32.51366 \\
\hline & YAL068C & 1 & 11 & 20 & 19.14683 & 36.51344 & -26.2931 & 57.10306 & 36.36364 \\
\hline RHK1 & YBL082C & 1 & 11 & 21 & 23.24195 & 59.61661 & -86.3071 & 31.79191 & 32.97872 \\
\hline \multirow[t]{2}{*}{ SEO1 } & YAL067C & 1 & 11 & 22 & 21.87845 & 72.08122 & 52.61044 & 34.93282 & 12.92135 \\
\hline & YBL081W & 1 & 11 & 23 & 37.10451 & 7.267222 & 20 & 55.38058 & 45.41752 \\
\hline HIS3 & YOR202W & 1 & 11 & 24 & 29.0267 & 28.59155 & -11.2426 & & \\
\hline HIS3 & YOR202W & 1 & 12 & 1 & 22.72025 & -38.0423 & -13.4807 & & \\
\hline YMC2 & YBR104W & 1 & 12 & 2 & 26.99436 & 1.623816 & -7.29783 & 46.66667 & 40.51383 \\
\hline NGR1 & YBR212W & 1 & 12 & 3 & 19.36508 & -43.4916 & 0 & 40.84249 & 29.17847 \\
\hline SIF2 & YBR103W & 1 & 12 & 4 & 40.38462 & 62.0332 & \#DIV/0! & 36.30705 & 29.78142 \\
\hline ERV15 & YBR210W & 1 & 12 & 5 & 21.24352 & 16.51652 & -22.5389 & 30.03597 & 24.4382 \\
\hline \multirow[t]{3}{*}{ FES1 } & YBR101C & 1 & 12 & 6 & 39.72746 & -35.7435 & -2.92398 & 59.22509 & 43.125 \\
\hline & YBR209W & 1 & 12 & 7 & 24.5614 & 12.08633 & -3.02376 & 32.05829 & 20.73171 \\
\hline & YBR100W & 1 & 12 & 8 & 31.58879 & 42.14876 & 33.70288 & 41.2 & 55 \\
\hline \multirow[t]{2}{*}{ DUR1,2 } & YBR208C & 1 & 12 & 9 & \#DIV/0! & \#DIV/0! & \#DIV/0! & 53.8003 & 37.5 \\
\hline & YBR099C & 1 & 12 & 10 & 19.59799 & 27.02952 & 0.21645 & 55.5 & 40 \\
\hline FTH1 & YBR207W & 1 & 12 & 11 & 23.04394 & 20.19584 & 9.935897 & 47.24919 & 38.46154 \\
\hline \multirow[t]{2}{*}{ MMS4 } & YBR098W & 1 & 12 & 12 & 13.30357 & 44.66546 & 19.67779 & 42.63094 & 32.1267 \\
\hline & YBR206W & 1 & 12 & 13 & 2.409639 & 24.3871 & 33.64888 & 63.84181 & 45.19481 \\
\hline RXT2 & YBR095C & 1 & 12 & 14 & 15.35714 & 52.51799 & 13.60078 & 23.2493 & 33.39041 \\
\hline \multirow[t]{3}{*}{ KTR3 } & YBR205W & 1 & 12 & 15 & 2.954755 & 21.9883 & 5.297297 & 45.87629 & 53.8695 \\
\hline & YBR094W & 1 & 12 & 16 & 53.50318 & 100 & \#DIV/0! & 46.41148 & 38.23529 \\
\hline & YBR204C & 1 & 12 & 17 & -50.2256 & 52.4836 & 20.88536 & 48.5 & 37.36559 \\
\hline PHO5 & YBR093C & 1 & 12 & 18 & -30.5847 & -2.45023 & 8.912037 & 36.01399 & 33.93782 \\
\hline $\cos 111$ & YBR203W & 1 & 12 & 19 & 33.81166 & 9.111111 & -26.6833 & 49.78723 & 42.6087 \\
\hline PHO3 & YBR092C & 1 & 12 & 20 & 53.10174 & 64.49843 & 0.522648 & 72.96137 & 41.94444 \\
\hline \multirow[t]{2}{*}{ DER1 } & YBR201W & 1 & 12 & 21 & 18.21862 & 21.68022 & 63.38983 & 63.68876 & 35.87786 \\
\hline & YBR090C-A & 1 & 12 & 22 & -5.01253 & 7.649513 & 24.20814 & 60.27027 & 34.68835 \\
\hline BEM1 & YBR200W & 1 & 12 & 23 & 28.60041 & 13.10345 & -5.67613 & 12.33244 & 31.63636 \\
\hline HIS3 & YOR202W & 1 & 12 & 24 & 25.14507 & 30.11583 & -1.16448 & & \\
\hline \multirow[t]{2}{*}{ HIS3 } & YOR202W & 1 & 13 & 1 & 0.817236 & 35.6094 & -8.7236 & & \\
\hline & YAR047C & 1 & 13 & 2 & 1.106771 & -8.49134 & -10.5128 & 32.53012 & 28.43823 \\
\hline
\end{tabular}




\begin{tabular}{|c|c|c|c|c|c|c|c|c|c|}
\hline NTH2 & YBR001C & 1 & 13 & 3 & 32.62712 & -30.777 & 3.333333 & 42.80374 & 39.40887 \\
\hline \multirow[t]{3}{*}{ OSH1 } & YAR044W & 1 & 13 & 4 & 16.28995 & -4.64602 & -14.1694 & 49.52741 & 43.84236 \\
\hline & YBL107C & 1 & 13 & 5 & 61.20527 & 89.20635 & \#DIV/0! & 47.98535 & 40.16393 \\
\hline & YAR043C & 1 & 13 & 6 & 34.41467 & 30.28391 & -8.66808 & 40.42934 & 33.52436 \\
\hline SRO77 & YBL106C & 1 & 13 & 7 & 37.95455 & 34.5679 & \#DIV/0! & 42.51825 & 54.8105 \\
\hline \multirow[t]{3}{*}{ SWH1 } & YAR042W & 1 & 13 & 8 & 8.745583 & 32.29665 & 20.73684 & 52.20588 & 49.85163 \\
\hline & YBL104C & 1 & 13 & 9 & 40.21871 & 22.39221 & -11.413 & 29.86248 & 47.32394 \\
\hline & YAR040C & 1 & 13 & 10 & 24.75685 & 7.063712 & 14.96674 & 47.90698 & 39.13043 \\
\hline \multirow[t]{2}{*}{ RTG3 } & YBL103C & 1 & 13 & 11 & 14.31111 & 18.35106 & 13.34895 & 48 & -9.09091 \\
\hline & YAR037W & 1 & 13 & 12 & 7.571802 & 16.84211 & 21.30872 & 23.6 & 22.44898 \\
\hline SFT2 & YBL102W & 1 & 13 & 13 & 3.443878 & -3.02521 & 17.56757 & 36.82171 & 45.96774 \\
\hline YAT1 & YAR035W & 1 & 13 & 14 & 29.31894 & 60.88154 & -26.9517 & 20.93496 & 24.19825 \\
\hline ECM21 & YBL101C & 1 & 13 & 15 & 19.48052 & 35.30259 & 47.39336 & 49.77974 & 34.10256 \\
\hline \multirow[t]{3}{*}{ PRM9 } & YAR031W & 1 & 13 & 16 & 51.10132 & 73.63344 & \#DIV/0! & 59.3343 & 42.82178 \\
\hline & YBL100C & 1 & 13 & 17 & 3.658537 & 49.89154 & \#DIV/0! & 44.74097 & 32.40223 \\
\hline & YAR030C & 1 & 13 & 18 & 8.899297 & 14.25517 & 31.76972 & 51.42857 & 43.05177 \\
\hline \multirow[t]{5}{*}{ BNA4 } & YBL098W & 1 & 13 & 19 & 30.83538 & 42.85714 & -38.835 & 60.77886 & 43.92765 \\
\hline & YAR029W & 1 & 13 & 20 & 26.27346 & 30.41667 & -4.89362 & 51.62524 & 41.37931 \\
\hline & YBL096C & 1 & 13 & 21 & 61.16959 & 32.27571 & -28.9694 & 64.30636 & 42.44792 \\
\hline & YAR028W & 1 & 13 & 22 & 32.97721 & 17.96157 & -0.69606 & 60.19152 & 30.09709 \\
\hline & YBL095W & 1 & 13 & 23 & 9.562212 & 14.03727 & 39.39394 & 36.95271 & 50.62657 \\
\hline HIS3 & YOR202W & 1 & 13 & 24 & 1.898188 & -16.3828 & 29.79592 & & \\
\hline HIS3 & YOR202W & 1 & 14 & 1 & 47.0852 & 37.691 & 16.09195 & & \\
\hline GRS1 & YBR121C & 1 & 14 & 2 & 11.96755 & 18.70504 & -31.3099 & 29.38144 & 18.54685 \\
\hline TDP1 & YBR223C & 1 & 14 & 3 & 73.36815 & -3.04569 & 36.68639 & 39.01961 & 43.00518 \\
\hline MUD1 & YBR119W & 1 & 14 & 4 & 62.78447 & 1.090909 & 29.21348 & 32.74162 & 35.50136 \\
\hline \multirow[t]{2}{*}{ PCS60 } & YBR222C & 1 & 14 & 5 & 16.55438 & 14.36314 & 6.828704 & 22.04611 & 28.04124 \\
\hline & YBR116C & 1 & 14 & 6 & 53.28185 & 46.25375 & -2.89331 & 47.77397 & 32.03343 \\
\hline PDB1 & YBR221C & 1 & 14 & 7 & 31.11639 & 34.77493 & 23.89381 & 59.87261 & 47.60705 \\
\hline \multirow[t]{2}{*}{ LYS2 } & YBR115C & 1 & 14 & 8 & 5.973025 & 33.52941 & 22.93987 & 35.50725 & 36.31124 \\
\hline & YBR220C & 1 & 14 & 9 & -3.91753 & 6.811989 & 9.147609 & 32.91405 & 45.52846 \\
\hline \multirow[t]{3}{*}{ RAD16 } & YBR114W & 1 & 14 & 10 & 0.302725 & 40.07989 & 4.526749 & 52.93132 & 42.24138 \\
\hline & YBR219C & 1 & 14 & 11 & 6.754772 & 7.120743 & 16.59919 & 46.67832 & 29.34132 \\
\hline & YBR113W & 1 & 14 & 12 & 19.35807 & -36.7331 & 9.416196 & 20.08457 & 19.17404 \\
\hline PYC2 & YBR218C & 1 & 14 & 13 & 20.71228 & 26.16202 & 12.30769 & 53.53383 & 30.8311 \\
\hline YSA1 & YBR111C & 1 & 14 & 14 & 12.40741 & 26.57658 & 10.31042 & 47.53788 & 41.6 \\
\hline \multirow[t]{2}{*}{ ATG12 } & YBR217W & 1 & 14 & 15 & 12.65509 & 8.476395 & 15.98131 & 48.13084 & 26.5252 \\
\hline & YBR108W & 1 & 14 & 16 & -74.5856 & 30.67485 & \#DIV/0! & 60.11004 & 35.73201 \\
\hline YBP1 & YBR216C & 1 & 14 & 17 & 17.64706 & 91.06529 & \#DIV/0! & 51.03093 & 44.52736 \\
\hline IML3 & YBR107C & 1 & 14 & 18 & 31.61765 & 10.79622 & 29.56204 & -11.1276 & 39.65142 \\
\hline HPC2 & YBR215W & 1 & 14 & 19 & 23.03665 & 11.37586 & 13.88235 & 56.58363 & 37.77174 \\
\hline PHO88 & YBR106W & 1 & 14 & 20 & 27.46536 & 7.756024 & 9.111617 & 13.36516 & 36.79245 \\
\hline SDS24 & YBR214W & 1 & 14 & 21 & 4.666667 & 27.95181 & 10.38961 & 61.60584 & 39.52255 \\
\hline
\end{tabular}




\begin{tabular}{|c|c|c|c|c|c|c|c|c|c|}
\hline VID24 & YBR105C & 1 & 14 & 22 & 16.1346 & 24.16268 & -41.9149 & 47.33333 & 26.73267 \\
\hline MET8 & YBR213W & 1 & 14 & 23 & 43.92804 & 44.43603 & 37.78626 & 61.71735 & 52.37154 \\
\hline HIS3 & YOR202W & 1 & 14 & 24 & -8.09477 & -1.11111 & -7.79944 & & \\
\hline HIS3 & YOR202W & 1 & 15 & 1 & 6.776316 & 5.237809 & -0.47664 & & \\
\hline \multirow[t]{2}{*}{$\mathrm{ACH} 1$} & YBL015W & 1 & 15 & 2 & 29.4699 & -1.93906 & 22.30624 & 30.45455 & 47.41379 \\
\hline & YBR016W & 1 & 15 & 3 & 41.50718 & -3.7962 & 34.13333 & 39.12176 & 53.57143 \\
\hline FMT1 & YBL013W & 1 & 15 & 4 & 34.71284 & -8.75274 & -16.8932 & 8.8 & 15.68627 \\
\hline MNN2 & YBR015C & 1 & 15 & 5 & 22.72727 & 100 & \#DIV/0! & $\begin{array}{l}34.14179 \\
\text { missing }\end{array}$ & 53.04878 \\
\hline \multirow[t]{6}{*}{ SCT1 } & YBL011W & 1 & 15 & 6 & 24.22259 & 1.71184 & -0.94787 & $\begin{array}{l}\text { colony } \\
\text { missing }\end{array}$ & missing colony \\
\hline & YBR014C & 1 & 15 & 7 & 43.62745 & 10.44944 & 26.15385 & colony & missing colony \\
\hline & YBL010C & 1 & 15 & 8 & 46.87208 & 13.7224 & -7.25624 & 46.77686 & 53.75587 \\
\hline & YBR013C & 1 & 15 & 9 & 1.049475 & 19.26514 & 16.71309 & 37.26496 & 29.97118 \\
\hline & YBL009W & 1 & 15 & 10 & 21.20536 & 63.46005 & 10.56662 & 38.03089 & 46.21027 \\
\hline & YBR012C & 1 & 15 & 11 & 30.5712 & -1.39958 & 20.94763 & 40.26403 & 42.36842 \\
\hline HIR1 & YBL008W & 1 & 15 & 12 & 18.94273 & 16.37795 & 12.92985 & 53.53038 & 47.08861 \\
\hline НHT1 & YBR010W & 1 & 15 & 13 & 22.73171 & 64.14698 & 33.20755 & 38.98917 & 38.96458 \\
\hline SLA1 & YBL007C & 1 & 15 & 14 & 90.29703 & 90.1178 & 85.11111 & 54.66448 & 89.87654 \\
\hline HHF1 & YBR009C & 1 & 15 & 15 & -4.50902 & -24.9622 & 8.018868 & 44.88304 & 24.69136 \\
\hline PDR3 & YBL005W & 1 & 15 & 16 & 14.92933 & 14.0553 & 6.797235 & 34.84574 & 25.41436 \\
\hline FLR1 & YBR008C & 1 & 15 & 17 & 38.0123 & 41.79756 & -10.2725 & 38.33333 & 31.98992 \\
\hline HTA2 & YBL003C & 1 & 15 & 18 & 20.23217 & 14.36135 & 5.067064 & 55.15239 & 28.43137 \\
\hline DSF2 & YBR007C & 1 & 15 & 19 & 52.67559 & 60.44444 & 36.80982 & 64.1791 & 45.16807 \\
\hline ECM15 & YBL001C & 1 & 15 & 20 & 40.15826 & 3.717827 & 61.18012 & 59.82143 & 45.59585 \\
\hline UGA2 & YBR006W & 1 & 15 & 21 & 35.68406 & 24.79893 & 33.33333 & 47.57576 & 47.35683 \\
\hline FLO1 & YAR050W & 1 & 15 & 22 & 17.2043 & 19.15052 & -4.60358 & 55.76923 & 45.5157 \\
\hline RCR1 & YBR005W & 1 & 15 & 23 & 23.24159 & 2.020202 & 16.82135 & 54.83405 & 30.25751 \\
\hline HIS3 & YOR202W & 1 & 15 & 24 & 15.63786 & -13.961 & -9.9187 & & \\
\hline HIS3 & YOR202W & 1 & 16 & 1 & 52.41568 & 13.53919 & -12.922 & & \\
\hline HIS3 & YOR202W & 1 & 16 & 2 & 51.28631 & 3.326544 & 7.18232 & & \\
\hline HIS3 & YOR202W & 1 & 16 & 3 & 51.16697 & -9.94109 & 1.538462 & & \\
\hline HIS3 & YOR202W & 1 & 16 & 4 & 8.333333 & -24.3536 & -26.5086 & & \\
\hline HIS3 & YOR202W & 1 & 16 & 5 & 22.44898 & 86.22754 & \#DIV/0! & & \\
\hline HIS3 & YOR202W & 1 & 16 & 6 & 25.23745 & -17.7845 & 13.69606 & & \\
\hline HIS3 & YOR202W & 1 & 16 & 7 & 7.429963 & 1.936872 & -25.5014 & & \\
\hline HIS3 & YOR202W & 1 & 16 & 8 & 32.81133 & 13.64591 & -7.62332 & & \\
\hline HIS3 & YOR202W & 1 & 16 & 9 & 31.37956 & 7.321131 & 39.69072 & & \\
\hline HIS3 & YOR202W & 1 & 16 & 10 & 54.19546 & 20.04381 & 15.96639 & & \\
\hline HIS3 & YOR202W & 1 & 16 & 11 & 33.06392 & -4.11227 & -0.88063 & & \\
\hline HIS3 & YOR202W & 1 & 16 & 12 & 38.05668 & 43.21503 & -43.8356 & & \\
\hline HIS3 & YOR202W & 1 & 16 & 13 & 36.1 & 31.69691 & -21 & & \\
\hline HIS3 & YOR202W & 1 & 16 & 14 & 26.96897 & 25.91823 & 41.81818 & & \\
\hline
\end{tabular}




\begin{tabular}{|c|c|c|c|c|c|c|c|c|c|}
\hline HIS3 & YOR202W & 1 & 16 & 15 & 38.96424 & 45.74468 & \#DIV/0! & & \\
\hline HIS3 & YOR202W & 1 & 16 & 16 & 38.80952 & 30.12367 & -8.98618 & & \\
\hline HIS3 & YOR202W & 1 & 16 & 17 & 22.62411 & 12.44387 & 25.58594 & & \\
\hline HIS3 & YOR202W & 1 & 16 & 18 & 33.63119 & 15.89347 & -4.25926 & & \\
\hline HIS3 & YOR202W & 1 & 16 & 19 & 20.1895 & 1.971609 & 14.70588 & & \\
\hline HIS3 & YOR202W & 1 & 16 & 20 & -27.339 & 30.46927 & -9.79021 & & \\
\hline HIS3 & YOR202W & 1 & 16 & 21 & 20.13311 & 31.60205 & -42 & & \\
\hline HIS3 & YOR202W & 1 & 16 & 22 & -8.36614 & 3.581461 & 18.62745 & & \\
\hline HIS3 & YOR202W & 1 & 16 & 23 & -15.7676 & -28.852 & 16.7364 & & \\
\hline HIS3 & YOR202W & 1 & 16 & 24 & 41.78874 & 9.51547 & 9.776833 & & \\
\hline HIS3 & YOR202W & 3 & 1 & 1 & 83.7464 & 24.79951 & 79.22794 & & \\
\hline HIS3 & YOR202W & 3 & 1 & 2 & 29.91851 & -88.3241 & 67.65957 & & \\
\hline HIS3 & YOR202W & 3 & 1 & 3 & 17.73547 & -53.1973 & 70.88256 & & \\
\hline HIS3 & YOR202W & 3 & 1 & 4 & 44.71469 & -86.7052 & 63.78738 & & \\
\hline HIS3 & YOR202W & 3 & 1 & 5 & -3.1941 & -46.0674 & 55.89569 & & \\
\hline HIS3 & YOR202W & 3 & 1 & 6 & 13.67061 & -89.7249 & 53.60531 & & \\
\hline HIS3 & YOR202W & 3 & 1 & 7 & -2.01005 & -36.6587 & 93.09623 & & \\
\hline HIS3 & YOR202W & 3 & 1 & 8 & 26.28399 & -28.8756 & 41.45078 & & \\
\hline HIS3 & YOR202W & 3 & 1 & 9 & 14.28571 & -21.2639 & 64.11111 & & \\
\hline HIS3 & YOR202W & 3 & 1 & 10 & 19.79045 & -30.4564 & 49.5814 & & \\
\hline HIS3 & YOR202W & 3 & 1 & 11 & 23.81477 & -31 & 71.2627 & & \\
\hline HIS3 & YOR202W & 3 & 1 & 12 & 18.48152 & -0.47885 & 67.67372 & & \\
\hline HIS3 & YOR202W & 3 & 1 & 13 & 19.8123 & -94.2669 & 83.30827 & & \\
\hline HIS3 & YOR202W & 3 & 1 & 14 & 20.72491 & 2.5417 & 47.87823 & & \\
\hline HIS3 & YOR202W & 3 & 1 & 15 & 22.73603 & -41.5288 & 50.45719 & & \\
\hline HIS3 & YOR202W & 3 & 1 & 16 & 30.62569 & 6.372935 & 65.9751 & & \\
\hline HIS3 & YOR202W & 3 & 1 & 17 & 24.55775 & -58.551 & 52.76273 & & \\
\hline HIS3 & YOR202W & 3 & 1 & 18 & 33.8091 & -48.933 & 36.36364 & & \\
\hline HIS3 & YOR202W & 3 & 1 & 19 & 36.98011 & -41.331 & 38.69892 & & \\
\hline HIS3 & YOR202W & 3 & 1 & 20 & 33.48375 & -46.9914 & 26.17622 & & \\
\hline HIS3 & YOR202W & 3 & 1 & 21 & 34.50947 & -55.2374 & 32.7853 & & \\
\hline HIS3 & YOR202W & 3 & 1 & 22 & -12.1127 & -36.9534 & 42.79902 & & \\
\hline HIS3 & YOR202W & 3 & 1 & 23 & -35.6269 & -15.1035 & 32.2093 & & \\
\hline HIS3 & YOR202W & 3 & 1 & 24 & -69.0323 & -4.50051 & 19.56364 & & \\
\hline \multirow[t]{2}{*}{ HIS3 } & YOR202W & 3 & 2 & 1 & 74.52229 & -10.5556 & 47.34694 & & \\
\hline & YDR199W & 3 & 2 & 2 & 28.08783 & -17.2314 & 57.70142 & 9.369369 & 52.02156 \\
\hline \multirow[t]{2}{*}{ OMS1 } & YDR316W & 3 & 2 & 3 & -13.4314 & 18.95161 & 34.62069 & 29.8419 & 41.66667 \\
\hline & YDR198C & 3 & 2 & 4 & 24.74804 & -0.41929 & 38.42975 & 37.33333 & 42.69972 \\
\hline \multirow[t]{3}{*}{ IPK1 } & YDR315C & 3 & 2 & 5 & 30.08226 & 17.08785 & 44 & 24.15094 & 45.42773 \\
\hline & YDR193W & 3 & 2 & 6 & 21.07482 & 8.113036 & 42.09968 & 3.687636 & 37.31778 \\
\hline & YDR314C & 3 & 2 & 7 & 23.19128 & 25.77475 & 37.90524 & 33.14176 & 67.04545 \\
\hline NUP42 & YDR192C & 3 & 2 & 8 & 25.80645 & 25.91025 & 25.15263 & 13.98176 & 44.33249 \\
\hline PIB1 & YDR313C & 3 & 2 & 9 & 2.631579 & 100 & 78.7037 & 14.09266 & 39.06706 \\
\hline
\end{tabular}




\begin{tabular}{|c|c|c|c|c|c|c|c|c|c|}
\hline HST4 & YDR191W & 3 & 2 & 10 & 26.87313 & 12.63158 & 57.80998 & -15 & 38.69347 \\
\hline \multirow[t]{2}{*}{ SSF2 } & YDR312W & 3 & 2 & 11 & 36.93271 & 44.60501 & 63.25301 & 33.22581 & 52.36908 \\
\hline & YDR186C & 3 & 2 & 12 & 17.08595 & 10.91703 & 22.13656 & 62.37037 & 41.62011 \\
\hline \multirow[t]{2}{*}{ SUM1 } & YDR310C & 3 & 2 & 13 & 25.46973 & 8.268482 & 53.90728 & 58.99865 & 27.20588 \\
\hline & YDR185C & 3 & 2 & 14 & 21.2963 & 1.604938 & 12.6294 & 46.53465 & 52.33918 \\
\hline GIC2 & YDR309C & 3 & 2 & 15 & 12.33906 & -12.5316 & 28.98696 & 63.15789 & 31.2336 \\
\hline \multirow[t]{2}{*}{ ATC1 } & YDR184C & 3 & 2 & 16 & 27.97619 & 16.15181 & 27.27273 & 49.36248 & 48.21002 \\
\hline & YDR307W & 3 & 2 & 17 & 17.62918 & 1.785714 & 1.877934 & 74.24658 & 46.83841 \\
\hline \multirow[t]{2}{*}{ PLP1 } & YDR183W & 3 & 2 & 18 & 16.02151 & 22.54791 & 56.86567 & 54.02685 & 45.71429 \\
\hline & YDR306C & 3 & 2 & 19 & 18.55874 & 4.181185 & 11.8665 & 55.35714 & 42.94294 \\
\hline SAS4 & YDR181C & 3 & 2 & 20 & 18.61862 & 42.59056 & 40.35674 & 45.47244 & 55.30726 \\
\hline \multirow[t]{3}{*}{ HNT2 } & YDR305C & 3 & 2 & 21 & -48.5114 & 52.72915 & 62.14405 & 8.163265 & 54.48578 \\
\hline & YDR179W- & & & & & & & & \\
\hline & A & 3 & 2 & 22 & 27.36842 & 15.54054 & 35.07463 & -2.91595 & 64.49275 \\
\hline CPR5 & YDR304C & 3 & 2 & 23 & 64.40678 & 100 & 100 & 64.682 & 51.65394 \\
\hline HIS3 & YOR202W & 3 & 2 & 24 & \#DIV/0! & \#DIV/0! & 100 & & \\
\hline \multirow[t]{3}{*}{ HIS3 } & YOR202W & 3 & 3 & 1 & 78.60765 & -49.7263 & 67.46371 & & \\
\hline & YDL199C & 3 & 3 & 2 & 29.1423 & 33.78092 & 72.39354 & 54.39056 & 52.18447 \\
\hline & YDR090C & 3 & 3 & 3 & -11.0599 & 16.97793 & 50.92251 & -11.5888 & 50.41783 \\
\hline \multirow[t]{2}{*}{ ASF2 } & YDL197C & 3 & 3 & 4 & 26.10526 & 36.12262 & 49.78102 & 44.46786 & 55.82524 \\
\hline & YDR089W & 3 & 3 & 5 & 23.36066 & -8.47222 & 42.83668 & 23.54369 & 49.20635 \\
\hline SNF3 & YDL194W & 3 & 3 & 6 & 20.92486 & 8.704253 & 68.53147 & 63.19896 & 57.10456 \\
\hline AFR1 & YDR085C & 3 & 3 & 7 & 43.9577 & 47.15219 & 60.34483 & 22.2973 & 32.62195 \\
\hline ARF1 & YDL192W & 3 & 3 & 8 & 12.38739 & -18.0778 & 41.79567 & 33.45865 & 50 \\
\hline TVP23 & YDR084C & 3 & 3 & 9 & 14.8265 & 8.300395 & 27.42317 & 34.41358 & 44.73684 \\
\hline RPL35A & YDL191W & 3 & 3 & 10 & 10.71429 & 20.15855 & 39.79136 & 73.39181 & 50.45872 \\
\hline RRP8 & YDR083W & 3 & 3 & 11 & 9.555855 & -1.54278 & 57.67635 & 64.52991 & 46.98413 \\
\hline UFD2 & YDL190C & 3 & 3 & 12 & 22.87048 & -9.6603 & 34.80243 & 66.02659 & 40.75067 \\
\hline VPS41 & YDR080W & 3 & 3 & 13 & 18.57765 & 0.682927 & 82.69231 & 71.07969 & 64.23174 \\
\hline RBS1 & YDL189W & 3 & 3 & 14 & 13.86243 & -3.96996 & 30.93023 & -13.215 & 36.15819 \\
\hline SED1 & YDR077W & 3 & 3 & 15 & 16.60859 & -6.0307 & 36.7801 & 70.01522 & 51.89504 \\
\hline PPH22 & YDL188C & 3 & 3 & 16 & 28.11861 & 12.13018 & 32.22683 & 72.71505 & 51.11111 \\
\hline \multirow[t]{2}{*}{ RAD55 } & YDR076W & 3 & 3 & 17 & 13.06991 & 72.64516 & 100 & 66.0312 & 51.5493 \\
\hline & YDL187C & 3 & 3 & 18 & 16.27635 & -10.4612 & 58.72576 & 78.85714 & 50.97493 \\
\hline \multirow[t]{2}{*}{ PPH3 } & YDR075W & 3 & 3 & 19 & 13.20166 & 6.840391 & 27.45902 & 62.68657 & 47.52747 \\
\hline & YDL186W & 3 & 3 & 20 & 15.71429 & -5.76923 & 49.77169 & 70.04539 & 51.01523 \\
\hline TPS2 & YDR074W & 3 & 3 & 21 & \#DIV/0! & 100 & 94.47853 & 11.16625 & 79.74359 \\
\hline TFP1 & YDL185W & 3 & 3 & 22 & 25.12438 & 19.10774 & 27.94918 & 52.22405 & 58.15085 \\
\hline SNF11 & YDR073W & 3 & 3 & 23 & -47.9076 & 28.85772 & 45.03043 & 23.89525 & 59.1002 \\
\hline HIS3 & YOR202W & 3 & 3 & 24 & -76.4124 & 4.887984 & 43.74226 & & \\
\hline HIS3 & YOR202W & 3 & 4 & 1 & 45.52048 & 1.278409 & 84.50363 & & \\
\hline ADR1 & YDR216W & 3 & 4 & 2 & -14.6701 & 25.56333 & 54.88166 & -0.5 & 44.67005 \\
\hline MSN5 & YDR335W & 3 & 4 & 3 & 71.99721 & 89.86829 & 73.59882 & 45.98214 & 86.95652 \\
\hline
\end{tabular}




\begin{tabular}{|c|c|c|c|c|c|c|c|c|c|}
\hline & YDR215C & 3 & 4 & 4 & 23.46263 & 16.08154 & 56.81558 & 5.642023 & 57.74648 \\
\hline SWR1 & YDR334W & 3 & 4 & 5 & 21.05263 & -31.934 & 62.00345 & 45.3831 & 49.5549 \\
\hline \multirow[t]{2}{*}{ AHA1 } & YDR214W & 3 & 4 & 6 & 23.73689 & -23.1293 & 35.00678 & 10.6383 & 40.87838 \\
\hline & YDR333C & 3 & 4 & 7 & 34.07022 & 13.46999 & 75.81699 & 12.45353 & 48.5119 \\
\hline \multirow[t]{3}{*}{ UPC2 } & YDR213W & 3 & 4 & 8 & 11.37856 & -11.9048 & 48.38275 & 51.89107 & 50.88235 \\
\hline & YDR332W & 3 & 4 & 9 & 24.91429 & -42.1801 & 50.85271 & 23.49823 & 49.23547 \\
\hline & YDR210W & 3 & 4 & 10 & 65.13089 & 67.06667 & 29.09091 & -2.07852 & 66.01671 \\
\hline \multirow[t]{2}{*}{ UBX5 } & YDR330W & 3 & 4 & 11 & 10.16043 & 14.97396 & 71.61905 & 66.94796 & 41.86747 \\
\hline & YDR209C & 3 & 4 & 12 & 20.89888 & -1.25945 & 47.69231 & 50.46584 & 65.28926 \\
\hline PEX3 & YDR329C & 3 & 4 & 13 & 10.74468 & -12.439 & 22.67038 & 52.98399 & 31.17284 \\
\hline UME6 & YDR207C & 3 & 4 & 14 & 36.19529 & 20.8981 & 63.29412 & 23.95023 & 65.43909 \\
\hline ASP1 & YDR321W & 3 & 4 & 15 & 58.88052 & 60.14286 & 40.52813 & 60.6264 & 60.19108 \\
\hline EBS1 & YDR206W & 3 & 4 & 16 & 60.39387 & 45.10067 & 61.7284 & 74.96183 & 52.89256 \\
\hline SWA2 & YDR320C & 3 & 4 & 17 & 29.93131 & 4.435058 & 45.81673 & 59.15721 & 53.35277 \\
\hline \multirow[t]{3}{*}{ MSC2 } & YDR205W & 3 & 4 & 18 & 32.51462 & 48.30189 & 58.34971 & 73.93484 & 50.12285 \\
\hline & YDR319C & 3 & 4 & 19 & 14.57663 & 14.24555 & 39.33518 & 47.64398 & 37.53666 \\
\hline & YDR203W & 3 & 4 & 20 & 18.8755 & 13.51607 & 9.9631 & 38.14433 & 51.88406 \\
\hline MCM21 & YDR318W & 3 & 4 & 21 & 14.27184 & 6.287683 & 26.79245 & 58.01187 & 50.98684 \\
\hline \multirow[t]{2}{*}{ RAV2 } & YDR202C & 3 & 4 & 22 & 13.95349 & 100 & -38.3178 & 45.06627 & 51.71429 \\
\hline & YDR317W & 3 & 4 & 23 & 42.42424 & 62.23565 & 82.01058 & 70.01195 & 50.76923 \\
\hline HIS3 & YOR202W & 3 & 4 & 24 & \#DIV/0! & \#DIV/0! & 100 & & \\
\hline HIS3 & YOR202W & 3 & 5 & 1 & 48.54607 & -38.1305 & 75.53406 & & \\
\hline \multirow[t]{2}{*}{ RRI1 } & YDL216C & 3 & 5 & 2 & 34.22131 & -12.9244 & 46.69261 & 46.97218 & 53.7037 \\
\hline & YDR102C & 3 & 5 & 3 & -7.29927 & 29.36047 & 47.2028 & 34.62838 & 55.11364 \\
\hline $\mathrm{GDH} 2$ & YDL215C & 3 & 5 & 4 & 18.0061 & -22.7632 & 65.06639 & 2.868852 & 46.96486 \\
\hline ARX1 & YDR101C & 3 & 5 & 5 & 3.581662 & -40.6934 & 86.73835 & 4.805492 & 58.25243 \\
\hline PRR2 & YDL214C & 3 & 5 & 6 & 17.71255 & 18.7251 & 42.42094 & 23.44045 & 46.98413 \\
\hline TVP15 & YDR100W & 3 & 5 & 7 & 11.73709 & 2.040816 & 32.26572 & 54.79452 & 48.19277 \\
\hline NOP6 & YDL213C & 3 & 5 & 8 & 14.30108 & 23.01255 & 21.59244 & 28.3054 & 57.53012 \\
\hline \multirow[t]{2}{*}{$\mathrm{BMH} 2$} & YDR099W & 3 & 5 & 9 & 20.24291 & 42.55608 & 49.28401 & 5.869074 & 56.89655 \\
\hline & YDL211C & 3 & 5 & 10 & 4.785276 & 32.76027 & 58.375 & 84.39532 & 53.33333 \\
\hline GRX3 & YDR098C & 3 & 5 & 11 & 10.98655 & 0.113766 & 45.1497 & 28.17204 & 52.10843 \\
\hline UGA4 & YDL210W & 3 & 5 & 12 & 10.79958 & -10.8597 & 27.60473 & 67.48092 & 44.96855 \\
\hline \multirow[t]{2}{*}{ MSH6 } & YDR097C & 3 & 5 & 13 & 16.25616 & -2.96192 & 57.2238 & 15.625 & 50.81522 \\
\hline & YDL206W & 3 & 5 & 14 & 16.7019 & -2.40175 & 41.40508 & 83.33333 & 55.95568 \\
\hline GIS1 & YDR096W & 3 & 5 & 15 & 19.63714 & -9.48537 & 34.43627 & -10.1617 & 37.03704 \\
\hline \multirow[t]{4}{*}{ RTN2 } & YDL204W & 3 & 5 & 16 & 12.14058 & -17.7215 & 44.9235 & 72.82322 & 51.84211 \\
\hline & YDR095C & 3 & 5 & 17 & 17.89038 & 27.77778 & 48.7882 & 50.76687 & 65.63467 \\
\hline & YDL203C & 3 & 5 & 18 & 13.63636 & 22.48858 & 38.2442 & 39.93453 & 44.74474 \\
\hline & YDR094W & 3 & 5 & 19 & 19.80932 & 17.9402 & 47.77595 & 66.15169 & 66.12466 \\
\hline TRM8 & YDL201W & 3 & 5 & 20 & 17.29469 & 18.4265 & 14.87779 & 59.43396 & 53.35366 \\
\hline DNF2 & YDR093W & 3 & 5 & 21 & 12.22329 & 6.82968 & 22.63099 & 18.52552 & 59.94318 \\
\hline MGT1 & YDL200C & 3 & 5 & 22 & 50.49505 & 63.64692 & 68.37607 & 58.84354 & 64.80263 \\
\hline
\end{tabular}




\begin{tabular}{|c|c|c|c|c|c|c|c|c|c|}
\hline UBC13 & YDR092W & 3 & 5 & 23 & 18.61648 & 27.80749 & 85.15152 & 63.22115 & 58.56833 \\
\hline HIS3 & YOR202W & 3 & 5 & 24 & 15.96899 & -14.4525 & 22.46094 & & \\
\hline HIS3 & YOR202W & 3 & 6 & 1 & 17.36478 & -18.9146 & 61.4192 & & \\
\hline RTN1 & YDR233C & 3 & 6 & 2 & 25.38531 & 7.008289 & 70.38328 & 28.4153 & 59.72603 \\
\hline TRP4 & YDR354W & 3 & 6 & 3 & 33.33333 & 30.30303 & 79.56044 & -5.53191 & 59.26966 \\
\hline \multirow[t]{2}{*}{ IVY1 } & YDR229W & 3 & 6 & 4 & 34.8513 & 28.30803 & 62.75395 & 25.76985 & 64.98674 \\
\hline & YDR352W & 3 & 6 & 5 & 33.57364 & 38.01874 & 44.83926 & 25 & 65.91549 \\
\hline SIR4 & YDR227W & 3 & 6 & 6 & 18.40088 & 35.18519 & 53.57562 & 59.45513 & 52.88754 \\
\hline SBE2 & YDR351W & 3 & 6 & 7 & 17.9669 & 48.3871 & 52.03915 & 65.35552 & 47.64706 \\
\hline HTA1 & YDR225W & 3 & 6 & 8 & 17.68018 & 3.020134 & 20.71331 & 6.654344 & 57.14286 \\
\hline YPS7 & YDR349C & 3 & 6 & 9 & 10.06289 & -19.3627 & 46.13124 & 25.85895 & 51.07527 \\
\hline \multirow[t]{3}{*}{ CRF1 } & YDR223W & 3 & 6 & 10 & 12.10256 & -27.1605 & 52.14385 & 17.11111 & 58.58586 \\
\hline & YDR348C & 3 & 6 & 11 & 15.43624 & -8.57143 & 60.80247 & 55.53571 & 60.17699 \\
\hline & YDR222W & 3 & 6 & 12 & 20.18927 & 23.04493 & 56.05381 & 69.02778 & 48.73239 \\
\hline \multirow[t]{2}{*}{ SVF1 } & YDR346C & 3 & 6 & 13 & 9.945946 & 0 & 36.43512 & 46.45161 & 46.58385 \\
\hline & YDR221W & 3 & 6 & 14 & 28.71287 & 39.83402 & 32.27633 & 74.30684 & 63.39869 \\
\hline \multirow[t]{5}{*}{ HXT3 } & YDR345C & 3 & 6 & 15 & 19.57619 & 19.70021 & 42.51012 & 60.25878 & 49.83607 \\
\hline & YDR220C & 3 & 6 & 16 & 22.67932 & -6.69344 & 29.45026 & 71.02362 & 64.34783 \\
\hline & YDR344C & 3 & 6 & 17 & 49.17492 & 100 & 100 & 45.49419 & 45.15235 \\
\hline & YDR219C & 3 & 6 & 18 & 22.14452 & -13.4181 & 46.6948 & -0.61983 & 47.2561 \\
\hline & YDR340W & 3 & 6 & 19 & 16.96607 & 13.75117 & 14.81132 & 56.44917 & 46.97802 \\
\hline \multirow[t]{2}{*}{ SPR28 } & YDR218C & 3 & 6 & 20 & 18.62643 & -4.79115 & 29.70414 & 44.39655 & 22.85714 \\
\hline & YDR338C & 3 & 6 & 21 & 6.962664 & 18.22079 & 32.78272 & 49.53271 & 59.30233 \\
\hline \multirow[t]{2}{*}{ RAD9 } & YDR217C & 3 & 6 & 22 & 17.51894 & 29.07762 & 42.83037 & 7.677543 & 43.64641 \\
\hline & YDR336W & 3 & 6 & 23 & -61.9512 & 100 & 59.30807 & -11.1336 & 50.49261 \\
\hline HIS3 & YOR202W & 3 & 6 & 24 & 27.70324 & -14.6753 & 48.36528 & & \\
\hline HIS3 & YOR202W & 3 & 7 & 1 & 55.76748 & -42.7083 & 57.15236 & & \\
\hline OST4 & YDL232W & 3 & 7 & 2 & 30.81155 & 23.26241 & 70.1087 & -30.5185 & 23.3945 \\
\hline RBF64 & YDR117C & 3 & 7 & 3 & 11.62362 & -5.00642 & 53.26504 & 61.63982 & 51.47059 \\
\hline BRE4 & YDL231C & 3 & 7 & 4 & 28.05836 & -22.2222 & 82.26221 & 0.759013 & 58.42697 \\
\hline MRPL1 & YDR116C & 3 & 7 & 5 & 16.51685 & 47.0073 & 50.18382 & 39.61268 & 57.00326 \\
\hline \multirow[t]{2}{*}{ PTP1 } & YDL230W & 3 & 7 & 6 & 14.62168 & 8.115183 & 43.2304 & 28.25342 & 58.09524 \\
\hline & YDR112W & 3 & 7 & 7 & 13.51661 & 1.544799 & 57.38725 & 67.86207 & 53.60231 \\
\hline SSB1 & YDL229W & 3 & 7 & 8 & 5.804111 & 33.54655 & 48.76616 & 16.80961 & 49.85507 \\
\hline ALT2 & YDR111C & 3 & 7 & 9 & 33.0656 & 28.05195 & 44.54343 & 30.2521 & 64.62585 \\
\hline $\mathrm{HO}$ & YDL227C & 3 & 7 & 10 & 15.14778 & 42.51358 & 56.33803 & 69.34097 & 41.31148 \\
\hline FOB1 & YDR110W & 3 & 7 & 11 & 11.28364 & -23.5294 & 27.82369 & 40.31496 & 55.82822 \\
\hline GCS1 & YDL226C & 3 & 7 & 12 & 83.81713 & 89.13428 & 73.644 & 87.53943 & 90.97222 \\
\hline STE5 & YDR103W & 3 & 7 & 13 & 12.7551 & 8.974359 & 40.42289 & 84.07461 & 55.17241 \\
\hline \multirow[t]{2}{*}{ WHI4 } & YDL224C & 3 & 7 & 14 & 20.71713 & 11.95652 & 19.68974 & 71.6763 & 48.87006 \\
\hline & YDR109C & 3 & 7 & 15 & 12.98969 & 16.95138 & 11.14865 & 75.52553 & 56.97674 \\
\hline HBT1 & YDL223C & 3 & 7 & 16 & 39.30693 & 26.17383 & 40.10417 & 88.67925 & 61.67147 \\
\hline GSG1 & YDR108W & 3 & 7 & 17 & 20.22472 & -2.40642 & 11.8984 & 70.66451 & 47.61905 \\
\hline
\end{tabular}




\begin{tabular}{|c|c|c|c|c|c|c|c|c|c|}
\hline \multirow[t]{2}{*}{ FMP45 } & YDL222C & 3 & 7 & 18 & 16.70282 & 19.26298 & 40.2439 & 63.12399 & 58.02469 \\
\hline & YDR107C & 3 & 7 & 19 & 15.89267 & -19.1667 & -5.73248 & 61.50307 & 54.10765 \\
\hline DTD1 & YDL219W & 3 & 7 & 20 & 20.11494 & 17.24453 & 40.92166 & 63.88889 & 46.53179 \\
\hline \multirow[t]{2}{*}{ TMS1 } & YDR105C & 3 & 7 & 21 & 10.30246 & 10.46788 & 14.91863 & 67.20827 & 53.8874 \\
\hline & YDL218W & 3 & 7 & 22 & 17.99629 & -6.17284 & 24.47699 & 64.84375 & 60.80692 \\
\hline SPO71 & YDR104C & 3 & 7 & 23 & 0.104275 & 55.14834 & 36.99634 & -33.3333 & 50.13928 \\
\hline HIS3 & YOR202W & 3 & 7 & 24 & -11.7984 & 48.75 & 66.52661 & & \\
\hline HIS3 & YOR202W & 3 & 8 & 1 & 46.3512 & -9.43513 & 48.48289 & & \\
\hline BTT1 & YDR252W & 3 & 8 & 2 & 17.84753 & 49.39911 & 53.89798 & 41.76373 & 53.31565 \\
\hline VPS74 & YDR372C & 3 & 8 & 3 & 21.24863 & 31.99195 & 71.7757 & 42.97521 & 64.86486 \\
\hline PAM1 & YDR251W & 3 & 8 & 4 & 20.39409 & 8.268156 & 63.35404 & $\begin{array}{l}21.94719 \\
\text { missing }\end{array}$ & 63.42857 \\
\hline \multirow[t]{4}{*}{ CTS2 } & YDR371W & 3 & 8 & 5 & 19.92188 & -21.7995 & 63.3557 & $\begin{array}{l}\text { colony } \\
\text { missing }\end{array}$ & missing colony \\
\hline & YDR250C & 3 & 8 & 6 & 4.089219 & 36.87544 & 45.03817 & colony & missing colony \\
\hline & YDR370C & 3 & 8 & 7 & 24.32778 & 3.268349 & 86.84211 & 26.45503 & 65.40698 \\
\hline & YDR249C & 3 & 8 & 8 & 20.4005 & 41.73307 & 71.65354 & 66.12903 & 53.89049 \\
\hline \multirow[t]{2}{*}{ XRS2 } & YDR369C & 3 & 8 & 9 & 0.806452 & 35.73807 & 29.41176 & 37.0892 & 61.20401 \\
\hline & YDR248C & 3 & 8 & 10 & 11.11111 & 5.340454 & 49.20635 & 46.52406 & 54.72313 \\
\hline YPR1 & YDR368W & 3 & 8 & 11 & -2.19058 & 54.79361 & 50.31847 & 49.92224 & 39.30818 \\
\hline VHS1 & $\begin{array}{l}\text { YDR247W } \\
\text { YDR363W- }\end{array}$ & 3 & 8 & 12 & 30.97643 & 43.49442 & 93.06931 & 38.57759 & 60.45752 \\
\hline SEM1 & $A$ & 3 & 8 & 13 & 71.98986 & 47.48804 & 66.42336 & -52.7273 & 38.06647 \\
\hline MNN10 & YDR245W & 3 & 8 & 14 & 32.56545 & 24.57213 & 70.73171 & 50.84444 & 70.23499 \\
\hline ESC2 & YDR363W & 3 & 8 & 15 & 60.59908 & 63.67089 & 77.3494 & 62.31061 & 73.48243 \\
\hline \multirow[t]{2}{*}{ PEX5 } & YDR244W & 3 & 8 & 16 & 13.56467 & 27.62605 & 64.05622 & 78.32586 & 57.14286 \\
\hline & YDR360W & 3 & 8 & 17 & 13.75126 & -7.67442 & 33.42105 & 69.50147 & 50.27933 \\
\hline BUD26 & YDR241W & 3 & 8 & 18 & 16.83968 & 17.31161 & 11.9898 & 57.86802 & 44.44444 \\
\hline \multirow[t]{2}{*}{ VID21 } & YDR359C & 3 & 8 & 19 & 60.9319 & 97.16157 & 100 & 18.4 & 68.59296 \\
\hline & YDR239C & 3 & 8 & 20 & 35.27815 & 45.46436 & 10.94771 & 40.71429 & 37.1875 \\
\hline GGA1 & YDR358W & 3 & 8 & 21 & 26.2218 & -0.13532 & 39.8524 & 30.96085 & 35.29412 \\
\hline \multirow[t]{2}{*}{ LYS4 } & YDR234W & 3 & 8 & 22 & 0.589971 & -0.10977 & 22.98682 & 16.57941 & 48.6413 \\
\hline & YDR357C & 3 & 8 & 23 & 14.07104 & 26.91415 & 36.12479 & 22.40437 & 62.62887 \\
\hline HIS3 & YOR202W & 3 & 8 & 24 & 30.48889 & -14.6958 & 31.14144 & & \\
\hline HIS3 & YOR202W & 3 & 9 & 1 & -3.50224 & -6.77083 & 77.41617 & & \\
\hline NTH1 & YDR001C & 3 & 9 & 2 & 18.76988 & -6.72646 & 89.58785 & 46.86469 & 59.53003 \\
\hline FIN1 & YDR130C & 3 & 9 & 3 & 20.86614 & -28.852 & 69.75666 & 2.028398 & 54.72222 \\
\hline \multirow[t]{3}{*}{ AAD4 } & YDL243C & 3 & 9 & 4 & 6.417112 & 35.37582 & 51.09677 & 58.19793 & 49.70238 \\
\hline & YDR128W & 3 & 9 & 5 & 11.71459 & 37.0447 & 58.68984 & 42.53968 & 62.07792 \\
\hline & YDL242W & 3 & 9 & 6 & 22.59488 & 6.451613 & 64.90566 & 42.27886 & 59.82906 \\
\hline \multirow[t]{2}{*}{ ARO1 } & YDR127W & 3 & 9 & 7 & 51.14943 & 64.5664 & 69.43069 & 33.89474 & 67.96407 \\
\hline & YDL241W & 3 & 9 & 8 & 1.459035 & 40.45521 & 79.57611 & 35.23654 & 59.71429 \\
\hline SWF1 & YDR126W & 3 & 9 & 9 & \#DIV/0! & \#DIV/0! & \#DIV/0! & 36.06557 & 63.86861 \\
\hline
\end{tabular}




\begin{tabular}{|c|c|c|c|c|c|c|c|c|c|}
\hline LRG1 & YDL240W & 3 & 9 & 10 & 10.78611 & -8.71413 & 44.97512 & 31.83453 & 37.07165 \\
\hline ECM18 & YDR125C & 3 & 9 & 11 & 16.72355 & 7.047279 & 63.6646 & 18.39286 & 59.15916 \\
\hline \multirow[t]{2}{*}{ ADY3 } & YDL239C & 3 & 9 & 12 & 2.763018 & 12.41791 & 38.33333 & 27.56849 & 45.75758 \\
\hline & YDR124W & 3 & 9 & 13 & 9.207921 & -19.8519 & 0.925926 & 73.78049 & 58.04598 \\
\hline GUD1 & YDL238C & 3 & 9 & 14 & 1.646091 & -17.6667 & 37.13714 & 41.46758 & 49.20128 \\
\hline \multirow[t]{2}{*}{ INO2 } & YDR123C & 3 & 9 & 15 & 19.69388 & 24.91309 & 87.82816 & 36.25954 & 51.25 \\
\hline & YDL237W & 3 & 9 & 16 & 23.52941 & 96.23397 & 95.52239 & 46.98997 & 42.05298 \\
\hline KIN1 & YDR122W & 3 & 9 & 17 & 17.29679 & 22.11302 & 34.73837 & 43.19328 & 50 \\
\hline PHO13 & YDL236W & 3 & 9 & 18 & 39.12176 & 71.25891 & 97.4026 & 76.72779 & 55.65476 \\
\hline DPB4 & YDR121W & 3 & 9 & 19 & 21.71123 & 15.42923 & 46.03854 & 67.01031 & 60.78431 \\
\hline GYP7 & YDL234C & 3 & 9 & 20 & 13.0352 & 17.16621 & 51.96771 & 67.46575 & 63.0303 \\
\hline \multirow[t]{3}{*}{ TRM1 } & YDR120C & 3 & 9 & 21 & 14.40501 & -5.78313 & 36.42857 & 52.32558 & 47.53086 \\
\hline & YDL233W & 3 & 9 & 22 & 5.665468 & -6.56013 & 24.97847 & 65.73117 & 39.32584 \\
\hline & YDR119W & 3 & 9 & 23 & 36.12975 & 42.57939 & 12.23103 & 14.13043 & 59.47368 \\
\hline HIS3 & YOR202W & 3 & 9 & 24 & 25.03817 & -18.2981 & 33.77483 & & \\
\hline HIS3 & YOR202W & 3 & 10 & 1 & 24.01264 & -52.6154 & 39.23166 & & \\
\hline \multirow[t]{3}{*}{ DIN7 } & YDR263C & 3 & 10 & 2 & 25.28363 & -32.1101 & 81.16147 & 40.76923 & 62.56545 \\
\hline & YDR387C & 3 & 10 & 3 & 17.66784 & -25.179 & 72.01835 & 62.10826 & 58.02817 \\
\hline & YDR262W & 3 & 10 & 4 & 36.59466 & 10.21635 & 72.93233 & 49.90792 & 52.64901 \\
\hline MUS81 & YDR386W & 3 & 10 & 5 & 21.50982 & -2.10396 & 74.48454 & 62.43386 & 60.68111 \\
\hline EXG2 & YDR261C & 3 & 10 & 6 & 46.12546 & 57.11501 & 87.64706 & 30.38585 & 66.28571 \\
\hline EFT2 & YDR385W & 3 & 10 & 7 & 1.793722 & -22.7586 & 52.27586 & 29.19255 & 57.14286 \\
\hline SWM1 & YDR260C & 3 & 10 & 8 & 7.432432 & 44.00373 & 50.16146 & 48.95666 & 56.09756 \\
\hline ATO3 & YDR384C & 3 & 10 & 9 & 8.531317 & -12.5598 & 28.07775 & 72.83142 & 44 \\
\hline YAP6 & YDR259C & 3 & 10 & 10 & 15.82524 & -0.95408 & 46.55462 & 21.92623 & 59.2233 \\
\hline NKP1 & YDR383C & 3 & 10 & 11 & 17.98299 & 24.13442 & 88.7218 & 57.08885 & 64.09396 \\
\hline HSP78 & YDR258C & 3 & 10 & 12 & 31.96061 & 49.19436 & 50.75075 & 48.35526 & 67.22222 \\
\hline RPP2B & YDR382W & 3 & 10 & 13 & 23.5687 & 49.55556 & 41.87726 & 68.68687 & 62.71186 \\
\hline SET7 & YDR257C & 3 & 10 & 14 & 38.23065 & -14.8038 & 50.0761 & 61.23235 & 49.73262 \\
\hline ARO10 & YDR380W & 3 & 10 & 15 & 13.4715 & -4.45183 & 69.03461 & 37.90614 & 52.79503 \\
\hline CTA1 & YDR256C & 3 & 10 & 16 & -25.2446 & 19.94048 & 16.47059 & 73.79913 & 51.88406 \\
\hline RGA2 & YDR379W & 3 & 10 & 17 & 25.1821 & -17.0213 & 41.36858 & 54.92958 & 49.71098 \\
\hline RMD5 & YDR255C & 3 & 10 & 18 & \#DIV/0! & 100 & 100 & 44.23676 & 44.66019 \\
\hline LSM6 & YDR378C & 3 & 10 & 19 & 83.10153 & 76.75765 & 88.43683 & 83.79747 & 82.27848 \\
\hline CHL4 & YDR254W & 3 & 10 & 20 & 9.778598 & 18.40844 & 51.40845 & 66.66667 & 58.3815 \\
\hline BCS1 & YDR375C & 3 & 10 & 21 & \#DIV/0! & \#DIV/0! & 100 & 46.25624 & 26.4214 \\
\hline \multirow[t]{2}{*}{ MET32 } & YDR253C & 3 & 10 & 22 & 11.36974 & 2.369338 & 57.72114 & 72.54623 & 55.16304 \\
\hline & YDR374C & 3 & 10 & 23 & 56.6443 & 0.414938 & 36.01896 & 1.321586 & 49.01961 \\
\hline HIS3 & YOR202W & 3 & 10 & 24 & -0.26954 & -5.52995 & 34.41926 & & \\
\hline \multirow[t]{2}{*}{ HIS3 } & YOR202W & 3 & 11 & 1 & 9.435552 & -15.2979 & 38.30334 & & \\
\hline & YDR015C & 3 & 11 & 2 & 20.58024 & 48.60853 & 53.03551 & 43.90244 & 40.16563 \\
\hline EKI1 & YDR147W & 3 & 11 & 3 & 25.13369 & 54.16991 & 64.15712 & 41.72794 & 58.80682 \\
\hline RAD61 & YDR014W & 3 & 11 & 4 & 1.849568 & -28.655 & 77.61807 & 45.79439 & 58.72093 \\
\hline
\end{tabular}




\begin{tabular}{|c|c|c|c|c|c|c|c|c|c|}
\hline SWI5 & YDR146C & 3 & 11 & 5 & -2.83019 & -8.17455 & 57.96703 & 34.7079 & 47.30878 \\
\hline SNQ2 & YDR011W & 3 & 11 & 6 & 3.444181 & 9.210526 & 53.90625 & 44.42413 & 60.11561 \\
\hline \multirow[t]{2}{*}{ MKC7 } & YDR144C & 3 & 11 & 7 & 53.97653 & 19.12 & 50.55556 & 55.57522 & 66.34921 \\
\hline & YDR010C & 3 & 11 & 8 & 67.97872 & 85.84906 & 67.04162 & 66.77686 & 82.18391 \\
\hline SAN1 & YDR143C & 3 & 11 & 9 & 7.596254 & 41.94348 & 20.40541 & 33.39921 & 57.27554 \\
\hline GAL3 & YDR009W & 3 & 11 & 10 & 8.548708 & 23.47762 & 22.28797 & 30.22847 & 50.8982 \\
\hline \multirow[t]{2}{*}{ PEX7 } & YDR142C & 3 & 11 & 11 & 12.04819 & -32.1144 & 22.40185 & 61.07872 & 48.52071 \\
\hline & YDR008C & 3 & 11 & 12 & -15.6676 & 17.50306 & 31.5528 & 23.01741 & 52.87009 \\
\hline RUB1 & YDR139C & 3 & 11 & 13 & 7.454017 & 21.09905 & 47.90823 & 75.77548 & 49 \\
\hline TRP1 & YDR007W & 3 & 11 & 14 & 45.99628 & -18.4597 & 46.99367 & 63.7084 & 57.14286 \\
\hline YCF1 & YDR135C & 3 & 11 & 15 & 43.25452 & -16.0592 & 49.62121 & 17.79026 & 43.0303 \\
\hline \multirow[t]{2}{*}{ SOK1 } & YDR006C & 3 & 11 & 16 & 57.15746 & 71.83521 & 56.93161 & 48.47162 & 72.48062 \\
\hline & YDR134C & 3 & 11 & 17 & 1.004304 & -48.0433 & 81.92771 & 37.81942 & 57.64706 \\
\hline \multirow[t]{2}{*}{ MAF1 } & YDR005C & 3 & 11 & 18 & 4.065041 & 37.81575 & 30.96774 & 68.0916 & 42.52199 \\
\hline & YDR133C & 3 & 11 & 19 & 20.34358 & -24.4681 & 32.40454 & -75.1724 & 42.18289 \\
\hline \multirow[t]{2}{*}{ RAD57 } & YDR004W & 3 & 11 & 20 & 15.23688 & 46.70051 & 56.58263 & 65.53398 & 55.35168 \\
\hline & YDR132C & 3 & 11 & 21 & 11.51079 & 24.58874 & 34.92754 & 72.61146 & 49.54128 \\
\hline \multirow[t]{2}{*}{ RCR2 } & YDR003W & 3 & 11 & 22 & 3.919781 & 25.32228 & 24.40476 & 67.89555 & 55.71429 \\
\hline & YDR131C & 3 & 11 & 23 & 67.80156 & 64.48692 & 52.9106 & 26.27451 & 56.65025 \\
\hline HIS3 & YOR202W & 3 & 11 & 24 & -37.9104 & 94.16667 & 94.41624 & & \\
\hline HIS3 & YOR202W & 3 & 12 & 1 & 8.801697 & -36.4562 & 42.78132 & & \\
\hline $\mathrm{RNH} 2 \mathrm{O} 2$ & YDR279W & 3 & 12 & 2 & 8.256881 & 44.35437 & 73.06434 & 35.18519 & 46.71533 \\
\hline \multirow[t]{2}{*}{ DIT1 } & YDR403W & 3 & 12 & 3 & 30.05025 & 54.22409 & 52.88368 & 31.19266 & 65.81197 \\
\hline & YDR278C & 3 & 12 & 4 & 8.756757 & -1.60321 & 58.77342 & 16.66667 & 35.71429 \\
\hline DIT2 & YDR402C & 3 & 12 & 5 & 12.15881 & 21.23348 & 91.46919 & 23.73541 & 61.95965 \\
\hline \multirow[t]{2}{*}{ MTH1 } & YDR277C & 3 & 12 & 6 & -8.25545 & -28.5849 & 74.26778 & 59.21288 & 55.22388 \\
\hline & YDR401W & 3 & 12 & 7 & -1.14286 & 37.96296 & 45.14563 & 51.2334 & 64.51613 \\
\hline PMP3 & YDR276C & 3 & 12 & 8 & 19.94135 & -14.2193 & 64.85671 & 38.95833 & 64.50617 \\
\hline URH1 & YDR400W & 3 & 12 & 9 & 8.856849 & -9.25291 & 37.7095 & 66.22074 & 53.77049 \\
\hline $\mathrm{BSC2}$ & YDR275W & 3 & 12 & 10 & 3.498542 & -22.2594 & 33.37629 & 76.09682 & 53.01587 \\
\hline \multirow[t]{2}{*}{ HPT1 } & YDR399W & 3 & 12 & 11 & -5.86667 & 28.31439 & 57.34127 & 48.6014 & 52.39617 \\
\hline & YDR274C & 3 & 12 & 12 & 100 & 100 & 84.48276 & 42.52199 & 67.20867 \\
\hline SXM1 & YDR395W & 3 & 12 & 13 & -5.91085 & 28.39216 & 35.04532 & 36.36364 & 51.72414 \\
\hline DON1 & YDR273W & 3 & 12 & 14 & 15.1277 & -8.76601 & 44.32432 & 64.7619 & 54.46429 \\
\hline SHE9 & YDR393W & 3 & 12 & 15 & -1.89702 & 9.073359 & 64.43089 & 54.3514 & 63.30275 \\
\hline GLO2 & YDR272W & 3 & 12 & 16 & 11.84573 & -18.3178 & 48.22955 & 56.28227 & 57.22892 \\
\hline SPT3 & YDR392W & 3 & 12 & 17 & 22.14212 & 15.28354 & 43.48485 & 21.27303 & 48.66469 \\
\hline \multirow[t]{3}{*}{$\mathrm{CCC} 2$} & YDR270W & 3 & 12 & 18 & 8.214286 & 25.68807 & 100 & 54.81481 & 48.14815 \\
\hline & YDR391C & 3 & 12 & 19 & 15.40516 & 17.64092 & 28.33517 & 68 & 49.56522 \\
\hline & YDR266C & 3 & 12 & 20 & 63.47107 & 75.09934 & 68.17043 & 59.80861 & 47.1875 \\
\hline SAC7 & YDR389W & 3 & 12 & 21 & 60.36585 & 63.48155 & 64.70588 & 81.46912 & 67.14697 \\
\hline PEX10 & YDR265W & 3 & 12 & 22 & -3.17893 & 45.04249 & 35.46284 & 19.02985 & 51.31195 \\
\hline RVS167 & YDR388W & 3 & 12 & 23 & 84.375 & 87.24761 & 90.84229 & 82.93769 & 92.66802 \\
\hline
\end{tabular}




\begin{tabular}{|c|c|c|c|c|c|c|c|c|c|}
\hline HIS3 & YOR202W & 3 & 12 & 24 & 64.49045 & -126.417 & 19.28783 & & \\
\hline \multirow[t]{2}{*}{ HIS3 } & YOR202W & 3 & 13 & 1 & 3.402062 & -11.2557 & 32.1883 & & \\
\hline & YDR056C & 3 & 13 & 2 & -8.87967 & -5.74034 & 52.25225 & 58.16024 & 51.87638 \\
\hline HOM2 & YDR158W & 3 & 13 & 3 & 14.375 & 48.01247 & 79.96109 & 53.30396 & 42.57426 \\
\hline \multirow[t]{3}{*}{ PST1 } & YDR055W & 3 & 13 & 4 & -12.1387 & -5.99051 & 63.12155 & 44.79718 & 50.2809 \\
\hline & YDR157W & 3 & 13 & 5 & -8.27225 & -9.42308 & 66.79058 & 63.50365 & 59.55056 \\
\hline & YDR051C & 3 & 13 & 6 & 54.51542 & 63.41935 & 76.45951 & 45.96273 & 71.17647 \\
\hline \multirow[t]{2}{*}{ RPA14 } & YDR156W & 3 & 13 & 7 & 65.27149 & 65.23469 & 62.66234 & 49.48454 & 70.39474 \\
\hline & YDR049W & 3 & 13 & 8 & 62.14286 & 67.10875 & 79.00943 & 74.49393 & 75.60976 \\
\hline \multirow[t]{3}{*}{ CPR1 } & YDR155C & 3 & 13 & 9 & 2.003817 & -14.3817 & 42.93981 & 44.40367 & 36.05442 \\
\hline & YDR048C & 3 & 13 & 10 & -21.9488 & 20.24117 & 46.57114 & 70.87227 & 52.32198 \\
\hline & YDR154C & 3 & 13 & 11 & 4 & 4.72973 & 28.77292 & 76.57807 & 59.74441 \\
\hline RPS11A & YDR025W & 3 & 13 & 12 & 78.63591 & 59.57728 & 60.19284 & 76.41326 & 69.69697 \\
\hline ENT5 & YDR153C & 3 & 13 & 13 & -16.6154 & 36.56286 & 48.38275 & 73.54196 & 65.11628 \\
\hline FYV1 & YDR024W & 3 & 13 & 14 & 4.049494 & -8.49571 & 74.02597 & 50.5176 & 68.09211 \\
\hline GIR2 & YDR152W & 3 & 13 & 15 & 14.32836 & 14.32927 & 16.39785 & 65.65495 & 60.40462 \\
\hline CIS1 & YDR022C & 3 & 13 & 16 & 11.60377 & -4.31803 & 48.95191 & 42.14286 & 50.96953 \\
\hline \multirow[t]{2}{*}{ CTH1 } & YDR151C & 3 & 13 & 17 & 36.82664 & 14.50243 & 10.10363 & 73.24159 & 60.69364 \\
\hline & YDR020C & 3 & 13 & 18 & 15.50947 & -8.63981 & 33.25031 & 23.25581 & 40.57508 \\
\hline NUM1 & YDR150W & 3 & 13 & 19 & 41.66667 & 43.026 & 55.69986 & 58.07692 & 52.2293 \\
\hline \multirow[t]{3}{*}{ GCV1 } & YDR019C & 3 & 13 & 20 & 46.88027 & 32.00389 & 65.64246 & 78.01527 & 69.12929 \\
\hline & YDR149C & 3 & 13 & 21 & 74.58034 & 67.37805 & 48.11321 & 79.74482 & 67.46988 \\
\hline & YDR018C & 3 & 13 & 22 & 3.030303 & 16.80585 & 38.74773 & 61.80124 & 55.29412 \\
\hline KGD2 & YDR148C & 3 & 13 & 23 & 57.3991 & -4.5292 & 36.51452 & 38.25043 & 49.60212 \\
\hline HIS3 & YOR202W & 3 & 13 & 24 & 63.66972 & -14.6435 & 49.09478 & & \\
\hline HIS3 & YOR202W & 3 & 14 & 1 & 5.623243 & -56.6908 & 55.34653 & & \\
\hline SUR2 & YDR297W & 3 & 14 & 2 & 23.78855 & 45.36082 & 85.92593 & 29.57746 & 45.87973 \\
\hline SIP1 & YDR422C & 3 & 14 & 3 & 59.90396 & -48.9879 & 82.38512 & 46.08031 & 45.42936 \\
\hline DPL1 & YDR294C & 3 & 14 & 4 & -11.2125 & 3.600655 & 78.7594 & 36.66667 & 60.72508 \\
\hline ARO80 & YDR421W & 3 & 14 & 5 & 7.172131 & 1.287303 & 56.96068 & 12.89063 & 38.32853 \\
\hline SSD1 & YDR293C & 3 & 14 & 6 & 19.66605 & 9.112855 & 79.84375 & 68.83562 & 59.17927 \\
\hline \multirow[t]{2}{*}{ HKR1 } & YDR420W & 3 & 14 & 7 & 0.567537 & -11.0236 & 74.31193 & 40 & 60.37152 \\
\hline & YDR291W & 3 & 14 & 8 & 59.13591 & 65.06986 & 59.9094 & 70.08264 & 66.5625 \\
\hline RAD30 & YDR419W & 3 & 14 & 9 & 63.27103 & 54.32024 & 65.71429 & 43.79699 & 66.14907 \\
\hline \multirow[t]{3}{*}{ RTT103 } & YDR289C & 3 & 14 & 10 & 90.06494 & 87.04503 & 88.98899 & 73.55372 & 86.34686 \\
\hline & YDR415C & 3 & 14 & 11 & 60.74219 & 61.39592 & 65.55024 & 48.61368 & 77.91411 \\
\hline & YDR287W & 3 & 14 & 12 & 43.4739 & 33.51605 & 63.09524 & 75.99388 & 60.71429 \\
\hline \multirow[t]{2}{*}{ ERD1 } & YDR414C & 3 & 14 & 13 & 13.07385 & -15.5924 & 78.81041 & 15.30782 & 51.75439 \\
\hline & YDR286C & 3 & 14 & 14 & 56.47558 & 58.31081 & 67.68559 & 56.80581 & 68.2243 \\
\hline DFM1 & YDR411C & 3 & 14 & 15 & 16 & 15.21036 & 42.40903 & 54.56204 & 60.05435 \\
\hline ZIP1 & YDR285W & 3 & 14 & 16 & -0.47304 & 32.18605 & 33.02264 & 52.94118 & 59.5092 \\
\hline STE14 & YDR410C & 3 & 14 & 17 & 59.65966 & 67.20613 & 57.31272 & 79.27565 & 77.00535 \\
\hline DPP1 & YDR284C & 3 & 14 & 18 & 14.50313 & -4.23012 & 45.71429 & 60.62718 & 57.68194 \\
\hline
\end{tabular}




\begin{tabular}{|c|c|c|c|c|c|c|c|c|c|}
\hline \multirow[t]{2}{*}{ SIZ1 } & YDR409W & 3 & 14 & 19 & -0.55741 & 26.82927 & 59.54106 & 61.97183 & 52.03252 \\
\hline & YDR282C & 3 & 14 & 20 & 8.22838 & 24.50728 & 45.22222 & 42.17687 & 53.65854 \\
\hline ADE8 & YDR408C & 3 & 14 & 21 & 80.49536 & 100 & 100 & 74.01316 & 72.63158 \\
\hline PHM6 & YDR281C & 3 & 14 & 22 & 7.039864 & 25.01961 & 37.684 & 72.40437 & 58.98058 \\
\hline PDR15 & YDR406W & 3 & 14 & 23 & 28.01418 & -68.5273 & 52.25564 & 48.24281 & 50.87719 \\
\hline HIS3 & YOR202W & 3 & 14 & 24 & 26.20482 & -31.5446 & 58.55263 & & \\
\hline HIS3 & YOR202W & 3 & 15 & 1 & -8.76804 & 50.12175 & 72.15412 & & \\
\hline IPT1 & YDR072C & 3 & 15 & 2 & 36.4539 & 14.57525 & 52.31092 & 16.04938 & 47.49403 \\
\hline CSN9 & YDR179C & 3 & 15 & 3 & -19.5426 & -26.1702 & 81.59851 & 48.49057 & 45.79208 \\
\hline PAA1 & YDR071C & 3 & 15 & 4 & 42.0904 & 40.96 & 100 & 43.28657 & 62.43781 \\
\hline $\mathrm{SDH} 4$ & YDR178W & 3 & 15 & 5 & 3.919694 & -9.84806 & 55.44218 & 36.66667 & 45.37313 \\
\hline FMP16 & YDR070C & 3 & 15 & 6 & 15.49784 & 52.61745 & 47.10221 & 34.85194 & 63.28358 \\
\hline HMO1 & YDR174W & 3 & 15 & 7 & 70.08475 & 77.34375 & 84.72222 & 37.75216 & 74.07407 \\
\hline DOS2 & YDR068W & 3 & 15 & 8 & 6.531049 & 7.54717 & 64.04908 & 62.98422 & 59.93691 \\
\hline \multirow[t]{2}{*}{ ARG82 } & YDR173C & 3 & 15 & 9 & -4.38596 & 53.28283 & 100 & 41.17647 & 33.51648 \\
\hline & YDR067C & 3 & 15 & 10 & -0.59701 & -14.4658 & 51.44828 & 42.81525 & 61.6 \\
\hline \multirow[t]{2}{*}{ HSP42 } & YDR171W & 3 & 15 & 11 & 1.548887 & -10.3862 & 44.80676 & 41.37931 & 54.70588 \\
\hline & YDR066C & 3 & 15 & 12 & 10.33569 & 9.109731 & 52.02232 & 64.03509 & 56.07477 \\
\hline \multirow[t]{2}{*}{ STB3 } & YDR169C & 3 & 15 & 13 & 17.46765 & 27.87698 & 53.63528 & 57.83972 & 68.09651 \\
\hline & YDR063W & 3 & 15 & 14 & 62.88933 & 59.19674 & 75.34538 & 81.14754 & 61.65803 \\
\hline \multirow[t]{2}{*}{ TRM82 } & YDR165W & 3 & 15 & 15 & 17.61724 & 21.81485 & 100 & 70.26627 & 57.33696 \\
\hline & YDR061W & 3 & 15 & 16 & 53.62938 & 63.13657 & 70.71353 & 63.19115 & 69.12568 \\
\hline CWC15 & YDR163W & 3 & 15 & 17 & 20.66728 & 33.13407 & 33.03685 & 31.32328 & 57.26027 \\
\hline UBC5 & YDR059C & 3 & 15 & 18 & 16.09799 & 34.68606 & 39.76608 & 75.65698 & 54.59459 \\
\hline NBP2 & YDR162C & 3 & 15 & 19 & 34.94898 & -0.92348 & 100 & 72.55689 & 60.9375 \\
\hline TGL2 & YDR058C & 3 & 15 & 20 & 5.996473 & 50.25018 & 74.17323 & 80.96591 & 73.84988 \\
\hline $\mathrm{TCl} 1$ & YDR161W & 3 & 15 & 21 & 63.33955 & 75 & 63.39492 & 35.09545 & 78.29978 \\
\hline YOS9 & YDR057W & 3 & 15 & 22 & 3.938907 & 25.25151 & 31.45161 & 67.93249 & 57.36842 \\
\hline SAC3 & YDR159W & 3 & 15 & 23 & 78.26087 & 100 & 100 & -8.01034 & 68.79699 \\
\hline HIS3 & YOR202W & 3 & 15 & 24 & 36.55914 & 5.575066 & 45.85987 & & \\
\hline HIS3 & YOR202W & 3 & 16 & 1 & -28.8398 & 11.937 & 68.88069 & & \\
\hline HIS3 & YOR202W & 3 & 16 & 2 & 99.07236 & -241.509 & 87.4552 & & \\
\hline HIS3 & YOR202W & 3 & 16 & 3 & 97.39583 & -91.6667 & 100 & & \\
\hline HIS3 & YOR202W & 3 & 16 & 4 & 53.44673 & 10.38062 & 73.05296 & & \\
\hline HIS3 & YOR202W & 3 & 16 & 5 & 2.475685 & -1.78311 & 70.03817 & & \\
\hline HIS3 & YOR202W & 3 & 16 & 6 & 12.19512 & -73.0132 & 56.79612 & & \\
\hline HIS3 & YOR202W & 3 & 16 & 7 & 13.52041 & -110.2 & 59.64286 & & \\
\hline HIS3 & YOR202W & 3 & 16 & 8 & -8.63821 & -3.15623 & 40.83333 & & \\
\hline HIS3 & YOR202W & 3 & 16 & 9 & 15.82979 & 36.89684 & 53.69198 & & \\
\hline HIS3 & YOR202W & 3 & 16 & 10 & 17.81451 & -26.3838 & 39.08046 & & \\
\hline HIS3 & YOR202W & 3 & 16 & 11 & 21.11111 & 17.93103 & 55.19031 & & \\
\hline HIS3 & YOR202W & 3 & 16 & 12 & 15.81818 & -1.48515 & 81.62252 & & \\
\hline HIS3 & YOR202W & 3 & 16 & 13 & 16.15385 & 19.47162 & 98.67987 & & \\
\hline
\end{tabular}




\begin{tabular}{|c|c|c|c|c|c|c|c|c|c|}
\hline HIS3 & YOR202W & 3 & 16 & 14 & -2.5227 & 10.42471 & 91.60714 & & \\
\hline HIS3 & YOR202W & 3 & 16 & 15 & 28.7187 & 46.57658 & 100 & & \\
\hline HIS3 & YOR202W & 3 & 16 & 16 & 2.581262 & -37.7968 & 89.92806 & & \\
\hline HIS3 & YOR202W & 3 & 16 & 17 & 9.003497 & -88.7192 & 60.76818 & & \\
\hline HIS3 & YOR202W & 3 & 16 & 18 & 81.69935 & 63.70192 & 96.42857 & & \\
\hline HIS3 & YOR202W & 3 & 16 & 19 & 24.16107 & -48.8698 & 63.85135 & & \\
\hline HIS3 & YOR202W & 3 & 16 & 20 & 15.66976 & -89.3269 & 66.29779 & & \\
\hline HIS3 & YOR202W & 3 & 16 & 21 & 20 & -4.23117 & 90.53156 & & \\
\hline HIS3 & YOR202W & 3 & 16 & 22 & 18.61371 & -126.435 & 33.33333 & & \\
\hline HIS3 & YOR202W & 3 & 16 & 23 & -17.2589 & 37.04819 & 96.05735 & & \\
\hline HIS3 & YOR202W & 3 & 16 & 24 & 31.37996 & -6.39078 & 40.2529 & & \\
\hline HIS3 & YOR202W & 4 & 1 & 1 & 47.00389 & -18.6188 & 51.75676 & & \\
\hline HIS3 & YOR202W & 4 & 1 & 2 & 75.84241 & 4.704876 & 33.6036 & & \\
\hline HIS3 & YOR202W & 4 & 1 & 3 & 42.92657 & 16.23506 & 5.930657 & & \\
\hline HIS3 & YOR202W & 4 & 1 & 4 & 72.7624 & 16.99883 & -6.3745 & & \\
\hline HIS3 & YOR202W & 4 & 1 & 5 & 32.69962 & 31.87043 & 41.77694 & & \\
\hline HIS3 & YOR202W & 4 & 1 & 6 & 9.779482 & 28.35979 & 52.51743 & & \\
\hline HIS3 & YOR202W & 4 & 1 & 7 & -2.12121 & 32.99799 & 29.24748 & & \\
\hline HIS3 & YOR202W & 4 & 1 & 8 & 18.85813 & 22.54005 & 17.0117 & & \\
\hline HIS3 & YOR202W & 4 & 1 & 9 & 25.76957 & 38.6562 & 52.57092 & & \\
\hline HIS3 & YOR202W & 4 & 1 & 10 & 3.668862 & 34.29571 & -13.2597 & & \\
\hline HIS3 & YOR202W & 4 & 1 & 11 & 11.80451 & 33.2211 & 50.39872 & & \\
\hline HIS3 & YOR202W & 4 & 1 & 12 & -18.3876 & 25.06938 & 45.72108 & & \\
\hline HIS3 & YOR202W & 4 & 1 & 13 & 1.442308 & 24.17582 & 59.35128 & & \\
\hline HIS3 & YOR202W & 4 & 1 & 14 & -16.3412 & 41.82789 & 76.18394 & & \\
\hline HIS3 & YOR202W & 4 & 1 & 15 & 14.73595 & 24.55404 & 53.89696 & & \\
\hline HIS3 & YOR202W & 4 & 1 & 16 & 15.74344 & 32.13645 & 43.85704 & & \\
\hline HIS3 & YOR202W & 4 & 1 & 17 & 9.325397 & 37.14556 & -11.053 & & \\
\hline HIS3 & YOR202W & 4 & 1 & 18 & 13.20755 & 25.05133 & 34.29724 & & \\
\hline HIS3 & YOR202W & 4 & 1 & 19 & 18.64126 & 28.06172 & 57.73969 & & \\
\hline HIS3 & YOR202W & 4 & 1 & 20 & 2.083333 & 20.57654 & 11.64221 & & \\
\hline HIS3 & YOR202W & 4 & 1 & 21 & 27.37255 & 59.19158 & 23.50978 & & \\
\hline HIS3 & YOR202W & 4 & 1 & 22 & 9.057301 & 50.73012 & 14.125 & & \\
\hline HIS3 & YOR202W & 4 & 1 & 23 & 18.90304 & 48.27255 & 14.32225 & & \\
\hline HIS3 & YOR202W & 4 & 1 & 24 & 48.021 & 48.72274 & 88.90593 & & \\
\hline HIS3 & YOR202W & 4 & 2 & 1 & 62.21353 & -5.10471 & 86.96517 & & \\
\hline \multirow[t]{2}{*}{ RPL34A } & YER056C-A & 4 & 2 & 2 & 58.00664 & 33.22185 & 82.5419 & 42.21825 & 31.23209 \\
\hline & YER158C & 4 & 2 & 3 & 54.08593 & 79.83539 & 61.48796 & 9.375 & 82.78689 \\
\hline \multirow[t]{2}{*}{$\mathrm{FCY} 2$} & YER056C & 4 & 2 & 4 & 48.84837 & 18.54839 & 43.23432 & -0.96386 & 28.75318 \\
\hline & YER156C & 4 & 2 & 5 & 12.55411 & 44.07713 & 44.44444 & 25.05176 & 44.91018 \\
\hline HIS1 & YER055C & 4 & 2 & 6 & -6.68724 & 46.80338 & 29.4964 & 3.623188 & 31.7757 \\
\hline BEM2 & YER155C & 4 & 2 & 7 & -3.68239 & 43.13454 & 60.71429 & 31.17117 & 39.74684 \\
\hline GIP2 & YER054C & 4 & 2 & 8 & -17.3436 & 31.87919 & 97.00599 & 41.35447 & 40.10152 \\
\hline
\end{tabular}




\begin{tabular}{|c|c|c|c|c|c|c|c|c|c|}
\hline PET122 & YER153C & 4 & 2 & 9 & 23.61111 & 33.46008 & 94.50549 & -12.2078 & 39.86014 \\
\hline \multirow[t]{2}{*}{ PIC2 } & YER053C & 4 & 2 & 10 & 28.33333 & 30.51044 & -12.9956 & -12.9252 & 11.62791 \\
\hline & YER152C & 4 & 2 & 11 & 17.7137 & 28.60104 & -11.6392 & 34.51677 & 52.04082 \\
\hline HOM3 & YER052C & 4 & 2 & 12 & 28.87651 & 16.98881 & 74.82517 & -6.86499 & 42.43243 \\
\hline \multirow[t]{2}{*}{ UBP3 } & YER151C & 4 & 2 & 13 & 28.38284 & 53.40659 & 10.05338 & 34.55285 & 31.83099 \\
\hline & YER051W & 4 & 2 & 14 & -11.9612 & 74.73583 & 41.19217 & 22.29885 & 49.47735 \\
\hline \multirow[t]{2}{*}{ SPI1 } & YER150W & 4 & 2 & 15 & 26.65953 & 69.61451 & 7.33945 & 1.851852 & 33.50923 \\
\hline & YER049W & 4 & 2 & 16 & -13.7584 & 40.49296 & 37.122 & 1.702128 & 40.71038 \\
\hline PEA2 & YER149C & 4 & 2 & 17 & 10.28684 & 25.55911 & 20.62827 & -15.367 & 24.03561 \\
\hline CAJ1 & YER048C & 4 & 2 & 18 & 45.73947 & 35.96491 & 63.56746 & 31.26253 & 61.94444 \\
\hline FTR1 & YER145C & 4 & 2 & 19 & 61.28457 & 44.79737 & 25.36585 & -37.9195 & 47.04225 \\
\hline SAP1 & YER047C & 4 & 2 & 20 & 24.11693 & 38.95985 & 55.77292 & -8.25472 & 42.55952 \\
\hline \multirow[t]{3}{*}{ UBP5 } & YER144C & 4 & 2 & 21 & -8.52713 & -0.46838 & -2.26284 & 39.52484 & 23.4957 \\
\hline & YER046W- & & & & & & & & \\
\hline & $A$ & 4 & 2 & 22 & 7.700205 & 37.84666 & 14.11665 & 15.81769 & 39.84962 \\
\hline DDI1 & YER143W & 4 & 2 & 23 & -22.2395 & 20.28302 & 0.981354 & 4.914934 & 40.2439 \\
\hline HIS3 & YOR202W & 4 & 2 & 24 & 18.25168 & 42.73595 & 89.84446 & & \\
\hline HIS3 & YOR202W & 4 & 3 & 1 & 47.62138 & 87.44395 & 26.08696 & & \\
\hline LRS4 & YDR439W & 4 & 3 & 2 & 58.9781 & 31.23288 & 67.9159 & 27.12477 & 44.3418 \\
\hline \multirow[t]{2}{*}{ VAC8 } & YEL013W & 4 & 3 & 3 & 33.26039 & 68.04065 & 56.82103 & 19.02834 & 48.91304 \\
\hline & YDR438W & 4 & 3 & 4 & 11.30346 & 14.05868 & 19.03553 & 28.24742 & 28.77698 \\
\hline UBC8 & YEL012W & 4 & 3 & 5 & -29.7461 & 24.41701 & 43.79371 & -3.65854 & 26.12903 \\
\hline PPZ2 & YDR436W & 4 & 3 & 6 & 2.356268 & 16.87429 & 100 & -4.94382 & 33.42318 \\
\hline GLC3 & YEL011W & 4 & 3 & 7 & 42.08754 & 21.23077 & \#DIV/0! & 27.38739 & 36.90476 \\
\hline \multirow[t]{4}{*}{ PPM1 } & YDR435C & 4 & 3 & 8 & 31.07477 & 30.85317 & 50.61263 & 13.86555 & 58.46154 \\
\hline & YEL010W & 4 & 3 & 9 & -7.46835 & 22.24797 & 38.01498 & 39.69298 & 52.54692 \\
\hline & YDR431W & 4 & 3 & 10 & -5.46512 & 20.90452 & 37.54717 & 13.99594 & 47.36842 \\
\hline & YEL008W & 4 & 3 & 11 & -12.8205 & 31.84031 & -5.7226 & 20.15504 & 51.03627 \\
\hline CYM1 & YDR430C & 4 & 3 & 12 & -18.9044 & 30.14218 & 24.47489 & 22.97297 & 45.50562 \\
\hline \multirow[t]{4}{*}{ TOS9 } & YEL007W & 4 & 3 & 13 & 39.26129 & 33.12183 & -12.3472 & 19.4211 & 37.14953 \\
\hline & YDR428C & 4 & 3 & 14 & 7.74578 & 29.6861 & 4.912664 & 22.90749 & 42.54144 \\
\hline & YEL006W & 4 & 3 & 15 & 26.31579 & 26.16339 & 41.96532 & 29.48718 & 43.53562 \\
\hline & YDR426C & 4 & 3 & 16 & 3.741153 & 46.7433 & 89.26554 & 40.67086 & 38.95487 \\
\hline VAB2 & YEL005C & 4 & 3 & 17 & 10.72961 & 50.52356 & 75.96154 & -10.7991 & 37.5 \\
\hline SNX41 & YDR425W & 4 & 3 & 18 & 7.469342 & 28.82096 & 58.47569 & 18.88298 & 35.23035 \\
\hline YEA4 & YEL004W & 4 & 3 & 19 & -47.1204 & -36.3636 & 47.27992 & -50.1511 & 34.97653 \\
\hline DYN2 & YDR424C & 4 & 3 & 20 & 2.122347 & 33.36414 & -62.3 & -59.8706 & 29.54545 \\
\hline GIM4 & YEL003W & 4 & 3 & 21 & 0.931099 & 5.660377 & -31.3237 & -98.0989 & 22.5 \\
\hline \multirow[t]{2}{*}{ CAD1 } & YDR423C & 4 & 3 & 22 & 19.39328 & 53.00752 & 52.74302 & 39.55224 & 48.83268 \\
\hline & YEL001C & 4 & 3 & 23 & 10.10309 & 45.51341 & 67.94326 & 2.857143 & 39.61456 \\
\hline HIS3 & YOR202W & 4 & 3 & 24 & 44.10256 & 55.06653 & 78.24238 & & \\
\hline \multirow[t]{2}{*}{ HIS3 } & YOR202W & 4 & 4 & 1 & 66.40954 & 25.72707 & 75.64297 & & \\
\hline & YER066W & 4 & 4 & 2 & 30.20408 & 66.18304 & 14.10658 & 19.09449 & 45.49266 \\
\hline
\end{tabular}




\begin{tabular}{|c|c|c|c|c|c|c|c|c|c|}
\hline \multirow[t]{2}{*}{ ECM32 } & YER176W & 4 & 4 & 3 & 29.07376 & 29.66507 & 72.28426 & 62.1345 & 61.63366 \\
\hline & YER066C-A & 4 & 4 & 4 & -16.0296 & 21.86495 & 86.85832 & 25.95978 & 40.90909 \\
\hline TMT1 & YER175C & 4 & 4 & 5 & 17.87026 & 26.40264 & 84.37884 & 20.65491 & 51.25786 \\
\hline ICL1 & YER065C & 4 & 4 & 6 & -9.55665 & 24.47307 & 27.76413 & 21.41328 & 61.51832 \\
\hline \multirow[t]{2}{*}{ GRX4 } & YER174C & 4 & 4 & 7 & 59.7449 & 34.80129 & 38.2199 & 27.21382 & 64.08046 \\
\hline & YER064C & 4 & 4 & 8 & -15.0171 & 49.21947 & 13.49372 & 16.08527 & 36.77812 \\
\hline RAD24 & YER173W & 4 & 4 & 9 & -4.26639 & 37.5 & 23.33736 & 18.5336 & 54.93333 \\
\hline THO1 & YER063W & 4 & 4 & 10 & 29.33962 & 67.43064 & 28.55556 & 20.625 & 40.29412 \\
\hline ADK2 & YER170W & 4 & 4 & 11 & 56.95306 & 12.9668 & 26.94878 & 19.76471 & 39.86928 \\
\hline HOR2 & YER062C & 4 & 4 & 12 & -21.7338 & 30.98592 & 22.94056 & 37.72455 & 49.7561 \\
\hline BCK2 & YER167W & 4 & 4 & 13 & 40.74749 & 33.82937 & -25.4351 & 34.19593 & 24.5098 \\
\hline CEM1 & YER061C & 4 & 4 & 14 & -9.50413 & 29.56705 & -15.7447 & 25.11013 & 28.74618 \\
\hline DNF1 & $\begin{array}{l}\text { YER166W } \\
\text { YER060W- }\end{array}$ & 4 & 4 & 15 & 23.73626 & 31.8872 & 83.77153 & 41.17647 & 62.25895 \\
\hline $\mathrm{FCY} 22$ & $A$ & 4 & 4 & 16 & 18.25095 & 41.37192 & 89.70448 & 25.80071 & 42.50681 \\
\hline CHD1 & YER164W & 4 & 4 & 17 & -9.50226 & 39.28215 & 2.478551 & 20.4797 & 51.35135 \\
\hline \multirow[t]{2}{*}{ FCY21 } & YER060W & 4 & 4 & 18 & 30.57176 & 30.39443 & -7.22772 & -33.0601 & 26.62722 \\
\hline & YER163C & 4 & 4 & 19 & 32.7451 & 49.5155 & 9.656453 & 34.67742 & 44.02174 \\
\hline PCL6 & YER059W & 4 & 4 & 20 & 24.80106 & 18.29405 & 60.14418 & 32.89037 & 42.52199 \\
\hline RAD4 & YER162C & 4 & 4 & 21 & 6.067678 & 39.54635 & 61.07784 & 7.802875 & 53.41772 \\
\hline HMF1 & YER057C & 4 & 4 & 22 & 26.63399 & 36.78756 & -8.33333 & 13.03191 & 37.15596 \\
\hline SPT2 & YER161C & 4 & 4 & 23 & -1.83099 & 40 & 24.98255 & 3.529412 & 44.55253 \\
\hline HIS3 & YOR202W & 4 & 4 & 24 & 7.042254 & 52.71238 & 47.26644 & & \\
\hline \multirow[t]{2}{*}{ HIS3 } & YOR202W & 4 & 5 & 1 & 70.61533 & 76.36917 & 91.39319 & & \\
\hline & YDR459C & 4 & 5 & 2 & 25.4689 & 49.10714 & 81.60443 & 5.010893 & 28.90855 \\
\hline \multirow[t]{2}{*}{ SPF1 } & YEL031W & 4 & 5 & 3 & -1.02669 & 33.56926 & -25.9104 & 26.86869 & 58.10056 \\
\hline & YDR458C & 4 & 5 & 4 & 14.48468 & 19.33775 & 37.36142 & 24.74227 & 50.40872 \\
\hline \multirow[t]{3}{*}{ ECM10 } & YEL030W & 4 & 5 & 5 & 20.8007 & 20.57522 & 47.48718 & 32.83262 & 65.36458 \\
\hline & YDR455C & 4 & 5 & 6 & 26.79466 & 32.92806 & -3.94456 & 31.73077 & 45.40943 \\
\hline & YEL028W & 4 & 5 & 7 & 61.17021 & 28.61668 & 53.14109 & 24.48513 & 66.47059 \\
\hline TSA2 & YDR453C & 4 & 5 & 8 & -15.7388 & 21.15183 & 76.76969 & 19.04762 & 25.1634 \\
\hline SRI1 & YEL025C & 4 & 5 & 9 & 58.37629 & 31.8593 & 77.31855 & 3.125 & 29.24528 \\
\hline \multirow[t]{2}{*}{ PPN1 } & YDR452W & 4 & 5 & 10 & -16.9897 & 32.55814 & 8.341811 & 24.26036 & 38.29114 \\
\hline & YEL023C & 4 & 5 & 11 & -9.3361 & 12.11477 & -3.55161 & 15.45624 & 40 \\
\hline \multirow[t]{2}{*}{ YHP1 } & YDR451C & 4 & 5 & 12 & 51.98556 & 16.27219 & -15.0802 & 26.74419 & 60.24845 \\
\hline & YEL020C & 4 & 5 & 13 & -0.35757 & 34.32203 & 21.04771 & 14.02439 & 24.17219 \\
\hline RPS17B & YDR447C & 4 & 5 & 14 & 35.34768 & 10.41389 & 42.94118 & 12.68293 & 63.66197 \\
\hline GTT3 & YEL017W & 4 & 5 & 15 & -12.1315 & 55.86797 & 28.06499 & $\begin{array}{l}39.4913 \\
\text { missing }\end{array}$ & 55.83756 \\
\hline ECM11 & YDR446W & 4 & 5 & 16 & -8.34236 & 57.36016 & 36.42322 & colony & missing colony \\
\hline \multirow[t]{2}{*}{ PMP2 } & YEL017C-A & 4 & 5 & 17 & -13.5897 & 63.30128 & 40.27149 & 21.82952 & 59.54198 \\
\hline & YDR445C & 4 & 5 & 18 & 39.51613 & -31.8182 & 26.12613 & 8.707124 & 54.75578 \\
\hline NPP2 & YEL016C & 4 & 5 & 19 & 64.9494 & 6.837607 & -15.8963 & 32.10084 & 55.08021 \\
\hline
\end{tabular}




\begin{tabular}{|c|c|c|c|c|c|c|c|c|c|}
\hline APT2 & YDR441C & 4 & 5 & 20 & 3.333333 & 64.84076 & 69.81318 & 42.07221 & 50.6812 \\
\hline EDC3 & YEL015W & 4 & 5 & 21 & 6.302021 & 40.44834 & 71.30996 & 43.96825 & 40.28777 \\
\hline & & & & & & & & missing & \\
\hline \multirow[t]{2}{*}{ DOT1 } & YDR440W & 4 & 5 & 22 & 12.73957 & 39.8892 & 26.92665 & $\begin{array}{l}\text { colony } \\
\text { missing }\end{array}$ & missing colony \\
\hline & YEL014C & 4 & 5 & 23 & 12.02783 & 43.83803 & 41.17205 & colony & missing colony \\
\hline HIS3 & YOR202W & 4 & 5 & 24 & 10.62147 & 62.23709 & 6.215084 & & \\
\hline \multirow[t]{7}{*}{ HIS3 } & YOR202W & 4 & 6 & 1 & 67.37912 & 13.74853 & 53.90585 & & \\
\hline & YER079W & 4 & 6 & 2 & 30.97983 & 14.068 & -14.2373 & 15.80042 & 53.62694 \\
\hline & YER187W & 4 & 6 & 3 & -12.5992 & 2.138365 & 15.98808 & -4.57516 & 47.44318 \\
\hline & YER078C & 4 & 6 & 4 & -20.6385 & 24.26901 & -12.0468 & 48.18898 & 53.9548 \\
\hline & YER186C & 4 & 6 & 5 & -19.4805 & 36.70374 & 19.43574 & 7.272727 & 33.24176 \\
\hline & YER077C & 4 & 6 & 6 & -33.3691 & 57.11785 & 30.68592 & -13.6891 & 3.311258 \\
\hline & YER185W & 4 & 6 & 7 & -25.4296 & 10.42105 & 25.3406 & 34.92366 & 59.45274 \\
\hline \multirow[t]{2}{*}{ PTP3 } & YER075C & 4 & 6 & 8 & -3.77541 & 27.12032 & -25.0305 & 21.07356 & 52.00946 \\
\hline & YER184C & 4 & 6 & 9 & 46.93069 & -4.65872 & 24.2126 & 33.56282 & 27.73973 \\
\hline RPS24A & YER074W & 4 & 6 & 10 & 67.79661 & 70.05076 & -33.8583 & 19.83914 & 43.81625 \\
\hline FAU1 & YER183C & 4 & 6 & 11 & 52.94651 & -1.34228 & 3.346457 & 24.44444 & 51.98864 \\
\hline ALD5 & YER073W & 4 & 6 & 12 & 18.51041 & 23.88831 & 30.70624 & 35.2 & 48.13953 \\
\hline FMP10 & YER182W & 4 & 6 & 13 & 2.250804 & 78.81874 & -25.5162 & 28.48948 & 52.72206 \\
\hline \multirow[t]{3}{*}{ VTC1 } & YER072W & 4 & 6 & 14 & 32.64971 & 77.2348 & 19.43794 & 39.01515 & 59.00783 \\
\hline & YER181C & 4 & 6 & 15 & -6.07662 & 62.2449 & 12.96061 & 27.54159 & 65.88542 \\
\hline & YER071C & 4 & 6 & 16 & 0 & 36.4073 & 7.128906 & 37.12687 & 67.25441 \\
\hline ISC10 & YER180C & 4 & 6 & 17 & 4.11985 & 5.863539 & 23.63782 & 18.18182 & 47.49263 \\
\hline ARG5,6 & YER069W & 4 & 6 & 18 & 36.97318 & 2.196382 & 36.88458 & 16.06765 & 60.66838 \\
\hline \multirow[t]{2}{*}{ DMC1 } & YER179W & 4 & 6 & 19 & -14.2526 & 80.41475 & 34.09091 & -17.7419 & 58.28729 \\
\hline & YER067W & 4 & 6 & 20 & 1.604938 & 18.41808 & 66.02349 & -78.1145 & 43.10345 \\
\hline \multirow[t]{2}{*}{ PDA1 } & YER178W & 4 & 6 & 21 & -10.7558 & 33.81783 & 46.49682 & 35.52124 & 33.50785 \\
\hline & YER067C-A & 4 & 6 & 22 & 17.69088 & 40.49793 & 48.26255 & 23.57895 & 59.29978 \\
\hline BMH1 & YER177W & 4 & 6 & 23 & 0.75188 & 41.47287 & 64.72632 & 34.77654 & 53.84615 \\
\hline HIS3 & YOR202W & 4 & 6 & 24 & 3.456221 & 46.08939 & 34.06088 & & \\
\hline HIS3 & YOR202W & 4 & 7 & 1 & 80.89021 & 32.92181 & 39.91131 & & \\
\hline DIG2 & YDR480W & 4 & 7 & 2 & 17.85174 & 27.62078 & 39.70588 & 8.458647 & 25.69061 \\
\hline PAU2 & YEL049W & 4 & 7 & 3 & 29.76 & 24.48378 & 12.30248 & 20.11834 & 54.59941 \\
\hline \multirow[t]{4}{*}{ PEX29 } & YDR479C & 4 & 7 & 4 & 15.69468 & 15.0289 & -5.20305 & 19.375 & 56.14035 \\
\hline & YEL048C & 4 & 7 & 5 & 16.03943 & 17.22973 & 45.56355 & 16.02434 & 51.56695 \\
\hline & YDR476C & 4 & 7 & 6 & 17.29921 & 28.1893 & 54.01015 & 19.07631 & 54.57143 \\
\hline & YEL047C & 4 & 7 & 7 & -16.8186 & 34.84556 & 65.5102 & 31.11111 & 41.44144 \\
\hline \multirow[t]{3}{*}{ JIP4 } & YDR475C & 4 & 7 & 8 & 13.03441 & 45 & 68.62197 & 39.35599 & 65.84767 \\
\hline & YEL043W & 4 & 7 & 9 & 46.26866 & 25.51963 & 2.803738 & 38.87097 & 52.35602 \\
\hline & YDR474C & 4 & 7 & 10 & 29.59685 & 34.64674 & 19.1022 & 35.96987 & 61.75711 \\
\hline GDA1 & YEL042W & 4 & 7 & 11 & 71.89752 & 71.15578 & 100 & 35.35912 & 61.85819 \\
\hline RPL27B & YDR471W & 4 & 7 & 12 & 7.18232 & 40.1662 & \#DIV/O! & 23.35766 & 48.78788 \\
\hline
\end{tabular}




\begin{tabular}{|c|c|c|c|c|c|c|c|c|c|}
\hline & YEL041W & 4 & 7 & 13 & -17.2973 & 1.234568 & 14.94755 & 33.55932 & 49.0566 \\
\hline SDC1 & YDR469W & 4 & 7 & 14 & -6.05565 & 3.759398 & -5.19878 & 0.683761 & 16.66667 \\
\hline \multirow[t]{2}{*}{ UTR2 } & YEL040W & 4 & 7 & 15 & 35.30633 & 28.6115 & 6.542056 & 25.7732 & 65.09186 \\
\hline & YDR467C & 4 & 7 & 16 & 43.04348 & 1.096224 & 35.82231 & 38.06452 & 52.1978 \\
\hline CYC7 & YEL039C & 4 & 7 & 17 & 3.3241 & 52.01794 & 37.82051 & 28.86364 & 52.18579 \\
\hline PKH3 & YDR466W & 4 & 7 & 18 & -21.4195 & 20.04111 & 32.62673 & 57.69746 & 57.27003 \\
\hline UTR4 & YEL038W & 4 & 7 & 19 & 36.83333 & 28.66593 & 32.41252 & 25 & 59.21788 \\
\hline RMT2 & YDR465C & 4 & 7 & 20 & 29.45129 & 27.08963 & 2.665441 & 24.00835 & 45.50898 \\
\hline RAD23 & YEL037C & 4 & 7 & 21 & 24.53855 & 25.38803 & 2.840909 & 16.46489 & 60.57143 \\
\hline \multirow[t]{2}{*}{ STP1 } & YDR463W & 4 & 7 & 22 & 20.99502 & 45.85586 & 19.80392 & 16.39344 & 57.30994 \\
\hline & YEL033W & 4 & 7 & 23 & 21.20867 & 16.88574 & 44.60285 & 12.29839 & 44.27646 \\
\hline HIS3 & YOR202W & 4 & 7 & 24 & 25.50053 & 52.38829 & 40.93087 & & \\
\hline HIS3 & YOR202W & 4 & 8 & 1 & 78.31764 & 25.22167 & 46.76923 & & \\
\hline IES5 & YER092W & 4 & 8 & 2 & 23.96761 & 33.48018 & 66.16279 & 25.8 & 47.38041 \\
\hline \multirow[t]{2}{*}{ BUD27 } & YFL023W & 4 & 8 & 3 & -141.896 & 35.21127 & -36.3636 & -47.4201 & 25.67568 \\
\hline & YER091C-A & 4 & 8 & 4 & -9.93184 & 65.19608 & 30.76923 & 13.7931 & 48.42767 \\
\hline GAT1 & YFL021W & 4 & 8 & 5 & -12.013 & 29.6861 & 61.28342 & 31.06061 & 44.77612 \\
\hline MET6 & YER091C & 4 & 8 & 6 & -16.4309 & 26.29162 & 39.32773 & 13.50211 & 44.07713 \\
\hline PAU5 & YFL020C & 4 & 8 & 7 & 9.519321 & 39.26219 & -37.8007 & 30.11811 & 54.52055 \\
\hline \multirow[t]{2}{*}{ TRP2 } & YER090W & 4 & 8 & 8 & -11.4815 & 29.43495 & 20.37267 & 10.88889 & 47.48603 \\
\hline & YFL019C & 4 & 8 & 9 & -21.7848 & 50.19157 & -26.053 & 18.08732 & 33.02181 \\
\hline DOT6 & YER088C & 4 & 8 & 10 & -5.08772 & 71.46341 & 37.29476 & 14.64355 & 46.875 \\
\hline \multirow[t]{4}{*}{ LPD1 } & YFL018C & 4 & 8 & 11 & 34.0535 & -16.9643 & 86.84417 & 16.66667 & 48.91304 \\
\hline & YER087C-A & 4 & 8 & 12 & 40.56848 & 24.65753 & 82.59325 & 32.14953 & 62.5323 \\
\hline & YFL015C & 4 & 8 & 13 & 56.17761 & -17.0951 & 15.87444 & 36.25498 & 50.52632 \\
\hline & YER085C & 4 & 8 & 14 & 54.08271 & -18.9189 & 10.57082 & 33.57664 & 54.73684 \\
\hline \multirow[t]{4}{*}{ HSP12 } & YFL014W & 4 & 8 & 15 & 5.263158 & 52.74725 & 11.08491 & 7.114625 & 48.29545 \\
\hline & YER084W & 4 & 8 & 16 & -14.7465 & 13.10345 & 21.34387 & 16.91649 & 48.5342 \\
\hline & YFL013W- & & & & & & & & \\
\hline & $A$ & 4 & 8 & 17 & 22.125 & 32.92434 & 4.362416 & 32.77467 & 50.2809 \\
\hline RMD7 & YER083C & 4 & 8 & 18 & -11.9178 & 51.36612 & 21.51724 & -7.173 & 45.63953 \\
\hline HXT10 & YFL011W & 4 & 8 & 19 & 14.67136 & 54.63415 & 42.86798 & -5.7554 & 55.52239 \\
\hline \multirow[t]{2}{*}{ SER3 } & YER081W & 4 & 8 & 20 & 13.52718 & 5.176471 & 25.39803 & 31.3912 & 53.64078 \\
\hline & YFL006W & 4 & 8 & 21 & 2.888087 & 31.41724 & -18.8641 & -4.23529 & 56.71233 \\
\hline \multirow[t]{2}{*}{ FMP29 } & YER080W & 4 & 8 & 22 & 33.75796 & 33.49802 & 43.64876 & -26.9022 & 50 \\
\hline & YER188W & 4 & 8 & 23 & 2.078775 & 31.18173 & 47.99371 & 36 & 42.82116 \\
\hline HIS3 & YOR202W & 4 & 8 & 24 & -4.82063 & 40.66047 & 66.27566 & & \\
\hline HIS3 & YOR202W & 4 & 9 & 1 & 60.13913 & 35.18687 & 50.9396 & & \\
\hline PUF6 & YDR496C & 4 & 9 & 2 & 49.05231 & 26.38623 & 28.79684 & -1.72414 & 54.1779 \\
\hline SIT1 & YEL065W & 4 & 9 & 3 & -19.196 & 28.34275 & 63.87795 & 34.29119 & 56.49547 \\
\hline RSM28 & YDR494W & 4 & 9 & 4 & 19.10569 & 63.70614 & 63.40206 & 27.63419 & 62.53687 \\
\hline AVT2 & YEL064C & 4 & 9 & 5 & -10.9312 & 24.08088 & 5.847374 & 25.28217 & 57.75862 \\
\hline IZH1 & YDR492W & 4 & 9 & 6 & -2.17177 & 12.51462 & 7.5 & 11.63265 & 46.03659 \\
\hline
\end{tabular}




\begin{tabular}{|c|c|c|c|c|c|c|c|c|c|}
\hline \multirow[t]{2}{*}{ CAN1 } & YEL063C & 4 & 9 & 7 & -23.0203 & 2.378593 & 84.90153 & 21.01167 & 57.55968 \\
\hline & YDR491C & 4 & 9 & 8 & -10.3093 & 43.85475 & 77.70598 & 3.27553 & 42.74406 \\
\hline NPR2 & YEL062W & 4 & 9 & 9 & -6.17284 & 26.90289 & -12.3177 & 34.20479 & 59.81873 \\
\hline PKH1 & YDR490C & 4 & 9 & 10 & 48.89217 & 38.04124 & 33.52657 & 35.90226 & 50 \\
\hline CIN8 & YEL061C & 4 & 9 & 11 & 59.91837 & -2.13483 & 37.3913 & 71.67139 & 66.97248 \\
\hline PAC11 & YDR488C & 4 & 9 & 12 & 0 & 46.02273 & 30.11078 & 18.82771 & 40.36697 \\
\hline PRB1 & YEL060C & 4 & 9 & 13 & 5.247525 & 40.4321 & -5.25253 & 27.16763 & 63.68421 \\
\hline \multirow[t]{2}{*}{ VPS60 } & YDR486C & 4 & 9 & 14 & 68.68178 & 36.08247 & 10.89682 & 21.31439 & 26.97368 \\
\hline & YEL059W & 4 & 9 & 15 & -3.79363 & 21.12403 & -2.14592 & 5.13347 & 49.11765 \\
\hline \multirow[t]{2}{*}{ VPS72 } & YDR485C & 4 & 9 & 16 & 1.090909 & 27.77086 & 67.01681 & 29.35421 & 52.05882 \\
\hline & YEL057C & 4 & 9 & 17 & 3.214286 & 58.5335 & 68.54271 & 40.9919 & 56.02837 \\
\hline KRE2 & YDR483W & 4 & 9 & 18 & 11.85567 & 32.26453 & 3.692615 & 20.68966 & 51.19048 \\
\hline HAT2 & YEL056W & 4 & 9 & 19 & -28.9256 & 28.21918 & -20.8205 & 28.39757 & 52.25225 \\
\hline CWC21 & YDR482C & 4 & 9 & 20 & 17.91831 & 13.50614 & 24.2141 & 22.98025 & 46.01227 \\
\hline MAK10 & YEL053C & 4 & 9 & 21 & 9.42883 & 53.35917 & 15.63981 & -13.5266 & 32.91139 \\
\hline $\mathrm{PHO} 8$ & YDR481C & 4 & 9 & 22 & 40.16736 & 28.34862 & -24.4065 & -3.08483 & 48.34025 \\
\hline AFG1 & YEL052W & 4 & 9 & 23 & 34.25414 & 13.91129 & 18.11263 & 50.05767 & 55.95745 \\
\hline HIS3 & YOR202W & 4 & 9 & 24 & 25.2193 & 31.54185 & 56.49351 & & \\
\hline \multirow[t]{2}{*}{ HIS3 } & YOR202W & 4 & 10 & 1 & 50.86745 & 42.17311 & 61.53846 & & \\
\hline & YER113C & 4 & 10 & 2 & -19.285 & 17.72031 & 65.88694 & 23.29247 & 58.41584 \\
\hline RPO41 & YFL036W & 4 & 10 & 3 & 41.08216 & 51.77305 & 75.24613 & 11.25541 & 55.62701 \\
\hline \multirow[t]{2}{*}{ SWI4 } & YER111C & 4 & 10 & 4 & 22.25806 & 47.74436 & -9.53058 & 12.98932 & 52 \\
\hline & YFL035C-B & 4 & 10 & 5 & -13.8756 & 66.73511 & 65.67164 & 19.04762 & 37.36264 \\
\hline \multirow[t]{3}{*}{ FLO8 } & YER109C & 4 & 10 & 6 & -4.64015 & 33.70661 & 20.0995 & 16.38298 & 57.70393 \\
\hline & YFL034W & 4 & 10 & 7 & -5.96095 & 30.30973 & 16.40625 & 17.99591 & 52.0436 \\
\hline & YER108C & 4 & 10 & 8 & -14.4309 & 52.68426 & 14.28571 & 15.20343 & 54.6875 \\
\hline RPL22B & YFL034C-A & 4 & 10 & 9 & 2.898551 & 17.9635 & -10.2926 & 43.36973 & 61.2987 \\
\hline \multirow[t]{2}{*}{ MAM1 } & YER106W & 4 & 10 & 10 & 3.739316 & 27.29026 & 6.567993 & 17.71429 & 57.58514 \\
\hline & YFL032W & 4 & 10 & 11 & 4.536489 & 37.22268 & 11.39742 & 23.67906 & 44.65753 \\
\hline AST2 & YER101C & 4 & 10 & 12 & 13.47785 & 61.91388 & 94.35666 & 17.87709 & 54.75504 \\
\hline HAC1 & YFL031W & 4 & 10 & 13 & 12.28758 & 76.26208 & 92.51269 & 30 & 53.38542 \\
\hline UBP9 & YER098W & 4 & 10 & 14 & 26.95167 & 37.43891 & -6.06827 & 22.04724 & 55.30726 \\
\hline \multirow[t]{2}{*}{ AGX1 } & YFL030W & 4 & 10 & 15 & 12.76371 & 39.38815 & 7.515152 & 13.35953 & 52.33161 \\
\hline & YER097W & 4 & 10 & 16 & 16.04343 & 5.030675 & 33.24492 & 29.30403 & 59.22865 \\
\hline CAF16 & YFL028C & 4 & 10 & 17 & 27.03349 & 18.85813 & -5.13274 & 24.81752 & 50.28571 \\
\hline SHC1 & YER096W & 4 & 10 & 18 & 61.43451 & 50.10438 & 24.68301 & 31.41264 & 51.29683 \\
\hline GYP8 & YFL027C & 4 & 10 & 19 & 43.12115 & 34.59621 & 72.63514 & 33.55482 & 61.73184 \\
\hline RAD51 & YER095W & 4 & 10 & 20 & 31.98483 & 58.17942 & 67.5 & 30.73286 & 61.7737 \\
\hline \multirow[t]{2}{*}{ STE2 } & YFL026W & 4 & 10 & 21 & 37.48309 & 25.52764 & 13.47352 & 17.8634 & 51.85185 \\
\hline & YER093C-A & 4 & 10 & 22 & 13.54467 & 30.4753 & 48.59729 & 1.096491 & 43.37349 \\
\hline BST1 & YFL025C & 4 & 10 & 23 & 30.3932 & 18.46154 & 43.89439 & -1.59091 & 69.11111 \\
\hline HIS3 & YOR202W & 4 & 10 & 24 & 14.81769 & 19.10891 & 42.22503 & & \\
\hline HIS3 & YOR202W & 4 & 11 & 1 & 50.67265 & 33.09735 & 34.21053 & & \\
\hline
\end{tabular}




\begin{tabular}{|c|c|c|c|c|c|c|c|c|c|}
\hline \multirow[t]{2}{*}{ EMI1 } & YDR512C & 4 & 11 & 2 & 49.3653 & 68.83886 & 56.39713 & 10.12346 & 41.09589 \\
\hline & YER010C & 4 & 11 & 3 & 17.89849 & 25.71711 & 34.03827 & 39.02878 & 56.5097 \\
\hline ACN9 & YDR511W & 4 & 11 & 4 & -18.4341 & 19.97608 & -11.828 & 4.852321 & 58.90805 \\
\hline \multirow[t]{2}{*}{ PAC2 } & YER007W & 4 & 11 & 5 & -11.6183 & 32.16783 & 77.09191 & 24.40945 & 50.71429 \\
\hline & YDR509W & 4 & 11 & 6 & 33.68321 & 25.12563 & 79.40447 & 18.03607 & 45.3125 \\
\hline RBF20 & YER007C-A & 4 & 11 & 7 & 23.79836 & 59.06563 & 35.36737 & 22.68519 & 62.71777 \\
\hline GNP1 & YDR508C & 4 & 11 & 8 & -8.07985 & 30.98592 & 7.420925 & 28.51563 & 56.08309 \\
\hline \multirow[t]{2}{*}{ YND1 } & YER005W & 4 & 11 & 9 & 4.424779 & 40.29268 & 6.982872 & 16.06119 & 43.1085 \\
\hline & YDR506C & 4 & 11 & 10 & -17.7802 & 61.45952 & 12.89398 & 16.17934 & 55.43478 \\
\hline FMP52 & YER004W & 4 & 11 & 11 & 0.992063 & 20.64777 & -17.2078 & 20.75848 & 57.26027 \\
\hline PSP1 & YDR505C & 4 & 11 & 12 & 15.54252 & 26.97517 & 23.67688 & 40.625 & 60.4712 \\
\hline NOP16 & YER002W & 4 & 11 & 13 & 13.74607 & 42.11618 & 46.25984 & -1.2285 & 51.72414 \\
\hline SPG3 & YDR504C & 4 & 11 & 14 & 11.33909 & 23.33664 & 5.934718 & 19.88304 & 52.02312 \\
\hline MNN1 & YER001W & 4 & 11 & 15 & -24.0994 & 60.78431 & -28.0462 & 39.46281 & 63.4058 \\
\hline LPP1 & YDR503C & 4 & 11 & 16 & 12.90323 & 32.46862 & 15.10673 & 16.2 & 61.40845 \\
\hline DLD3 & YEL071W & 4 & 11 & 17 & 15.10791 & 34.27992 & -26.7892 & 10.23622 & 32.20859 \\
\hline \multirow[t]{2}{*}{ PLM2 } & YDR501W & 4 & 11 & 18 & -6.07735 & -19.9074 & 0.228833 & 10.02132 & 55.59322 \\
\hline & YEL068C & 4 & 11 & 19 & -27.9762 & 30.13699 & 63.48358 & 12.5 & 43.62018 \\
\hline \multirow[t]{2}{*}{ RPL37B } & YDR500C & 4 & 11 & 20 & 34.70375 & 42.34592 & 61.16751 & 14.58774 & 36.56958 \\
\hline & YEL067C & 4 & 11 & 21 & 46.23172 & 27.64538 & 73.36726 & 17.46988 & 37.42857 \\
\hline ITR1 & YDR497C & 4 & 11 & 22 & 18.54584 & 45.10638 & 72.01426 & 27.89784 & 52.85714 \\
\hline HPA3 & YEL066W & 4 & 11 & 23 & 31.41684 & 38.11252 & 1.477833 & 4.283054 & 47.70408 \\
\hline HIS3 & YOR202W & 4 & 11 & 24 & -24.7059 & 43.82239 & 70.16667 & & \\
\hline HIS3 & YOR202W & 4 & 12 & 1 & 51.58255 & 31.79396 & 56.50759 & & \\
\hline \multirow[t]{2}{*}{ DSE1 } & YER124C & 4 & 12 & 2 & 25.93134 & 58.42199 & 79.25 & 13.33333 & 47.42857 \\
\hline & YFL051C & 4 & 12 & 3 & 42.3913 & 28.09917 & 31.53846 & 16.7002 & 56.72043 \\
\hline YCK3 & YER123W & 4 & 12 & 4 & 24.91349 & 42.9703 & 82.59669 & $\begin{array}{l}2.886598 \\
\text { missing }\end{array}$ & 31.49847 \\
\hline \multirow[t]{3}{*}{ ALR2 } & YFL050C & 4 & 12 & 5 & -13.6232 & 25.34949 & -19.6453 & colony & missing colony \\
\hline & YER121W & 4 & 12 & 6 & -15.493 & 25.45455 & 44.18872 & -3.4 & 53.00261 \\
\hline & YFL049W & 4 & 12 & 7 & -17.2483 & 44.0678 & -56.7797 & 27.1719 & 54.01662 \\
\hline SCS2 & YER120W & 4 & 12 & 8 & -4.02235 & 46.47713 & -36.5854 & 18.59956 & 46.24625 \\
\hline \multirow[t]{2}{*}{ EMP47 } & YFL048C & 4 & 12 & 9 & -9.11885 & 35.29412 & 34.39364 & 30.76923 & 57.52212 \\
\hline & YER119C-A & 4 & 12 & 10 & -25.93 & 38.8309 & 1.969697 & 24.06716 & 47.94521 \\
\hline RGD2 & YFL047W & 4 & 12 & 11 & 13.28273 & 42.12638 & 1.538462 & 16.46341 & 52.02492 \\
\hline AVT6 & YER119C & 4 & 12 & 12 & 14.14822 & 45.41485 & -50.078 & 23.02026 & 51.04478 \\
\hline FMP32 & YFL046W & 4 & 12 & 13 & 7.755102 & 56.70103 & -5.30697 & 15.32417 & 47.10526 \\
\hline SHO1 & YER118C & 4 & 12 & 14 & 7.723577 & 63.59712 & -8.11881 & 22.20183 & 51.36778 \\
\hline YOD1 & YFL044C & 4 & 12 & 15 & 12.89662 & 31.8018 & 21.00917 & 24.36647 & 49.18919 \\
\hline \multirow[t]{2}{*}{ RPL23B } & YER117W & 4 & 12 & 16 & 11.61357 & 35.50792 & 20.50985 & 24.49355 & 43.9528 \\
\hline & YFL043C & 4 & 12 & 17 & 15.13859 & 31.15488 & 34.59854 & 31.05263 & 54.69613 \\
\hline \multirow[t]{2}{*}{ SLX8 } & YER116C & 4 & 12 & 18 & 17.05336 & 64.57801 & -56 & 41.73355 & 55 \\
\hline & YFL042C & 4 & 12 & 19 & 20.74264 & -7.02782 & 40.93567 & 23.64991 & 52.78515 \\
\hline
\end{tabular}




\begin{tabular}{|c|c|c|c|c|c|c|c|c|c|}
\hline SPR6 & YER115C & 4 & 12 & 20 & 31.68539 & 18.20866 & -4.34419 & 22.06897 & 46.58754 \\
\hline FET5 & YFL041W & 4 & 12 & 21 & 45.83772 & 33.03835 & 16.46586 & 23.81786 & 53.27314 \\
\hline \multirow[t]{2}{*}{$\mathrm{BOI} 2$} & YER114C & 4 & 12 & 22 & 37.89238 & 31.919 & -14.2171 & 14.80144 & 43.82716 \\
\hline & YFL040W & 4 & 12 & 23 & 35.06944 & 33.04264 & 11.57718 & 9.407666 & 43.08943 \\
\hline HIS3 & YOR202W & 4 & 12 & 24 & -25.9115 & 5.995475 & 69.03409 & & \\
\hline HIS3 & YOR202W & 4 & 13 & 1 & 63.89481 & 35.22415 & 21.54255 & & \\
\hline HLR1 & YDR528W & 4 & 13 & 2 & 12.35741 & 1.853659 & 68.84368 & 13.75 & 54.68354 \\
\hline FIR1 & $\begin{array}{l}\text { YER032W } \\
\text { YDR525W- }\end{array}$ & 4 & 13 & 3 & 50.67751 & 22.63451 & 49.19441 & 21.20658 & 55.29716 \\
\hline SNA2 & $A$ & 4 & 13 & 4 & -25.5396 & 29.44497 & 41.80887 & 27.01149 & 55.80737 \\
\hline YРT31 & YER031C & 4 & 13 & 5 & 46.59091 & 68.56095 & 61.63194 & 32.61649 & 72.97297 \\
\hline \multirow[t]{2}{*}{ API2 } & YDR525W & 4 & 13 & 6 & -34.189 & 31.15942 & 18.37184 & 19.03915 & 39.03904 \\
\hline & YER030W & 4 & 13 & 7 & 11.60799 & 23.99173 & 86.0262 & 40.22556 & 41.63701 \\
\hline AGE1 & YDR524C & 4 & 13 & 8 & 38.58425 & 41.95223 & 80.0638 & 47.23618 & 62.67123 \\
\hline MIG3 & YER028C & 4 & 13 & 9 & -2.81442 & 19.33842 & 45.30744 & 19.23077 & 51.01351 \\
\hline SPS2 & YDR522C & 4 & 13 & 10 & 13.18052 & 31.66144 & 86.58892 & 26.80608 & 66.85552 \\
\hline \multirow[t]{2}{*}{ GAL83 } & YER027C & 4 & 13 & 11 & 29.88166 & 32.57143 & 55.84551 & 35.65217 & 47.1519 \\
\hline & YDR520C & 4 & 13 & 12 & 9.210526 & 35.81267 & 27.21519 & 51.65945 & 57.06806 \\
\hline YAT2 & YER024W & 4 & 13 & 13 & 15.34604 & 40.89636 & 35.53488 & 0.458716 & 45.03546 \\
\hline FPR2 & YDR519W & 4 & 13 & 14 & 8.070866 & 3.9783 & 27.5766 & 51.0917 & 50.74627 \\
\hline GPA2 & YER020W & 4 & 13 & 15 & 17.67442 & 58.83669 & 30.78292 & 4.960836 & 58.97436 \\
\hline GRH1 & YDR517W & 4 & 13 & 16 & 13.70656 & 51.43113 & 86.49374 & 27.7972 & 57.34463 \\
\hline ISC1 & YER019W & 4 & 13 & 17 & 35.96392 & 58.93588 & 88.32449 & 19.34827 & 62.53776 \\
\hline EMI2 & YDR516C & 4 & 13 & 18 & 17.95918 & 30.75221 & 23.23462 & 17.81609 & 61.95652 \\
\hline \multirow[t]{2}{*}{$\mathrm{SBH} 2$} & YER019C-A & 4 & 13 & 19 & -17.1983 & 45.92902 & 13.60996 & -0.97847 & 39.70149 \\
\hline & YDR514C & 4 & 13 & 20 & 28.87113 & 34.55357 & 21.13353 & 37.16338 & 50.75529 \\
\hline BIM1 & YER016W & 4 & 13 & 21 & 49.38272 & 19.60094 & 36.76923 & 51.53846 & 46.73913 \\
\hline TTR1 & YDR513W & 4 & 13 & 22 & 27.8534 & 35.89512 & 6.114551 & 53.82309 & 52.48042 \\
\hline TIR1 & YER011W & 4 & 13 & 23 & 26.25251 & 39.33464 & 17.36282 & 24.8227 & 56.52174 \\
\hline HIS3 & YOR202W & 4 & 13 & 24 & 15.7377 & 24.97551 & 51.44566 & & \\
\hline HIS3 & YOR202W & 4 & 14 & 1 & 72.54545 & 34.72851 & -16.3449 & & \\
\hline \multirow[t]{3}{*}{ MAG1 } & YER142C & 4 & 14 & 2 & 43.36043 & 22.66527 & 84.74388 & 15.93928 & 48.69792 \\
\hline & YFR011C & 4 & 14 & 3 & 80.51146 & 14.38596 & 73.88535 & 33.05085 & 66.00567 \\
\hline & YER140W & 4 & 14 & 4 & 33.06808 & 26.12782 & 62.5 & 15.47085 & 46.75676 \\
\hline \multirow[t]{2}{*}{ UBP6 } & YFR010W & 4 & 14 & 5 & 79.61433 & 87.11712 & 86.26016 & 48.12968 & 91.17647 \\
\hline & YER139C & 4 & 14 & 6 & -9.33192 & 19.67213 & 28.52853 & -0.47393 & 57.76398 \\
\hline \multirow[t]{2}{*}{ GCN20 } & YFR009W & 4 & 14 & 7 & -3.85701 & 61.78571 & 34.72803 & 32.08333 & 66.56977 \\
\hline & YER137C & 4 & 14 & 8 & 7.785642 & 35.97884 & 78.02817 & 46.81208 & 52.81899 \\
\hline \multirow[t]{5}{*}{ FAR7 } & YFR008W & 4 & 14 & 9 & 7.692308 & 71.98748 & 80.10336 & 16.92015 & 57.18563 \\
\hline & YER135C & 4 & 14 & 10 & 71.10266 & 59.23345 & 73.36815 & 70.11129 & 73.59155 \\
\hline & YFR007W & 4 & 14 & 11 & 5.079962 & 55.01406 & -64.3002 & 24.73118 & 50.92025 \\
\hline & YER134C & 4 & 14 & 12 & 19.08326 & 26.64418 & 46.54088 & 19.8895 & 60.89385 \\
\hline & YFR006W & 4 & 14 & 13 & 14.44653 & 44.20485 & 59.25926 & 31.25 & 53.125 \\
\hline
\end{tabular}




\begin{tabular}{|c|c|c|c|c|c|c|c|c|c|}
\hline PMD1 & YER132C & 4 & 14 & 14 & 18.64245 & 45.26219 & 5.371094 & 25.4 & 64.36782 \\
\hline AAD6 & YFL056C & 4 & 14 & 15 & 12.67327 & 33.05989 & 43.00412 & 33.72681 & 47.53623 \\
\hline RPS26B & YER131W & 4 & 14 & 16 & 12.61352 & 40.26095 & 57.31895 & 55.40741 & 59.71831 \\
\hline \multirow[t]{3}{*}{ AGP3 } & YFL055W & 4 & 14 & 17 & 21.40733 & 71.09458 & 6.671839 & 56.81818 & 32.80255 \\
\hline & YER130C & 4 & 14 & 18 & 12.82932 & 47.59036 & 3.078203 & 6.438632 & 51.76152 \\
\hline & YFL054C & 4 & 14 & 19 & -1.57593 & 51.66994 & 62.0155 & 61.10363 & 51.73333 \\
\hline PAK1 & YER129W & 4 & 14 & 20 & 28.85246 & 17.49436 & 43.91192 & 16.89861 & 49.36387 \\
\hline \multirow[t]{3}{*}{ DAK2 } & YFL053W & 4 & 14 & 21 & 14.06571 & 31.56398 & -29.6199 & 48.8408 & 53.33333 \\
\hline & YER128W & 4 & 14 & 22 & 39.42505 & 38.08219 & 65.27919 & 13.38028 & 50.25381 \\
\hline & YFL052W & 4 & 14 & 23 & 25.07375 & 37.88634 & 38.08664 & 27.5641 & 60.43478 \\
\hline HIS3 & YOR202W & 4 & 14 & 24 & 10.07634 & 19.41748 & -38.0866 & & \\
\hline \multirow[t]{2}{*}{ HIS3 } & YOR202W & 4 & 15 & 1 & 78.71377 & 41.06176 & 8.16092 & & \\
\hline & YDR541C & 4 & 15 & 2 & 49.51644 & 29.07735 & 84.41397 & 30.41958 & 57.03883 \\
\hline \multirow[t]{2}{*}{ SPO73 } & YER046W & 4 & 15 & 3 & 76.44444 & 28.29028 & 58.69565 & 44.21053 & 59.88701 \\
\hline & YDR540C & 4 & 15 & 4 & 23.7037 & 33.98169 & 56.6719 & 29.10663 & 64.56954 \\
\hline \multirow[t]{2}{*}{ ACA1 } & YER045C & 4 & 15 & 5 & 46.62111 & -31.3758 & 95.03106 & 17.03854 & 63.11475 \\
\hline & YDR539W & 4 & 15 & 6 & -26.6892 & 47.57282 & 95.20124 & 14.31579 & 52.06612 \\
\hline MEI4 & YER044C-A & 4 & 15 & 7 & 16.65229 & 44.20677 & 84.47205 & 35.04587 & 48.46797 \\
\hline PAD1 & YDR538W & 4 & 15 & 8 & 50.13699 & 44.56825 & 89.11968 & 41.52542 & 61.69492 \\
\hline \multirow[t]{3}{*}{ MXR1 } & YER042W & 4 & 15 & 9 & 23.30468 & 3.521127 & 10.81349 & 43.07958 & 58.22785 \\
\hline & YDR537C & 4 & 15 & 10 & 25.13089 & 28.90335 & 12.31803 & 24.23729 & 56.86275 \\
\hline & YER039C-A & 4 & 15 & 11 & 22.12149 & 28.68852 & 79.28844 & 24.16667 & 48.25871 \\
\hline STL1 & YDR536W & 4 & 15 & 12 & 39.25104 & 62.41331 & 60.76696 & 61.48556 & 71.21535 \\
\hline \multirow[t]{2}{*}{ YEN1 } & YER041W & 4 & 15 & 13 & 16.0043 & 5.677868 & -2.5641 & 33.76623 & 68.59903 \\
\hline & YDR535C & 4 & 15 & 14 & 16.23762 & -2.27577 & 35.33398 & 49.22118 & 60.13986 \\
\hline HVG1 & YER039C & 4 & 15 & 15 & 44.26692 & 59.88201 & 80.29851 & 54.17867 & 67.58794 \\
\hline FIT1 & $\begin{array}{l}\text { YDR534C } \\
\text { YER038W- }\end{array}$ & 4 & 15 & 16 & 32.50729 & 53.49345 & 69.13357 & 5.032468 & 55.95506 \\
\hline FMP49 & $A$ & 4 & 15 & 17 & 16.8254 & 55.86957 & 31.0089 & 25.09363 & 63.8191 \\
\hline HSP31 & YDR533C & 4 & 15 & 18 & 23.22457 & 36.4564 & -41.3589 & 51.72932 & 56.2963 \\
\hline \multirow[t]{3}{*}{ EDC2 } & YER035W & 4 & 15 & 19 & 35.38306 & 23.33916 & 64.47917 & 48.49354 & 47.97136 \\
\hline & YDR532C & 4 & 15 & 20 & 11.95219 & 56.43225 & 65.9919 & 50.35461 & 29.64824 \\
\hline & YER034W & 4 & 15 & 21 & 61.36842 & 28.22581 & 97.9571 & 25.6651 & 56.86813 \\
\hline APA2 & YDR530C & 4 & 15 & 22 & 64.83051 & 61.5917 & 96.01386 & 25.89577 & 46.85864 \\
\hline ZRG8 & YER033C & 4 & 15 & 23 & 25.33557 & 11.86017 & 42.88681 & 26.64577 & 62.84585 \\
\hline HIS3 & YOR202W & 4 & 15 & 24 & -28.5068 & 38.68678 & 59.8759 & & \\
\hline HIS3 & YOR202W & 4 & 16 & 1 & 76.24882 & 43.31088 & -8.72642 & & \\
\hline HIS3 & YOR202W & 4 & 16 & 2 & 73.40191 & 35.43307 & 60.56338 & & \\
\hline HIS3 & YOR202W & 4 & 16 & 3 & 85.46017 & 37.27811 & 65.13158 & & \\
\hline HIS3 & YOR202W & 4 & 16 & 4 & 54.89007 & 32.95255 & 83.27103 & & \\
\hline HIS3 & YOR202W & 4 & 16 & 5 & 49.09311 & 25.84071 & 81.96393 & & \\
\hline HIS3 & YOR202W & 4 & 16 & 6 & 39.18495 & 35.70402 & 32.37052 & & \\
\hline HIS3 & YOR202W & 4 & 16 & 7 & 41.14114 & 18.72146 & -10.7353 & & \\
\hline
\end{tabular}




\begin{tabular}{|c|c|c|c|c|c|c|c|c|c|}
\hline HIS3 & YOR202W & 4 & 16 & 8 & 39.29619 & 24.97626 & -34.5646 & & \\
\hline HIS3 & YOR202W & 4 & 16 & 9 & -6.45905 & -4.31472 & 59.96132 & & \\
\hline HIS3 & YOR202W & 4 & 16 & 10 & -9.69305 & 13.75 & 62.31618 & & \\
\hline HIS3 & YOR202W & 4 & 16 & 11 & 25.3886 & 30.40936 & 42.75093 & & \\
\hline HIS3 & YOR202W & 4 & 16 & 12 & 14.62113 & 14.037 & -29.8361 & & \\
\hline HIS3 & YOR202W & 4 & 16 & 13 & 5.700713 & 37.63608 & 30.80242 & & \\
\hline HIS3 & YOR202W & 4 & 16 & 14 & 5.369928 & 22.62931 & 48.04422 & & \\
\hline HIS3 & YOR202W & 4 & 16 & 15 & 3.250975 & 22.93497 & 54.85294 & & \\
\hline HIS3 & YOR202W & 4 & 16 & 16 & -5.28967 & 19.06542 & 52.61221 & & \\
\hline HIS3 & YOR202W & 4 & 16 & 17 & 9.248555 & 27.83985 & 88.73457 & & \\
\hline HIS3 & YOR202W & 4 & 16 & 18 & 23.27311 & 28.67036 & 93.81107 & & \\
\hline HIS3 & YOR202W & 4 & 16 & 19 & 6.28866 & 31.51261 & -5.86319 & & \\
\hline HIS3 & YOR202W & 4 & 16 & 20 & 16.94915 & 40 & -0.92593 & & \\
\hline HIS3 & YOR202W & 4 & 16 & 21 & 7.619048 & 38.63636 & 21.24905 & & \\
\hline HIS3 & YOR202W & 4 & 16 & 22 & 18.32979 & 42.84428 & 31.51341 & & \\
\hline HIS3 & YOR202W & 4 & 16 & 23 & 41.41274 & 37.24023 & 54.49403 & & \\
\hline HIS3 & YOR202W & 4 & 16 & 24 & -11.8782 & 47.06912 & 61.57697 & & \\
\hline HIS3 & YOR202W & 5 & 1 & 1 & 63.5383 & 37.87086 & 14.76346 & & \\
\hline HIS3 & YOR202W & 5 & 1 & 2 & 30.96903 & 63.42466 & -1.17117 & & \\
\hline HIS3 & YOR202W & 5 & 1 & 3 & 71.5075 & 99.22481 & -9.83051 & & \\
\hline HIS3 & YOR202W & 5 & 1 & 4 & 29.24071 & -10.1073 & 3.948667 & & \\
\hline HIS3 & YOR202W & 5 & 1 & 5 & 41.39591 & 48.73164 & -2.99252 & & \\
\hline HIS3 & YOR202W & 5 & 1 & 6 & 31.73554 & 6.067678 & 17.67677 & & \\
\hline HIS3 & YOR202W & 5 & 1 & 7 & 30.75031 & 58.28571 & 22.75242 & & \\
\hline HIS3 & YOR202W & 5 & 1 & 8 & 38.42869 & 4.672897 & 26.71186 & & \\
\hline HIS3 & YOR202W & 5 & 1 & 9 & 36.40777 & 67.40331 & 8.061154 & & \\
\hline HIS3 & YOR202W & 5 & 1 & 10 & -7.43155 & 54.81481 & 18.14904 & & \\
\hline HIS3 & YOR202W & 5 & 1 & 11 & 23.65064 & 43.66544 & 43.43972 & & \\
\hline HIS3 & YOR202W & 5 & 1 & 12 & -11.1702 & -38.7352 & 8.496732 & & \\
\hline HIS3 & YOR202W & 5 & 1 & 13 & 4.104903 & 58.14978 & 36.67426 & & \\
\hline HIS3 & YOR202W & 5 & 1 & 14 & 12.19239 & 57.59494 & 18.54305 & & \\
\hline HIS3 & YOR202W & 5 & 1 & 15 & 25.40984 & 54.79876 & 32.99663 & & \\
\hline HIS3 & YOR202W & 5 & 1 & 16 & 1.948052 & 66.08 & 25.41828 & & \\
\hline HIS3 & YOR202W & 5 & 1 & 17 & 12.68293 & 70 & 31.46796 & & \\
\hline HIS3 & YOR202W & 5 & 1 & 18 & 21.34039 & 49.29972 & 28.25301 & & \\
\hline HIS3 & YOR202W & 5 & 1 & 19 & 37.85211 & -26.6667 & 35.82575 & & \\
\hline HIS3 & YOR202W & 5 & 1 & 20 & 30.919 & 27.25173 & -19.0217 & & \\
\hline HIS3 & YOR202W & 5 & 1 & 21 & 6.137866 & 63.08806 & 27.75974 & & \\
\hline HIS3 & YOR202W & 5 & 1 & 22 & 29.62588 & 53.29114 & 16.88858 & & \\
\hline HIS3 & YOR202W & 5 & 1 & 23 & 28.09717 & 48.80503 & 25.62418 & & \\
\hline HIS3 & YOR202W & 5 & 1 & 24 & 36.11394 & -35.3073 & 58.22785 & & \\
\hline HIS3 & YOR202W & 5 & 2 & 1 & 74.37326 & 59.13201 & 7.142857 & & \\
\hline CLG1 & YGL215W & 5 & 2 & 2 & -19.1702 & 98.7152 & -17.1053 & 57.91962 & 45.33898 \\
\hline
\end{tabular}


SKI8

SCM4

VAM7

NCS6

RME1

YPT32

MIG2

SIP2

BUD9

POX1

KSS1

KEX1

ARO8

ORM1

HIS3

HIS3

SCY1

ATG18

FAB1

FMP37

DBP3

HNM1

GSY1

NPY1

CMK1

SGF73

IOC3

PYC1
YGR051C

YGL214W

YGR050C

YGL213C

YGR049W

YGL212W

YGR045C

YGL211W

YGR044C

YGL210W

YGR043C

YGL209W

YGR042W

YGL208W

YGR041W

YGL205W

YGR040W

YGL203C

YGR039W

YGL202W

YGR038W

YOR202W

YOR202W

YFR022W

YGL083W

YFR021W

YGL082W

YFR020W

YGL081W

YFR019W

YGL080W

YFR018C

YGL079W

YFR017C

YGL078C

YFR016C

YGLO77C

YFR015C

YGL067W

YFR014C

YGL066W

YFR013W

YGL062W $\begin{array}{lllll}3 & 47.05144 & 67.33524 & 32.23767\end{array}$

$\begin{array}{lllll}4 & 9.803922 & 50.95541 & -19.5652\end{array}$

$\begin{array}{lllll}5 & -5.22152 & 7.427056 & 78.80795\end{array}$

$\begin{array}{lllll}6 & 38.37838 & 70.25761 & 4.098361\end{array}$

$\begin{array}{lllll}7 & 54.02456 & 64.04494 & 2.727273\end{array}$

$\begin{array}{lllll}8 & 10.18711 & 33.41584 & 28.50746\end{array}$

$\begin{array}{lllll}9 & 26.67364 & -15.6863 & 20.70099\end{array}$

$\begin{array}{llll}10 & 42.35437 & 21.72414 & 6.841339\end{array}$

$\begin{array}{llll}11 & 19.11221 & 21.34831 & 19.01984\end{array}$

$\begin{array}{lllll}12 & -0.65147 & 28.30189 & 70.55151\end{array}$

$\begin{array}{lllll}13 & -11.1502 & -43.2099 & -3.95968\end{array}$

$14 \quad-5.82011$

$15 \quad 50.95541$

$\begin{array}{ll}16 & -46.3576\end{array}$

$\begin{array}{ll}17 & 46.14537\end{array}$

$18 \quad 27.68421$

$19 \quad 37.53609$

$20 \quad 58.83392$

$21 \quad 57.90458$

$22 \quad 48.73354$

$23 \quad 54.45205$

$24 \quad 27.91552$

145.4955

$2-17.8445$

$3 \quad-19.6881$

$\begin{array}{ll}4 & -4.7619\end{array}$

$5 \quad-27.6451$

$\begin{array}{ll}6 & 14.04494\end{array}$

7

$-0.48$

$\begin{array}{ll}8 & 77.16535\end{array}$

$9 \quad 13.38583$

$\begin{array}{ll}10 & 6.439854\end{array}$

$\begin{array}{ll}11 & 27.82037\end{array}$

12

21.2932

48.9301

13

14

15

16

17

$18 \quad 54.28571$

23.13167

42.2718

$-3.41176$

-3.4116
-828671

$37.5 \quad 17.15839$

$0.923077 \quad 5.160662$

$-20.8333-2.88363$

$49.17492-11.5591$

$44.03846 \quad 38.79142$

$57.48663 \quad 28.61469$

$\begin{array}{ll}-11 & 16.92456\end{array}$

$2.063492 \quad 24.08602$

$31.28713-1.21581$

$57.22656-19.4286$

$51.21951 \quad 75.20436$

$\begin{array}{ll}41.84028 & -25.7554\end{array}$

$58.04067 \quad 26.28625$

$\begin{array}{lll}48.11828 & 4.371217\end{array}$

$56.28415 \quad 1.076813$

$100 \quad 9.69697$

$\begin{array}{ll}13.99177 & -8.67526\end{array}$

$\begin{array}{ll}17.0068 \quad 27.62063 \\ -37.1795 & 73.82502\end{array}$

$\begin{array}{lll}-37.1795 & 73.82502\end{array}$

$32.85458-11.9335$

$\begin{array}{ll}-30.0781 & -33.5354\end{array}$

$-34.6154-7.21221$

$-35.1682-11.9159$

\begin{tabular}{ll}
$-26.1745 \quad 9.275731$ \\
\hline
\end{tabular}

$\begin{array}{ll}7.317073 & -16.0289\end{array}$

$\begin{array}{ll}40.04283 & -20.9344\end{array}$

$\begin{array}{lllll}19 & 62.38274 & 55.21472 & 3.611738\end{array}$

$20 \quad 61.65865$

$64.77273 \quad 3.874539$

$21 \quad 37.74704$

$20.42553-15.3114$

44.94118

35.10896

65.87112

37.65823

63.21839

43.10777

57.31343

22.50712

27.0073

52.48042

33.33333

24.40191

48.11594

35.38813

22.71762

29.38389

16.82927

23.42342

33.61884

24.48037

28.6031

21.57303

20.60086

24.67532

36.22047

54.06872

61.48492

48.125

49.3865

64.48598

78.60465

46.83908

59.95423

65.70743

58.50144

48.8189

61.22995

59.63061

62.39554

44.63158

50

78.4

50.14749

25.78125

48.76033

17.63341

37.84355

28.61357

38.4778

38.44156

30.90024

16.79389

34.77089

31.57895

23.18841

28.67647

22.22222

21.98953

11.27451

30.62645

33.11111

25.51253

19.35484

26.73031

28.44639

25 


\begin{tabular}{|c|c|c|c|c|c|c|c|c|c|}
\hline & YFR012W & 5 & 3 & 22 & 49.07787 & 15.96639 & 10.49841 & 49.11591 & 25.72062 \\
\hline YBP2 & YGL060W & 5 & 3 & 23 & 40.1005 & 33.11258 & 0 & 41.39651 & 33.25581 \\
\hline HIS3 & YOR202W & 5 & 3 & 24 & 1.66113 & -6.69371 & 9.32858 & & \\
\hline HIS3 & YOR202W & 5 & 4 & 1 & -23.3645 & -0.17986 & 55.07874 & & \\
\hline SHE10 & YGL228W & 5 & 4 & 2 & 41.01911 & 4.144144 & 29.19501 & 58.61183 & 76.37741 \\
\hline PEX8 & YGR077C & 5 & 4 & 3 & 8.431703 & -4.05797 & -8.46954 & 72.37852 & 36.88312 \\
\hline VID30 & YGL227W & 5 & 4 & 4 & 6.605223 & 17.27642 & -28.586 & 42.1875 & 20.90909 \\
\hline \multirow[t]{3}{*}{ UPF3 } & YGR072W & 5 & 4 & 5 & 30.8 & 41.54818 & 95.14416 & 67.46725 & 30.36212 \\
\hline & YGL226W & 5 & 4 & 6 & 31.74274 & 28.05755 & 39.44591 & 77.07182 & 27.4359 \\
\hline & YGR071C & 5 & 4 & 7 & 52.2673 & 41.47982 & 35.69682 & 80.22222 & 29.84615 \\
\hline OST5 & YGL226C-A & 5 & 4 & 8 & 8.97909 & 28.48101 & -2.80728 & 71.49758 & 26.6129 \\
\hline ROM1 & YGR070W & 5 & 4 & 9 & 15.27027 & 28.90365 & -11.3687 & 75.72614 & 15.68123 \\
\hline \multirow[t]{2}{*}{ SDT1 } & YGL224C & 5 & 4 & 10 & 21.76471 & 47.73519 & 80.05115 & 59.71429 & 10.92637 \\
\hline & YGR069W & 5 & 4 & 11 & -15.6812 & 61.24314 & 63.71859 & 55.07614 & 18.35106 \\
\hline \multirow[t]{2}{*}{ EDC1 } & YGL222C & 5 & 4 & 12 & 34.43396 & -19 & 89.2822 & 50.5176 & 21.18451 \\
\hline & YGR066C & 5 & 4 & 13 & 4.376368 & 50.25126 & 68.86145 & 47.27626 & 29.90196 \\
\hline NIF3 & YGL221C & 5 & 4 & 14 & 34.65909 & -27.6762 & 5.321782 & 80.45326 & 18 \\
\hline SPR3 & YGR059W & 5 & 4 & 15 & 12.74175 & -5.82707 & 19.57746 & 74.32763 & 20.81218 \\
\hline \multirow[t]{6}{*}{ MDM34 } & YGL219C & 5 & 4 & 16 & 47.97927 & 43.41253 & 12.00807 & 20.64516 & 18.93204 \\
\hline & YGR058W & 5 & 4 & 17 & 56.46512 & 30.18868 & 4.815574 & 37.88151 & 19.07692 \\
\hline & YGL218W & 5 & 4 & 18 & 65.83333 & 64.65517 & 64.68647 & -21.1905 & -5.39216 \\
\hline & YGR054W & 5 & 4 & 19 & 69.89796 & 69.5122 & \#DIV/0! & 61.32075 & 24.67532 \\
\hline & YGL217C & 5 & 4 & 20 & -4.3578 & 56.45695 & 25.76531 & 12.62626 & 19.08213 \\
\hline & YGR053C & 5 & 4 & 21 & 27.25118 & 28.03532 & 5.136986 & 50.23256 & 27.08333 \\
\hline KIP3 & YGL216W & 5 & 4 & 22 & 39.38992 & -8.67052 & -17.9268 & 39.10891 & 24.42396 \\
\hline FMP48 & YGR052W & 5 & 4 & 23 & 54.86348 & -33.9726 & 0.800801 & 36.34686 & 31.80873 \\
\hline HIS3 & YOR202W & 5 & 4 & 24 & 14.16185 & 50 & -4.02047 & & \\
\hline HIS3 & YOR202W & 5 & 5 & 1 & 41.21115 & -36.3014 & 45.5623 & & \\
\hline \multirow[t]{2}{*}{ PHO4 } & YFR034C & 5 & 5 & 2 & -6.79612 & -10.199 & 44.03993 & 55.34442 & 33.2574 \\
\hline & YGL109W & 5 & 5 & 3 & 19.77401 & -35.9551 & -13.1479 & 73.07692 & 27.02703 \\
\hline \multirow[t]{2}{*}{ QCR6 } & YFR033C & 5 & 5 & 4 & -21.5267 & 32.24138 & 44.32773 & 67.76119 & 11.23919 \\
\hline & YGL108C & 5 & 5 & 5 & 32.31084 & 23.8477 & 56.03544 & 72.94118 & 18.58407 \\
\hline RPL29 & YFR032C-A & 5 & 5 & 6 & 38.01843 & 25.8547 & 12.32877 & 34.55497 & 12.5 \\
\hline \multirow[t]{2}{*}{ ARC1 } & YGL105W & 5 & 5 & 7 & 49.4382 & 44.25887 & 12.73885 & 72.4359 & 44.03974 \\
\hline & YFR032C & 5 & 5 & 8 & -8.62275 & 50 & 28.11565 & 60.86957 & 27.7439 \\
\hline VPS73 & YGL104C & 5 & 5 & 9 & 32.01285 & -14.7727 & 11.58645 & 66.2531 & 13.86139 \\
\hline \multirow[t]{2}{*}{ RPL2A } & YFR031C-A & 5 & 5 & 10 & 19.79167 & 20.23256 & -4.62107 & 70.61856 & 21.42857 \\
\hline & YGL101W & 5 & 5 & 11 & 14.58094 & 31.44105 & 63.95028 & 62.61682 & 20.8 \\
\hline MET10 & YFR030W & 5 & 5 & 12 & 41.75506 & 7.317073 & -7.46269 & 73.95833 & 17.64706 \\
\hline \multirow[t]{2}{*}{ LIF1 } & YGL090W & 5 & 5 & 13 & 17.99163 & 61.17647 & \#DIV/0! & 78.08564 & 26.22951 \\
\hline & YFR026C & 5 & 5 & 14 & 10.97285 & 5.596107 & -2.06718 & 65.68627 & 16.72474 \\
\hline $\operatorname{MF}(\mathrm{ALPHA}) 2$ & YGL089C & 5 & 5 & 15 & 21.24352 & 50 & -14.6588 & 66.43026 & 14.2132 \\
\hline HIS2 & YFR025C & 5 & 5 & 16 & 39.86014 & -38.4393 & 6.270997 & 79.8005 & 25.323 \\
\hline
\end{tabular}




\begin{tabular}{|c|c|c|c|c|c|c|c|c|c|}
\hline MMS2 & YGL087C & 5 & 5 & 17 & 52.83401 & -18.3673 & 74.4879 & 62.02186 & 25.76531 \\
\hline LSB3 & YFR024C-A & 5 & 5 & 18 & 54.82728 & -18.4343 & 64.16153 & 47.35202 & 10.41096 \\
\hline \multirow[t]{3}{*}{ MAD1 } & YGL086W & 5 & 5 & 19 & 54.33476 & 40.65282 & 0 & 52.59434 & 30.54893 \\
\hline & YFR024C & 5 & 5 & 20 & 58.37321 & 50.50847 & 47.86585 & 44.65812 & 35.61321 \\
\hline & YGL085W & 5 & 5 & 21 & -14.1531 & -17.1141 & -4.03826 & 33.09693 & 27.57848 \\
\hline PES4 & YFR023W & 5 & 5 & 22 & 35.98938 & -0.53476 & -11.4043 & 40.71429 & 32.94664 \\
\hline GUP1 & YGL084C & 5 & 5 & 23 & 62.51172 & 20.70588 & 29.40447 & 47.14038 & 43.69369 \\
\hline HIS3 & YOR202W & 5 & 5 & 24 & 49.76303 & 58.41584 & -22.8037 & & \\
\hline HIS3 & YOR202W & 5 & 6 & 1 & 32.24461 & 65.95745 & -24.8616 & & \\
\hline TAD1 & YGL243W & 5 & 6 & 2 & 19.09722 & -8.13008 & -11.7932 & 60.86957 & 42.50681 \\
\hline \multirow[t]{2}{*}{ DBF2 } & YGR092W & 5 & 6 & 3 & 22.86689 & 39.20973 & 70.03891 & 46.03774 & 39.14286 \\
\hline & YGL242C & 5 & 6 & 4 & 26.2069 & 42.9078 & 49.40855 & 79.08497 & 56.07477 \\
\hline NNF2 & YGR089W & 5 & 6 & 5 & 46.51584 & 25.93703 & 74.69459 & 71.9917 & 20.69892 \\
\hline KAP114 & YGL241W & 5 & 6 & 6 & 15.68627 & -19.6667 & 31.72952 & 74.73958 & 19.07357 \\
\hline CTT1 & YGR088W & 5 & 6 & 7 & 40.73276 & 3.178484 & -22.13 & 68.30467 & 13.02326 \\
\hline HAP2 & YGL237C & 5 & 6 & 8 & 26.87704 & 35.33569 & 72.74436 & 72.09877 & 5.396825 \\
\hline PDC6 & YGR087C & 5 & 6 & 9 & 31.4258 & 4.205607 & 30.35936 & 74.52575 & 23.39181 \\
\hline MTO1 & YGL236C & 5 & 6 & 10 & 31.89474 & 51.0467 & 100 & 49.59677 & 8.108108 \\
\hline \multirow[t]{2}{*}{ PIL1 } & YGR086C & 5 & 6 & 11 & 19.1766 & -14.0171 & 89.81938 & 73.25905 & 24.27746 \\
\hline & YGL235W & 5 & 6 & 12 & 32.50774 & 36.47235 & 100 & 39.87342 & 22.9798 \\
\hline RPL11B & YGR085C & 5 & 6 & 13 & 23.26622 & 68.88489 & 26.0479 & 67.47967 & 12.23242 \\
\hline ADE5,7 & YGL234W & 5 & 6 & 14 & 89.53975 & 85.07463 & 30.36936 & 74.80315 & 42.07317 \\
\hline MRP13 & YGR084C & 5 & 6 & 15 & 47.60449 & 37.77403 & 29.15129 & 16.39723 & 17.12707 \\
\hline TAN1 & YGL232W & 5 & 6 & 16 & 37.17277 & 28.57143 & -4.38871 & 32.80543 & 21.93732 \\
\hline \multirow[t]{2}{*}{ SLX9 } & YGR081C & 5 & 6 & 17 & 45.31616 & 41.07486 & 23.35116 & 13.40206 & 21.98142 \\
\hline & YGL231C & 5 & 6 & 18 & 40.03813 & -7.3107 & 9.012346 & 53.33333 & 24.8062 \\
\hline \multirow[t]{3}{*}{ TWF1 } & YGR080W & 5 & 6 & 19 & 37.08415 & 5.645161 & 77.94823 & 30.26634 & 29.25659 \\
\hline & YGL230C & 5 & 6 & 20 & 41.75824 & 22.32143 & -5.72917 & 47.36842 & 30.2381 \\
\hline & YGR079W & 5 & 6 & 21 & 51.20733 & 14.65893 & 3.93401 & 51.0582 & 30.91787 \\
\hline SAP4 & YGL229C & 5 & 6 & 22 & 33.90171 & 59.02439 & 8.540541 & 34.21687 & 21.78899 \\
\hline PAC10 & YGR078C & 5 & 6 & 23 & 0.234742 & -20.8211 & 10.20408 & 26.79426 & 24.42922 \\
\hline HIS3 & YOR202W & 5 & 6 & 24 & 11.37488 & 11.8797 & -41.7352 & & \\
\hline HIS3 & YOR202W & 5 & 7 & 1 & 46.54481 & 55.02392 & 0.560897 & & \\
\hline RMD8 & YFR048W & 5 & 7 & 2 & -11.0985 & 9.166667 & -28.5349 & 67.35219 & 27.87286 \\
\hline SNT2 & YGL131C & 5 & 7 & 3 & 40.15826 & 26.69173 & -2.48714 & 64.78873 & 33.23263 \\
\hline BNA6 & YFR047C & 5 & 7 & 4 & 12.93996 & 47.44646 & -25.7078 & 76 & 28.49315 \\
\hline SOH1 & YGL127C & 5 & 7 & 5 & 33.10105 & 38.02817 & 76.75941 & 43.46505 & 7.272727 \\
\hline CNN1 & YFR046C & 5 & 7 & 6 & 36.35417 & 23.48703 & 16.82497 & 78 & 23.07692 \\
\hline \multirow[t]{2}{*}{ SCS3 } & YGL126W & 5 & 7 & 7 & 12.53792 & -16.2393 & -18.5861 & 64.94118 & 14.23841 \\
\hline & YFR045W & 5 & 7 & 8 & 28.6631 & 32.28621 & -3.73563 & 66.17647 & 31.53153 \\
\hline \multirow[t]{2}{*}{ MET13 } & YGL125W & 5 & 7 & 9 & 11.40025 & 29.74052 & 57.29443 & 61.89024 & 24.44444 \\
\hline & YFR044C & 5 & 7 & 10 & 1.35468 & -14.135 & -22.1154 & 56.77419 & 23.85621 \\
\hline MON1 & YGL124C & 5 & 7 & 11 & 33.33333 & 6.032483 & 81.17359 & 12.20159 & 10.48387 \\
\hline
\end{tabular}




\begin{tabular}{|c|c|c|c|c|c|c|c|c|c|}
\hline & YFR043C & 5 & 7 & 12 & 0.431499 & 47.30047 & 31.98594 & 70.41801 & 26.62539 \\
\hline GPG1 & YGL121C & 5 & 7 & 13 & 38.82236 & -25.8312 & -13.7255 & 69.11392 & 29.15663 \\
\hline \multirow[t]{2}{*}{ ERJ5 } & YFR041C & 5 & 7 & 14 & 13.7817 & 61.11975 & -17.3333 & 93.55932 & 44.19263 \\
\hline & YGL118C & 5 & 7 & 15 & 34.01222 & 12.8692 & -24.2026 & 62.60388 & 26.91293 \\
\hline \multirow[t]{3}{*}{ SAP155 } & YFR040W & 5 & 7 & 16 & 66.53061 & 37.81095 & -24.4681 & 49.87893 & 35.65217 \\
\hline & YGL117W & 5 & 7 & 17 & 53.58527 & -30.7927 & 71.18246 & 46.71202 & 34.08451 \\
\hline & YFR039C & 5 & 7 & 18 & 46.89716 & -7.21868 & 32.72506 & 59.02439 & 38.17664 \\
\hline \multirow[t]{4}{*}{ SNF4 } & YGL115W & 5 & 7 & 19 & 43.26087 & 31.37996 & 86.69302 & 18.67816 & 16.87898 \\
\hline & YFR038W & 5 & 7 & 20 & 28.119 & 57.87172 & 1.970803 & 46.13583 & 38.79093 \\
\hline & YGL114W & 5 & 7 & 21 & 40.62947 & 37.64259 & 72.73277 & 24.42159 & 26.29108 \\
\hline & YFR035C & 5 & 7 & 22 & 65.28792 & -28.8235 & 77.14497 & 34.05276 & 37.90524 \\
\hline CUE3 & YGL110C & 5 & 7 & 23 & 64.77941 & -29.0976 & 0 & 49.3014 & 45.78834 \\
\hline HIS3 & YOR202W & 5 & 7 & 24 & 28.28185 & 50.14327 & 16.85714 & & \\
\hline HIS3 & YOR202W & 5 & 8 & 1 & 23.73042 & 50.96899 & 46.34968 & & \\
\hline \multirow[t]{2}{*}{ MNT2 } & YGL257C & 5 & 8 & 2 & 43.10954 & 51.94553 & 14.27194 & 82.09302 & 37.18487 \\
\hline & YGR110W & 5 & 8 & 3 & 17.05499 & 6.774194 & 27.897 & 86.88047 & 33.05556 \\
\hline $\mathrm{ADH} 4$ & YGL256W & 5 & 8 & 4 & 45.02203 & -19.8157 & 2.467811 & 78.93617 & 47.73333 \\
\hline CLB6 & YGR109C & 5 & 8 & 5 & 77.86458 & 94.18605 & 39.58333 & 71.07692 & 30.95238 \\
\hline ZRT1 & YGL255W & 5 & 8 & 6 & 48.04104 & 4.841402 & 51.94805 & 84.86647 & 25.12315 \\
\hline CLB1 & YGR108W & 5 & 8 & 7 & 40.50633 & 63.76812 & -12.0066 & 84.96042 & 30.42254 \\
\hline \multirow[t]{2}{*}{ FZF1 } & YGL254W & 5 & 8 & 8 & 49.17749 & -96.7033 & 87.59358 & 78.65169 & 26.72673 \\
\hline & YGR107W & 5 & 8 & 9 & 48.33492 & -16.2706 & -22.1675 & 83.5 & 29.69121 \\
\hline \multirow[t]{2}{*}{ HXK2 } & YGL253W & 5 & 8 & 10 & 37.48474 & -25.6962 & 15.84699 & 65.29563 & 25.22796 \\
\hline & YGR106C & 5 & 8 & 11 & 36.36364 & -31.4763 & 52.5641 & 77.94118 & 37.82235 \\
\hline RTG2 & YGL252C & 5 & 8 & 12 & 52.55941 & 8.528428 & 28.95323 & 45.51845 & 40.05305 \\
\hline VMA21 & YGR105W & 5 & 8 & 13 & 100 & \#DIV/0! & 87.14286 & 39.06706 & 33.45455 \\
\hline HFM1 & YGL251C & 5 & 8 & 14 & 27.47475 & -34.9174 & -3.61011 & 73.18436 & 37.10074 \\
\hline \multirow[t]{2}{*}{ PCP1 } & YGR101W & 5 & 8 & 15 & 100 & \#DIV/0! & 100 & 74.77745 & 24.63054 \\
\hline & YGL250W & 5 & 8 & 16 & 39.38144 & 33.48315 & 67.295 & 52.08791 & 46.80233 \\
\hline MDR1 & YGR100W & 5 & 8 & 17 & 28.15846 & 59.23393 & 3.602484 & 75.5814 & 37.84461 \\
\hline ZIP2 & YGL249W & 5 & 8 & 18 & 48.54651 & 22.91667 & 38.59155 & 35.6962 & 36.50386 \\
\hline ASK10 & YGR097W & 5 & 8 & 19 & 53.5685 & 10.47431 & 61.86579 & 74.27293 & 54.97512 \\
\hline PDE1 & YGL248W & 5 & 8 & 20 & 5.319149 & 77.26737 & 53.71094 & 30.32581 & 38.72549 \\
\hline TPC1 & YGR096W & 5 & 8 & 21 & 43.09252 & -19.9195 & -18.6012 & 38.31325 & 42.18362 \\
\hline \multirow[t]{2}{*}{ RTF1 } & YGL244W & 5 & 8 & 22 & 53.9823 & 16.49485 & 15.15152 & -22.4265 & 30.41958 \\
\hline & YGR093W & 5 & 8 & 23 & 57.95364 & 29.79003 & 0.3125 & 40.12739 & 41.45833 \\
\hline HIS3 & YOR202W & 5 & 8 & 24 & 43.55644 & 7.676768 & 17.61518 & & \\
\hline \multirow[t]{2}{*}{ HIS3 } & YOR202W & 5 & 9 & 1 & 46.54545 & 95.99271 & 36.43385 & & \\
\hline & YGL007W & 5 & 9 & 2 & 45.93114 & 97.52747 & 8.467742 & 57.40741 & 12.56684 \\
\hline ARO2 & YGL148W & 5 & 9 & 3 & 48.81266 & 67.91569 & 13.7931 & 90.61489 & 28.60759 \\
\hline PMC1 & YGL006W & 5 & 9 & 4 & 41.66667 & 61.12601 & 34 & 67.17557 & 31.66144 \\
\hline RPL9A & YGL147C & 5 & 9 & 5 & 50.37675 & -20.2643 & 81.34557 & 45.21531 & 21.72702 \\
\hline COG7 & YGL005C & 5 & 9 & 6 & 51.78408 & -18.6709 & 50.3428 & 75.36946 & 22.08955 \\
\hline
\end{tabular}




\begin{tabular}{|c|c|c|c|c|c|c|c|c|c|}
\hline & YGL146C & 5 & 9 & 7 & 22.53521 & 53.6 & 1.946108 & 71 & 33.92857 \\
\hline RPN14 & YGL004C & 5 & 9 & 8 & -1.55587 & 34.95935 & -36.7347 & 53.06122 & 27.19298 \\
\hline ROG1 & YGL144C & 5 & 9 & 9 & 39.23611 & -9.2511 & 33.80282 & 49.74093 & 34.09091 \\
\hline ERP6 & YGL002W & 5 & 9 & 10 & 41.7124 & 51.10701 & 42.32143 & 46.84685 & 35.83333 \\
\hline \multirow[t]{8}{*}{ HUL5 } & YGL141W & 5 & 9 & 11 & 43.19654 & 20.22472 & 12.32877 & 70.7989 & 33.97436 \\
\hline & YFR057W & 5 & 9 & 12 & 39.51012 & 64.56808 & -12.3539 & 43.0622 & 42.54144 \\
\hline & YGL140C & 5 & 9 & 13 & 36.95421 & 28.96175 & 71.9603 & 51.49051 & 41.86047 \\
\hline & YFR056C & 5 & 9 & 14 & 39.54858 & 11.27451 & -22.9665 & 49.02808 & 51.32275 \\
\hline & YGL139W & 5 & 9 & 15 & 51.6525 & -12.7193 & 61.30435 & 28.60125 & 35.58897 \\
\hline & YFR055W & 5 & 9 & 16 & 31.78295 & 61.44144 & 2.945736 & 50.67385 & 50.41096 \\
\hline & YGL138C & 5 & 9 & 17 & 48.99522 & 6.456954 & 57.58276 & 37.67123 & 44.41687 \\
\hline & YFR054C & 5 & 9 & 18 & 24.10314 & -37.4194 & 12.75168 & 39.82869 & 31.75966 \\
\hline MRM2 & YGL136C & 5 & 9 & 19 & 41.07744 & -24.2623 & 34.89933 & 43.88298 & 38.63636 \\
\hline HXK1 & YFR053C & 5 & 9 & 20 & 27.66631 & 14.46078 & 4.914371 & 39.46731 & 52.21932 \\
\hline ITC1 & YGL133W & 5 & 9 & 21 & 50.21277 & 61.90476 & 14.50079 & 48.26733 & 50.12788 \\
\hline \multirow[t]{2}{*}{ YMR31 } & YFR049W & 5 & 9 & 22 & 35.6701 & 64.94192 & 14.42642 & 15.42056 & 36.66667 \\
\hline & YGL132W & 5 & 9 & 23 & 60.9375 & 49.51456 & 19.77226 & 35.71429 & 45.78059 \\
\hline HIS3 & YOR202W & 5 & 9 & 24 & 62.27273 & 63.69427 & -4.51189 & & \\
\hline HIS3 & YOR202W & 5 & 10 & 1 & 51.79718 & 72.01087 & 2.735711 & & \\
\hline \multirow[t]{2}{*}{ STF2 } & YGR008C & 5 & 10 & 2 & 32.89474 & 86.13281 & -11.5942 & 44.3609 & 28.79464 \\
\hline & YGR130C & 5 & 10 & 3 & 10.41445 & 100 & 12.64368 & 34.09669 & 12.99094 \\
\hline MUQ1 & YGR007W & 5 & 10 & 4 & 38.59649 & -8.58586 & 31.92534 & 82.20859 & 35.18519 \\
\hline SYF2 & YGR129W & 5 & 10 & 5 & 26.90802 & 83.92226 & 16.30537 & 69.83696 & 22.89474 \\
\hline \multirow[t]{2}{*}{ PEX31 } & YGR004W & 5 & 10 & 6 & 28.35113 & 20.83333 & 0.448029 & 75.70621 & 33.44262 \\
\hline & YGR127W & 5 & 10 & 7 & 44.45813 & 44.52975 & -22.2372 & 81.52493 & 29.72973 \\
\hline \multirow[t]{4}{*}{ CUL3 } & YGR003W & 5 & 10 & 8 & 23.2859 & 56.35179 & 45.04692 & 78.00512 & 35.53719 \\
\hline & YGR126W & 5 & 10 & 9 & 36.92308 & 13.13321 & 29.09091 & 47.41144 & 31.98847 \\
\hline & YGR001C & 5 & 10 & 10 & 47.14785 & 41.69742 & -26.4344 & 38.99083 & 43.14286 \\
\hline & YGR125W & 5 & 10 & 11 & 44.80519 & -12.4161 & 19.10275 & 46.17225 & 37.2549 \\
\hline $\cos 12$ & YGL263W & 5 & 10 & 12 & 39.14849 & -10.6904 & 50.33557 & 31.12583 & 31.78808 \\
\hline \multirow[t]{2}{*}{ ASN2 } & YGR124W & 5 & 10 & 13 & 48.3871 & 10.05587 & 27.33224 & 27.59494 & 35.27919 \\
\hline & YGL262W & 5 & 10 & 14 & 53.04762 & 68.65672 & 66.21005 & 87.5 & 65.56962 \\
\hline \multirow[t]{2}{*}{ PPT1 } & YGR123C & 5 & 10 & 15 & 32.2449 & 1.832994 & 14.41224 & 49.19786 & 49.05149 \\
\hline & YGL261C & 5 & 10 & 16 & 44.28008 & -2.71429 & 79.8951 & 40.46512 & 54.09429 \\
\hline \multirow[t]{2}{*}{ MEP1 } & YGR121C & 5 & 10 & 17 & 33.65385 & -33.0864 & \#DIV/0! & 33.69176 & 46.63212 \\
\hline & YGL260W & 5 & 10 & 18 & 36.2254 & -25.2669 & 60.56818 & 17.0088 & 47.19764 \\
\hline RPS23A & YGR118W & 5 & 10 & 19 & 29.54545 & 13.35807 & \#DIV/0! & 46.13181 & 48.94737 \\
\hline \multirow[t]{2}{*}{ YPS5 } & YGL259W & 5 & 10 & 20 & 38.4778 & -25.7336 & 51.33283 & 29.28349 & 42.2043 \\
\hline & YGR117C & 5 & 10 & 21 & 37.3 & -21.9638 & 35.63025 & 55.29716 & 57.21649 \\
\hline \multirow[t]{2}{*}{ VEL1 } & YGL258W & 5 & 10 & 22 & 44.82759 & -2.33281 & -14.3905 & 11.26374 & 44.93827 \\
\hline & YGR111W & 5 & 10 & 23 & 45.71704 & -4.72279 & -1.01523 & 53.79147 & 52.26824 \\
\hline HIS3 & YOR202W & 5 & 10 & 24 & 53.48837 & 38.24604 & 3.340595 & & \\
\hline HIS3 & YOR202W & 5 & 11 & 1 & 19.6648 & 69.06615 & -0.44958 & & \\
\hline
\end{tabular}




\begin{tabular}{|c|c|c|c|c|c|c|c|c|c|}
\hline PIB2 & YGL023C & 5 & 11 & 2 & 28.19672 & 100 & 25.97201 & 35.82996 & 75.05155 \\
\hline YIP5 & YGL161C & 5 & 11 & 3 & 42.73504 & 99.25 & 3.130755 & 67.90123 & 35.87571 \\
\hline \multirow[t]{2}{*}{ ALK1 } & YGL021W & 5 & 11 & 4 & 26.74419 & 99.03846 & 3.033006 & 57.29167 & 32.94461 \\
\hline & YGL160W & 5 & 11 & 5 & 33.55336 & 95.72954 & 20.89762 & 35.43956 & 30.93093 \\
\hline \multirow[t]{2}{*}{ MDM39 } & YGL020C & 5 & 11 & 6 & 17.43243 & 48.3209 & 7.272727 & 44.93506 & 17.24138 \\
\hline & YGL159W & 5 & 11 & 7 & 21.84532 & -37.3673 & 15.29657 & 35.94378 & 26.24672 \\
\hline CKB1 & YGL019W & 5 & 11 & 8 & 22.96196 & 14.86928 & 34.10405 & 87.22045 & 37.82383 \\
\hline RCK1 & YGL158W & 5 & 11 & 9 & 41.96078 & 18.57585 & -4.86976 & 57.72152 & 30.37249 \\
\hline \multirow[t]{2}{*}{ ATE1 } & YGL017W & 5 & 11 & 10 & 38.90187 & 40 & 26.21167 & 40.09217 & 35.1145 \\
\hline & YGL157W & 5 & 11 & 11 & 47.03344 & 39.68872 & 57.52637 & 70.2011 & 61.34831 \\
\hline KAP122 & YGL016W & 5 & 11 & 12 & 64.91228 & 78.53881 & 69.43005 & 35.01094 & 47.92244 \\
\hline \multirow[t]{2}{*}{ AMS1 } & YGL156W & 5 & 11 & 13 & 40.64865 & -45.8248 & 34.36426 & 48.37758 & 48.55491 \\
\hline & YGL015C & 5 & 11 & 14 & 44.04517 & -7.14286 & 17.32474 & 58.62832 & 40.45226 \\
\hline LYS5 & YGL154C & 5 & 11 & 15 & 35.91294 & -8.68687 & 35.93909 & 29.50311 & 43.84615 \\
\hline PUF4 & YGL014W & 5 & 11 & 16 & 34.71933 & -3.46154 & 52.67686 & 23.93617 & 33.72922 \\
\hline PEX14 & YGL153W & 5 & 11 & 17 & 42.8997 & 14.47619 & 21.875 & 24.24242 & 49.64029 \\
\hline \multirow[t]{3}{*}{ PDR1 } & YGL013C & 5 & 11 & 18 & 62.88557 & 56.59341 & 62.60081 & 48.23529 & 65.54404 \\
\hline & YGL152C & 5 & 11 & 19 & 38.76698 & 22.79693 & -17.8672 & 34.78261 & 50.91743 \\
\hline & YGL010W & 5 & 11 & 20 & 28.0837 & -7.89474 & 51.21317 & 2.432432 & 32 \\
\hline NUT1 & YGL151W & 5 & 11 & 21 & 62.0614 & 58.19975 & 77.70461 & 34.57711 & 67.28972 \\
\hline \multirow[t]{2}{*}{ LEU1 } & YGL009C & 5 & 11 & 22 & 46.1165 & 47.411 & -14.6835 & 37.82235 & 40.18692 \\
\hline & YGL149W & 5 & 11 & 23 & 54.3662 & -8.57143 & 59.83067 & 55.55556 & 56.50407 \\
\hline HIS3 & YOR202W & 5 & 11 & 24 & 64.8398 & 39.50057 & 7.620818 & & \\
\hline \multirow[t]{2}{*}{ HIS3 } & YOR202W & 5 & 12 & 1 & -8.81671 & 44.68085 & 4.387417 & & \\
\hline & YGR022C & 5 & 12 & 2 & -22.5725 & 52.82714 & 29.55801 & 47.69231 & 36.27639 \\
\hline \multirow[t]{2}{*}{ BTN2 } & YGR142W & 5 & 12 & 3 & 21.02804 & 96.01677 & 21.24277 & 66.66667 & 40.10695 \\
\hline & YGR021W & 5 & 12 & 4 & 28.48752 & 70.4501 & -8.65103 & 24.08602 & 14.98708 \\
\hline VPS62 & YGR141W & 5 & 12 & 5 & 19.91643 & 100 & 79.22705 & 71.90722 & 26.03878 \\
\hline \multirow[t]{3}{*}{ UGA1 } & YGR019W & 5 & 12 & 6 & 18.56925 & 53.90071 & 9.249084 & 73.91304 & 35.17588 \\
\hline & YGR139W & 5 & 12 & 7 & 15.55233 & -8.47953 & -5.85366 & 72.74827 & 41.19171 \\
\hline & YGR018C & 5 & 12 & 8 & 32.1519 & 28.37209 & 38.95447 & 68.96552 & 26.37363 \\
\hline \multirow[t]{4}{*}{ TPO2 } & YGR138C & 5 & 12 & 9 & 48.99103 & 24.72222 & -7.33083 & 31.60173 & 35.30864 \\
\hline & YGR017W & 5 & 12 & 10 & 41.90178 & -0.39604 & 65.46185 & 52.64228 & 45.81281 \\
\hline & YGR137W & 5 & 12 & 11 & 42.01681 & 73.54497 & 7.801418 & 47.52252 & 49.2823 \\
\hline & YGR016W & 5 & 12 & 12 & 48.39045 & 12.5 & -13.5933 & 61.58038 & 41.46919 \\
\hline \multirow[t]{2}{*}{ LSB1 } & YGR136W & 5 & 12 & 13 & 34.19913 & 13.80417 & 43.73057 & 64.80447 & 43.55972 \\
\hline & YGR015C & 5 & 12 & 14 & 43.09955 & -17.094 & 20.66225 & 44.22111 & 55.63549 \\
\hline PRE9 & YGR135W & 5 & 12 & 15 & 40.24691 & 22.56214 & 13.88368 & 27.35849 & 32.82051 \\
\hline MSB2 & YGR014W & 5 & 12 & 16 & 40.29703 & -8.27206 & -3.125 & 41.50485 & 46.875 \\
\hline \multirow[t]{2}{*}{ CAF130 } & YGR134W & 5 & 12 & 17 & 40.8998 & 49.06015 & 44.69112 & 54.16667 & 61.64021 \\
\hline & YGR012W & 5 & 12 & 18 & 28.37259 & 19.73333 & 23.87665 & 31.31068 & 46.09756 \\
\hline \multirow[t]{2}{*}{ PEX4 } & YGR133W & 5 & 12 & 19 & 26.54691 & -2.99625 & -11.3318 & 53.26371 & 55.03513 \\
\hline & YGR011W & 5 & 12 & 20 & 44.7944 & 45.82593 & 3.737113 & 41.40625 & 55.932 \\
\hline
\end{tabular}




\begin{tabular}{|c|c|c|c|c|c|c|c|c|c|}
\hline PHB1 & YGR132C & 5 & 12 & 21 & 42.07709 & 16.60777 & 8.11044 & 31.78082 & 40.63205 \\
\hline \multirow[t]{2}{*}{ NMA2 } & YGR010W & 5 & 12 & 22 & 63.83588 & 25.2149 & 22.83272 & 38.2263 & 47.51773 \\
\hline & YGR131W & 5 & 12 & 23 & 62.41007 & -13.0435 & & 44.1989 & 63.48449 \\
\hline HIS3 & YOR202W & 5 & 12 & 24 & 64.4898 & 39.26097 & 7.443683 & & \\
\hline \multirow[t]{2}{*}{ HIS3 } & YOR202W & 5 & 13 & 1 & 33.38997 & -35.8491 & 11.02629 & & \\
\hline & YGL041C & 5 & 13 & 2 & 58.04848 & -20.3165 & 45.18188 & 35.22013 & 40.40179 \\
\hline \multirow[t]{2}{*}{ SAE2 } & YGL175C & 5 & 13 & 3 & 0.583658 & 100 & 8.108108 & 40.43011 & 42.64706 \\
\hline & YGL039W & 5 & 13 & 4 & -18.6944 & 100 & 5.166375 & 69.38202 & 35.34483 \\
\hline \multirow[t]{2}{*}{ BUD13 } & YGL174W & 5 & 13 & 5 & -3.73692 & -9.79667 & 35.51724 & 34.19355 & 15.49708 \\
\hline & YGL036W & 5 & 13 & 6 & 24.25107 & 100 & 43.6553 & 56.38767 & 34.31953 \\
\hline KEM1 & YGL173C & 5 & 13 & 7 & 72.79693 & 100 & 100 & 85.03401 & 80.83067 \\
\hline MIG1 & YGL035C & 5 & 13 & 8 & 45.2381 & 100 & 37.67857 & 74.93917 & 57.83784 \\
\hline \multirow[t]{2}{*}{ SPO74 } & YGL170C & 5 & 13 & 9 & 51.29803 & -14.8241 & 27.12054 & 53.28084 & 45.54707 \\
\hline & YGL034C & 5 & 13 & 10 & 50 & 63.09795 & -0.661 & 32.31441 & 47.59494 \\
\hline HUR1 & YGL168W & 5 & 13 & 11 & 43.43029 & 47.06868 & 14.0884 & -21.2202 & 17.38149 \\
\hline AGA2 & YGL032C & 5 & 13 & 12 & 51.01833 & 93.61233 & 4.415372 & 76.1039 & 55 \\
\hline PMR1 & YGL167C & 5 & 13 & 13 & 37.5 & 73.5786 & 43.27917 & 9.904153 & 23.5732 \\
\hline RPL24A & YGL031C & 5 & 13 & 14 & 47.82609 & 44.08759 & 37.2449 & 23.83901 & 40.54054 \\
\hline CUP2 & YGL166W & 5 & 13 & 15 & 27.68763 & 63.70023 & -13.309 & 22.72727 & 54.56674 \\
\hline \multirow[t]{2}{*}{ SCW11 } & YGL028C & 5 & 13 & 16 & 43.05949 & 38.91798 & -24.0402 & 19.87179 & 49.76526 \\
\hline & YGL165C & 5 & 13 & 17 & 41.69041 & 77.27273 & -7.72358 & 40.98765 & 58.09524 \\
\hline CWH41 & YGL027C & 5 & 13 & 18 & 42.85714 & 17.66562 & 39.06103 & 27.88671 & 54.19664 \\
\hline YRB30 & YGL164C & 5 & 13 & 19 & 38.15385 & 60.27875 & -19.6544 & 17.64706 & 41.50943 \\
\hline TRP5 & YGL026C & 5 & 13 & 20 & 33.12565 & 35.2071 & 55.58565 & 65.57789 & 79.25 \\
\hline \multirow[t]{2}{*}{ RAD54 } & YGL163C & 5 & 13 & 21 & 40.71856 & 95.91281 & \#DIV/0! & 36.5651 & 57.25594 \\
\hline & YGL024W & 5 & 13 & 22 & 90.02876 & 91.06628 & 85.81979 & 58.39243 & 83.66834 \\
\hline SUT1 & YGL162W & 5 & 13 & 23 & 66.58692 & 35.59322 & 29.26337 & 40.84821 & 61.44578 \\
\hline HIS3 & YOR202W & 5 & 13 & 24 & 18.18182 & 74.34679 & 9.032258 & & \\
\hline HIS3 & YOR202W & 5 & 14 & 1 & -1.35593 & 17.06587 & 6.490566 & & \\
\hline ACB1 & YGR037C & 5 & 14 & 2 & -16.6911 & 29.29936 & 27.44887 & 47.43875 & 42.36559 \\
\hline \multirow[t]{2}{*}{ RTS3 } & YGR161C & 5 & 14 & 3 & -47.4874 & 100 & 16.97417 & 54.29553 & 48.33837 \\
\hline & YGR035C & 5 & 14 & 4 & -1.61054 & 100 & 25.57781 & 57.277 & 49.61832 \\
\hline $\mathrm{CHO} 2$ & YGR157W & 5 & 14 & 5 & 45.125 & 100 & 72.72727 & 51.47929 & 38.79518 \\
\hline \multirow[t]{2}{*}{ RPL26B } & YGR034W & 5 & 14 & 6 & 28.58617 & 100 & 16.64168 & 61.95122 & 54.43038 \\
\hline & YGR154C & 5 & 14 & 7 & 40.74505 & 100 & 45.69288 & 31.81818 & 20 \\
\hline FMP17 & YGR033C & 5 & 14 & 8 & 46.90832 & 100 & -6.56934 & 62.35294 & 53.96825 \\
\hline TOS10 & YGR153W & 5 & 14 & 9 & 49.45598 & 100 & 9.355247 & 72.52747 & 45.83333 \\
\hline GSC2 & YGR032W & 5 & 14 & 10 & 53.41556 & 100 & -8.59649 & 72.90323 & 61.83575 \\
\hline \multirow[t]{3}{*}{ RSR1 } & YGR152C & 5 & 14 & 11 & 46.04605 & 51.92308 & 11.37088 & 47.50542 & 59.47242 \\
\hline & YGR031W & 5 & 14 & 12 & 54.31034 & 72.9783 & 8.62069 & 50.45249 & 42.98441 \\
\hline & YGR151C & 5 & 14 & 13 & 41.92186 & 25.62396 & -3.75865 & 37.87529 & 45.08929 \\
\hline \multirow[t]{2}{*}{ MSP1 } & YGR028W & 5 & 14 & 14 & 35.30895 & 26.0101 & 22.90749 & 47.25537 & 59.95526 \\
\hline & YGR149W & 5 & 14 & 15 & 54.35185 & 29.53737 & 5.125977 & 42.23433 & 55.37757 \\
\hline
\end{tabular}




\begin{tabular}{|c|c|c|c|c|c|c|c|c|c|}
\hline RPS25A & YGR027C & 5 & 14 & 16 & 61.53846 & 68.08036 & 59.91649 & 74.27885 & 72.90749 \\
\hline \multirow[t]{4}{*}{ RPL24B } & YGR148C & 5 & 14 & 17 & 44.12331 & 73.40426 & 58.16062 & 28.91892 & 58.00464 \\
\hline & YGR026W & 5 & 14 & 18 & 52.06136 & 79.59641 & 22.86357 & 34.69388 & 61.74497 \\
\hline & YGR146C & 5 & 14 & 19 & 52.14133 & 66.56977 & 60.07533 & 28.44639 & 64.35644 \\
\hline & YGR025W & 5 & 14 & 20 & 40.05525 & -1.88679 & 51.79487 & 20.73864 & 45.51887 \\
\hline THI4 & YGR144W & 5 & 14 & 21 & 41.39265 & 25.97087 & -12.4894 & 33.95722 & 61.0245 \\
\hline MTL1 & YGR023W & 5 & 14 & 22 & 58.28516 & -20.4724 & -7.42534 & 33.42105 & 60.56034 \\
\hline SKN1 & YGR143W & 5 & 14 & 23 & 45.10121 & -28.25 & 4.060018 & -9.93228 & 75.44248 \\
\hline HIS3 & YOR202W & 5 & 14 & 24 & 44.54638 & -29.0484 & 2.361005 & & \\
\hline \multirow[t]{3}{*}{ HIS3 } & YOR202W & 5 & 15 & 1 & 58.97436 & -9.74265 & -14.6943 & & \\
\hline & YGL059W & 5 & 15 & 2 & 54.10628 & 22.49489 & 25.02317 & & \\
\hline & YGL199C & 5 & 15 & 3 & 62.32508 & 100 & 17.03704 & & \\
\hline RAD6 & YGL058W & 5 & 15 & 4 & 46.8948 & 25.37313 & 24.4314 & & \\
\hline \multirow[t]{2}{*}{ YIP4 } & YGL198W & 5 & 15 & 5 & 24.31259 & 100 & 19.42369 & & \\
\hline & YGL057C & 5 & 15 & 6 & 26.54746 & 100 & -19.914 & & \\
\hline MDS3 & YGL197W & 5 & 15 & 7 & 33.65517 & 98.5119 & 18.36327 & & \\
\hline \multirow[t]{2}{*}{ SDS23 } & YGL056C & 5 & 15 & 8 & 41.04046 & 70.94474 & 6.946984 & & \\
\hline & YGL196W & 5 & 15 & 9 & 59.03285 & 73.29377 & 14.53901 & & \\
\hline ERV14 & YGL054C & 5 & 15 & 10 & 49.57447 & 72.1374 & 52.84916 & & \\
\hline GCN1 & YGL195W & 5 & 15 & 11 & 47.60614 & 67.61062 & 17.52464 & & \\
\hline PRM8 & YGL053W & 5 & 15 & 12 & 56.61626 & 68.96552 & 71.52406 & & \\
\hline HOS2 & YGL194C & 5 & 15 & 13 & 51.4947 & -19.0099 & 28.79098 & & \\
\hline MST27 & YGL051W & 5 & 15 & 14 & 46.7425 & 72.85714 & 17.60204 & & \\
\hline \multirow[t]{2}{*}{ GTS1 } & YGL181W & 5 & 15 & 15 & 60.43643 & 73.61702 & 58.60374 & & \\
\hline & YGL050W & 5 & 15 & 16 & 43.35206 & 33.26531 & 44.88501 & & \\
\hline \multirow[t]{2}{*}{ ATG1 } & YGL180W & 5 & 15 & 17 & 40.97087 & 44.16404 & -18.4091 & & \\
\hline & YGL046W & 5 & 15 & 18 & 47.88214 & 65.20376 & 76.16633 & & \\
\hline TOS3 & YGL179C & 5 & 15 & 19 & 52.62636 & 9.160305 & 3.71308 & & \\
\hline \multirow[t]{2}{*}{ RIM8 } & YGL045W & 5 & 15 & 20 & 77.09924 & 75.13043 & 69.07124 & & \\
\hline & YGL177W & 5 & 15 & 21 & 61.68133 & 74.4836 & 61.12703 & & \\
\hline \multirow[t]{2}{*}{ DST1 } & YGL043W & 5 & 15 & 22 & 62.36162 & 65.60976 & 68.93204 & & \\
\hline & YGL176C & 5 & 15 & 23 & 37.08738 & 5.21327 & 3.435115 & & \\
\hline HIS3 & YOR202W & 5 & 15 & 24 & -28.7356 & 17.46231 & 32.45357 & & \\
\hline HIS3 & YOR202W & 5 & 16 & 1 & 44.44444 & 8.67052 & 42.10526 & & \\
\hline HIS3 & YOR202W & 5 & 16 & 2 & 30.75769 & 41.79266 & 40.11142 & & \\
\hline HIS3 & YOR202W & 5 & 16 & 3 & 11.3089 & 30.08739 & 53.60231 & & \\
\hline HIS3 & YOR202W & 5 & 16 & 4 & -17.5627 & 53.74532 & 21.43541 & & \\
\hline HIS3 & YOR202W & 5 & 16 & 5 & -1.6 & 15.43274 & 13.41571 & & \\
\hline HIS3 & YOR202W & 5 & 16 & 6 & -1.80624 & 41.29721 & 72.40705 & & \\
\hline HIS3 & YOR202W & 5 & 16 & 7 & 12.82468 & -0.26212 & 24.52555 & & \\
\hline HIS3 & YOR202W & 5 & 16 & 8 & 15.41916 & 26.48352 & 26.03627 & & \\
\hline HIS3 & YOR202W & 5 & 16 & 9 & 56.31529 & 37.91946 & 38.5214 & & \\
\hline HIS3 & YOR202W & 5 & 16 & 10 & 22.60749 & 9.810875 & 29.08623 & & \\
\hline
\end{tabular}




\begin{tabular}{|c|c|c|c|c|c|c|c|c|c|}
\hline HIS3 & YOR202W & 5 & 16 & 11 & 43.20594 & 20.57522 & 59.84556 & & \\
\hline HIS3 & YOR202W & 5 & 16 & 12 & 36.84677 & 39.77798 & 0.991736 & & \\
\hline HIS3 & YOR202W & 5 & 16 & 13 & 60.77547 & 60.77441 & 41.14471 & & \\
\hline HIS3 & YOR202W & 5 & 16 & 14 & 54.96922 & 56.61047 & 41.24597 & & \\
\hline HIS3 & YOR202W & 5 & 16 & 15 & 34.22877 & 21.75141 & 10.55276 & & \\
\hline HIS3 & YOR202W & 5 & 16 & 16 & 42.06081 & 60.46512 & -1.18343 & & \\
\hline HIS3 & YOR202W & 5 & 16 & 17 & 58.82908 & 62.5947 & 34.48873 & & \\
\hline HIS3 & YOR202W & 5 & 16 & 18 & 30.41988 & 48.4767 & 38.28571 & & \\
\hline HIS3 & YOR202W & 5 & 16 & 19 & 36.21399 & 61.92171 & 55.55556 & & \\
\hline HIS3 & YOR202W & 5 & 16 & 20 & 49.16388 & 69.87296 & 20.76765 & & \\
\hline HIS3 & YOR202W & 5 & 16 & 21 & 54.4757 & 67.08438 & 51.3465 & & \\
\hline HIS3 & YOR202W & 5 & 16 & 22 & 60.84071 & 19.79638 & 35.44415 & & \\
\hline HIS3 & YOR202W & 5 & 16 & 23 & 47.14612 & 42.73084 & 6.105263 & & \\
\hline HIS3 & YOR202W & 5 & 16 & 24 & -11.3797 & 20.47325 & 16.15691 & & \\
\hline HIS3 & YOR202W & 6 & 1 & 1 & 69.05291 & 77.73689 & & & \\
\hline HIS3 & YOR202W & 6 & 1 & 2 & 48.08044 & -8.41622 & & & \\
\hline HIS3 & YOR202W & 6 & 1 & 3 & \#DIV/0! & 15.70319 & & & \\
\hline HIS3 & YOR202W & 6 & 1 & 4 & 25.19824 & 35.3187 & & & \\
\hline HIS3 & YOR202W & 6 & 1 & 5 & 7.403315 & 14.0625 & & & \\
\hline HIS3 & YOR202W & 6 & 1 & 6 & 11.9403 & 26.12482 & & & \\
\hline HIS3 & YOR202W & 6 & 1 & 7 & 39.91597 & 0.927835 & & & \\
\hline HIS3 & YOR202W & 6 & 1 & 8 & 12.69841 & 22.14567 & & & \\
\hline HIS3 & YOR202W & 6 & 1 & 9 & 82.72251 & 10.04785 & & & \\
\hline HIS3 & YOR202W & 6 & 1 & 10 & 8.759757 & 2 & & & \\
\hline HIS3 & YOR202W & 6 & 1 & 11 & 92.74673 & 24.29501 & & & \\
\hline HIS3 & YOR202W & 6 & 1 & 12 & 40.86511 & -20.4706 & & & \\
\hline HIS3 & YOR202W & 6 & 1 & 13 & 3.618421 & 1.940639 & & & \\
\hline HIS3 & YOR202W & 6 & 1 & 14 & 7.508834 & -20.0328 & & & \\
\hline HIS3 & YOR202W & 6 & 1 & 15 & -16.4397 & 0.220994 & & & \\
\hline HIS3 & YOR202W & 6 & 1 & 16 & 45.65702 & 8.011583 & & & \\
\hline HIS3 & YOR202W & 6 & 1 & 17 & 26.36684 & 16.03289 & & & \\
\hline HIS3 & YOR202W & 6 & 1 & 18 & 30.39486 & 19.9 & & & \\
\hline HIS3 & YOR202W & 6 & 1 & 19 & 4.193548 & 12.73743 & & & \\
\hline HIS3 & YOR202W & 6 & 1 & 20 & 55.07777 & 26.24647 & & & \\
\hline HIS3 & YOR202W & 6 & 1 & 21 & 32.39203 & 13.56899 & & & \\
\hline HIS3 & YOR202W & 6 & 1 & 22 & 49.70356 & 10.81731 & & & \\
\hline HIS3 & YOR202W & 6 & 1 & 23 & 13.64929 & 13.36538 & & & \\
\hline HIS3 & YOR202W & 6 & 1 & 24 & -11.1836 & 22.24797 & & & \\
\hline HIS3 & YOR202W & 6 & 2 & 1 & 41.95545 & 66.19718 & & & \\
\hline SSF1 & YHR066W & 6 & 2 & 2 & 57.93991 & 15.54227 & & -16.9412 & 13.26531 \\
\hline SVP26 & YHR181W & 6 & 2 & 3 & 89.3617 & \#DIV/0! & & 16.27358 & 26.68394 \\
\hline \multirow[t]{2}{*}{ GIC1 } & YHR061C & 6 & 2 & 4 & \#DIV/0! & 24.88038 & & 25 & 23.52941 \\
\hline & YHR180W & 6 & 2 & 5 & 36.46724 & 17.14286 & & 45.75835 & 63.08725 \\
\hline
\end{tabular}




\begin{tabular}{|c|c|c|c|c|c|c|c|c|}
\hline FYV4 & YHR059W & 6 & 2 & 6 & 44.40497 & 8.16092 & 31.97115 & -1.2945 \\
\hline OYE2 & YHR179W & 6 & 2 & 7 & 48.69029 & 16.44399 & 49.22737 & 18.93491 \\
\hline CPR2 & YHR057C & 6 & 2 & 8 & 17.23108 & 7.369403 & 67.37213 & 25.68306 \\
\hline STB5 & YHR178W & 6 & 2 & 9 & 98.17008 & \#DIV/0! & 50.10571 & 29.13165 \\
\hline SMF2 & YHR050W & 6 & 2 & 10 & 59.75774 & 19.04287 & 58.97921 & 23.5589 \\
\hline FMO1 & YHR176W & 6 & 2 & 11 & 100 & \#DIV/0! & 55.35354 & 6.944444 \\
\hline FSH1 & YHR049W & 6 & 2 & 12 & 8.547889 & -13.9032 & 67.52768 & 19.21053 \\
\hline \multirow[t]{2}{*}{ ATG7 } & YHR171W & 6 & 2 & 13 & 31.19171 & 21.22699 & 64.01515 & 14.73684 \\
\hline & YHR049C-A & 6 & 2 & 14 & 23.7911 & 8.833922 & 2.01005 & -6.68693 \\
\hline \multirow[t]{2}{*}{ THP2 } & YHR167W & 6 & 2 & 15 & 25.74917 & 1.666667 & 64.40972 & 3.480278 \\
\hline & YHR048W & 6 & 2 & 16 & 65.6644 & 27.95181 & 57.65306 & 20.05208 \\
\hline SOL3 & YHR163W & 6 & 2 & 17 & 42.23035 & 30.84261 & 69.52381 & 9.405941 \\
\hline \multirow[t]{2}{*}{ AAP1' } & YHR047C & 6 & 2 & 18 & 41 & 35.08772 & 66.22889 & 14.87805 \\
\hline & YHR162W & 6 & 2 & 19 & 45.86729 & -0.29851 & 56.27451 & 45.28796 \\
\hline INM1 & YHR046C & 6 & 2 & 20 & 61.2069 & 36.32075 & 37.84278 & 22.87234 \\
\hline \multirow[t]{2}{*}{ YAP1801 } & YHR161C & 6 & 2 & 21 & 47.02653 & 21.58155 & 59.28854 & 28.76344 \\
\hline & YHR045W & 6 & 2 & 22 & 34.31517 & 4.249292 & 71.92225 & 13.05638 \\
\hline PEX18 & YHR160C & 6 & 2 & 23 & -16.1602 & 5.08326 & 58.83534 & 17.75457 \\
\hline HIS3 & YOR202W & 6 & 2 & 24 & 26.75159 & -10.4242 & & \\
\hline HIS3 & YOR202W & 6 & 3 & 1 & -31.906 & 23.6417 & & \\
\hline PBP1 & YGR178C & 6 & 3 & 2 & 29.64492 & 11.95559 & 43.3584 & 23.27189 \\
\hline SHU1 & YHLO06C & 6 & 3 & 3 & 87.19677 & 93.65854 & 45.4955 & 26.44628 \\
\hline \multirow[t]{3}{*}{ ATF2 } & YGR177C & 6 & 3 & 4 & \#DIV/0! & \#DIV/0! & 33.19756 & 14.72684 \\
\hline & YHLO05C & 6 & 3 & 5 & -5.6338 & 1.360544 & -52.9412 & 2.588997 \\
\hline & YGR176W & 6 & 3 & 6 & 42.46285 & -20.7442 & 61.76471 & 26.85714 \\
\hline LAG1 & YHL003C & 6 & 3 & 7 & 70.51836 & 6.923838 & 47.7396 & 31.15727 \\
\hline CBP4 & YGR174C & 6 & 3 & 8 & 16.14679 & 21.9419 & 24.27184 & 16.30769 \\
\hline HSE1 & YHL002W & 6 & 3 & 9 & \#DIV/0! & -0.71795 & 32.00795 & 11.45251 \\
\hline RBG2 & YGR173W & 6 & 3 & 10 & 79.38144 & 33.13783 & 46.39175 & 18.09524 \\
\hline $\cos 6$ & YGR295C & 6 & 3 & 11 & -18.8906 & 6.461864 & 69.5122 & 20.88773 \\
\hline \multirow[t]{2}{*}{ PSD2 } & YGR170W & 6 & 3 & 12 & -26.6881 & 5.681818 & 30.93023 & 26.91131 \\
\hline & YGR290W & 6 & 3 & 13 & 14.42516 & 3.657363 & 64.79592 & 10 \\
\hline PUS6 & YGR169C & 6 & 3 & 14 & 15.07538 & 1.214953 & 54.67197 & 0.842697 \\
\hline \multirow[t]{3}{*}{ MAL13 } & YGR288W & 6 & 3 & 15 & 45.14388 & 23.37917 & 54.36893 & 18.95604 \\
\hline & YGR168C & 6 & 3 & 16 & 94.77521 & 0.269058 & 46.55532 & 11.2532 \\
\hline & YGR287C & 6 & 3 & 17 & 91.54286 & -5.73689 & 68.9013 & 8.28877 \\
\hline KRE11 & YGR166W & 6 & 3 & 18 & 62.3431 & 43.37748 & 56.31769 & 8.461538 \\
\hline \multirow[t]{2}{*}{$\mathrm{BIO2}$} & YGR286C & 6 & 3 & 19 & 65.88889 & 35.94937 & 44.71058 & 22.58065 \\
\hline & YGR164W & 6 & 3 & 20 & 13.47737 & -2.61194 & 32.67327 & 53.82883 \\
\hline ERV29 & YGR284C & 6 & 3 & 21 & 3.496503 & 1.888772 & 54.56204 & 13.11475 \\
\hline \multirow[t]{2}{*}{ GTR2 } & YGR163W & 6 & 3 & 22 & 45.91837 & -7.86207 & 35.141 & -4.5045 \\
\hline & YGR283C & 6 & 3 & 23 & 30.90316 & -4.24374 & 29.69072 & 38.25 \\
\hline HIS3 & YOR202W & 6 & 3 & 24 & 21.07574 & -6.82635 & & \\
\hline
\end{tabular}




\begin{tabular}{|c|c|c|c|c|c|c|c|c|}
\hline HIS3 & YOR202W & 6 & 4 & 1 & -19.7389 & 14.52785 & & \\
\hline KSP1 & YHR082C & 6 & 4 & 2 & 21.62791 & 37.9646 & -1.62602 & 24.73118 \\
\hline RPN10 & YHR200W & 6 & 4 & 3 & 44.13462 & -10.1545 & 45.12821 & 28.73563 \\
\hline LRP1 & YHR081W & 6 & 4 & 4 & 92.48705 & \#DIV/0! & 26.88442 & 37.24832 \\
\hline \multirow[t]{2}{*}{ FMP34 } & YHR199C & 6 & 4 & 5 & 100 & \#DIV/0! & 32.61339 & 31.75074 \\
\hline & YHR080C & 6 & 4 & 6 & 15.70681 & 1.451906 & 61.28364 & 34.52381 \\
\hline \multirow[t]{2}{*}{ FMP22 } & YHR198C & 6 & 4 & 7 & 84.18757 & 43.77682 & 8.130081 & 51.58501 \\
\hline & YHR079C-B & 6 & 4 & 8 & 21.26126 & 0.267618 & 60.18735 & 33.69176 \\
\hline NVJ1 & YHR195W & 6 & 4 & 9 & 100 & \#DIV/0! & 17.1831 & 20.92308 \\
\hline IRE1 & YHR079C & 6 & 4 & 10 & 18.03885 & 23.33333 & 41.68514 & 25.7971 \\
\hline \multirow[t]{2}{*}{ MDM31 } & YHR194W & 6 & 4 & 11 & 40.15152 & -11.8519 & 56.68317 & 24.07407 \\
\hline & YHR078W & 6 & 4 & 12 & 31.52174 & 8.290816 & 70.88608 & 19.37173 \\
\hline EGD2 & YHR193C & 6 & 4 & 13 & 19.67681 & -2.58621 & 48.59611 & 20.35398 \\
\hline NMD2 & YHR077C & 6 & 4 & 14 & 80.64919 & 1.06383 & 81.02041 & 34.68635 \\
\hline CTF8 & YHR191C & 6 & 4 & 15 & 79.90148 & -27.8195 & 72.26415 & 37.76435 \\
\hline PTC7 & YHR076W & 6 & 4 & 16 & 100 & \#DIV/0! & 31.67421 & 34.12463 \\
\hline PTH1 & YHR189W & 6 & 4 & 17 & 100 & \#DIV/0! & 77.02448 & 33.61345 \\
\hline PPE1 & YHR075C & 6 & 4 & 18 & 90.02822 & 25 & 59.95976 & 47.12991 \\
\hline PFS1 & YHR185C & 6 & 4 & 19 & 47.50378 & 23.65196 & 64.22893 & 42.15686 \\
\hline OSH3 & YHR073W & 6 & 4 & 20 & 71.91393 & -0.99404 & 23.92344 & 39.04762 \\
\hline SSP1 & YHR184W & 6 & 4 & 21 & 71.0076 & -28.7356 & 33.56481 & 42.12034 \\
\hline \multirow[t]{2}{*}{ HTD2 } & YHR067W & 6 & 4 & 22 & 40.14156 & 56.32066 & 79.72973 & 56.54596 \\
\hline & YHR182W & 6 & 4 & 23 & 45.1497 & 2.09205 & 60 & 49.50249 \\
\hline HIS3 & YOR202W & 6 & 4 & 24 & 10.92657 & -11.6481 & & \\
\hline HIS3 & YOR202W & 6 & 5 & 1 & -23.1638 & 15.10554 & & \\
\hline FYV8 & YGR196C & 6 & 5 & 2 & -10.5263 & 24.12109 & 0 & 27.00297 \\
\hline OPI1 & YHLO20C & 6 & 5 & 3 & 68.10229 & 60.4333 & 16.43836 & 73.40426 \\
\hline XKS1 & YGR194C & 6 & 5 & 4 & 62.88515 & -0.41841 & 66.66667 & 30.61224 \\
\hline APM2 & YHL019C & 6 & 5 & 5 & -11.8943 & -4.15512 & 46.08879 & 30.59937 \\
\hline \multirow[t]{2}{*}{ PDX1 } & YGR193C & 6 & 5 & 6 & 86.79962 & 9.9631 & 70.72165 & 38.29114 \\
\hline & YHL017W & 6 & 5 & 7 & -5.98291 & -24.9022 & 48.49785 & 51.25786 \\
\hline TDH3 & YGR192C & 6 & 5 & 8 & 38.80478 & 8.534323 & 45.06726 & 34.35374 \\
\hline DUR3 & YHL016C & 6 & 5 & 9 & 38.40206 & -1.2 & 24.94172 & 35.52239 \\
\hline $\mathrm{CRH} 1$ & YGR189C & 6 & 5 & 10 & 14.64531 & -14.1388 & 17.85064 & 40.70796 \\
\hline YLF2 & YHL014C & 6 & 5 & 11 & 14.75054 & -22.7418 & 52.55624 & 46.73913 \\
\hline \multirow[t]{2}{*}{ BUB1 } & YGR188C & 6 & 5 & 12 & 51.89137 & 27.44681 & 66.7992 & 43.42508 \\
\hline & YHL013C & 6 & 5 & 13 & 17.74976 & 0.726141 & 64.02027 & 43.54839 \\
\hline \multirow[t]{2}{*}{$\mathrm{HGH} 1$} & YGR187C & 6 & 5 & 14 & 82.50319 & 73.91304 & 25.10917 & 41.23077 \\
\hline & YHL012W & 6 & 5 & 15 & 15.55334 & -4.96454 & 43.31984 & 34.97268 \\
\hline \multirow[t]{2}{*}{ UBR1 } & YGR184C & 6 & 5 & 16 & -25.7396 & 0.675024 & 67.65625 & 54.92228 \\
\hline & YHL010C & 6 & 5 & 17 & 0.775194 & -3.39943 & 74.43869 & 45.32164 \\
\hline QCR9 & YGR183C & 6 & 5 & 18 & 3.901437 & -1.19826 & 54.04255 & 35.09934 \\
\hline YAP3 & YHL009C & 6 & 5 & 19 & 82.12121 & 81.84615 & 71.90635 & 45.66929 \\
\hline
\end{tabular}




\begin{tabular}{|c|c|c|c|c|c|c|c|c|}
\hline & YGR182C & 6 & 5 & 20 & 89.11368 & 24.4898 & 37.0892 & 44.1791 \\
\hline & YHL008C & 6 & 5 & 21 & 41.74136 & -5.72687 & 63.98601 & 53.03867 \\
\hline TIM13 & YGR181W & 6 & 5 & 22 & -15.4126 & 1.944444 & 58.7473 & 41.96429 \\
\hline STE2O & YHL007C & 6 & 5 & 23 & 74.44238 & -4.19486 & 54.34298 & 59.61538 \\
\hline HIS3 & YOR202W & 6 & 5 & 24 & 15.5642 & -18.3673 & & \\
\hline HIS3 & YOR202W & 6 & 6 & 1 & 64.8824 & 28.09524 & & \\
\hline \multirow[t]{2}{*}{ YPT35 } & YHR105W & 6 & 6 & 2 & 45.37037 & -7.18085 & 40.11194 & 37.20317 \\
\hline & YIL006W & 6 & 6 & 3 & 22.057 & 4.251012 & 22.86325 & 55.08685 \\
\hline GRE3 & YHR104W & 6 & 6 & 4 & 62.27545 & 13.35505 & 60.72144 & 41.61491 \\
\hline EPS1 & YIL005W & 6 & 6 & 5 & 74.25101 & 23.1441 & 55.37525 & 46.41745 \\
\hline SBE22 & YHR103W & 6 & 6 & 6 & 31.97625 & -14.8284 & 74.53184 & 42.94118 \\
\hline \multirow[t]{3}{*}{ INP51 } & YIL002C & 6 & 6 & 7 & 63.02521 & 30.02833 & 67.51969 & 47.23926 \\
\hline & YHR097C & 6 & 6 & 8 & -4.14129 & 12.94886 & 49.71963 & 41.73913 \\
\hline & YIL001W & 6 & 6 & 9 & 25.69948 & -26.6487 & 20.76372 & 40.44586 \\
\hline \multirow[t]{4}{*}{ HXT5 } & YHR096C & 6 & 6 & 10 & 35.16854 & 8.213552 & 74.14966 & 44.94048 \\
\hline & YHR210C & 6 & 6 & 11 & 10.26667 & -1.86916 & 64.67066 & 40.25157 \\
\hline & YHR095W & 6 & 6 & 12 & 63.15789 & 28.85431 & 58.37838 & 35.26171 \\
\hline & YHR209W & 6 & 6 & 13 & 22.43902 & -14.3606 & 58.33333 & 42.10526 \\
\hline HXT1 & YHR094C & 6 & 6 & 14 & 38.96761 & 22.95552 & 51.6129 & 44.57478 \\
\hline SET5 & YHR207C & 6 & 6 & 15 & 13.14103 & 1.533019 & 65.64626 & 34.22619 \\
\hline АHT1 & YHR093W & 6 & 6 & 16 & 44.3609 & 12.42604 & 38.94009 & 46.74556 \\
\hline SKN7 & YHR206W & 6 & 6 & 17 & 38.18182 & 8.143939 & 48.4787 & 42.31884 \\
\hline HXT4 & YHR092C & 6 & 6 & 18 & -4.3956 & -9.73558 & 30.20833 & 54.6875 \\
\hline \multirow[t]{2}{*}{ MNL1 } & YHR204W & 6 & 6 & 19 & 50.27563 & 1.559454 & 24.81928 & 48.52459 \\
\hline & YHR087W & 6 & 6 & 20 & 25.83784 & 6.638567 & 49.87775 & 57.1875 \\
\hline RPS4B & YHR203C & 6 & 6 & 21 & 57.74648 & 12.89474 & -8.75706 & 47.09898 \\
\hline \multirow[t]{2}{*}{ NAM8 } & YHR086W & 6 & 6 & 22 & -16.3842 & -15.7044 & 14.52514 & 47.05882 \\
\hline & YHR202W & 6 & 6 & 23 & 9.463415 & 4.612707 & 54.18502 & 51.26263 \\
\hline HIS3 & YOR202W & 6 & 6 & 24 & 15.70482 & -8.11765 & & \\
\hline HIS3 & YOR202W & 6 & 7 & 1 & 48.34846 & 24.70893 & & \\
\hline TRX2 & YGR209C & 6 & 7 & 2 & 12.10191 & 5.590717 & 29.87013 & 37.61468 \\
\hline GOS1 & YHL031C & 6 & 7 & 3 & 69.85075 & 9.667674 & 37.91946 & 30.96927 \\
\hline SER2 & YGR208W & 6 & 7 & 4 & 19.68504 & 24.08377 & 40.96774 & 52.85088 \\
\hline \multirow[t]{4}{*}{ ECM29 } & YHL030W & 6 & 7 & 5 & -16.2762 & 14.97093 & 53.79747 & 41.07486 \\
\hline & YGR207C & 6 & 7 & 6 & 24.63519 & 1.864407 & 49.70414 & 63.74502 \\
\hline & YHL029C & 6 & 7 & 7 & 46.06227 & -3.21678 & 43.23529 & 62.5 \\
\hline & YGR206W & 6 & 7 & 8 & 28.53567 & -14.5119 & 41.74455 & 41.93548 \\
\hline \multirow[t]{2}{*}{ WSC4 } & YHL028W & 6 & 7 & 9 & 48.46491 & 35.99034 & 38.58521 & 47.17742 \\
\hline & YGR205W & 6 & 7 & 10 & 54.29122 & 5.392912 & 36.21262 & 59.67413 \\
\hline \multirow[t]{3}{*}{ RIM101 } & YHL027W & 6 & 7 & 11 & 52.64516 & -9.67742 & 36.07038 & 51.35699 \\
\hline & YGR203W & 6 & 7 & 12 & 34.42623 & 6.666667 & 40.52478 & 45.81818 \\
\hline & YHL026C & 6 & 7 & 13 & 1.802885 & -5.62771 & 42.02899 & 51.86567 \\
\hline РCT1 & YGR202C & 6 & 7 & 14 & 9.600863 & 2.164948 & 35.62092 & 27.15517 \\
\hline
\end{tabular}




\begin{tabular}{|c|c|c|}
\hline \multirow[t]{2}{*}{ SNF6 } & YHL025W & 6 \\
\hline & YGR201C & 6 \\
\hline RIM4 & YHL024W & 6 \\
\hline ELP2 & YGR200C & 6 \\
\hline RMD11 & YHL023C & 6 \\
\hline PMT6 & YGR199W & 6 \\
\hline SPO11 & YHL022C & 6 \\
\hline SNG1 & YGR197C & 6 \\
\hline FMP12 & YHL021C & 6 \\
\hline HIS3 & YOR202W & 6 \\
\hline HIS3 & YOR202W & 6 \\
\hline TOM71 & YHR117W & 6 \\
\hline SNL1 & YIL016W & 6 \\
\hline $\operatorname{cox} 23$ & YHR116W & 6 \\
\hline BAR1 & YIL015W & 6 \\
\hline \multirow[t]{2}{*}{ DMA1 } & YHR115C & 6 \\
\hline & YIL015C-A & 6 \\
\hline BZZ1 & YHR114W & 6 \\
\hline \multirow[t]{2}{*}{ MNT3 } & YIL014W & 6 \\
\hline & YHR113W & 6 \\
\hline \multirow[t]{3}{*}{ PDR11 } & YIL013C & 6 \\
\hline & YHR112C & 6 \\
\hline & YIL012W & 6 \\
\hline UBA4 & YHR111W & 6 \\
\hline TIR3 & YIL011W & 6 \\
\hline ERP5 & YHR110W & 6 \\
\hline DOT5 & YIL010W & 6 \\
\hline CTM1 & YHR109W & 6 \\
\hline EST3 & YIL009C-A & 6 \\
\hline GGA2 & YHR108W & 6 \\
\hline URM1 & YIL008W & 6 \\
\hline TRR2 & YHR106W & 6 \\
\hline NAS2 & YIL007C & 6 \\
\hline HIS3 & YOR202W & 6 \\
\hline HIS3 & YOR202W & 6 \\
\hline DIE2 & YGR227W & 6 \\
\hline \multirow[t]{3}{*}{ ECM34 } & YHL043W & 6 \\
\hline & YGR226C & 6 \\
\hline & YHL042W & 6 \\
\hline \multirow[t]{2}{*}{ AMA1 } & YGR225W & 6 \\
\hline & YHL041W & 6 \\
\hline AZR1 & YGR224W & 6 \\
\hline ARN1 & YHL040C & 6 \\
\hline
\end{tabular}

$\begin{array}{rrr}15 & -3.51563 & -4.00891 \\ 16 & -28.3862 & -4.36548 \\ 17 & 15.67308 & -2.43094 \\ 18 & 36.12418 & 9.906292 \\ 19 & 81.81818 & -37.5635 \\ 20 & -11.5337 & -1.93966 \\ 21 & -10.9091 & -9.7561 \\ 22 & -0.33557 & -8.49315 \\ 23 & 4.873803 & -0.19029 \\ 24 & 72.30624 & -18.7586 \\ 1 & 47.11286 & 33.79924 \\ 2 & 25.02575 & -4.22535 \\ 3 & 78.89273 & 56.72515 \\ 4 & 51.89003 & 70.6422 \\ 5 & 47.64919 & -26.0606 \\ 6 & 53.32569 & -21.4824 \\ 7 & 36.13527 & 16.70702 \\ 8 & -13.2208 & 22.34637 \\ 9 & 38.12677 & 7.126168 \\ 10 & 29.53092 & 5.493274 \\ 11 & 43.71585 & -10.6178 \\ 12 & 84.97738 & 27.7551 \\ 13 & 40.45207 & 36.03276 \\ 14 & 20.48872 & 3.596404 \\ 15 & -0.29028 & 5.927552 \\ 16 & 22.62846 & -3.11005 \\ 17 & 48.8172 & -10.4794 \\ 18 & 49.77578 & -10.1307 \\ 19 & 100 & \# D I V / 0 ! \\ 20 & 24.4 & 3.02714 \\ 21 & \# D I V / 0 ! & 3.157895 \\ 22 & 93.01533 & 35.97561 \\ 23 & 9.613657 & 7.784986 \\ 24 & 56.48352 & 28.25607 \\ 1 & -31.8363 & 11.50362 \\ 2 & 28.32661 & 9.160305 \\ 3 & 78.88493 & 29.96255 \\ 4 & -30.312 & 35.84071 \\ 5 & -12.5691 & 10.30928 \\ 6 & 69.80256 & 24.46237 \\ 7 & 27.17241 & 19.85816 \\ 8 & 45.54538 & 11.2426 \\ 9 & 59.47631 & 6.605923\end{array}$

\begin{tabular}{|c|c|}
\hline 55.88235 & 70.05254 \\
\hline 58.7766 & 25.68627 \\
\hline 38.4 & 37.99213 \\
\hline 25.66038 & 20.10724 \\
\hline 26.08696 & 20.82324 \\
\hline 52.78592 & 55.34709 \\
\hline 52 & 58.93471 \\
\hline 58.85714 & 28.50972 \\
\hline 59.18919 & 54.89796 \\
\hline 31.61905 & 45.50265 \\
\hline 51.05042 & 37.66234 \\
\hline 2.222222 & 5.660377 \\
\hline 56.41548 & 44.7619 \\
\hline 56.71343 & 41.40127 \\
\hline 58.14889 & 52.88462 \\
\hline 62.92776 & 45.58824 \\
\hline 37.63441 & 45.99407 \\
\hline 53.24165 & 41.81818 \\
\hline 71.11111 & 50.97403 \\
\hline 76.37655 & 58.78963 \\
\hline 12.55924 & 28.66044 \\
\hline 63.12741 & 39.78873 \\
\hline 69.85646 & 40.12346 \\
\hline 38.5439 & 41.6185 \\
\hline 60.66116 & 29.20354 \\
\hline 49.60784 & 42.49292 \\
\hline 72.43948 & 55.95568 \\
\hline 43.85027 & 41.12676 \\
\hline-0.23474 & 33.44156 \\
\hline 68.75 & 46.36872 \\
\hline 60.30928 & 49.08616 \\
\hline 9.677419 & 52.41379 \\
\hline 50.73529 & 48.49398 \\
\hline 47.58221 & 38.9881 \\
\hline 46.04462 & 40.25559 \\
\hline 57.14286 & 45.39877 \\
\hline 66.60156 & 46.82779 \\
\hline 51.66667 & 43.38462 \\
\hline 67.55647 & 46.05263 \\
\hline
\end{tabular}




$\begin{array}{llll}\text { HSV2 } & \text { YGR223C } & 6 & 9 \\ & \text { YHL039W } & 6 & 9 \\ \text { TOS2 } & \text { YGR221C } & 6 & 9 \\ & \text { YHL037C } & 6 & 9 \\ \text { CCH1 } & \text { YGR217W } & 6 & 9 \\ \text { MUP3 } & \text { YHL036W } & 6 & 9 \\ \text { RPSOA } & \text { YGR214W } & 6 & 9 \\ \text { VMR1 } & \text { YHL035C } & 6 & 9 \\ \text { RTA1 } & \text { YGR213C } & 6 & 9 \\ \text { SBP1 } & \text { YHL034C } & 6 & 9 \\ \text { SLI1 } & \text { YGR212W } & 6 & 9 \\ \text { RPL8A } & \text { YHL033C } & 6 & 9 \\ & \text { YGR210C } & 6 & 9 \\ \text { GUT1 } & \text { YHL032C } & 6 & 9 \\ \text { HIS3 } & \text { YOR202W } & 6 & 9 \\ \text { HIS3 } & \text { YOR202W } & 6 & 10 \\ & \text { YHR132W- } & & \end{array}$

$$
\begin{array}{rrr}
10 & 4.749568 & 7.092199 \\
11 & 60.54827 & 64.17749 \\
12 & 44.88 & 2.384106 \\
13 & 42.55319 & 2.320888 \\
14 & 37.33234 & 0.684932 \\
15 & 48.92704 & 24.78992 \\
16 & 20.17893 & -13.9594 \\
17 & 51.08593 & 18.84058 \\
18 & 68.09701 & 18.78981 \\
19 & 25.49203 & -4.29864 \\
20 & -6.62577 & -2.5974 \\
21 & 100 & 31.2566 \\
22 & 73.52342 & -8.84692 \\
23 & 40.12876 & -7.40375 \\
24 & 15.83333 & -4.54087 \\
1 & 3.56212 & 7.082153
\end{array}
$$

CAP2

A

ECM14

YIL034C

YHR132C

YIL032C

YHR131C

SSM4

YILO30C

YHR130C

YIL029C

ARP1

YHR129C

YIL028W

YHR127W

KRE27 YIL027C

YHR126C

YIL025C

YHR125W

YIL024C

NDT80

YHR124W

YIL023C

EPT1

YHR123W

HIS6

YIL020C

LSM12

YHR121W

VID28

HIS3

YIL017C

YOR202W

$2 \quad 72.89314-25.4206$

$\begin{array}{lll}23 & 86.88525 & 49.35622\end{array}$

$\begin{array}{lll}24 & 2.325581 & -33.1852\end{array}$

YOR202W

YGR238C

YHR012W

KEL2

VPS29 $\begin{array}{lll}1 & -27.1 & -3.33333\end{array}$

$\begin{array}{lll}2 & 64.15981 & 40.92769\end{array}$

$\begin{array}{lll}3 & -11.4311 & 3.889458\end{array}$
63.90977

24.47552

51.17773

68.09211

20.59497

0

64.98195

28.31858

55.20833

29.32166

63.3218

44.25887

66.10738

64.80263

37.01431

49.01961

51.69173

41.98312

52.23881

43.36449

57.17092

43.96728

53.70705

42.64392

64.07285

38.88025

40.81633

35.36804

55.96026

63.81818

58.669

52.72408

35.9408

11.71717

40.26217

58.46457

44.68085

64.98516

59.61538

42.38806

58.30721

62.7451

55.76923

41.00295

46

44.72934

44.32432

62.25806

36.36364

50.13405

41.16095

46.83544

41.73913

35.19737

37.6947

41.4557

43.73089

42.42424

56.28931

40.28986

45.06173

32.14286

40.9375

51.93548

38.16568

33.22884

38.46154

62.07865

45.4023

54.54545

54.57227

52.97158

50.41186

62.6506

40.625

49.74874 


\begin{tabular}{|c|c|c|c|c|c|c|c|c|}
\hline & YGR237C & 6 & 11 & 4 & 33.87097 & 9.346847 & 66.19385 & 59.84556 \\
\hline & YHR009C & 6 & 11 & 5 & 32.1883 & -9.15805 & 48.94068 & 22.86689 \\
\hline SPG1 & YGR236C & 6 & 11 & 6 & 55.39335 & 26.07595 & 32.021 & 38.97059 \\
\hline \multirow[t]{2}{*}{ STP2 } & YHR006W & 6 & 11 & 7 & 16.49602 & -1.88679 & 15.31915 & 46.05678 \\
\hline & YGR235C & 6 & 11 & 8 & 42.68657 & -14.2195 & 51.55039 & 46.90265 \\
\hline GPA1 & YHR005C & 6 & 11 & 9 & 71.01449 & 38.30334 & 64.41948 & 38.53821 \\
\hline YHB1 & YGR234W & 6 & 11 & 10 & 0 & -24.8109 & 49.6124 & 54.5977 \\
\hline NEM1 & YHR004C & 6 & 11 & 11 & -9.85507 & -4.46009 & 46.84838 & 32.43243 \\
\hline \multirow[t]{2}{*}{ PHO81 } & YGR233C & 6 & 11 & 12 & 27.5405 & \#DIV/0! & -42.4157 & 31.27036 \\
\hline & YHR003C & 6 & 11 & 13 & 9.025916 & -6.17647 & 22.28682 & 33.73494 \\
\hline PHB2 & $\begin{array}{l}\text { YGR231C } \\
\text { YHR001W- }\end{array}$ & 6 & 11 & 14 & 10.41041 & 35.71429 & 67.87879 & 51.50502 \\
\hline QCR10 & $A$ & 6 & 11 & 15 & 27.30085 & 49.12281 & 48.55196 & 33.73494 \\
\hline NAS6 & YGR232W & 6 & 11 & 16 & 7.897241 & -1.88679 & 62.81834 & 43.40836 \\
\hline ARN2 & YHL047C & 6 & 11 & 17 & 12.10677 & -17.6275 & 69.61806 & 50.2924 \\
\hline \multirow[t]{2}{*}{ BNS1 } & YGR230W & 6 & 11 & 18 & 57.24346 & 12.1118 & 52.77778 & 35.96215 \\
\hline & YHL046C & 6 & 11 & 19 & 32.46445 & -6.22797 & 47.25086 & 48.31461 \\
\hline \multirow[t]{4}{*}{ SMI1 } & YGR229C & 6 & 11 & 20 & 42.53028 & -11.1237 & 21.97531 & 45.63107 \\
\hline & YHL045W & 6 & 11 & 21 & 32.03215 & -13.5103 & 36.14232 & 38.8724 \\
\hline & YGR228W & 6 & 11 & 22 & 79.96255 & -13.2231 & 39.81308 & 43.71429 \\
\hline & YHL044W & 6 & 11 & 23 & 30.03831 & -3.81041 & 66.56805 & 52.92683 \\
\hline HIS3 & YOR202W & 6 & 11 & 24 & 55.14365 & -9.23567 & & \\
\hline HIS3 & YOR202W & 6 & 12 & 1 & 60.13687 & 3.11042 & & \\
\hline DSE2 & YHR143W & 6 & 12 & 2 & 85.13514 & 7.755102 & 42.7027 & 53.22196 \\
\hline PIG2 & YIL045W & 6 & 12 & 3 & 15.54455 & -5.55556 & 30.49793 & 45.01511 \\
\hline CHS7 & YHR142W & 6 & 12 & 4 & 41.09712 & -9.62025 & 63.47032 & 76.08069 \\
\hline \multirow[t]{2}{*}{ AGE2 } & YIL044C & 6 & 12 & 5 & \#DIV/0! & 55.38462 & 49.38525 & 53.59281 \\
\hline & YHR140W & 6 & 12 & 6 & 11.4053 & 5.957447 & 40.38835 & 33.33333 \\
\hline \multirow[t]{3}{*}{ CBR1 } & YIL043C & 6 & 12 & 7 & 55.75365 & -15.8596 & 56.06362 & 48.46416 \\
\hline & YHR139C-A & 6 & 12 & 8 & 32.13078 & -7.43034 & 31.56733 & 52.42718 \\
\hline & YIL042C & 6 & 12 & 9 & -20 & -6.98198 & 69.65649 & 58.14696 \\
\hline SPS100 & YHR139C & 6 & 12 & 10 & 62.81938 & 37.5 & 24.28198 & 44.3769 \\
\hline \multirow[t]{2}{*}{ GVP36 } & YIL041W & 6 & 12 & 11 & 76.82171 & 23.77622 & 12.64108 & 45.02924 \\
\hline & YHR138C & 6 & 12 & 12 & 14.49407 & 3.852459 & 62.37942 & 52.85285 \\
\hline APQ12 & YIL040W & 6 & 12 & 13 & -74.4361 & 30.74074 & -53.6232 & 31.26935 \\
\hline \multirow[t]{2}{*}{ ARO9 } & YHR137W & 6 & 12 & 14 & 79.83725 & 61.82857 & 69.51788 & 50.3012 \\
\hline & YIL039W & 6 & 12 & 15 & 62.1988 & 61.21437 & 44.66801 & 70.79646 \\
\hline SPL2 & YHR136C & 6 & 12 & 16 & 7.6 & -15.711 & 3.023758 & 44.57143 \\
\hline NOT3 & YIL038C & 6 & 12 & 17 & 13.76518 & -5.16039 & 53.79061 & 50.28409 \\
\hline YCK1 & YHR135C & 6 & 12 & 18 & 31.96315 & -24.4159 & 30.16949 & 45.39749 \\
\hline PRM2 & YIL037C & 6 & 12 & 19 & -0.51813 & 7.692308 & 30.67961 & 38.55422 \\
\hline WSS1 & YHR134W & 6 & 12 & 20 & 65.53819 & 6.627018 & 55.99251 & 40.84967 \\
\hline CST6 & YIL036W & 6 & 12 & 21 & 67.29249 & -14.5522 & 26.89487 & 38.71951 \\
\hline
\end{tabular}




\begin{tabular}{|c|c|c|c|c|c|c|c|c|}
\hline NSG1 & YHR133C & 6 & 12 & 22 & 37.52809 & -7.38758 & 29.6146 & 41.98251 \\
\hline CKA1 & YIL035C & 6 & 12 & 23 & 70.33986 & -1.80905 & 0.316957 & 49.19169 \\
\hline HIS3 & YOR202W & 6 & 12 & 24 & -22.0798 & -15.7764 & & \\
\hline \multirow[t]{3}{*}{ HIS3 } & YOR202W & 6 & 13 & 1 & -11.167 & 8.347245 & & \\
\hline & YGR259C & 6 & 13 & 2 & -12.5804 & 19.33934 & 51.55807 & 55.16432 \\
\hline & YHR029C & 6 & 13 & 3 & -3.10078 & 3.460621 & 65.14806 & 65.87302 \\
\hline GND2 & YGR256W & 6 & 13 & 4 & -57.8415 & 2.047782 & 49.27835 & 47.53086 \\
\hline \multirow[t]{2}{*}{ DAP2 } & YHR028C & 6 & 13 & 5 & 100 & \#DIV/0! & 51.52749 & 47.86885 \\
\hline & YGR250C & 6 & 13 & 6 & \#DIV/0! & -9.63665 & 44.03292 & 35.87302 \\
\hline THR1 & YHR025W & 6 & 13 & 7 & 80.90999 & 30.54662 & 48.36272 & 40.78947 \\
\hline \multirow[t]{2}{*}{ MGA1 } & YGR249W & 6 & 13 & 8 & 65.26994 & 25.97403 & 54.37616 & 39.0625 \\
\hline & YHR022C & 6 & 13 & 9 & 57.69882 & 2.636917 & 67.55408 & 51.35135 \\
\hline \multirow[t]{2}{*}{ SOL4 } & YGR248W & 6 & 13 & 10 & 24.65347 & -6.74286 & 72.6297 & 49.4012 \\
\hline & YHR021W- & & & & & & & \\
\hline ECM12 & $A$ & 6 & 13 & 11 & 15.301 & -16.7695 & 64.62094 & 43.76812 \\
\hline CPD1 & YGR247W & 6 & 13 & 12 & 37.35726 & 23.84259 & 57.38832 & 34.21829 \\
\hline RPS27B & YHR021C & 6 & 13 & 13 & 23.219 & 53.35277 & 58.78525 & 62.9771 \\
\hline LSC2 & YGR244C & 6 & 13 & 14 & 31.88291 & 1.339829 & 32.29814 & 44.09222 \\
\hline ARG4 & YHR018C & 6 & 13 & 15 & 94.6213 & 23.31341 & 35.95238 & 56.8915 \\
\hline FMP43 & YGR243W & 6 & 13 & 16 & 100 & \#DIV/0! & 38.22394 & 51.26582 \\
\hline \multirow[t]{2}{*}{ YSC83 } & YHR017W & 6 & 13 & 17 & 58.06452 & 27.14127 & 14.11531 & 43.94464 \\
\hline & YGR242W & 6 & 13 & 18 & 13.57702 & 13.67807 & 47.67025 & 49.84709 \\
\hline YSC84 & YHR016C & 6 & 13 & 19 & 63.7644 & 5.711423 & 56.50685 & 40.36939 \\
\hline YAP1802 & YGR241C & 6 & 13 & 20 & 75.33742 & 35.54987 & 21.55172 & 38.55799 \\
\hline MIP6 & YHR015W & 6 & 13 & 21 & 32.36575 & -12.531 & 51.65794 & 41.69014 \\
\hline PEX21 & YGR239C & 6 & 13 & 22 & 52.7551 & -13.4783 & 48.93617 & 55.5205 \\
\hline SPO13 & YHR014W & 6 & 13 & 23 & 93.62055 & -3.8835 & 13.42685 & 44.90358 \\
\hline HIS3 & YOR202W & 6 & 13 & 24 & 62.41921 & -25.2 & & \\
\hline \multirow[t]{3}{*}{ HIS3 } & YOR202W & 6 & 14 & 1 & 57.57813 & 6.226415 & & \\
\hline & YHR159W & 6 & 14 & 2 & 6.633166 & 14.14254 & 52.53333 & 56.25 \\
\hline & YIL059C & 6 & 14 & 3 & \#DIV/0! & 48.74851 & 48.27586 & 46.7354 \\
\hline \multirow[t]{2}{*}{ KEL1 } & YHR158C & 6 & 14 & 4 & 77.95656 & 46.23116 & 48.57143 & 49.21466 \\
\hline & YIL058W & 6 & 14 & 5 & 4.505814 & 4.756243 & 52.41779 & 57.65766 \\
\hline \multirow[t]{2}{*}{ REC104 } & YHR157W & 6 & 14 & 6 & 71.949 & 37.5 & 62.42661 & 47.12644 \\
\hline & YIL057C & 6 & 14 & 7 & 43.27765 & -13.719 & 21.9457 & 45.06173 \\
\hline \multirow[t]{2}{*}{ LIN1 } & YHR156C & 6 & 14 & 8 & \#DIV/0! & 1.85567 & 43.75 & 38.36858 \\
\hline & YIL056W & 6 & 14 & 9 & 96.53751 & 43.03797 & 7.758621 & 39.11765 \\
\hline \multirow[t]{2}{*}{ YSP1 } & YHR155W & 6 & 14 & 10 & 66.16541 & 60.38961 & 53.22581 & 69.00585 \\
\hline & YIL055C & 6 & 14 & 11 & 42.30263 & -19.7733 & 24.46809 & 58.28402 \\
\hline \multirow[t]{2}{*}{ RTT107 } & YHR154W & 6 & 14 & 12 & 29.38596 & 13.28502 & 61.46435 & 32.13213 \\
\hline & YIL054W & 6 & 14 & 13 & 76.92308 & 13.80597 & 57.87671 & 56.50888 \\
\hline SPO16 & YHR153C & 6 & 14 & 14 & \#DIV/0! & -0.42052 & 19.36937 & 58.07365 \\
\hline RHR2 & YIL053W & 6 & 14 & 15 & 100 & 29.82456 & 78.25397 & 67.53623 \\
\hline
\end{tabular}




\begin{tabular}{|c|c|c|c|c|c|c|c|c|}
\hline SPO12 & YHR152W & 6 & 14 & 16 & -24.97 & 12.30487 & -3.28283 & 40.89636 \\
\hline \multirow[t]{2}{*}{ RPL34B } & YIL052C & 6 & 14 & 17 & 80.47445 & 25.55066 & 65.35836 & 48.11594 \\
\hline & YHR151C & 6 & 14 & 18 & 5.98389 & -2.92135 & 37.17949 & 46.0452 \\
\hline PCL7 & YIL050W & 6 & 14 & 19 & 22.37762 & 21.91304 & 66.77852 & 48.36066 \\
\hline PEX28 & YHR150W & 6 & 14 & 20 & 4.141104 & -9.5986 & 67.58621 & 51.64557 \\
\hline DFG10 & YIL049W & 6 & 14 & 21 & 90.14658 & -19.5402 & 38.84892 & 52 \\
\hline CRP1 & YHR146W & 6 & 14 & 22 & \#DIV/0! & 20 & 60.81871 & 46.8599 \\
\hline SYG1 & YIL047C & 6 & 14 & 23 & \#DIV/0! & -12.0213 & 72.02473 & 54.35294 \\
\hline HIS3 & YOR202W & 6 & 14 & 24 & 52.14881 & -16.775 & & \\
\hline HIS3 & YOR202W & 6 & 15 & 1 & 17.64081 & 17.45201 & & \\
\hline BGL2 & YGR282C & 6 & 15 & 2 & 61.8202 & -11.0353 & $\begin{array}{l}\text { missing } \\
\text { colony }\end{array}$ & missing colony \\
\hline DOG1 & YHR044C & 6 & 15 & 3 & 100 & 52.34899 & 57.47126 & 61.45833 \\
\hline YOR1 & YGR281W & 6 & 15 & 4 & 78.36134 & 55.42169 & 35.16068 & 49.50739 \\
\hline DOG2 & YHR043C & 6 & 15 & 5 & 72.25 & 34.62322 & 26.28866 & 60.06006 \\
\hline SCW4 & YGR279C & 6 & 15 & 6 & 81.51476 & 22.72727 & 52.80172 & 59.64912 \\
\hline SRB2 & YHR041C & 6 & 15 & 7 & 44.45554 & 75.77143 & 63.87283 & 67.23164 \\
\hline RTT102 & YGR275W & 6 & 15 & 8 & 100 & \#DIV/0! & 47.66537 & 53.65854 \\
\hline MSC7 & YHR039C & 6 & 15 & 9 & 54.98812 & -7.98226 & 33.19058 & 59.93789 \\
\hline YTA7 & YGR270W & 6 & 15 & 10 & 46.26209 & 2.196532 & 39.9061 & 49.58678 \\
\hline \multirow[t]{3}{*}{ PUT2 } & YHR037W & 6 & 15 & 11 & 61.00094 & 47.83737 & 51.69811 & 62.42775 \\
\hline & YGR269W & 6 & 15 & 12 & 86.69468 & 27.79661 & 60.77058 & 55.09642 \\
\hline & YHR035W & 6 & 15 & 13 & 85.7248 & 54.35606 & 34.43983 & 58.82353 \\
\hline HUA1 & YGR268C & 6 & 15 & 14 & 100 & \#DIV/0! & 56.90789 & 52.30352 \\
\hline \multirow[t]{5}{*}{ PIH1 } & YHR034C & 6 & 15 & 15 & 100 & \#DIV/0! & 71.10187 & 49.34211 \\
\hline & YGR266W & 6 & 15 & 16 & 59.30851 & 42.41908 & 50 & 48.57143 \\
\hline & YHR033W & 6 & 15 & 17 & 13.0674 & 6.315789 & 47.62846 & 56.23342 \\
\hline & YGR263C & 6 & 15 & 18 & 9.134615 & 28.28746 & 54.80944 & 40.67278 \\
\hline & YHR032W & 6 & 15 & 19 & 60.0551 & 29.44039 & 75.07788 & 54.95283 \\
\hline APL6 & YGR261C & 6 & 15 & 20 & 100 & 91.93548 & 61.1479 & 45.01608 \\
\hline RRM3 & YHR031C & 6 & 15 & 21 & -19.5046 & 46.95898 & 68.18182 & 50.79787 \\
\hline TNA1 & YGR260W & 6 & 15 & 22 & 100 & 57 & 45.37572 & 62.31579 \\
\hline SLT2 & YHR030C & 6 & 15 & 23 & 100 & 100 & 96 & 93.0446 \\
\hline HIS3 & YOR202W & 6 & 15 & 24 & 27.96781 & 30.81264 & & \\
\hline HIS3 & YOR202W & 6 & 16 & 1 & -5.54124 & 45.27151 & & \\
\hline HIS3 & YOR202W & 6 & 16 & 2 & -31.9066 & -14.8315 & & \\
\hline HIS3 & YOR202W & 6 & 16 & 3 & 98.8792 & -10.1064 & & \\
\hline HIS3 & YOR202W & 6 & 16 & 4 & 100 & \#DIV/0! & & \\
\hline HIS3 & YOR202W & 6 & 16 & 5 & 100 & 57.89474 & & \\
\hline HIS3 & YOR202W & 6 & 16 & 6 & -16.9649 & 5.070657 & & \\
\hline HIS3 & YOR202W & 6 & 16 & 7 & -5.51181 & 38.19302 & & \\
\hline HIS3 & YOR202W & 6 & 16 & 8 & -42.6065 & 22.54408 & & \\
\hline HIS3 & YOR202W & 6 & 16 & 9 & -30.5215 & -10.7943 & & \\
\hline
\end{tabular}




\begin{tabular}{|c|c|c|c|c|c|c|c|c|}
\hline HIS3 & YOR202W & 6 & 16 & 10 & -10.3371 & 50.20188 & & \\
\hline HIS3 & YOR202W & 6 & 16 & 11 & 1.058201 & 82.18487 & & \\
\hline HIS3 & YOR202W & 6 & 16 & 12 & -26.8437 & -12.5749 & & \\
\hline HIS3 & YOR202W & 6 & 16 & 13 & 76.1079 & -4.39147 & & \\
\hline HIS3 & YOR202W & 6 & 16 & 14 & 67.21854 & 29.96778 & & \\
\hline HIS3 & YOR202W & 6 & 16 & 15 & 40.94755 & -35.9322 & & \\
\hline HIS3 & YOR202W & 6 & 16 & 16 & -6.23946 & -47.7801 & & \\
\hline HIS3 & YOR202W & 6 & 16 & 17 & -25.7895 & 21.68421 & & \\
\hline HIS3 & YOR202W & 6 & 16 & 18 & -62.5 & 42.48366 & & \\
\hline HIS3 & YOR202W & 6 & 16 & 19 & -28.6232 & 4.231975 & & \\
\hline HIS3 & YOR202W & 6 & 16 & 20 & -16.6052 & -15.6627 & & \\
\hline HIS3 & YOR202W & 6 & 16 & 21 & -14 & 8.359133 & & \\
\hline HIS3 & YOR202W & 6 & 16 & 22 & 73.93526 & 3.61991 & & \\
\hline HIS3 & YOR202W & 6 & 16 & 23 & 81.84855 & 19.15285 & & \\
\hline HIS3 & YOR202W & 6 & 16 & 24 & -17.1533 & 11.75373 & & \\
\hline HIS3 & YOR202W & 7 & 1 & 1 & 67.21992 & 28.32168 & & \\
\hline HIS3 & YOR202W & 7 & 1 & 2 & 86.4392 & -5.6713 & & \\
\hline HIS3 & YOR202W & 7 & 1 & 3 & 23.47458 & 3.846154 & & \\
\hline HIS3 & YOR202W & 7 & 1 & 4 & 48.44961 & -3.59521 & & \\
\hline HIS3 & YOR202W & 7 & 1 & 5 & 42.67327 & 57.10145 & & \\
\hline HIS3 & YOR202W & 7 & 1 & 6 & -17.0441 & 16.20553 & & \\
\hline HIS3 & YOR202W & 7 & 1 & 7 & 28.16635 & 14.77273 & & \\
\hline HIS3 & YOR202W & 7 & 1 & 8 & -27.2727 & 9.08059 & & \\
\hline HIS3 & YOR202W & 7 & 1 & 9 & -12.5561 & 54.16667 & & \\
\hline HIS3 & YOR202W & 7 & 1 & 10 & 39.39064 & 34.0564 & & \\
\hline HIS3 & YOR202W & 7 & 1 & 11 & -30.599 & 13.88565 & & \\
\hline HIS3 & YOR202W & 7 & 1 & 12 & 20.63679 & 1.340861 & & \\
\hline HIS3 & YOR202W & 7 & 1 & 13 & -8.43882 & 6.535948 & & \\
\hline HIS3 & YOR202W & 7 & 1 & 14 & 24.51759 & 4.500703 & & \\
\hline HIS3 & YOR202W & 7 & 1 & 15 & 6.367583 & 84.41558 & & \\
\hline HIS3 & YOR202W & 7 & 1 & 16 & 19.00826 & 11.20081 & & \\
\hline HIS3 & YOR202W & 7 & 1 & 17 & -3.27422 & 14.81481 & & \\
\hline HIS3 & YOR202W & 7 & 1 & 18 & 1.708279 & 6.289308 & & \\
\hline HIS3 & YOR202W & 7 & 1 & 19 & 8.425721 & -7.38636 & & \\
\hline HIS3 & YOR202W & 7 & 1 & 20 & -6.74847 & 13.92857 & & \\
\hline HIS3 & YOR202W & 7 & 1 & 21 & 31.10285 & 26.09542 & & \\
\hline HIS3 & YOR202W & 7 & 1 & 22 & 13.66006 & 14.59035 & & \\
\hline HIS3 & YOR202W & 7 & 1 & 23 & 45.5665 & 51.51515 & & \\
\hline HIS3 & YOR202W & 7 & 1 & 24 & 9.128205 & 66.82627 & & \\
\hline HIS3 & YOR202W & 7 & 2 & 1 & 96.8254 & 52.81501 & & \\
\hline \multirow[t]{2}{*}{ GZF3 } & YJL110C & 7 & 2 & 2 & 56.41256 & 12.08791 & 50.7722 & 44.44444 \\
\hline & YJL215C & 7 & 2 & 3 & 40.04044 & 30.33088 & 48.50746 & 30.3653 \\
\hline PRM10 & YJL108C & 7 & 2 & 4 & 46.50699 & 13.48206 & 27.04918 & 29.52646 \\
\hline
\end{tabular}




\begin{tabular}{|c|c|c|c|c|c|c|}
\hline HXT8 & YJL214W & 7 & 2 & 5 & 53.22425 & 13.00992 \\
\hline \multirow[t]{2}{*}{ SET4 } & YJL105W & 7 & 2 & 6 & 28.25651 & 3.69863 \\
\hline & YJL213W & 7 & 2 & 7 & 27.58242 & 21.74888 \\
\hline GSH1 & YJL101C & 7 & 2 & 8 & 85.38539 & 11.92053 \\
\hline OPT1 & YJL212C & 7 & 2 & 9 & 12.80992 & 59.89011 \\
\hline \multirow[t]{2}{*}{ LSB6 } & YJL100W & 7 & 2 & 10 & 62.1978 & 22.4261 \\
\hline & YJL211C & 7 & 2 & 11 & 23.29193 & 62.19839 \\
\hline CHS6 & YJL099W & 7 & 2 & 12 & -22.0228 & 100 \\
\hline PEX2 & YJL210W & 7 & 2 & 13 & -8.76963 & 20.84592 \\
\hline SAP185 & YJL098W & 7 & 2 & 14 & \#DIV/0! & 9.722222 \\
\hline NUC1 & YJL208C & 7 & 2 & 15 & 14.57831 & 13.15789 \\
\hline \multirow[t]{2}{*}{ BCK1 } & YJL095W & 7 & 2 & 16 & 14.63845 & -12.1027 \\
\hline & YJL207C & 7 & 2 & 17 & 4.887715 & -6.28571 \\
\hline \multirow[t]{2}{*}{ KHA1 } & YJL094C & 7 & 2 & 18 & \#DIV/0! & 52.30769 \\
\hline & YJL206C-A & 7 & 2 & 19 & 1.081081 & 20.92652 \\
\hline \multirow[t]{2}{*}{ TOK1 } & YJL093C & 7 & 2 & 20 & 14.37309 & 31.67203 \\
\hline & YJL206C & 7 & 2 & 21 & 77.19101 & 9.939148 \\
\hline SIP4 & YJL089W & 7 & 2 & 22 & 2.115159 & 6.122449 \\
\hline RCY1 & YJL204C & 7 & 2 & 23 & -3.40237 & 10.05435 \\
\hline HIS3 & YOR202W & 7 & 2 & 24 & -20.7024 & 0.859107 \\
\hline HIS3 & YOR202W & 7 & 3 & 1 & \#DIV/0! & 41.46341 \\
\hline \multirow[t]{2}{*}{ SEC28 } & YIL076W & 7 & 3 & 2 & 46.1 & 26.05459 \\
\hline & YIR020C & 7 & 3 & 3 & 76 & 39.46309 \\
\hline SER33 & YIL074C & 7 & 3 & 4 & -1.98675 & 0.641026 \\
\hline MUC1 & YIR019C & 7 & 3 & 5 & 54.36433 & -3.23213 \\
\hline SPO22 & YIL073C & 7 & 3 & 6 & 25.62241 & 13.48548 \\
\hline YAP5 & YIR018W & 7 & 3 & 7 & 9.766926 & 15.08951 \\
\hline HOP1 & YIL072W & 7 & 3 & 8 & 23.60319 & 18.85014 \\
\hline MET28 & YIR017C & 7 & 3 & 9 & 23.65591 & -0.24213 \\
\hline \multirow[t]{2}{*}{ PCl8 } & YIL071C & 7 & 3 & 10 & 81.13208 & -7.40741 \\
\hline & YIR016W & 7 & 3 & 11 & 9.009009 & -8.93417 \\
\hline \multirow[t]{3}{*}{ MAM33 } & YIL070C & 7 & 3 & 12 & 18.93096 & 36.64773 \\
\hline & YIR014W & 7 & 3 & 13 & 0.743494 & 21.59091 \\
\hline & YIL067C & 7 & 3 & 14 & 72.12121 & 40 \\
\hline GAT4 & YIR013C & 7 & 3 & 15 & -2.31405 & 6.389452 \\
\hline RNR3 & YIL066C & 7 & 3 & 16 & 12.11656 & 18.31426 \\
\hline MSL1 & YIR009W & 7 & 3 & 17 & 10.98131 & 1.799486 \\
\hline \multirow[t]{3}{*}{ FIS1 } & YIL065C & 7 & 3 & 18 & -9.37913 & 6.457926 \\
\hline & YIR007W & 7 & 3 & 19 & 35.72241 & 61.1465 \\
\hline & YIL064W & 7 & 3 & 20 & 56.47059 & 12.58741 \\
\hline \multirow[t]{3}{*}{ IST3 } & YIR005W & 7 & 3 & 21 & 21.17962 & 22.48804 \\
\hline & YIL060W & 7 & 3 & 22 & 61.57205 & 12.5 \\
\hline & YIR003W & 7 & 3 & 23 & 2.461899 & 8.888889 \\
\hline
\end{tabular}

\begin{tabular}{rr}
58.88031 & 48.52652 \\
57.94393 & 21.26437 \\
-49.7908 & 21.55172 \\
42.89277 & 25.08591 \\
-2.5 & 21.843 \\
3.921569 & 25.23659 \\
44.03893 & 19.72789 \\
64.02116 & 51.84049 \\
64.09736 & 21.05263 \\
6.546275 & 13.55311 \\
4.450262 & 43.80403 \\
42.93629 & 51.44928 \\
48.54369 & 11.00324 \\
23.42733 & 37.65432 \\
7.673267 & 35.59322 \\
33.66337 & 26.17647 \\
25.85366 & 19.66759 \\
45.26112 & 42.03297 \\
45.05208 & -16.5584 \\
& \\
\hline 5.219
\end{tabular}




\begin{tabular}{|c|c|c|c|c|c|c|c|c|}
\hline HIS3 & YOR202W & 7 & 3 & 24 & 10.28249 & -2.43056 & & \\
\hline \multirow[t]{3}{*}{ HIS3 } & YOR202W & 7 & 4 & 1 & 91.22894 & 44.98186 & & \\
\hline & YJL131C & 7 & 4 & 2 & 40.34582 & 40.41237 & 55.23013 & 31.54762 \\
\hline & YJR011C & 7 & 4 & 3 & 63.83363 & 41.67852 & 65.8 & 50.73171 \\
\hline URA2 & YJL130C & 7 & 4 & 4 & 34.96933 & -5.12821 & 24.34988 & 11.26761 \\
\hline MET3 & YJR010W & 7 & 4 & 5 & 30.52738 & 32.48882 & 38.75862 & 31.00304 \\
\hline PBS2 & YJL128C & 7 & 4 & 6 & 16.52893 & 26.27986 & 38.57143 & 13.55932 \\
\hline SPC1 & YJR010C-A & 7 & 4 & 7 & 36.55324 & 21.97036 & 53.99645 & 29.90937 \\
\hline NIT2 & YJL126W & 7 & 4 & 8 & 5.477032 & 15.48387 & 3.916449 & 33.44262 \\
\hline $\mathrm{TDH} 2$ & YJR009C & 7 & 4 & 9 & 28.49741 & 4.021938 & 21.05263 & 23.3463 \\
\hline \multirow[t]{3}{*}{ LSM1 } & YJL124C & 7 & 4 & 10 & 24.08066 & 32.89474 & 56.33188 & 53.05164 \\
\hline & YJR008W & 7 & 4 & 11 & 17.26619 & 10.42471 & 38.8309 & 45.2381 \\
\hline & YJL123C & 7 & 4 & 12 & 22.93465 & 5.741627 & 52.69565 & -1.41343 \\
\hline \multirow[t]{3}{*}{ APL1 } & YJR005W & 7 & 4 & 13 & 12.9751 & 14.92537 & 15.29126 & 43.90244 \\
\hline & YJL122W & 7 & 4 & 14 & 50.07299 & 23.17708 & 57.99087 & 21.59468 \\
\hline & YJR003C & 7 & 4 & 15 & 63.1728 & 60.86106 & 66.95502 & 51.89504 \\
\hline RPE1 & YJL121C & 7 & 4 & 16 & 18.78669 & 18.04511 & -18.4049 & 33.71648 \\
\hline AVT1 & YJR001W & 7 & 4 & 17 & 10.99476 & 25.61757 & 33.57843 & 27.96053 \\
\hline \multirow[t]{2}{*}{ NCA3 } & YJL116C & 7 & 4 & 18 & 59.79381 & 63.91639 & 45.80234 & 69.75806 \\
\hline & YJL218W & 7 & 4 & 19 & 47.35849 & 20.72539 & 43.57143 & 17.61006 \\
\hline \multirow[t]{2}{*}{ ASF1 } & YJL115W & 7 & 4 & 20 & 98.2906 & 46.06061 & 32.02247 & 38.59649 \\
\hline & YJL217W & 7 & 4 & 21 & 27.66571 & 15.75342 & 2.933985 & 46.88427 \\
\hline \multirow[t]{2}{*}{ MDV1 } & YJL112W & 7 & 4 & 22 & 26.49573 & 2.667893 & 36.17512 & 40 \\
\hline & YJL216C & 7 & 4 & 23 & 60.93294 & 21.47239 & 62.83019 & 36.60714 \\
\hline HIS3 & YOR202W & 7 & 4 & 24 & 10.53985 & 13.31776 & & \\
\hline HIS3 & YOR202W & 7 & 5 & 1 & 94.75728 & 59.76744 & & \\
\hline PRK1 & YIL095W & 7 & 5 & 2 & 78.75116 & 7.55287 & -25 & 27.75229 \\
\hline MGA2 & YIR033W & 7 & 5 & 3 & 36.9287 & 23.72671 & 35.71429 & 26.22951 \\
\hline RSM25 & YIL093C & 7 & 5 & 4 & 32.08661 & 55.48098 & 51.53374 & 37.82991 \\
\hline \multirow[t]{2}{*}{ DAL3 } & YIR032C & 7 & 5 & 5 & 35.91481 & 42.51884 & 16.15854 & 36.02694 \\
\hline & YIL092W & 7 & 5 & 6 & 29.00609 & 53.43915 & 7.537688 & 29.73856 \\
\hline DAL7 & YIR031C & 7 & 5 & 7 & 20.44855 & 56.68016 & 54.48113 & 32.46269 \\
\hline ICE2 & YIL090W & 7 & 5 & 8 & 34.70874 & 0.4914 & 58.01527 & 59.46844 \\
\hline \multirow[t]{2}{*}{ DCG1 } & YIR030C & 7 & 5 & 9 & 19.6986 & 19.79167 & 23.52941 & 27.04626 \\
\hline & YIL089W & 7 & 5 & 10 & 63.23988 & 47.27744 & 76.43885 & 37.58865 \\
\hline DAL2 & YIR029W & 7 & 5 & 11 & 14.18919 & 8.040201 & 13.59773 & 50.61728 \\
\hline AVT7 & YIL088C & 7 & 5 & 12 & 62.25681 & 37.7551 & 63.12057 & 52.25225 \\
\hline \multirow[t]{2}{*}{ DAL4 } & YIR028W & 7 & 5 & 13 & 16.35688 & 31.03448 & 50.73684 & 33.67347 \\
\hline & YIL087C & 7 & 5 & 14 & -5.01393 & 20.21403 & 46.15385 & 47.03704 \\
\hline \multirow[t]{2}{*}{ DAL1 } & YIR027C & 7 & 5 & 15 & -5.39629 & 17.64168 & 27.89256 & 30.14184 \\
\hline & YIL086C & 7 & 5 & 16 & -7.90155 & 36.33721 & 28.18792 & 38.52941 \\
\hline MND2 & YIR025W & 7 & 5 & 17 & 10 & 10.29963 & 54.19708 & 41.15756 \\
\hline SDS3 & YIL084C & 7 & 5 & 18 & 52.07101 & 59.25926 & 25.54745 & 50.34014 \\
\hline
\end{tabular}




\begin{tabular}{|c|c|c|c|c|c|c|c|c|}
\hline GIF1 & YIR024C & 7 & 5 & 19 & 50.1827 & 24.02402 & -16.9935 & 39.03904 \\
\hline AIR1 & YIL079C & 7 & 5 & 20 & 13.18807 & 9.850107 & 43.81551 & 42.54658 \\
\hline \multirow[t]{3}{*}{ DAL81 } & YIR023W & 7 & 5 & 21 & 19.95104 & 7.34767 & 3.669725 & 43.9759 \\
\hline & YIL077C & 7 & 5 & 22 & 51.28866 & 8.558559 & 57.16912 & 48.21429 \\
\hline & YIR020W-B & 7 & 5 & 23 & \#DIV/0! & 9.756098 & 19.23077 & 32.34624 \\
\hline HIS3 & YOR202W & 7 & 5 & 24 & 41.2037 & 23.25581 & & \\
\hline \multirow[t]{2}{*}{ HIS3 } & YOR202W & 7 & 6 & 1 & 78.79836 & 14.47903 & & \\
\hline & YJL144W & 7 & 6 & 2 & 61.86441 & 15.66485 & 43.37349 & 25.28409 \\
\hline \multirow[t]{2}{*}{ CPR7 } & YJR032W & 7 & 6 & 3 & 50.3937 & 28.07018 & 20.25316 & 55.58739 \\
\hline & YJL142C & 7 & 6 & 4 & 28.24287 & -5.76923 & 46.52632 & 47.52475 \\
\hline GEA1 & YJR031C & 7 & 6 & 5 & 24.57879 & -0.51635 & 45.20833 & 48.83721 \\
\hline \multirow[t]{2}{*}{ YAK1 } & YJL141C & 7 & 6 & 6 & 26.79739 & 48.78049 & 56.35593 & 35.37906 \\
\hline & YJR030C & 7 & 6 & 7 & 10.24793 & 14.2132 & 53.45912 & 25 \\
\hline \multirow[t]{2}{*}{ YUR1 } & YJL139C & 7 & 6 & 8 & 23.71245 & -8.40517 & 50.5137 & 29.01024 \\
\hline & YJR026W & 7 & 6 & 9 & 24.07045 & 9.837545 & 8.277405 & 44.98382 \\
\hline TIF2 & YJL138C & 7 & 6 & 10 & 69.2221 & 91.07143 & 10.84337 & 26.12903 \\
\hline BNA1 & YJR025C & 7 & 6 & 11 & -3.47107 & 7.522124 & 38.26923 & 27.77778 \\
\hline \multirow[t]{2}{*}{ GLG2 } & YJL137C & 7 & 6 & 12 & 14.7644 & 17.56007 & 53.01418 & 35.36977 \\
\hline & YJR024C & 7 & 6 & 13 & 4.563233 & 17.64005 & 25.30713 & 52.38095 \\
\hline RPS21B & YJL136C & 7 & 6 & 14 & 15.54054 & 17.26027 & 39.82301 & 57.00935 \\
\hline \multirow[t]{3}{*}{ REC107 } & YJR021C & 7 & 6 & 15 & -3.98352 & 13.03763 & 35.57312 & 45.30744 \\
\hline & YJL135W & 7 & 6 & 16 & 21 & 42.25352 & 31.82898 & 42.35669 \\
\hline & YJR020W & 7 & 6 & 17 & 8.734177 & 26.09442 & 25.79853 & 41.69279 \\
\hline LCB3 & YJL134W & 7 & 6 & 18 & 2.214022 & 11.20219 & -3.60825 & 37.94212 \\
\hline TES1 & YJR019C & 7 & 6 & 19 & 17.50292 & 6.057692 & 40.20202 & 32.27848 \\
\hline \multirow[t]{3}{*}{ MRS3 } & YJL133W & 7 & 6 & 20 & 40 & 32.39437 & 22.22222 & 31.86441 \\
\hline & YJR015W & 7 & 6 & 21 & 3.021978 & -5.71429 & 22.76786 & 39.49843 \\
\hline & YJL132W & 7 & 6 & 22 & 84.32203 & -2.7027 & 11.95122 & 40.39548 \\
\hline RBF22 & YJR014W & 7 & 6 & 23 & 28.60169 & -3.20856 & 22.42991 & 36.29032 \\
\hline HIS3 & YOR202W & 7 & 6 & 24 & -1.16686 & 13.48624 & & \\
\hline \multirow[t]{4}{*}{ HIS3 } & YOR202W & 7 & 7 & 1 & 70.38627 & 100 & & \\
\hline & YIL110W & 7 & 7 & 2 & 71.19658 & 61.49068 & 55.59772 & 46.34761 \\
\hline & YJL007C & 7 & 7 & 3 & 93.60465 & 6.986028 & 44.80151 & 50.16077 \\
\hline & YIL108W & 7 & 7 & 4 & 41.16251 & 35.38999 & 32.42507 & 40.96386 \\
\hline SYS1 & YJL004C & 7 & 7 & 5 & 38.02682 & 31.0559 & 12.1447 & 33.87755 \\
\hline \multirow[t]{2}{*}{ PFK26 } & YIL107C & 7 & 7 & 6 & 25.89499 & 26.93878 & 36.96099 & 50.55762 \\
\hline & YIR044C & 7 & 7 & 7 & 15.69966 & 13.33333 & 40.49904 & 28.51711 \\
\hline \multirow[t]{2}{*}{ LIT2 } & YIL105C & 7 & 7 & 8 & -19.9382 & 24.18646 & 61.99616 & 52.02703 \\
\hline & YIR043C & 7 & 7 & 9 & 16.64753 & 0.80429 & 16.44144 & 36.39576 \\
\hline \multirow[t]{2}{*}{ DPH1 } & YIL103W & 7 & 7 & 10 & 45.90495 & 3.808354 & 20.75472 & 33.45588 \\
\hline & YIR042C & 7 & 7 & 11 & 40.05305 & 37.74955 & 19.16667 & 27.42475 \\
\hline XBP1 & YIL101C & 7 & 7 & 12 & 45.02427 & 52.66525 & 29.51807 & 41.875 \\
\hline YPS6 & YIR039C & 7 & 7 & 13 & 47.30159 & 1.06296 & 52.513 & 45.67901 \\
\hline
\end{tabular}




\begin{tabular}{|c|c|c|c|c|c|c|c|c|}
\hline & YIL100W & 7 & 7 & 14 & 9.791332 & 40.59701 & 27.0073 & 45.58824 \\
\hline GTT1 & YIR038C & 7 & 7 & 15 & 33.33333 & 27.8481 & 19.34732 & 45.31722 \\
\hline SGA1 & YIL099W & 7 & 7 & 16 & 58.51064 & 43.41148 & 21.0084 & 42.31975 \\
\hline HYR1 & YIR037W & 7 & 7 & 17 & 58.72443 & 6.591337 & 33.09693 & 40.06309 \\
\hline \multirow[t]{2}{*}{ FMC1 } & YIL098C & 7 & 7 & 18 & 40.58524 & 26.81704 & 44.05458 & 48.26498 \\
\hline & YIR036C & 7 & 7 & 19 & 59.33962 & 54.50942 & 52.19298 & 57.14286 \\
\hline \multirow[t]{3}{*}{ FYV10 } & YIL097W & 7 & 7 & 20 & 39.49704 & 20.38043 & 44.54756 & 54.02299 \\
\hline & YIR035C & 7 & 7 & 21 & 35.95506 & 12.08499 & 34.39024 & 50.14006 \\
\hline & YIL096C & 7 & 7 & 22 & 72.13303 & 1.52439 & 31.73913 & 50.63694 \\
\hline LYS1 & YIR034C & 7 & 7 & 23 & 44.08602 & 15.04425 & 30.27027 & 51.65394 \\
\hline HIS3 & YOR202W & 7 & 7 & 24 & 7.416268 & -5.85586 & & \\
\hline HIS3 & YOR202W & 7 & 8 & 1 & 18.69723 & 39.24269 & & \\
\hline FBP26 & YJL155C & 7 & 8 & 2 & \#DIV/0! & 15.38462 & 38.06228 & 26.37076 \\
\hline ISY1 & YJR050W & 7 & 8 & 3 & 36.39876 & 18.91892 & 39.03226 & 26.58228 \\
\hline VPS35 & YJL154C & 7 & 8 & 4 & 74.57627 & 13.48315 & 46.95122 & 37.64906 \\
\hline UTR1 & YJR049C & 7 & 8 & 5 & 39.43503 & 7.02275 & 56.20915 & 53.15985 \\
\hline INO1 & YJL153C & 7 & 8 & 6 & 25.37764 & 7.416268 & 33.89831 & 35.59322 \\
\hline \multirow[t]{2}{*}{ CYC1 } & YJR048W & 7 & 8 & 7 & 18.87006 & 19.17808 & 43.2526 & 0.755668 \\
\hline & YJL152W & 7 & 8 & 8 & 36.88525 & 14.54545 & 39.54984 & 53.83305 \\
\hline ANB1 & YJR047C & 7 & 8 & 9 & 6.464646 & 17.52669 & 33.33333 & 12.7182 \\
\hline SNA3 & YJL151C & 7 & 8 & 10 & 25.24138 & 32.75862 & 54.54545 & 55.51895 \\
\hline \multirow[t]{2}{*}{ POL32 } & YJR043C & 7 & 8 & 11 & 21.93732 & 45.77465 & 35.04823 & 30.26585 \\
\hline & YJL150W & 7 & 8 & 12 & 4.289544 & 47.69231 & 44.27245 & 27.93792 \\
\hline \multirow[t]{3}{*}{ GEF1 } & YJR040W & 7 & 8 & 13 & 29.36963 & 13.41463 & 32.97101 & 38.88889 \\
\hline & YJL149W & 7 & 8 & 14 & 11.68289 & 19.42509 & 44.55446 & 63.77246 \\
\hline & YJR038C & 7 & 8 & 15 & 4.30839 & 12.39669 & 38.36478 & 11.59794 \\
\hline \multirow[t]{3}{*}{ RPA34 } & YJL148W & 7 & 8 & 16 & 7.035928 & 24.81645 & 54.07166 & 44.25532 \\
\hline & YJR037W & 7 & 8 & 17 & 3.609626 & 5.263158 & 42.43243 & 19.87179 \\
\hline & YJL147C & 7 & 8 & 18 & 1.108374 & 23.77778 & 37.93103 & 31.13006 \\
\hline HUL4 & YJR036C & 7 & 8 & 19 & 4.098361 & 15.64626 & 52.90698 & 43.52031 \\
\hline IDS2 & YJL146W & 7 & 8 & 20 & 74.18033 & 8.759124 & 39.75904 & 23.2816 \\
\hline RAD26 & YJR035W & 7 & 8 & 21 & 46.36943 & 16.57658 & 37.65432 & 15.38462 \\
\hline SFH5 & YJL145W & 7 & 8 & 22 & 37.78111 & 1.440922 & 42.5 & 52.66055 \\
\hline RAV1 & YJR033C & 7 & 8 & 23 & 4.76731 & 7.637232 & 40.95745 & 4.457364 \\
\hline HIS3 & YOR202W & 7 & 8 & 24 & 25.1087 & 19.5258 & & \\
\hline HIS3 & YOR202W & 7 & 9 & 1 & 18.58887 & 25.14178 & & \\
\hline \multirow[t]{2}{*}{ GIN1 } & YIL130W & 7 & 9 & 2 & 37.02943 & -1.93452 & 43.68932 & 40 \\
\hline & YJL028W & 7 & 9 & 3 & 30.58712 & 6.45463 & 48.1409 & 54.7619 \\
\hline \multirow[t]{2}{*}{ MET18 } & YIL128W & 7 & 9 & 4 & 32.09607 & 5.882353 & 17.40088 & 30.83004 \\
\hline & YJL027C & 7 & 9 & 5 & 25.81281 & 41.72494 & 15.18987 & 31.74603 \\
\hline AYR1 & YIL124W & 7 & 9 & 6 & -3.74332 & 20.3252 & 23.16258 & 31.04693 \\
\hline APS3 & YJL024C & 7 & 9 & 7 & 20.49037 & -7.86517 & 19.01709 & 24.54874 \\
\hline SIM1 & YIL123W & 7 & 9 & 8 & 16.05839 & 19.26606 & 8.333333 & 37.54153 \\
\hline
\end{tabular}




\begin{tabular}{|c|c|c|c|c|c|c|c|c|}
\hline PET130 & YJL023C & 7 & 9 & 9 & \#DIV/0! & 9.89011 & 58.95652 & 53.87205 \\
\hline \multirow[t]{2}{*}{ QDR1 } & YIL120W & 7 & 9 & 10 & -4.57604 & 19.21739 & 56.73077 & 46.1039 \\
\hline & YJL022W & 7 & 9 & 11 & 28.85572 & 23.63636 & 26.81913 & 37.63066 \\
\hline \multirow[t]{2}{*}{ RPI1 } & YIL119C & 7 & 9 & 12 & -3.10559 & 6.392694 & 41.45383 & 50 \\
\hline & YJL021C & 7 & 9 & 13 & -8.75332 & 13.46154 & 14.35768 & 36.91275 \\
\hline PRM5 & YIL117C & 7 & 9 & 14 & 28.86836 & 20.67736 & 54.32526 & 39.93174 \\
\hline BBC1 & YJL020C & 7 & 9 & 15 & 3.195739 & 37.98257 & 20.66667 & 37 \\
\hline \multirow[t]{2}{*}{ HIS5 } & YIL116W & 7 & 9 & 16 & 2.056555 & -7.05394 & 43.33959 & 56.231 \\
\hline & YJL017W & 7 & 9 & 17 & 0.550964 & 17.93928 & 32.37251 & 53.07443 \\
\hline \multirow[t]{2}{*}{ POR2 } & YIL114C & 7 & 9 & 18 & -6.98856 & 44.09769 & 9.375 & 46.70487 \\
\hline & YJL016W & 7 & 9 & 19 & 18.33856 & 23.64486 & 26.18026 & 49.2163 \\
\hline SDP1 & YIL113W & 7 & 9 & 20 & 26.1157 & 21.40762 & 61.51079 & 49.56012 \\
\hline MAD3 & YJL013C & 7 & 9 & 21 & 11.37539 & 5.876393 & 36.83128 & 50 \\
\hline HOS4 & YIL112W & 7 & 9 & 22 & 13.04348 & 54.0797 & 63.40426 & 43.80952 \\
\hline VTC4 & YJL012C & 7 & 9 & 23 & 3.567447 & 17.21212 & 11.90053 & 44.64286 \\
\hline HIS3 & YOR202W & 7 & 9 & 24 & 7.015458 & 18.43532 & & \\
\hline \multirow[t]{2}{*}{ HIS3 } & YOR202W & 7 & 10 & 1 & 2.817824 & 16.18759 & & \\
\hline & YJL169W & 7 & 10 & 2 & 50 & 25.0726 & 40 & 52.9274 \\
\hline LIA1 & YJR070C & 7 & 10 & 3 & 45.42484 & 47.19101 & 24.79784 & 28.57143 \\
\hline SET2 & YJL168C & 7 & 10 & 4 & 10.79235 & 38.93805 & 50 & 37.2434 \\
\hline HAM1 & YJR069C & 7 & 10 & 5 & 23.88202 & 8.042895 & 46.12476 & 46.10169 \\
\hline HAL5 & YJL165C & 7 & 10 & 6 & 4.98615 & 15.86777 & 56.02968 & 54.01929 \\
\hline NTA1 & YJR062C & 7 & 10 & 7 & 38.91892 & 12.80603 & 16.15925 & 40.58442 \\
\hline \multirow[t]{3}{*}{ TPK1 } & YJL164C & 7 & 10 & 8 & 59.27505 & 9.509658 & 30.52632 & 43.49206 \\
\hline & YJR061W & 7 & 10 & 9 & 6.242775 & 53.78378 & 51.68142 & 34.96732 \\
\hline & YJL163C & 7 & 10 & 10 & 17.86284 & 41.70854 & 40.88176 & 46.33333 \\
\hline CBF1 & YJR060W & 7 & 10 & 11 & 6.970509 & 43.51504 & 24.22907 & 31.76101 \\
\hline JJJ2 & YJL162C & 7 & 10 & 12 & 35.06787 & 2.045455 & 44.72656 & 33.91003 \\
\hline PTK2 & YJR059W & 7 & 10 & 13 & 3.985056 & 23.36283 & 44.27609 & 20.95588 \\
\hline FMP33 & YJL161W & 7 & 10 & 14 & 10.46512 & 22.45763 & 25.29274 & 44.96855 \\
\hline \multirow[t]{3}{*}{ APS2 } & YJR058C & 7 & 10 & 15 & 0.796813 & 18.4573 & 42.7957 & 52.75362 \\
\hline & YJL160C & 7 & 10 & 16 & 14.71653 & 1.836394 & 66.99029 & 54.79452 \\
\hline & YJR054W & 7 & 10 & 17 & 2.008032 & 21.5072 & 29.17505 & 50.64935 \\
\hline HSP150 & YJL159W & 7 & 10 & 18 & 6.367041 & 3.427419 & 46.54378 & 57.44048 \\
\hline BFA1 & YJR053W & 7 & 10 & 19 & 25.34884 & 12.39745 & 44.87395 & 59.29919 \\
\hline CIS3 & YJL158C & 7 & 10 & 20 & 34.88 & 18.85626 & 54.72062 & 36.9186 \\
\hline RAD7 & YJR052W & 7 & 10 & 21 & 1.3269 & 4.094828 & 48.61407 & 50.82508 \\
\hline FAR1 & YJL157C & 7 & 10 & 22 & 7.888889 & 15.49421 & 53.23475 & 44.57143 \\
\hline OSM1 & YJR051W & 7 & 10 & 23 & 60.32609 & 64.51204 & 15.12915 & 48.52547 \\
\hline HIS3 & YOR202W & 7 & 10 & 24 & 49.94325 & 9.752322 & & \\
\hline HIS3 & YOR202W & 7 & 11 & 1 & 30.50847 & 28.31683 & & \\
\hline ЕСМ37 & YIL146C & 7 & 11 & 2 & 66.66667 & 8.396947 & 56.37664 & 52.20264 \\
\hline UBX6 & YJL048C & 7 & 11 & 3 & 43.94213 & -14.5631 & 39.87342 & 45.01718 \\
\hline
\end{tabular}




\begin{tabular}{|c|c|c|c|c|c|c|c|c|}
\hline PAN6 & YIL145C & 7 & 11 & 4 & -52.9321 & 16.48649 & 42.85714 & 40.13378 \\
\hline \multirow[t]{3}{*}{ RTT101 } & YJL047C & 7 & 11 & 5 & 8.873239 & 19.58763 & 31.97674 & 45.52239 \\
\hline & YIL141W & 7 & 11 & 6 & 29.06611 & -23.5131 & 40.18349 & 43.47826 \\
\hline & YJL046W & 7 & 11 & 7 & 20.70646 & 37.72955 & 37.27506 & 31.34921 \\
\hline \multirow[t]{2}{*}{ AXL2 } & YIL140W & 7 & 11 & 8 & 22.20828 & -4.35556 & 38.35878 & 38.98305 \\
\hline & YJL045W & 7 & 11 & 9 & 16.02339 & 40.38128 & 49.57265 & 39.13043 \\
\hline REV7 & YIL139C & 7 & 11 & 10 & 13.7931 & 28.13688 & 56.81004 & 40 \\
\hline GYP6 & YJL044C & 7 & 11 & 11 & 14.2268 & 13.57309 & 40.33264 & 36.33441 \\
\hline \multirow[t]{2}{*}{ TPM2 } & YIL138C & 7 & 11 & 12 & 2.329193 & 28.07512 & 51.57116 & 40.46053 \\
\hline & YJL043W & 7 & 11 & 13 & 9.243697 & 29.13817 & 26.94878 & 53.63636 \\
\hline RBF108 & YIL137C & 7 & 11 & 14 & 23.73114 & 36.82008 & 25.22936 & 43.05085 \\
\hline MHP1 & YJL042W & 7 & 11 & 15 & 10.98765 & 4.494382 & 32.22958 & 51.18343 \\
\hline \multirow[t]{2}{*}{ VHS2 } & YIL135C & 7 & 11 & 16 & 28.66407 & 34.38045 & 36.13861 & 61.53846 \\
\hline & YJL038C & 7 & 11 & 17 & 8.75 & 26.32933 & $\begin{array}{l}\text { missing } \\
\text { colony } \\
\text { missing }\end{array}$ & missing colony \\
\hline \multirow[t]{2}{*}{ FLX1 } & YIL134W & 7 & 11 & 18 & -2.87443 & 56.10302 & colony & missing colony \\
\hline & YJL037W & 7 & 11 & 19 & -1.25 & 56.14035 & 36.6 & 53.74677 \\
\hline RPL16A & YIL133C & 7 & 11 & 20 & 53.61596 & 35.44304 & 33.75315 & 50.60606 \\
\hline SNX4 & YJL036W & 7 & 11 & 21 & 7.957245 & 19.58763 & 45.62842 & 55.23466 \\
\hline CSM2 & YIL132C & 7 & 11 & 22 & 2.603037 & 8.495146 & 60.04016 & 54.74255 \\
\hline MAD2 & YJL030W & 7 & 11 & 23 & 40.09547 & 8.908046 & 41.54676 & 49.25 \\
\hline HIS3 & YOR202W & 7 & 11 & 24 & 50.7772 & 17.08483 & & \\
\hline HIS3 & YOR202W & 7 & 12 & 1 & 29.54866 & 5.320946 & & \\
\hline \multirow[t]{3}{*}{ MNN5 } & YJL186W & 7 & 12 & 2 & 42.78261 & 27.27273 & 34.19811 & 55.47074 \\
\hline & YJR087W & 7 & 12 & 3 & 42.30769 & 4.819277 & 27.76413 & 48.36601 \\
\hline & YJL185C & 7 & 12 & 4 & 2.597403 & 1.571709 & 22.64706 & 55.69231 \\
\hline CSN12 & YJR084W & 7 & 12 & 5 & 30.37037 & 62.37288 & 39.34426 & 45.63107 \\
\hline MNN11 & YJL183W & 7 & 12 & 6 & 30.12232 & 11.91336 & 16.53944 & 27.65957 \\
\hline \multirow[t]{2}{*}{ ACF4 } & YJR083C & 7 & 12 & 7 & 7.162534 & 26.88525 & 45.48944 & 44.40559 \\
\hline & YJL182C & 7 & 12 & 8 & 11.82654 & 2.619048 & 54.37616 & 46.93878 \\
\hline \multirow[t]{2}{*}{ EAF6 } & YJR082C & 7 & 12 & 9 & 72.72727 & 21.2766 & 17.76765 & 26.23574 \\
\hline & YJL181W & 7 & 12 & 10 & 11.87739 & 40.3393 & 24.62527 & 35.11706 \\
\hline FMP26 & YJR080C & 7 & 12 & 11 & 14.0118 & 42.43176 & 47.46094 & 28.57143 \\
\hline \multirow[t]{2}{*}{ ATG27 } & YJL178C & 7 & 12 & 12 & -4.06291 & 6.024096 & 64.56559 & 47.1875 \\
\hline & YJR079W & 7 & 12 & 13 & 17.31669 & 15.95547 & 8.796296 & 38.76923 \\
\hline SWI3 & YJL176C & 7 & 12 & 14 & -2.23152 & 14.03941 & 47.60994 & 52.3511 \\
\hline \multirow[t]{2}{*}{ BNA2 } & YJR078W & 7 & 12 & 15 & 6.06488 & 7.85124 & 43.61314 & 50.15674 \\
\hline & YJL175W & 7 & 12 & 16 & 24.26128 & 16.17162 & $\begin{array}{l}55.66038 \\
\text { missing }\end{array}$ & 54.7619 \\
\hline MIR1 & YJR077C & 7 & 12 & 17 & 15.14243 & 11.15174 & $\begin{array}{l}\text { colony } \\
\text { missing }\end{array}$ & missing colony \\
\hline CPS1 & YJL172W & 7 & 12 & 18 & 9.447674 & 7.366483 & colony & missing colony \\
\hline \multirow[t]{2}{*}{ HOC1 } & YJR075W & 7 & 12 & 19 & 20.68966 & 13.39713 & 37.16667 & 18.83289 \\
\hline & & & & & & & & 241 \\
\hline
\end{tabular}




\begin{tabular}{|c|c|c|c|c|c|c|c|c|}
\hline & YJL171C & 7 & 12 & 20 & 10.09296 & 1.532567 & 66.06852 & 55.55556 \\
\hline MOG1 & YJR074W & 7 & 12 & 21 & 100 & 18.64407 & 45.03817 & 36.74121 \\
\hline ASG7 & YJL170C & 7 & 12 & 22 & 24.42334 & 12.29743 & 46.24277 & 50.96774 \\
\hline OPI3 & YJR073C & 7 & 12 & 23 & 11.94379 & 27.77778 & 45.43762 & 52 \\
\hline HIS3 & YOR202W & 7 & 12 & 24 & 41.21532 & 5.319149 & & \\
\hline \multirow[t]{2}{*}{ HIS3 } & YOR202W & 7 & 13 & 1 & 40.48494 & 11.17021 & & \\
\hline & YIL161W & 7 & 13 & 2 & 15.14286 & 26.77643 & 52.83019 & 51.95531 \\
\hline DLS1 & YJL065C & 7 & 13 & 3 & \#DIV/0! & 29.6875 & 45.93407 & 57.58427 \\
\hline \multirow[t]{2}{*}{ РOT1 } & YIL160C & 7 & 13 & 4 & 45.6872 & 31.10368 & 36.60377 & 40.60914 \\
\hline & YJL064W & 7 & 13 & 5 & 28.26667 & 5.202312 & 47.57282 & 45.27027 \\
\hline BNR1 & YIL159W & 7 & 13 & 6 & -1.84843 & 8.832808 & 42.05817 & 53.94737 \\
\hline LAS21 & YJL062W & 7 & 13 & 7 & 23.75519 & 11.98428 & 33.81038 & 32.6087 \\
\hline FMP35 & YIL157C & 7 & 13 & 8 & 14.28571 & 33.42466 & 47.92453 & 38.32753 \\
\hline BNA3 & YJL060W & 7 & 13 & 9 & -24.032 & 29.97481 & 22.64529 & 39.53488 \\
\hline UBP7 & YIL156W & 7 & 13 & 10 & -11.6386 & 37.63654 & 23.79913 & 45.19231 \\
\hline YHC3 & YJL059W & 7 & 13 & 11 & -17.4221 & 17.51527 & 38.55721 & 43.01471 \\
\hline GUT2 & YIL155C & 7 & 13 & 12 & 17.3913 & 11.9403 & 44.33594 & 49.67105 \\
\hline BIT61 & YJL058C & 7 & 13 & 13 & -11.0795 & 29.35065 & 28.27443 & 42.08861 \\
\hline IMP2' & YIL154C & 7 & 13 & 14 & 4.047218 & 39.75904 & 54.08163 & 50.49505 \\
\hline IKS1 & YJL057C & 7 & 13 & 15 & 18.25581 & 26.50602 & 50.18587 & 51.1976 \\
\hline \multirow[t]{3}{*}{ RRD1 } & YIL153W & 7 & 13 & 16 & 0.729927 & 24.69136 & 60.57143 & 55.52239 \\
\hline & YJL055W & 7 & 13 & 17 & 14.20205 & 41.94286 & 68.68538 & 52.58216 \\
\hline & YIL152W & 7 & 13 & 18 & 2.796272 & 8.505747 & 54.38871 & 50.40431 \\
\hline PEP8 & YJL053W & 7 & 13 & 19 & 18.78863 & 11 & 34.35374 & 49.86877 \\
\hline \multirow[t]{2}{*}{ MLP2 } & YIL149C & 7 & 13 & 20 & 12.11031 & 8.390646 & 61.02564 & 54.61957 \\
\hline & YJL051W & 7 & 13 & 21 & 1.589103 & 14.63687 & -9.74155 & 33.85827 \\
\hline \multirow[t]{2}{*}{ RPL40A } & YIL148W & 7 & 13 & 22 & 53.26877 & 21.3245 & 64.02878 & 58.21326 \\
\hline & YJL049W & 7 & 13 & 23 & 50.18821 & 29.16031 & 35.88342 & 57.3201 \\
\hline HIS3 & YOR202W & 7 & 13 & 24 & 58.17061 & 4.829211 & & \\
\hline HIS3 & YOR202W & 7 & 14 & 1 & 63.00872 & 58.33333 & & \\
\hline ECM25 & YJL201W & 7 & 14 & 2 & 61.20219 & 57.51295 & $\begin{array}{l}\text { missing } \\
\text { colony }\end{array}$ & missing colony \\
\hline YUH1 & YJR099W & 7 & 14 & 3 & 87.64045 & 45.97701 & 47.90528 & 78.50877 \\
\hline \multirow[t]{2}{*}{ MBB1 } & YJL199C & 7 & 14 & 4 & 54.54545 & 14.28571 & 41.07143 & 43.77358 \\
\hline & YJR098C & 7 & 14 & 5 & 35.07463 & 31.21019 & 42.96675 & 47.4359 \\
\hline PHO90 & YJL198W & 7 & 14 & 6 & 39.6 & 23.25581 & 36.54971 & 50.32468 \\
\hline JJJ3 & YJR097W & 7 & 14 & 7 & 46.01367 & 33.06983 & 46.85714 & 53.89831 \\
\hline \multirow[t]{2}{*}{ UBP12 } & YJL197W & 7 & 14 & 8 & 28.5881 & 35.40252 & 52.11864 & 37.62712 \\
\hline & YJR096W & 7 & 14 & 9 & 48.27586 & 18.48739 & 11.38952 & 40.20619 \\
\hline ELO1 & YJL196C & 7 & 14 & 10 & 40.72165 & 14.98881 & 52.9623 & 55.72755 \\
\hline \multirow[t]{2}{*}{ SFC1 } & YJR095W & 7 & 14 & 11 & 30.42105 & 44.85777 & 55.57491 & 55.79937 \\
\hline & YJL193W & 7 & 14 & 12 & 15.91187 & 58.02752 & 63.87435 & 50.7837 \\
\hline
\end{tabular}


RPL43B

SOP4

IME1

RPS14B

BUD4

RPS22A

JSN1

BUD19

GRR1

SWE1

HIS3

HIS3

$\mathrm{MPH} 1$

ARG3

SGN1

VTH1

TAX4

HXT12

PRY3

SDL1

ICS3

SDL1

JEM1

ARG2

NIT1

SUC2

MPM1

HIS3

HIS3

HIS3

HIS3

HIS3

HIS3
YJR094W-

$$
\text { A }
$$

YJL192C

YJR094C

YJL191W

YJR092W

YJL190C

YJR091C

YJL188C

YJR090C

YJL187C

YJR088C

YOR202W

YOR202W

YIR002C

YJL088W

YIR001C

YJL084C

YIL173W

YJL083W

YIL170W

YJL078C

YIL168W

YJL077C

YIL167W

YJL073W

YIL166C

YJL071W

YIL165C

YJL070C

YIL164C

YJL068C

YIL163C

YJL067W

YIL162W

YJL066C

YOR202W

YOR202W

YOR202W

YOR202W

YOR202W

YOR202W $\begin{array}{lll}13 & 24.39024 & 17.30337\end{array}$

$\begin{array}{lll}14 & 19.07969 & 28.98172\end{array}$

$\begin{array}{lll}15 & 20.5157 & -19.4969\end{array}$

$\begin{array}{lll}16 & 17.88991 & 21.91781\end{array}$

$\begin{array}{lll}17 & 0.566572 & 37.80822\end{array}$

$\begin{array}{lll}18 & 16.21074 & 62.95652\end{array}$

$\begin{array}{lll}19 & 2.93772 & 9.213483\end{array}$

$20 \quad 25.9434 \quad 47.61905$

$\begin{array}{lll}21 & 2.5 & 17.51152\end{array}$

$\begin{array}{lll}22 & 9.116809 & 36.12663\end{array}$

$\begin{array}{lll}23 & 24.08759 & 21.21849\end{array}$

$\begin{array}{lll}24 & 41.84615 & 26.16408\end{array}$

1 \#DIV/0! 59.25926

$\begin{array}{lll}2 & 87.13137 & 92.6813\end{array}$

$3 \quad 85.71429 \quad 13.63636$

$\begin{array}{lll}4 & 67.16004 & 18.35443\end{array}$

$\begin{array}{lll}5 & 15.33835 & 1.257862\end{array}$

$\begin{array}{lll}6 & 50.81967 & 3.539823\end{array}$

$\begin{array}{lll}7 & 100 & 14.44444\end{array}$

$\begin{array}{lll}8 & 3.655914 & 38.61386\end{array}$

$\begin{array}{lll}9 & 43.82434 & 9.034908\end{array}$

$\begin{array}{lll}10 & 27.82783 & 1.866667\end{array}$

1150 \#DIV/0!

$\begin{array}{lll}12 & 49.10979 \quad 37.65625\end{array}$

$\begin{array}{lll}13 & 39.58944 \quad 34.4186\end{array}$

$\begin{array}{lll}14 & 26.89655 & 10.10753\end{array}$

$\begin{array}{lll}15 & 46.58019 & 20.70588\end{array}$

$\begin{array}{lll}16 & 8.586526 & 29.61117\end{array}$

17 \#DIV/0! \#DIV/0!

$\begin{array}{lll}18 & 16.57382 & 43.51464\end{array}$

$\begin{array}{lll}19 & 15.83893 & 25.38803\end{array}$

$\begin{array}{lll}20 & 29.8005 & 12.72523\end{array}$

$\begin{array}{lll}21 & 83.15789 & 12.82051\end{array}$

$\begin{array}{lll}22 & 62.38938 & 58.09399\end{array}$

$\begin{array}{lll}23 & 100 \quad 52.39295\end{array}$

$\begin{array}{lll}24 & 26.41682 & 19.68864\end{array}$

$1 \quad 64.19375 \quad 60.17634$

$2 \quad 53.26223 \quad 52.27503$

$\begin{array}{lll}3 & 66.0177 & 50.63731\end{array}$

$\begin{array}{lll}4 & 79.47598 & 28.82414\end{array}$

$\begin{array}{lll}5 & 64.34464 \quad 13.6612\end{array}$

$\begin{array}{rr}41.0148 & 57.47508 \\ 58.31776 & 47.76119 \\ 40.23904 & 44.72843 \\ 44.6184 & 43.0303 \\ 2.941176 & 39.88764 \\ 34.81153 & 44.44444 \\ 68.98396 & 51.55807 \\ 61.65541 & 62.23404 \\ \text { missing } & \end{array}$

colony

43.20298

24.59677

missing colony

57.57576

57.83366

67.81768

$-4.4843$

78.38384

71.42857

27.95181

30.42453

46.43678

48.85932

56.79214

43.47826

44.86957

61.26984

30.5668

43.57639

42.25806

58.60735

43.79562

36.57143

50.97276

51.06762

56.30081

24.70817
86.75079

47.82609

56.63265

50.88235

44.01294

45.50898

49.20128

51.81518

55.23256

47.05882

37.12375

51.62242

51.31195

55.25606

34.35754

52.38095

42.85714

52.48042

39.57055

51.23967

71.37014

52.08791 


\begin{tabular}{|c|c|c|c|c|c|c|c|}
\hline HIS3 & YOR202W & 7 & 16 & 6 & 47.18693 & 68.96552 & \\
\hline HIS3 & YOR202W & 7 & 16 & 7 & 45.80645 & 13.23077 & \\
\hline HIS3 & YOR202W & 7 & 16 & 8 & 18.11847 & 12.21221 & \\
\hline HIS3 & YOR202W & 7 & 16 & 9 & 18.42697 & 40.94955 & \\
\hline HIS3 & YOR202W & 7 & 16 & 10 & 28.69565 & 3.991131 & \\
\hline HIS3 & YOR202W & 7 & 16 & 11 & 7.125 & 11.6129 & \\
\hline HIS3 & YOR202W & 7 & 16 & 12 & 14.28571 & 34.80519 & \\
\hline HIS3 & YOR202W & 7 & 16 & 13 & 18.98017 & 4.093567 & \\
\hline HIS3 & YOR202W & 7 & 16 & 14 & 34.59119 & 7.060334 & \\
\hline HIS3 & YOR202W & 7 & 16 & 15 & 28.6732 & 3.71179 & \\
\hline HIS3 & YOR202W & 7 & 16 & 16 & 14.25577 & 16.6419 & \\
\hline HIS3 & YOR202W & 7 & 16 & 17 & 13.66525 & 7.082631 & \\
\hline HIS3 & YOR202W & 7 & 16 & 18 & 13.37793 & 12.91793 & \\
\hline HIS3 & YOR202W & 7 & 16 & 19 & 10.91549 & 7.083333 & \\
\hline HIS3 & YOR202W & 7 & 16 & 20 & 18.45765 & 10.89744 & \\
\hline HIS3 & YOR202W & 7 & 16 & 21 & 45.99542 & 12.16216 & \\
\hline HIS3 & YOR202W & 7 & 16 & 22 & 10.17544 & 41.80704 & \\
\hline HIS3 & YOR202W & 7 & 16 & 23 & 27.27273 & 7.017544 & \\
\hline HIS3 & YOR202W & 7 & 16 & 24 & 45.1782 & 26.19664 & \\
\hline HIS3 & YOR202W & 8 & 1 & 1 & 45.05229 & -6.91106 & 14.50665 \\
\hline HIS3 & YOR202W & 8 & 1 & 2 & 44.46154 & -9.84916 & -5.20059 \\
\hline HIS3 & YOR202W & 8 & 1 & 3 & 43.85867 & -39.9306 & 32.7318 \\
\hline HIS3 & YOR202W & 8 & 1 & 4 & 53.1339 & 3.206997 & 52.21429 \\
\hline HIS3 & YOR202W & 8 & 1 & 5 & 12.36147 & 2.769231 & 24.53862 \\
\hline HIS3 & YOR202W & 8 & 1 & 6 & -5.35714 & 55.84 & 32.94753 \\
\hline HIS3 & YOR202W & 8 & 1 & 7 & 15.19126 & 36.60856 & 41.71512 \\
\hline HIS3 & YOR202W & 8 & 1 & 8 & 42.17604 & 56.35088 & 29.60463 \\
\hline HIS3 & YOR202W & 8 & 1 & 9 & 67.53247 & 56.43777 & 12.43926 \\
\hline HIS3 & YOR202W & 8 & 1 & 10 & 67.56757 & 18.77667 & 35.83062 \\
\hline HIS3 & YOR202W & 8 & 1 & 11 & 53.53818 & 37.39946 & 27.3743 \\
\hline HIS3 & YOR202W & 8 & 1 & 12 & 15.06631 & 34.0035 & 29.27046 \\
\hline HIS3 & YOR202W & 8 & 1 & 13 & 3.79494 & 26.90355 & 13.40206 \\
\hline HIS3 & YOR202W & 8 & 1 & 14 & 40.56818 & 11.87335 & 22.51121 \\
\hline HIS3 & YOR202W & 8 & 1 & 15 & 57.78894 & 38.27655 & 35.64155 \\
\hline HIS3 & YOR202W & 8 & 1 & 16 & 27.92422 & 35.07326 & 9.867629 \\
\hline HIS3 & YOR202W & 8 & 1 & 17 & 20.88452 & 22.66895 & -6.84262 \\
\hline HIS3 & YOR202W & 8 & 1 & 18 & 54.81728 & 81.04089 & -19.0647 \\
\hline HIS3 & YOR202W & 8 & 1 & 19 & 34.74903 & 39.80848 & 52.96443 \\
\hline HIS3 & YOR202W & 8 & 1 & 20 & 71.2963 & 42.34496 & 16.11526 \\
\hline HIS3 & YOR202W & 8 & 1 & 21 & 59.15493 & 26.96477 & 18.91892 \\
\hline HIS3 & YOR202W & 8 & 1 & 22 & 40.06999 & 28.73167 & 14.99033 \\
\hline HIS3 & YOR202W & 8 & 1 & 23 & 34.61538 & 34.23729 & 4.540764 \\
\hline HIS3 & YOR202W & 8 & 1 & 24 & 65.3211 & -3.96104 & 41.8251 \\
\hline
\end{tabular}




\begin{tabular}{|c|c|c|c|c|c|c|c|c|c|}
\hline \multirow[t]{2}{*}{ HIS3 } & YOR202W & 8 & 2 & 1 & 81.83242 & -2.57681 & 1.926445 & & \\
\hline & YKL187C & 8 & 2 & 2 & 50.58078 & 44.42029 & 39.39962 & 32.48299 & 44.89796 \\
\hline TIF1 & YKR059W & 8 & 2 & 3 & 51.63934 & 26.20087 & 22.35023 & 58.14607 & 45.72193 \\
\hline ASH1 & YKL185W & 8 & 2 & 4 & 56.95652 & 20.05676 & 34.98294 & 32.81787 & 68.09211 \\
\hline GLG1 & YKR058W & 8 & 2 & 5 & 73.33333 & 39.39394 & 77.90927 & 51.82927 & 63.26531 \\
\hline SPE1 & YKL184W & 8 & 2 & 6 & 64.65781 & 44.07039 & 28.23834 & 40.97117 & 48.14815 \\
\hline RPS21A & YKR057W & 8 & 2 & 7 & -16.8397 & 33.8756 & 19.0027 & -5.33981 & 41.48936 \\
\hline LOT5 & YKL183W & 8 & 2 & 8 & 3.497758 & 25.78829 & -10.8668 & 8.490566 & 29.12621 \\
\hline TRM2 & YKR056W & 8 & 2 & 9 & 2.605571 & 42.49783 & -16.3735 & 54.75819 & 24.41472 \\
\hline COY1 & YKL179C & 8 & 2 & 10 & -6.31164 & 24.84277 & -28.0047 & 9.145129 & 11.51316 \\
\hline RHO4 & YKR055W & 8 & 2 & 11 & 12.2 & 92.5641 & 34.71074 & 19.84283 & -3.84615 \\
\hline STE3 & YKL178C & 8 & 2 & 12 & -17.9798 & 39.556 & -7.59878 & 50 & 24.3553 \\
\hline \multirow[t]{2}{*}{ DYN1 } & YKR054C & 8 & 2 & 13 & -6.94215 & -23.7864 & -24.4395 & 57.23077 & 31.43713 \\
\hline & YKL177W & 8 & 2 & 14 & 24.5977 & 51.96687 & -6.63212 & 55.64356 & 21.13208 \\
\hline YSR3 & YKR053C & 8 & 2 & 15 & 51.58879 & 59.70149 & 19.23077 & 18.08696 & 8.67052 \\
\hline LST4 & YKL176C & 8 & 2 & 16 & -17.1159 & 8.982036 & 22.17054 & 53.5545 & 12.3839 \\
\hline MRS4 & YKR052C & 8 & 2 & 17 & -4.2654 & 32.95347 & -22.9345 & -26.1391 & 6.158358 \\
\hline \multirow[t]{2}{*}{ ZRT3 } & YKL175W & 8 & 2 & 18 & -11.8896 & 41.41791 & -15.71 & 44.09836 & 21.75325 \\
\hline & YKR051W & 8 & 2 & 19 & 53.87205 & 52.01794 & -8.44063 & 31.91057 & 30.27211 \\
\hline TPO5 & YKL174C & 8 & 2 & 20 & 11.27168 & -26.4085 & -21.2121 & 42.27769 & 29.12088 \\
\hline \multirow[t]{2}{*}{ TRK2 } & YKR050W & 8 & 2 & 21 & 52.22635 & -52.6316 & 1.92 & 66.35514 & 19.70075 \\
\hline & YKL171W & 8 & 2 & 22 & 55.52268 & 18.6863 & 24.49923 & -14.0873 & 33.89356 \\
\hline FMP46 & YKR049C & 8 & 2 & 23 & 23.45013 & 17.15439 & -7.00637 & 53.99408 & 29.41176 \\
\hline HIS3 & YOR202W & 8 & 2 & 24 & -4.61394 & 45.74257 & 5.292479 & & \\
\hline HIS3 & YOR202W & 8 & 3 & 1 & 77.77053 & 43.18026 & 54.49707 & & \\
\hline STE24 & YJR117W & 8 & 3 & 2 & 37.9737 & 52.46964 & 15.62168 & 31.94707 & 48 \\
\hline \multirow[t]{2}{*}{ LHS1 } & YKL073W & 8 & 3 & 3 & 41.35338 & 14.43299 & 37.30018 & 45.63107 & 66.759 \\
\hline & YJR116W & 8 & 3 & 4 & 67.26519 & 43.68231 & 13.57466 & 48.85621 & 59.13621 \\
\hline \multirow[t]{5}{*}{ STB6 } & YKL072W & 8 & 3 & 5 & 17.01932 & -3.53753 & 8.333333 & 48.22804 & 55.7047 \\
\hline & YJR115W & 8 & 3 & 6 & 40.6015 & 47.41306 & 35.41985 & 27.12177 & 49.68153 \\
\hline & YKL071W & 8 & 3 & 7 & -17.4377 & 27.1831 & 19.38895 & 32.08191 & 47.45223 \\
\hline & YJR111C & 8 & 3 & 8 & -1.29983 & 34.45378 & 11.57233 & 21.37405 & 33.78378 \\
\hline & YKL070W & 8 & 3 & 9 & 53.76 & 34.73227 & 14.22222 & 41.41252 & 44.34251 \\
\hline \multirow[t]{2}{*}{ YMR1 } & YJR110W & 8 & 3 & 10 & 24.3 & 51.57438 & 10.53412 & 45.55556 & 30.59937 \\
\hline & YKL069W & 8 & 3 & 11 & 29.15129 & 73.34184 & 84.73804 & 47.43833 & 34.53237 \\
\hline CPA2 & YJR109C & 8 & 3 & 12 & 50.0998 & 46.16322 & 11.73709 & 39.9287 & 52.53165 \\
\hline NUP100 & YKL068W & 8 & 3 & 13 & -13.8372 & 49.23201 & -3.20334 & 45.6964 & 41.98251 \\
\hline ABM1 & YJR108W & 8 & 3 & 14 & 21.16402 & -8.8359 & -19.5751 & 11.97917 & 45.97701 \\
\hline \multirow[t]{3}{*}{ YNK1 } & YKL067W & 8 & 3 & 15 & 18.12401 & 13.67713 & 40.66667 & 29.72536 & 36.61202 \\
\hline & YJR107W & 8 & 3 & 16 & 53.07856 & 13.24111 & 20.84691 & 51.36364 & 34.27762 \\
\hline & YKL066W & 8 & 3 & 17 & 14.43609 & 33.36291 & -19.4444 & 22.62357 & 25.37764 \\
\hline ECM27 & YJR106W & 8 & 3 & 18 & 46.16588 & 32.03704 & 44.31487 & 38.4252 & 4.80226 \\
\hline YET1 & YKL065C & 8 & 3 & 19 & 18.92523 & 16.64932 & -15.5303 & 63.85135 & 21.32132 \\
\hline
\end{tabular}




\begin{tabular}{|c|c|c|c|c|c|c|c|c|c|}
\hline URA8 & YJR103W & 8 & 3 & 20 & 44.63337 & 16.42764 & -3.05206 & 30.64516 & 28.01205 \\
\hline \multirow[t]{3}{*}{ MNR2 } & YKL064W & 8 & 3 & 21 & 53.80282 & 72.75748 & 23.89381 & 55.33662 & 30.09404 \\
\hline & YJR100C & 8 & 3 & 22 & 86.74242 & 82.75862 & -38.0952 & -12.1519 & 21.17347 \\
\hline & YKL063C & 8 & 3 & 23 & 29.91612 & 34.69388 & -1.86199 & 10.93474 & 22.12121 \\
\hline HIS3 & YOR202W & 8 & 3 & 24 & -1.27157 & 30.16241 & 18.58491 & & \\
\hline HIS3 & YOR202W & 8 & 4 & 1 & 55.04152 & 26.84022 & 92.16462 & & \\
\hline LOS1 & YKL205W & 8 & 4 & 2 & 20.96944 & 10.60743 & 13.97616 & 58.05947 & 50.73314 \\
\hline ECM4 & YKR076W & 8 & 4 & 3 & 40.02134 & -3.88802 & 89.98211 & 35.02538 & 39.93056 \\
\hline \multirow[t]{3}{*}{ EAP1 } & YKL204W & 8 & 4 & 4 & 63.63636 & 63.32046 & 68.16479 & 50.5423 & 61.48649 \\
\hline & YKR074W & 8 & 4 & 5 & 18.34711 & 13.78866 & -21.6718 & 21.55689 & 38.67596 \\
\hline & YKL202W & 8 & 4 & 6 & 68.52497 & 39.60703 & 49.83713 & 44.55285 & 60.625 \\
\hline SIS2 & YKR072C & 8 & 4 & 7 & 49.41374 & 43.75 & 5.211726 & 17.44966 & 54.03509 \\
\hline \multirow[t]{3}{*}{ MNN4 } & YKL201C & 8 & 4 & 8 & 31.61094 & 33.33333 & 11.06195 & 50 & 44.8505 \\
\hline & YKR070W & 8 & 4 & 9 & 36.0799 & 56.79724 & 52.63158 & 43.44123 & 26.96246 \\
\hline & YKL200C & 8 & 4 & 10 & 12.30159 & -29.5259 & 39.11472 & 13.78556 & 53.96226 \\
\hline MET1 & YKR069W & 8 & 4 & 11 & -8.92308 & -60.4683 & -16.7598 & 53.52113 & 39.52802 \\
\hline YKT9 & YKL199C & 8 & 4 & 12 & -8.76993 & 19.39759 & -8.48057 & 33.27172 & 30.22599 \\
\hline GPT2 & YKR067W & 8 & 4 & 13 & 8.901099 & 25.97403 & -5.58036 & 62.43981 & 45.37037 \\
\hline PTK1 & YKL198C & 8 & 4 & 14 & 7.765957 & -104.685 & 16.81196 & 53.44 & 41.12903 \\
\hline CCP1 & YKR066C & 8 & 4 & 15 & 11.76471 & -33.9876 & 54.31655 & 14.14538 & 48.71795 \\
\hline PEX1 & YKL197C & 8 & 4 & 16 & 39.92322 & 9.364909 & 15.25424 & 51.05008 & 46.45161 \\
\hline FMP18 & YKR065C & 8 & 4 & 17 & 7.863696 & -19.9422 & -10.1563 & 48.40764 & 31.76101 \\
\hline \multirow[t]{2}{*}{ DPH2 } & YKL191W & 8 & 4 & 18 & 10.31866 & -41.8564 & 25.42694 & 62.98246 & 43.91892 \\
\hline & YKR064W & 8 & 4 & 19 & 60.96257 & 43.52792 & 14.04255 & 40.76539 & 36.93182 \\
\hline CNB1 & YKL190W & 8 & 4 & 20 & 62.97094 & 36.52393 & 25.433 & 44.5993 & 42.38411 \\
\hline KTR2 & YKR061W & 8 & 4 & 21 & 58.00712 & 13.30561 & -9.11602 & 54.01929 & 28.18182 \\
\hline PXA2 & YKL188C & 8 & 4 & 22 & 35.51968 & 28.81773 & 21.79837 & 60.33287 & 32.96703 \\
\hline UTP30 & YKR060W & 8 & 4 & 23 & 27.14552 & 64.75196 & 18.79699 & 17.24138 & 42 \\
\hline HIS3 & YOR202W & 8 & 4 & 24 & 3.157895 & 41.8738 & -8.17236 & & \\
\hline HIS3 & YOR202W & 8 & 5 & 1 & 40.67914 & 10.03135 & 73.37017 & & \\
\hline \multirow[t]{3}{*}{ STR2 } & YJR130C & 8 & 5 & 2 & -18.018 & 11.35242 & 69.62025 & 36.64688 & 52.6455 \\
\hline & YKL091C & 8 & 5 & 3 & 17.26354 & 33.45227 & 71.01911 & 51.24611 & 68.62745 \\
\hline & YJR129C & 8 & 5 & 4 & 17.38516 & 45.05777 & 7.047619 & 43.04267 & 66.8693 \\
\hline \multirow[t]{2}{*}{ CUE2 } & YKL090W & 8 & 5 & 5 & 68.21429 & 76.63043 & 77.35849 & 53.01205 & 65.16854 \\
\hline & YJR128W & 8 & 5 & 6 & 9.198813 & -4.51128 & 26.82927 & 51.52488 & 55.36913 \\
\hline SRX1 & YKL086W & 8 & 5 & 7 & 36.52778 & 28.06324 & 11.36364 & 37.33583 & 66.88742 \\
\hline ZMS1 & YJR127C & 8 & 5 & 8 & 45.99057 & -13.6364 & 28.89096 & 64.297 & 57.96178 \\
\hline MDH1 & YKL085W & 8 & 5 & 9 & 1.984127 & 21.48481 & 21.45624 & 47.61092 & 30.10753 \\
\hline VPS70 & YJR126C & 8 & 5 & 10 & 21.26068 & 53.43008 & 36.52393 & 56.27063 & 53.92857 \\
\hline HOT13 & YKL084W & 8 & 5 & 11 & 3.282532 & 28.00954 & 5.555556 & 64.61295 & 41.86747 \\
\hline ENT3 & YJR125C & 8 & 5 & 12 & 24.79339 & 44.0625 & -29.3706 & 58.13953 & 37.62376 \\
\hline \multirow[t]{2}{*}{ TEF4 } & YKL081W & 8 & 5 & 13 & 16.76587 & 38.96499 & -5.88235 & -9.69388 & 48.14815 \\
\hline & YJR124C & 8 & 5 & 14 & -9.36073 & 22.56236 & 32.5779 & 60.5 & 46.25407 \\
\hline
\end{tabular}




\begin{tabular}{|c|c|c|c|c|c|c|c|c|c|}
\hline SMY1 & YKL079W & 8 & 5 & 15 & 12.48721 & 36.84211 & -13.3333 & 20.33272 & 48.97361 \\
\hline \multirow[t]{3}{*}{ ATP2 } & YJR121W & 8 & 5 & 16 & 14.69501 & 36.41364 & 45.84352 & 50.83682 & 47.74436 \\
\hline & YKL077W & 8 & 5 & 17 & 44.24084 & 62.60237 & 59.59916 & 33.92857 & 33.61702 \\
\hline & YJR120W & 8 & 5 & 18 & 18.13677 & 48.06867 & 1.362862 & 55.64388 & 56.69782 \\
\hline \multirow[t]{3}{*}{ PSY1 } & YKL076C & 8 & 5 & 19 & 34.68586 & 91.72662 & 55.4007 & 51.14504 & 45.32578 \\
\hline & YJR119C & 8 & 5 & 20 & 66.50485 & 76.90722 & 6.521739 & 37.4 & 27.68166 \\
\hline & YKL075C & 8 & 5 & 21 & 6.634499 & 47.14687 & -6.27306 & 13.46499 & 39.88095 \\
\hline ILM1 & YJR118C & 8 & 5 & 22 & 29.50237 & 38.79121 & 21.6895 & 8.163265 & 45.86207 \\
\hline MUD2 & YKL074C & 8 & 5 & 23 & 21.60714 & 62.55792 & -12.6834 & 12.33333 & 45.28302 \\
\hline HIS3 & YOR202W & 8 & 5 & 24 & 2.07502 & 28.04995 & 22.24317 & & \\
\hline HIS3 & YOR202W & 8 & 6 & 1 & -5.01529 & -39.916 & 81.5864 & & \\
\hline SRY1 & YKL218C & 8 & 6 & 2 & 67.26384 & 35.19313 & 71.949 & 64.22018 & 80.19802 \\
\hline SRP40 & YKR092C & 8 & 6 & 3 & 40.38005 & 18.73199 & 46.54378 & 26.21564 & 60.16949 \\
\hline JEN1 & YKL217W & 8 & 6 & 4 & 24.26966 & 24.91429 & 62.89308 & 45.46952 & 68.50649 \\
\hline SRL3 & YKR091W & 8 & 6 & 5 & 41.68337 & 6.400966 & 16.11511 & 4.195804 & 63.31169 \\
\hline URA1 & YKL216W & 8 & 6 & 6 & 26.48026 & 2.755454 & 54.58996 & 39.28013 & 47.04142 \\
\hline \multirow[t]{2}{*}{ PXL1 } & YKR090W & 8 & 6 & 7 & 62.13275 & 61.05611 & 76.14424 & 58.96226 & 64.55696 \\
\hline & YKL215C & 8 & 6 & 8 & 16.93811 & 14.74978 & 8.575198 & 15.7563 & 76.64671 \\
\hline STC1 & YKR089C & 8 & 6 & 9 & -7.02055 & 50.63898 & 8.265425 & 61.85065 & 59.62963 \\
\hline YRA2 & YKL214C & 8 & 6 & 10 & 16.96721 & 34.81196 & 57.03704 & 27.94118 & 55.97668 \\
\hline TVP38 & YKR088C & 8 & 6 & 11 & 16.7263 & 39.44444 & 23.20819 & 55.99369 & 54.48916 \\
\hline DOA1 & YKL213C & 8 & 6 & 12 & 0 & 53.57143 & -5.35714 & 69.8 & 48.97959 \\
\hline OMA1 & YKR087C & 8 & 6 & 13 & 39.05429 & 19.10966 & 71.35462 & 56.73077 & 66.10169 \\
\hline SAC1 & YKL212W & 8 & 6 & 14 & 44.57143 & 98.8345 & 59.01639 & 41.42857 & 5 \\
\hline HBS1 & YKR084C & 8 & 6 & 15 & 14.92674 & 39.17647 & -3.53698 & -4.97835 & 56.50685 \\
\hline TRP3 & YKL211C & 8 & 6 & 16 & 7.715431 & 33.20988 & 12.32877 & 57.84469 & 55.21472 \\
\hline NUP133 & YKR082W & 8 & 6 & 17 & 6.404494 & 18.87227 & 9.351145 & 25.97968 & 57.83133 \\
\hline CBT1 & YKL208W & 8 & 6 & 18 & -1.7257 & 35.41667 & 78.51047 & 62.54682 & 28.0303 \\
\hline \multirow[t]{5}{*}{ MTD1 } & YKR080W & 8 & 6 & 19 & 15.69921 & 23.91094 & 58.32583 & 59.96785 & 35.24096 \\
\hline & YKL207W & 8 & 6 & 20 & 65.61372 & 5.24109 & 68.79293 & 11.53846 & 40.83333 \\
\hline & YKR078W & 8 & 6 & 21 & 2.983139 & 39.43355 & 66.99376 & -17.6 & 36.54485 \\
\hline & YKL206C & 8 & 6 & 22 & 17.5814 & 31.81395 & 13.19623 & 18.13285 & 61.87845 \\
\hline & YKR077W & 8 & 6 & 23 & 12.41643 & 38.921 & 23.40234 & 55.35957 & 46.01542 \\
\hline HIS3 & YOR202W & 8 & 6 & 24 & -3.86304 & 23.60122 & 13.77304 & & \\
\hline HIS3 & YOR202W & 8 & 7 & 1 & 12.87247 & 56.2568 & 94.42834 & & \\
\hline HMS2 & YJR147W & 8 & 7 & 2 & 20.21277 & 24.54175 & 60.43084 & 10.54545 & 62.27545 \\
\hline \multirow[t]{3}{*}{ LAP4 } & YKL103C & 8 & 7 & 3 & 61.24352 & 78.57848 & 34.47537 & 60.55046 & 85.47297 \\
\hline & YJR146W & 8 & 7 & 4 & 66.29213 & 61.9403 & 64.97696 & 43.17343 & 71.09375 \\
\hline & YKL102C & 8 & 7 & 5 & 11.17764 & 50.39474 & 20.9622 & 56.43879 & 69.33333 \\
\hline RPS4A & YJR145C & 8 & 7 & 6 & 46.36234 & -3.73027 & 59.68 & 50.16234 & 63.12057 \\
\hline \multirow[t]{3}{*}{ HSL1 } & YKL101W & 8 & 7 & 7 & 78.71287 & 57.43175 & 60.96738 & 61.5894 & 79.76654 \\
\hline & YJR142W & 8 & 7 & 8 & 23.73596 & 55.44676 & 41.05392 & 43.73089 & 69.77492 \\
\hline & YKL100C & 8 & 7 & 9 & 45.78488 & 30 & 67.04663 & 62.7095 & 62.2222 \\
\hline
\end{tabular}




\begin{tabular}{|c|c|c|c|c|c|c|c|c|c|}
\hline \multirow[t]{2}{*}{ HIR3 } & YJR140C & 8 & 7 & 10 & 22.78978 & 9.049774 & 65.4023 & 33.26226 & 45.59271 \\
\hline & YKL098W & 8 & 7 & 11 & 19.78764 & 49.6904 & 47.82097 & 27.93834 & 58.80282 \\
\hline \multirow[t]{2}{*}{ HOM6 } & YJR139C & 8 & 7 & 12 & 28.65672 & 54.16316 & 62.36125 & 71.9457 & 71.25 \\
\hline & YKL097C & 8 & 7 & 13 & 15.85366 & 27.77778 & 73.87802 & 50.91514 & 46.59498 \\
\hline ECM17 & $\begin{array}{l}\text { YJR137C } \\
\text { YKL096W- }\end{array}$ & 8 & 7 & 14 & 10.3664 & 44.67178 & 14.57431 & 63.68794 & 56.81063 \\
\hline CWP2 & $A$ & 8 & 7 & 15 & 37.02703 & 74.50658 & 69.56522 & 68.42105 & 66.01942 \\
\hline MCM22 & YJR135C & 8 & 7 & 16 & -3.44186 & 7.191448 & 60.89194 & 49.92413 & 57.71605 \\
\hline CWP1 & YKL096W & 8 & 7 & 17 & 21.97962 & 20.54507 & 16.26928 & -58.3535 & 28.79257 \\
\hline SGM1 & YJR134C & 8 & 7 & 18 & 9.090909 & 30.58608 & 22.92645 & 36.08903 & 42.71186 \\
\hline YJU3 & YKL094W & 8 & 7 & 19 & 11.46667 & 44.23841 & 67.42671 & 54.48505 & 59.43396 \\
\hline XPT1 & YJR133W & 8 & 7 & 20 & -4.63918 & 46.21212 & 22.77121 & 52.26586 & 53.73134 \\
\hline MBR1 & YKL093W & 8 & 7 & 21 & 76.68213 & 84.30657 & 79.70353 & 58.80682 & 82.97101 \\
\hline MNS1 & YJR131W & 8 & 7 & 22 & 30.15707 & 33.90805 & 24.24917 & -16.4179 & 52.86195 \\
\hline BUD2 & YKL092C & 8 & 7 & 23 & 45.51724 & 53.22751 & 41.35189 & 43.31984 & 62.11699 \\
\hline HIS3 & YOR202W & 8 & 7 & 24 & 7.840532 & 38.31672 & -9.48396 & & \\
\hline \multirow[t]{2}{*}{ HIS3 } & YOR202W & 8 & 8 & 1 & 22.15909 & 40.68413 & 45.10903 & & \\
\hline & YKR012C & 8 & 8 & 2 & 37.81513 & 16.13162 & 16.10979 & 24.37137 & 67.68293 \\
\hline NFT1 & YKR103W & 8 & 8 & 3 & 81.69717 & 75.99068 & 63.39286 & 63.7037 & 72.90323 \\
\hline TOS5 & YKR011C & 8 & 8 & 4 & 75.07163 & 58.78788 & 65.94203 & -3.98323 & 68.06452 \\
\hline FLO10 & YKR102W & 8 & 8 & 5 & 46.72775 & 19.86628 & 75.78397 & 32.76596 & 64.64286 \\
\hline TOF2 & YKR010C & 8 & 8 & 6 & 44.27673 & 10.68323 & 6.146179 & 3.448276 & 66.77215 \\
\hline SIR1 & YKR101W & 8 & 8 & 7 & 52.87234 & 63.83285 & 61.57025 & 36.36364 & 70.06803 \\
\hline FOX2 & YKR009C & 8 & 8 & 8 & 31.85185 & 54.43841 & 30.12295 & 40.92593 & 68.51211 \\
\hline SKG1 & YKR100C & 8 & 8 & 9 & 62.80255 & 59.45355 & 66.29213 & 5.62249 & 73.95498 \\
\hline MEH1 & YKR007W & 8 & 8 & 10 & 15.98712 & 16.37487 & 26.14476 & 36.93878 & 66.79245 \\
\hline \multirow[t]{2}{*}{ BAS1 } & YKR099W & 8 & 8 & 11 & 55.18717 & 62.5 & 74.42573 & 22.01646 & 69.57929 \\
\hline & YKR005C & 8 & 8 & 12 & 17.87565 & 31.8612 & 65.75 & 45.56575 & 51.5625 \\
\hline UBP11 & YKR098C & 8 & 8 & 13 & 62.35078 & 70.13783 & 66.34921 & -13.5468 & 66.46154 \\
\hline OSH6 & YKR003W & 8 & 8 & 14 & 15.38462 & 32.19557 & 4.101839 & 49.03537 & 48.17276 \\
\hline PCK1 & YKR097W & 8 & 8 & 15 & 17.34961 & 32.02545 & 69.25373 & 50.79595 & 63.47305 \\
\hline \multirow[t]{3}{*}{ VPS1 } & YKR001C & 8 & 8 & 16 & 33.55049 & 48.22804 & 52.11268 & 28.71452 & 53.90071 \\
\hline & YKR096W & 8 & 8 & 17 & 13.30604 & 22.02797 & 23.12312 & 25.78125 & 55.58739 \\
\hline & YKL222C & 8 & 8 & 18 & 21.88217 & 43.18182 & 31.06436 & 55.55556 & 57.27273 \\
\hline MLP1 & YKR095W & 8 & 8 & 19 & 21.23162 & 31.95777 & 53.28767 & 50.08 & 64.60674 \\
\hline $\mathrm{MCH} 2$ & YKL221W & 8 & 8 & 20 & 39.75535 & 83.41121 & 54.74138 & 56.39731 & 54.24242 \\
\hline RPL40B & YKR094C & 8 & 8 & 21 & 20.71636 & 46.46878 & 15.36313 & 66.76971 & 65.78947 \\
\hline FRE2 & YKL220C & 8 & 8 & 22 & 61.89918 & 20.39711 & 18.18182 & 53.59661 & 63.27684 \\
\hline PTR2 & YKR093W & 8 & 8 & 23 & 42.91667 & 43.89562 & 17.2144 & 57.12329 & 63.1016 \\
\hline HIS3 & YOR202W & 8 & 8 & 24 & 29.00763 & 13.87407 & 21.50644 & & \\
\hline HIS3 & YOR202W & 8 & 9 & 1 & 22.96374 & 18.66567 & 83.00349 & 42.37013 & 67.61006 \\
\hline LAC1 & YKL008C & 8 & 9 & 2 & 25.13312 & 80.61674 & 32.28571 & 18.75 & 77.55102 \\
\hline OAC1 & YKL120W & 8 & 9 & 3 & 51.17878 & 72.31638 & 66.57224 & 5.934066 & 72.22222 \\
\hline
\end{tabular}




\begin{tabular}{|c|c|c|c|c|c|c|c|c|c|}
\hline CAP1 & YKL007W & 8 & 9 & 4 & 49.40476 & 62.62815 & 37.70779 & 46.47239 & 65.9176 \\
\hline SBA1 & YKL117W & 8 & 9 & 5 & 21.65109 & 78.23303 & 32.75862 & 46.68008 & 56.38767 \\
\hline RPL14A & YKL006W & 8 & 9 & 6 & 56.65829 & 52.41546 & 10.66667 & 48.70968 & 76.36364 \\
\hline PRR1 & YKL116C & 8 & 9 & 7 & 41.51309 & 34.42164 & 41.99815 & 19.54733 & 50 \\
\hline \multirow[t]{2}{*}{ BYE1 } & YKL005C & 8 & 9 & 8 & 27.27273 & 33.12883 & \#DIV/0! & 62.7907 & 73.8806 \\
\hline & YKL115C & 8 & 9 & 9 & 57.11086 & 61.65803 & 65.78947 & 59.81595 & 48.13559 \\
\hline MET14 & YKL001C & 8 & 9 & 10 & 13.7163 & 0.755668 & 76.26582 & 62.01991 & 67.30769 \\
\hline \multirow[t]{2}{*}{ APN1 } & YKL114C & 8 & 9 & 11 & 27.07182 & 44.09302 & 53.61446 & 57.3913 & 61.95965 \\
\hline & YJR154W & 8 & 9 & 12 & 33.55394 & 22.34469 & 16.18382 & 34.79125 & 57.97665 \\
\hline RAD27 & YKL113C & 8 & 9 & 13 & 58.82353 & \#DIV/0! & 49.0566 & 53.61272 & 62.53776 \\
\hline PGU1 & YJR153W & 8 & 9 & 14 & 75.08772 & 50.94017 & 69 & -33.9483 & 65.19824 \\
\hline KTI12 & YKL110C & 8 & 9 & 15 & 35.35354 & 56.16798 & 7.929515 & 6.535948 & 53.7415 \\
\hline DAL5 & YJR152W & 8 & 9 & 16 & 24.821 & 32.23481 & 69.02738 & 65.2439 & 47.12644 \\
\hline HAP4 & YKL109W & 8 & 9 & 17 & 15.74864 & 20.74789 & 28 & 50.40388 & 50.3125 \\
\hline \multirow[t]{3}{*}{ DAN1 } & YJR150C & 8 & 9 & 18 & 18.15642 & \#DIV/0! & 19.25926 & 61.7284 & 58.28221 \\
\hline & YKL107W & 8 & 9 & 19 & 39.68254 & 72.72727 & 40.54054 & 42.65176 & 67.17791 \\
\hline & YJR149W & 8 & 9 & 20 & 59.1 & 60.99585 & 61.95373 & -18.9796 & 59.61538 \\
\hline AAT1 & YKL106W & 8 & 9 & 21 & 33.16541 & 52.63158 & 71.04796 & 46.2203 & 67.69231 \\
\hline \multirow[t]{2}{*}{ BAT2 } & YJR148W & 8 & 9 & 22 & 46.19883 & 52.10793 & 97.36842 & -8.8015 & 55.94595 \\
\hline & YKL105C & 8 & 9 & 23 & 12.64957 & 58.07478 & -1.36054 & & \\
\hline HIS3 & YOR202W & 8 & 9 & 24 & -2.65423 & 9.518717 & 16.16702 & & \\
\hline HIS3 & YOR202W & 8 & 10 & 1 & 64.07821 & 60.78886 & 94.79354 & & \\
\hline DBP7 & YKR024C & 8 & 10 & 2 & 17.55319 & 2.845528 & 9.333333 & 62.39837 & 55.75221 \\
\hline \multirow[t]{2}{*}{ PUF3 } & YLL013C & 8 & 10 & 3 & 51.65563 & 1.671891 & 33.24709 & 34.14179 & 57.19178 \\
\hline & YKR023W & 8 & 10 & 4 & 6.068163 & 21.55689 & 31.81149 & 36.55706 & 74.0625 \\
\hline \multirow[t]{2}{*}{ YEH1 } & YLL012W & 8 & 10 & 5 & 58.11856 & 72.35294 & 63.81381 & 54.31711 & 73.77049 \\
\hline & YKR021W & 8 & 10 & 6 & 0.256191 & 3.963012 & 52.21893 & 46.89542 & 66.55738 \\
\hline PSR1 & YLL010C & 8 & 10 & 7 & 5.284016 & 32.61164 & 76.13883 & 38.48987 & 65.56291 \\
\hline VPS51 & YKR020W & 8 & 10 & 8 & 24.0865 & 23.64934 & 12.2871 & -4.9763 & 43.7931 \\
\hline cox17 & YLL009C & 8 & 10 & 9 & 17.6 & 7.619048 & 100 & 53.09568 & 19.15709 \\
\hline \multirow[t]{3}{*}{ IRS4 } & YKR019C & 8 & 10 & 10 & 8.589512 & 28.85246 & 70.74031 & -15.5388 & 40 \\
\hline & YLL007C & 8 & 10 & 11 & 14.66049 & 8.584687 & 14.47178 & 0.520833 & 42.3221 \\
\hline & YKR018C & 8 & 10 & 12 & 15.02976 & 7.430998 & 21.85592 & 53.33333 & 55.87302 \\
\hline \multirow[t]{2}{*}{ MMM1 } & YLL006W & 8 & 10 & 13 & 63.53093 & \#DIV/0! & 60.69364 & 59.77528 & 65.59633 \\
\hline & YKR017C & 8 & 10 & 14 & 36.34185 & 28.90792 & 74.93495 & 41.43731 & 54.77707 \\
\hline SPO75 & YLL005C & 8 & 10 & 15 & 20.35623 & 20.81218 & 67.76492 & -2.1322 & 49.12791 \\
\hline FMP13 & YKR016W & 8 & 10 & 16 & 16.2465 & 18.39827 & 4.506699 & 44.70939 & 48.66667 \\
\hline \multirow[t]{2}{*}{ RTT109 } & YLL002W & 8 & 10 & 17 & 27.93988 & 41.41104 & 33.47548 & 16.55773 & 57.14286 \\
\hline & YKR015C & 8 & 10 & 18 & 54.24354 & 34.03042 & 66.54135 & 49.46728 & 35.9736 \\
\hline DNM1 & YLL001W & 8 & 10 & 19 & 13.02083 & 17.19128 & 64.17489 & 11.97605 & 45.37037 \\
\hline \multirow[t]{2}{*}{ YPT52 } & YKR014C & 8 & 10 & 20 & -28.658 & 32.7654 & 39.65245 & 59.65463 & 50.64103 \\
\hline & YKR106W & 8 & 10 & 21 & 6.701807 & 30.16043 & 32.56433 & 29.37743 & 45.33333 \\
\hline PRY2 & YKR013W & 8 & 10 & 22 & 42.1466 & 35.3229 & 47.29858 & 53.69596 & 58.72576 \\
\hline
\end{tabular}




\begin{tabular}{|c|c|c|c|c|c|c|c|c|c|}
\hline & YKR105C & 8 & 10 & 23 & 61.39241 & 66.56627 & 58.82353 & 63.10078 & 73.50427 \\
\hline HIS3 & YOR202W & 8 & 10 & 24 & 27.15947 & 61.05769 & 4.042806 & & \\
\hline \multirow[t]{3}{*}{ HIS3 } & YOR202W & 8 & 11 & 1 & 51.20773 & 19.88389 & 69.8908 & & \\
\hline & YKL030W & 8 & 11 & 2 & 68.35222 & 41.9181 & 61.6095 & 32.37925 & 76.12903 \\
\hline & YKL136W & 8 & 11 & 3 & 50.17341 & 19.45744 & 51.60256 & 53.22339 & 62.45955 \\
\hline \multirow[t]{3}{*}{ MAE1 } & YKL029C & 8 & 11 & 4 & 7.721519 & -4.102 & 11.57456 & 19.84127 & 38.67596 \\
\hline & YKL133C & 8 & 11 & 5 & 23.67788 & 77.57437 & 55.03597 & 30.97514 & 44.44444 \\
\hline & YKL027W & 8 & 11 & 6 & 11.52263 & 0.915751 & 74.20635 & 4.117647 & 35.57692 \\
\hline RMA1 & YKL132C & 8 & 11 & 7 & 13.80186 & 60.61121 & 51.26263 & 34.12564 & 61.27946 \\
\hline \multirow[t]{2}{*}{ GPX1 } & YKL026C & 8 & 11 & 8 & 21.96796 & 37.91209 & 25.89499 & 52.39726 & 44.91228 \\
\hline & YKL131W & 8 & 11 & 9 & 9.896324 & 32.30922 & 50.08547 & 39.29766 & 42.10526 \\
\hline PAN3 & YKL025C & 8 & 11 & 10 & -9.2668 & 17.42331 & 38.94231 & 53.2925 & 51.63399 \\
\hline \multirow[t]{2}{*}{ SHE2 } & YKL130C & 8 & 11 & 11 & -5.12273 & 18.95262 & 87.43068 & -3.46535 & 48.05195 \\
\hline & YKL023W & 8 & 11 & 12 & 38.80464 & 35.77465 & 55.0706 & 34.42623 & 64.40129 \\
\hline MYO3 & YKL129C & 8 & 11 & 13 & 6.48824 & 31.78893 & 45.37445 & 37.14734 & 44.40895 \\
\hline SPT23 & YKL020C & 8 & 11 & 14 & 21.73547 & 24.28571 & 53.43511 & 0.456621 & 44.59016 \\
\hline PMU1 & YKL128C & 8 & 11 & 15 & 37.94466 & 87.86232 & 3.422053 & -10.9504 & 47.78481 \\
\hline HCS1 & YKL017C & 8 & 11 & 16 & 16.66667 & 0 & 67.18081 & 14.16122 & 61.32813 \\
\hline PGM1 & YKL127W & 8 & 11 & 17 & 0.817996 & 52.03883 & 39.58333 & -7.44921 & 40.55728 \\
\hline PUT3 & YKL015W & 8 & 11 & 18 & 28.40195 & 28.40671 & 74.58647 & -0.38835 & 43.45238 \\
\hline $\mathrm{SSH} 4$ & YKL124W & 8 & 11 & 19 & 14.40443 & 17.54933 & 54.35644 & -3.80313 & 61.37072 \\
\hline \multirow[t]{2}{*}{ UFD4 } & YKL010C & 8 & 11 & 20 & 14.90978 & 41.25874 & 21.26697 & 13.2948 & 63.60947 \\
\hline & YKL123W & 8 & 11 & 21 & 15.05824 & 41.90141 & 74.5167 & 3.761062 & 60.17699 \\
\hline \multirow[t]{2}{*}{ MRT4 } & YKL009W & 8 & 11 & 22 & 66.15385 & 52.85714 & 58.55131 & 23.64641 & 69.77273 \\
\hline & YKL121W & 8 & 11 & 23 & 12.00397 & 64.52991 & 53.83023 & 39.40256 & 63.14496 \\
\hline HIS3 & YOR202W & 8 & 11 & 24 & -8.97833 & 19.43867 & 41.56425 & & \\
\hline HIS3 & $\begin{array}{l}\text { YOR202W } \\
\text { YKR035W- }\end{array}$ & 8 & 12 & 1 & 12.67829 & 43.69565 & 58.82353 & & \\
\hline DID2 & $A$ & 8 & 12 & 2 & 8.690745 & 66.77815 & 69.76744 & 32.38866 & 52.44755 \\
\hline \multirow[t]{3}{*}{ HSP104 } & YLL026W & 8 & 12 & 3 & 45.36391 & 23.30435 & 54.92662 & 54.73856 & 61.18421 \\
\hline & YKR035C & 8 & 12 & 4 & 32.60542 & 44.92754 & 39.29314 & 39.67972 & 62.31884 \\
\hline & YLL025W & 8 & 12 & 5 & -2.6943 & 0.736377 & 13.53887 & 25.83643 & 17.10526 \\
\hline DAL80 & YKR034W & 8 & 12 & 6 & 16.06392 & 25.04817 & 19.07895 & 54.2811 & 61.96721 \\
\hline \multirow[t]{4}{*}{ SSA2 } & YLL024C & 8 & 12 & 7 & 16.68138 & 56.66667 & -5.4917 & 54.62555 & 65.04425 \\
\hline & YKR033C & 8 & 12 & 8 & 15.625 & 7.756813 & 42.83966 & 54.43038 & 55.87189 \\
\hline & YLL023C & 8 & 12 & 9 & 16.92047 & 9.742441 & 57.34649 & 55.13595 & 56.37982 \\
\hline & YKR032W & 8 & 12 & 10 & 15.92115 & 21.05832 & 35.39916 & 43.20388 & 55.21472 \\
\hline SPA2 & YLL021W & 8 & 12 & 11 & 58.92587 & 84.46727 & 58.62377 & 50.84485 & 62.02091 \\
\hline \multirow[t]{2}{*}{ SPO14 } & YKR031C & 8 & 12 & 12 & 18.13765 & -14.2259 & 12.7566 & 50 & 40.92527 \\
\hline & YLL020C & 8 & 12 & 13 & 29.04564 & 34.92063 & 6.806283 & 40.25765 & 42.99363 \\
\hline GMH1 & YKR030W & 8 & 12 & 14 & 10.4717 & 24.65409 & 82.11382 & 4.918033 & 47.26688 \\
\hline KNS1 & YLL019C & 8 & 12 & 15 & 10.92801 & 42.69231 & 61.77215 & 44.83986 & 40.13378 \\
\hline SET3 & YKR029C & 8 & 12 & 16 & 3.11245 & 42.72727 & \#DIV/0! & 43.87569 & 65.01548 \\
\hline
\end{tabular}




$\begin{array}{llll}\text { SDC25 } & \text { YLL017W } & 8 & 12 \\ \text { SAP190 } & \text { YKR028W } & 8 & 12 \\ \text { SDC25 } & \text { YLL016W } & 8 & 12 \\ \text { FMP50 } & \text { YKR027W } & 8 & 12 \\ \text { BPT1 } & \text { YLL015W } & 8 & 12 \\ \text { GCN3 } & \text { YKR026C } & 8 & 12 \\ & \text { YLL014W } & 8 & 12 \\ \text { HIS3 } & \text { YOR202W } & 8 & 12 \\ \text { HIS3 } & \text { YOR202W } & 8 & 13 \\ & \text { YKL044W } & 8 & 13 \\ \text { RPS27A } & \text { YKL156W } & 8 & 13 \\ \text { PHD1 } & \text { YKL043W } & 8 & 13 \\ & \text { YKL151C } & 8 & 13 \\ \text { VPS24 } & \text { YKL041W } & 8 & 13 \\ \text { MCR1 } & \text { YKL150W } & 8 & 13 \\ \text { NFU1 } & \text { YKL040C } & 8 & 13 \\ \text { DBR1 } & \text { YKL149C } & 8 & 13 \\ \text { PTM1 } & \text { YKL039W } & 8 & 13 \\ \text { SDH1 } & \text { YKL148C } & 8 & 13 \\ \text { RGT1 } & \text { YKL038W } & 8 & 13 \\ & \text { YKL147C } & 8 & 13 \\ & \text { YKL037W } & 8 & 13 \\ \text { AVT3 } & \text { YKL146W } & 8 & 13 \\ \text { TUL1 } & \text { YKL034W } & 8 & 13 \\ \text { MRP8 } & \text { YKL142W } & 8 & 13 \\ & \text { YKL033W- } & & \\ & \text { A } & 8 & 13\end{array}$

$\begin{array}{lll}\text { A } & 8 & 13\end{array}$

TGL1

$\begin{array}{lll}\text { YKL140W } & 8 & 13\end{array}$

$\begin{array}{lll}\text { YKL032C } & 8 & 13\end{array}$

CTK1

YKL139W

YKL031W

YKL137W

HIS3

YOR202W

HIS3

NAP1

YOR202W

RPL8B

YKR048C

YLL045C

YKR047W

YLL044W

PET10

YKR046C

FPS1

ATG10

YLL043W

YKR045C

YLL042C

\section{3}

\section{3}

13

\section{3}

\section{4}

14

14

14

14

14

14

14

14

$\begin{array}{rrrrr}17 & -13.0435 & 14.02062 & 9.392265 & 44.12214 \\ 18 & -1.02516 & 30.2521 & -7.97546 & 27.12871 \\ 19 & 17.94311 & 40.42781 & 29.97481 & -10.2473 \\ 20 & 23.05296 & \text { \#DIV/O! } & 19.26606 & 19.2389 \\ 21 & 20.8221 & 35.69231 & 55.92748 & 33.05785 \\ 22 & 56.52681 & 39.14027 & 67.64706 & -2.63736 \\ 23 & 12.40524 & -5.55556 & 34.55497 & 36.41457 \\ 24 & 48.48485 & 83.06998 & 43.3908 & \\ 1 & 28.53448 & 17.30463 & 80.95238 & \end{array}$

35.39519

39.96383

50.08757

35.55957

32.78956

43.66472

47.09898

48.16667

$-45.4338$

21.63355

53.24675

34.70948

26.34731

37.51914

7.415254

41.64306

missing

$\begin{array}{llll}18 & 16.54902 & 16.30435 & 79.12773\end{array}$

$\begin{array}{llll}19 & 20.4953 & 29.12621 & 82.27848\end{array}$

colony

missing

$\begin{array}{llll}20 & 17.02722 & 53.45774 & 69.32907\end{array}$

colony

$21 \quad 52.40328$

$41.70543 \quad 53.63322$

2219.79695

$50.42644 \quad 13.51767$

$\begin{array}{llll}23 & -8.05176 & 25.58635 & 44.3433\end{array}$

$\begin{array}{llll}24 & 52.82093 & 6.829897 & 23.94775\end{array}$

$\begin{array}{llll}1 & 52 & 9.857904 & 81.69336\end{array}$

$\begin{array}{llll}2 & 51.26728 & 54.73684 & 37.24138\end{array}$

30.05391

37.75322

58.74126

53.58423

49.74271

65.2452

39.30636

26.36054
53.125

67.93003

39.94169

47.14286

56.67575

61.83432

56.88889

37.46313

29.85612

36.54485

40.24768

32.50883

28.47222

33.33333

46.6899

29.43038

27.30159

68.35443

45.51495

27.77778

61.00629

43.03406

55.52699

missing colony

missing colony

52.96053

41.71598

66.54804

38.10888

38.15385

40

64.78261

51.1254

43.34365

76.92308

33.96226

40.41298 


\begin{tabular}{|c|c|c|c|c|c|c|c|c|c|}
\hline UIP5 & YKR044W & 8 & 14 & 10 & 19.31649 & 10.84337 & 30.55215 & 41.23077 & 38.03681 \\
\hline \multirow[t]{2}{*}{ SDH2 } & YLL041C & 8 & 14 & 11 & 7.266436 & 36.71971 & -2.574 & 17.94872 & 19.11765 \\
\hline & YKR043C & 8 & 14 & 12 & 26.51633 & 32.51121 & 14.16667 & 48.80113 & 57.46479 \\
\hline VPS13 & YLLO4OC & 8 & 14 & 13 & 12.5 & 49.12281 & 20.02924 & 31.07511 & 42.23433 \\
\hline UTH1 & YKR042W & 8 & 14 & 14 & 14.6383 & 37.86848 & 12.43339 & 1.351351 & 30.89005 \\
\hline \multirow[t]{2}{*}{ UBI4 } & YLL039C & 8 & 14 & 15 & 48.98785 & 40.43993 & 19.59799 & 43.30986 & 57.55627 \\
\hline & YKR041W & 8 & 14 & 16 & 26.78063 & 5.964215 & 15.97796 & 30 & 54.17957 \\
\hline \multirow[t]{3}{*}{ ENT4 } & YLL038C & 8 & 14 & 17 & 12.26321 & 46.75877 & 66.85304 & $\begin{array}{l}34.26471 \\
\text { missing }\end{array}$ & 60.08316 \\
\hline & YKR040C & 8 & 14 & 18 & 9.708738 & 40.27778 & 59.77011 & $\begin{array}{l}\text { colony } \\
\text { missing }\end{array}$ & missing colony \\
\hline & YLL032C & 8 & 14 & 19 & 24.94817 & 28.31858 & 72.82502 & colony & missing colony \\
\hline \multirow[t]{2}{*}{ GAP1 } & YKR039W & 8 & 14 & 20 & 18.9166 & 14.89104 & 18.69783 & 55.7072 & 61.28319 \\
\hline & YLL029W & 8 & 14 & 21 & -4.59393 & 25.92975 & 12.2905 & 14.67269 & 50.87719 \\
\hline CAF4 & YKR036C & 8 & 14 & 22 & 36.72161 & 34.40367 & 85.38462 & 18.48907 & 62.96296 \\
\hline TPO1 & YLL028W & 8 & 14 & 23 & 18.67058 & 43.93305 & 35.41033 & 12.32639 & 58.27338 \\
\hline HIS3 & YOR202W & 8 & 14 & 24 & 30.40238 & 24.50787 & 9.8 & & \\
\hline HIS3 & YOR202W & 8 & 15 & 1 & 47.48441 & 34.76466 & 85.71429 & & \\
\hline MSN4 & YKL062W & 8 & 15 & 2 & 25.62066 & 16.93841 & 29.29032 & 37.68595 & 55.43767 \\
\hline \multirow[t]{2}{*}{ KKQ8 } & YKL168C & 8 & 15 & 3 & 31.51198 & 23.55769 & 79.59698 & 49.52077 & 40.72948 \\
\hline & YKL061W & 8 & 15 & 4 & 60.5939 & 38.93376 & 18.10237 & 37.32639 & 40.37433 \\
\hline MRP49 & YKL167C & 8 & 15 & 5 & 22.23543 & 36.85259 & 42.87245 & 49.42308 & 11.11111 \\
\hline RBF18 & YKL056C & 8 & 15 & 6 & 4.56503 & 60.6524 & 60.26365 & 64.20582 & 54.61255 \\
\hline TPK3 & YKL166C & 8 & 15 & 7 & 14.16208 & 23.02569 & 19.7561 & 33.83838 & 34.24658 \\
\hline OAR1 & YKL055C & 8 & 15 & 8 & 15.89896 & -19.6375 & 13.62089 & 11.01695 & 26.72956 \\
\hline \multirow[t]{2}{*}{ PIR1 } & YKL164C & 8 & 15 & 9 & 13.52814 & 58.1058 & 6.40608 & 39.60396 & 28.88889 \\
\hline & YKL053W & 8 & 15 & 10 & -2.9035 & 16.4794 & 27.95699 & 44.81328 & 50.75758 \\
\hline PIR3 & YKL163W & 8 & 15 & 11 & 27.88226 & 40.98837 & 57.41325 & 23.00885 & 45.38835 \\
\hline \multirow[t]{2}{*}{ MDM35 } & YKL053C-A & 8 & 15 & 12 & 92.00195 & 34.87179 & 22.37001 & 28.60125 & 42.68775 \\
\hline & YKL162C & 8 & 15 & 13 & 9.753788 & 31.19891 & 38.36018 & 0.642398 & 47.34043 \\
\hline \multirow[t]{3}{*}{ SFK1 } & YKL051W & 8 & 15 & 14 & 10.47463 & -19.3591 & 69.83333 & 36.65224 & 55.75448 \\
\hline & YKL161C & 8 & 15 & 15 & 24.3079 & 18.44758 & 7.399794 & 57.20461 & 55.38058 \\
\hline & YKLO50C & 8 & 15 & 16 & 3.189793 & 38.58115 & 33.18872 & -7.43802 & 56.70103 \\
\hline ELF1 & YKL160W & 8 & 15 & 17 & 29.24694 & 42.01313 & 73.88003 & $\begin{array}{l}9.587379 \\
\text { missing }\end{array}$ & 63.89351 \\
\hline ELM1 & YKL048C & 8 & 15 & 18 & 4.98155 & 46.4752 & 12.16216 & colony & missing colony \\
\hline \multirow[t]{3}{*}{ RCN1 } & YKL159C & 8 & 15 & 19 & 21.98002 & 52.8914 & 58.15217 & 37.76882 & 70.10676 \\
\hline & YKL047W & 8 & 15 & 20 & 0.920598 & 19.50207 & 51.05348 & 32.80757 & 65.30172 \\
\hline & YKL158W & 8 & 15 & 21 & 24.3299 & 34.94737 & 70.04608 & 43.19728 & 60.43011 \\
\hline DCW1 & YKL046C & 8 & 15 & 22 & 7.771536 & 40.6953 & 63.09091 & 62.03822 & 59.65665 \\
\hline APE2 & YKL157W & 8 & 15 & 23 & 76.8559 & 49.13151 & 60.66482 & 18.21782 & 65.77778 \\
\hline HIS3 & YOR202W & 8 & 15 & 24 & 26.00596 & 37.01357 & 13.07902 & & \\
\hline HIS3 & YOR202W & 8 & 16 & 1 & 70.11086 & 34.34629 & 78.61771 & & \\
\hline
\end{tabular}




\begin{tabular}{|c|c|c|c|c|c|c|c|}
\hline HIS3 & YOR202W & 8 & 16 & 2 & 62.36374 & 26.64736 & 7.865169 \\
\hline HIS3 & YOR202W & 8 & 16 & 3 & 69.35791 & 0.207039 & 25.74032 \\
\hline HIS3 & YOR202W & 8 & 16 & 4 & 41.49346 & -13.8856 & 4.142012 \\
\hline HIS3 & YOR202W & 8 & 16 & 5 & -10.084 & 25.10823 & 17.55952 \\
\hline HIS3 & YOR202W & 8 & 16 & 6 & -23.8252 & 33.48924 & 8.165548 \\
\hline HIS3 & YOR202W & 8 & 16 & 7 & 48.23732 & 45.58522 & 34.57627 \\
\hline HIS3 & YOR202W & 8 & 16 & 8 & 6.210466 & -24.7536 & 10.93394 \\
\hline HIS3 & YOR202W & 8 & 16 & 9 & 16.10942 & -13.8095 & 48.69359 \\
\hline HIS3 & YOR202W & 8 & 16 & 10 & -31.1013 & 19.63071 & 25.63081 \\
\hline HIS3 & YOR202W & 8 & 16 & 11 & -43.3993 & -14.133 & 7.178841 \\
\hline HIS3 & YOR202W & 8 & 16 & 12 & -28.4436 & -6.27753 & 6.435644 \\
\hline HIS3 & YOR202W & 8 & 16 & 13 & 33.77097 & -24.2877 & -1.68831 \\
\hline HIS3 & YOR202W & 8 & 16 & 14 & -31.8773 & -29.8851 & 13.29268 \\
\hline HIS3 & YOR202W & 8 & 16 & 15 & -2.38322 & 45.42553 & -9.3985 \\
\hline HIS3 & YOR202W & 8 & 16 & 16 & 36.34497 & 22.26891 & \\
\hline HIS3 & YOR202W & 8 & 16 & 17 & 26.81818 & 26.34352 & 36.96172 \\
\hline HIS3 & YOR202W & 8 & 16 & 18 & -12.0879 & -8.71287 & 8.342023 \\
\hline HIS3 & YOR202W & 8 & 16 & 19 & -9.67742 & -22.1461 & 25.7868 \\
\hline HIS3 & YOR202W & 8 & 16 & 20 & 16.74107 & 51.71103 & -31.7821 \\
\hline HIS3 & YOR202W & 8 & 16 & 21 & 24.63585 & -1.36437 & 32.35083 \\
\hline HIS3 & YOR202W & 8 & 16 & 22 & 38.73659 & 41.72099 & -13.9535 \\
\hline HIS3 & YOR202W & 8 & 16 & 23 & 14.06366 & -15.4463 & 2.59901 \\
\hline HIS3 & YOR202W & 8 & 16 & 24 & 33.02064 & 52.20484 & 37.73585 \\
\hline HIS3 & YOR202W & 9 & 1 & 1 & 52.38817 & 64.99367 & 54.99455 \\
\hline HIS3 & YOR202W & 9 & 1 & 2 & 23.30097 & 22.46234 & 17.42066 \\
\hline HIS3 & YOR202W & 9 & 1 & 3 & 22.16288 & 19.57576 & 59.89884 \\
\hline HIS3 & YOR202W & 9 & 1 & 4 & 51.87713 & 18.16901 & 50.5278 \\
\hline HIS3 & YOR202W & 9 & 1 & 5 & 10.06061 & -9.49477 & 55.84843 \\
\hline HIS3 & YOR202W & 9 & 1 & 6 & 10.06897 & 23.3087 & 36.03708 \\
\hline HIS3 & YOR202W & 9 & 1 & 7 & -16.315 & 6.64 & 56.1326 \\
\hline HIS3 & YOR202W & 9 & 1 & 8 & 10.14686 & 23.15922 & 52.35205 \\
\hline HIS3 & YOR202W & 9 & 1 & 9 & 18.83803 & 26.30174 & 52.52387 \\
\hline HIS3 & YOR202W & 9 & 1 & 10 & 14.92329 & 13.67054 & 59.72851 \\
\hline HIS3 & YOR202W & 9 & 1 & 11 & 4.882813 & 11.66667 & 48.75149 \\
\hline HIS3 & YOR202W & 9 & 1 & 12 & 9.558824 & 30.15964 & 51.75439 \\
\hline HIS3 & YOR202W & 9 & 1 & 13 & 23.59249 & 14.45405 & 60.54913 \\
\hline HIS3 & YOR202W & 9 & 1 & 14 & 29.16667 & 9.160305 & 67.37531 \\
\hline HIS3 & YOR202W & 9 & 1 & 15 & 14.13238 & 12.85386 & 45.26485 \\
\hline HIS3 & YOR202W & 9 & 1 & 16 & 73.29412 & 13.33333 & 28.86029 \\
\hline HIS3 & YOR202W & 9 & 1 & 17 & 11.08462 & 11.36364 & 29.88314 \\
\hline HIS3 & YOR202W & 9 & 1 & 18 & 22.08238 & 23.14887 & 58.97981 \\
\hline HIS3 & YOR202W & 9 & 1 & 19 & 23.90572 & 12.73292 & 41.43095 \\
\hline HIS3 & YOR202W & 9 & 1 & 20 & 17.7305 & 15.12859 & 56.60881 \\
\hline
\end{tabular}




\begin{tabular}{|c|c|c|c|c|c|c|c|c|c|}
\hline HIS3 & YOR202W & 9 & 1 & 21 & 4.980843 & 9.070295 & 57.24534 & & \\
\hline HIS3 & YOR202W & 9 & 1 & 22 & 1.29199 & 5.584129 & 44.28384 & & \\
\hline HIS3 & YOR202W & 9 & 1 & 23 & 16.56977 & -48.6364 & 13.88102 & & \\
\hline HIS3 & YOR202W & 9 & 1 & 24 & 1.325758 & -16.0957 & 35.3431 & & \\
\hline \multirow[t]{2}{*}{ HIS3 } & YOR202W & 9 & 2 & 1 & 47.79412 & 31.81395 & 75.25711 & & \\
\hline & YLR217W & 9 & 2 & 2 & 12.85714 & 19.14305 & 40.85433 & 66.44205 & 41.96429 \\
\hline CHS5 & YLR330W & 9 & 2 & 3 & 6.165414 & 8.132045 & 40.55513 & 31.50074 & 38.62069 \\
\hline CPR6 & YLR216C & 9 & 2 & 4 & -26.2609 & 3.042434 & 21.70732 & -5.28541 & 35.8396 \\
\hline REC102 & YLR329W & 9 & 2 & 5 & 18.93939 & 22.92994 & 12.20779 & 39.0411 & 27.7937 \\
\hline FRE1 & YLR214W & 9 & 2 & 6 & 4.251386 & 29.61072 & 60.90573 & 12.99304 & 31.08614 \\
\hline NMA1 & YLR328W & 9 & 2 & 7 & 11.46384 & 87.01754 & 58.7517 & 15.03268 & 28.97727 \\
\hline CRR1 & YLR213C & 9 & 2 & 8 & 25.92105 & 15.37324 & 62.5 & -8.86918 & 22.27979 \\
\hline \multirow[t]{3}{*}{ RBF9 } & YLR327C & 9 & 2 & 9 & 8.501119 & 10.38168 & 67.13287 & 28.13688 & 19.04762 \\
\hline & YLR211C & 9 & 2 & 10 & 19.07984 & 21.36401 & 77.2846 & 58.76133 & 26.27551 \\
\hline & YLR326W & 9 & 2 & 11 & 15.46961 & 13.06576 & 67.00899 & 7.905983 & 18.76923 \\
\hline CLB4 & YLR210W & 9 & 2 & 12 & 86.41371 & 10.72531 & 73.02564 & 77.80749 & 30.56995 \\
\hline RPL38 & YLR325C & 9 & 2 & 13 & 19.58569 & 14.54948 & 1.866883 & 17.72152 & 23.30383 \\
\hline PNP1 & YLR209C & 9 & 2 & 14 & 36.56481 & 19.33086 & 17.41655 & 51.03858 & 22.51309 \\
\hline PEX30 & YLR324W & 9 & 2 & 15 & 29.87198 & 8.287293 & 4.296875 & 30.49793 & 21.14883 \\
\hline HRD3 & YLR207W & 9 & 2 & 16 & 29.97712 & 34.90054 & 12.5222 & -41.0112 & 19.375 \\
\hline MMS22 & YLR320W & 9 & 2 & 17 & 16.20172 & 8.013937 & 92.04545 & 18.12865 & 13.63636 \\
\hline ENT2 & YLR206W & 9 & 2 & 18 & 37.51387 & 27.99043 & 33.27645 & 32.98033 & 34.5679 \\
\hline BUD6 & YLR319C & 9 & 2 & 19 & 33.06727 & 0.6639 & 6.204136 & -22.1983 & 14.28571 \\
\hline HMX1 & YLR205C & 9 & 2 & 20 & 8.530806 & 3.291536 & 34.72949 & 58.51064 & 14.95601 \\
\hline EST2 & YLR318W & 9 & 2 & 21 & 7.894737 & 31.32716 & 31.71642 & 12.06497 & 6.642066 \\
\hline YKE2 & YLR200W & 9 & 2 & 22 & 7.64526 & -20.0803 & 6.935333 & 59.51904 & 34.83146 \\
\hline NKP2 & YLR315W & 9 & 2 & 23 & 31.74603 & 7.627765 & 63.68243 & 51.40962 & 32.52427 \\
\hline HIS3 & YOR202W & 9 & 2 & 24 & 2.202643 & 17.25626 & 1.605505 & & \\
\hline HIS3 & YOR202W & 9 & 3 & 1 & 18.66667 & 19.76967 & 70.97878 & & \\
\hline JLP1 & YLL057C & 9 & 3 & 2 & 19.31818 & 26.42694 & 41.27358 & 36.71875 & 32.82937 \\
\hline \multirow[t]{2}{*}{$\mathrm{CHA} 4$} & YLR098C & 9 & 3 & 3 & 29.11534 & 9.577677 & 37.47253 & 26.94497 & 32.87356 \\
\hline & YLL056C & 9 & 3 & 4 & 17.01571 & 16.55431 & 46.16667 & 48.93993 & 38.51852 \\
\hline \multirow[t]{2}{*}{ HRT3 } & YLR097C & 9 & 3 & 5 & 36.07595 & 12.65337 & 33.52941 & 74.49585 & 30.82902 \\
\hline & YLL055W & 9 & 3 & 6 & 12.53776 & 18.19699 & 26.47569 & 1.118568 & 33.25359 \\
\hline \multirow[t]{2}{*}{ KIN2 } & YLR096W & 9 & 3 & 7 & 21.41176 & 9.553641 & 29.02973 & 54.81833 & 30.86735 \\
\hline & YLL054C & 9 & 3 & 8 & \#DIV/0! & 13.43168 & 9.895833 & 19.80583 & 30.02545 \\
\hline \multirow[t]{2}{*}{ IOC2 } & YLR095C & 9 & 3 & 9 & 20.05731 & 22.49657 & -1.41612 & 6.418219 & 27.34584 \\
\hline & YLL053C & 9 & 3 & 10 & 14.88372 & 15.33695 & -5.57621 & 59.74441 & 12.97935 \\
\hline GIS3 & YLR094C & 9 & 3 & 11 & 2.359882 & 34.93078 & 3.981797 & 59.77809 & 28.8 \\
\hline AQY2 & YLL052C & 9 & 3 & 12 & 36.46532 & 15.20062 & 9.49094 & 14.22594 & 17.86834 \\
\hline NYV1 & YLR093C & 9 & 3 & 13 & 15.26163 & 7.986689 & 4.417671 & 18.20041 & 27.92553 \\
\hline FRE6 & YLL051C & 9 & 3 & 14 & 35.49696 & 15.77008 & -8.84718 & 30.76923 & 20.66116 \\
\hline SUL2 & YLR092W & 9 & 3 & 15 & 51.23711 & 37.29309 & 15.78947 & 49.62687 & 20.50473 \\
\hline
\end{tabular}


YBT1

XDJ1

ALTI

RNP1

CSF1

HIS3

HIS3

TOP3

EST1

RPL26A

GAS2

BNA5

FKS1

ECM22

SPO77

VRP1

RSA3

NUP2

CCC1

MSC3

RPS25B

MID2

HIS3

HIS3

LOT6

SSK1

HOG1
YLL049W

YLR091W

YLL048C

YLR090W

YLL047W

YLR089C

YLL046C

YLR087C

YOR202W

YOR202W

YLR234W

YLR345W

YLR233C

YLR344W

YLR232W

YLR343W

YLR231C

YLR342W

YLR228C

YLR341W

YLR225C

YLR338W

YLR224W

YLR337C

YLR221C

YLR335W

YLR220W

YLR334C

YLR219W

YLR333C

YLR218C

YLR332W

YOR202W

YOR202W

YLR011W

YLR114C

YLR006C

YLR113W

YLR004C

YLR112W

YLR003C

YLR111W
16

$17 \quad 43.30855$

5.877342

26.65765

28.24268

40.38232

$\begin{array}{ll}18 & 26.23377\end{array}$

25.79505

52.02232

$19 \quad 32.20974$

8.78553

15.89825

$20 \quad 11.31448$

22.11139

48.72727

$21 \quad 6.614786$

22.53743

40.32922

$22 \quad 15.60062$

1.28

19.58302

56.64452

$23 \quad 42.18062$

$\begin{array}{ll}24 & 19.48658\end{array}$

11.79856

22.19731

14.658385

2.736842

8.156499

$2-2.94906$

$3 \quad 22.22222$

16.10032

71.26168

$\begin{array}{ll}4 & 34.78261\end{array}$

14.24125

10.69723

12.92024

5.907173

14.28571

$\begin{array}{ll}5 & 6.027397\end{array}$

4.483283

20.27972

$\begin{array}{ll}6 & 7.929515\end{array}$

14.80111

61.15965

$\begin{array}{ll}7 & 31.49284\end{array}$

42.57261

21.09276

$\begin{array}{ll}8 & 18.01802\end{array}$

15.86314

4.737295

$9 \quad 23.71134$

$10 \quad 25.11521$

$10.42312-10.2477$

13.29787

15.25424

$11 \quad 27.68879$

10.8378

10.55156

$12 \quad 21.0101$

$13 \quad 34.44816$

11.11111

$-9.70378$

$6.547619 \quad 22.60343$

$14 \quad 57.14286$

$-15.2659-4.57256$

1544.61046

32.97872

100

$16 \quad 31.45161$

$\begin{array}{ll}5.245902 & 0.091408\end{array}$

$\begin{array}{ll}17 & 18.53211\end{array}$

12.06349

$-2.98507$

$\begin{array}{ll}18 & 31.29371\end{array}$

11.43594

0.181818

$\begin{array}{ll}19 & 25.63238\end{array}$

$20 \quad 21.53846$

$32.87327 \quad 65.59633$

$17.17842 \quad 46.48829$

$21 \quad 26.08696$

29.86301

39.92172

$22 \quad 11.30952$

25.58747

70.13889

$\begin{array}{ll}23 & 38.37072\end{array}$

3.72093

60.77266

$\begin{array}{ll}24 & 2.706767\end{array}$

$-14.1357$

47.9021

$1 \quad 27.87751$

$6.520247 \quad 47.19815$

242.71845

$19.85612 \quad 53.51598$

38.130081

\#DIV/0

\#DIV/0!

4 \#DIV/0!

14.735237 .258065

$\begin{array}{ll}5 & 41.17647\end{array}$

$62.82974 \quad 33.40909$

$\begin{array}{ll}6 & 0.611621\end{array}$

17.92319

46.68346

$\begin{array}{ll}7 & 16.13833\end{array}$

17.88756

8.255159

$\begin{array}{ll}8 & 9.605489\end{array}$

9.32971

28.30189

$9 \quad 17.51269$

17.25626
77.95527

49.10277

33.21234

40.68966

63.51351

40.31936

51.36876

45.36377

32.90043

34.4942

$-1.76991$

22.64151

26.69492

47.36842

13.25301

34.05276

57.50422

34.55882

30.75435

10.82024

63.97415

73.70968

64.70588

52.58765

69.05222

58.28221

63.20755

61.49533

9.144543

62.62626

65.34392

45.58559

29.59698

missing

colony

15.76087

14.12067

74.45483

53.46216
29.08587

17.12707

27.15736

24.82517

27.54342

41.95122

43.12977

42.10526

39.819

35.7631

16.42229

23.23944

26.96629

34.44109

15.89404

20.57878

14.95601

24.26901

25.3406

30.2521

20.91153

70.32641

10.14925

17.6781

25.14793

27.08934

22.8739

24.7093

10.66667

36.79458

36.27049

9.160305

47.98387

missing colony

51.11111

34.60317

25.94752

22.67442 


\begin{tabular}{|c|c|c|c|c|c|c|c|c|c|}
\hline & YLR001C & 9 & 5 & 10 & 14.64435 & 18.66667 & -2.51092 & 41.09312 & 33.78747 \\
\hline CCW12 & YLR110C & 9 & 5 & 11 & 31.71558 & 13.40206 & 9.586276 & -5.10441 & 31.22924 \\
\hline AYT1 & YLL063C & 9 & 5 & 12 & 73.08161 & 17.79359 & -1.69332 & 77.5837 & 22.34957 \\
\hline AHP1 & YLR109W & 9 & 5 & 13 & -3.57143 & 10.16548 & -2.94643 & 29.41176 & 20.21661 \\
\hline \multirow[t]{2}{*}{ MHT1 } & YLL062C & 9 & 5 & 14 & 35.04983 & 19.17326 & 9.276249 & 63.76812 & 20.4611 \\
\hline & YLR108C & 9 & 5 & 15 & 13.18052 & 12.75338 & 2.085506 & 14.59227 & 10.54131 \\
\hline MMP1 & YLL061W & 9 & 5 & 16 & 31.48426 & 5.494505 & 16.14035 & 54.69613 & 5.357143 \\
\hline REX3 & YLR107W & 9 & 5 & 17 & 15.88089 & 43.06473 & 17.80673 & 31.76044 & 27.60736 \\
\hline \multirow[t]{3}{*}{ GTT2 } & YLL060C & 9 & 5 & 18 & 27.34375 & 25.50282 & 5.566219 & 56.68359 & 23.45013 \\
\hline & YLR104W & 9 & 5 & 19 & -0.96774 & 15.54404 & 24.40678 & 37.24395 & 14.89362 \\
\hline & YLL059C & 9 & 5 & 20 & 23.68421 & 21.38728 & 41.70213 & 41.15646 & 19.5122 \\
\hline \multirow[t]{2}{*}{ APC9 } & YLR102C & 9 & 5 & 21 & 9.090909 & 16.7847 & 41.49909 & 55.9792 & 26.34409 \\
\hline & YLL058W & 9 & 5 & 22 & 29.92611 & 100 & 13.33333 & 20.68966 & 27.7027 \\
\hline ICT1 & YLR099C & 9 & 5 & 23 & 16.23489 & 34.76563 & 16.01423 & 74.45255 & 27.16049 \\
\hline HIS3 & YOR202W & 9 & 5 & 24 & 5.490849 & 12.09677 & 1.814647 & & \\
\hline HIS3 & YOR202W & 9 & 6 & 1 & 44.31818 & 13.92663 & 1.526286 & & \\
\hline SYM1 & YLR251W & 9 & 6 & 2 & 29.73621 & 100 & 52.02156 & 60.74499 & 42.55319 \\
\hline VPS38 & YLR360W & 9 & 6 & 3 & -3.65297 & 15.45584 & 31.80016 & -1.68067 & 6.921241 \\
\hline SSP120 & YLR250W & 9 & 6 & 4 & 14.98471 & 6.70304 & 6.125971 & $\begin{array}{l}53.29513 \\
\text { missing }\end{array}$ & 22.01835 \\
\hline $\mathrm{RSC2}$ & YLR357W & 9 & 6 & 5 & 25.55831 & 47.4359 & 5.555556 & colony & missing colony \\
\hline \multirow[t]{3}{*}{ RCK2 } & YLR248W & 9 & 6 & 6 & 11.89591 & 10.37376 & -3.02782 & 48.13754 & 35.94937 \\
\hline & YLR356W & 9 & 6 & 7 & 18.59558 & 19.07216 & 20.50164 & 41.71779 & 22.42991 \\
\hline & YLR247C & 9 & 6 & 8 & 23.2288 & 9.053156 & 4.340124 & 67.46627 & 29.9169 \\
\hline TAL1 & YLR354C & 9 & 6 & 9 & 20.41522 & 7.794362 & 9.419152 & 75.03876 & 19.70588 \\
\hline ERF2 & YLR246W & 9 & 6 & 10 & 13.30472 & 6.260032 & 4.914005 & 54.92958 & 17.97101 \\
\hline BUD8 & YLR353W & 9 & 6 & 11 & 27.39018 & -4.26439 & 2.58427 & 43.86282 & 36.33803 \\
\hline \multirow[t]{3}{*}{ ARV1 } & YLR242C & 9 & 6 & 12 & 28.99329 & 8.285714 & 10.8871 & 15.67291 & 17.23164 \\
\hline & YLR352W & 9 & 6 & 13 & 27.98595 & 8.092486 & 9.134158 & 51.09612 & 13.35616 \\
\hline & YLR241W & 9 & 6 & 14 & 13.49036 & -6.12423 & 1.530153 & 77.64165 & 31.56342 \\
\hline NIT3 & YLR351C & 9 & 6 & 15 & 34.11079 & 6.660666 & -3.94432 & 44.92255 & 11.78248 \\
\hline LIP2 & YLR239C & 9 & 6 & 16 & 34.67656 & 16.87631 & 20.4431 & 38.11659 & 9.584665 \\
\hline ORM2 & YLR350W & 9 & 6 & 17 & 23.07692 & 5.981595 & 2.053571 & 68.57585 & 24.50704 \\
\hline \multirow[t]{3}{*}{ THI7 } & YLR237W & 9 & 6 & 18 & 37.64581 & 88.79552 & 13.39174 & 74.60317 & 31.07345 \\
\hline & YLR349W & 9 & 6 & 19 & 38.82353 & 9.318378 & 13.07359 & 77.79553 & 23.03207 \\
\hline & YLR236C & 9 & 6 & 20 & 31.80662 & 10.40268 & 0 & 51.44804 & 29.56522 \\
\hline \multirow[t]{3}{*}{ DIC1 } & YLR348C & 9 & 6 & 21 & 14.73214 & -33.3692 & 57.24907 & 41.47727 & 28.35366 \\
\hline & YLR235C & 9 & 6 & 22 & 29.27928 & 15.31612 & 27.13936 & 49.46921 & 30.79268 \\
\hline & YLR346C & 9 & 6 & 23 & 34.07821 & 5.970149 & 63.14399 & 78.8712 & 36.61327 \\
\hline HIS3 & YOR202W & 9 & 6 & 24 & 5.639615 & 9.81241 & 38.3915 & & \\
\hline HIS3 & YOR202W & 9 & 7 & 1 & 17.31025 & 15.58785 & 13.74309 & & \\
\hline $\mathrm{IZH} 3$ & YLR023C & 9 & 7 & 2 & 11.28049 & 10.53795 & 44.99205 & 22.67081 & 35.45455 \\
\hline ZRT2 & YLR130C & 9 & 7 & 3 & 25.45455 & 11.52959 & 26.771 & 72.79006 & 36.71875 \\
\hline
\end{tabular}


DCN1

YLR021W

YEH3

PSR2

POM34

MEU1

BRE2

YPS3

PPR1

YPS1

GAT3

SRN2

HIS3

HIS3

RPS28B

SUR4

RED1

ROM2

RBF7

ARC18

YPT6

MDM30

RPS22B

GSY2

NDL1 NMD4
YLR128W

YLRO20C

YLR126C

YLR019W

YLR125W

YLR018C

YLR124W

YLR017W

YLR123C

YLR016C

YLR122C

YLR015W

YLR121C

YLR014C

YLR120C

YLR013W

YLR119W

YLR012C

YLR118C

YOR202W

YOR202W

YLR264W

YLR372W

YLR263W

YLR371W

YLR262C-A

YLR370C

YLR262C

YLR368W

YLR261C

YLR367W

YLR258W

YLR366W

YLR257W

YLR365W

YLR255C

YLR364W

YLR254C

YLR363C $\begin{array}{llll}4 & 27.37643 & 27.82194 & 30.03731\end{array}$

$\begin{array}{lllll}5 & 19.25466 & 16.27146 & 10.04785\end{array}$

$\begin{array}{llll}6 & 30.4878 & 2.33564 & 3.866906\end{array}$

$\begin{array}{llll}7 & 25.30488 & 14.65969 & 10.17102\end{array}$

$\begin{array}{lllll}8 & 29.38719 & 4.888152 & -0.55504\end{array}$

$\begin{array}{lllll}9 & 39.10733 & 7.620818 & 17.47285\end{array}$

$10 \quad 43.02214$

6.267806

4.837117

$\begin{array}{ll}11 & 25.98425 \\ 11 & 1\end{array}$

11.80077

0.454133

$\begin{array}{ll}12 & 74.86188\end{array}$

26.0355

14.49631

$\begin{array}{ll}13 & 30.37037\end{array}$

15.75203

8.275174

$14 \quad 35.0365$

0.831025

$-3.00107$

$\begin{array}{lll}15 & 4.201681 \\ 14 & 0\end{array}$

6.573276

11.66837

$\begin{array}{ll}16 & 39.60827 \\ 11 & 2\end{array}$

29.15567

4.516129

$\begin{array}{ll}17 & 11.91919\end{array}$

14.17134

3.127793

27.657

7.610475

5.31401

$19 \quad 24.03382$

$\begin{array}{lll}5.663567 & 7.870754\end{array}$

$20 \quad 22.69129$

11.35802

46.39279

$\begin{array}{ll}21 & 17.55618\end{array}$

18.47826

32.80423

$\begin{array}{ll}22 & 3.472222\end{array}$

$23 \quad 2.578797$

$-51.5015 \quad 37.94643$

$\begin{array}{ll}24 & 4.411765\end{array}$

9.974425

119.31624

26.77761

0.073099

2 -13.0841

36.99907

22.8739

13.14414

$4 \quad 60.51136$

$5 \quad 30.83333$

16.20746

631.19266

$18.39465 \quad 25.65789$

$7 \quad 32.2132$

$\begin{array}{ll}7.077856 & 0.913706\end{array}$

$\begin{array}{ll}8 & 22.63158\end{array}$

9.770115

15.26823

$\begin{array}{ll}9 & 27.88586\end{array}$

$\begin{array}{ll}-3.00752 & 6.073567\end{array}$

$10 \quad 8.179959$

13.51792

14.41125

$\begin{array}{ll}11 & 17.40088\end{array}$

2.539683

10.89682

$12 \quad 35.16854$

11.59901

13.86861

$\begin{array}{ll}13 & 5.652174\end{array}$

10.4503

5.57554

$14 \quad 11.2069$

5.762082

$-0.10406$

15

7.58928

$-2.40856$

$-8.66602$

$\begin{array}{llll}16 & 30.82353 & 24.84663 & 24.31791\end{array}$

$\begin{array}{lllll}17 & 27.94293 & 22.59721 & 3.977798\end{array}$

18

19
77.67988

missing

colony

75.02945

55.75221

47.65101

62.47723

80.63439

77.33918

66.23134

73.32293

27.62951

33.97028

42.78846

33.33333

67.79935

44.4

43.7037

69.48905

38.88889

26.46104

49.19499

53.64372

39.31777

62.2973

missing

colony missing

colony

48.97959

65.48536

69.69697

42.18415

64.54006

42.83154

67.1916

69.96381

55.13748

63.38798

75.16556

65.89506
49.57983

missing colony

39.72286

29.41176

28.44037

22.89562

32.06349

31.10465

26.71233

25.55205

19.25287

22.76657

11.20944

29.39633

22.22222

22.95597

24.63343

22.58953

17.06587

28.35052

36.84211

37.00306

37.53425

61.42857

missing colony

missing colony

35.90909

33.04094

37.54153

25.65789

31.46067

15.74074

32.36842

26.37076

29.41176

10.3125

22.7882

29.36288 


\begin{tabular}{|c|c|c|c|c|c|c|c|c|c|}
\hline & YLR253W & 9 & 8 & 20 & -9.44351 & 8.706468 & 45.08547 & -34.3902 & 24.50142 \\
\hline \multirow[t]{2}{*}{ STE11 } & YLR362W & 9 & 8 & 21 & 33.19328 & 14.30868 & 48.65526 & 49.03846 & 6.603774 \\
\hline & YLR252W & 9 & 8 & 22 & 15.35948 & 30.06834 & 20.24648 & 35.14938 & 32.81654 \\
\hline DCR2 & YLR361C & 9 & 8 & 23 & 33.73626 & 14.97396 & 67.32588 & 63.30014 & 32.43243 \\
\hline HIS3 & YOR202W & 9 & 8 & 24 & 7.972028 & 2.7146 & 29.09091 & & \\
\hline HIS3 & YOR202W & 9 & 9 & 1 & 16.66667 & 14.69799 & 2.094241 & & \\
\hline RIC1 & YLR039C & 9 & 9 & 2 & 10.59603 & 24.25665 & 30.26316 & 43.87001 & 49.7549 \\
\hline SPE4 & YLR146C & 9 & 9 & 3 & 0 & 28.59885 & 50 & 63.03237 & 37.53149 \\
\hline $\operatorname{cox} 12$ & YLR038C & 9 & 9 & 4 & 13.42857 & 27.50716 & 30.59937 & 9.897611 & 5.154639 \\
\hline ACF2 & YLR144C & 9 & 9 & 5 & 14.42308 & 8.624535 & 15.10574 & $\begin{array}{l}44.29224 \\
\text { missing }\end{array}$ & 52.27964 \\
\hline \multirow[t]{3}{*}{ DAN2 } & YLR037C & 9 & 9 & 6 & 11.72249 & -2.7439 & 21.57795 & $\begin{array}{l}\text { colony } \\
\text { missing }\end{array}$ & missing colony \\
\hline & YLR143W & 9 & 9 & 7 & 22.68657 & 1.571092 & -5.62448 & colony & missing colony \\
\hline & YLR036C & 9 & 9 & 8 & -3.65854 & -0.59423 & 1.507937 & 47.61905 & 41.83381 \\
\hline PUT1 & YLR142W & 9 & 9 & 9 & 24.69775 & -4.004 & 39.83531 & 85.31469 & 58.04416 \\
\hline MLH2 & YLR035C & 9 & 9 & 10 & 16.03053 & 20.79439 & 2.021277 & 46.34146 & 45.92834 \\
\hline NHA1 & YLR138W & 9 & 9 & 11 & 59.61538 & 13.15519 & 6.536585 & 36.09272 & 20.42553 \\
\hline \multirow[t]{2}{*}{ SMF3 } & YLR034C & 9 & 9 & 12 & 24.27822 & 7.347876 & 7.045216 & 79.23729 & 37.53425 \\
\hline & YLR137W & 9 & 9 & 13 & 13.85768 & -4.94728 & 13.08204 & $\begin{array}{l}34.23567 \\
\text { missing }\end{array}$ & 22.94618 \\
\hline RAD5 & YLR032W & 9 & 9 & 14 & 2.728227 & 18.89503 & 1.666667 & $\begin{array}{l}\text { colony } \\
\text { missing }\end{array}$ & missing colony \\
\hline \multirow[t]{2}{*}{ TIS11 } & YLR136C & 9 & 9 & 15 & 28.45395 & 10.56388 & 0.500417 & $\begin{array}{l}\text { colony } \\
\text { missing }\end{array}$ & missing colony \\
\hline & YLR031W & 9 & 9 & 16 & -4.36508 & 6.523765 & 8.384279 & colony & missing colony \\
\hline \multirow[t]{2}{*}{ SLX4 } & YLR135W & 9 & 9 & 17 & 33.53151 & 10.0894 & 2.37849 & 57.54339 & 25.13228 \\
\hline & YLR030W & 9 & 9 & 18 & 9.809264 & -2.41187 & 9.88075 & 67.44186 & 32.62032 \\
\hline PDC5 & YLR134W & 9 & 9 & 19 & 24.45255 & 0 & 4.524887 & 53.55191 & 26.74419 \\
\hline ADE16 & YLR028C & 9 & 9 & 20 & 27.63006 & -2.92842 & 7.314974 & 19.79167 & 35.22388 \\
\hline CKI1 & YLR133W & 9 & 9 & 21 & 100 & -0.18553 & 23.17757 & 19.96047 & 14.77987 \\
\hline UBR2 & YLR024C & 9 & 9 & 22 & 26.04712 & -0.39761 & 15.89825 & 27.25118 & 39.62848 \\
\hline ACE2 & YLR131C & 9 & 9 & 23 & 49.57746 & 9.903792 & 15.47619 & -1.68634 & 50.59382 \\
\hline HIS3 & YOR202W & 9 & 9 & 24 & 9.036145 & 18.32179 & 40.33058 & & \\
\hline \multirow[t]{2}{*}{ HIS3 } & YOR202W & 9 & 10 & 1 & 5.718954 & 11.88811 & 23.16968 & & \\
\hline & YLR281C & 9 & 10 & 2 & 12.90323 & 7.878315 & 38.94118 & 53.60624 & 34.13978 \\
\hline \multirow[t]{2}{*}{ REH1 } & YLR387C & 9 & 10 & 3 & 12.8169 & 14.17966 & 42.42424 & 57.56522 & 38.44086 \\
\hline & YLR280C & 9 & 10 & 4 & 15.51095 & 16.61238 & 31.77648 & 57.19008 & 42.04204 \\
\hline \multirow[t]{2}{*}{ VAC14 } & YLR386W & 9 & 10 & 5 & 13.60544 & 22.17742 & 18.43778 & 55.07246 & 22.06304 \\
\hline & YLR279W & 9 & 10 & 6 & 21.9917 & 7.723036 & 6.896552 & 38.92734 & 45.78313 \\
\hline \multirow[t]{2}{*}{ SWC7 } & YLR385C & 9 & 10 & 7 & 15.47779 & 8.333333 & 0.290698 & 49.08836 & 32.05882 \\
\hline & YLR278C & 9 & 10 & 8 & 52.08333 & 0.905797 & 10.44235 & 68.08199 & 40.75145 \\
\hline IKI3 & YLR384C & 9 & 10 & 9 & 12.32227 & 100 & \#DIV/0! & 49.79839 & 22.02166 \\
\hline PIG1 & YLR273C & 9 & 10 & 10 & 19.2 & 14.49735 & 6.740614 & 66.46586 & 33.33333 \\
\hline
\end{tabular}




\begin{tabular}{|c|c|c|c|c|c|c|c|c|c|}
\hline \multirow[t]{2}{*}{ CTF3 } & YLR381W & 9 & 10 & 11 & 25.82524 & 2.777778 & 5.709625 & 49 & 30.21148 \\
\hline & YLR271W & 9 & 10 & 12 & 40.73252 & 13.6255 & 3.294893 & 58.26514 & 40.99379 \\
\hline \multirow[t]{2}{*}{ CSR1 } & YLR380W & 9 & 10 & 13 & 18.75 & 4.894578 & 3.849115 & 42.90598 & 44.11028 \\
\hline & YLR269C & 9 & 10 & 14 & 28.14238 & 48.92086 & 43.13043 & $\begin{array}{l}\text { missing } \\
\text { colony } \\
\text { missing }\end{array}$ & missing colony \\
\hline FBP1 & YLR377C & 9 & 10 & 15 & 33.08176 & 9.561129 & 15.34351 & $\begin{array}{l}\text { colony } \\
\text { missing }\end{array}$ & missing colony \\
\hline SEC22 & YLR268W & 9 & 10 & 16 & 5.555556 & 0.901713 & 11.45564 & colony & missing colony \\
\hline PSY3 & YLR376C & 9 & 10 & 17 & 33.96825 & 2.684564 & 8.976661 & 27.65957 & 25.40984 \\
\hline BOP2 & YLR267W & 9 & 10 & 18 & 33.11258 & 15.4519 & 0.492126 & 51.52979 & 29.80501 \\
\hline STP3 & YLR375W & 9 & 10 & 19 & 32.86385 & 4.780564 & 3.504482 & 52.52708 & 21.22186 \\
\hline \multirow[t]{2}{*}{ PDR8 } & YLR266C & 9 & 10 & 20 & 34.50882 & 7.517084 & 6.781193 & 76.50696 & 33.50254 \\
\hline & YLR374C & 9 & 10 & 21 & 20.11834 & 14.00438 & 41.30106 & 45.6 & 13.04348 \\
\hline NEJ1 & YLR265C & 9 & 10 & 22 & 18.09816 & 31.11511 & 13.60137 & 56.19048 & 20.17544 \\
\hline VID22 & YLR373C & 9 & 10 & 23 & 26.42487 & 31.63265 & 18.88298 & 8.838384 & 28.4153 \\
\hline HIS3 & YOR202W & 9 & 10 & 24 & 15.52419 & 18.22064 & 41.59445 & & \\
\hline \multirow[t]{4}{*}{ HIS3 } & YOR202W & 9 & 11 & 1 & 29.95595 & 3.761062 & 19.33702 & & \\
\hline & YLR053C & 9 & 11 & 2 & 10.51753 & 22.67574 & 6.300813 & 58.46599 & 50.88106 \\
\hline & YLR171W & 9 & 11 & 3 & 33.20755 & 12.79492 & 3.519313 & 47.65478 & 36.57143 \\
\hline & YLR050C & 9 & 11 & 4 & 31.23167 & 20.20997 & 22.00903 & 61.14551 & 51.17647 \\
\hline \multirow[t]{3}{*}{ APS1 } & YLR170C & 9 & 11 & 5 & 27.11111 & 21.80952 & 16.95473 & 69.93243 & 55.55556 \\
\hline & YLR049C & 9 & 11 & 6 & 0.718133 & 2.742616 & 0.81448 & 68.37456 & 34.91124 \\
\hline & YLR169W & 9 & 11 & 7 & 17.44966 & 13.05326 & 15.09769 & 34.02256 & 47.89916 \\
\hline \multirow[t]{3}{*}{ RPSOB } & YLR048W & 9 & 11 & 8 & 15.27778 & 1.18505 & 9.044369 & 60.39823 & 36.14458 \\
\hline & YLR168C & 9 & 11 & 9 & 20.91503 & 40.66074 & 24.47307 & 53.71622 & 22.14765 \\
\hline & YLR047C & 9 & 11 & 10 & 50 & 5.482815 & 1.486698 & 94.31818 & 40.16064 \\
\hline \multirow[t]{3}{*}{ PUS5 } & YLR165C & 9 & 11 & 11 & 44.98747 & 29.77941 & 37.98283 & 50.16502 & 36.97479 \\
\hline & YLR046C & 9 & 11 & 12 & 31.98324 & 1.426025 & 12.05393 & 64.84375 & 37.06897 \\
\hline & YLR164W & 9 & 11 & 13 & 36.50075 & 7.916182 & 11.45176 & 17.87521 & 19.94135 \\
\hline PDC1 & YLR044C & 9 & 11 & 14 & 0 & 20.23692 & 19.45813 & 7.738095 & 35.52239 \\
\hline $\mathrm{RNH} 203$ & YLR154C & 9 & 11 & 15 & 6.578947 & 8.316832 & 7.061901 & 71.59763 & 33.76289 \\
\hline \multirow[t]{3}{*}{ TRX1 } & YLR043C & 9 & 11 & 16 & 27.23147 & 42.36311 & 28.09365 & 45.78512 & 40.625 \\
\hline & YLR152C & 9 & 11 & 17 & 18.13187 & 4.377432 & 6.433678 & 73.35165 & 26.23377 \\
\hline & YLR042C & 9 & 11 & 18 & 43.29787 & 14.21421 & 11.72353 & 13.58025 & 31.87135 \\
\hline \multirow[t]{2}{*}{ PCD1 } & YLR151C & 9 & 11 & 19 & 8.561644 & 2.129719 & 11.68724 & 70.83333 & 33.87978 \\
\hline & YLR041W & 9 & 11 & 20 & 69.48424 & 13.04348 & 7.334109 & 39.61606 & 12.03438 \\
\hline \multirow[t]{3}{*}{ STM1 } & YLR150W & 9 & 11 & 21 & 98.39357 & 13.52236 & 40.47244 & 58.9198 & 32.52688 \\
\hline & YLR040C & 9 & 11 & 22 & 16.43836 & 14.43662 & 33.23442 & 29.18919 & 25.49575 \\
\hline & YLR149C & 9 & 11 & 23 & \#DIV/0! & 18.02326 & 5.547337 & 29.69984 & 34.90991 \\
\hline HIS3 & YOR202W & 9 & 11 & 24 & 4.957265 & 47.59259 & 29.93103 & & \\
\hline \multirow[t]{2}{*}{ HIS3 } & YOR202W & 9 & 12 & 1 & 31.26551 & 12.19681 & 43.31897 & & \\
\hline & YLR294C & 9 & 12 & 2 & 12.47863 & 20.28436 & 26.26064 & 23.43434 & 42.82353 \\
\hline
\end{tabular}




\begin{tabular}{|c|c|c|c|c|c|c|c|c|c|}
\hline & YLR400W & 9 & 12 & 3 & -3.07692 & 14.23948 & 22.36842 & 67.29131 & 54.65116 \\
\hline SEC72 & YLR292C & 9 & 12 & 4 & 58.52783 & 31.42857 & 27.30435 & 13.51889 & 25.625 \\
\hline \multirow[t]{2}{*}{ SKI2 } & YLR398C & 9 & 12 & 5 & 52.65018 & 3.423237 & 21.74281 & 66.73077 & 62.41379 \\
\hline & YLR290C & 9 & 12 & 6 & 21.98492 & 31.74274 & 7.329843 & 50.61296 & 40.99379 \\
\hline cox8 & YLR395C & 9 & 12 & 7 & 33.33333 & -4.82823 & 9.725316 & 23.19149 & 44.8505 \\
\hline GUF1 & YLR289W & 9 & 12 & 8 & 4.580153 & 29.11796 & 8.115419 & 64.54352 & 54.34783 \\
\hline CST9 & YLR394W & 9 & 12 & 9 & 41.27907 & 35.2861 & 28.30189 & 75.9375 & 56.66667 \\
\hline RPS30A & YLR287C-A & 9 & 12 & 10 & 23.61111 & 13.95706 & 5.367965 & 63.7149 & 40.13841 \\
\hline \multirow[t]{3}{*}{ ATP10 } & YLR393W & 9 & 12 & 11 & 22.43976 & 39.50233 & 13.81156 & 64.99133 & 42.48366 \\
\hline & YLR287C & 9 & 12 & 12 & 30.22636 & 4.90566 & 5.667702 & 48.82459 & 50.42017 \\
\hline & YLR392C & 9 & 12 & 13 & 5.604203 & 4.147465 & 0.443262 & 58.85714 & 30.44693 \\
\hline \multirow[t]{2}{*}{ CTS1 } & YLR286C & 9 & 12 & 14 & 19.8324 & 12.6447 & 5.75 & 40.78212 & 32.0802 \\
\hline & YLR391W & 9 & 12 & 15 & 35.41018 & 7.149404 & 13.69969 & 60.43046 & 46.00551 \\
\hline NNT1 & $\begin{array}{l}\text { YLR285W } \\
\text { YLR390W- }\end{array}$ & 9 & 12 & 16 & 7.5 & 10.88546 & -2.87121 & 47.42424 & 28.18428 \\
\hline CCW14 & $A$ & 9 & 12 & 17 & 33.02217 & 15.55352 & 31.32743 & 48.39319 & 35.52239 \\
\hline $\mathrm{ECl} 1$ & YLR284C & 9 & 12 & 18 & 33.11432 & 7.592593 & -7.32797 & 27.91878 & 21.00592 \\
\hline \multirow[t]{2}{*}{ ECM19 } & YLR390W & 9 & 12 & 19 & 35.06494 & 9.082969 & -4.5933 & 34.52915 & 27.54098 \\
\hline & YLR283W & 9 & 12 & 20 & 26.93997 & 17.52848 & 2.740864 & 65.99379 & 33.5958 \\
\hline \multirow[t]{2}{*}{ STE23 } & YLR389C & 9 & 12 & 21 & 30.18373 & 1.574803 & 5.060729 & 76.24521 & 38.83162 \\
\hline & YLR282C & 9 & 12 & 22 & 83.66935 & 12.21374 & 5.711987 & -0.89087 & 52.72727 \\
\hline RPS29A & YLR388W & 9 & 12 & 23 & 38.81335 & 40.25045 & 23.40426 & -1.2474 & 29.27461 \\
\hline HIS3 & YOR202W & 9 & 12 & 24 & 34.16667 & 15.44901 & 37.20238 & & \\
\hline \multirow[t]{2}{*}{ HIS3 } & YOR202W & 9 & 13 & 1 & 21.42857 & 12.97641 & 6.813627 & & \\
\hline & YLR065C & 9 & 13 & 2 & 27.33447 & 3.516174 & 48.42767 & 29.25532 & 43.09524 \\
\hline \multirow[t]{2}{*}{ TOS4 } & YLR183C & 9 & 13 & 3 & 43.69973 & 27.67296 & 15.96821 & 59.17722 & 54.81928 \\
\hline & YLR064W & 9 & 13 & 4 & 14.28571 & 10.13728 & 18.74491 & 41.58215 & 51.52355 \\
\hline \multirow[t]{2}{*}{ SWI6 } & YLR182W & 9 & 13 & 5 & 10.6383 & 8.298755 & 14.48598 & 49.01186 & 40.11976 \\
\hline & YLR063W & 9 & 13 & 6 & 47.10145 & 32.66055 & 2.658228 & 50.58005 & 63.80597 \\
\hline VTA1 & YLR181C & 9 & 13 & 7 & 9.51586 & 49.5122 & 42.35075 & 40.38462 & 46.01227 \\
\hline BUD28 & YLR062C & 9 & 13 & 8 & 19.91215 & 17.81377 & -0.67969 & 34.57031 & 32.97872 \\
\hline SAM1 & YLR180W & 9 & 13 & 9 & 2.857143 & 26.94805 & -8.58209 & 30.9434 & 46.62162 \\
\hline \multirow[t]{2}{*}{ RPL22A } & YLR061W & 9 & 13 & 10 & 10.80196 & 100 & 89.04762 & 52.66667 & 38.83929 \\
\hline & YLR179C & 9 & 13 & 11 & 28.37838 & -1.00853 & -0.55249 & 55.16605 & 31.3099 \\
\hline REX2 & YLR059C & 9 & 13 & 12 & 28.65412 & 42.68943 & 10.28037 & 63 & 43.84858 \\
\hline TFS1 & YLR178C & 9 & 13 & 13 & 35.05155 & 10.19173 & -7.31707 & 77.44946 & 48.96755 \\
\hline \multirow[t]{3}{*}{ SHM2 } & YLR058C & 9 & 13 & 14 & 68.38535 & 52.99807 & -5.33333 & 40.30837 & 42.76923 \\
\hline & YLR177W & 9 & 13 & 15 & 24.86911 & 17.02128 & 30.66202 & -0.33058 & 7.788162 \\
\hline & YLR057W & 9 & 13 & 16 & 22.14286 & 28.27586 & -2.63381 & 65.4321 & 27.98834 \\
\hline RFX1 & YLR176C & 9 & 13 & 17 & 38.46154 & 11.71171 & 9.413708 & 57.19921 & 24.41472 \\
\hline ERG3 & YLR056W & 9 & 13 & 18 & 27.6131 & 92.73743 & 38.20225 & 32.76836 & 36.12167 \\
\hline IDP2 & YLR174W & 9 & 13 & 19 & 35.8047 & 91.15308 & -0.67179 & 28.157 & 35.73201 \\
\hline SPT8 & YLR055C & 9 & 13 & 20 & 24.53988 & 46.17737 & 4.195804 & 11.07872 & 19.34307 \\
\hline
\end{tabular}




\begin{tabular}{|c|c|c|c|c|c|c|c|c|c|}
\hline & YLR173W & 9 & 13 & 21 & 42.59472 & 23.91858 & 0.669643 & -3.21782 & 22.54902 \\
\hline OSW2 & YLR054C & 9 & 13 & 22 & 53.16239 & 42.3676 & -6.4114 & 58.22981 & 37.59398 \\
\hline DPH5 & YLR172C & 9 & 13 & 23 & 39.7878 & 4.316547 & -11.6129 & 66.0626 & 39.03614 \\
\hline HIS3 & YOR202W & 9 & 13 & 24 & 44.26752 & 26.37704 & 40.98361 & & \\
\hline HIS3 & YOR202W & 9 & 14 & 1 & 44.0367 & 13.81818 & 3.224013 & & \\
\hline \multirow[t]{6}{*}{ SPH1 } & YLR313C & 9 & 14 & 2 & 2.2222222 & 10.62335 & 47.42857 & 44.64945 & 43.20388 \\
\hline & YLR414C & 9 & 14 & 3 & 11.05263 & 100 & 9.6 & 47.67932 & 44.19355 \\
\hline & YLR312C & 9 & 14 & 4 & 16.37931 & 5.602241 & -3.53753 & 37.2549 & 51.14007 \\
\hline & YLR413W & 9 & 14 & 5 & 17.77509 & 7.831901 & 4.607509 & 45.11278 & 36.9281 \\
\hline & YLR311C & 9 & 14 & 6 & 6.263499 & 16.54198 & 8.77193 & 48.00664 & 52.52809 \\
\hline & YLR412W & 9 & 14 & 7 & 19.32773 & 8.779443 & 1.963865 & 40.37736 & 28.4345 \\
\hline $\mathrm{IMH1}$ & YLR309C & 9 & 14 & 8 & 10 & 17.82946 & 29.47658 & 45.25547 & 35.64669 \\
\hline VIP1 & YLR410W & 9 & 14 & 9 & -10.846 & 12.77778 & 55.58252 & 74.29467 & 57.26496 \\
\hline \multirow[t]{2}{*}{ CDA1 } & YLR307W & 9 & 14 & 10 & 2.712477 & 26.04651 & 2.887139 & 57.11864 & 42.29607 \\
\hline & YLR408C & 9 & 14 & 11 & 7.092199 & 18.15154 & 13.48228 & 43.19328 & 38.55072 \\
\hline \multirow[t]{2}{*}{ UBC12 } & YLR306W & 9 & 14 & 12 & 27.33119 & 6.004619 & 9.69697 & 46.12676 & 48.05556 \\
\hline & YLR407W & 9 & 14 & 13 & 23.09859 & 9.63481 & 17.97337 & 30.81285 & 32.71605 \\
\hline MET17 & YLR303W & 9 & 14 & 14 & 31.37255 & 16.80851 & 14.862 & 47.23618 & 53.40599 \\
\hline RPL31B & YLR406C & 9 & 14 & 15 & 21.90942 & 12.65823 & 34.00335 & 51.22549 & 43.21767 \\
\hline EXG1 & YLR300W & 9 & 14 & 16 & 55.80229 & 23.17518 & 3.111111 & 71.32018 & 47.26027 \\
\hline DUS4 & YLR405W & 9 & 14 & 17 & 15.61997 & 13.95349 & 2.603664 & 68.43854 & 36.29032 \\
\hline \multirow[t]{5}{*}{ ECM38 } & YLR299W & 9 & 14 & 18 & 26.25954 & 16.7397 & 4.64455 & 47.15719 & 35.46911 \\
\hline & YLR404W & 9 & 14 & 19 & 3.231598 & 20.31008 & 14.38356 & 60.60127 & 36.05634 \\
\hline & YLR297W & 9 & 14 & 20 & 100 & 17.66862 & 8.980583 & 57.64526 & 31.54034 \\
\hline & YLR402W & 9 & 14 & 21 & 73.36198 & 14.28571 & 40.82569 & 67.71037 & 34.30421 \\
\hline & YLR296W & 9 & 14 & 22 & 32.31441 & 22.27378 & 11.72996 & 60.06885 & 33.83838 \\
\hline DUS3 & YLR401C & 9 & 14 & 23 & 16.66667 & 15.13218 & 33.16977 & 58.62944 & 55.11022 \\
\hline HIS3 & YOR202W & 9 & 14 & 24 & 35.93429 & 19.27512 & 26.26387 & & \\
\hline HIS3 & YOR202W & 9 & 15 & 1 & 38.13869 & 22.95082 & -34.6154 & & \\
\hline \multirow[t]{2}{*}{ ARP6 } & YLR085C & 9 & 15 & 2 & 24.91166 & 15.48077 & 57.84884 & & \\
\hline & YLR199C & 9 & 15 & 3 & 24.91803 & 36.56148 & 22.2488 & & \\
\hline \multirow[t]{2}{*}{ RAX2 } & YLR084C & 9 & 15 & 4 & 58.49923 & 8.98138 & 12.10247 & & \\
\hline & YLR194C & 9 & 15 & 5 & 64.44444 & 10.55227 & -3.46715 & & \\
\hline \multirow[t]{2}{*}{ EMP70 } & YLR083C & 9 & 15 & 6 & 5.604203 & 22.79349 & -11.7586 & & \\
\hline & YLR193C & 9 & 15 & 7 & 41.75824 & 23.48485 & 20.41367 & & \\
\hline SRL2 & YLR082C & 9 & 15 & 8 & 73.78641 & 40.67548 & -10.2041 & & \\
\hline HCR1 & YLR192C & 9 & 15 & 9 & 38.76812 & 43.24895 & 49.08062 & & \\
\hline GAL2 & YLR081W & 9 & 15 & 10 & 94.23868 & 54.4086 & 23.86555 & & \\
\hline PEX13 & YLR191W & 9 & 15 & 11 & 51.75439 & 21.79487 & 44.11765 & & \\
\hline EMP46 & YLR080W & 9 & 15 & 12 & 12.57606 & 54.15385 & 43.50797 & & \\
\hline MMR1 & YLR190W & 9 & 15 & 13 & 27.77778 & 13.18052 & 37.20038 & & \\
\hline SIC1 & YLR079W & 9 & 15 & 14 & 17.0579 & 100 & 98.31933 & & \\
\hline ATG26 & YLR189C & 9 & 15 & 15 & 5.882353 & 22.41546 & 11.8838 & & \\
\hline
\end{tabular}




\begin{tabular}{|c|c|c|c|c|c|c|c|}
\hline FMP25 & YLR077W & 9 & 15 & 16 & 6.477733 & 37.86408 & 29.5122 \\
\hline \multirow[t]{2}{*}{ MDL1 } & YLR188W & 9 & 15 & 17 & 10.71429 & 21.45305 & 4.962779 \\
\hline & YLR073C & 9 & 15 & 18 & -3.64372 & 10.99612 & 6.09319 \\
\hline \multirow[t]{2}{*}{ SKG3 } & YLR187W & 9 & 15 & 19 & 28.35249 & 8.436725 & 7.136564 \\
\hline & YLR072W & 9 & 15 & 20 & -7.61421 & 19.33116 & 6.909789 \\
\hline RPL37A & YLR185W & 9 & 15 & 21 & 65.08475 & 16.16509 & 15.2657 \\
\hline \multirow[t]{2}{*}{ XYL2 } & YLR070C & 9 & 15 & 22 & 63.80368 & 4.856895 & 14.27239 \\
\hline & YLR184W & 9 & 15 & 23 & 17.78169 & 24.45141 & 29.1939 \\
\hline HIS3 & YOR202W & 9 & 15 & 24 & -4.67572 & 26.41509 & 31.40028 \\
\hline HIS3 & YOR202W & 9 & 16 & 1 & 2.507837 & 35.19553 & 0.942761 \\
\hline HIS3 & YOR202W & 9 & 16 & 2 & 3.235294 & 41.22353 & 59.29329 \\
\hline HIS3 & YOR202W & 9 & 16 & 3 & -8.16993 & 36.90531 & 50.99085 \\
\hline HIS3 & YOR202W & 9 & 16 & 4 & 6.169297 & 46.63114 & 23.39413 \\
\hline HIS3 & YOR202W & 9 & 16 & 5 & 12.39936 & 14.96599 & 45.01718 \\
\hline HIS3 & YOR202W & 9 & 16 & 6 & 27.49676 & 0.616808 & 10.9437 \\
\hline HIS3 & YOR202W & 9 & 16 & 7 & 64.41006 & 25.29343 & 30.30075 \\
\hline HIS3 & YOR202W & 9 & 16 & 8 & 47.76632 & -0.60377 & 16.97793 \\
\hline HIS3 & YOR202W & 9 & 16 & 9 & 23.65931 & 3.276836 & 36.41618 \\
\hline HIS3 & YOR202W & 9 & 16 & 10 & 76.13365 & 8.87522 & 9.31677 \\
\hline HIS3 & YOR202W & 9 & 16 & 11 & 10.41456 & 28.12339 & -12.2517 \\
\hline HIS3 & YOR202W & 9 & 16 & 12 & 53.63735 & 13.49845 & 2.197802 \\
\hline HIS3 & YOR202W & 9 & 16 & 13 & 72.34043 & 48.56512 & -1.5748 \\
\hline HIS3 & YOR202W & 9 & 16 & 14 & 73.95349 & 66.30435 & 20.30744 \\
\hline HIS3 & YOR202W & 9 & 16 & 15 & 18.591 & 9.005376 & 2.166477 \\
\hline HIS3 & YOR202W & 9 & 16 & 16 & 16.0221 & 1.888489 & -2.93132 \\
\hline HIS3 & YOR202W & 9 & 16 & 17 & 63.15789 & -12.2823 & -4.01929 \\
\hline HIS3 & YOR202W & 9 & 16 & 18 & 33.5084 & 25.51471 & 12.57806 \\
\hline HIS3 & YOR202W & 9 & 16 & 19 & 40.26187 & 1.450231 & -5.78176 \\
\hline HIS3 & YOR202W & 9 & 16 & 20 & 40.54054 & 11.11987 & -6.71642 \\
\hline HIS3 & YOR202W & 9 & 16 & 21 & 52.27273 & -3.60679 & 19.37086 \\
\hline HIS3 & YOR202W & 9 & 16 & 22 & 61.35266 & 8 & 19.40639 \\
\hline HIS3 & YOR202W & 9 & 16 & 23 & 49.53271 & 16.12466 & 42.05519 \\
\hline HIS3 & YOR202W & 9 & 16 & 24 & & & 28.7844 \\
\hline HIS3 & YOR202W & 10 & 1 & 1 & 46.5587 & 57.56771 & 24.38434 \\
\hline HIS3 & YOR202W & 10 & 1 & 2 & 48.1896 & 35.79109 & 58.15684 \\
\hline HIS3 & YOR202W & 10 & 1 & 3 & 69.50355 & -4.3257 & 69.36416 \\
\hline HIS3 & YOR202W & 10 & 1 & 4 & 72.12766 & 54.9126 & 51.97368 \\
\hline HIS3 & YOR202W & 10 & 1 & 5 & 19.0061 & -14.0909 & 63.92456 \\
\hline HIS3 & YOR202W & 10 & 1 & 6 & 27.69517 & -51.5517 & 70.06069 \\
\hline HIS3 & YOR202W & 10 & 1 & 7 & 45.77703 & 10.12793 & 71.2216 \\
\hline HIS3 & YOR202W & 10 & 1 & 8 & 15.73546 & 10.2402 & 57.07178 \\
\hline HIS3 & YOR202W & 10 & 1 & 9 & 8.604651 & 32.19107 & 64.51224 \\
\hline HIS3 & YOR202W & 10 & 1 & 10 & 38.70968 & 3.656821 & 51.75182 \\
\hline
\end{tabular}




\begin{tabular}{|c|c|c|c|c|c|c|c|c|c|}
\hline HIS3 & YOR202W & 10 & 1 & 11 & 48.69993 & -14.4578 & 64.43265 & & \\
\hline HIS3 & YOR202W & 10 & 1 & 12 & 40.99585 & 10.76582 & 67.8487 & & \\
\hline HIS3 & YOR202W & 10 & 1 & 13 & 38.3378 & 14.58128 & 74.60692 & & \\
\hline HIS3 & YOR202W & 10 & 1 & 14 & 39.35811 & 21.06244 & 65.16245 & & \\
\hline HIS3 & YOR202W & 10 & 1 & 15 & 35.9204 & -11.3235 & 69.27651 & & \\
\hline HIS3 & YOR202W & 10 & 1 & 16 & 35.46638 & -13.2275 & 64.52145 & & \\
\hline HIS3 & YOR202W & 10 & 1 & 17 & 38.8303 & 19.15789 & 72.60594 & & \\
\hline HIS3 & YOR202W & 10 & 1 & 18 & 43.88985 & 17.09402 & 60.63947 & & \\
\hline HIS3 & YOR202W & 10 & 1 & 19 & 29.39481 & -4.86726 & 63.39458 & & \\
\hline HIS3 & YOR202W & 10 & 1 & 20 & 44.43626 & 9.263658 & 65.71114 & & \\
\hline HIS3 & YOR202W & 10 & 1 & 21 & 35.79952 & 1.242236 & 72.49376 & & \\
\hline HIS3 & YOR202W & 10 & 1 & 22 & 33.1 & 13.34149 & 64.15908 & & \\
\hline HIS3 & YOR202W & 10 & 1 & 23 & 29.02564 & 2.186589 & 61.66754 & & \\
\hline HIS3 & YOR202W & 10 & 1 & 24 & 62.7323 & 69.46265 & 69.86766 & & \\
\hline HIS3 & YOR202W & 10 & 2 & 1 & 61.68421 & 38.75339 & 55.12412 & & \\
\hline \multirow[t]{2}{*}{$10 C 4$} & $\begin{array}{l}\text { YMR044W } \\
\text { YMR158C- }\end{array}$ & 10 & 2 & 2 & 25.80645 & -16.5877 & 49.64739 & $\begin{array}{l}\text { missing } \\
\text { colony }\end{array}$ & missing colony \\
\hline & B & 10 & 2 & 3 & 13.60691 & 28.00609 & 59.6875 & 20.28986 & 31.46552 \\
\hline ARG80 & YMR042W & 10 & 2 & 4 & 14.86611 & -59.2437 & 57.7634 & 57.71605 & 29.03981 \\
\hline \multirow[t]{2}{*}{ FMP39 } & YMR157C & 10 & 2 & 5 & 19.24399 & 25.52011 & 62.56299 & 18.91026 & 27.45098 \\
\hline & YMR041C & 10 & 2 & 6 & 21.11984 & -18.543 & 53.85757 & 34.36293 & 12.07729 \\
\hline TPP1 & YMR156C & 10 & 2 & 7 & 11.3164 & -20.4082 & 40.05806 & 47.00704 & 12.43781 \\
\hline \multirow[t]{2}{*}{ YET2 } & YMR040W & 10 & 2 & 8 & 18.71768 & 32.41955 & 61.44715 & 44.96124 & 20.19704 \\
\hline & YMR155W & 10 & 2 & 9 & 18.01802 & 18.77193 & 59.35728 & 55.83483 & 16.79012 \\
\hline SUB1 & YMR039C & 10 & 2 & 10 & 19.46309 & 61.79245 & 58.58676 & 71.4795 & 35.21505 \\
\hline RIM13 & YMR154C & 10 & 2 & 11 & 66.18986 & 55.49669 & 62.65985 & 52.64957 & 19.7861 \\
\hline LYS7 & YMR038C & 10 & 2 & 12 & 30.96085 & 36.88312 & 69.50549 & 52.24417 & 34.96333 \\
\hline NUP53 & YMR153W & 10 & 2 & 13 & 58.45697 & 70 & 64.67768 & 50.9839 & -0.59172 \\
\hline \multirow[t]{3}{*}{ MSN2 } & YMR037C & 10 & 2 & 14 & 2.557201 & 71.09635 & 65.20446 & 55.60821 & 11.5493 \\
\hline & YMR153C- & & & & & & & & \\
\hline & $A$ & 10 & 2 & 15 & 56.06796 & 32.22836 & 63.66348 & 3.038674 & 13.52657 \\
\hline MIH1 & YMR036C & 10 & 2 & 16 & 26.85083 & 67.04197 & 61.93428 & 60.51437 & 10.21505 \\
\hline YIM1 & YMR152W & 10 & 2 & 17 & 13.74745 & 17.1875 & 63.17949 & 68.24926 & 15.89242 \\
\hline \multirow[t]{5}{*}{ IMP2 } & YMR035W & 10 & 2 & 18 & 13.19444 & 28.80143 & 65.95128 & 56.32582 & 9.217877 \\
\hline & YMR148W & 10 & 2 & 19 & 13.31237 & 34.48276 & 64.10768 & -11.9826 & 1.072386 \\
\hline & YMR034C & 10 & 2 & 20 & 11.58798 & 6.788512 & 59.61686 & -1.99005 & 7.228916 \\
\hline & $\begin{array}{l}\text { YMR147W } \\
\text { YMR031W- }\end{array}$ & 10 & 2 & 21 & 15.58855 & 21.981 & 57.57403 & 63.92857 & 24.66368 \\
\hline & A & 10 & 2 & 22 & 26.28931 & 17.81377 & 68.85246 & 46.47676 & 24.32432 \\
\hline NDE1 & YMR145C & 10 & 2 & 23 & 41.32139 & 31.46552 & 59.27835 & 40.64626 & 27.56892 \\
\hline HIS3 & YOR202W & 10 & 2 & 24 & 34.29158 & 17.1875 & 60.02468 & & \\
\hline \multirow[t]{2}{*}{ HIS3 } & YOR202W & 10 & 3 & 1 & -24.165 & 33.84956 & 57.74336 & & \\
\hline & YLR428C & 10 & 3 & 2 & 24.87844 & -15.852 & 50.65584 & 41.97349 & 40.94203 \\
\hline
\end{tabular}




\begin{tabular}{|c|c|c|c|c|c|c|c|c|c|}
\hline MFT1 & YML062C & 10 & 3 & 3 & 6.392199 & -23.2432 & 59.92063 & 41.89406 & 23.95062 \\
\hline MAG2 & YLR427W & 10 & 3 & 4 & 31.39653 & 55.06466 & 51.24125 & 14.31925 & 33.24397 \\
\hline \multirow[t]{2}{*}{ OGG1 } & YML060W & 10 & 3 & 5 & 36.20885 & -54.4118 & 43.7058 & 38.58521 & 16.93002 \\
\hline & YLR426W & 10 & 3 & 6 & 15.76087 & 28.55228 & 60.14235 & 37.84355 & 19.89101 \\
\hline NTE1 & YML059C & 10 & 3 & 7 & 26.30597 & 55.19077 & 56.15711 & 47.54386 & 20.55138 \\
\hline TUS1 & YLR425W & 10 & 3 & 8 & 29.02299 & 48.13478 & 66.72932 & 58.21138 & 45.49878 \\
\hline SML1 & YML058W & 10 & 3 & 9 & 14.50578 & 26.49842 & 62.42127 & 50.79618 & 12.92876 \\
\hline \multirow[t]{4}{*}{ ATG17 } & YLR423C & 10 & 3 & 10 & 27.9661 & 43.9781 & 60.88517 & 50.44444 & 22.93763 \\
\hline & YML058C- & & & & & & & & \\
\hline & $A$ & 10 & 3 & 11 & 14.7651 & 55.42986 & 63.49047 & 68.50829 & 23.23009 \\
\hline & YLR422W & 10 & 3 & 12 & 24.31243 & 63.22778 & 60.80526 & 36.25806 & 11.30435 \\
\hline CMP2 & YML057W & 10 & 3 & 13 & 24.45415 & 5.300353 & 72.12944 & 72.39165 & 21.14883 \\
\hline RPN13 & YLR421C & 10 & 3 & 14 & 27.52497 & 76.84918 & 59.84848 & 13.7218 & 16.75532 \\
\hline IMD4 & YML056C & 10 & 3 & 15 & 33.91204 & 63.10345 & 59.46602 & 50.83207 & 14.86146 \\
\hline URA4 & YLR420W & 10 & 3 & 16 & 22.03742 & 52.58824 & 59.06788 & 45.88235 & 13.31719 \\
\hline SPC2 & YML055W & 10 & 3 & 17 & 36.39706 & 18.28794 & 66.43836 & 50.26643 & 30.60748 \\
\hline CDC73 & YLR418C & 10 & 3 & 18 & 18.65772 & -9.11765 & 67.54564 & 64.20582 & 12.0915 \\
\hline \multirow[t]{4}{*}{ CYB2 } & YML054C & 10 & 3 & 19 & 15.96916 & 45.89878 & 58.65604 & 63.48123 & 16.70886 \\
\hline & YLR416C & 10 & 3 & 20 & 59.1029 & 59.60265 & 69.23077 & 55.5938 & 21.5 \\
\hline & YML053C & 10 & 3 & 21 & 73.16103 & -9.84127 & 65.26909 & 46.65523 & 22.38806 \\
\hline & YLR415C & 10 & 3 & 22 & 23.81413 & 37.27634 & 68.06283 & 16.59292 & 29.57111 \\
\hline SUR7 & YML052W & 10 & 3 & 23 & 19.6984 & 19.13978 & 67.37463 & 40.53156 & 32.02765 \\
\hline HIS3 & YOR202W & 10 & 3 & 24 & 46.64603 & -0.45317 & 66.25659 & & \\
\hline HIS3 & YOR202W & 10 & 4 & 1 & -12.3457 & -1.24014 & 57.19298 & & \\
\hline RIM9 & YMR063W & 10 & 4 & 2 & -13.7755 & 27.41935 & 38.04348 & 42.20532 & 32.71605 \\
\hline ALD2 & YMR170C & 10 & 4 & 3 & 15.17691 & 32.32759 & 51.26582 & 55.4295 & 36.74541 \\
\hline TOM37 & YMR060C & 10 & 4 & 4 & -5.54855 & 68.47947 & 56.8152 & -7.78443 & 7.178218 \\
\hline ALD3 & YMR169c & 10 & 4 & 5 & 15.78354 & 47.70115 & 67.28281 & 59.02778 & 16.71159 \\
\hline FET3 & YMR058W & 10 & 4 & 6 & 28.24176 & -43.5083 & 69.62923 & 41.30841 & 13.7931 \\
\hline \multirow[t]{3}{*}{ MLH1 } & YMR167W & 10 & 4 & 7 & 41.84149 & 23.05825 & 63.97985 & 87.76224 & 35.61644 \\
\hline & YMR057C & 10 & 4 & 8 & 12.82609 & -11.0092 & 69.21087 & 60.52174 & 26.08696 \\
\hline & YMR166C & 10 & 4 & 9 & 5.731707 & 35.55195 & 62.48349 & $\begin{array}{l}54.12131 \\
\text { missing }\end{array}$ & 11.76471 \\
\hline AAC1 & YMR056C & 10 & 4 & 10 & 20.02488 & 34.80826 & 61.32964 & $\begin{array}{l}\text { colony } \\
\text { missing }\end{array}$ & missing colony \\
\hline MSS11 & YMR164C & 10 & 4 & 11 & 16.55556 & -49.7717 & 56.53358 & $\begin{array}{l}\text { colony } \\
\text { missing }\end{array}$ & missing colony \\
\hline \multirow[t]{2}{*}{ BUB2 } & YMR055C & 10 & 4 & 12 & 24.4186 & 2.702703 & 66.51115 & colony & missing colony \\
\hline & YMR163C & 10 & 4 & 13 & 19.97875 & -29.0429 & 66.72582 & 61.91136 & 31.08808 \\
\hline STV1 & YMR054W & 10 & 4 & 14 & 55.61497 & 66.14786 & 69.22608 & 16.94915 & 0.539084 \\
\hline DNF3 & YMR162C & 10 & 4 & 15 & 35.36896 & -3.52941 & 66.03212 & 61.3963 & 14.67577 \\
\hline STB2 & YMR053C & 10 & 4 & 16 & 43.34601 & 13.97059 & 63.43923 & 46.80851 & 18.97436 \\
\hline HL1 & YMR161W & 10 & 4 & 17 & 10.40975 & -67.462 & 62.2544 & 58.63309 & 28.22384 \\
\hline FAR3 & YMR052W & 10 & 4 & 18 & 29.24312 & 64.3401 & 71.84901 & 52.45902 & 17.2619 \\
\hline
\end{tabular}


ATG16

CSM3

HIS3

HIS3

SIR3

ATP18

RPS1A

WAR1

CAR2

HMG1

FPR3

ECM30

TCB3

TSR2

COG8

DAK1

CNA1

ITT1

IMD3

ERV41

ATG23

SMA2

CRN1

RPS1B

HIS3

HIS3

SPT21

NAM7

MMT1

CTF18
YMR160W
YMR052C-

A

10

10

YMR159C

YMR048W

YMR158W-

A

YOR202W

YOR202W

YLR442C

YML081C-

A

YLR441C

YML076C

YLR438W

YML075C

YLR437C

YML074C

YLR436C

YML072C

YLR435W

YML071C

YLR434C

YML070W

YLR433C

YML068W

YLR432W

YML067C

YLR431C

YML066C

YLR429W

YML063W

YOR202W

YOR202W

YMR086C-

A

YMR179W

YMR085W

YMR178W

YMR080C

YMR177W

YMR078C

10
$19 \quad 23.37079$

37.72242

57.99127

$20 \quad 22.63798$

$46.68335 \quad 59.97286$

$21-13.3436$

6.70157163 .14348

$22 \quad-9.60699$

$61.959 \quad 56.42458$

$23 \quad 19.31106$

$47.71372 \quad 66.6326$

$24 \quad 23.82705$

$-11.2434$

72.72727

$1 \quad 36.51877$

11.03753

75.96542

$2 \quad 22.3092$

45.01953

55.77596

32.520046

$4 \quad 13.00505$

$50.12438 \quad 46.81015$

$35.84475 \quad 40.21622$

$\begin{array}{ll}5 & 20.80808\end{array}$

50.35714

60.1687

$\begin{array}{ll}6 & 16.40798\end{array}$

$54.01015 \quad 65.10353$

$\begin{array}{ll}7 & 9.961686\end{array}$

36.84211

81.32296

$\begin{array}{ll}8 & 17.66784\end{array}$

$47.61905 \quad 61.04046$

$9 \quad 13.2336$

$-9.53678 \quad 57.48098$

1018.21472

$54.11298 \quad 59.69856$

$11 \quad 16.24424$

28.4722275 .05995

$12 \quad 66.81223$

$64.85714 \quad 54.04556$

$\begin{array}{ll}13 & 3.071672\end{array}$

64.4831

64.74279

$14 \quad 36.10755$

2.572347

66.73065

$15 \quad 30.67633$

63.56757

63.6731

$16 \quad 28.98904$

$60.34173 \quad 55.27177$

$17 \quad 17.51553$

$18 \quad 33.78378$

$60.07853 \quad 74.19836$

$\begin{array}{ll}0 & 68.92285\end{array}$

$\begin{array}{ll}19 & 17.74554\end{array}$

$20 \quad 17.76251$

$21 \quad 66.49746$

$22 \quad 27.84689$

$\begin{array}{ll}23 & 25.22388\end{array}$

$24 \quad 21.83794$

$1 \quad 84.3413$

63.57553

62.91955

$-4.52962 \quad 62.31648$

68.1274963 .10559

$-9.2970569 .80983$

$-21.3087$

62.16867

31.37255

59.56522

$21.5625 \quad 81.5047$

$2 \quad 22.47191$

$3 \quad 28.0303$

41.0569

39.1494

55.44

26.7275151 .66772

$4 \quad 11.3369$

54.06137

57.65633

$-22.708 \quad 74.33743$

$-15.6934 \quad 65.18519$

$-39.5631$

58.74027
73.17073

57.37123

45.50669

63.49481

63.19648

60.36415

55.86081

66.32479

58.87372

49.12892

54.35203

63.32117

13.45646

47.28261

missing

colony

missing

colony

48.64865

48.54202

52.85235

53.45133

55.40541

72.44733

$-60.6952$

51.03448

54.36364

47.36842

56.78119

43.66438

39.15094

64.9635

26.51822

52.63158

35.70128

65.24954
29.90431

22.63158

13.25648

26.78571

28.92562

37.88187

31.11888

38.83792

27

28.98551

14.13428

25.1928

3.333333

16.77419

missing colony

missing colony

25.44529

9.935897

21.89974

20.94972

18.97106

26.75676

27.46114

28.45304

26.13982

35.92677

30.38961

33.10811

45.953

32.96089

28.07018

15.70248

22.22222

16.92308 


\begin{tabular}{|c|c|c|c|c|c|c|c|c|c|}
\hline ECM5 & YMR176W & 10 & 6 & 9 & 13.10734 & 43.85321 & 62.94416 & 58.2038 & 22.16359 \\
\hline RCO1 & YMR075W & 10 & 6 & 10 & 13.34094 & -9.27357 & 65.74394 & 15.38462 & 49.43311 \\
\hline \multirow[t]{3}{*}{ SIP18 } & YMR175w & 10 & 6 & 11 & 26.18758 & 48.1665 & 67.56919 & 35.45966 & 30.30303 \\
\hline & YMR075C- & & & & & & & & \\
\hline & $A$ & 10 & 6 & 12 & 13.37209 & 0.917431 & 73.24324 & 2.931596 & 44.79638 \\
\hline \multirow[t]{4}{*}{ PAI3 } & YMR174C & 10 & 6 & 13 & -4.90308 & 15.70732 & 77.37705 & 51.89655 & 48.20359 \\
\hline & YMR073C & 10 & 6 & 14 & 41.60401 & 48.88124 & 63.43391 & 37.31656 & 25.09653 \\
\hline & YMR173W- & & & & & & & & \\
\hline & $A$ & 10 & 6 & 15 & 36.65835 & 37.38019 & 69.7173 & 50.84746 & 25.76177 \\
\hline Мот3 & YMR070W & 10 & 6 & 16 & -3.5309 & 23.1068 & 66.9863 & 40.28777 & 22.01592 \\
\hline DDR48 & YMR173W & 10 & 6 & 17 & 21.13636 & -18.1818 & 62.4374 & 71.96721 & 28.46154 \\
\hline AVO2 & YMR068W & 10 & 6 & 18 & 30.99548 & -11.2285 & 71.14094 & 65.4321 & 31.65829 \\
\hline НОТ1 & YMR172W & 10 & 6 & 19 & 3.068182 & -14.9451 & 61.02362 & 58.19398 & 28.125 \\
\hline \multirow[t]{3}{*}{ UBX4 } & YMR067C & 10 & 6 & 20 & 19.33702 & 63.64594 & 67.79266 & 50.08403 & 21.95846 \\
\hline & YMR172C- & & & & & & & & \\
\hline & $A$ & 10 & 6 & 21 & 21.87803 & 56.87569 & 63.43724 & 51.60772 & 34.78261 \\
\hline \multirow[t]{2}{*}{ KAR5 } & YMR065W & 10 & 6 & 22 & 55.07766 & 39.87654 & 68.74725 & 69.07776 & 24.75962 \\
\hline & YMR171C & 10 & 6 & 23 & 22.83885 & 65.06899 & 71.422 & 44.9635 & 43.85593 \\
\hline HIS3 & YOR202W & 10 & 6 & 24 & 17.08428 & -38.5214 & 91.28065 & & \\
\hline HIS3 & YOR202W & 10 & 7 & 1 & 78.07167 & 21.51899 & 50 & & \\
\hline FMP27 & YLR454W & 10 & 7 & 2 & 37.77778 & 19.2582 & 43.90582 & 41.94313 & 59.78091 \\
\hline CAC2 & YML102W & 10 & 7 & 3 & 14.12935 & 8.983957 & 63.12275 & 41.22807 & -24.8366 \\
\hline \multirow[t]{3}{*}{ RIF2 } & YLR453C & 10 & 7 & 4 & 39.37888 & 17.27159 & 57.19274 & 28.61736 & 51.3465 \\
\hline & YML012C- & & & & & & & & \\
\hline & $A$ & 10 & 7 & 5 & 2.44898 & -15.9091 & 73.80531 & 31.94444 & 46.48148 \\
\hline SST2 & YLR452C & 10 & 7 & 6 & 15.96548 & 40 & 69.49153 & 21.17904 & 54.04475 \\
\hline CUE4 & YML101C & 10 & 7 & 7 & 31.01415 & 56.41646 & 61.34831 & 32.14286 & 63.01824 \\
\hline \multirow[t]{3}{*}{ LEU3 } & YLR451W & 10 & 7 & 8 & 23.28431 & 27.96093 & 61.3968 & 36.9906 & 67.38411 \\
\hline & YML100W- & & & & & & & & \\
\hline & $A$ & 10 & 7 & 9 & 13.19797 & 59.09091 & 64.85569 & 35.79235 & 69.87741 \\
\hline HMG2 & YLR450W & 10 & 7 & 10 & 20.8238 & 62.83784 & 73.81387 & 35.64955 & 73.82876 \\
\hline TSL1 & YML100W & 10 & 7 & 11 & 14.78953 & 60.30612 & 73.60179 & 37.59791 & 64.95327 \\
\hline FPR4 & YLR449W & 10 & 7 & 12 & 14.41441 & -35.9155 & 75.25692 & 29.62963 & 60.25039 \\
\hline ARG81 & YML099C & 10 & 7 & 13 & 5.231144 & -13.9752 & 67.8681 & 32.66476 & -33.0484 \\
\hline RPL6B & YLR448W & 10 & 7 & 14 & 40.33149 & 65.34653 & 63.23887 & 63.60759 & 78.77907 \\
\hline \multirow[t]{4}{*}{ VPS9 } & YML097C & 10 & 7 & 15 & \#DIV/0! & 95.16129 & 65.11628 & 28.88889 & 54.02098 \\
\hline & YLR446W & 10 & 7 & 16 & 18.2598 & 26.95214 & 73.97356 & 36 & 57.4359 \\
\hline & YML096W & 10 & 7 & 17 & 41.30982 & -3.93701 & 63.12133 & 26.4631 & 60.65858 \\
\hline & YLR445W & 10 & 7 & 18 & 8.691099 & 51.79426 & 66.69866 & 35.32934 & 60.52632 \\
\hline \multirow[t]{2}{*}{ RAD10 } & YML095C & 10 & 7 & 19 & 11.72333 & 2.460203 & 66.66667 & 39.41176 & 40.75342 \\
\hline & YLR444C & 10 & 7 & 20 & 25.44554 & 73.77919 & 66.27027 & 34.55657 & 49.66216 \\
\hline GIM5 & YML094W & 10 & 7 & 21 & 40.05935 & 29.24528 & 59.54524 & 38.65337 & -0.97324 \\
\hline \multirow[t]{2}{*}{ ECM7 } & YLR443W & 10 & 7 & 22 & 40.79422 & -10.4027 & 68.39506 & 44.66501 & 2.189781 \\
\hline & YML090W & 10 & 7 & 23 & 11.58537 & 53.90374 & 69.77291 & 34.94505 & 68.69436 \\
\hline
\end{tabular}




\begin{tabular}{|c|c|c|c|c|c|c|c|c|c|}
\hline HIS3 & YOR202W & 10 & 7 & 24 & 20.49808 & 26.04938 & 70.63622 & & \\
\hline \multirow[t]{2}{*}{ HIS3 } & YOR202W & 10 & 8 & 1 & 83.26026 & 3.703704 & 42.28487 & & \\
\hline & YMR103C & 10 & 8 & 2 & 48.5936 & 8.495822 & 62.41935 & 39.0925 & 49.09091 \\
\hline \multirow[t]{2}{*}{ APP2 } & YMR192W & 10 & 8 & 3 & 14.2255 & -20.7951 & 63.32952 & 67.72277 & 40.99217 \\
\hline & YMR102C & 10 & 8 & 4 & -25.0663 & 69.88485 & 60.18433 & 32.26415 & 35.79677 \\
\hline SPG5 & YMR191W & 10 & 8 & 5 & 22.4158 & 41.91344 & 55.11364 & 56.13718 & 29.26136 \\
\hline SRT1 & YMR101C & 10 & 8 & 6 & 44.74053 & 69.85699 & 63.32398 & 65.10319 & 23.8342 \\
\hline SGS1 & YMR190C & 10 & 8 & 7 & 13.1934 & 30.73529 & 66.20267 & 55.2017 & 36.53251 \\
\hline MUB1 & YMR100W & 10 & 8 & 8 & 4.851158 & 40.60606 & 66.63914 & 23.01038 & 41.96185 \\
\hline \multirow[t]{2}{*}{ GCV2 } & YMR189W & 10 & 8 & 9 & 22.22222 & -7.93651 & 77.81155 & 62.54613 & 32.1519 \\
\hline & YMR099C & 10 & 8 & 10 & 20.1995 & 37.0945 & 65.72188 & 64.63415 & 37.2449 \\
\hline MRPS17 & YMR188C & 10 & 8 & 11 & 19.95104 & 49.15254 & 71.23153 & 56.82968 & 38.00539 \\
\hline \multirow[t]{2}{*}{ SNZ1 } & YMR096W & 10 & 8 & 12 & 26.6756 & 27.55418 & 63.90041 & 53.7037 & 27.40113 \\
\hline & YMR187C & 10 & 8 & 13 & -29.0625 & 34.96732 & 64.48802 & 54.79876 & 35.60209 \\
\hline SNO1 & YMR095C & 10 & 8 & 14 & 14.34426 & 35.90604 & 66.6025 & 59.80707 & 37.15847 \\
\hline HSC82 & YMR186W & 10 & 8 & 15 & 13.17568 & 3.918495 & 58.83495 & 65.0655 & 37.6 \\
\hline AIP1 & YMR092C & 10 & 8 & 16 & 9.513274 & 27.67154 & 75.83333 & 37.15415 & 33.0303 \\
\hline $\mathrm{SSO} 2$ & YMR183C & 10 & 8 & 17 & 0.813008 & 15.95442 & 69.62916 & 72.08955 & 34.8285 \\
\hline VBA1 & YMR088C & 10 & 8 & 18 & 20.48458 & 49.8452 & 58.85226 & 9.88075 & 27.02703 \\
\hline \multirow[t]{4}{*}{ RGM1 } & YMR182C & 10 & 8 & 19 & 20.89844 & 59.57711 & 75.43071 & 75.47771 & 44.02174 \\
\hline & YMR087W & 10 & 8 & 20 & 10.63158 & 6.332139 & 62.3301 & 53.24459 & 38.82979 \\
\hline & YMR181C & 10 & 8 & 21 & 11.11111 & 59.79899 & 62.26623 & 50 & 37.33333 \\
\hline & YMR086W & 10 & 8 & 22 & -29.4425 & -35.8885 & 70.08634 & 50.31348 & 44.97354 \\
\hline CTL1 & YMR180C & 10 & 8 & 23 & 9.962049 & -20.078 & 79.53368 & 80.70652 & 52.62055 \\
\hline HIS3 & YOR202W & 10 & 8 & 24 & 28.69641 & 5.625 & 71.33127 & & \\
\hline HIS3 & YOR202W & 10 & 9 & 1 & 83.43531 & -15.1329 & 82.09769 & & \\
\hline YAP1 & YML007W & 10 & 9 & 2 & 55.03792 & 16 & 63.05201 & 57.81759 & 33.15927 \\
\hline NGL3 & YML118W & 10 & 9 & 3 & -10.8374 & -15.5354 & 60.34483 & 15.98985 & 29.17847 \\
\hline \multirow[t]{3}{*}{ GIS4 } & YML006C & 10 & 9 & 4 & 28.04171 & 55.91286 & 64.70588 & 62.60297 & 41.38889 \\
\hline & YML117W- & & & & & & & & \\
\hline & $A$ & 10 & 9 & 5 & 5.839416 & 59.51417 & 63.88889 & 55.42636 & 33.43373 \\
\hline TRM12 & YML005W & 10 & 9 & 6 & 10.34078 & 63.10573 & 64.49735 & 46.09929 & 38.48485 \\
\hline NAB6 & YML117W & 10 & 9 & 7 & 1.234568 & 6.843575 & 50.85537 & 61.12116 & 34.05405 \\
\hline GLO1 & YML004C & 10 & 9 & 8 & 19.88304 & -46.7391 & 72.71073 & 33.09222 & 40.34582 \\
\hline \multirow[t]{2}{*}{ ATR1 } & YML116W & 10 & 9 & 9 & 22.02462 & 55.70971 & 63.24324 & 55.79323 & 36.44068 \\
\hline & YML003W & 10 & 9 & 10 & 23.58491 & 55.39499 & 63.49643 & 60.27165 & 41.66667 \\
\hline \multirow[t]{2}{*}{ DAT1 } & YML113W & 10 & 9 & 11 & 28.15249 & 61.06195 & 48.84591 & 62.81407 & 44.28969 \\
\hline & YML002W & 10 & 9 & 12 & 49.27152 & 69.207 & 67.68448 & 65.7277 & 37.53501 \\
\hline ZDS2 & YML109W & 10 & 9 & 13 & 18.06283 & 44.18367 & 67.20895 & 55.14019 & 37.17579 \\
\hline \multirow[t]{2}{*}{ YPT7 } & YML001W & 10 & 9 & 14 & 4.479283 & 38.7931 & 55.81854 & 41.08216 & 40.26403 \\
\hline & YML108W & 10 & 9 & 15 & 2.587322 & 50.4451 & 73.28605 & 3.183024 & 33.09353 \\
\hline \multirow[t]{2}{*}{ PAU4 } & YLR461W & 10 & 9 & 16 & 5.350318 & 62.70677 & 57.23125 & 59.93691 & 45.30831 \\
\hline & YML107C & 10 & 9 & 17 & 4.055944 & 17.59379 & 64.52917 & 11.27168 & 49.19094 \\
\hline
\end{tabular}




\begin{tabular}{|c|c|c|c|c|c|c|c|c|c|}
\hline & YLR460C & 10 & 9 & 18 & 15.2381 & 28.72996 & 65.38776 & 56.2599 & 45.75 \\
\hline \multirow[t]{2}{*}{ URA5 } & YML106W & 10 & 9 & 19 & 2.145411 & 9.04325 & 76.24848 & -14.5503 & 40.34582 \\
\hline & YLR456W & 10 & 9 & 20 & 41.54653 & 16.37108 & 64.19214 & 70.50243 & 53.0303 \\
\hline \multirow[t]{2}{*}{ MDM1 } & YML104C & 10 & 9 & 21 & 0.923077 & 6.855792 & 64.42364 & 59.27152 & 47.61905 \\
\hline & YLR455W & 10 & 9 & 22 & 35.08772 & 46.7344 & 69.11488 & 61.70886 & 51.62907 \\
\hline NUP188 & YML103C & 10 & 9 & 23 & 72.25806 & -1.26126 & 63.02083 & 47.38676 & 49.54955 \\
\hline HIS3 & YOR202W & 10 & 9 & 24 & 11.54381 & 6.16622 & 77.37705 & & \\
\hline HIS3 & YOR202W & 10 & 10 & 1 & 94.35897 & 45.3125 & 78.79158 & & \\
\hline \multirow[t]{2}{*}{ ASI1 } & YMR119W & 10 & 10 & 2 & 66.84073 & 54.09253 & 59.24113 & 54.43609 & 44.71545 \\
\hline & YMR206W & 10 & 10 & 3 & 32.21851 & 25.06329 & 57.82038 & 52.91829 & 35.71429 \\
\hline ASC1 & YMR116C & 10 & 10 & 4 & 63.21467 & 20.47414 & 84.15033 & 68.91089 & 51.42857 \\
\hline PFK2 & YMR205C & 10 & 10 & 5 & 52.25806 & 15.9204 & 65.12141 & 66.66667 & 44.21365 \\
\hline \multirow[t]{3}{*}{ FMP24 } & YMR115W & 10 & 10 & 6 & -6.78851 & 62.05788 & 53.45036 & 47.33096 & 39.28571 \\
\hline & YMR204C & 10 & 10 & 7 & 9.485714 & -13.0797 & 55.36268 & 52.9511 & 37.79762 \\
\hline & YMR114C & 10 & 10 & 8 & 8.826816 & 33.67347 & 62.85112 & 59.18699 & 44.15888 \\
\hline \multirow[t]{2}{*}{ ERG2 } & YMR202W & 10 & 10 & 9 & 13.92904 & 14.24658 & 75.57058 & 60 & 40.51447 \\
\hline & YMR111C & 10 & 10 & 10 & 11.75694 & 50.93085 & 64.51613 & 53.7415 & 45.50562 \\
\hline \multirow[t]{2}{*}{ RAD14 } & YMR201C & 10 & 10 & 11 & 32.42134 & 51.14155 & 61.43216 & 33.4471 & 46.38554 \\
\hline & YMR110C & 10 & 10 & 12 & 60.0243 & 64.65116 & 65.30612 & 91.19171 & 74.61774 \\
\hline CLN1 & YMR199W & 10 & 10 & 13 & 18.83657 & 35.59322 & 68.58805 & 13.24786 & 43.12668 \\
\hline MYO5 & YMR109W & 10 & 10 & 14 & 12.4487 & 24.59499 & 59.35974 & 25.12998 & 38.01653 \\
\hline CIK1 & YMR198W & 10 & 10 & 15 & 11.05193 & 11.06501 & 68.23064 & 10.91954 & 43.95604 \\
\hline \multirow[t]{2}{*}{ SPG4 } & YMR107W & 10 & 10 & 16 & 22.48603 & 34.57627 & 61.42778 & 69.95885 & 44.59103 \\
\hline & YMR196W & 10 & 10 & 17 & 6.849315 & 10.42524 & 76.43098 & 57.57098 & 42.23602 \\
\hline YKU80 & YMR106C & 10 & 10 & 18 & -1.68472 & -8.37989 & 74.8924 & 47.08819 & 48.65591 \\
\hline ICY1 & YMR195W & 10 & 10 & 19 & 4.265403 & 16.75063 & 58.80109 & 61.59052 & 40.84507 \\
\hline PGM2 & YMR105C & 10 & 10 & 20 & 16.61891 & 17.24138 & 81.7817 & 76.78275 & 48.00995 \\
\hline RPL36A & YMR194W & 10 & 10 & 21 & 2.756892 & 4.72103 & 78.6106 & -9.5672 & 38.74172 \\
\hline \multirow[t]{3}{*}{ YPK2 } & YMR104C & 10 & 10 & 22 & 53.5503 & 18.20128 & 42.07317 & 64.5768 & 47.72182 \\
\hline & YMR193C- & & & & & & & & \\
\hline & $A$ & 10 & 10 & 23 & 43.47357 & 37.9668 & 66.02604 & 51.01721 & 54.03423 \\
\hline HIS3 & YOR202W & 10 & 10 & 24 & 40.47393 & 35.04274 & 68.30572 & & \\
\hline HIS3 & YOR202W & 10 & 11 & 1 & 73.84732 & -9.20044 & 75.4856 & & \\
\hline OST6 & YML019W & 10 & 11 & 2 & 71.89409 & 15.86476 & 68.8478 & 50.66667 & 47.97441 \\
\hline \multirow[t]{3}{*}{ MVP1 } & YMR004W & 10 & 11 & 3 & 43.89189 & 10.55684 & 56.33142 & 27.05314 & 25.13514 \\
\hline & YML018C & 10 & 11 & 4 & 38.98305 & 50.52879 & 52.65511 & 58.08383 & 35.02674 \\
\hline & YMR003W & 10 & 11 & 5 & 28.125 & 20.04154 & 55.67851 & 53.02548 & 46.66667 \\
\hline \multirow[t]{2}{*}{ PSP2 } & YML017W & 10 & 11 & 6 & 1.692866 & -3.16456 & 54.65884 & 46.72269 & 37.89174 \\
\hline & YMR002W & 10 & 11 & 7 & 27.42616 & 20.95808 & 60.17094 & 56.31659 & 47.63231 \\
\hline \multirow[t]{2}{*}{ PPZ1 } & YML016C & 10 & 11 & 8 & 2.371542 & 47.27273 & 62.64397 & 26.54867 & 41.97183 \\
\hline & YML131W & 10 & 11 & 9 & 0.127065 & 19.46472 & 58.35616 & 39.96448 & 41.83673 \\
\hline SEL1 & YML013W & 10 & 11 & 10 & 6.134094 & 19.85816 & 66.92587 & 32.6087 & 23.54497 \\
\hline MSC1 & YML128C & 10 & 11 & 11 & 8.059024 & 16.45367 & 64.97765 & 49.59742 & 44.23077 \\
\hline
\end{tabular}


YML013C-

\section{TUB3}

ERV25

PHO84

GTR1

MRPL39

NDI1

ERG6

HIS3

HIS3

JLP2

MRE11

UBP8

POM152

$\mathrm{FSH} 2$

SAS2

FMP42

DLT1

ESC1

SKY1

PKR1

GAS3

SCJ1

RPL15B

ADE17

HFA1

HIS3

HIS3

SRC1
A

YML124C

YML012W

YML123C

YML011C

YML122C

YML010C-

B

YML121W

YML009C

YML120C

YML008C

YML119W

YOR202W

YOR202W

YMR132C

YMR224C

YMR130W

YMR223W

YMR129W

YMR222C

YMR127C

YMR221C

YMR126C

YMR219W

YMR124W

YMR216C

YMR123W

YMR215W

YMR122C

YMR214W

YMR121C

YMR210W

YMR120C

YMR209C

YMR119W-

A

YMR207C

YOR202W

YOR202W

YML034W

YMR018W
10

$10 \quad 11$

10

10

10

10

10

10

10

10

10

10

10

10

10

10

10

10

10

10

10

10

10

10

10

10

10

10

10

10

10

10

10

10

10

10

10

10

10

10
11

11

11

11

11

11

11

11

11

11

11

11

12

12

12

12

12

12

12

12

12

12

12

12

12

12

12

12

12

12

12

12

12

\section{2}

12

12

13

13

13

$\begin{array}{rrrrr}12 & 62.67696 & 55.63549 & 67.6913 & 76.91002 \\ 13 & 16.86747 & 26.15658 & 69.19255 & 25.45788 \\ 14 & -13.6525 & 3.571429 & 59.27483 & 58.05471 \\ 15 & 6.268222 & 29.44785 & 58.25985 & 55.68513 \\ 16 & 5.30504 & -3.04878 & 73.05977 & 4.030227 \\ 17 & -12.8726 & 11.57347 & 59.7367 & 69.6\end{array}$

$\begin{array}{lllll}23 & 8.551069 & 16.50794 & 90.66524\end{array}$

$\begin{array}{lllll}24 & -42.8571 & 33.69697 & 73.32985\end{array}$

$\begin{array}{lllll}1 & 56.26186 & 35.90244 & 54.37788\end{array}$

$\begin{array}{llll}2 & 83.64718 & 24.39024 & 58.0511\end{array}$

$\begin{array}{lllll}3 & 59.86486 & 60.94276 & 50.16892\end{array}$

$\begin{array}{lllll}4 & 52.22073 & 53.12955 & 51.29288\end{array}$

$\begin{array}{lllll}5 & 34.81928 & 17.87995 & 85.20115\end{array}$

$\begin{array}{lllll}6 & 35.73487 & 34.33071 & 63.51626\end{array}$

$\begin{array}{lllll}7 & 9.476662 & 16.31579 & 65.08408\end{array}$

$\begin{array}{lllll}8 & 44.01198 & 67.10875 & 70.77244\end{array}$

$\begin{array}{lllll}9 & 2.060606 & 15.07177 & 60.51081\end{array}$

$10-1.76101$

12.15753

70

$\begin{array}{ll}11 & -7.7455\end{array}$

17.35537

62.69663

$12 \quad 5.707196$

$21.91781 \quad 69.64785$

$\begin{array}{lllll}13 & -3.16056 & 2.033037 & 56.99627\end{array}$

$\begin{array}{llll}14 & 9.042553 & -0.92593 & 68.28165\end{array}$

$\begin{array}{lllll}15 & 5.086705 & -5.78662 & 51.23457\end{array}$

$\begin{array}{lllll}16 & 7.570621 & -4.22125 & 54.83871\end{array}$

$\begin{array}{lllll}17 & 6.919946 & -18.7692 & 57.01408\end{array}$

$\begin{array}{lllll}18 & 4.819277 & -1.20482 & 62.2108\end{array}$

$\begin{array}{llll}19 & -15.2956 & -3.26705 & 61.04294\end{array}$

$\begin{array}{llll}20 & -12.2525 & -26.2626 & 59.89305\end{array}$

$\begin{array}{lllll}21 & -6.2982 & -0.15576 & 65.92689\end{array}$

$\begin{array}{llll}22 & 2.378256 & 34.31818 & 79.38788\end{array}$

$\begin{array}{lllll}23 & 46.01399 & 63.40206 & 74.9531\end{array}$

$\begin{array}{llll}24 & 20.79022 & 24.82168 & 70.62187\end{array}$

$\begin{array}{lllll}1 & 52.09924 & 23.71706 & 79.03226\end{array}$

$\begin{array}{lllll}2 & 78.15722 & 27.01987 & 53.95841 & 53.60825\end{array}$

$\begin{array}{lllll}3 & 70.01621 & 29.19075 & 54.3335 & 61.15214\end{array}$
39.30958

73.79032

53.98671

74.16546

78.61386

68.75

65.48387

38.65031

49.0085

41.16162

42.22874

48.09783

37.19677

50.77882

34.21927

56.6586

78.11448

53.20665

55.43478

58.15324

24.16452

7.29927

61.13074

37.36462

64.51613

53.38208

35.20833

55.55556

52.29202

6.387226

48.26255

20.50817

12.94821

47.66506

$-10.0304$

44.29012

$-1.15207$

64.63768

31.69014

29.274

51.53664

54.27252

36.40167

42.96482 


\begin{tabular}{|c|c|c|c|c|c|c|c|c|c|}
\hline & YML033W & 10 & 13 & 4 & 58.7156 & 32.66833 & 52.50583 & 50.89723 & 39.90025 \\
\hline SPO20 & YMR017W & 10 & 13 & 5 & 49.31846 & 20.46005 & 49.77099 & 52.26537 & 34.01015 \\
\hline RAD52 & YML032C & 10 & 13 & 6 & 53.66162 & 4.868914 & 73.10443 & 56.18321 & 57.53425 \\
\hline \multirow[t]{2}{*}{ SOK2 } & YMR016C & 10 & 13 & 7 & 39.64706 & 12.78539 & 79.69849 & 43.97163 & 43.03797 \\
\hline & YML030W & 10 & 13 & 8 & -10.2144 & 2.38379 & 53.47692 & 47.25664 & 40.70081 \\
\hline ERG5 & YMR015C & 10 & 13 & 9 & -5.9126 & 16.66667 & 64.89362 & 58.33333 & 49.43182 \\
\hline USA1 & YML029W & 10 & 13 & 10 & -3.42936 & 33.50318 & 69.53028 & -2.33645 & 50 \\
\hline CLU1 & YMR012W & 10 & 13 & 11 & 4.680365 & 10.45296 & 64.64461 & 59.87362 & 48.6413 \\
\hline TSA1 & YML028W & 10 & 13 & 12 & -3.39367 & 13.1291 & 68.06387 & 60.67961 & 52.07756 \\
\hline HXT2 & YMR011W & 10 & 13 & 13 & 5.794948 & 12.14689 & 66.82283 & 46.86998 & 41.35338 \\
\hline \multirow[t]{2}{*}{ YOX1 } & YML027W & 10 & 13 & 14 & 5.675369 & 12.51799 & 53.03226 & 69.21899 & 47.5827 \\
\hline & YMR010W & 10 & 13 & 15 & 36.57351 & 57.32861 & 71.20456 & 68.20988 & 48.91566 \\
\hline \multirow[t]{2}{*}{ RPS18B } & YML026C & 10 & 13 & 16 & 2.673147 & 16.06426 & 51.00503 & 44.21053 & 50.27624 \\
\hline & YMR009W & 10 & 13 & 17 & 17.17922 & 35.8209 & 69.18489 & 48.04469 & 51.0101 \\
\hline APT1 & YML022W & 10 & 13 & 18 & 95.33333 & 67.5 & 62.14286 & 58.74016 & 54.0201 \\
\hline PLB1 & YMR008C & 10 & 13 & 19 & -14.3054 & 21.41901 & 55.20833 & 47.74306 & 43.81443 \\
\hline \multirow[t]{3}{*}{ UNG1 } & YML021C & 10 & 13 & 20 & 41.63043 & 54.37853 & 73.9816 & 68.17391 & 60.82949 \\
\hline & YMR007W & 10 & 13 & 21 & -29.0715 & 58.58931 & 57.494 & 29.82143 & 38.46154 \\
\hline & YML020W & 10 & 13 & 22 & 16.54762 & 36.99732 & 51.25839 & 62.8866 & 47.38292 \\
\hline PLB2 & YMR006C & 10 & 13 & 23 & 35.89212 & 16.93548 & 57.92541 & 81.65761 & 55.65032 \\
\hline HIS3 & YOR202W & 10 & 13 & 24 & 69.47464 & 33.28632 & 71.01329 & & \\
\hline \multirow[t]{3}{*}{ HIS3 } & YOR202W & 10 & 14 & 1 & 46.97674 & 28.17734 & 61.47496 & & \\
\hline & $\begin{array}{l}\text { YMR144W } \\
\text { YMR244C- }\end{array}$ & 10 & 14 & 2 & 17.25616 & 13.55748 & 68.5907 & 42.98507 & 40.30172 \\
\hline & $A$ & 10 & 14 & 3 & 99.27361 & 0.32 & 73.91304 & 31.69643 & 27.06767 \\
\hline RPS16A & YMR143W & 10 & 14 & 4 & 69.69274 & 25.68966 & 68.70813 & 43.50797 & 42.6087 \\
\hline \multirow[t]{2}{*}{ ZRC1 } & YMR243C & 10 & 14 & 5 & 69.0205 & 5.735661 & 58.21745 & 43.69115 & 46.08501 \\
\hline & YMR141C & 10 & 14 & 6 & 60.54591 & 27.15895 & 59.75359 & 64.81224 & 54.87528 \\
\hline YHM2 & YMR241W & 10 & 14 & 7 & 36.01583 & 56.86275 & 59.65281 & $\begin{array}{l}51.88834 \\
\text { missing }\end{array}$ & 46.78899 \\
\hline SIP5 & YMR140W & 10 & 14 & 8 & 26.6756 & -12.6806 & 59.39553 & colony & missing colony \\
\hline DFG5 & YMR238W & 10 & 14 & 9 & -1.5873 & -7.09135 & 59.43396 & 23.80952 & 48.47059 \\
\hline RIM11 & YMR139W & 10 & 14 & 10 & 8.738938 & -11.9342 & 63.65947 & 59.46372 & 44.47592 \\
\hline $\mathrm{BCH} 1$ & YMR237W & 10 & 14 & 11 & 1.990632 & -20.1309 & 63.67738 & 58.69219 & 45.21739 \\
\hline CIN4 & YMR138W & 10 & 14 & 12 & 6.455399 & 4.347826 & 38.37342 & 76.2406 & 54.82353 \\
\hline RNH1 & YMR234W & 10 & 14 & 13 & 2.393617 & 26.93333 & 57.5939 & 28.62595 & 39.89501 \\
\hline PSO2 & YMR137C & 10 & 14 & 14 & 6.823028 & 6.625 & 50.13902 & 58.85023 & 51.35135 \\
\hline TRI1 & YMR233W & 10 & 14 & 15 & 7.255139 & 34.19619 & 51.82927 & 41.72414 & 58.13397 \\
\hline GAT2 & YMR136W & 10 & 14 & 16 & 6.666667 & 15.13514 & 64.21053 & 66.56442 & 44.18605 \\
\hline \multirow[t]{2}{*}{ FUS2 } & $\begin{array}{l}\text { YMR232W } \\
\text { YMR135W- }\end{array}$ & 10 & 14 & 17 & 10.69182 & 54.75862 & 60.39755 & 65.07463 & 52.95567 \\
\hline & $A$ & 10 & 14 & 18 & 9.662921 & 17.60243 & 60.81871 & 56.28141 & 49.29907 \\
\hline RPS10B & YMR230W & 10 & 14 & 19 & -16.7862 & 67.44433 & 66.66667 & 51.13788 & 52.75779 \\
\hline
\end{tabular}




\begin{tabular}{|c|c|c|c|c|c|c|c|c|c|}
\hline \multirow[t]{2}{*}{ GID8 } & YMR135C & 10 & 14 & 20 & -14.157 & -6.56566 & 57.55452 & 36.62551 & 44.59459 \\
\hline & YMR226C & 10 & 14 & 21 & 3.870162 & 51.97452 & 52.72851 & 69.78967 & 55.59105 \\
\hline REC114 & YMR133W & 10 & 14 & 22 & -2.91508 & 51.51515 & 61.8172 & -38.5802 & 46.36364 \\
\hline MRPL44 & YMR225C & 10 & 14 & 23 & -112.15 & 14.11531 & 57.88656 & 4.057279 & 43.6853 \\
\hline HIS3 & YOR202W & 10 & 14 & 24 & -6.80804 & 27.22949 & 92.44012 & & \\
\hline HIS3 & YOR202W & 10 & 15 & 1 & 12.75328 & 5.315615 & 88.76404 & & \\
\hline \multirow[t]{3}{*}{ GAL80 } & YML051W & 10 & 15 & 2 & 41.86603 & -14.1141 & 64.67722 & & \\
\hline & YMR031C & 10 & 15 & 3 & 78.7796 & 9.585799 & 70.17692 & & \\
\hline & $\begin{array}{l}\text { YML048W- } \\
\text { A }\end{array}$ & 10 & 15 & 4 & 85.05155 & 24.91228 & 60.60976 & & \\
\hline RSF1 & YMR030W & 10 & 15 & 5 & 68.35443 & 74.26901 & 57.46556 & & \\
\hline GSF2 & YML048W & 10 & 15 & 6 & 76.5445 & 12.20196 & 78.53736 & & \\
\hline FAR8 & YMR029C & 10 & 15 & 7 & 45.27607 & 37.97619 & 61.38329 & & \\
\hline PRM6 & YML047C & 10 & 15 & 8 & 44.90482 & -15.2203 & 46.44608 & & \\
\hline HRT2 & YMR027W & 10 & 15 & 9 & 4.885993 & 40.58537 & 65.84906 & & \\
\hline CAT2 & YML042W & 10 & 15 & 10 & 8.422175 & 50.55165 & 59.00415 & & \\
\hline PEX12 & YMR026C & 10 & 15 & 11 & -1.65877 & -8.59873 & 63.20593 & & \\
\hline VPS71 & YML041C & 10 & 15 & 12 & 2.714932 & -31.8182 & 53.73721 & & \\
\hline CSI1 & YMR025W & 10 & 15 & 13 & 15.5351 & 21.57773 & 50.98548 & & \\
\hline YMD8 & YML038C & 10 & 15 & 14 & 63.56707 & 76.0139 & 63.62876 & & \\
\hline \multirow[t]{2}{*}{ MSS1 } & YMR023C & 10 & 15 & 15 & 4.079498 & 18.83746 & 64.57971 & & \\
\hline & YML037C & 10 & 15 & 16 & 6.73516 & 55.29595 & 44.36424 & & \\
\hline \multirow[t]{2}{*}{ QRI8 } & YMR022W & 10 & 15 & 17 & 2.576112 & 52.34234 & 52.6284 & & \\
\hline & YML036W & 10 & 15 & 18 & 4.892966 & 45.57235 & 51.9337 & & \\
\hline \multirow[t]{3}{*}{ MAC1 } & YMR021C & 10 & 15 & 19 & 7.403315 & -9.57854 & 55 & & \\
\hline & YML035C- & & & & & & & & \\
\hline & A & 10 & 15 & 20 & 1.287554 & -2.8169 & 52.22615 & & \\
\hline FMS1 & YMR020W & 10 & 15 & 21 & -3.27684 & -17.6634 & 53.69844 & & \\
\hline AMD1 & YML035C & 10 & 15 & 22 & \#DIV/O! & \#DIV/0! & 3.896104 & & \\
\hline STB4 & YMR019W & 10 & 15 & 23 & 50.27747 & -235.211 & 53.59043 & & \\
\hline HIS3 & YOR202W & 10 & 15 & 24 & 30.66955 & 12.62554 & 100 & & \\
\hline HIS3 & YOR202W & 10 & 16 & 1 & 1.420455 & -8.3739 & 56.43564 & & \\
\hline HIS3 & YOR202W & 10 & 16 & 2 & 51.26582 & -34.1748 & 42.5184 & & \\
\hline HIS3 & YOR202W & 10 & 16 & 3 & 45.39249 & -54.6233 & 60 & & \\
\hline HIS3 & YOR202W & 10 & 16 & 4 & 75.8034 & -32.7797 & 59.31454 & & \\
\hline HIS3 & YOR202W & 10 & 16 & 5 & 84.53782 & -63.6986 & 51.28505 & & \\
\hline HIS3 & YOR202W & 10 & 16 & 6 & 78.27076 & -20.591 & 40.82988 & & \\
\hline HIS3 & YOR202W & 10 & 16 & 7 & 58.28313 & -38.9552 & 43.18182 & & \\
\hline HIS3 & YOR202W & 10 & 16 & 8 & 66.95652 & -19.4305 & 59.78618 & & \\
\hline HIS3 & YOR202W & 10 & 16 & 9 & 42.96296 & -41.3965 & 57.58755 & & \\
\hline HIS3 & YOR202W & 10 & 16 & 10 & 48.03695 & -28.0172 & 62.38532 & & \\
\hline HIS3 & YOR202W & 10 & 16 & 11 & 30.45455 & -3.02572 & 44.87555 & & \\
\hline HIS3 & YOR202W & 10 & 16 & 12 & 12.97108 & -43.433 & 75.79162 & & \\
\hline
\end{tabular}




\begin{tabular}{|c|c|c|c|c|c|c|c|c|c|}
\hline HIS3 & YOR202W & 10 & 16 & 13 & 15.24202 & -24.0521 & 65.56082 & & \\
\hline HIS3 & YOR202W & 10 & 16 & 14 & 4.952381 & -45.6349 & 77.99325 & & \\
\hline HIS3 & YOR202W & 10 & 16 & 15 & 1.168969 & -42.4552 & 68.16648 & & \\
\hline HIS3 & YOR202W & 10 & 16 & 16 & 2.544769 & -24.533 & 68.11398 & & \\
\hline HIS3 & YOR202W & 10 & 16 & 17 & 12.7182 & 57.70283 & 53.35943 & & \\
\hline HIS3 & YOR202W & 10 & 16 & 18 & 4.855372 & -14.3739 & 64.0625 & & \\
\hline HIS3 & YOR202W & 10 & 16 & 19 & 7.777778 & -9.91736 & 66.69196 & & \\
\hline HIS3 & YOR202W & 10 & 16 & 20 & 8.333333 & 31.35135 & 54.65549 & & \\
\hline HIS3 & YOR202W & 10 & 16 & 21 & 5.933118 & -2.30179 & 51.98883 & & \\
\hline HIS3 & YOR202W & 10 & 16 & 22 & 51.6129 & 24.4511 & 67.57044 & & \\
\hline HIS3 & YOR202W & 10 & 16 & 23 & 77.40964 & -24.2079 & 71.50924 & & \\
\hline HIS3 & YOR202W & 10 & 16 & 24 & 43.6478 & 48.49921 & 67.80735 & & \\
\hline HIS3 & YOR202W & 11 & 1 & 1 & 53.38983 & 26.13883 & 61.62141 & & \\
\hline HIS3 & YOR202W & 11 & 1 & 2 & 19.08284 & 66.09131 & 66.66667 & & \\
\hline HIS3 & YOR202W & 11 & 1 & 3 & 9.42029 & 48.36472 & 58.69565 & & \\
\hline HIS3 & YOR202W & 11 & 1 & 4 & 13.12057 & 48.8266 & 10.32548 & & \\
\hline HIS3 & YOR202W & 11 & 1 & 5 & -16.8157 & 54.05157 & 58.46645 & & \\
\hline HIS3 & YOR202W & 11 & 1 & 6 & -7.85714 & 62.00632 & 66.56379 & & \\
\hline HIS3 & YOR202W & 11 & 1 & 7 & 6.515152 & 61.65011 & 37.85489 & & \\
\hline HIS3 & YOR202W & 11 & 1 & 8 & -20.2756 & 60.90604 & 53.17139 & & \\
\hline HIS3 & YOR202W & 11 & 1 & 9 & -4.56942 & 37.94212 & 91.15282 & & \\
\hline HIS3 & YOR202W & 11 & 1 & 10 & -1.4652 & 50.80559 & 64.34911 & & \\
\hline HIS3 & YOR202W & 11 & 1 & 11 & 3.065693 & 31.26615 & 88.0916 & & \\
\hline HIS3 & YOR202W & 11 & 1 & 12 & 5.331179 & 44.06605 & 68.44758 & & \\
\hline HIS3 & YOR202W & 11 & 1 & 13 & -12.2288 & 30.87719 & 92.72727 & & \\
\hline HIS3 & YOR202W & 11 & 1 & 14 & 30.44248 & 64.67277 & 78.29545 & & \\
\hline HIS3 & YOR202W & 11 & 1 & 15 & 29.09408 & 53.66242 & 65.81986 & & \\
\hline HIS3 & YOR202W & 11 & 1 & 16 & 28.54641 & 42.79428 & 48.34906 & & \\
\hline HIS3 & YOR202W & 11 & 1 & 17 & 32.89474 & 46.63992 & 69.24035 & & \\
\hline HIS3 & YOR202W & 11 & 1 & 18 & 11.30064 & 37.14286 & 52.66904 & & \\
\hline HIS3 & YOR202W & 11 & 1 & 19 & 40.0545 & 51.35952 & 53.68421 & & \\
\hline HIS3 & YOR202W & 11 & 1 & 20 & 26.51007 & 28.97297 & 54.51467 & & \\
\hline HIS3 & YOR202W & 11 & 1 & 21 & 25.96899 & 45.69055 & 34.5496 & & \\
\hline HIS3 & YOR202W & 11 & 1 & 22 & 64.43662 & 37.61658 & 54.82955 & & \\
\hline HIS3 & YOR202W & 11 & 1 & 23 & 44.86957 & 41.46341 & 34.62069 & & \\
\hline HIS3 & YOR202W & 11 & 1 & 24 & -22.2543 & 59.84252 & 12.19065 & & \\
\hline \multirow[t]{3}{*}{ HIS3 } & YOR202W & 11 & 2 & 1 & 84.88278 & -19.086 & -6.18982 & & \\
\hline & YNL146W & 11 & 2 & 2 & -33.1288 & 25.16129 & 76.28362 & $\begin{array}{l}\text { missing } \\
\text { colony }\end{array}$ & missing colony \\
\hline & YNL274C & 11 & 2 & 3 & -44.6991 & 3.580563 & 79.7153 & 45.81749 & 36.29764 \\
\hline MFA2 & YNL145W & 11 & 2 & 4 & -20.0883 & 41.37353 & 76.25571 & 50.9165 & 12.69841 \\
\hline \multirow[t]{2}{*}{ TOF1 } & YNL273W & 11 & 2 & 5 & -13.8107 & 1.111111 & 69.68085 & 23.63636 & 27.66497 \\
\hline & YNL144C & 11 & 2 & 6 & -15.6863 & 33.57934 & 86.56388 & 30.4878 & 15.22199 \\
\hline
\end{tabular}




\begin{tabular}{|c|c|c|c|c|c|c|c|c|c|}
\hline \multirow[t]{2}{*}{ BNI1 } & YNL271C & 11 & 2 & 7 & 62.26708 & 73.77432 & 75.08772 & 38.43844 & 65.09901 \\
\hline & YNL143C & 11 & 2 & 8 & 37.0915 & 16.41949 & 45.02762 & 38.35294 & 30.73593 \\
\hline ALP1 & YNL270C & 11 & 2 & 9 & 51.76909 & -23.7192 & 88.84462 & 29.73761 & 35.35912 \\
\hline MEP2 & YNL142W & 11 & 2 & 10 & 17.94872 & 23.5514 & 47.65714 & 32.1513 & 35.72939 \\
\hline LYP1 & YNL268W & 11 & 2 & 11 & 36.42987 & 36.80297 & 51.67702 & 30.0885 & 33.42175 \\
\hline \multirow[t]{3}{*}{ AAH1 } & YNL141W & 11 & 2 & 12 & 33.50785 & -23.0143 & 88.2793 & 63.65639 & 38.37209 \\
\hline & YNL266W & 11 & 2 & 13 & 34.38045 & -29.0323 & 41.33333 & 11.16883 & 26.96629 \\
\hline & YNL140C & 11 & 2 & 14 & 32.54438 & 6.582278 & 57.28814 & 14.76923 & 3.71517 \\
\hline IST1 & YNL265C & 11 & 2 & 15 & 32.98969 & -46.7167 & 46.8661 & 56.40449 & 40.44321 \\
\hline EAF7 & YNL136W & 11 & 2 & 16 & 33.90411 & -56.5947 & 48.57571 & 16 & 11.32686 \\
\hline ATX1 & YNL259C & 11 & 2 & 17 & 26.95652 & 51.89456 & 86.66667 & 42.92343 & 37.37374 \\
\hline FPR1 & YNL135C & 11 & 2 & 18 & -39.0023 & -19.7895 & 63.13646 & -28.5714 & 11.1437 \\
\hline \multirow[t]{2}{*}{ SIP3 } & YNL257C & 11 & 2 & 19 & 29.93421 & -25.3863 & 67.41071 & 36 & 36.31436 \\
\hline & YNL134C & 11 & 2 & 20 & 14.18685 & -24.1584 & 53.81944 & 32.47423 & 42.42424 \\
\hline GIS2 & YNL255C & 11 & 2 & 21 & -36.2934 & -27.9597 & 78.4106 & -2.80899 & 31.51862 \\
\hline \multirow[t]{2}{*}{ CPT1 } & YNL130C & 11 & 2 & 22 & 55.50661 & 38.96552 & 84.46115 & 29.75207 & 19.03409 \\
\hline & YNL254C & 11 & 2 & 23 & 49.56522 & 36.89567 & 37.01997 & 25.86667 & 35.89744 \\
\hline HIS3 & YOR202W & 11 & 2 & 24 & 11.04762 & 24.07615 & 71.17904 & & \\
\hline HIS3 & YOR202W & 11 & 3 & 1 & 62.81157 & -31.5668 & 15.97938 & & \\
\hline \multirow[t]{5}{*}{ GFD1 } & YMR255W & 11 & 3 & 2 & 9.268293 & 78.65353 & 9.090909 & 53.65449 & 31.52709 \\
\hline & YNL035C & 11 & 3 & 3 & -2.41546 & 54.48343 & 72.09705 & 45.81395 & 24.36441 \\
\hline & YMR254C & 11 & 3 & 4 & -22.2707 & 63.47483 & 50.6237 & 57.31707 & 38.26087 \\
\hline & YNL034W & 11 & 3 & 5 & 37.42138 & 48.2238 & 80.20833 & 39.8374 & 31 \\
\hline & YMR253C & 11 & 3 & 6 & -16.8582 & 43.02508 & 80.67416 & 24.67192 & 37.81321 \\
\hline \multirow[t]{2}{*}{ SIW14 } & YNL032W & 11 & 3 & 7 & -26.9488 & 0.415512 & 81.40097 & 50 & 31.23732 \\
\hline & YMR252C & 11 & 3 & 8 & 7.692308 & 19.26606 & 39.95609 & 29.42584 & 34.14634 \\
\hline HHT2 & $\begin{array}{l}\text { YNL031C } \\
\text { YMR251W- }\end{array}$ & 11 & 3 & 9 & -24.0437 & -10.7422 & 75.67568 & -25.6484 & 39.67391 \\
\hline HOR7 & A & 11 & 3 & 10 & 1.858736 & 62.8447 & 86.76471 & 58.53081 & 41.57549 \\
\hline \multirow[t]{2}{*}{ HHF2 } & YNL030W & 11 & 3 & 11 & 35.80645 & 3.111111 & 66.05317 & 25.05855 & 38.02083 \\
\hline & YMR251W & 11 & 3 & 12 & 35.60477 & 30.56769 & 67.51773 & 21.8593 & 33.13253 \\
\hline KTR5 & YNL029C & 11 & 3 & 13 & 25 & \#DIV/0! & 75.61983 & 37.38095 & 38.64865 \\
\hline \multirow[t]{3}{*}{ GAD1 } & YMR250W & 11 & 3 & 14 & 38.06452 & 18.01242 & 80.45977 & 21.48148 & 27.74869 \\
\hline & YNL028W & 11 & 3 & 15 & -42.5791 & 46.78077 & 80.89286 & 43.40659 & 34.08451 \\
\hline & YMR247C & 11 & 3 & 16 & 39.9654 & -3.71567 & 77.40175 & 61.95652 & 53.63128 \\
\hline CRZ1 & YNL027W & 11 & 3 & 17 & 35.19856 & 64.59627 & 30.56027 & 17.16621 & 32.24852 \\
\hline \multirow[t]{3}{*}{ FAA4 } & YMR246W & 11 & 3 & 18 & 31.52709 & 6.060606 & 48.42681 & -23.8235 & 23.31288 \\
\hline & YNL024C & 11 & 3 & 19 & 29.43633 & -1.6055 & 48.35329 & 23.92473 & 25.91623 \\
\hline & YMR245W & 11 & 3 & 20 & 19.17808 & -32.4675 & 92.61084 & -30.5921 & 30.06329 \\
\hline \multirow[t]{3}{*}{ FAP1 } & YNL023C & 11 & 3 & 21 & 7.296137 & -41.8655 & 87.67606 & 51.35135 & 54.34174 \\
\hline & YMR244W & 11 & 3 & 22 & 56.66667 & -22.9675 & 52.79255 & 12.7027 & 36.19792 \\
\hline & YNL022C & 11 & 3 & 23 & 26.34855 & 30.91483 & 78.24859 & -89.8263 & 32.58065 \\
\hline HIS3 & YOR202W & 11 & 3 & 24 & -22.3427 & 27.29384 & -26.7568 & & \\
\hline
\end{tabular}




\begin{tabular}{|c|c|c|c|c|c|c|c|c|c|}
\hline HIS3 & YOR202W & 11 & 4 & 1 & 41.22658 & 39.80154 & 46.95864 & & \\
\hline SKO1 & YNL167C & 11 & 4 & 2 & -6.08519 & 64.33628 & 48.80319 & 49.375 & 29.81744 \\
\hline PCL1 & YNL289W & 11 & 4 & 3 & -24.356 & 71.66667 & 63.10484 & 51.43488 & 32.82937 \\
\hline BNI5 & YNL166C & 11 & 4 & 4 & 8.136483 & 60.61606 & 81.46853 & 31.11111 & 40.92409 \\
\hline \multirow[t]{2}{*}{ CAF40 } & YNL288W & 11 & 4 & 5 & 30.41237 & 39.3578 & 68.24584 & 58.62832 & 45.95843 \\
\hline & YNL165W & 11 & 4 & 6 & -29.8013 & 23.94366 & 50.81967 & 9.917355 & 28.45953 \\
\hline CUS2 & YNL286W & 11 & 4 & 7 & 33.77386 & 37.21973 & 68.76574 & 36.65835 & 49.48718 \\
\hline \multirow[t]{2}{*}{ IBD2 } & YNL164C & 11 & 4 & 8 & 81.96721 & 6.301824 & 45.94286 & -6.89655 & 18.09816 \\
\hline & YNL285W & 11 & 4 & 9 & 43.11688 & 22.11155 & 43.58601 & 34.25693 & 28.57143 \\
\hline RPL42A & YNL162W & 11 & 4 & 10 & -8.5758 & 5.976676 & -50.9836 & -45.9649 & 18.77551 \\
\hline WSC2 & YNL283C & 11 & 4 & 11 & 40.71661 & 19.41924 & 54.65253 & 32.90155 & 35.90504 \\
\hline ASI2 & YNL159C & 11 & 4 & 12 & 3.345725 & 28.49916 & 83.12757 & 15.54404 & 37.03704 \\
\hline \multirow[t]{2}{*}{$\mathrm{HCH} 1$} & YNL281W & 11 & 4 & 13 & 22.81879 & 9.966216 & 79.31034 & 16.71309 & 39.30818 \\
\hline & YNL157W & 11 & 4 & 14 & 14.03509 & 54.62555 & 84.67433 & -42.2619 & 19.26606 \\
\hline ERG24 & YNL280C & 11 & 4 & 15 & 45.48552 & -23.2906 & 55.87097 & 28.72063 & 39.40299 \\
\hline NSG2 & YNL156C & 11 & 4 & 16 & -53.5519 & 20.6089 & 78.99543 & 4.497354 & 24.92918 \\
\hline \multirow[t]{2}{*}{ PRM1 } & YNL279W & 11 & 4 & 17 & -62.6703 & 32.23322 & 29.28571 & 24.92997 & 37.76435 \\
\hline & YNL155W & 11 & 4 & 18 & -45.1039 & 53.63372 & 90.36827 & 33.79888 & 40.74074 \\
\hline CAF120 & YNL278W & 11 & 4 & 19 & -49.6386 & 28.40779 & 76.17925 & 10.89385 & 43.13187 \\
\hline YCK2 & YNL154C & 11 & 4 & 20 & -2.42718 & 27.72074 & 70.7457 & 34.49275 & 55.30086 \\
\hline MET2 & YNL277W & 11 & 4 & 21 & 59.72461 & -62.954 & 81.84049 & 18.39378 & 39.94413 \\
\hline GIM3 & YNL153C & 11 & 4 & 22 & -25.8486 & 20.75055 & -36.9863 & 19.35484 & 34.31953 \\
\hline BOR1 & YNL275W & 11 & 4 & 23 & 18.51852 & -27.1676 & 27.82835 & 45.59471 & 52.32274 \\
\hline HIS3 & YOR202W & 11 & 4 & 24 & -39.3023 & 20.04479 & 40.55172 & & \\
\hline HIS3 & YOR202W & 11 & 5 & 1 & 37.88546 & 31.91721 & 66.21136 & & \\
\hline URA10 & YMR271C & 11 & 5 & 2 & 3.658537 & 43.01887 & 77.25322 & 44.68547 & 26.69584 \\
\hline \multirow[t]{2}{*}{ COX5A } & YNL052W & 11 & 5 & 3 & -11.7647 & -7.51566 & 60.45576 & -40.8571 & 24.5509 \\
\hline & YMR269W & 11 & 5 & 4 & 29.98028 & 36.20474 & 84.04255 & -15.8249 & 22.18543 \\
\hline COG5 & YNL051W & 11 & 5 & 5 & 63.46801 & 24.23581 & 66.4311 & -21.2366 & 14.57726 \\
\hline \multirow[t]{3}{*}{ RSN1 } & YMR266W & 11 & 5 & 6 & 37.22045 & 46.64926 & 58.28877 & 37.23404 & 36.09467 \\
\hline & YNL050C & 11 & 5 & 7 & 34.43918 & 33.6688 & 30.63063 & 10.25641 & 35.38012 \\
\hline & YMR265C & 11 & 5 & 8 & -6.59898 & -7.35632 & 62.2276 & 18.62464 & 24.52229 \\
\hline SFB2 & YNL049C & 11 & 5 & 9 & 43.63636 & 44.06355 & 47.29064 & 25.12821 & 34.8285 \\
\hline \multirow[t]{2}{*}{ CUE1 } & YMR264W & 11 & 5 & 10 & 54.2029 & 42.90822 & 7.279693 & 21.28205 & 21.38728 \\
\hline & YNL046W & 11 & 5 & 11 & 40.39146 & 54.96536 & 85.95166 & 26.84932 & 34.26184 \\
\hline \multirow[t]{3}{*}{ SAP30 } & YMR263W & 11 & 5 & 12 & 39.74152 & 9.572301 & 56.58602 & 17.15818 & 19.93355 \\
\hline & YNL045W & 11 & 5 & 13 & 41.91063 & 26.39029 & 79.77099 & 0.892857 & 39.52096 \\
\hline & YMR262W & 11 & 5 & 14 & 35.76052 & -7.90861 & 80.07663 & 35.67251 & 33.62573 \\
\hline YIP3 & YNL044W & 11 & 5 & 15 & 32.07547 & 54.45545 & 73.09645 & -10.7345 & 28.27381 \\
\hline \multirow[t]{3}{*}{ TPS3 } & YMR261C & 11 & 5 & 16 & 14.08228 & 15.86957 & 68.04124 & 25 & 41.17647 \\
\hline & YNL043C & 11 & 5 & 17 & -10.4816 & 37.77293 & 76.54321 & 14.24149 & 42.15116 \\
\hline & YMR259C & 11 & 5 & 18 & -17.5711 & 62.1447 & 79.77778 & 12.29947 & 45.38043 \\
\hline CoG6 & YNL041C & 11 & 5 & 19 & -22.4719 & 51.53374 & 78.92857 & -0.83333 & 40.37433 \\
\hline
\end{tabular}




\begin{tabular}{|c|c|c|c|c|c|c|c|c|c|}
\hline & YMR258C & 11 & 5 & 20 & 54.17476 & 22.29437 & 90.57816 & 35.79235 & 50 \\
\hline & YNL040W & 11 & 5 & 21 & 55.19231 & 38.44857 & 100 & 24.27184 & 51.31965 \\
\hline cox7 & YMR256C & 11 & 5 & 22 & 57.11462 & -43.346 & 52.85253 & -65.2542 & 16.94215 \\
\hline IDH1 & YNL037C & 11 & 5 & 23 & -31.6017 & -23.7154 & 34.57944 & -12.9032 & 21.99413 \\
\hline HIS3 & YOR202W & 11 & 5 & 24 & -6.60377 & 23.3809 & 41.42661 & & \\
\hline \multirow[t]{2}{*}{ HIS3 } & YOR202W & 11 & 6 & 1 & 46.25796 & 36.6055 & 23.00725 & & \\
\hline & YNL190W & 11 & 6 & 2 & -18.4416 & 54.25868 & 72.35772 & 61.21212 & 35.18887 \\
\hline \multirow[t]{2}{*}{ RPL18B } & YNL301C & 11 & 6 & 3 & -10.7759 & -36.3636 & 78.54406 & 16.12091 & 31.09049 \\
\hline & YNL187W & 11 & 6 & 4 & -35.446 & 36.79612 & 62.51366 & 16.89815 & 31.75487 \\
\hline TOS6 & YNL300W & 11 & 6 & 5 & -37.7528 & 49.44882 & 59.23379 & 2.105263 & 21.32565 \\
\hline NPR1 & YNL183C & 11 & 6 & 6 & 20.16349 & 54.2811 & 70.99237 & -3.53982 & 44.56522 \\
\hline TRF5 & YNL299W & 11 & 6 & 7 & 9.708738 & 37.53815 & 89.91597 & 61.18252 & 40.30769 \\
\hline SRF6 & YNL179C & 11 & 6 & 8 & 46.00326 & 55.21886 & 64.39206 & 9.142857 & 32.35294 \\
\hline \multirow[t]{2}{*}{ CLA4 } & YNL298W & 11 & 6 & 9 & 25.57545 & -6.03448 & 84.2 & 48.80952 & missing colony \\
\hline & YNL176C & 11 & 6 & 10 & 39.41842 & 0.964187 & 76.53061 & 39.39394 & 41.14441 \\
\hline MON2 & YNL297C & 11 & 6 & 11 & 35.16874 & 8.513932 & 79.00763 & 11.74377 & 39.85765 \\
\hline \multirow[t]{2}{*}{ NOP13 } & YNL175C & 11 & 6 & 12 & 41.45078 & 52.26131 & 51.96507 & -18.5714 & -2.05479 \\
\hline & YNL296W & 11 & 6 & 13 & -60 & 52.40642 & 67.90123 & -9.36556 & 18.4466 \\
\hline \multirow[t]{3}{*}{ MDG1 } & YNL173C & 11 & 6 & 14 & -48.0198 & 47.88732 & 92 & 22.73902 & 41.31737 \\
\hline & YNL295W & 11 & 6 & 15 & 31.05802 & -44.469 & 64.69072 & -14.3382 & 25.37879 \\
\hline & YNL171C & 11 & 6 & 16 & 30.67332 & -6.95652 & 33.125 & 22.02797 & 37.80919 \\
\hline \multirow[t]{2}{*}{ RIM21 } & YNL294C & 11 & 6 & 17 & 30.37975 & -20.4 & 57.33831 & -3.97727 & 27.54098 \\
\hline & YNL170W & 11 & 6 & 18 & -9.17874 & 47.60638 & 100 & -9.34579 & 24.6988 \\
\hline MSB3 & YNL293W & 11 & 6 & 19 & -10.3659 & 49.52681 & 76.31579 & -3.69393 & 31.96721 \\
\hline PSD1 & YNL169C & 11 & 6 & 20 & -2.50896 & 38.69732 & 60.39604 & -11.5385 & 44.01294 \\
\hline PUS4 & YNL292W & 11 & 6 & 21 & -39.5652 & 30.74581 & 83.9851 & 24.17582 & 49.85755 \\
\hline FMP41 & YNL168C & 11 & 6 & 22 & -12.5806 & -38.0457 & 33.33333 & -21.148 & 37.4269 \\
\hline MID1 & YNL291C & 11 & 6 & 23 & 35.22013 & 36.48208 & 61.21495 & 51.97368 & 57.00483 \\
\hline HIS3 & YOR202W & 11 & 6 & 24 & -9.25508 & 27.84399 & 61.03352 & & \\
\hline HIS3 & YOR202W & 11 & 7 & 1 & 46.08379 & 66.42468 & 72.50787 & & \\
\hline YKU70 & YMR284W & 11 & 7 & 2 & 25.81169 & 34.03933 & 57.77778 & 53.45912 & 33.06878 \\
\hline FKH2 & YNL068C & 11 & 7 & 3 & -9.58409 & 37.6914 & 70.37702 & 25.8794 & 37.76596 \\
\hline RIT1 & YMR283C & 11 & 7 & 4 & 38.48739 & 37.21925 & 51.6632 & 36.43216 & 40 \\
\hline RPL9B & YNL067W & 11 & 7 & 5 & 37.89649 & 42.38833 & 81.37803 & 38.22844 & 66.09195 \\
\hline AEP2 & YMR282C & 11 & 7 & 6 & 34.14211 & 21.88139 & 63.67816 & 9.677419 & 41.15523 \\
\hline SUN4 & YNL066W & 11 & 7 & 7 & 37.57862 & 41.77632 & 79.07407 & 27.73537 & 37.04735 \\
\hline CAT8 & YMR280C & 11 & 7 & 8 & 40.99822 & 19.36759 & 62.98077 & 7.03125 & 19.04762 \\
\hline \multirow[t]{2}{*}{ AQR1 } & YNL065W & 11 & 7 & 9 & 44.82759 & 33.8843 & 81.30252 & 37.27034 & 54.80226 \\
\hline & YMR279C & 11 & 7 & 10 & 44.79167 & 49.35733 & 15.05017 & 23.8806 & 36.47416 \\
\hline \multirow[t]{2}{*}{ CPA2 } & YJR109C & 11 & 7 & 11 & 41.99346 & 25.83065 & 65.6051 & 31.28342 & 44.27711 \\
\hline & YMR278W & 11 & 7 & 12 & 37.33333 & 26.13122 & 31.30904 & 33.42175 & 49.56522 \\
\hline YDJ1 & YNL064C & 11 & 7 & 13 & 38.00384 & 46.38783 & 64.0599 & 2.826855 & 41.00719 \\
\hline DSK2 & YMR276W & 11 & 7 & 14 & 25.27233 & 64.41281 & 75.23452 & -10.3064 & 31.51515 \\
\hline
\end{tabular}




\begin{tabular}{|c|c|c|c|c|c|c|c|c|c|}
\hline MTQ1 & YNL063W & 11 & 7 & 15 & 38.35404 & 28.89138 & 34.58647 & 9.477124 & 35.86207 \\
\hline \multirow[t]{2}{*}{ BUL1 } & YMR275C & 11 & 7 & 16 & 22.03065 & 45.36465 & 78.11705 & 35.08287 & 47.68786 \\
\hline & YNL058C & 11 & 7 & 17 & -24.5431 & 48.24281 & 58.96806 & 19.87768 & 41.78273 \\
\hline \multirow[t]{2}{*}{ RCE1 } & YMR274C & 11 & 7 & 18 & 55.26316 & 36.06103 & 73.83178 & 33.79888 & 47.60563 \\
\hline & YNL057W & 11 & 7 & 19 & 0.911854 & 34.76298 & 46.27767 & -38.2184 & 43.30709 \\
\hline \multirow[t]{2}{*}{ ZDS1 } & YMR273C & 11 & 7 & 20 & 30.20408 & -6.34921 & 31.56966 & 39.94083 & 47.10983 \\
\hline & YNL056W & 11 & 7 & 21 & 9.621993 & 3.099174 & 55.39568 & -9.45513 & 53.56371 \\
\hline SCS7 & YMR272C & 11 & 7 & 22 & -14.4105 & -13.9037 & 36.33136 & 7.594937 & 49.38272 \\
\hline VAC7 & YNL054W & 11 & 7 & 23 & -6.4588 & 51.29108 & 73.12661 & 7.904412 & 42.15457 \\
\hline HIS3 & YOR202W & 11 & 7 & 24 & -4.60177 & 30.98434 & 63.45934 & & \\
\hline HIS3 & YOR202W & 11 & 8 & 1 & -5.19126 & 27.13615 & 79.98489 & & \\
\hline PSY2 & YNL201C & 11 & 8 & 2 & 36.9637 & 59.86717 & 57.69231 & 69.87952 & 45.671 \\
\hline \multirow[t]{2}{*}{ HXT14 } & YNL318C & 11 & 8 & 3 & 10.76642 & 60.33058 & 53.41923 & 45.65217 & 42.75362 \\
\hline & YNL200C & 11 & 8 & 4 & -25.8824 & 24.08759 & 63.23988 & 35.7513 & 36.07427 \\
\hline PHA2 & YNL316C & 11 & 8 & 5 & 37.71626 & 48.29396 & 39.68825 & 6.284153 & 30.39216 \\
\hline GCR2 & YNL199C & 11 & 8 & 6 & 27.03704 & 40.74941 & 68.27309 & -8.94855 & 14.12104 \\
\hline \multirow[t]{2}{*}{ ATP11 } & YNL315C & 11 & 8 & 7 & 40.25045 & 37.18861 & 90.82031 & 30.71161 & 32.05128 \\
\hline & YNL198C & 11 & 8 & 8 & 26.0274 & 54.9763 & 53.5 & 37.81513 & 47.92332 \\
\hline DAL82 & YNL314W & 11 & 8 & 9 & 34.62158 & 41.37339 & 73.84615 & -27.6353 & 24.24242 \\
\hline \multirow[t]{2}{*}{ WHI3 } & YNL197C & 11 & 8 & 10 & 38.80866 & -21.5889 & 72.8125 & 43.10345 & 50.63694 \\
\hline & YNL311C & 11 & 8 & 11 & 40.63604 & 16.13793 & 60.10855 & 10.03135 & 38.5906 \\
\hline SLZ1 & YNL196C & 11 & 8 & 12 & 62.43496 & 34.53318 & -33.5821 & 1.944444 & 40.42553 \\
\hline \multirow[t]{2}{*}{ STB1 } & YNL309W & 11 & 8 & 13 & 48.11881 & -2.1028 & 35.43401 & 8.059701 & 33.73494 \\
\hline & YNL195C & 11 & 8 & 14 & 34.07643 & 15.33333 & 57.93201 & 34.75936 & 44.70588 \\
\hline \multirow[t]{4}{*}{ MCK1 } & YNL307C & 11 & 8 & 15 & 33.11828 & 44.02332 & 84.94624 & 45.09202 & 36.76012 \\
\hline & YNL194C & 11 & 8 & 16 & 56.63475 & 43.68771 & 79.94792 & 8.139535 & 41.74174 \\
\hline & YNL305C & 11 & 8 & 17 & 30.26706 & 33.01775 & 84.43114 & -14.7222 & 41.81287 \\
\hline & YNL193W & 11 & 8 & 18 & 24.34457 & 49.77376 & 95.45455 & 12.53731 & 46.27329 \\
\hline YPT11 & YNL304W & 11 & 8 & 19 & 18.71227 & 64.48864 & 74.31694 & -5.59006 & 31.13208 \\
\hline \multirow[t]{3}{*}{ CHS1 } & YNL192W & 11 & 8 & 20 & 54.4164 & 34.86842 & 76.27119 & 23.3677 & 58.36177 \\
\hline & YNL303W & 11 & 8 & 21 & 44.86301 & -30.2428 & 42.73595 & 6.845238 & 42.24138 \\
\hline & YNL191W & 11 & 8 & 22 & -8.49858 & 39.43894 & 100 & 21.06825 & 42.98507 \\
\hline RPS19B & YNL302C & 11 & 8 & 23 & 11.29032 & 45.56962 & 72.64574 & -31.2183 & 44.44444 \\
\hline HIS3 & YOR202W & 11 & 8 & 24 & 44.31599 & 32.86788 & 38.91509 & & \\
\hline HIS3 & YOR202W & 11 & 9 & 1 & -11.68 & 43.23432 & 53.23713 & & \\
\hline PRP12 & YMR302C & 11 & 9 & 2 & -17.5824 & 31.11814 & 75.87035 & 51.66994 & 71.96078 \\
\hline SWS2 & YNL081C & 11 & 9 & 3 & 18.18182 & 28.08989 & -72.1311 & -101.567 & -0.83682 \\
\hline \multirow[t]{2}{*}{ ADE4 } & YMR300C & 11 & 9 & 4 & 35.48387 & 31.63507 & 61.86709 & 23.76238 & 53.57143 \\
\hline & YNL080C & 11 & 9 & 5 & 21.07728 & 64.99417 & 56.52742 & 10.9215 & 28.68217 \\
\hline DYN3 & YMR299C & 11 & 9 & 6 & -22.1719 & 48.37728 & 52.75288 & 33.42466 & 36.68639 \\
\hline TPM1 & YNL079C & 11 & 9 & 7 & 40 & \#DIV/0! & 50 & 12.26994 & 19.01639 \\
\hline PRC1 & YMR297W & 11 & 9 & 8 & 46.86347 & 49.14934 & 46.75497 & 48.70466 & 32.27666 \\
\hline NIS1 & YNL078W & 11 & 9 & 9 & -54.6559 & 33.44828 & \#DIV/0! & 9.363296 & 40.99617 \\
\hline
\end{tabular}




\begin{tabular}{|c|c|c|c|c|c|c|c|c|c|}
\hline & YMR295C & 11 & 9 & 10 & -57.7465 & 35.58824 & 47.41276 & 16.20112 & 44.29967 \\
\hline \multirow[t]{2}{*}{ APJ1 } & $\begin{array}{l}\text { YNL077W } \\
\text { YMR294W- }\end{array}$ & 11 & 9 & 11 & 39.38356 & 8.154506 & 56.72269 & 32.68156 & 58.89571 \\
\hline & $A$ & 11 & 9 & 12 & 35.23622 & -8.13772 & 83.12808 & 35.77023 & 51.76471 \\
\hline MKS1 & YNL076W & 11 & 9 & 13 & 35.9375 & 36.11473 & 33.80282 & -14.956 & 41.26506 \\
\hline JNM1 & YMR294W & 11 & 9 & 14 & -6.08466 & 26.27551 & 65.10264 & -14.1509 & 24.11348 \\
\hline MLF3 & YNL074C & 11 & 9 & 15 & 18.7067 & 34.13015 & 62.23278 & 21.15942 & 52.02312 \\
\hline GOT1 & YMR292W & 11 & 9 & 16 & -0.98765 & 30.16086 & 84.63768 & 13.71429 & 48.33837 \\
\hline \multirow[t]{2}{*}{ RNH201 } & YNL072W & 11 & 9 & 17 & -28.3154 & 38.38384 & 70.05348 & 9.818182 & 51.23675 \\
\hline & YMR291W & 11 & 9 & 18 & 56.38507 & 55.74913 & 70.39911 & 18.31832 & 52.7439 \\
\hline LAT1 & YNL071W & 11 & 9 & 19 & 16.15385 & 40.50822 & 100 & 29.78723 & 52.61438 \\
\hline ABZ2 & YMR289W & 11 & 9 & 20 & 52.05811 & 34.48276 & 55.42522 & -16.1058 & 44.71744 \\
\hline TOM7 & YNL070W & 11 & 9 & 21 & -15.2091 & 42.13483 & 69.11447 & 15.32847 & 51.76056 \\
\hline NGL2 & YMR285C & 11 & 9 & 22 & -42.0824 & -16.8739 & 36.8306 & 17.04545 & 47.44318 \\
\hline RPL16B & YNL069C & 11 & 9 & 23 & 35.8156 & 34.96503 & 12.96928 & 54.56853 & 63.29114 \\
\hline HIS3 & YOR202W & 11 & 9 & 24 & 69.28675 & 30.89888 & 72.76786 & & \\
\hline \multirow[t]{2}{*}{ HIS3 } & YOR202W & 11 & 10 & 1 & -7.11921 & 44.85356 & -27.1864 & & \\
\hline & YNL217W & 11 & 10 & 2 & -39.2578 & 55.24412 & 61.10553 & 37.41497 & 50.50251 \\
\hline PEX6 & YNL329C & 11 & 10 & 3 & -28.178 & 58.1265 & 64.14882 & 53.30739 & 30.46218 \\
\hline IES2 & YNL215W & 11 & 10 & 4 & -17.2043 & 57.66807 & 54.70085 & 37.45928 & 38.57567 \\
\hline MDJ2 & YNL328C & 11 & 10 & 5 & 34.07155 & 26.44135 & 68.13725 & 37.2796 & 37.31778 \\
\hline PEX17 & YNL214W & 11 & 10 & 6 & 56.85096 & 1.557632 & 72.81879 & 8.136483 & 30.3681 \\
\hline EGT2 & YNL327W & 11 & 10 & 7 & 39.89362 & 26.10294 & 71.09557 & -19.837 & 32.38095 \\
\hline VID27 & YNL212W & 11 & 10 & 8 & 35.71429 & 60.91743 & 78.92074 & 20.50562 & 33.97436 \\
\hline \multirow[t]{2}{*}{ PFA3 } & YNL326C & 11 & 10 & 9 & 32.55361 & 15.6682 & 54.35953 & 16.52661 & 40.77381 \\
\hline & YNL211C & 11 & 10 & 10 & 40.73427 & 20.06543 & 65 & 13.61111 & 42.94671 \\
\hline \multirow[t]{3}{*}{ FIG4 } & YNL325C & 11 & 10 & 11 & 40.23179 & -5.09383 & 76.41357 & 37.20379 & 33.12693 \\
\hline & YNL208W & 11 & 10 & 12 & 43.69085 & 0.632911 & 81.33562 & -16.0763 & 46.89266 \\
\hline & YNL324W & 11 & 10 & 13 & 43.01242 & 44.23963 & 71.0443 & 47.17445 & 48.91641 \\
\hline RTT106 & YNL206C & 11 & 10 & 14 & -53.8251 & 13.11953 & 79.32862 & -0.28249 & 38.69048 \\
\hline \multirow[t]{2}{*}{ LEM3 } & YNL323W & 11 & 10 & 15 & 48.23695 & -10.3011 & 84.75936 & -21.8023 & 12.2449 \\
\hline & YNL205C & 11 & 10 & 16 & 12.65823 & 36.25954 & 64.88095 & 18.9415 & 50.8982 \\
\hline KRE1 & YNL322C & 11 & 10 & 17 & 22.85251 & 43.47826 & 88.43813 & 18.05556 & 36.85637 \\
\hline \multirow[t]{4}{*}{ SPS18 } & YNL204C & 11 & 10 & 18 & -1.77936 & 27.833 & 70.58824 & 25.83333 & 49.68944 \\
\hline & YNL321W & 11 & 10 & 19 & -8.79828 & 53.08642 & 78.78289 & -4.89297 & 47.09677 \\
\hline & YNL203C & 11 & 10 & 20 & -5.85106 & -38.7368 & 17.34694 & -75.2119 & 38.22785 \\
\hline & YNL320W & 11 & 10 & 21 & 33.125 & 46.54255 & 49.24875 & 33.52436 & 47.64706 \\
\hline \multirow[t]{2}{*}{ SPS19 } & YNL202W & 11 & 10 & 22 & 30 & 13.71191 & 32.76983 & 22.92264 & 47.67123 \\
\hline & YNL319W & 11 & 10 & 23 & 24.71591 & 22.38164 & 39.18919 & 36.59218 & 45.53314 \\
\hline HIS3 & YOR202W & 11 & 10 & 24 & -18.3486 & 32.01804 & 29.37337 & & \\
\hline HIS3 & YOR202W & 11 & 11 & 1 & -17.1076 & 10.69519 & 24.00706 & & \\
\hline \multirow[t]{2}{*}{ TGL3 } & YMR313C & 11 & 11 & 2 & 26.22642 & 60.57386 & 69.56522 & 43.94904 & 35.73201 \\
\hline & YNL095C & 11 & 11 & 3 & -19.6464 & 63.71681 & 64.53089 & 31.56627 & 40.15345 \\
\hline
\end{tabular}




\begin{tabular}{|c|c|c|c|c|c|c|c|c|c|}
\hline ELP6 & YMR312W & 11 & 11 & 4 & -30.3226 & 36.83036 & 87.93774 & 28.52459 & 52.32975 \\
\hline APP1 & YNL094W & 11 & 11 & 5 & 35.05155 & -3.28467 & 65.20424 & 44.93506 & 34.62687 \\
\hline GLC8 & YMR311C & 11 & 11 & 6 & 38.55856 & 38.72378 & 33.15217 & 29.35323 & 38.0805 \\
\hline \multirow[t]{3}{*}{ YPT53 } & YNL093W & 11 & 11 & 7 & 39.03654 & 43.98798 & 80 & 10.27778 & 43.33333 \\
\hline & YMR310C & 11 & 11 & 8 & 31.01045 & -9.2869 & 36.92308 & 20.82192 & 26.77419 \\
\hline & YNL092W & 11 & 11 & 9 & -15.6089 & 48.09228 & 57.69231 & -40.8719 & 35.16129 \\
\hline GAS1 & YMR307W & 11 & 11 & 10 & -31.9489 & -23.1465 & 53.17726 & -121.184 & 54.97076 \\
\hline NST1 & YNL091W & 11 & 11 & 11 & 35.20833 & 42.13075 & 79.05138 & -25.2252 & 18.67089 \\
\hline FKS3 & YMR306W & 11 & 11 & 12 & 34.88806 & 51.16279 & 62.97199 & -5.9322 & 42.23602 \\
\hline \multirow[t]{3}{*}{$\mathrm{RHO} 2$} & $\begin{array}{l}\text { YNL090W } \\
\text { YMR306C- }\end{array}$ & 11 & 11 & 13 & \#DIV/0! & \#DIV/0! & 68.78307 & 4.398827 & 20.71429 \\
\hline & $A$ & 11 & 11 & 14 & 27.25599 & 29.56927 & 41.52652 & 21.73913 & 44.54545 \\
\hline & YNL089C & 11 & 11 & 15 & 37.02213 & 51.00671 & 70.61611 & 17.79449 & 48.45938 \\
\hline SCW10 & YMR305C & 11 & 11 & 16 & 43.65079 & 37.17827 & 85.97938 & -18.2825 & 41.36808 \\
\hline TCB2 & YNL087W & 11 & 11 & 17 & 34.01254 & 39.61772 & 74.30998 & -7.44337 & 29.59184 \\
\hline UBP15 & YMR304W & 11 & 11 & 18 & -32.2251 & 25.02744 & 71.09974 & -11.1782 & 37.79264 \\
\hline \multirow[t]{2}{*}{ MKT1 } & $\begin{array}{l}\text { YNL085W } \\
\text { YMR304C- }\end{array}$ & 11 & 11 & 19 & 56.30027 & 64.22947 & 66.48352 & 47.12644 & 63.33333 \\
\hline & $A$ & 11 & 11 & 20 & 59.40054 & 41.20763 & 57.65125 & -7.12074 & 35.47401 \\
\hline SAL1 & YNL083W & 11 & 11 & 21 & 25.61576 & 9.445277 & 69.47162 & 15.34247 & 43.75 \\
\hline $\mathrm{ADH} 2$ & YMR303C & 11 & 11 & 22 & -30.3297 & -3.28571 & 37.97468 & -21.3622 & 32.19178 \\
\hline PMS1 & YNL082W & 11 & 11 & 23 & 8.045977 & 35.90604 & 58.08824 & 30.86681 & 60.96386 \\
\hline HIS3 & YOR202W & 11 & 11 & 24 & 27.91461 & 31.59645 & 72.98387 & & \\
\hline HIS3 & YOR202W & 11 & 12 & 1 & 15.26104 & 14.06699 & 16.92308 & & \\
\hline \multirow[t]{2}{*}{ BNI4 } & YNL233W & 11 & 12 & 2 & 23.47505 & 59.30851 & 58.65031 & 32.25806 & 46.24697 \\
\hline & YNR004W & 11 & 12 & 3 & -23.5421 & 13.71528 & 62.11699 & 41.50943 & 30.95238 \\
\hline PDR16 & YNL231C & 11 & 12 & 4 & 39.49731 & 13.99177 & 36.01695 & 21.69576 & 31.85596 \\
\hline FUN34 & YNR002C & 11 & 12 & 5 & 36.72727 & 43.62745 & 78.125 & 29.31442 & 43.26648 \\
\hline ELA1 & YNL230C & 11 & 12 & 6 & 34.47684 & 43.52283 & 64.32432 & -6.30372 & 16.24549 \\
\hline CIT1 & YNR001C & 11 & 12 & 7 & 35.10638 & 36.64596 & 30.81571 & 24.47368 & 39.69697 \\
\hline URE2 & YNL229C & 11 & 12 & 8 & 38.00738 & 49.33206 & 87.66447 & 19.34426 & 29.96255 \\
\hline \multirow[t]{3}{*}{ DOM34 } & YNL001W & 11 & 12 & 9 & 38.81188 & 50.92402 & 76.25 & 59.72696 & 60.58091 \\
\hline & YNL228W & 11 & 12 & 10 & 34.65784 & 49.53464 & 79.80226 & 1.271186 & 41.91919 \\
\hline & YNL338W & 11 & 12 & 11 & 36.39775 & 32.93333 & 42.66304 & 31.05134 & 48.61111 \\
\hline JJJ1 & YNL227C & 11 & 12 & 12 & 36.36364 & -25.817 & 67.42712 & 33.44828 & 47.31183 \\
\hline \multirow[t]{4}{*}{$\cos 1$} & YNL336W & 11 & 12 & 13 & -53.9773 & 18.13953 & 44.31525 & 42.25352 & 52.97297 \\
\hline & YNL226W & 11 & 12 & 14 & 26.65441 & 18.05825 & 53.51351 & 27.42616 & 46.88995 \\
\hline & YNL335W & 11 & 12 & 15 & 38.85918 & 42.13007 & 56.79542 & 21.81818 & 54.41176 \\
\hline & YNL224C & 11 & 12 & 16 & 31.45455 & 42.38603 & 79.5756 & 27.58621 & 47.03947 \\
\hline $\mathrm{SNO} 2$ & YNL334C & 11 & 12 & 17 & 36.08059 & 31.95548 & 37.08861 & -0.88235 & 46.15385 \\
\hline ATG4 & YNL223W & 11 & 12 & 18 & 42.38683 & -35.2941 & 66.00985 & 17.05202 & 40.44586 \\
\hline SNZ2 & YNL333W & 11 & 12 & 19 & -29.3963 & 43.69427 & 74.24242 & 18.80597 & 48.4058 \\
\hline ALG9 & YNL219C & 11 & 12 & 20 & 23.52941 & 18.98527 & 67.93651 & 40.33613 & 55.58739 \\
\hline
\end{tabular}




\begin{tabular}{|c|c|c|c|c|c|c|c|c|c|}
\hline THI12 & YNL332W & 11 & 12 & 21 & 24.91166 & 31.82266 & -50.2128 & 14.86486 & 51.52355 \\
\hline MGS1 & YNL218W & 11 & 12 & 22 & -20 & 30.79961 & 53.05514 & 44.94624 & 52.73224 \\
\hline RPD3 & YNL330C & 11 & 12 & 23 & -11.245 & 41.60942 & 10.32746 & 32.66332 & 55.33708 \\
\hline HIS3 & YOR202W & 11 & 12 & 24 & 65.37217 & 27.15376 & 10.24182 & & \\
\hline HIS3 & YOR202W & 11 & 13 & 1 & -8.80649 & 34.38914 & 23.14709 & & \\
\hline \multirow[t]{4}{*}{ YRF1-6 } & YNL339C & 11 & 13 & 2 & 28.44639 & 33.92769 & 48.17293 & 26.71395 & 31.04265 \\
\hline & YNL115C & 11 & 13 & 3 & 26.08696 & 20 & 68.83721 & 39.39394 & 34.07202 \\
\hline & YMR326C & 11 & 13 & 4 & 42.53247 & 33.17536 & 60.11976 & 25.92593 & 43.85475 \\
\hline & YNL108C & 11 & 13 & 5 & 45.7478 & 11.26761 & 44.09722 & 25.06667 & 43.59756 \\
\hline SNO4 & YMR322C & 11 & 13 & 6 & -41.1184 & 50.34602 & 85.04673 & 25.1269 & 46.78899 \\
\hline \multirow[t]{2}{*}{ YAF9 } & YNL107W & 11 & 13 & 7 & 35.90664 & 30.65187 & 0 & 45.76271 & 56.36364 \\
\hline & YMR320W & 11 & 13 & 8 & 30.10563 & 38.94501 & 54.48276 & 15.81769 & 32.22892 \\
\hline INP52 & YNL106C & 11 & 13 & 9 & 31.07143 & 2.296451 & 73.89163 & 2.216066 & 13.20755 \\
\hline \multirow[t]{2}{*}{ FET4 } & YMR319C & 11 & 13 & 10 & -51.1173 & 7.522698 & 55.49296 & 46.72304 & 40.11299 \\
\hline & YNL105W & 11 & 13 & 11 & -49.1525 & 49.03934 & 60.53512 & 37.53086 & 50.73314 \\
\hline ADH6 & YMR318C & 11 & 13 & 12 & -57.8125 & 41.60584 & 22.66667 & 7.848837 & 19.23077 \\
\hline \multirow[t]{2}{*}{ LEU4 } & YNL104C & 11 & 13 & 13 & -50 & 60.96866 & \#DIV/0! & 21.5847 & 47.02194 \\
\hline & YMR317W & 11 & 13 & 14 & -54.6835 & 2.118003 & 52.10084 & 31.50685 & 41.1215 \\
\hline AVT4 & YNL101W & 11 & 13 & 15 & -35.1899 & 47.91252 & -1.97628 & 16.84211 & 49.31129 \\
\hline \multirow[t]{3}{*}{ DIA1 } & YMR316W & 11 & 13 & 16 & -39.5939 & 30.71749 & 33.9726 & 38.70968 & 55.04323 \\
\hline & $\begin{array}{l}\text { YNL100W } \\
\text { YMR316C- }\end{array}$ & 11 & 13 & 17 & -49.1228 & 45.07389 & -148.077 & 25.61983 & 57.27273 \\
\hline & B & 11 & 13 & 18 & -43.8692 & 20.74689 & 27.73208 & -34.2029 & 30.03663 \\
\hline \multirow[t]{2}{*}{ OCA1 } & $\begin{array}{l}\text { YNL099C } \\
\text { YNR16C }\end{array}$ & 11 & 13 & 19 & -46.2006 & 9.965636 & 68.16479 & 20.68966 & 42.74194 \\
\hline & A & 11 & 13 & 20 & 40.18349 & 44.46721 & 43.309 & 6.818182 & 43.66667 \\
\hline \multirow[t]{2}{*}{ RAS2 } & YNL098C & 11 & 13 & 21 & -22.7273 & 10.46154 & 15.18987 & 27.07182 & 32.74021 \\
\hline & YMR315W & 11 & 13 & 22 & 58.48303 & 77.12177 & 77.72727 & 25 & 72.48062 \\
\hline $\mathrm{PHO} 23$ & YNL097C & 11 & 13 & 23 & -27.8689 & 39.1844 & 58.94309 & 17.79497 & 28.64865 \\
\hline HIS3 & YOR202W & 11 & 13 & 24 & 65.06747 & 27.58242 & 33.60768 & & \\
\hline HIS3 & YOR202W & 11 & 14 & 1 & 34 & 59.14684 & 67.6259 & & \\
\hline \multirow[t]{2}{*}{ TEX1 } & YNL253W & 11 & 14 & 2 & 42.35727 & 24.31641 & 54.53515 & 43.06723 & 16.85649 \\
\hline & YNR018W & 11 & 14 & 3 & 81.05437 & 78.27298 & 64.7603 & -12.0253 & 29.4702 \\
\hline MPA43 & YNL249C & 11 & 14 & 4 & -13.4831 & 48.3871 & 65.69343 & 37.35763 & 43.90935 \\
\hline SMM1 & YNR015W & 11 & 14 & 5 & 32.9331 & 3.601695 & 63.48425 & 18.03279 & 43.49112 \\
\hline \multirow[t]{2}{*}{ VPS75 } & YNL246W & 11 & 14 & 6 & 31.11546 & 3.826955 & -13.3929 & 12.5964 & 20.19544 \\
\hline & YNR014W & 11 & 14 & 7 & -50.9695 & 24.7093 & 47.55662 & 20 & 23.97476 \\
\hline ATG2 & YNL242W & 11 & 14 & 8 & -45.8221 & 12.75441 & 68.63354 & 30.60109 & 48.50746 \\
\hline PHO91 & YNR013C & 11 & 14 & 9 & -30.6569 & 5.30303 & 48.32215 & 24.0099 & 45.60907 \\
\hline ZWF1 & YNL241C & 11 & 14 & 10 & -42.6316 & -20.6439 & 53.7594 & 40.82126 & 54.71125 \\
\hline URK1 & YNR012W & 11 & 14 & 11 & -59.1304 & 46.21479 & 26.11684 & 27.3399 & 25.66372 \\
\hline LAP3 & YNL239W & 11 & 14 & 12 & -38.3632 & 35.58394 & 36.55172 & 34.38257 & 33.96226 \\
\hline CSE2 & YNR010W & 11 & 14 & 13 & -29.0155 & 39.62264 & 79.52218 & 24.59016 & 48.19672 \\
\hline
\end{tabular}




\begin{tabular}{|c|c|c|c|c|c|c|c|c|c|}
\hline \multirow[t]{2}{*}{ KEX2 } & YNL238W & 11 & 14 & 14 & \#DIV/0! & \#DIV/0! & \#DIV/0! & -33.452 & 8.270677 \\
\hline & YNR009W & 11 & 14 & 15 & -36.7397 & 64.7452 & 44.63625 & 32.5779 & 27.06271 \\
\hline YTP1 & YNL237W & 11 & 14 & 16 & -32.3607 & 46.96545 & 17.62712 & 37.68473 & 46.84932 \\
\hline LRO1 & YNR008W & 11 & 14 & 17 & -36.5591 & 42.71938 & \#DIV/0! & 39.66942 & 55.3719 \\
\hline SIN4 & YNL236W & 11 & 14 & 18 & 35.61873 & -23.3333 & 62.41007 & -245.968 & 15.38462 \\
\hline \multirow[t]{2}{*}{ ATG3 } & YNR007C & 11 & 14 & 19 & 63.9313 & 36.64773 & -37.4046 & 22.72727 & 56.87831 \\
\hline & YNL235C & 11 & 14 & 20 & 12.39669 & 55.96026 & 64.12556 & 25.13369 & 33.47107 \\
\hline \multirow[t]{3}{*}{ VPS27 } & YNR006W & 11 & 14 & 21 & \#DIV/0! & \#DIV/0! & 63.93443 & -185 & 37.46702 \\
\hline & YNL234W & 11 & 14 & 22 & 60.18519 & 48.20144 & 60.75472 & -1.1925 & 64.84211 \\
\hline & YNR005C & 11 & 14 & 23 & 11.2532 & 43.19149 & 20.26862 & 37.09677 & 52.34742 \\
\hline HIS3 & YOR202W & 11 & 14 & 24 & 47.52475 & 33.65269 & 44.12214 & & \\
\hline HIS3 & YOR202W & 11 & 15 & 1 & 61.19403 & 37.99472 & 46.38298 & & \\
\hline HDA1 & YNL021W & 11 & 15 & 2 & 82.39203 & -39.2799 & 59.94526 & & \\
\hline NRK1 & YNL129W & 11 & 15 & 3 & 62.21837 & -31.3253 & 60.39604 & & \\
\hline ARK1 & YNL020C & 11 & 15 & 4 & 27.70898 & 25.87917 & 57.17647 & & \\
\hline TEP1 & YNL128W & 11 & 15 & 5 & -36.5957 & 29.84456 & 73.78641 & & \\
\hline PUB1 & YNL016W & 11 & 15 & 6 & -36.8557 & -30.6818 & 58.89029 & & \\
\hline FAR11 & YNL127W & 11 & 15 & 7 & -31.5315 & 62.96975 & 70.73171 & & \\
\hline PBI2 & YNL015W & 11 & 15 & 8 & 74.02235 & -11.2853 & 61.59822 & & \\
\hline \multirow[t]{3}{*}{ ESBP6 } & YNL125C & 11 & 15 & 9 & 25.29711 & -9.04685 & 79.49674 & & \\
\hline & YNL013C & 11 & 15 & 10 & 64.40678 & 62.26115 & 78.90205 & & \\
\hline & YNL123W & 11 & 15 & 11 & -18.8406 & -23.4837 & 75.41322 & & \\
\hline \multirow[t]{3}{*}{ SPO1 } & YNL012W & 11 & 15 & 12 & -1.69492 & 0 & 57.21854 & & \\
\hline & YNL122C & 11 & 15 & 13 & -30.9973 & 55.66038 & 23.17881 & & \\
\hline & YNL010W & 11 & 15 & 14 & -12.6338 & -25.5853 & 60.19656 & & \\
\hline TOM70 & YNL121C & 11 & 15 & 15 & -18.6047 & 46.1165 & 72.92929 & & \\
\hline \multirow[t]{2}{*}{ IDP3 } & YNL009W & 11 & 15 & 16 & -31.9809 & 24.2126 & 76.78019 & & \\
\hline & YNL120C & 11 & 15 & 17 & -17.3729 & 35.46911 & \#DIV/0! & & \\
\hline ASI3 & YNL008C & 11 & 15 & 18 & -17.6871 & 33.9981 & 4.374057 & & \\
\hline NCS2 & YNL119W & 11 & 15 & 19 & -35.3801 & 35.59871 & 57.31497 & & \\
\hline HRB1 & YNL004W & 11 & 15 & 20 & 34.84252 & 24.73684 & 19.5122 & & \\
\hline MLS1 & YNL117W & 11 & 15 & 21 & -24.0964 & 36.74367 & 27.63338 & & \\
\hline PET8 & YNL003C & 11 & 15 & 22 & -30 & 28.30882 & 58.83015 & & \\
\hline DMA2 & YNL116W & 11 & 15 & 23 & 28.98936 & 71.02086 & 50.77793 & & \\
\hline HIS3 & YOR202W & 11 & 15 & 24 & 61.15326 & 25.52301 & 44.55446 & & \\
\hline HIS3 & YOR202W & 11 & 16 & 1 & 55.41126 & 24.7191 & 50.56604 & & \\
\hline HIS3 & YOR202W & 11 & 16 & 2 & 39.24915 & 30.99328 & 43.73233 & & \\
\hline HIS3 & YOR202W & 11 & 16 & 3 & 82.99156 & 50.77658 & 51.86275 & & \\
\hline HIS3 & YOR202W & 11 & 16 & 4 & 73.98844 & 20.0495 & 56.72414 & & \\
\hline HIS3 & YOR202W & 11 & 16 & 5 & 43.4629 & -7.75047 & 71.07438 & & \\
\hline HIS3 & YOR202W & 11 & 16 & 6 & 77.24868 & 22.0339 & 63.29114 & & \\
\hline HIS3 & YOR202W & 11 & 16 & 7 & 81.71021 & 6.74264 & 65.91928 & & \\
\hline HIS3 & YOR202W & 11 & 16 & 8 & 76.86567 & -17.3623 & 80.21761 & & \\
\hline
\end{tabular}


HIS3

HIS3

HIS3

HIS3

HIS3

HIS3

HIS3

HIS3

HIS3

HIS3

HIS3

HIS3

HIS3

HIS3

HIS3

HIS3

HIS3

HIS3

HIS3

HIS3

HIS3

HIS3

HIS3

HIS3

HIS3

HIS3

HIS3

HIS3

HIS3

HIS3

HIS3

HIS3

HIS3

HIS3

HIS3

HIS3

HIS3

HIS3

HIS3

HIS3

HIS3

$\begin{array}{lll}\text { YOR202W } & 11 & 16 \\ \text { YOR202W } & 11 & 16 \\ \text { YOR202W } & 11 & 16 \\ \text { YOR202W } & 11 & 16 \\ \text { YOR202W } & 11 & 16 \\ \text { YOR202W } & 11 & 16 \\ \text { YOR202W } & 11 & 16 \\ \text { YOR202W } & 11 & 16 \\ \text { YOR202W } & 11 & 16 \\ \text { YOR202W } & 11 & 16 \\ \text { YOR202W } & 11 & 16 \\ \text { YOR202W } & 11 & 16 \\ \text { YOR202W } & 11 & 16 \\ \text { YOR202W } & 11 & 16 \\ \text { YOR202W } & 11 & 16 \\ \text { YOR202W } & 11 & 16 \\ \text { YOR202W } & 12 & 1 \\ \text { YOR202W } & 12 & 1 \\ \text { YOR202W } & 12 & 1 \\ \text { YOR202W } & 12 & 1 \\ \text { YOR202W } & 12 & 1 \\ \text { YOR202W } & 12 & 1 \\ \text { YOR202W } & 12 & 1 \\ \text { YOR202W } & 12 & 1 \\ \text { YOR202W } & 12 & 1 \\ \text { YOR202W } & 12 & 1 \\ \text { YOR202W } & 12 & 1 \\ \text { YOR202W } & 12 & 1 \\ \text { YOR202W } & 12 & 1 \\ \text { YOR202W } & 12 & 1 \\ \text { YOR202W } & 12 & 1 \\ \text { YOR202W } & 12 & 1 \\ \text { YOR202W } & 12 & 1 \\ \text { YOR202W } & 12 & 1 \\ \text { YOR202W } & 12 & 1 \\ \text { YOR202W } & 12 & 1 \\ \text { YOR202W } & 12 & 1 \\ \text { YOR202W } & 12 & 1 \\ \text { YOR202W } & 12 & 1 \\ \text { YOR202W } & 12 & 1 \\ \text { YOR202W } & 12 & 2 \\ \text { YOR006C } & 12 & 2 \\ \text { YOR097C } & 12 & 2 \\ \text { YO } & & \end{array}$

$\begin{array}{rrrr}9 & -43.7647 & 33.45656 & 84.10714 \\ 10 & 58.81326 & 21.6681 & 66.66667 \\ 11 & 63.7931 & 25.6162 & 68.53312 \\ 12 & -13.6095 & 49.12959 & 89.7177 \\ 13 & 40.68182 & 30.7767 & 91\end{array}$

$\begin{array}{llll}14 & -16.9192 & -9.98632 & 77.60814\end{array}$

$\begin{array}{lllll}15 & -7.93201 & 33.89356 & 78.39898\end{array}$

$\begin{array}{llll}16 & 39.1369 & 10.76512 & 57.52961\end{array}$

$\begin{array}{lllll}17 & 31.2253 & 20.61856 & 67.4581\end{array}$

$\begin{array}{llll}18 & 25.65543 & 19.59248 & 60.8333\end{array}$

$\begin{array}{lllll}19 & 25.87992 & 28.91566 & 85.19108\end{array}$

$\begin{array}{llll}20 & 39.70588 & 11.15772 & 68.45124\end{array}$

$\begin{array}{lllll}21 & 41.34165 & 10.40856 & 46.59686\end{array}$

$\begin{array}{lllll}22 & -7.91209 & 21.39831 & 49.69843\end{array}$

$\begin{array}{llll}23 & -13.7079 & 11.73959 & 32.43824\end{array}$

$\begin{array}{llll}24 & 35.07042 & 10.46065 & 47.35812\end{array}$

$\begin{array}{lllll}1 & 83.7464 & 23.68301 & 69.73579\end{array}$

$\begin{array}{llll}2 & 90.10601 & 31.52709 & 100\end{array}$

3 \#DIV/0! $67.83784 \quad 86.51163$

$\begin{array}{lllll}4 & 58.30153 & 67.82609 & 74.04306\end{array}$

$\begin{array}{lllll}5 & 73.92405 & 36.03925 & 68.1606\end{array}$

$\begin{array}{lllll}6 & 1.291513 & 50.09747 & 77.51479\end{array}$

$\begin{array}{lllll}7 & -2034.78 & 45.54759 & 97.56944\end{array}$

$\begin{array}{llll}8 & -58.3333 & -11.413 & 100\end{array}$

$\begin{array}{llll}9 & -20.9979 & 26.45429 & 100\end{array}$

$\begin{array}{lllll}10 & 8.522727 & 38.77791 & 90.5868\end{array}$

$\begin{array}{lllll}11 & 20.70064 & 47.32221 & 100\end{array}$

$\begin{array}{llll}12 & 8.401977 & 5.136437 & 100\end{array}$

$\begin{array}{lllll}13 & 5.769231 & 38.61284 & 63.31361\end{array}$

$\begin{array}{lllll}14 & 2.03125 & 30.96976 & 27.87743\end{array}$

$\begin{array}{lllll}15 & -24.812 & -2.70677 & 43.25758\end{array}$

$\begin{array}{llll}16 & -0.18182 & 35.33835 & 100\end{array}$

$\begin{array}{llll}17 & -23.3471 & 31.36095 & 48.8\end{array}$

$\begin{array}{llll}18 & 43.43303 & 44.17778 & 100\end{array}$

$\begin{array}{llll}19 & 50.40323 & 48.16557 & 100\end{array}$

$\begin{array}{llll}20 & 73.39181 & 28.9172 & 92.38411\end{array}$

$\begin{array}{lllll}21 & 40.78431 & 24.75369 & 17.02586\end{array}$

$\begin{array}{lllll}22 & 42.06897 & 20.93301 & 0.248344\end{array}$

$\begin{array}{lllll}23 & 59.05849 & 1.634877 & 2.923977\end{array}$

$\begin{array}{llll}24 & 77.66032 & 14.96681 & -2.35792\end{array}$

$\begin{array}{llll}1 & -18.8679 & 66.38452 & 34.07407\end{array}$

2 \#DIV/0! $38.94602 \quad-8.78505$

$\begin{array}{llllll}3 & 80.66528 & -23.3333 & 29.18396 & 60.33898\end{array}$

38.09524

31.03448 


\begin{tabular}{|c|c|c|c|c|c|c|c|c|c|}
\hline DNL4 & YOR005C & 12 & 2 & 4 & 69.44444 & 28.24427 & 44.67409 & 37.8119 & 25.10204 \\
\hline ARF3 & YOR094W & 12 & 2 & 5 & 74.68354 & 40.61489 & 100 & 74.19355 & 33.63636 \\
\hline \multirow[t]{2}{*}{ YSP3 } & YOR003W & 12 & 2 & 6 & 72.47863 & 1.513241 & 73.50746 & 8.615819 & 21.28906 \\
\hline & YOR093C & 12 & 2 & 7 & 7.495741 & 52.56757 & 96.10984 & 38.50688 & 20.81218 \\
\hline ALG6 & YOR002W & 12 & 2 & 8 & 57.95587 & -8.33333 & 24.60317 & 50.47393 & 35.2518 \\
\hline ECM3 & YOR092W & 12 & 2 & 9 & 47.10253 & 32.2963 & 19.91828 & 48.14815 & 32.33083 \\
\hline RRP6 & YOR001W & 12 & 2 & 10 & -3.94089 & 39.60217 & 20.39911 & 36.88312 & 34.57944 \\
\hline \multirow[t]{2}{*}{ RBF46 } & YOR091W & 12 & 2 & 11 & -49.7512 & 35.6623 & -3.58272 & 61.08871 & 35.68075 \\
\hline & YOL163W & 12 & 2 & 12 & -9.44559 & 38.46154 & 1.461988 & 64.28571 & 41.9802 \\
\hline \multirow[t]{2}{*}{ PTC5 } & YOR090C & 12 & 2 & 13 & 10.43339 & 19.17404 & 25.30949 & 35.53922 & 11.14865 \\
\hline & YOL162W & 12 & 2 & 14 & 4.940375 & 37.39237 & 21.73315 & 62.89308 & 31.42251 \\
\hline \multirow[t]{4}{*}{ VPS21 } & YOR089C & 12 & 2 & 15 & 32.68072 & -0.73529 & 100 & 6.6313 & 6.360424 \\
\hline & YOL160W & 12 & 2 & 16 & -38.9831 & 37.24227 & 8.181818 & 18.13285 & 55.67282 \\
\hline & YOR088W & 12 & 2 & 17 & 13.36336 & 27.62284 & 1.901566 & 48.40637 & 31.40097 \\
\hline & YOL159C & 12 & 2 & 18 & 98.39572 & 29.85075 & 100 & 59.96241 & 15.7377 \\
\hline YVC1 & YOR087W & 12 & 2 & 19 & 64 & 4.372355 & 70.8502 & 11.47541 & 28.80184 \\
\hline ENB1 & YOL158C & 12 & 2 & 20 & 14.63415 & 34.41467 & 33.25608 & 64.55446 & 35.68282 \\
\hline \multirow[t]{2}{*}{ TCB1 } & YOR086C & 12 & 2 & 21 & 32.39609 & -23.1263 & 49.41022 & 55.30612 & 39.40092 \\
\hline & YOL155C & 12 & 2 & 22 & 31.63265 & -33.0153 & 100 & 63.18786 & 36.42691 \\
\hline OST3 & YOR085W & 12 & 2 & 23 & 0.58651 & 2.932551 & 71.86207 & 48.54518 & 38.06306 \\
\hline HIS3 & YOR202W & 12 & 2 & 24 & 57.1965 & 0.156986 & 100 & & \\
\hline HIS3 & YOR202W & 12 & 3 & 1 & 74.17453 & 20.91837 & 49.23077 & & \\
\hline SSK2 & YNR031C & 12 & 3 & 2 & 83.87372 & 39.93435 & 37.06422 & 42.9477 & 19.66102 \\
\hline THI20 & YOL055C & 12 & 3 & 3 & 85.57951 & -38.1395 & -14.1864 & 63.30827 & 29.61538 \\
\hline ECM39 & YNR030W & 12 & 3 & 4 & 75.18797 & 62.29117 & 100 & 26.87225 & 25.99532 \\
\hline \multirow[t]{3}{*}{ PSH1 } & YOL054W & 12 & 3 & 5 & 85.33773 & -8.25083 & 79.40741 & 53.37302 & 25.84746 \\
\hline & YNR029C & 12 & 3 & 6 & 63.65546 & 56.69824 & 100 & 54.35203 & 32.77662 \\
\hline & YOL053W & 12 & 3 & 7 & 65.37102 & 30.03534 & 64.0625 & 38.70246 & 28.75536 \\
\hline \multirow[t]{2}{*}{ CPR8 } & YNR028W & 12 & 3 & 8 & -23.7245 & 16.35135 & 63.59918 & 47.38155 & 34.5898 \\
\hline & YOL053C-A & 12 & 3 & 9 & -46.1347 & 35.26971 & 27.06693 & 69.17178 & 63.52201 \\
\hline BUD17 & YNR027W & 12 & 3 & 10 & -47.2779 & 26.92998 & 9.90991 & 39.74082 & 39.12037 \\
\hline \multirow[t]{4}{*}{ SPE2 } & YOL052C & 12 & 3 & 11 & 11.14865 & 19.96269 & -9.66667 & 45.59387 & 17.29958 \\
\hline & YNR025C & 12 & 3 & 12 & -14.8883 & 40.91487 & 100 & 62.21805 & 32.67813 \\
\hline & YOL050C & 12 & 3 & 13 & 30.66202 & 28.7902 & 56.25 & 59.22921 & 35.15284 \\
\hline & YNR024W & 12 & 3 & 14 & -21.1397 & 40.02404 & 24.46043 & 58.99772 & 29.90654 \\
\hline GSH2 & YOL049W & 12 & 3 & 15 & -7.00637 & -40.458 & 32.84356 & 60.91954 & 30.24523 \\
\hline \multirow[t]{6}{*}{ MRPL50 } & YNR022C & 12 & 3 & 16 & 72.47475 & 36.40449 & 100 & 74.27184 & 29.44162 \\
\hline & YOL048C & 12 & 3 & 17 & 50.24077 & 11.75337 & 28.91986 & 33.91813 & 26.31579 \\
\hline & YNR021W & 12 & 3 & 18 & 38.88889 & 43.05382 & 100 & 69.11519 & 34.5679 \\
\hline & YOL047C & 12 & 3 & 19 & 10.4918 & 26.14379 & 45.87459 & 39.09287 & 34.19023 \\
\hline & YNR020C & 12 & 3 & 20 & 38.66667 & 10.34483 & 32.96 & 38.05104 & 22.81369 \\
\hline & YOL046C & 12 & 3 & 21 & 44.11215 & -6.38298 & 52.64586 & 56.47059 & 30.81395 \\
\hline ARE2 & YNR019W & 12 & 3 & 22 & 60.53748 & -34.413 & 97.74011 & 66.12319 & 48.53556 \\
\hline
\end{tabular}




\begin{tabular}{|c|c|c|c|c|c|c|c|c|c|}
\hline PSK2 & YOL045W & 12 & 3 & 23 & -15.3986 & -38.3838 & 33.56643 & 39.30754 & 40.25641 \\
\hline HIS3 & YOR202W & 12 & 3 & 24 & 9.831824 & -12.3794 & 19.78866 & & \\
\hline HIS3 & YOR202W & 12 & 4 & 1 & 6.716418 & 73.17732 & 32.11111 & & \\
\hline \multirow[t]{4}{*}{ ERP4 } & YOR016C & 12 & 4 & 2 & 92.76423 & 3.338392 & 1.835915 & 69.34307 & 31.42329 \\
\hline & YOR112W & 12 & 4 & 3 & 26.43678 & -46.8447 & 52.91902 & 50.89286 & 24.61538 \\
\hline & YOR015W & 12 & 4 & 4 & -24.8744 & 43.08943 & 39.48339 & 62.41379 & 33.33333 \\
\hline & YOR111W & 12 & 4 & 5 & -1.19225 & 46.42857 & 64.57726 & 63.55311 & 29.83193 \\
\hline RTS1 & YOR014W & 12 & 4 & 6 & 20 & 61.66667 & 51.40187 & 29.15493 & 42.85714 \\
\hline \multirow[t]{2}{*}{ INP53 } & YOR109W & 12 & 4 & 7 & \#DIV/0! & 37.5 & 43.94184 & 65.87436 & 41.39535 \\
\hline & YOR013W & 12 & 4 & 8 & -50 & 32.29706 & 37.66049 & 63.78987 & 29.36709 \\
\hline \multirow[t]{2}{*}{ LEU9 } & YOR108W & 12 & 4 & 9 & -0.40241 & 12.42604 & 24.19798 & 67.88991 & 35.83535 \\
\hline & YOR012W & 12 & 4 & 10 & -26.0304 & -31.7073 & -18.5087 & 52.09302 & 31.10048 \\
\hline RGS2 & YOR107W & 12 & 4 & 11 & -29.6218 & 42.62295 & -3.62401 & 45.89615 & 20.4793 \\
\hline AUS1 & YOR011W & 12 & 4 & 12 & 16.26248 & 37.01754 & 9.553571 & 57.54885 & 23.40426 \\
\hline VAM3 & YOR106W & 12 & 4 & 13 & 41.8956 & -28.1008 & 99.46237 & 71.31783 & 42.89044 \\
\hline \multirow[t]{2}{*}{ TIR2 } & YOR010C & 12 & 4 & 14 & 14.55399 & 9.490334 & 100 & 34.61538 & 23.75887 \\
\hline & YOR105W & 12 & 4 & 15 & 12.93532 & 5.175983 & 99.19192 & 76.49254 & 32.04134 \\
\hline TIR4 & YOR009W & 12 & 4 & 16 & 14.28571 & -1.04167 & 63.23319 & 73.86364 & 30.74713 \\
\hline \multirow[t]{3}{*}{ PIN2 } & YOR104W & 12 & 4 & 17 & 22.94807 & 16.88034 & 100 & 67.90353 & 34.35115 \\
\hline & YOR008C- & & & & & & & & \\
\hline & $A$ & 12 & 4 & 18 & 49.77238 & 23.26333 & 91.96787 & 66.9627 & 27.65273 \\
\hline RAS1 & YOR101W & 12 & 4 & 19 & -3.62473 & 9.389671 & 45.29915 & 57.05128 & 35.67073 \\
\hline SLG1 & YOR008C & 12 & 4 & 20 & 48.52151 & -31.6397 & 89.96212 & 52.31388 & 46.47436 \\
\hline CRC1 & YOR100C & 12 & 4 & 21 & -1.34454 & 1.091703 & 91.11111 & 71.28028 & 46.08696 \\
\hline SGT2 & YOR007C & 12 & 4 & 22 & 14.11765 & -26.5625 & 75.30675 & 59.60265 & 45.15235 \\
\hline KTR1 & YOR099W & 12 & 4 & 23 & 19.97455 & 30.38147 & 51.72414 & 46.78819 & 49.2986 \\
\hline HIS3 & YOR202W & 12 & 4 & 24 & -1.39276 & 2.503682 & 33.44948 & & \\
\hline HIS3 & YOR202W & 12 & 5 & 1 & 88.27773 & 20.14303 & -3.88199 & & \\
\hline LYS9 & YNR050C & 12 & 5 & 2 & 63.57827 & 32.59587 & 36.88073 & $\begin{array}{l}47.53289 \\
\text { missing }\end{array}$ & 32.20721 \\
\hline RTG1 & YOL067C & 12 & 5 & 3 & 77.12177 & -18.5501 & 16.43836 & colony & missing colony \\
\hline MSO1 & YNR049C & 12 & 5 & 4 & -16.9231 & -15.8763 & 100 & 68.76122 & 34.17367 \\
\hline \multirow[t]{2}{*}{ INP54 } & YOL065C & 12 & 5 & 5 & -36.0825 & 36.59004 & 47.28878 & 53.08151 & 41.35514 \\
\hline & YNR048W & 12 & 5 & 6 & 85.73333 & 21.5971 & 46.75325 & 53.09568 & 27.11443 \\
\hline \multirow[t]{2}{*}{ MET22 } & YOL064C & 12 & 5 & 7 & -34.6626 & 9.237875 & -1.12994 & 37.16216 & 26.15385 \\
\hline & YNR047W & 12 & 5 & 8 & 72.64151 & 54.2328 & 100 & 32.56785 & 25.24917 \\
\hline MOR1 & YOL063C & 12 & 5 & 9 & 21.31902 & -32.8 & 32.35294 & 52.35602 & 28.80259 \\
\hline PET494 & YNR045W & 12 & 5 & 10 & -9.45455 & 22.08333 & 34.53125 & 56.66667 & 21.92308 \\
\hline \multirow[t]{2}{*}{ APM4 } & YOL062C & 12 & 5 & 11 & 11.54499 & 17.26079 & 48.14815 & 69.21606 & 39.68958 \\
\hline & YNR042W & 12 & 5 & 12 & -23.8462 & -21.3198 & -13.1073 & 74.53626 & 32.89474 \\
\hline \multirow[t]{2}{*}{ PRS5 } & YOL061W & 12 & 5 & 13 & 32.00549 & -22.7166 & 50.27701 & 59.84043 & 33.23263 \\
\hline & YNR040W & 12 & 5 & 14 & 10.47957 & -22.4837 & 30.14587 & 64.25993 & 35.71429 \\
\hline MAM3 & YOL060C & 12 & 5 & 15 & -2.46046 & -7.32026 & 24.34498 & 47.26891 & 39.89501 \\
\hline
\end{tabular}




\begin{tabular}{|c|c|c|c|c|c|c|c|c|c|}
\hline ZRG17 & YNR039C & 12 & 5 & 16 & 14.26593 & -9.02367 & 65.4321 & 72.56318 & 43.79562 \\
\hline GPD2 & YOL059W & 12 & 5 & 17 & 25.14881 & 23.21792 & 62.07827 & 74.51923 & 46.71916 \\
\hline SOL1 & YNR034W & 12 & 5 & 18 & 21.35177 & -6.19469 & 100 & 65.68627 & 38.09524 \\
\hline ARG1 & YOL058W & 12 & 5 & 19 & 35.72543 & 39.40594 & 23.78121 & 54.14258 & 36.58537 \\
\hline \multirow[t]{2}{*}{ PPG1 } & YNR032W & 12 & 5 & 20 & -6.45756 & 17.75362 & 67.92763 & 43.46895 & 34.14634 \\
\hline & $\begin{array}{l}\text { YOL057W } \\
\text { YNR032C- }\end{array}$ & 12 & 5 & 21 & 35.90734 & -41.0628 & 34.34226 & 69.89619 & 48.88889 \\
\hline HUB1 & $A$ & 12 & 5 & 22 & -4.49251 & 51.57068 & 87.01595 & 40.5959 & 28.81944 \\
\hline GPM3 & YOL056W & 12 & 5 & 23 & 46.55556 & -10.4015 & 82.19178 & 63.43154 & 53.16973 \\
\hline HIS3 & YOR202W & 12 & 5 & 24 & -20.3704 & 30.25568 & 83.70044 & & \\
\hline HIS3 & YOR202W & 12 & 6 & 1 & 84.375 & -21.9048 & 32.86467 & & \\
\hline \multirow[t]{2}{*}{ CIN5 } & YOR028C & 12 & 6 & 2 & 23.66288 & 53.62903 & 0.844595 & 58.85023 & 40.11858 \\
\hline & YOR129C & 12 & 6 & 3 & -40.5594 & -29.3011 & 80.92105 & 60.47486 & 37.67821 \\
\hline STI1 & YOR027W & 12 & 6 & 4 & -6.76157 & 23.29247 & 56.32184 & 52.81553 & 33.96739 \\
\hline RGA1 & YOR127W & 12 & 6 & 5 & 65.89862 & 45.0899 & 46.54378 & 38.07615 & 28.39506 \\
\hline BUB3 & YOR026W & 12 & 6 & 6 & -7.63158 & 11.83295 & -9.51417 & 25.05133 & 41.79104 \\
\hline $\mathrm{IAH} 1$ & YOR126C & 12 & 6 & 7 & -44.4444 & -1.55709 & 40.78498 & 60.56106 & 40.13304 \\
\hline HST3 & YOR025W & 12 & 6 & 8 & -54 & 6.6787 & 26.93157 & 37.27506 & 30.95238 \\
\hline \multirow[t]{2}{*}{ UBP2 } & YOR124C & 12 & 6 & 9 & 11.65414 & 5.843072 & 100 & 36.82984 & 39.53488 \\
\hline & YOR024W & 12 & 6 & 10 & -34.3137 & 17.65957 & 14.92386 & $\begin{array}{l}59.10494 \\
\text { missing }\end{array}$ & 39.13978 \\
\hline LEO1 & YOR123C & 12 & 6 & 11 & 36.27845 & 35.35503 & 60 & colony & missing colony \\
\hline \multirow[t]{3}{*}{$\mathrm{AHC1}$} & YOR023C & 12 & 6 & 12 & -42.8854 & 1.778656 & -32.1053 & 74.35159 & 42.31738 \\
\hline & YOR121C & 12 & 6 & 13 & 19.02778 & -20.1695 & 75.86912 & 55.13393 & 45.85987 \\
\hline & YOR022C & 12 & 6 & 14 & -0.2907 & 11.53846 & 14.02908 & 49.30556 & 33.69565 \\
\hline \multirow[t]{4}{*}{ GCY1 } & YOR120W & 12 & 6 & 15 & 45.9926 & 33.42898 & 100 & 74.12587 & 53.25843 \\
\hline & YOR021C & 12 & 6 & 16 & 34.09836 & 28.71287 & 70.98446 & 48.78049 & 24.82759 \\
\hline & YOR118W & 12 & 6 & 17 & -59.7561 & -16.849 & 42.48447 & 49.12281 & 50.96618 \\
\hline & YOR019W & 12 & 6 & 18 & 17.61658 & 3.342618 & 52.72953 & 38.42975 & 36.71642 \\
\hline TRS33 & YOR115C & 12 & 6 & 19 & 18.98212 & 48.05195 & 100 & 53.45455 & 33.23615 \\
\hline \multirow[t]{2}{*}{ ROD1 } & YOR018W & 12 & 6 & 20 & 19.02098 & 3.183024 & 10.11905 & 64.40329 & 45.55256 \\
\hline & YOR114W & 12 & 6 & 21 & 40.08208 & 37.06294 & 18.23105 & 63.81462 & 38.81402 \\
\hline PET127 & YOR017W & 12 & 6 & 22 & -1.96429 & 43.97245 & -11.7008 & 30.38229 & 40 \\
\hline AZF1 & YOR113W & 12 & 6 & 23 & 48.91304 & 42.47788 & 57.14286 & 36.76732 & 41.33949 \\
\hline HIS3 & YOR202W & 12 & 6 & 24 & 12.42079 & -3.76068 & 24.76923 & & \\
\hline \multirow[t]{4}{*}{ HIS3 } & YOR202W & 12 & 7 & 1 & 60.72874 & -17.2161 & 39.85793 & & \\
\hline & YNR064C & 12 & 7 & 2 & 32.14936 & 15.0165 & 7.119741 & 62.15722 & 33.16456 \\
\hline & YOL085C & 12 & 7 & 3 & 21.40381 & -31.1015 & 60.64757 & 37.3673 & 33.80682 \\
\hline & YNR063W & 12 & 7 & 4 & 4.12844 & -7.40741 & 38.5 & 58.53211 & 27.81457 \\
\hline \multirow[t]{4}{*}{ PHM7 } & YOL084W & 12 & 7 & 5 & 68.18182 & 72.70876 & 62.5 & 47.83505 & 38.59649 \\
\hline & YNR062C & 12 & 7 & 6 & -18.4143 & 55.75658 & 41.3442 & 52.4109 & 31.66667 \\
\hline & YOL083W & 12 & 7 & 7 & -13.8739 & 7.76699 & 16.79317 & 30.71429 & 26.1745 \\
\hline & YNR061C & 12 & 7 & 8 & -15.9794 & 3.949731 & 32.1238 & 60.78067 & 43.0622 \\
\hline
\end{tabular}




\begin{tabular}{|c|c|c|c|c|c|c|c|c|c|}
\hline ATG19 & YOL082W & 12 & 7 & 9 & -22.5434 & 31.86583 & 14.58333 & 26.65331 & 31.72414 \\
\hline FRE4 & YNR060W & 12 & 7 & 10 & -45.2539 & -37.4245 & 73.27766 & 72.86184 & 49.49239 \\
\hline IRA2 & YOL081W & 12 & 7 & 11 & 99.60317 & 60.62874 & 100 & 44.42013 & 28.57143 \\
\hline MNT4 & YNR059W & 12 & 7 & 12 & -13.4948 & -47.7798 & 80.57554 & 62.5 & 43.30144 \\
\hline REX4 & YOL080C & 12 & 7 & 13 & 22.78646 & -16.6951 & 48.04688 & 75.78947 & 52.50836 \\
\hline \multirow[t]{2}{*}{$\mathrm{BIO3}$} & YNR058W & 12 & 7 & 14 & 0.475436 & -14.6018 & 59.9681 & 48.99598 & 24.18301 \\
\hline & YOL079W & 12 & 7 & 15 & 14.1844 & 24.71655 & 76.32312 & 75.46875 & 44.47174 \\
\hline \multirow[t]{2}{*}{$\mathrm{BIO4}$} & YNR057C & 12 & 7 & 16 & 16.75824 & 47.1555 & 24.39516 & 64.1791 & 40.20888 \\
\hline & YOL075C & 12 & 7 & 17 & 29.84869 & 51.68662 & 17.33333 & 71.79024 & 50.78947 \\
\hline $\mathrm{BIO5}$ & YNR056C & 12 & 7 & 18 & 10.24793 & -57.1942 & 31.65761 & 65.49912 & 36.17647 \\
\hline EMI5 & YOL071W & 12 & 7 & 19 & 29.95595 & 44.5977 & 29.76879 & 41.10429 & 30 \\
\hline \multirow[t]{2}{*}{ HOL1 } & YNR055C & 12 & 7 & 20 & 36 & 26.73611 & 81.90476 & 69.01141 & 47.44318 \\
\hline & YOL070C & 12 & 7 & 21 & 10.24465 & 35.8349 & 33.46203 & 44.41805 & 53.25779 \\
\hline BRE5 & YNR051C & 12 & 7 & 22 & 58.66667 & -2.49169 & 66.56581 & 49.36061 & 59.80583 \\
\hline HST1 & YOL068C & 12 & 7 & 23 & 29.43548 & 27.95858 & 30.28846 & 64.9919 & 50.79007 \\
\hline HIS3 & YOR202W & 12 & 7 & 24 & 10.99021 & -11.8971 & 19.15888 & & \\
\hline HIS3 & YOR202W & 12 & 8 & 1 & 78.23409 & -30.8017 & -18.5185 & & \\
\hline GLO4 & YOR040W & 12 & 8 & 2 & -8.64917 & 28.89984 & 30.12048 & 57.1912 & 33.49057 \\
\hline ARP8 & YOR141C & 12 & 8 & 3 & -20.5052 & 32.52336 & 29.67033 & 5.864198 & 23.6246 \\
\hline CKB2 & YOR039W & 12 & 8 & 4 & 29.3772 & 36.96858 & 47.49263 & 39.13978 & 24.46809 \\
\hline SFL1 & YOR140W & 12 & 8 & 5 & 6.394316 & 6.832298 & -0.23015 & 46.50924 & 37.87879 \\
\hline \multirow[t]{2}{*}{ HIR2 } & YOR038C & 12 & 8 & 6 & -25.5034 & -27.1462 & 28.4153 & 29.04884 & 48.79518 \\
\hline & YOR139C & 12 & 8 & 7 & 26.40523 & 44.28795 & 69.8941 & 70.55336 & 52.82555 \\
\hline CYC2 & YOR037W & 12 & 8 & 8 & 10.21021 & -12.9032 & -7.44921 & 40.97561 & 25.5814 \\
\hline RUP1 & YOR138C & 12 & 8 & 9 & -19.5364 & -33.1349 & 100 & 63.18681 & 43.79562 \\
\hline SHE4 & YOR035C & 12 & 8 & 10 & 28.44639 & 22.17899 & 81.91682 & 24.80499 & 33.73494 \\
\hline SIA1 & YOR137C & 12 & 8 & 11 & 25.57252 & -11.7293 & 100 & 59.89209 & 35.06944 \\
\hline AKR2 & YOR034C & 12 & 8 & 12 & -11.9601 & 18.41004 & 49.85714 & 57.14286 & 40.44665 \\
\hline IDH2 & YOR136W & 12 & 8 & 13 & 20.45455 & -15.368 & 44.23077 & 58.69121 & 45.52632 \\
\hline \multirow[t]{2}{*}{ EXO1 } & YOR033C & 12 & 8 & 14 & 100 & \#DIV/0! & 100 & 48.60335 & 41.44144 \\
\hline & YOR135C & 12 & 8 & 15 & \#DIV/0! & 3.143418 & 56.75676 & 46.72304 & 15 \\
\hline HMS1 & YOR032C & 12 & 8 & 16 & 16.52661 & 31.27341 & 38.82212 & 71.26806 & 55.9194 \\
\hline BAG7 & YOR134W & 12 & 8 & 17 & 13.07471 & 21.02497 & 65.12097 & 65.61181 & 31.19266 \\
\hline CRS5 & YOR031W & 12 & 8 & 18 & 22.28047 & -18.6441 & 15.9228 & 67.45027 & 32.86119 \\
\hline EFT1 & YOR133W & 12 & 8 & 19 & 29.7568 & -3.85542 & 82.25256 & 65.61798 & 48.52547 \\
\hline DFG16 & YOR030W & 12 & 8 & 20 & 42.25166 & -9.95146 & 45.56041 & 55.86481 & 51.92308 \\
\hline \multirow[t]{3}{*}{ VPS17 } & YOR132W & 12 & 8 & 21 & 12.22057 & -25.8065 & 14.23077 & 55.07559 & 46.61247 \\
\hline & YOR029W & 12 & 8 & 22 & 95.83893 & 34.11978 & 27.5 & 57.91667 & 39.50276 \\
\hline & YOR131C & 12 & 8 & 23 & \#DIV/0! & 34.32155 & 64.53674 & 70.27027 & 55.14223 \\
\hline HIS3 & YOR202W & 12 & 8 & 24 & -57.5198 & 6.595365 & 12.87016 & & \\
\hline HIS3 & YOR202W & 12 & 9 & 1 & 76.7209 & 20.48193 & -10.7062 & & \\
\hline IZH2 & YOL002C & 12 & 9 & 2 & 42.44444 & -42.3611 & 56.93878 & 44.46429 & 33.74083 \\
\hline $\mathrm{IZH} 4$ & YOL101C & 12 & 9 & 3 & -39.6624 & -21.0843 & 34.21053 & 46.86275 & 15.51155 \\
\hline
\end{tabular}




\begin{tabular}{|c|c|c|c|c|c|c|c|c|c|}
\hline \multirow[t]{2}{*}{ PHO80 } & YOL001W & 12 & 9 & 4 & -15.3361 & 4.972376 & 95.61753 & 16.09195 & 38.40206 \\
\hline & YOL099C & 12 & 9 & 5 & 3.927492 & -39.375 & 40.59041 & 28.95833 & 19.02985 \\
\hline \multirow[t]{2}{*}{$\cos 10$} & YNR075W & 12 & 9 & 6 & -3.35097 & -14.3095 & 61.18421 & 53.20911 & 33.75796 \\
\hline & YOL098C & 12 & 9 & 7 & -29.3785 & 19.34673 & 72.1519 & 28.93983 & 25.16129 \\
\hline AlF1 & YNR074C & 12 & 9 & 8 & -60.8295 & 9.833024 & 89.43662 & 44.12417 & 33.03571 \\
\hline \multirow[t]{2}{*}{ HMI1 } & YOL095C & 12 & 9 & 9 & 21.73913 & 56.84211 & 70.09346 & 39.13043 & 31.05023 \\
\hline & YNR073C & 12 & 9 & 10 & \#DIV/0! & -18.8034 & 31.45009 & 27.77778 & 23.9726 \\
\hline TRM10 & YOL093W & 12 & 9 & 11 & -19.0583 & 33.75 & 76.87861 & 54.92701 & 41.25874 \\
\hline \multirow[t]{3}{*}{ HXT17 } & YNR072W & 12 & 9 & 12 & -22.807 & -29.4004 & 100 & 59.29204 & 36.33803 \\
\hline & YOL092W & 12 & 9 & 13 & 10.58315 & 44.95413 & 88.72404 & 71.7803 & 34.18079 \\
\hline & YNR071C & 12 & 9 & 14 & -29.202 & 2.505219 & -10.5778 & 54.89443 & 46.71916 \\
\hline SPO21 & YOL091W & 12 & 9 & 15 & 41.21532 & 41.32104 & \#DIV/0! & 59.61538 & 49.88864 \\
\hline BSC5 & YNR069C & 12 & 9 & 16 & -4.78723 & 43.79786 & 91.96676 & 62.58352 & 59.53079 \\
\hline $\mathrm{MSH} 2$ & YOL090W & 12 & 9 & 17 & 70.12195 & 48.0303 & 98.4375 & 82.79221 & 65.27778 \\
\hline DSE4 & YNR067C & 12 & 9 & 18 & 36.08871 & 36.90476 & 100 & 55.6719 & 46.46925 \\
\hline \multirow[t]{2}{*}{ HAL9 } & YOL089C & 12 & 9 & 19 & 28.37573 & -3.31126 & 7.253886 & 60.03717 & 29.29293 \\
\hline & YNR066C & 12 & 9 & 20 & 79.40741 & 44.7191 & 64.32432 & 70.93236 & 43.04813 \\
\hline MPD2 & YOL088C & 12 & 9 & 21 & 59.0625 & 52.20339 & 84.33735 & 66.66667 & 49.60422 \\
\hline \multirow[t]{2}{*}{ YSN1 } & YNR065C & 12 & 9 & 22 & 21.33152 & 30.71895 & 4.223433 & 62.92373 & 56.61914 \\
\hline & YOL087C & 12 & 9 & 23 & 20.10114 & 42.34801 & 70.27027 & 54.74777 & 56.59472 \\
\hline HIS3 & YOR202W & 12 & 9 & 24 & 29.1411 & 13.20182 & -9.08131 & & \\
\hline \multirow[t]{2}{*}{ HIS3 } & YOR202W & 12 & 10 & 1 & -13.2107 & 28.29582 & 25.13904 & & \\
\hline & YOR053W & 12 & 10 & 2 & -4.91667 & 32.31198 & -16.9118 & 33.63533 & 58.7007 \\
\hline \multirow[t]{4}{*}{ SEY1 } & YOR165W & 12 & 10 & 3 & -53.9583 & 46.71053 & 76.9697 & 50.38911 & 27.12329 \\
\hline & YOR052C & 12 & 10 & 4 & 89.47368 & -20.5011 & 81.76101 & 14.31452 & 23.03523 \\
\hline & YOR164C & 12 & 10 & 5 & 42.92453 & 19.86532 & 100 & 32.86385 & 10.14493 \\
\hline & YOR051C & 12 & 10 & 6 & 27.95699 & -3.22034 & 20.07001 & 45.80645 & 36.88312 \\
\hline \multirow[t]{2}{*}{ DDP1 } & YOR163W & 12 & 10 & 7 & 8.598726 & -7.81955 & 76.2533 & 59.88142 & 40.83333 \\
\hline & YOR050C & 12 & 10 & 8 & -18.5273 & -0.21786 & -7.58684 & 55.97723 & 36.29442 \\
\hline YRR1 & YOR162C & 12 & 10 & 9 & -32.8267 & 4.364326 & 84.35252 & 58.28877 & 44.3609 \\
\hline RSB1 & YOR049C & 12 & 10 & 10 & 30.74141 & 51.5625 & 83.8785 & 49.06716 & 30.06536 \\
\hline PNS1 & YOR161C & 12 & 10 & 11 & -31.016 & 15.86998 & 16.53846 & 62.61023 & 34.10405 \\
\hline STD1 & YOR047C & 12 & 10 & 12 & -12.8302 & 37.17421 & 55.87349 & 53.63458 & 27.77778 \\
\hline NFI1 & YOR156C & 12 & 10 & 13 & 27.45536 & 0 & 95.07576 & 43.32724 & 35.39519 \\
\hline \multirow[t]{3}{*}{ TOM6 } & YOR045W & 12 & 10 & 14 & 16.62338 & 39.4314 & 44.71649 & 69.98224 & 42.78481 \\
\hline & YOR154W & 12 & 10 & 15 & 57.43855 & 47.29194 & 100 & 61.45833 & 47.86967 \\
\hline & YOR044W & 12 & 10 & 16 & -19.1489 & -28.6082 & -4.12698 & 65.08227 & 37.86667 \\
\hline PDR5 & YOR153W & 12 & 10 & 17 & 100 & 52.45536 & 100 & 100 & 61.94226 \\
\hline \multirow[t]{2}{*}{ WHI2 } & YOR043W & 12 & 10 & 18 & -31.3636 & -17.0259 & 87.80488 & 66.22074 & 32.80255 \\
\hline & YOR152C & 12 & 10 & 19 & -36.9369 & 33.82353 & -1.02171 & 46.9112 & 36.77249 \\
\hline CUE5 & YOR042W & 12 & 10 & 20 & 19.37984 & 39.63211 & -6.57016 & 52.89575 & 32.25806 \\
\hline ELG1 & YOR144C & 12 & 10 & 21 & 56.76606 & -11.9433 & 5.068226 & 39.20973 & 42.1875 \\
\hline SRF5 & YOR041C & 12 & 10 & 22 & 28.31858 & -36.4562 & 100 & 55.33088 & 55.85366 \\
\hline
\end{tabular}




\begin{tabular}{|c|c|c|c|c|c|c|c|c|c|}
\hline LSC1 & YOR142W & 12 & 10 & 23 & 83.23782 & 41.85083 & \#DIV/0! & 57.43671 & 44.03471 \\
\hline HIS3 & YOR202W & 12 & 10 & 24 & \#DIV/0! & 11.40496 & -30.4976 & & \\
\hline \multirow[t]{2}{*}{ HIS3 } & YOR202W & 12 & 11 & 1 & 28.23649 & 18.09955 & 5.508475 & & \\
\hline & YOL014W & 12 & 11 & 2 & 12.24961 & -21.6458 & 7.50469 & 47.00855 & 33.33333 \\
\hline \multirow[t]{2}{*}{ SKM1 } & $\begin{array}{l}\text { YOL113W } \\
\text { YOL013W- }\end{array}$ & 12 & 11 & 3 & 24.86388 & -1.33843 & 100 & 39.93174 & 18.0203 \\
\hline & $A$ & 12 & 11 & 4 & 10.62699 & -3.84615 & 100 & 30.8046 & 15.87302 \\
\hline MSB4 & YOL112W & 12 & 11 & 5 & 86.32813 & 37.88546 & 100 & 67.2093 & 63.81323 \\
\hline \multirow[t]{2}{*}{ HRD1 } & YOL013C & 12 & 11 & 6 & 27 & 10.13917 & -4.44444 & -3.57995 & -11.7834 \\
\hline & YOL111C & 12 & 11 & 7 & -1.95313 & 11.4053 & 52.80899 & 46.64032 & 23.31461 \\
\hline HTZ1 & YOL012C & 12 & 11 & 8 & -21.8855 & 11.65049 & 56.84647 & 34.90364 & 3.557312 \\
\hline SHR5 & YOL110W & 12 & 11 & 9 & 26.0771 & -18.0617 & 82.45931 & 65.25424 & 41.39651 \\
\hline PLB3 & YOL011W & 12 & 11 & 10 & 8.992806 & -35.31 & 43.38942 & 70.02096 & 46.49351 \\
\hline ZEO1 & YOL109W & 12 & 11 & 11 & 69.57447 & 49.43639 & 100 & 56.6474 & 33.02181 \\
\hline MDM12 & YOL009C & 12 & 11 & 12 & -59.3373 & 13.26531 & 98.03063 & 33.89356 & 27.51092 \\
\hline \multirow[t]{5}{*}{ INO4 } & YOL108C & 12 & 11 & 13 & 43.28657 & 47.84946 & 94.2446 & 53.73913 & 51.17925 \\
\hline & YOL008W & 12 & 11 & 14 & -12.9436 & 8.536585 & 27.14932 & 49.16468 & 23.86831 \\
\hline & YOL107W & 12 & 11 & 15 & -8.48375 & -18.2292 & 31.81818 & 60.55846 & 40.55416 \\
\hline & YOL007C & 12 & 11 & 16 & -32.6121 & 12.3348 & 1.154957 & 58.79828 & 26.49842 \\
\hline & YOL106W & 12 & 11 & 17 & 24.32432 & 27.98165 & 4.659498 & 50.73996 & 29.50392 \\
\hline TOP1 & YOL006C & 12 & 11 & 18 & 100 & 55.51232 & 100 & 18.46523 & 18.51852 \\
\hline WSC3 & YOL105C & 12 & 11 & 19 & \#DIV/0! & 25.24005 & 76.49573 & 57.02128 & 46.69927 \\
\hline SIN3 & YOL004W & 12 & 11 & 20 & 100 & 53.59395 & 70.46632 & 55.7971 & 2.803738 \\
\hline \multirow[t]{2}{*}{ NDJ1 } & YOL104C & 12 & 11 & 21 & 49.37107 & -2.12014 & 79.01786 & 31.09756 & 48.8665 \\
\hline & YOL003C & 12 & 11 & 22 & 57.44681 & 62.96296 & 79.2381 & 71.56309 & 74.27885 \\
\hline ITR2 & YOL103W & 12 & 11 & 23 & 43.50282 & -18.4366 & 59.55056 & 59.34579 & 46.88172 \\
\hline HIS3 & YOR202W & 12 & 11 & 24 & 28.37573 & -19.0071 & 60 & & \\
\hline HIS3 & YOR202W & 12 & 12 & 1 & 77.63401 & -13.8032 & 79.97179 & & \\
\hline VPS5 & YOR069W & 12 & 12 & 2 & 23.68728 & -25.2232 & -20.8855 & -7.14286 & 6.684492 \\
\hline FYV12 & YOR183W & 12 & 12 & 3 & 72.88136 & 18.01948 & -102.89 & 40.91904 & 3.773585 \\
\hline VAM10 & YOR068C & 12 & 12 & 4 & 34.53237 & 12.56932 & 65.13761 & 33.33333 & -2.81124 \\
\hline RPS30B & YOR182C & 12 & 12 & 5 & 23.23232 & 63.42525 & 37.95918 & 41.17647 & 18.29268 \\
\hline ALG8 & YOR067C & 12 & 12 & 6 & -27.4953 & 36.33136 & 36.26168 & 49.4577 & 15.71429 \\
\hline \multirow[t]{2}{*}{ GAC1 } & YOR178C & 12 & 12 & 7 & -1.2 & 3.802281 & 84.18491 & 58.62069 & 22.71186 \\
\hline & YOR066W & 12 & 12 & 8 & -7.35786 & 43.58621 & 11.00917 & 52.06612 & 14.80263 \\
\hline MPC54 & YOR177C & 12 & 12 & 9 & -33.8496 & 3.752039 & 41.83007 & 49.69325 & 29.11392 \\
\hline \multirow[t]{2}{*}{ CYT1 } & YOR065W & 12 & 12 & 10 & 100 & 48.47059 & 100 & 58.87446 & 34.55497 \\
\hline & YOR175C & 12 & 12 & 11 & \#DIV/0! & -23.6196 & 94.01709 & 52.7163 & 31.5493 \\
\hline YNG1 & YOR064C & 12 & 12 & 12 & -30.6288 & -23.2484 & 12.26415 & 70.73955 & 44.78873 \\
\hline \multirow[t]{2}{*}{ DCS2 } & YOR173W & 12 & 12 & 13 & 12.89782 & 29.51542 & 13.73708 & 55.09761 & 37.94212 \\
\hline & YOR062C & 12 & 12 & 14 & 4.690432 & 24.19929 & 6.330749 & 49.59016 & 48.96074 \\
\hline YRM1 & YOR172W & 12 & 12 & 15 & 19.7724 & 39.90461 & -1.507 & 42.57028 & 45.54217 \\
\hline CKA2 & YOR061W & 12 & 12 & 16 & 32 & 34.45513 & 36.97719 & 50.52192 & 50.25773 \\
\hline
\end{tabular}




\begin{tabular}{|c|c|c|c|c|c|c|c|c|c|}
\hline \multirow[t]{3}{*}{ LCB4 } & YOR171C & 12 & 12 & 17 & 32.44382 & 14.16667 & 54.81611 & 60.24306 & 44.01914 \\
\hline & YOR059C & 12 & 12 & 18 & -25.9003 & -17.7215 & 0.331858 & 39.44954 & 31.9797 \\
\hline & YOR170W & 12 & 12 & 19 & 58.4507 & 24.72826 & 19.14324 & 53.41959 & 45.47564 \\
\hline ASE1 & YOR058C & 12 & 12 & 20 & -37.2038 & 2.966102 & 17.9941 & 54.32099 & 49.65986 \\
\hline \multirow[t]{3}{*}{ RPS28A } & YOR167C & 12 & 12 & 21 & 47.79661 & 34.40678 & -6.65635 & 53.97924 & 41.81034 \\
\hline & YOR055W & 12 & 12 & 22 & 13.02876 & 34.13258 & -12.8843 & 21.62162 & 38.34951 \\
\hline & YOR166C & 12 & 12 & 23 & 30.02257 & 29.87377 & 27.36486 & 37.38739 & 48.80478 \\
\hline HIS3 & YOR202W & 12 & 12 & 24 & 13.95062 & 12.17257 & 4.12234 & & \\
\hline \multirow[t]{2}{*}{ HIS3 } & YOR202W & 12 & 13 & 1 & 61.59306 & 4.718693 & -7.83242 & & \\
\hline & YOL029C & 12 & 13 & 2 & 73.25 & 74.56873 & 68.16269 & 41.66667 & 29.0795 \\
\hline YGK3 & YOL128C & 12 & 13 & 3 & 57.53968 & 5.34188 & 25.76271 & 41.36292 & 14.42543 \\
\hline YAP7 & YOL028C & 12 & 13 & 4 & -29.1822 & 13.48315 & 9.950249 & 24.81061 & 8.982036 \\
\hline $\mathrm{MDH} 2$ & YOL126C & 12 & 13 & 5 & 60.28369 & -26.9581 & 44.32624 & 43.00626 & 28.33333 \\
\hline \multirow[t]{2}{*}{ MDM38 } & YOL027C & 12 & 13 & 6 & 20.18244 & 3.860294 & 100 & 13.26316 & 14.23611 \\
\hline & YOL124C & 12 & 13 & 7 & 41.71705 & 33.94683 & 63.45609 & 49.59184 & 23.2 \\
\hline LAG2 & YOL025W & 12 & 13 & 8 & 21.70088 & -10.9445 & 84.43272 & 51.94444 & 42.65734 \\
\hline \multirow[t]{2}{*}{ SMF1 } & YOL122C & 12 & 13 & 9 & 39.16914 & -39.2157 & 25.3493 & 50.82781 & 28.74693 \\
\hline & YOL024W & 12 & 13 & 10 & 20.67449 & 22.64463 & 40.68493 & 46 & 23.52941 \\
\hline RPS19A & YOL121C & 12 & 13 & 11 & -29.698 & -26.1084 & 28.08564 & 60.73394 & 32.99233 \\
\hline TAT2 & YOL020W & 12 & 13 & 12 & 23.9531 & -4.69012 & 100 & 59.04366 & 42.3445 \\
\hline $\mathrm{MCH} 4$ & YOL119C & 12 & 13 & 13 & 27.43802 & 23.98844 & 100 & 73.02839 & 40 \\
\hline \multirow[t]{2}{*}{ TOS7 } & YOL019W & 12 & 13 & 14 & -5.69106 & 33.92857 & 79.34783 & 54.6789 & 43.66516 \\
\hline & YOL118C & 12 & 13 & 15 & 41.63265 & 37.58865 & 100 & 55.4386 & 34.21053 \\
\hline TLG2 & YOL018C & 12 & 13 & 16 & 45.77465 & 44.28858 & 100 & 26.76471 & 9.606987 \\
\hline RRI2 & YOL117W & 12 & 13 & 17 & 32.63757 & 44.97768 & 100 & 64.88812 & 50.11186 \\
\hline ESC8 & YOL017W & 12 & 13 & 18 & -48.8599 & -15.5629 & 91.03139 & 48.30339 & 47.26841 \\
\hline MSN1 & YOL116W & 12 & 13 & 19 & 100 & 64.19214 & 39.27765 & 40.06849 & 46.55172 \\
\hline CMK2 & YOL016C & 12 & 13 & 20 & \#DIV/0! & -5.88235 & 100 & 35.55094 & 34.10405 \\
\hline \multirow[t]{3}{*}{ TRF4 } & YOL115W & 12 & 13 & 21 & 42.10526 & 44.82249 & 11.89979 & 40.81633 & 34.5679 \\
\hline & YOL015W & 12 & 13 & 22 & 76.38436 & 25.48571 & 53.65854 & 35.1711 & 32.19178 \\
\hline & YOL114C & 12 & 13 & 23 & 64.73988 & 56.58915 & 100 & 44.26471 & 46.42857 \\
\hline HIS3 & YOR202W & 12 & 13 & 24 & 71.10553 & 33.49693 & 45.88665 & & \\
\hline \multirow[t]{2}{*}{ HIS3 } & YOR202W & 12 & 14 & 1 & 64.41441 & 50.7109 & 39.28571 & & \\
\hline & YOR084W & 12 & 14 & 2 & -24.2669 & 38.08438 & -15.7806 & 23.08917 & 15.20343 \\
\hline LIP5 & YOR196C & 12 & 14 & 3 & 56.41361 & -23.2014 & 40.45584 & 14.61101 & 18.4466 \\
\hline WHI5 & YOR083W & 12 & 14 & 4 & 4 & 58.83777 & 40.78947 & 31.11455 & 27.88204 \\
\hline \multirow[t]{2}{*}{ SLK19 } & YOR195W & 12 & 14 & 5 & 23.54772 & -12.585 & -37.8035 & 42.13938 & 28.50356 \\
\hline & YOR082C & 12 & 14 & 6 & 42.63364 & 16.61017 & 8.064516 & 36.33028 & 24.73684 \\
\hline PEX27 & YOR193W & 12 & 14 & 7 & -1.77515 & 35.90078 & 24.52632 & 38.90063 & 20.81081 \\
\hline \multirow[t]{2}{*}{ STC2 } & YOR081C & 12 & 14 & 8 & 18.27815 & 30.88803 & 10.23102 & 42.4812 & 24.6875 \\
\hline & YOR192C & 12 & 14 & 9 & 18.85246 & 48.6653 & 48.68651 & 56.00815 & 16.0221 \\
\hline DIA2 & YOR080W & 12 & 14 & 10 & 12.12121 & 3.784861 & 95.28302 & -19.5035 & 33.93502 \\
\hline RIS1 & YOR191W & 12 & 14 & 11 & 4.869565 & 36.27717 & 100 & 36.91684 & 31.15183 \\
\hline
\end{tabular}




\begin{tabular}{|c|c|c|c|c|c|c|c|c|c|}
\hline ATX2 & YOR079C & 12 & 14 & 12 & 7.33945 & 20.74074 & 53.70166 & 58.15603 & 29.8103 \\
\hline SPR1 & YOR190W & 12 & 14 & 13 & 12.66491 & 13.47709 & 50.25227 & 46.80451 & 33.53474 \\
\hline BUD21 & YOR078W & 12 & 14 & 14 & 35.19062 & 38.58785 & 87.81095 & 44.89164 & 6.220096 \\
\hline IES4 & YOR189W & 12 & 14 & 15 & 10.30928 & 66.57754 & 1.5625 & 32.99595 & 42.52874 \\
\hline SKI7 & YOR076C & 12 & 14 & 16 & 22.79635 & 32.94798 & 2.099448 & 69.58175 & 49.19169 \\
\hline \multirow[t]{4}{*}{ MSB1 } & YOR188W & 12 & 14 & 17 & 2.790698 & 87.29352 & 100 & 66.46884 & 51.00223 \\
\hline & YOR072W & 12 & 14 & 18 & 39.48171 & 19.28571 & 100 & 51.75781 & 37.1134 \\
\hline & YOR186W & 12 & 14 & 19 & 11.49228 & 85.89342 & 83.88626 & 44.86874 & 46.95431 \\
\hline & YOR071C & 12 & 14 & 20 & 1.422475 & 52.25653 & -3.24254 & 60.15779 & 43.87755 \\
\hline GSP2 & YOR185C & 12 & 14 & 21 & 75.60664 & 0 & \#DIV/0! & 54.34783 & 43.5443 \\
\hline GYP1 & YOR070C & 12 & 14 & 22 & -20.6349 & 53.51171 & 100 & 32.41525 & 47.65957 \\
\hline SER1 & YOR184W & 12 & 14 & 23 & 43.25301 & 41.51786 & 81.79272 & 44.05286 & 53.21888 \\
\hline HIS3 & YOR202W & 12 & 14 & 24 & 30.74468 & 20.30848 & 22.38548 & & \\
\hline HIS3 & YOR202W & 12 & 15 & 1 & 39.03177 & 42.09591 & 50.06676 & & \\
\hline PEX15 & YOL044W & 12 & 15 & 2 & \#DIV/0! & 100 & -11.3043 & & \\
\hline FRE7 & YOL152W & 12 & 15 & 3 & 20.85366 & 24 & 100 & & \\
\hline NTG2 & YOL043C & 12 & 15 & 4 & 6.666667 & 24.75248 & 6.521739 & & \\
\hline GRE2 & YOL151W & 12 & 15 & 5 & -21.2714 & 6.25 & 94.73684 & & \\
\hline \multirow[t]{2}{*}{ NGL1 } & YOL042W & 12 & 15 & 6 & -10.2655 & -27.8431 & 44.22383 & & \\
\hline & YOL150C & 12 & 15 & 7 & -31.9298 & 34.97053 & 14.89362 & & \\
\hline NOP12 & YOLO41C & 12 & 15 & 8 & -48.9899 & 48.17391 & 74.84277 & & \\
\hline PEX11 & YOL147C & 12 & 15 & 9 & 100 & 58.32148 & 54.73684 & & \\
\hline RPP2A & YOL039W & 12 & 15 & 10 & 34.06897 & 40.80882 & 79.41176 & & \\
\hline \multirow[t]{4}{*}{ PPM2 } & YOL141W & 12 & 15 & 11 & -25.1685 & 29.88048 & 4.244694 & & \\
\hline & YOL037C & 12 & 15 & 12 & -5.86466 & 60.30534 & 20.09901 & & \\
\hline & YOL138C & 12 & 15 & 13 & 29.83725 & 47.96547 & 90.47619 & & \\
\hline & YOL036W & 12 & 15 & 14 & 28.33333 & 44.29224 & 63.08725 & & \\
\hline \multirow[t]{2}{*}{ BSC6 } & YOL137W & 12 & 15 & 15 & 100 & 62.95547 & 100 & & \\
\hline & YOLO35C & 12 & 15 & 16 & -53.528 & 34.56973 & 44.66667 & & \\
\hline \multirow[t]{2}{*}{ PFK27 } & YOL136C & 12 & 15 & 17 & 11.20448 & 45.50562 & 99.55654 & & \\
\hline & YOL032W & 12 & 15 & 18 & 13.8482 & 43.08094 & 10.99888 & & \\
\hline GAS4 & YOL132W & 12 & 15 & 19 & 91.25152 & 30.99042 & 100 & & \\
\hline \multirow[t]{2}{*}{ SIL1 } & YOLO31C & 12 & 15 & 20 & 49.81818 & 21.82254 & 100 & & \\
\hline & YOL131W & 12 & 15 & 21 & -23.2558 & -15.2542 & 100 & & \\
\hline GAS5 & YOL030W & 12 & 15 & 22 & -31.5789 & 21.85339 & 100 & & \\
\hline VPS68 & YOL129W & 12 & 15 & 23 & -29.4355 & 26.57431 & 50.90909 & & \\
\hline HIS3 & YOR202W & 12 & 15 & 24 & 9.52381 & 16.66667 & 82.14286 & & \\
\hline HIS3 & YOR202W & 12 & 16 & 1 & -28.8462 & 2.009274 & 65.02242 & & \\
\hline HIS3 & YOR202W & 12 & 16 & 2 & -22.3629 & 0.894188 & 38.79957 & & \\
\hline HIS3 & YOR202W & 12 & 16 & 3 & 17.31669 & 28.8785 & 15.41566 & & \\
\hline HIS3 & YOR202W & 12 & 16 & 4 & -2.62626 & -20.1893 & 32.72201 & & \\
\hline HIS3 & YOR202W & 12 & 16 & 5 & -30.625 & -2.08817 & 92.67857 & & \\
\hline HIS3 & YOR202W & 12 & 16 & 6 & 10.15228 & 10.5548 & 46.40259 & & \\
\hline
\end{tabular}




\begin{tabular}{|c|c|c|c|c|c|c|c|}
\hline HIS3 & YOR202W & 12 & 16 & 7 & -55.8577 & 22.70341 & 99.38462 \\
\hline HIS3 & YOR202W & 12 & 16 & 8 & 81.65007 & 17.477 & 100 \\
\hline HIS3 & YOR202W & 12 & 16 & 9 & -27.9528 & 31.88776 & 91.7197 \\
\hline HIS3 & YOR202W & 12 & 16 & 10 & 2.850877 & 10.1473 & 55.36398 \\
\hline HIS3 & YOR202W & 12 & 16 & 11 & -16.9054 & 10.32078 & 37.11507 \\
\hline HIS3 & YOR202W & 12 & 16 & 12 & 16.08833 & 29.98713 & 72.89562 \\
\hline HIS3 & YOR202W & 12 & 16 & 13 & -16.7568 & 16.22807 & 66.29956 \\
\hline HIS3 & YOR202W & 12 & 16 & 14 & 36.41536 & 42.02561 & 82.10526 \\
\hline HIS3 & YOR202W & 12 & 16 & 15 & -7.16667 & 16.07516 & 38.48721 \\
\hline HIS3 & YOR202W & 12 & 16 & 16 & 99.7365 & 13.10212 & 77.42947 \\
\hline HIS3 & YOR202W & 12 & 16 & 17 & -19.9623 & 35.38051 & 84.30584 \\
\hline HIS3 & YOR202W & 12 & 16 & 18 & 33.39921 & -101.716 & 62.1118 \\
\hline HIS3 & YOR202W & 12 & 16 & 19 & -30.8176 & -34.275 & 49.28131 \\
\hline HIS3 & YOR202W & 12 & 16 & 20 & -30.9633 & -16.4134 & 24.46237 \\
\hline HIS3 & YOR202W & 12 & 16 & 21 & 52.7897 & 34.78894 & 13.22314 \\
\hline HIS3 & YOR202W & 12 & 16 & 22 & 55.57351 & -2.87278 & 32.13058 \\
\hline HIS3 & YOR202W & 12 & 16 & 23 & 68.20175 & -0.1297 & 31.8743 \\
\hline HIS3 & YOR202W & 12 & 16 & 24 & 82.36246 & 8.280255 & 65.63307 \\
\hline HIS3 & YOR202W & 13 & 1 & 1 & 22.89294 & 30.08008 & \\
\hline HIS3 & YOR202W & 13 & 1 & 2 & 23.02158 & -7.88758 & \\
\hline HIS3 & YOR202W & 13 & 1 & 3 & 36.18234 & 33.40174 & \\
\hline HIS3 & YOR202W & 13 & 1 & 4 & 40.715 & 32.20666 & \\
\hline HIS3 & YOR202W & 13 & 1 & 5 & 40.84507 & 7.756949 & \\
\hline HIS3 & YOR202W & 13 & 1 & 6 & 37.85714 & 10.96369 & \\
\hline HIS3 & YOR202W & 13 & 1 & 7 & -20.7526 & -10.7997 & \\
\hline HIS3 & YOR202W & 13 & 1 & 8 & 5.052632 & 18.98239 & \\
\hline HIS3 & YOR202W & 13 & 1 & 9 & -4.93562 & 12.41319 & \\
\hline HIS3 & YOR202W & 13 & 1 & 10 & 0.274474 & 12.92091 & \\
\hline HIS3 & YOR202W & 13 & 1 & 11 & -41.8577 & 17.05298 & \\
\hline HIS3 & YOR202W & 13 & 1 & 12 & 2.23072 & 4.868674 & \\
\hline HIS3 & YOR202W & 13 & 1 & 13 & -1.68067 & -10.4793 & \\
\hline HIS3 & YOR202W & 13 & 1 & 14 & 12.66846 & 12.57622 & \\
\hline HIS3 & YOR202W & 13 & 1 & 15 & 15.84906 & 19.12752 & \\
\hline HIS3 & YOR202W & 13 & 1 & 16 & 8.76494 & 17.53247 & \\
\hline HIS3 & YOR202W & 13 & 1 & 17 & 2.35546 & 14.89879 & \\
\hline HIS3 & YOR202W & 13 & 1 & 18 & 21.68285 & 28.95928 & \\
\hline HIS3 & YOR202W & 13 & 1 & 19 & 10.55495 & 1.873536 & \\
\hline HIS3 & YOR202W & 13 & 1 & 20 & 12.55646 & -9.09091 & \\
\hline HIS3 & YOR202W & 13 & 1 & 21 & 12.03791 & -28.8497 & \\
\hline HIS3 & YOR202W & 13 & 1 & 22 & 10.02921 & 1.434426 & \\
\hline HIS3 & YOR202W & 13 & 1 & 23 & 7.981221 & 8.745583 & \\
\hline HIS3 & YOR202W & 13 & 1 & 24 & -6.91358 & 13.86861 & \\
\hline HIS3 & YOR202W & 13 & 2 & 1 & 22.92818 & 66.94511 & \\
\hline
\end{tabular}




\begin{tabular}{|c|c|c|c|c|c|c|c|c|}
\hline LPE10 & YPL060W & 13 & 2 & 2 & 41.20172 & 45.35519 & 68.4435 & 39.30348 \\
\hline DAP1 & YPL170W & 13 & 2 & 3 & 36.97219 & 48.68132 & 50.08787 & 33.70288 \\
\hline \multirow[t]{2}{*}{ PDR12 } & YPL058C & 13 & 2 & 4 & 46.7354 & 23.89078 & 53.663 & 36.75 \\
\hline & YPL168W & 13 & 2 & 5 & 32.93839 & 12.60199 & 35.48387 & 29.87805 \\
\hline SUR1 & YPL057C & 13 & 2 & 6 & -2.60664 & 20.97107 & 30.6391 & 29.82885 \\
\hline \multirow[t]{3}{*}{ REV3 } & YPL167C & 13 & 2 & 7 & 6.271777 & 20.24922 & 19.71067 & 17.79141 \\
\hline & YPL056C & 13 & 2 & 8 & 9.052183 & 44.37663 & 74.74048 & 41.29464 \\
\hline & YPL166W & 13 & 2 & 9 & 12.52874 & 40.07561 & 59.92509 & 36.31714 \\
\hline LGE1 & YPL055C & 13 & 2 & 10 & 14.91935 & 39.97555 & 40.64171 & 34.75783 \\
\hline SET6 & YPL165C & 13 & 2 & 11 & 35.18519 & 30.76923 & 44.32432 & 29.01408 \\
\hline LEE1 & YPL054W & 13 & 2 & 12 & 15.77825 & 25.93361 & 90.15919 & 33.67983 \\
\hline MLH3 & YPL164C & 13 & 2 & 13 & 7.475317 & 35.48851 & 45.8256 & 37.14953 \\
\hline KTR6 & YPL053C & 13 & 2 & 14 & 14.31793 & 28.47153 & 30.98859 & 34.47293 \\
\hline SVS1 & YPL163C & 13 & 2 & 15 & 12.99127 & 16.4604 & -10.3448 & 29.56522 \\
\hline \multirow[t]{2}{*}{ OAZ1 } & YPL052W & 13 & 2 & 16 & 8.222222 & 18.31627 & 20.8589 & 21.16992 \\
\hline & YPL162C & 13 & 2 & 17 & 10.74919 & 42.42424 & 16.62269 & 41.64306 \\
\hline ARL3 & YPL051W & 13 & 2 & 18 & 11.48325 & 9.259259 & 6.479482 & 28.57143 \\
\hline BEM4 & YPL161C & 13 & 2 & 19 & 24.5098 & 8.544304 & 37.38318 & 37.12121 \\
\hline CAM1 & YPL048W & 13 & 2 & 20 & 23.35404 & 11.43345 & 25.6262 & 45.38259 \\
\hline PET20 & YPL159C & 13 & 2 & 21 & 16.23109 & -1.59681 & 36.99789 & 28.42893 \\
\hline SGF11 & YPL047W & 13 & 2 & 22 & 16.86616 & 3.917301 & 66.51917 & 34.03141 \\
\hline TGS1 & YPL157W & 13 & 2 & 23 & -0.35885 & 1.604938 & 20.30568 & 29.30108 \\
\hline HIS3 & YOR202W & 13 & 2 & 24 & 9.269663 & 7.706946 & & \\
\hline HIS3 & YOR202W & 13 & 3 & 1 & 14.84099 & 37.5927 & & \\
\hline MCT1 & YOR221C & 13 & 3 & 2 & 7.084785 & 29.29448 & 39.30754 & 30.2267 \\
\hline SNC2 & YOR327C & 13 & 3 & 3 & -1.22358 & 33.03099 & 22.41681 & 43.01994 \\
\hline STE13 & YOR219C & 13 & 3 & 4 & 2.696366 & 13.94528 & 33.05785 & 35.08772 \\
\hline FRT1 & YOR324C & 13 & 3 & 5 & 8.809524 & 38.66775 & 24.54212 & 41.94444 \\
\hline \multirow[t]{3}{*}{ RUD3 } & YOR216C & 13 & 3 & 6 & 18.6019 & 37.85311 & 50.98468 & 18.4573 \\
\hline & YOR322C & 13 & 3 & 7 & 16.13995 & -0.55249 & 39.24915 & 28.46442 \\
\hline & YOR215C & 13 & 3 & 8 & 13.48066 & 23.99527 & 42.79661 & 23.59551 \\
\hline \multirow[t]{2}{*}{ РMT3 } & YOR321W & 13 & 3 & 9 & 7.866508 & 27.29412 & 35.75221 & 48.32905 \\
\hline & YOR214C & 13 & 3 & 10 & 11.88811 & 34.47537 & 65.98837 & 28.99729 \\
\hline GNT1 & YOR320C & 13 & 3 & 11 & 8.748616 & 23.48718 & 69.09414 & 40.10152 \\
\hline \multirow[t]{2}{*}{ SAS5 } & YOR213C & 13 & 3 & 12 & 21.37143 & 45.92834 & 75.53517 & 34.95441 \\
\hline & YOR318C & 13 & 3 & 13 & 12.36203 & 21.71362 & 17.12474 & 24.36548 \\
\hline STE4 & YOR212W & 13 & 3 & 14 & 6.588735 & 16.06335 & 43.94904 & 29.5858 \\
\hline FAA1 & YOR317W & 13 & 3 & 15 & 11.28668 & 14.59391 & 4.70297 & 35.47401 \\
\hline NPT1 & YOR209C & 13 & 3 & 16 & 18.0602 & 18.0798 & 24.61538 & 32.16783 \\
\hline сOT1 & YOR316C & 13 & 3 & 17 & 15.27237 & 9.988901 & 20.6089 & 35.30997 \\
\hline \multirow[t]{2}{*}{ PTP2 } & YOR208W & 13 & 3 & 18 & 14.72709 & 9.215017 & 2.97619 & 31.75853 \\
\hline & YOR315W & 13 & 3 & 19 & 11.70732 & -1.4157 & 35.44304 & 6.344411 \\
\hline HIS3 & YOR202W & 13 & 3 & 20 & 15.91376 & 3.424658 & 27.52688 & 26.77165 \\
\hline
\end{tabular}




\begin{tabular}{|c|c|c|c|c|c|c|c|c|}
\hline & YOR314W & 13 & 3 & 21 & 10.30177 & 12.09767 & 36.50255 & 41.37931 \\
\hline MCA1 & YOR197W & 13 & 3 & 22 & 20.80402 & -11.5519 & 27.21519 & 52.80374 \\
\hline SPS4 & YOR313C & 13 & 3 & 23 & 14.44444 & 2.275078 & 42.03187 & 42.18362 \\
\hline HIS3 & YOR202W & 13 & 3 & 24 & 7.265774 & 2.316213 & 27.41379 & \\
\hline HIS3 & YOR202W & 13 & 4 & 1 & 40 & \#DIV/0! & & \\
\hline \multirow[t]{5}{*}{ YTA6 } & YPL074W & 13 & 4 & 2 & 1.16129 & -7.20339 & 60.79027 & 53.9604 \\
\hline & YPL183C & 13 & 4 & 3 & 40.61931 & 30.39216 & 10.15385 & 34.98233 \\
\hline & YPL073C & 13 & 4 & 4 & 13.14103 & 27.41047 & 58.36237 & 53.90625 \\
\hline & YPL182C & 13 & 4 & 5 & 9.583333 & -5.97826 & 37.00935 & 43.59756 \\
\hline & YPL071C & 13 & 4 & 6 & -0.22624 & 22.90616 & 41.24424 & 50.8427 \\
\hline CTI6 & YPL181W & 13 & 4 & 7 & -11.7878 & 46.02273 & 48.43424 & 47.90997 \\
\hline MUK1 & YPL070W & 13 & 4 & 8 & 13.11111 & 7.76699 & 23.37917 & 55.45977 \\
\hline TCO89 & YPL180W & 13 & 4 & 9 & 11.00917 & -1.35635 & -1.03359 & 34.47205 \\
\hline BTS1 & YPL069C & 13 & 4 & 10 & 0.440044 & -15.4848 & 47.2103 & 46.85315 \\
\hline \multirow[t]{2}{*}{ PPQ1 } & YPL179W & 13 & 4 & 11 & 15.97826 & 24.75149 & 6.625259 & 50.5848 \\
\hline & YPL068C & 13 & 4 & 12 & 12.6506 & 25.18837 & 23.69792 & 31.64179 \\
\hline \multirow[t]{2}{*}{$\mathrm{CBC2}$} & YPL178W & 13 & 4 & 13 & 68.10673 & 43.01221 & 50.54945 & 66.25 \\
\hline & YPL067C & 13 & 4 & 14 & 16.1435 & 22.32258 & 25.15337 & 37.09273 \\
\hline \multirow[t]{3}{*}{ CUP9 } & YPL177C & 13 & 4 & 15 & 13.64583 & 9.54023 & 41.66667 & 36.33333 \\
\hline & YPL066W & 13 & 4 & 16 & 9.90676 & 6.782107 & 55.4007 & 35.38961 \\
\hline & YPL176C & 13 & 4 & 17 & 0 & 10.97884 & 50.07924 & 35.97561 \\
\hline CWC27 & YPL064C & 13 & 4 & 18 & 14.42623 & 17.24599 & 67.96875 & 45.05814 \\
\hline \multirow[t]{2}{*}{ NIP100 } & YPL174C & 13 & 4 & 19 & 18.38317 & 19.30502 & 71.08844 & 59.66667 \\
\hline & YPL062W & 13 & 4 & 20 & 30 & 24.28884 & 21.79177 & 44.29967 \\
\hline cox10 & YPL172C & 13 & 4 & 21 & -17.8571 & 100 & -36.7542 & 7.168459 \\
\hline ALD6 & YPL061W & 13 & 4 & 22 & 14.25641 & 2.389486 & -8.03859 & 42.64706 \\
\hline OYE3 & YPL171C & 13 & 4 & 23 & 20.82139 & 5.800215 & 22.80992 & 29.80296 \\
\hline HIS3 & YOR202W & 13 & 4 & 24 & 16.66667 & 9.090909 & & \\
\hline HIS3 & YOR202W & 13 & 5 & 1 & 33.30349 & 25.46374 & & \\
\hline RPL33B & YOR234C & 13 & 5 & 2 & -23.9892 & 50.60514 & 56.68359 & 42.09184 \\
\hline PUT4 & YOR348C & 13 & 5 & 3 & -0.33186 & 20.26627 & 28.29828 & 23.5474 \\
\hline KIN4 & YOR233W & 13 & 5 & 4 & 3.09633 & 23.3209 & 26.41084 & 56.11702 \\
\hline PYK2 & YOR347C & 13 & 5 & 5 & 19.14414 & 24.39024 & 51.04364 & 61.33333 \\
\hline MKK1 & YOR231W & 13 & 5 & 6 & 10.01054 & 39.29236 & 69.27803 & 61.76471 \\
\hline REV1 & YOR346W & 13 & 5 & 7 & 10.79365 & -4.30233 & 60.06768 & 52.97806 \\
\hline WTM1 & YOR230W & 13 & 5 & 8 & 12.19512 & -3.65714 & 14.38515 & 37.42138 \\
\hline TYE7 & YOR344C & 13 & 5 & 9 & 2.844639 & 0.951374 & 2.428256 & 51.71429 \\
\hline \multirow[t]{5}{*}{ WTM2 } & YOR229W & 13 & 5 & 10 & 48.84454 & 48.0198 & 33.39921 & 63.04348 \\
\hline & YOR343C & 13 & 5 & 11 & 1.843818 & -5.39648 & 47.62712 & 44.54545 \\
\hline & YOR228C & 13 & 5 & 12 & 14.25314 & -12.5206 & 28.82096 & 35.06944 \\
\hline & YOR342C & 13 & 5 & 13 & 6.008584 & 9.927611 & 17.96407 & 39.76945 \\
\hline & YOR227W & 13 & 5 & 14 & 3.347732 & 29.9591 & 56.23003 & 50.63694 \\
\hline UBC11 & YOR339C & 13 & 5 & 15 & 11.78414 & 12.17064 & -22.5519 & 53.96825 \\
\hline
\end{tabular}




\begin{tabular}{|c|c|c|c|c|c|c|c|c|}
\hline \multirow[t]{3}{*}{ ISU2 } & YOR226C & 13 & 5 & 16 & 1.612903 & 0.895141 & 70 & 55.16129 \\
\hline & YOR338W & 13 & 5 & 17 & 14.61287 & 30.39092 & 45.02924 & 47.46835 \\
\hline & YOR225W & 13 & 5 & 18 & 17.86075 & 16.56943 & 71.15942 & 50.97493 \\
\hline \multirow[t]{2}{*}{ TEA1 } & YOR337W & 13 & 5 & 19 & 7.526882 & 14.01649 & 4.80167 & 41.51436 \\
\hline & YOR223W & 13 & 5 & 20 & 32.5 & 11.97183 & 24.09639 & 42.9078 \\
\hline MRS2 & YOR334W & 13 & 5 & 21 & 9.052183 & 6.580645 & -15.4028 & 28.35366 \\
\hline ODC2 & YOR222W & 13 & 5 & 22 & 21.81604 & 5.182073 & 9.894737 & 26.4881 \\
\hline PDR10 & YOR328W & 13 & 5 & 23 & 21.24884 & 5.43131 & 61.01083 & 51.39319 \\
\hline HIS3 & YOR202W & 13 & 5 & 24 & 9.027169 & 9.268293 & & \\
\hline \multirow[t]{2}{*}{ HIS3 } & YOR202W & 13 & 6 & 1 & 8.810069 & 28.29582 & & \\
\hline & YPL095C & 13 & 6 & 2 & 2.494577 & 31.98624 & 53.90625 & 55.76471 \\
\hline OXR1 & YPL196W & 13 & 6 & 3 & -4.14053 & -30.72 & 52.7027 & 42.99517 \\
\hline SSU1 & YPL092W & 13 & 6 & 4 & 2.024747 & 25.06266 & 45.07772 & 43.49315 \\
\hline APL5 & YPL195W & 13 & 6 & 5 & 7.367281 & -16.3539 & 31.00189 & 44.83776 \\
\hline GLR1 & YPL091W & 13 & 6 & 6 & 12.22571 & 1.454139 & 67.50789 & 58.57143 \\
\hline DDC1 & YPL194W & 13 & 6 & 7 & -4.86188 & 19.37567 & 61.74497 & 48.65672 \\
\hline RPS6A & YPL090C & 13 & 6 & 8 & 5.570292 & 29.56989 & 58.57143 & 53.82263 \\
\hline PRM3 & YPL192C & 13 & 6 & 9 & 17.70285 & -15.4653 & 40.75286 & 38.53211 \\
\hline \multirow[t]{3}{*}{ RLM1 } & YPL089C & 13 & 6 & 10 & -6.74449 & 35.59322 & 15.4661 & 60.28571 \\
\hline & YPL191C & 13 & 6 & 11 & 7.049608 & -4.6595 & 51.36986 & 39.05724 \\
\hline & YPL088W & 13 & 6 & 12 & 9.384776 & 17.79324 & 23.61111 & 60.66066 \\
\hline GUP2 & YPL189W & 13 & 6 & 13 & 12.96892 & 31.8097 & 51.60796 & 53.54839 \\
\hline YDC1 & YPL087W & 13 & 6 & 14 & 60.82803 & 65.94203 & 41.26016 & 73.39744 \\
\hline MF(ALPHA) 1 & YPL187W & 13 & 6 & 15 & 14.42663 & 19.97245 & 39.30269 & 38.15385 \\
\hline ELP3 & YPL086C & 13 & 6 & 16 & 10.9589 & 15.61514 & -17.0418 & 43.7247 \\
\hline UIP4 & YPL186C & 13 & 6 & 17 & 12.33886 & 8.322981 & 20.31524 & 51.32743 \\
\hline \multirow[t]{4}{*}{ RPS9A } & YPL081W & 13 & 6 & 18 & 38.57868 & 29.36508 & 38.07018 & 44.91803 \\
\hline & YPL185W & 13 & 6 & 19 & 14.52111 & 10.74114 & 12.99559 & 48.29721 \\
\hline & YPL080C & 13 & 6 & 20 & 10.64553 & 0.555556 & 68.73857 & 58.47176 \\
\hline & YPL184C & 13 & 6 & 21 & 17.75026 & 8.084577 & 7.341772 & 57.34767 \\
\hline RPL21B & $\begin{array}{l}\text { YPL079W } \\
\text { YPL183W- }\end{array}$ & 13 & 6 & 22 & 11.61369 & 0.138504 & 31.03448 & 55.92105 \\
\hline GON5 & $A$ & 13 & 6 & 23 & 12.23709 & -3.97074 & -0.20243 & 40.54878 \\
\hline HIS3 & YOR202W & 13 & 6 & 24 & 9.171076 & 10.98266 & & \\
\hline HIS3 & YOR202W & 13 & 7 & 1 & 7.220823 & -4.5658 & & \\
\hline RBF17 & YOR252W & 13 & 7 & 2 & -3.1556 & 25.82583 & 65.53398 & 42.8934 \\
\hline \multirow[t]{2}{*}{ PDE2 } & YOR360C & 13 & 7 & 3 & -13.8122 & 34.12322 & 41.76776 & 46.31902 \\
\hline & YOR251C & 13 & 7 & 4 & -1.52838 & 21.31287 & 31.20438 & 40.13158 \\
\hline VTS1 & YOR359W & 13 & 7 & 5 & 11.38614 & -5.08287 & -2.71493 & 40.98361 \\
\hline SRL1 & YOR247W & 13 & 7 & 6 & 4.0625 & 4.6 & -11.1905 & 49.20635 \\
\hline \multirow[t]{2}{*}{ HAP5 } & YOR358W & 13 & 7 & 7 & 10.36789 & -31.4079 & 57.99649 & 29.45736 \\
\hline & YOR246C & 13 & 7 & 8 & 6.165099 & 3.512195 & 44.09449 & 46.88645 \\
\hline GRD19 & YOR357C & 13 & 7 & 9 & 1.482326 & 22.65006 & 41.65436 & 48.04805 \\
\hline
\end{tabular}




\begin{tabular}{|c|c|c|c|c|c|c|c|c|}
\hline \multirow[t]{2}{*}{ DGA1 } & YOR245C & 13 & 7 & 10 & 0.524109 & 13.22751 & 22.78719 & 58.30986 \\
\hline & YOR356W & 13 & 7 & 11 & 10.96838 & -24.5833 & 73.82876 & 46.52568 \\
\hline PUS7 & YOR243C & 13 & 7 & 12 & 64.87907 & 57.82537 & 76.42857 & 67.25146 \\
\hline GDS1 & YOR355W & 13 & 7 & 13 & 53.02083 & 61.00478 & 42.96482 & 62.31343 \\
\hline SSP2 & YOR242C & 13 & 7 & 14 & 9.657321 & -1.17925 & 42.43986 & 44.09938 \\
\hline MSC6 & YOR354C & 13 & 7 & 15 & 10.91476 & 10.42129 & 29.60152 & 14.96815 \\
\hline \multirow[t]{3}{*}{ ABP140 } & YOR239W & 13 & 7 & 16 & 6.166495 & 7.692308 & 14.66165 & 43.4375 \\
\hline & YOR352W & 13 & 7 & 17 & 4.980469 & 0.701754 & 19.44444 & 54.8105 \\
\hline & YOR238W & 13 & 7 & 18 & 14.27115 & 8.435208 & 26.50177 & 44.66667 \\
\hline MEK1 & YOR351C & 13 & 7 & 19 & 22.87893 & 4.859335 & 73.48609 & 59.67742 \\
\hline HES1 & YOR237W & 13 & 7 & 20 & 26.44788 & 11.64021 & 60.73211 & 57.69231 \\
\hline \multirow[t]{2}{*}{ MNE1 } & YOR350C & 13 & 7 & 21 & -1.7758 & 1.415701 & 20.98214 & 55.67376 \\
\hline & YOR235W & 13 & 7 & 22 & 41.50943 & 25.77093 & 12.06544 & 62.62295 \\
\hline CIN1 & YOR349W & 13 & 7 & 23 & 13.72351 & -3.23974 & 42.83439 & 51.25348 \\
\hline HIS3 & YOR202W & 13 & 7 & 24 & 21.22862 & 19.30147 & & \\
\hline \multirow[t]{5}{*}{ HIS3 } & YOR202W & 13 & 8 & 1 & 10.34696 & 45.14851 & & \\
\hline & YPL108W & 13 & 8 & 2 & 1.590909 & -23.3096 & & \\
\hline & YPL208W & 13 & 8 & 3 & -23.7323 & 43.91144 & 53.71622 & 43.05177 \\
\hline & YPL107W & 13 & 8 & 4 & 1.92926 & 11.97034 & 22.3491 & 34.90566 \\
\hline & YPL207W & 13 & 8 & 5 & 2.159091 & 10.28971 & -5.59441 & 54.66667 \\
\hline \multirow[t]{4}{*}{ SSE1 } & YPL106C & 13 & 8 & 6 & 3.342618 & -33.7398 & 27.29211 & 60.99707 \\
\hline & YPL206C & 13 & 8 & 7 & 13.60825 & 7.607608 & 30.98237 & 50.93633 \\
\hline & YPL105C & 13 & 8 & 8 & 11.67464 & -4.96304 & -2.09302 & 53.23529 \\
\hline & YPL205C & 13 & 8 & 9 & -1.1236 & -2.05198 & 21.71946 & 48.83721 \\
\hline FMP30 & YPL103C & 13 & 8 & 10 & 9.070796 & -21.5017 & 37.05263 & 42.12219 \\
\hline \multirow[t]{2}{*}{ TPK2 } & YPL203W & 13 & 8 & 11 & 8.626974 & 0.903614 & 44.33164 & 43.81625 \\
\hline & YPL102C & 13 & 8 & 12 & \#DIV/0! & \#DIV/0! & 61.92893 & 50.16502 \\
\hline AFT2 & YPL202C & 13 & 8 & 13 & 6.404959 & 1.438053 & 38.8412 & 40 \\
\hline \multirow[t]{2}{*}{ ELP4 } & YPL101W & 13 & 8 & 14 & \#DIV/0! & \#DIV/0! & 59.92509 & 57.82313 \\
\hline & YPL201C & 13 & 8 & 15 & 15.93291 & 6.918239 & 26.26728 & 36.41026 \\
\hline ATG21 & YPL100W & 13 & 8 & 16 & 7.281001 & 27.06044 & 27.68031 & 48.58044 \\
\hline CSM4 & YPL200W & 13 & 8 & 17 & 4.612159 & -0.6865 & -10.8939 & 47.93103 \\
\hline \multirow[t]{3}{*}{ FMP14 } & YPL099C & 13 & 8 & 18 & 7.531866 & 16.56051 & 47.55906 & 48.47561 \\
\hline & YPL199C & 13 & 8 & 19 & 19.31818 & 5.263158 & -6.86499 & 52.2293 \\
\hline & YPL098C & 13 & 8 & 20 & 15.73705 & -38.8321 & 55.0813 & 58.71886 \\
\hline RPL7B & YPL198W & 13 & 8 & 21 & 19.00109 & 25.50232 & 46.03482 & 50.35461 \\
\hline \multirow[t]{2}{*}{ PNG1 } & YPL096W & 13 & 8 & 22 & 5.504587 & 2.422611 & 60.06547 & 57.30659 \\
\hline & YPL197C & 13 & 8 & 23 & 4.220779 & 26.45455 & 56.71642 & 43.90244 \\
\hline HIS3 & YOR202W & 13 & 8 & 24 & 3.497758 & 8.381839 & 8.033827 & 50.24038 \\
\hline \multirow[t]{3}{*}{ HIS3 } & YOR202W & 13 & 9 & 1 & -27.5199 & 10.33311 & & \\
\hline & YOR271C & 13 & 9 & 2 & -9.57447 & 3.540773 & 57.18157 & 69.65099 \\
\hline & YOR378W & 13 & 9 & 3 & -11.0112 & 4.264392 & 53.0504 & 52.75591 \\
\hline VPH1 & YOR270C & 13 & 9 & 4 & 2.320442 & -39.51 & 43.04636 & 36.76149 \\
\hline
\end{tabular}




\begin{tabular}{|c|c|c|c|c|c|c|c|c|}
\hline ATF1 & YOR377W & 13 & 9 & 5 & -4.94624 & -1.48368 & 37.2093 & 29.94836 \\
\hline \multirow[t]{3}{*}{ PAC1 } & YOR269W & 13 & 9 & 6 & 22.77657 & 27.12238 & 61.875 & 20.96774 \\
\hline & YOR376W & 13 & 9 & 7 & 4.725898 & -32.3185 & 48.8746 & 38.24451 \\
\hline & YOR268C & 13 & 9 & 8 & -2.41758 & -5.11111 & 41.6185 & 21.4527 \\
\hline GDH1 & YOR375C & 13 & 9 & 9 & 2.33298 & 6.372549 & 41.54412 & 3.1477 \\
\hline HRK1 & YOR267C & 13 & 9 & 10 & 11.89024 & 21.21807 & 53.87324 & 43.28859 \\
\hline ALD4 & YOR374W & 13 & 9 & 11 & 8.64745 & 6.286837 & 45.45455 & 16.08911 \\
\hline PNT1 & YOR266W & 13 & 9 & 12 & 15.11018 & 36.04853 & 53.89049 & 70.47619 \\
\hline GPB1 & YOR371C & 13 & 9 & 13 & 6.609808 & 28.08146 & 52.32198 & -10.628 \\
\hline RBL2 & YOR265W & 13 & 9 & 14 & 6.788247 & 17.55266 & 47.01987 & -7.57576 \\
\hline RAD17 & YOR368W & 13 & 9 & 15 & 8.248473 & 17.58105 & 56.81063 & 27.66885 \\
\hline DSE3 & YOR264W & 13 & 9 & 16 & 41.37931 & 11.18963 & 62.17949 & 56.6719 \\
\hline \multirow[t]{3}{*}{ SCP1 } & YOR367W & 13 & 9 & 17 & 48.0916 & 53.86338 & 63.5514 & 58.41424 \\
\hline & YOR263C & 13 & 9 & 18 & 3.379722 & 14.87179 & 47.61905 & 59.80231 \\
\hline & YOR365C & 13 & 9 & 19 & 16.01602 & -24.6334 & 53.99361 & -9.68468 \\
\hline \multirow[t]{2}{*}{ OSW1 } & YOR255W & 13 & 9 & 20 & 17.67578 & 11.34235 & 48.4472 & 41.75627 \\
\hline & YOR364W & 13 & 9 & 21 & 14.01274 & 26.4631 & 52.80236 & 64.94845 \\
\hline NAT5 & YOR253W & 13 & 9 & 22 & 19.9806 & 2.007528 & 52.27273 & 37.24138 \\
\hline PIP2 & YOR363C & 13 & 9 & 23 & 6.666667 & 4.265873 & 31.95266 & -26.988 \\
\hline HIS3 & YOR202W & 13 & 9 & 24 & 5.752212 & 19.86063 & & \\
\hline HIS3 & YOR202W & 13 & 10 & 1 & -10.6963 & 18.29537 & & \\
\hline \multirow[t]{2}{*}{ MEI5 } & YPL121C & 13 & 10 & 2 & 20.11385 & 30.07519 & 50.74627 & 20.20202 \\
\hline & YPL225W & 13 & 10 & 3 & -13.4845 & 6.732673 & 62.14511 & 44.2623 \\
\hline VPS30 & YPL120W & 13 & 10 & 4 & -20 & 31.29123 & -23.6318 & 54.3554 \\
\hline МMT2 & YPL224C & 13 & 10 & 5 & 2.542373 & 48.42271 & 12.4183 & 56.40244 \\
\hline DBP1 & YPL119C & 13 & 10 & 6 & 7.488987 & 11.97917 & 68.63354 & 59.05045 \\
\hline GRE1 & YPL223C & 13 & 10 & 7 & 5.280899 & 13.63163 & -12.5731 & 51.52542 \\
\hline HOS3 & YPL116W & 13 & 10 & 8 & 36.56793 & 38.1295 & 67.38132 & 62.42604 \\
\hline FMP40 & YPL222W & 13 & 10 & 9 & 3.811659 & 10.05639 & 28.48837 & 40.48443 \\
\hline BEM3 & YPL115C & 13 & 10 & 10 & 14.25462 & -8.89328 & 18.6551 & 59.13462 \\
\hline \multirow[t]{2}{*}{ BOP1 } & YPL221W & 13 & 10 & 11 & 6.820567 & -5.24064 & 29.98267 & 52.40793 \\
\hline & YPL114W & 13 & 10 & 12 & 18.02444 & 19.22269 & 57.58017 & 55.35168 \\
\hline \multirow[t]{2}{*}{ RPL1A } & YPL220W & 13 & 10 & 13 & 9.737418 & -14.6697 & 43.29502 & 61.11111 \\
\hline & YPL113C & 13 & 10 & 14 & 18.95206 & 26.60996 & 68.76972 & 62.05882 \\
\hline PCL8 & YPL219W & 13 & 10 & 15 & -5.72641 & 8.23389 & -11.5288 & 57.10227 \\
\hline \multirow[t]{2}{*}{ PEX25 } & YPL112C & 13 & 10 & 16 & 8.025343 & 5.856354 & 45.67474 & 41.69611 \\
\hline & YPL216W & 13 & 10 & 17 & 0.323625 & 12.54276 & 61.17851 & 56.31868 \\
\hline CAR1 & YPL111W & 13 & 10 & 18 & 23.02158 & 29.56879 & 47.58735 & 55.90062 \\
\hline \multirow[t]{2}{*}{ THI6 } & YPL214C & 13 & 10 & 19 & 8.872458 & -12.6531 & 55.79196 & 54.88215 \\
\hline & YPL110C & 13 & 10 & 20 & 80.13245 & 22.09302 & -17.217 & 41.06667 \\
\hline \multirow[t]{2}{*}{ LEA1 } & YPL213W & 13 & 10 & 21 & 21.9917 & 50.64599 & 28.63071 & 55.35714 \\
\hline & YPL109C & 13 & 10 & 22 & 12.67748 & 10.88757 & 72.56372 & 53.86905 \\
\hline PUS1 & YPL212C & 13 & 10 & 23 & 3.915663 & 14.95778 & 13.97206 & 57.97101 \\
\hline
\end{tabular}




\begin{tabular}{|c|c|c|c|c|c|c|c|c|}
\hline HIS3 & YOR202W & 13 & 10 & 24 & 14.29706 & 13.28344 & & \\
\hline HIS3 & YOR202W & 13 & 11 & 1 & 82.96164 & 20.48417 & & \\
\hline FMP31 & YOR286W & 13 & 11 & 2 & -12.294 & 5.840072 & 40.45936 & 48.58491 \\
\hline \multirow[t]{2}{*}{ CHL1 } & YPL008W & 13 & 11 & 3 & -13.2653 & 20.13423 & 38.25243 & 53.27635 \\
\hline & YOR285W & 13 & 11 & 4 & 3.383897 & 22.18468 & 59.30931 & 55.95238 \\
\hline LSP1 & YPL004C & 13 & 11 & 5 & -5.55556 & 4.115226 & 41.31944 & 47.23926 \\
\hline HUA2 & YOR284W & 13 & 11 & 6 & -11.9256 & 8.145766 & 48.16667 & 52 \\
\hline \multirow[t]{2}{*}{ ULA1 } & YPL003W & 13 & 11 & 7 & -1.49083 & 8.054523 & 27.41617 & 37.29373 \\
\hline & YOR283W & 13 & 11 & 8 & 12.55144 & 14.19558 & 43.18182 & 59.93789 \\
\hline HAT1 & YPL001W & 13 & 11 & 9 & -1.85387 & 24.48537 & 52.47934 & 43.04124 \\
\hline FSH3 & YOR280C & 13 & 11 & 10 & 13.99776 & -27.9141 & 37.45247 & 42.01183 \\
\hline PHR1 & YOR386W & 13 & 11 & 11 & 8.158765 & 9.080963 & 70.35256 & 56.17647 \\
\hline \multirow[t]{3}{*}{ RFM1 } & YOR279C & 13 & 11 & 12 & 11.21076 & 40.10753 & 11.1588 & 50.74184 \\
\hline & YOR385W & 13 & 11 & 13 & 10 & -7.8125 & 63.59223 & 50.30864 \\
\hline & YOR277C & 13 & 11 & 14 & 19.97805 & 14 & 42.68078 & 50.50847 \\
\hline FRE5 & YOR384W & 13 & 11 & 15 & 6.5075922 & 22.69417 & 66.50641 & 52.55682 \\
\hline CAF20 & YOR276W & 13 & 11 & 16 & -4.02174 & 28.88329 & 73.3234 & 37.86667 \\
\hline FIT3 & YOR383C & 13 & 11 & 17 & 0.608519 & -14.5478 & 53.83361 & 48.63388 \\
\hline RIM20 & YOR275C & 13 & 11 & 18 & 10.82474 & 20.83793 & 40.93567 & 51.05105 \\
\hline FIT2 & YOR382W & 13 & 11 & 19 & 6.635514 & -24.1189 & 66.79035 & 53.7859 \\
\hline MOD5 & YOR274W & 13 & 11 & 20 & 13.86593 & -19.2731 & 28.82448 & 29.0411 \\
\hline FRE3 & YOR381W & 13 & 11 & 21 & 14.93333 & -4.02062 & 38.02395 & 58.68056 \\
\hline TPO4 & YOR273C & 13 & 11 & 22 & 7.900208 & 12.06381 & 72.5832 & 44.94118 \\
\hline RDR1 & YOR380W & 13 & 11 & 23 & 10.2981 & 12.44589 & 59.62733 & 43.64896 \\
\hline HIS3 & YOR202W & 13 & 11 & 24 & 7.105719 & 22.39859 & & \\
\hline HIS3 & YOR202W & 13 & 12 & 1 & -13.0319 & 19.37716 & & \\
\hline \multirow[t]{2}{*}{ UME1 } & YPL139C & 13 & 12 & 2 & -6.00601 & 17.27273 & 50.83333 & 33.41232 \\
\hline & YPL245W & 13 & 12 & 3 & -3.62637 & 19.47115 & 54.36573 & 47.67123 \\
\hline SPP1 & YPL138C & 13 & 12 & 4 & -27.3418 & 11.93756 & 3.459119 & 46.52015 \\
\hline \multirow[t]{2}{*}{ HUT1 } & YPL244C & 13 & 12 & 5 & -13.9111 & 20.55215 & 34.68635 & 53.15315 \\
\hline & YPL137C & 13 & 12 & 6 & 0 & 54.1806 & -12.3457 & 44.44444 \\
\hline \multirow[t]{2}{*}{ CIN2 } & YPL241C & 13 & 12 & 7 & -16.198 & 15.77575 & 11.37339 & 41.15523 \\
\hline & YPL136W & 13 & 12 & 8 & -14.1615 & 22.8473 & 3.301887 & 55.19481 \\
\hline HSP82 & YPL240C & 13 & 12 & 9 & -4.15335 & 22.30523 & 72.66553 & 55.92105 \\
\hline ISU1 & YPL135W & 13 & 12 & 10 & -0.10741 & 18.94866 & 59.96441 & 35.29412 \\
\hline YAR1 & YPL239W & 13 & 12 & 11 & 13.04348 & 40.26667 & 40.30354 & 10.13514 \\
\hline \multirow[t]{2}{*}{ ODC1 } & YPL134C & 13 & 12 & 12 & -2.59301 & -10.7143 & 59.03226 & 53.04878 \\
\hline & YPL236C & 13 & 12 & 13 & -2.45902 & -18.9769 & 48.03002 & 50.15773 \\
\hline RDS2 & YPL133C & 13 & 12 & 14 & -8.83028 & 21.43865 & -9.53437 & 39.93506 \\
\hline SSO1 & YPL232W & 13 & 12 & 15 & -7.47253 & -2.37248 & 65.95745 & 58.95765 \\
\hline \multirow[t]{2}{*}{ SPO19 } & YPL130W & 13 & 12 & 16 & -3.51648 & 0.875274 & 11.3082 & 42.18289 \\
\hline & YPL230W & 13 & 12 & 17 & 11.87699 & 26.07407 & 45.99628 & 55.89226 \\
\hline HHO1 & YPL127C & 13 & 12 & 18 & 7.475728 & 21.35802 & 74.44089 & 52.29111 \\
\hline
\end{tabular}




\begin{tabular}{|c|c|c|c|c|c|c|c|c|}
\hline & YPL229W & 13 & 12 & 19 & 3.433476 & 17.10171 & 69.00685 & 52.73159 \\
\hline KAP120 & YPL125W & 13 & 12 & 20 & \#DIV/0! & \#DIV/0! & 44.18182 & 45.14286 \\
\hline ALG5 & YPL227C & 13 & 12 & 21 & \#DIV/0! & \#DIV/0! & 27.59924 & 37.77778 \\
\hline RNY1 & YPL123C & 13 & 12 & 22 & 14.31227 & 23.52941 & 72.27564 & 40.74074 \\
\hline NEW1 & YPL226W & 13 & 12 & 23 & 14.30193 & 8.557845 & 49.43396 & 49.06667 \\
\hline HIS3 & YOR202W & 13 & 12 & 24 & 5.676856 & 17.60741 & & \\
\hline \multirow[t]{2}{*}{ HIS3 } & YOR202W & 13 & 13 & 1 & -8.62069 & 14.19308 & & \\
\hline & YOR300W & 13 & 13 & 2 & -3.24939 & 17.67742 & -3.04569 & 33.84279 \\
\hline SKS1 & YPL026C & 13 & 13 & 3 & 0.607903 & 29.15225 & 43.0605 & 42.17877 \\
\hline \multirow[t]{2}{*}{ BUD7 } & YOR299W & 13 & 13 & 4 & -11.971 & 9.581646 & 63.02652 & 56.32184 \\
\hline & YPL025C & 13 & 13 & 5 & -7.33728 & 12.4105 & 55.64784 & 50.79365 \\
\hline MUM3 & YOR298W & 13 & 13 & 6 & 39.84064 & 40.23194 & 32.6226 & 62.53776 \\
\hline NCE4 & $\begin{array}{l}\text { YPL024W } \\
\text { YOR298C- }\end{array}$ & 13 & 13 & 7 & -10.5834 & 1.501155 & 63.51351 & 53.35689 \\
\hline MBF1 & $A$ & 13 & 13 & 8 & -16.568 & 18.21053 & 60.82474 & 58.35866 \\
\hline MET12 & YPL023C & 13 & 13 & 9 & -13.8856 & 17.9089 & 48.09689 & 41.01695 \\
\hline TIM18 & YOR297C & 13 & 13 & 10 & -8.53392 & 28.92562 & 14.35294 & 38.29787 \\
\hline \multirow[t]{2}{*}{ RAD1 } & YPL022W & 13 & 13 & 11 & 62.5 & 60.29233 & 54.59272 & 62.98343 \\
\hline & YOR296W & 13 & 13 & 12 & 6.732673 & 29.22869 & 71.84943 & 51.75879 \\
\hline ECM23 & YPL021W & 13 & 13 & 13 & -2.98913 & 29.85685 & 75.72965 & 56.54762 \\
\hline RPS10A & YOR293W & 13 & 13 & 14 & -21.2257 & 12.98246 & -44.4444 & 36.39144 \\
\hline \multirow[t]{2}{*}{ VTC3 } & YPL019C & 13 & 13 & 15 & -15.8798 & 5.273632 & 74.33071 & 59.78836 \\
\hline & YOR292C & 13 & 13 & 16 & -3.65726 & -11.1223 & 54.97835 & 39.07692 \\
\hline \multirow[t]{5}{*}{ CTF19 } & YPL018W & 13 & 13 & 17 & -4.79303 & 14.36464 & 53.2646 & 43.1085 \\
\hline & YOR291W & 13 & 13 & 18 & -14.2857 & 6.976744 & 50 & 38.81119 \\
\hline & YPL017C & 13 & 13 & 19 & -2.12766 & 19.29982 & 75.21968 & 48.96552 \\
\hline & YOR289W & 13 & 13 & 20 & \#DIV/0! & \#DIV/0! & 58.99814 & 40.53333 \\
\hline & YPL014W & 13 & 13 & 21 & \#DIV/0! & \#DIV/0! & 78.16092 & 37.5 \\
\hline \multirow[t]{2}{*}{ MPD1 } & YOR288C & 13 & 13 & 22 & 13.57649 & 19.72921 & 82.45083 & 55.43767 \\
\hline & YPL009C & 13 & 13 & 23 & 13.55276 & 12.36506 & 77.50716 & 54.34783 \\
\hline HIS3 & YOR202W & 13 & 13 & 24 & 14.58733 & 27.84572 & & \\
\hline HIS3 & YOR202W & 13 & 14 & 1 & -11.4754 & 28.46596 & & \\
\hline \multirow[t]{2}{*}{ PRM4 } & YPL156C & 13 & 14 & 2 & -26.9565 & 10.27593 & 29.86513 & 45.84178 \\
\hline & YPL260W & 13 & 14 & 3 & 33.46457 & 38.9049 & 22.52964 & 42.76923 \\
\hline KIP2 & YPL155C & 13 & 14 & 4 & 1.090343 & 3.609626 & 45.97701 & 49.7093 \\
\hline APM1 & YPL259C & 13 & 14 & 5 & -4.82955 & 16.23932 & 29.77867 & 33.25359 \\
\hline PEP4 & YPL154C & 13 & 14 & 6 & 26.23457 & 52.89673 & 15.68627 & 32.77027 \\
\hline THI21 & YPL258C & 13 & 14 & 7 & -31.8182 & -0.59737 & 9.82906 & 47.71429 \\
\hline \multirow[t]{3}{*}{ RRD2 } & YPL152W & 13 & 14 & 8 & -10.2343 & -3.83973 & 70.3154 & 56.52174 \\
\hline & YPL257W & 13 & 14 & 9 & -23.6782 & 13.1257 & 13.25581 & 59.59302 \\
\hline & YPL150W & 13 & 14 & 10 & 40.12158 & 29.63753 & 56.25 & 41.93548 \\
\hline CLN2 & YPL256C & 13 & 14 & 11 & -24.4104 & 12.52772 & 61.79966 & 43.27957 \\
\hline ATG5 & YPL149W & 13 & 14 & 12 & 31.72589 & 41.02564 & -29.2169 & 37.04819 \\
\hline
\end{tabular}




\begin{tabular}{|c|c|c|c|c|c|c|c|c|}
\hline VIK1 & YPL253C & 13 & 14 & 13 & -11.1864 & -19.1958 & 43.58974 & 55.72917 \\
\hline PXA1 & YPL147W & 13 & 14 & 14 & -15.5074 & 10.43129 & 80.56872 & 57.2973 \\
\hline ICY2 & YPL250C & 13 & 14 & 15 & -12.5561 & 11.84211 & 71.02941 & 44.1247 \\
\hline KES1 & YPL145C & 13 & 14 & 16 & 30.60029 & 9.649123 & 99.80119 & 48.06763 \\
\hline \multirow[t]{2}{*}{ GYP5 } & YPL249C & 13 & 14 & 17 & -7.09147 & -25.4282 & -13.9013 & 39.60114 \\
\hline & YPL144W & 13 & 14 & 18 & -2.63736 & -34.7945 & 32.78689 & 44.52736 \\
\hline \multirow[t]{3}{*}{ GAL4 } & YPL248C & 13 & 14 & 19 & -8.61345 & -0.83632 & 10.64426 & 53.06604 \\
\hline & YPL141C & 13 & 14 & 20 & -6.16216 & 21.65049 & 32.69639 & 46.53938 \\
\hline & YPL247C & 13 & 14 & 21 & -5.64263 & 9.44206 & 48.20717 & 58.88594 \\
\hline MKK2 & YPL140C & 13 & 14 & 22 & 9.90099 & -14.6845 & -5.06912 & 43.99038 \\
\hline RBD2 & YPL246C & 13 & 14 & 23 & 24.33555 & 20.31915 & 41.25874 & 43.13305 \\
\hline HIS3 & YOR202W & 13 & 14 & 24 & 26.4412 & 26.89784 & & \\
\hline HIS3 & YOR202W & 13 & 15 & 1 & -3.94191 & 19.27602 & & \\
\hline RPL20B & YOR312C & 13 & 15 & 2 & -18.0723 & 24.18112 & & \\
\hline ELC1 & YPL046C & 13 & 15 & 3 & -9.90338 & 19.25926 & & \\
\hline \multirow[t]{4}{*}{ HSD1 } & YOR311C & 13 & 15 & 4 & 4.074074 & 24.52999 & & \\
\hline & YPL041C & 13 & 15 & 5 & -9.10596 & 18.49057 & & \\
\hline & YOR309C & 13 & 15 & 6 & 16.20469 & 45.30271 & & \\
\hline & YPL039W & 13 & 15 & 7 & -15.0229 & 3.165939 & & \\
\hline SNU66 & YOR308C & 13 & 15 & 8 & -7.16332 & -5.51039 & & \\
\hline MET31 & YPL038W & 13 & 15 & 9 & -18.6592 & 14.85356 & & \\
\hline SLY41 & YOR307C & 13 & 15 & 10 & 34.55189 & 30.31579 & & \\
\hline EGD1 & YPL037C & 13 & 15 & 11 & 64.2132 & 31.97674 & & \\
\hline MCH5 & YOR306C & 13 & 15 & 12 & -15.0628 & 11.00592 & & \\
\hline PMA2 & YPL036W & 13 & 15 & 13 & -21.6931 & 26.54076 & & \\
\hline \multirow[t]{2}{*}{ CPA1 } & YOR303W & 13 & 15 & 14 & -18.5799 & 29.4971 & & \\
\hline & YPL034W & 13 & 15 & 15 & -12.5527 & 8.747045 & & \\
\hline \multirow[t]{3}{*}{ ISW2 } & YOR304W & 13 & 15 & 16 & 54.41595 & 9.680639 & & \\
\hline & YPL033C & 13 & 15 & 17 & 9.788654 & 6.766917 & & \\
\hline & A & 13 & 15 & 18 & 31.48576 & 1.336074 & & \\
\hline \multirow[t]{3}{*}{ SVL3 } & YPL032C & 13 & 15 & 19 & -1.26459 & 48.50107 & & \\
\hline & YOR302W & 13 & 15 & 20 & 2.45821 & 15.14806 & & \\
\hline & YPL030W & 13 & 15 & 21 & 6.733668 & 24.97274 & & \\
\hline RAX1 & YOR301W & 13 & 15 & 22 & 24.79564 & 13.33333 & & \\
\hline SMA1 & YPL027W & 13 & 15 & 23 & -11.2886 & 18.07359 & & \\
\hline HIS3 & YOR202W & 13 & 15 & 24 & 28.03514 & 12.28426 & & \\
\hline HIS3 & YOR202W & 13 & 16 & 1 & -0.39139 & 15.35615 & & \\
\hline HIS3 & YOR202W & 13 & 16 & 2 & 61.93772 & 24.92212 & & \\
\hline HIS3 & YOR202W & 13 & 16 & 3 & 16.76647 & 21.5428 & & \\
\hline HIS3 & YOR202W & 13 & 16 & 4 & -15.407 & 26.17978 & & \\
\hline HIS3 & YOR202W & 13 & 16 & 5 & 1.237113 & -6.88488 & & \\
\hline HIS3 & YOR202W & 13 & 16 & 6 & -24.1007 & 29.01745 & & \\
\hline
\end{tabular}




\begin{tabular}{|c|c|c|c|c|c|c|c|}
\hline HIS3 & YOR202W & 13 & 16 & 7 & 1.134522 & 29.12621 & \\
\hline HIS3 & YOR202W & 13 & 16 & 8 & 63.12 & 21.80995 & \\
\hline HIS3 & YOR202W & 13 & 16 & 9 & 63.09148 & 28.88707 & \\
\hline HIS3 & YOR202W & 13 & 16 & 10 & 42.27941 & 31.71188 & \\
\hline HIS3 & YOR202W & 13 & 16 & 11 & 32.109 & 17.57895 & \\
\hline HIS3 & YOR202W & 13 & 16 & 12 & -11.9588 & 37.87167 & \\
\hline HIS3 & YOR202W & 13 & 16 & 13 & 33.06452 & 26.32184 & \\
\hline HIS3 & YOR202W & 13 & 16 & 14 & 19.03114 & 9.485816 & \\
\hline HIS3 & YOR202W & 13 & 16 & 15 & -1.69651 & 14.94904 & \\
\hline HIS3 & YOR202W & 13 & 16 & 16 & 0.221566 & 32.98701 & \\
\hline HIS3 & YOR202W & 13 & 16 & 17 & 47.1875 & 14.0107 & \\
\hline HIS3 & YOR202W & 13 & 16 & 18 & 5.71066 & 9.698682 & \\
\hline HIS3 & YOR202W & 13 & 16 & 19 & 43.47826 & 6.805808 & \\
\hline HIS3 & YOR202W & 13 & 16 & 20 & 65.84383 & 8.839779 & \\
\hline HIS3 & YOR202W & 13 & 16 & 21 & -3.15361 & 5.073801 & \\
\hline HIS3 & YOR202W & 13 & 16 & 22 & 20.40998 & 11.62791 & \\
\hline HIS3 & YOR202W & 13 & 16 & 23 & 28.35005 & 16.00753 & \\
\hline HIS3 & YOR202W & 13 & 16 & 24 & 14.6771 & 10.24155 & \\
\hline HIS3 & YOR202W & 14 & 1 & 1 & 27.388 & 30.91257 & 52.43634 \\
\hline HIS3 & YOR202W & 14 & 1 & 2 & 60.90604 & 9.272997 & 20.3424 \\
\hline HIS3 & YOR202W & 14 & 1 & 3 & 80.68077 & 42.49548 & 30.75758 \\
\hline HIS3 & YOR202W & 14 & 1 & 4 & 88.37209 & 60.54545 & -5.9719 \\
\hline HIS3 & YOR202W & 14 & 1 & 5 & 51.65829 & 40.625 & 24.33173 \\
\hline HIS3 & YOR202W & 14 & 1 & 6 & 77.66393 & 4.587156 & -21.4582 \\
\hline HIS3 & YOR202W & 14 & 1 & 7 & 43.42105 & 38.41991 & 2.684049 \\
\hline HIS3 & YOR202W & 14 & 1 & 8 & 58.88889 & 39.79475 & 6.716418 \\
\hline HIS3 & YOR202W & 14 & 1 & 9 & 43.26087 & 38.78282 & 27.62097 \\
\hline HIS3 & YOR202W & 14 & 1 & 10 & 52.83798 & 43.7247 & -27.1663 \\
\hline HIS3 & YOR202W & 14 & 1 & 11 & 84.97653 & -29.434 & -25.5814 \\
\hline HIS3 & YOR202W & 14 & 1 & 12 & 64.65563 & 54.14488 & 27.55556 \\
\hline HIS3 & YOR202W & 14 & 1 & 13 & 75.3631 & 35.40373 & 10.22806 \\
\hline HIS3 & YOR202W & 14 & 1 & 14 & 60.08969 & 12.54181 & -17.2949 \\
\hline HIS3 & YOR202W & 14 & 1 & 15 & 56.71785 & 13.8535 & -10.3691 \\
\hline HIS3 & YOR202W & 14 & 1 & 16 & 60.28846 & 11.84433 & -6.72414 \\
\hline HIS3 & YOR202W & 14 & 1 & 17 & 52.29885 & 18.94563 & 39.22414 \\
\hline HIS3 & YOR202W & 14 & 1 & 18 & 58.78453 & 41.08659 & 25 \\
\hline HIS3 & YOR202W & 14 & 1 & 19 & 72.26891 & 63.77079 & 34.45783 \\
\hline HIS3 & YOR202W & 14 & 1 & 20 & 53.77517 & 45.60261 & -26.7303 \\
\hline HIS3 & YOR202W & 14 & 1 & 21 & 68.51026 & 31.25778 & -16.1099 \\
\hline HIS3 & YOR202W & 14 & 1 & 22 & 54.22222 & 31.59066 & 27.37597 \\
\hline HIS3 & YOR202W & 14 & 1 & 23 & 83.20091 & 37.38192 & 32.39308 \\
\hline HIS3 & YOR202W & 14 & 1 & 24 & 40.09336 & 8.792924 & -17.9678 \\
\hline HIS3 & YOR202W & 14 & 2 & 1 & 25.18968 & -20.57 & -5.35714 \\
\hline
\end{tabular}




\begin{tabular}{|c|c|c|c|c|c|c|c|c|c|}
\hline TIF4632 & YGL049C & 14 & 2 & 2 & 48.56361 & -36.514 & 15.47619 & 48.08126 & 46.21514 \\
\hline \multirow[t]{3}{*}{ IES1 } & YFL013C & 14 & 2 & 3 & 95.35398 & -10.101 & 68.64111 & 30.5483 & 39.95381 \\
\hline & YGL042C & 14 & 2 & 4 & 87.3065 & 25.77833 & 44.80519 & 58.95372 & 59.75904 \\
\hline & YFL012W & 14 & 2 & 5 & 56.62949 & 26.23986 & 28.09816 & 31.81818 & 42.05128 \\
\hline ECM33 & $\begin{array}{l}\text { YBR078W } \\
\text { YFL010W- }\end{array}$ & 14 & 2 & 6 & 81.20133 & 23.22404 & 7.077626 & 12.5 & 26.66667 \\
\hline AUA1 & $A$ & 14 & 2 & 7 & 43.90244 & 21.95946 & 24.78166 & 61.48796 & 54.98721 \\
\hline RPL17B & YJL177W & 14 & 2 & 8 & 51.11896 & 0.497512 & 2.072539 & 54.77583 & 41.00877 \\
\hline \multirow[t]{2}{*}{ WWM1 } & YFL010C & 14 & 2 & 9 & 90.61033 & 26.78186 & -1.21359 & 39.74592 & 35.33654 \\
\hline & YGR122W & 14 & 2 & 10 & 48.28061 & 17.90123 & 34.52915 & 76.21622 & 51.34576 \\
\hline \multirow[t]{2}{*}{ BLM3 } & YFL007W & 14 & 2 & 11 & 55.13158 & 25.35354 & 2.094241 & 58.38926 & 40.87193 \\
\hline & YGR068C & 14 & 2 & 12 & 51.85644 & 17.11823 & 24.44772 & 62.9981 & 34.28571 \\
\hline \multirow[t]{2}{*}{ VTC2 } & YFL004W & 14 & 2 & 13 & 46.0719 & 12.46684 & -24.1017 & 65.11628 & 40.11142 \\
\hline & YGR067C & 14 & 2 & 14 & 40.21305 & 22.62693 & 29.65019 & 67.81377 & 40.65041 \\
\hline MSH4 & YFL003C & 14 & 2 & 15 & 60.80283 & 2.237762 & -8.21662 & 15.96517 & 30.78652 \\
\hline ADE6 & YGR061C & 14 & 2 & 16 & 62.2807 & 0.247525 & 100 & 30.39514 & 59.56284 \\
\hline DEG1 & YFL001W & 14 & 2 & 17 & 41.44893 & 37.90123 & -17.6786 & 51.07527 & 16.1194 \\
\hline \multirow[t]{2}{*}{ MUP1 } & YGR055W & 14 & 2 & 18 & 70.38674 & -143.522 & -11.5897 & 44.73684 & 24.21652 \\
\hline & YGR255C & 14 & 2 & 19 & 61.89427 & 53.85514 & 5.050505 & 25.98039 & 27.56757 \\
\hline RCS1 & YGL071W & 14 & 2 & 20 & 39.17004 & 58.69297 & -2.7933 & 57.94769 & 43.45404 \\
\hline ENO1 & YGR254W & 14 & 2 & 21 & 32.82675 & 31.11111 & 10.05917 & 73.21814 & 41.35802 \\
\hline \multirow[t]{2}{*}{ PUS2 } & YGL063W & 14 & 2 & 22 & 45.08566 & 29.60373 & 53.30882 & 72.50923 & 41.86047 \\
\hline & YGR122C-A & 14 & 2 & 23 & 27.31893 & 14.54294 & 8.602151 & 69.54128 & 38.46154 \\
\hline HIS3 & YOR202W & 14 & 2 & 24 & 95.15306 & 28.33583 & -22.4698 & & \\
\hline HIS3 & YOR202W & 14 & 3 & 1 & 34.48276 & 18.99907 & -9.22722 & & \\
\hline SAM3 & YPL274W & 14 & 3 & 2 & 63.91892 & 28.73563 & -12.6973 & 41.61491 & 45.16129 \\
\hline CLB5 & YPR120C & 14 & 3 & 3 & 60.58302 & -35.7143 & 9.215017 & 51.63934 & 36.19303 \\
\hline SAM4 & YPL273W & 14 & 3 & 4 & 100 & 41.02564 & 46.42857 & 46.05263 & 45.71429 \\
\hline \multirow[t]{3}{*}{ CLB2 } & YPR119W & 14 & 3 & 5 & 45.49296 & 3.787879 & -20.625 & 29.09931 & 42.69663 \\
\hline & YPL272C & 14 & 3 & 6 & 63.8985 & 34.83557 & -1.84874 & 35.27697 & 45.20548 \\
\hline & YPR117W & 14 & 3 & 7 & 65.82598 & 26.87861 & 0.362757 & 25.20661 & 39.19308 \\
\hline \multirow[t]{2}{*}{ MDL2 } & YPL270W & 14 & 3 & 8 & 51.18203 & 32.69231 & -23.4252 & 38.52814 & 46.84466 \\
\hline & YPR115W & 14 & 3 & 9 & 46.09005 & -23.7721 & 37.7907 & 77.32794 & 52.70936 \\
\hline \multirow[t]{2}{*}{ KAR9 } & YPL269W & 14 & 3 & 10 & 48.90411 & 54.99182 & -26.9113 & 48.33006 & 45.16129 \\
\hline & YPR114W & 14 & 3 & 11 & 64 & 54.56885 & 5.882353 & 76.14679 & 54.14508 \\
\hline ACM1 & YPL267W & 14 & 3 & 12 & 54.94881 & 45.72193 & 30.0716 & 50 & 54.78723 \\
\hline DBF20 & YPR111W & 14 & 3 & 13 & 60.16355 & 43.88889 & 30 & 16.96429 & 43.43164 \\
\hline \multirow[t]{3}{*}{ DIP5 } & YPL265W & 14 & 3 & 14 & 50.67485 & 51.6129 & -28.394 & 55.18591 & 27.42857 \\
\hline & YPR109W & 14 & 3 & 15 & 51.39949 & 32.96854 & -19.0596 & 59.38144 & 46.68508 \\
\hline & YPL264C & 14 & 3 & 16 & 58.63991 & 40.36939 & -22.0302 & 76.04167 & 49.04459 \\
\hline ISR1 & YPR106W & 14 & 3 & 17 & 59.1133 & 43.67347 & -32.5901 & 23.01255 & 39.26174 \\
\hline \multirow[t]{2}{*}{ KEL3 } & YPL263C & 14 & 3 & 18 & 47.33475 & 65.36965 & -13.6697 & 40.49774 & 27.97203 \\
\hline & YPR098C & 14 & 3 & 19 & 44.42771 & 29.72973 & 1.612903 & 44.8 & 46.44068 \\
\hline
\end{tabular}




\begin{tabular}{|c|c|c|c|c|c|c|c|c|c|}
\hline \multirow[t]{4}{*}{ FUM1 } & YPL262W & 14 & 3 & 20 & 50.82569 & -26.5918 & 51.21951 & 28.08765 & 44.14063 \\
\hline & YPR097W & 14 & 3 & 21 & 36.73709 & 39.12363 & -8.24053 & 31.43418 & 52.34899 \\
\hline & YPL261C & 14 & 3 & 22 & 63.08806 & -22.089 & 27.15517 & 68.11321 & 50.87282 \\
\hline & YPR096C & 14 & 3 & 23 & 100 & 88.29787 & -12.1212 & 49.40577 & 40.65282 \\
\hline HIS3 & YOR202W & 14 & 3 & 24 & 71.12118 & -30.2817 & -27.7457 & & \\
\hline HIS3 & YOR202W & 14 & 4 & 1 & 6.262042 & -10.6045 & 64.20017 & & \\
\hline ISN1 & YOR155C & 14 & 4 & 2 & 50 & -35.8071 & 58.91089 & 69.89051 & 43.07692 \\
\hline TOS8 & YGL096W & 14 & 4 & 3 & 95.70815 & 57.57576 & 46.88797 & 43.49593 & 41.80791 \\
\hline VHS3 & YOR054C & 14 & 4 & 4 & 71.42857 & 28.69458 & 1.117318 & 70.85106 & 59.02965 \\
\hline PAN2 & YGL094C & 14 & 4 & 5 & 49.03382 & 45.62584 & 9.195402 & 63.65639 & 52.78515 \\
\hline RHO5 & YNL180C & 14 & 4 & 6 & 83.94277 & -7.06714 & -10.7955 & 34.66667 & 41.17647 \\
\hline PNC1 & YGL037C & 14 & 4 & 7 & 54.64824 & -26.5774 & -24.75 & 52.66525 & 47.88732 \\
\hline BOP3 & YNL042W & 14 & 4 & 8 & 54.37262 & 66.48352 & 66.31079 & 77.40492 & 74.08537 \\
\hline CGR1 & YGL029W & 14 & 4 & 9 & 52.05479 & 57.65766 & 27.70149 & 75.16199 & 54.15473 \\
\hline CYT2 & YKL087C & 14 & 4 & 10 & 27.08601 & 61.29754 & 25.51528 & 28.84615 & 41.68937 \\
\hline MAL12 & YGR292W & 14 & 4 & 11 & 53.48525 & 34.16537 & -15.1685 & 72.74368 & 57.10526 \\
\hline \multirow[t]{2}{*}{ KGD1 } & YIL125W & 14 & 4 & 12 & 23.8255 & 37.32504 & 11.82744 & 61.13208 & 53.22034 \\
\hline & YGR291C & 14 & 4 & 13 & 60.11494 & 33.53116 & -52.2876 & 65.99634 & 59.81873 \\
\hline GEM1 & YAL048C & 14 & 4 & 14 & 100 & 72.35294 & 28.88889 & 26.78063 & 24.51613 \\
\hline \multirow[t]{2}{*}{ MAL11 } & YGR289C & 14 & 4 & 15 & 63.33333 & 53.53846 & 26.78046 & 70.60998 & 56.75676 \\
\hline & YOR248W & 14 & 4 & 16 & 61.62528 & 67.09585 & 13.13869 & 58.90909 & 63.17365 \\
\hline \multirow[t]{3}{*}{ RNH70 } & YGR276C & 14 & 4 & 17 & 52.85118 & 57.1644 & -20.8301 & 23.71365 & 47.98535 \\
\hline & YOR220W & 14 & 4 & 18 & 56.33958 & 59.62441 & 13.59649 & 57.72647 & 53.07443 \\
\hline & YGR273C & 14 & 4 & 19 & 56.17834 & 55.53797 & -16.1725 & 29.10891 & 36.88761 \\
\hline \multirow[t]{2}{*}{$\mathrm{DCl} 1$} & YOR180C & 14 & 4 & 20 & 51.89456 & -37.1429 & -19.4915 & 56.06936 & 57.66667 \\
\hline & YGR272C & 14 & 4 & 21 & 100 & -14.3969 & 9.116809 & 9.825328 & 54.87365 \\
\hline SYC1 & YOR179C & 14 & 4 & 22 & 49.56971 & 43.10345 & 23.52941 & 40.39301 & 43.41085 \\
\hline SLH1 & YGR271W & 14 & 4 & 23 & 78.54711 & 51.63728 & 20.0627 & 59.5122 & 55.42169 \\
\hline HIS3 & YOR202W & 14 & 4 & 24 & 29.22318 & -26.9978 & -2.52828 & & \\
\hline \multirow[t]{2}{*}{ HIS3 } & YOR202W & 14 & 5 & 1 & -8.23077 & 27.02479 & -94.0895 & & \\
\hline & YPR013C & 14 & 5 & 2 & 50.13699 & 52.24072 & -11.6667 & 50.76923 & 36.04396 \\
\hline \multirow[t]{2}{*}{ MSS18 } & YPR134W & 14 & 5 & 3 & 46.85573 & 18.99441 & 25.25399 & 24.06639 & 43.58108 \\
\hline & YPR012W & 14 & 5 & 4 & 49.71942 & -6.33947 & -25.2216 & 55 & 48.8959 \\
\hline RPS23B & YPR132W & 14 & 5 & 5 & 49.06323 & 54.48954 & 31.65138 & 41.7795 & 43.37748 \\
\hline \multirow[t]{2}{*}{ SUT2 } & YPR009W & 14 & 5 & 6 & 51.50376 & 27.99378 & -26.8971 & 33.40564 & 38.65031 \\
\hline & YPR130C & 14 & 5 & 7 & 53.19149 & 33.71901 & -21.9569 & 65.30249 & 65.08876 \\
\hline HAA1 & YPR008W & 14 & 5 & 8 & 55.13017 & 24.22803 & -0.90381 & 62.749 & 57.52688 \\
\hline SCD6 & YPR129W & 14 & 5 & 9 & 59.13155 & 55.12996 & 9.516837 & 58.16514 & 58.18182 \\
\hline REC8 & YPR007C & 14 & 5 & 10 & 39.47037 & 70 & -1.96078 & 75 & 57.5 \\
\hline ANT1 & YPR128C & 14 & 5 & 11 & 32.85303 & 53.58306 & -26.3728 & 35.74219 & 50 \\
\hline \multirow[t]{2}{*}{ ICL2 } & YPR006C & 14 & 5 & 12 & 33.37969 & 62.95754 & -30.2469 & 66.15385 & 51.24224 \\
\hline & YPR127W & 14 & 5 & 13 & 48.93333 & 64.24419 & -15.2062 & 38.24228 & 46.51811 \\
\hline HAL1 & YPR005C & 14 & 5 & 14 & 55.55556 & 59.92509 & 24.57757 & 66.24549 & 54.39189 \\
\hline
\end{tabular}


PDH1

AXL1

CIT3

THI22

HIS3

HIS3

PET123

DJP1

$\mathrm{MRH} 1$

PST2

OM45

RAD28

FKH1

POG1

COX5B

ADY4

LYS12

KTR7

BAP3

HIS3

HIS3

YOP1

ATH1

NCE102

YME1
YPR126C

YPRO04C

YPR125W

YPR003C

YPR123C

YPR002W

YPR122W

YPR001W

YPR121W

YOR202W

YOR202W

YOR158W

YIR004W

YDR033W

YIL158W

YDR032C

YIL151C

YDR031W

YIL136W

YDR030C

YIL131C

YDR029W

YIL122W

YDR026C

YIL111W

YAL046C

YIL102C

YLR227C

YIL094C

YKR104W

YIL085C

YDR046C

YMR074C

YOR202W

YOR202W

YPR028W

YPR152C

YPR027C

YPR150W

YPR026W

YPR149W

YPR024W

YPR148C
14

14

14

14

14

14

14

14

14

14

14

14

14

14

14

14

14

14

14

14

14

14

14

14

14

14

14

14

14

14

14

14

14

14

14

14

14

14

14

14

14

14

14 $\begin{array}{llll}15 & 64.56422 & 30.56604 & 7.168459\end{array}$

$16 \quad 53.75723$

$17 \quad 61.3198$

$18 \quad 55.05747$

1941.57509

$20 \quad 38.91951$

$21 \quad 49.94524$

$22 \quad 45.09346$

$23 \quad 87.0187$

$24 \quad 42.85714$

144.61679

$2 \quad 53.875$

$3 \quad 36.06755$

454.00485

$5 \quad 55.81683$

$6 \quad 56.37019$

$7 \quad 53.7594$

844.50262

$9 \quad 45.96577$

$10 \quad 49.74026$

$11 \quad 35.7453$

$12 \quad 28.34758$

$13 \quad 50.58518$

$14 \quad 54.59184$

15

16

17

17

18

19

20

21

22

23

23

10.712831

248.56802

353.82883

$4 \quad 34.37908$

$5 \quad 100$

100

647.24221

$\begin{array}{ll}7 & 56.17716\end{array}$

$\begin{array}{ll}8 & 58.59473\end{array}$

$\begin{array}{ll}9 & 53.73333\end{array}$ $\begin{array}{ll}54.10095 & -28.0507\end{array}$

$60.92715-31.0828$

$62.44784-23.1594$

$51.10594-30.6799$

$43.12057-10.8974$

$42.69006-17.2685$

$-19.678 \quad 11.47541$

$64.97175 \quad 70.45045$

$38.20862 \quad 23.4375$

2.78787967 .90083

$14.16465 \quad 25.78462$

$43.61949 \quad 19.40812$

$35.57858 \quad 25.57652$

$-10.1523-14.1946$

$50.30864-8.28516$

$24.4373 \quad 31.94444$

$33.96524 \quad 25.75342$

$64.39483-19.5342$

$\begin{array}{lll}59.53608 & -11.0849\end{array}$

$46.80135-13.9271$

$53.39506-21.7514$

$23.39744 \quad 19.89529$

$54.0636 \quad 1.503759$

$46.18056-26.5461$

$57.8869-21.8601$

$59.65834 \quad 23.03406$

$62.55572-28.3186$

$\begin{array}{ll}-8.68545 & 18.7251\end{array}$

$48.40849 \quad 27.16763$

$\begin{array}{ll}57.88668 & 4.229607\end{array}$

$\begin{array}{lll}5.657709 & 7.100977\end{array}$

$8.408797 \quad 60.3363$

$-13.6126-20.1681$

$26.98864 \quad 37.6569$

$27.69819-24.3221$

$46.90265-17.9331$

$-5.41958 \quad 30.37383$

$62.72727 \quad 27.12766$

$37.16535-22.2405$

$35.98862-15.4966$

$44.41417-23.6842$

$41.96141-25.3385$
30.76923

58.34933

4.241071

68.11092

27.79661

60.38095

59.49821

47.15447

44.9115

47.20721

60

50.78125

46.13779

61.03647

48.90656

46.4455

37.8

66.66667

39.63039

51.41343

45.5914

27.71855

58.56237

32.47423

33.98821

43.14815

32.57732

60.60071

13.49036

34.5

33.96584

62.96992

49.7076

63.53383

19.36275

25.98253

51.41612

31.58915

58.11088
53.60231

35.45455

43.82022

50.60606

21.96721

60.82803

31.8328

41.53846

69.40063

43.67816

48.61751

55.05051

45.59271

57.67327

53.84615

48.54111

34.00673

53.21782

48.10997

34.17722

60.66176

50.34722

38.37209

56.47059

43.26923

46.28378

61.04294

46.10592

49.30556

47.8022

41.15854

48.68735

43.65782

60.48193

42.20779

48.48485

55.98802

40.33898

52.28758 


\begin{tabular}{|c|c|c|c|c|c|c|c|c|c|}
\hline \multirow[t]{4}{*}{ EAF3 } & YPR023C & 14 & 7 & 10 & 47.12644 & 57.70654 & 69.46387 & 31.35593 & 81.30841 \\
\hline & YPR147C & 14 & 7 & 11 & 51.84211 & 60.30989 & -13.5349 & 44.35484 & 41.29693 \\
\hline & YPR022C & 14 & 7 & 12 & 50.7371 & 63.9881 & -9.72973 & 54.01575 & 48.8024 \\
\hline & YPR146C & 14 & 7 & 13 & 48.77384 & 62.84722 & -14.9474 & 42.03455 & 31.15727 \\
\hline ATP20 & YPR020W & 14 & 7 & 14 & 69.33842 & 46.12188 & -1.13493 & 46 & 47.58621 \\
\hline ASN1 & YPR145W & 14 & 7 & 15 & 55.07246 & 69.05072 & 5.415861 & 38.60759 & 71.77914 \\
\hline RLF2 & YPR018W & 14 & 7 & 16 & 39.92147 & 64.7619 & -27.9489 & -35.9701 & 27.58621 \\
\hline KAR3 & YPR141C & 14 & 7 & 17 & 63.30532 & -9.6 & 50.58824 & 27.46914 & 50.70423 \\
\hline DSS4 & YPR017C & 14 & 7 & 18 & 85.12241 & 59.67213 & -16.7742 & 12.19512 & 52.91971 \\
\hline \multirow[t]{2}{*}{ TAZ1 } & YPR140W & 14 & 7 & 19 & 59.07473 & 66.66667 & -26.7658 & 14.3469 & 53.76344 \\
\hline & YPR015C & 14 & 7 & 20 & 65.98837 & 77.14581 & 82.29531 & 52.71318 & 81.49351 \\
\hline \multirow[t]{2}{*}{ MEP3 } & YPR138C & 14 & 7 & 21 & 53.29787 & 46.20155 & 44.97992 & 68.89693 & 59.93691 \\
\hline & YPR014C & 14 & 7 & 22 & 43.37812 & 46.95035 & 15.20645 & 42.31537 & 54.60317 \\
\hline CTF4 & YPR135W & 14 & 7 & 23 & 72 & 68.86447 & 52.73775 & 53.19549 & 76.99681 \\
\hline HIS3 & YOR202W & 14 & 7 & 24 & 62.87215 & 31.71355 & 24.9742 & & \\
\hline HIS3 & YOR202W & 14 & 8 & 1 & 48.16248 & 5.201699 & 50.98991 & & \\
\hline \multirow[t]{3}{*}{ NRG1 } & YDR043C & 14 & 8 & 2 & 57.93103 & 27.45665 & 39.06706 & 39.9568 & 30.54475 \\
\hline & YLR455W & 14 & 8 & 3 & 48.81101 & 41.33504 & -14.7203 & 49.74747 & 39.80198 \\
\hline & YDR042C & 14 & 8 & 4 & 60.1741 & 52.0202 & 8.813264 & 46.80307 & 62.9562 \\
\hline TOR1 & YJR066W & 14 & 8 & 5 & 56.84848 & 41.48061 & 5.706268 & 58.25545 & 61.17851 \\
\hline EHD3 & YDR036C & 14 & 8 & 6 & 100 & 77.52809 & 25.13661 & 47.89082 & 45.41667 \\
\hline VPS55 & YJR044C & 14 & 8 & 7 & 100 & 48.37209 & 26.15385 & 23.90572 & 31.82674 \\
\hline \multirow[t]{2}{*}{ ARO3 } & YDR035W & 14 & 8 & 8 & 90.39735 & 9.543568 & 3.603604 & 57.18391 & 28.52349 \\
\hline & YJR039W & 14 & 8 & 9 & 58.30303 & 49.85423 & 26.17261 & 62.22222 & 30.23715 \\
\hline \multirow[t]{3}{*}{ LYS14 } & YDR034C & 14 & 8 & 10 & 60.43956 & 51.02286 & 0 & 57.55814 & 39.02439 \\
\hline & YJL103C & 14 & 8 & 11 & 54.73684 & 59.54488 & 8.077994 & 47.75641 & 50.75188 \\
\hline & YPR039W & 14 & 8 & 12 & 56.15697 & 59.61332 & 8.399366 & 58.88594 & 64 \\
\hline VPS53 & YJL029C & 14 & 8 & 13 & 43.48387 & 57.28571 & 51.84466 & 13.7457 & -13.0252 \\
\hline \multirow[t]{3}{*}{ UBP16 } & YPL072W & 14 & 8 & 14 & 43.00126 & 11.32438 & 10.2719 & 47.58621 & 40.4814 \\
\hline & YNL086W & 14 & 8 & 15 & 32.56131 & 72.07944 & -21.4118 & 45.48387 & 56.57371 \\
\hline & YPL035C & 14 & 8 & 16 & 80.9322 & -5.02092 & 4.255319 & 30.50193 & 15.45254 \\
\hline MSG5 & YNL053W & 14 & 8 & 17 & 40.65934 & 70.37975 & 19.85185 & 43.18182 & 68.13725 \\
\hline HST2 & YPL015C & 14 & 8 & 18 & 53.13609 & 58.71688 & -9.15348 & 53.92157 & 58.08824 \\
\hline \multirow[t]{2}{*}{ SLM2 } & YNL047C & 14 & 8 & 19 & 44.4564 & 43.35106 & 25.80645 & 53.19149 & 48.06338 \\
\hline & YOR240W & 14 & 8 & 20 & 48.82629 & 54.60123 & 1.759531 & 53.29154 & 19.58525 \\
\hline \multirow[t]{3}{*}{ HEF3 } & $\begin{array}{l}\text { YNL014W } \\
\text { YMR194C- }\end{array}$ & 14 & 8 & 21 & 43.60087 & 19.71631 & 16.91094 & 60.91549 & 28.47682 \\
\hline & $A$ & 14 & 8 & 22 & 61.86047 & 53.5443 & 2.732707 & 52.27273 & 14.15313 \\
\hline & YNL011C & 14 & 8 & 23 & 60.19971 & 32.70142 & -10.5263 & 58.44875 & 65.90909 \\
\hline HIS3 & YOR202W & 14 & 8 & 24 & 55.24345 & 41.19048 & -17.2932 & & \\
\hline \multirow[t]{2}{*}{ HIS3 } & YOR202W & 14 & 9 & 1 & 44.15855 & 21.30841 & 4.337349 & & \\
\hline & YPR045C & 14 & 9 & 2 & 61.54971 & 40.91904 & -17.602 & 59.33014 & 33.56643 \\
\hline BSP1 & YPR171W & 14 & 9 & 3 & 41.65637 & 53.75494 & -42.0317 & 42.34043 & 51.45631 \\
\hline
\end{tabular}




\begin{tabular}{|c|c|c|c|c|c|c|c|c|c|}
\hline & YPR044C & 14 & 9 & 4 & 64.72303 & 24.66844 & 54.65116 & 44.44444 & 59.79021 \\
\hline & YPR170C & 14 & 9 & 5 & 63.65385 & 45.39007 & -42.2535 & 44.21252 & 60.26316 \\
\hline RPL43A & YPR043W & 14 & 9 & 6 & 100 & -4.59016 & \#DIV/0! & 3.395062 & 25.27076 \\
\hline MET16 & YPR167C & 14 & 9 & 7 & 63.61923 & 47.1066 & -13.8134 & 58.80077 & 59.14286 \\
\hline PUF2 & YPR042C & 14 & 9 & 8 & 54.98866 & 41.1415 & -12.8434 & 34.28571 & 66.74877 \\
\hline MMS1 & YPR164W & 14 & 9 & 9 & 65.62054 & 54.24929 & 0.154321 & 50.81206 & 57.66423 \\
\hline TIP41 & YPR040W & 14 & 9 & 10 & 100 & 75.26882 & -21.0843 & 48.99598 & 48.08917 \\
\hline \multirow[t]{3}{*}{ GPH1 } & YPR160W & 14 & 9 & 11 & 100 & 100 & 37.60684 & 31.40496 & 63.20346 \\
\hline & YPR038W & 14 & 9 & 12 & 40.56338 & 54.79951 & 19.79576 & 52.91577 & 61.33333 \\
\hline & YPR158W & 14 & 9 & 13 & -45.0626 & -4.60922 & -23.233 & 58.66426 & 52.63158 \\
\hline \multirow[t]{2}{*}{ ERV2 } & YPR037C & 14 & 9 & 14 & 47.33258 & 56.65914 & 44.02332 & 64.27289 & 60.33333 \\
\hline & YPR157W & 14 & 9 & 15 & 17.01534 & 75.14079 & -10.2724 & 69.34579 & 47.27891 \\
\hline SRO7 & YPR032W & 14 & 9 & 16 & 58.82353 & 37.93103 & 9.174312 & 36.13054 & 58.08581 \\
\hline TPO3 & YPR156C & 14 & 9 & 17 & 27.65957 & 16.49175 & 35.02994 & 26.69492 & 42.65734 \\
\hline NTO1 & YPR031W & 14 & 9 & 18 & 18.637 & 17.46575 & 32.76596 & 43.31066 & 49.27007 \\
\hline NCA2 & YPR155C & 14 & 9 & 19 & 43.65854 & 12.14848 & 23.86364 & 44.75248 & 67.10526 \\
\hline CSR2 & YPR030W & 14 & 9 & 20 & 52.42291 & 70.06726 & 25.04 & 38.88889 & 64.65517 \\
\hline PIN3 & YPR154W & 14 & 9 & 21 & 63.41256 & 76.23762 & 71.29278 & 79.8374 & 82.03593 \\
\hline \multirow[t]{2}{*}{ APL4 } & YPR029C & 14 & 9 & 22 & 58.876 & 8.458864 & 6.346272 & 72.91667 & 65.74394 \\
\hline & YPR153W & 14 & 9 & 23 & 44.25163 & 65.47619 & 1.958384 & 24.66281 & 55.2381 \\
\hline HIS3 & YOR202W & 14 & 9 & 24 & 8.152174 & -1.1984 & -8.85734 & & \\
\hline HIS3 & YOR202W & 14 & 10 & 1 & 22.44202 & 6.593407 & -76.7241 & & \\
\hline cox14 & YML129C & 14 & 10 & 2 & -33.3333 & 18 & -26.3566 & 51.36268 & 35.0211 \\
\hline SMP2 & YMR165C & 14 & 10 & 3 & 7.246377 & 3.846154 & -33.913 & 23.76068 & 47.4359 \\
\hline CTK3 & YML112W & 14 & 10 & 4 & 50.16797 & -122.399 & 1.452282 & 4.347826 & 46.70659 \\
\hline ASI1 & YMR119W & 14 & 10 & 5 & 60.16598 & 3.320562 & -11.0493 & 44.36494 & 60.84788 \\
\hline \multirow[t]{2}{*}{ BUL2 } & YML111W & 14 & 10 & 6 & 55.69918 & 61.87683 & 23.38028 & 47.90528 & 67.91444 \\
\hline & YMR118C & 14 & 10 & 7 & 58.15348 & 40.02294 & -14.6324 & 32.41252 & 45.27778 \\
\hline FAR10 & YLR238W & 14 & 10 & 8 & 61.2542 & 72.14612 & 10.67236 & 58.95522 & 39.62264 \\
\hline CAT5 & YOR125C & 14 & 10 & 9 & 0 & 0 & -17.7083 & -19.6759 & 16.58768 \\
\hline \multirow[t]{2}{*}{ RXT2 } & YBR095C & 14 & 10 & 10 & 51.48148 & 44.96644 & -2.41657 & 53.56415 & 41.72932 \\
\hline & YOL153C & 14 & 10 & 11 & 57.70701 & 57.87909 & 14.28571 & 40 & 58.47458 \\
\hline \multirow[t]{2}{*}{ IST2 } & YBR086C & 14 & 10 & 12 & 35.58673 & 46.59091 & 36.68831 & 50.08787 & 47.72036 \\
\hline & YOL073C & 14 & 10 & 13 & 49.43182 & 69.87847 & 7.989228 & 24.63768 & 49.12281 \\
\hline AAC3 & YBR085W & 14 & 10 & 14 & 53.29032 & 55.56858 & 34.24658 & 61.42035 & 47.54098 \\
\hline AGA1 & YNR044W & 14 & 10 & 15 & 34.98871 & 59.09091 & 51.72414 & 46.47619 & 54.73373 \\
\hline UBC4 & YBR082C & 14 & 10 & 16 & 32.4967 & 25.13514 & 46.71053 & 21.42857 & 59.85915 \\
\hline \multirow[t]{2}{*}{ ABZ1 } & YNR033W & 14 & 10 & 17 & 35.64356 & 32.27207 & 49.22049 & 43.17789 & 50.90361 \\
\hline & YBR075W & 14 & 10 & 18 & 21.69014 & 52.80778 & 1.357466 & 56.05787 & 58.25243 \\
\hline MRPL10 & YNL284C & 14 & 10 & 19 & 22.25859 & 28.11621 & -0.37594 & 39.11368 & 48.12287 \\
\hline TRM7 & YBR061C & 14 & 10 & 20 & 35.2075 & 69.75037 & 9.904762 & 50.49116 & 51.16279 \\
\hline \multirow[t]{2}{*}{ LSM7 } & YNL147W & 14 & 10 & 21 & 45.13158 & 29.64119 & 31.65761 & -7.75623 & 43.10345 \\
\hline & YBR020W & 14 & 10 & 22 & 38.20225 & -18.4094 & 0 & 45.55985 & 56.65722 \\
\hline
\end{tabular}




\begin{tabular}{|c|c|c|c|c|c|c|c|c|c|}
\hline CYB5 & YNL111C & 14 & 10 & 23 & 31.42857 & 42.92379 & -23.5632 & 36.57025 & 53.19149 \\
\hline HIS3 & YOR202W & 14 & 10 & 24 & 63.46783 & 35.54502 & -1.30039 & & \\
\hline HIS3 & YOR202W & 14 & 11 & 1 & 47.11637 & 4.889299 & 40.8284 & & \\
\hline JID1 & YPR061C & 14 & 11 & 2 & 62.89753 & 35.95506 & 1.359517 & 16.17934 & 24.23529 \\
\hline HPA2 & YPR193C & 14 & 11 & 3 & 52.89532 & 1.825558 & -31.6964 & 52.97398 & 55.58824 \\
\hline ARO7 & YPR060C & 14 & 11 & 4 & 44.10828 & 35.13514 & -21.1247 & 45.4343 & 54.96894 \\
\hline \multirow[t]{2}{*}{ AQY1 } & YPR192W & 14 & 11 & 5 & 43.24918 & -35.8712 & 6.220984 & 36.57505 & 46.73913 \\
\hline & YPR059C & 14 & 11 & 6 & 45.26167 & 53.55505 & 11.06061 & 53.62319 & 46.31268 \\
\hline QCR2 & YPR191W & 14 & 11 & 7 & 45.07227 & 29.25278 & 27.30375 & -17.4877 & 39.14591 \\
\hline YMC1 & YPR058W & 14 & 11 & 8 & 55.38642 & 27.77778 & -2.10526 & 33.33333 & 50.30488 \\
\hline SKI3 & YPR189W & 14 & 11 & 9 & 58.23129 & -16.2413 & 13.20475 & 62.80088 & 59.60265 \\
\hline BRR1 & YPR057W & 14 & 11 & 10 & 40.10767 & -6.40569 & -8.63986 & 41.56479 & 39.56386 \\
\hline MLC2 & YPR188C & 14 & 11 & 11 & 50.27933 & 43.44176 & -12.2995 & 76.65441 & 57.41758 \\
\hline SMK1 & YPR054W & 14 & 11 & 12 & 49.13218 & 52.41379 & 16.57459 & 38.15514 & 54.27729 \\
\hline \multirow[t]{2}{*}{ ATG13 } & YPR185W & 14 & 11 & 13 & 43.01075 & 32.78237 & 50 & 35.02935 & 51.79641 \\
\hline & YPR053C & 14 & 11 & 14 & 58.02469 & 72.87582 & -7.53941 & 15.55076 & 34.60076 \\
\hline GDB1 & YPR184W & 14 & 11 & 15 & 55.35714 & 47.72277 & 46.5847 & 49.91453 & 43.13725 \\
\hline NHP6A & YPR052C & 14 & 11 & 16 & 1.344086 & -19.3133 & 2.977667 & 43.77682 & 68.45238 \\
\hline HDA3 & YPR179C & 14 & 11 & 17 & 32.72981 & 38.46154 & 18.94934 & 34.89209 & 43.96783 \\
\hline \multirow[t]{3}{*}{ MAK3 } & YPR051W & 14 & 11 & 18 & 6.284658 & 78.80539 & -12.6488 & 3.674541 & 50 \\
\hline & YPR174C & 14 & 11 & 19 & -11.9107 & 69.82846 & -2.73151 & 59.50096 & 50.1385 \\
\hline & YPR050C & 14 & 11 & 20 & 13.43826 & 64.54653 & 39.06977 & 47.80876 & 60.96096 \\
\hline VPS4 & YPR173C & 14 & 11 & 21 & 86.42534 & -1.21212 & 94.73684 & -5.69476 & 19.24528 \\
\hline \multirow[t]{2}{*}{ MCM16 } & YPR046W & 14 & 11 & 22 & 49.07025 & 13.0621 & 27.91489 & 12.98969 & 40.70796 \\
\hline & YPR172W & 14 & 11 & 23 & 38.08463 & 24.63606 & 49.55224 & 69.33116 & 64.22764 \\
\hline HIS3 & YOR202W & 14 & 11 & 24 & 28.64583 & -16.2571 & 29.36763 & & \\
\hline HIS3 & YOR202W & 14 & 12 & 1 & 87.28956 & 18.0068 & 3.978052 & & \\
\hline \multirow[t]{2}{*}{ RTG3 } & YBL103C & 14 & 12 & 2 & 100 & 19.71831 & -36.6906 & 59.27273 & 61.13744 \\
\hline & YNL109W & 14 & 12 & 3 & 53.11751 & -27.1752 & 55.38922 & 46.63573 & 43.58108 \\
\hline LTE1 & YAL024C & 14 & 12 & 4 & 40.24226 & -32.3442 & 28.69898 & 51.58287 & 59.77337 \\
\hline \multirow[t]{5}{*}{ RPS7B } & YNL096C & 14 & 12 & 5 & 34.28944 & -24.8355 & 17.32283 & 46.39831 & 55.55556 \\
\hline & YDR271C & 14 & 12 & 6 & 38.84198 & -187.5 & 44.92188 & 35.71429 & 50.94851 \\
\hline & YOR379C & 14 & 12 & 7 & 41.47813 & 56.40745 & 14.11638 & 56.16943 & 60.28169 \\
\hline & YDR269C & 14 & 12 & 8 & 55.39972 & 24.44134 & 5.418719 & 47.30539 & 61.33721 \\
\hline & YOR366W & 14 & 12 & 9 & 55.87393 & -4.33884 & 16.33146 & 49.25926 & 50.8982 \\
\hline \multirow[t]{2}{*}{ AMD2 } & YDR242W & 14 & 12 & 10 & 46.22426 & 38.00905 & 2.777778 & 44.89796 & 63.48315 \\
\hline & YOR345C & 14 & 12 & 11 & 43.05556 & 23.28431 & -54.386 & 53.45528 & 44.1358 \\
\hline \multirow[t]{2}{*}{ BRE1 } & YDL074C & 14 & 12 & 12 & 50.91743 & 60.44864 & 41.21306 & -25.5578 & 27.09163 \\
\hline & YOR333C & 14 & 12 & 13 & 46.21969 & 71.13734 & 14.96954 & 56.00858 & 55.26316 \\
\hline \multirow[t]{2}{*}{ AAD3 } & YCR107W & 14 & 12 & 14 & 72.08413 & 9.060403 & 9.793814 & 60.94033 & 54.76923 \\
\hline & YOR325W & 14 & 12 & 15 & 62.23338 & 72.19512 & 10.88435 & 58.93186 & 55.24476 \\
\hline \multirow[t]{2}{*}{ MAL32 } & YBR299W & 14 & 12 & 16 & 49.81862 & 33.15603 & \#DIV/0! & 8.974359 & 46.66667 \\
\hline & YOR309C & 14 & 12 & 17 & 100 & 7.407407 & 63.33333 & 43.62745 & 51.89394 \\
\hline
\end{tabular}




\begin{tabular}{|c|c|c|c|c|c|c|c|c|c|}
\hline UBS1 & YBR165W & 14 & 12 & 18 & 23.60515 & 38.64447 & 37.7551 & 50.75885 & 57.02247 \\
\hline MCH5 & YOR306C & 14 & 12 & 19 & 16.75192 & 24.94305 & 45.24207 & 49.53271 & 42.93948 \\
\hline \multirow[t]{2}{*}{ TEF2 } & YBR118W & 14 & 12 & 20 & 91.59664 & 75 & \#DIV/0! & 50.43103 & 49.66443 \\
\hline & YOR300W & 14 & 12 & 21 & 18.60465 & -21.6162 & 18.44729 & 30 & 54.32526 \\
\hline LYS2 & YBR115C & 14 & 12 & 22 & 21.05927 & 65.14286 & 23.22835 & 63.7457 & 57.83664 \\
\hline ADE2 & YOR128C & 14 & 12 & 23 & 100 & 100 & \#DIV/0! & 62.58993 & 65 \\
\hline HIS3 & YOR202W & 14 & 12 & 24 & 10.03717 & 36.76248 & 22.24852 & & \\
\hline HIS3 & YOR202W & 14 & 13 & 1 & 62.28611 & 25.80372 & 19.31818 & & \\
\hline TKL1 & YPR074C & 14 & 13 & 2 & 20.62893 & -3.44619 & -11.5302 & 71.875 & 67.28395 \\
\hline IML2 & YJL082W & 14 & 13 & 3 & 40.97902 & 23.47267 & 28.85906 & 52.01401 & 56.70391 \\
\hline LTP1 & YPR073C & 14 & 13 & 4 & 5.384615 & 32.50774 & 12.45928 & 57.53425 & 60.6414 \\
\hline \multirow[t]{2}{*}{ PRY1 } & YJL079C & 14 & 13 & 5 & 12.30329 & -14.7303 & 8.892482 & 50.44248 & 53.89049 \\
\hline & YPR071W & 14 & 13 & 6 & 19.73899 & 46.72586 & -10.2962 & 45.33074 & 55.48589 \\
\hline SOD2 & YHR008C & 14 & 13 & 7 & 40.3183 & -12.9848 & 59.1954 & 45.95661 & 56.39344 \\
\hline MED1 & & 14 & 13 & 8 & 59.18885 & 3.816794 & 20.02002 & 28.07692 & 44.08602 \\
\hline ARR3 & YPR201W & 14 & 13 & 9 & 48.25436 & -9.46166 & 21.50171 & 53.30739 & 55.98802 \\
\hline SPE3 & YPR069C & 14 & 13 & 10 & 46.76674 & -1.9697 & 40.90909 & 54.19223 & 55.49451 \\
\hline ARR2 & YPR200C & 14 & 13 & 11 & 51.51515 & -25.3933 & -12.1374 & 55.81818 & 47.00855 \\
\hline HOS1 & YPR068C & 14 & 13 & 12 & 51.88794 & 32.10526 & 38.90215 & 70.46632 & 66.83292 \\
\hline ARR1 & YPR199C & 14 & 13 & 13 & 54.23024 & 64.44223 & 2.155172 & 60.18018 & 43.15068 \\
\hline UBA3 & YPR066W & 14 & 13 & 14 & 62.01456 & 93.71462 & 11.94732 & 10.06424 & 44.55446 \\
\hline SGE1 & YPR198W & 14 & 13 & 15 & 40.78624 & 41.64811 & 37.62376 & 71.63636 & 60.39326 \\
\hline \multirow[t]{6}{*}{ ROX1 } & YPR065W & 14 & 13 & 16 & 61.06195 & 49.0625 & 61.20996 & 69.08463 & 59.8916 \\
\hline & YPR197C & 14 & 13 & 17 & 68.84876 & 15.12915 & 61.51545 & 57.22656 & 54.48718 \\
\hline & YPR064W & 14 & 13 & 18 & 56.50645 & -34.1794 & 32.90598 & 58.76686 & 61.04651 \\
\hline & YPR196W & 14 & 13 & 19 & 21.94805 & 75.59581 & 19.03987 & 48.14815 & 45.09804 \\
\hline & YPR063C & 14 & 13 & 20 & 30.14493 & 55.07246 & 23.84259 & 61.66365 & 61.76471 \\
\hline & YPR195C & 14 & 13 & 21 & 30.67568 & 60.88517 & 76.1811 & 54.01302 & 53.41463 \\
\hline FCY1 & YPR062W & 14 & 13 & 22 & 48.49885 & 25.1816 & 26.73267 & 62.94326 & 65.69579 \\
\hline OPT2 & YPR194C & 14 & 13 & 23 & 41.19782 & 41.80645 & 91.42857 & 55.88752 & 65.85366 \\
\hline HIS3 & YOR202W & 14 & 13 & 24 & 45.97586 & -5.12821 & -27.8607 & & \\
\hline HIS3 & YOR202W & 14 & 14 & 1 & 79.7568 & -7.70142 & 40.54054 & & \\
\hline PTC2 & YER089C & 14 & 14 & 2 & 15.20958 & -57.4297 & 29.32692 & -6.16016 & 45.02618 \\
\hline SUE1 & YPR151C & 14 & 14 & 3 & \#DIV/0! & \#DIV/0! & 80.95238 & 9.306931 & 52.45399 \\
\hline \multirow[t]{2}{*}{ SLF1 } & YDR515W & 14 & 14 & 4 & 23.62093 & -18.6199 & 32.86252 & 42.85714 & 49.54407 \\
\hline & YPR118W & 14 & 14 & 5 & 37.64115 & 49.78587 & 46.94836 & 51.5873 & 58.48564 \\
\hline \multirow[t]{2}{*}{ SAM2 } & YDR502C & 14 & 14 & 6 & 31.47353 & 2.873563 & 25.89792 & 48.1203 & 54.79042 \\
\hline & YPR091C & 14 & 14 & 7 & 47.67596 & 72.07373 & 36.72055 & 14.71861 & 44.44444 \\
\hline FMP36 & YDR493W & 14 & 14 & 8 & 49.50912 & 75.73801 & 6.666667 & 50.60484 & 51.69231 \\
\hline MDM36 & YPR083W & 14 & 14 & 9 & 24.71627 & 2.007299 & 26.19403 & 58.68465 & 52.17391 \\
\hline KRE2 & YDR483W & 14 & 14 & 10 & 58.59951 & -14.6712 & 18.45494 & 40 & 62.67806 \\
\hline AGC1 & YPR021C & 14 & 14 & 11 & 56.7602 & -15.9593 & 0 & 50.17668 & 50.14006 \\
\hline SNF1 & YDR477W & 14 & 14 & 12 & 42.44121 & -35 & 76.08069 & 5.684755 & 34.24125 \\
\hline
\end{tabular}




\begin{tabular}{|c|c|c|c|c|c|c|c|c|}
\hline YPR011C & 14 & 14 & 13 & 57.44921 & 69.11608 & 28.21369 & 57.09091 & 45.68966 \\
\hline YDR444W & 14 & 14 & 14 & 55.7047 & 70.6044 & 51.38158 & 30.94688 & 66.11111 \\
\hline YPL158C & 14 & 14 & 15 & 45.78896 & -13.8381 & 64.41718 & 42.47967 & 61.11111 \\
\hline YDR326C & 14 & 14 & 16 & 56.74699 & 57.24816 & 5.190931 & 47.75862 & 50.70423 \\
\hline YML050W & 14 & 14 & 17 & 35.00557 & 31.01952 & 38.57143 & 69.46429 & 65.65097 \\
\hline YDR290W & 14 & 14 & 18 & 36.70034 & 35.44304 & 100 & 18.16038 & 65.78249 \\
\hline YLR308W & 14 & 14 & 19 & 49.5356 & -21.6274 & 70.33248 & 57.94769 & 47.69648 \\
\hline YFL063W & 14 & 14 & 20 & 3.6 & 31.36 & 13.11475 & -14.4 & 43.35664 \\
\hline YOL086C & 14 & 14 & 21 & 59.60347 & 68.71239 & 56.62393 & 40 & 59.01639 \\
\hline YFL033C & 14 & 14 & 22 & \#DIV/0! & \#DIV/0! & 41.32231 & 23.35329 & 51.75202 \\
\hline YDR461W & 14 & 14 & 23 & 55.33742 & 40.29228 & 96.14035 & 68.28885 & 67.90123 \\
\hline YOR202W & 14 & 14 & 24 & 54.72837 & 36.13861 & -25.5814 & & \\
\hline YOR202W & 14 & 15 & 1 & 90.40541 & -6.33147 & 27.51817 & & \\
\hline YPR095C & 14 & 15 & 2 & 56.15305 & 1.333333 & 83.46457 & & \\
\hline YOR202W & 14 & 15 & 3 & 10.54348 & -3.09989 & 100 & & \\
\hline YPR093C & 14 & 15 & 4 & 29.45205 & 30.61651 & 29.10714 & & \\
\hline YOR202W & 14 & 15 & 5 & 60.83521 & 65.11278 & 63.74134 & & \\
\hline YPR092W & 14 & 15 & 6 & 55.09434 & -0.82919 & 46.89042 & & \\
\hline YOR202W & 14 & 15 & 7 & 37.13188 & 7.745505 & 32.49759 & & \\
\hline YPR090W & 14 & 15 & 8 & 57.08502 & 42.27994 & 44.41088 & & \\
\hline YNR041C & 14 & 15 & 9 & \#DIV/0! & \#DIV/0! & \#DIV/0! & & \\
\hline YPR089W & 14 & 15 & 10 & 65.59428 & -23.6476 & 48.63388 & & \\
\hline YJR034W & 14 & 15 & 11 & 54.19689 & -26.8765 & 31.50685 & & \\
\hline YPR084W & 14 & 15 & 12 & 69.41515 & -32.0755 & 53.00146 & & \\
\hline YJL120W & 14 & 15 & 13 & 88.18182 & 91.85049 & 90.73615 & & \\
\hline YPR079W & 14 & 15 & 14 & 41.32918 & -10.566 & 54.03226 & & \\
\hline YJL119C & 14 & 15 & 15 & 55.16529 & 46.45833 & 27.8481 & & \\
\hline YPR078C & 14 & 15 & 16 & 98.32776 & 74.84663 & 42.06897 & & \\
\hline YJL117W & 14 & 15 & 17 & 37.75811 & 51.15766 & 50.61728 & & \\
\hline YPR077C & 14 & 15 & 18 & 56.5625 & 25.12376 & 28.71917 & & \\
\hline YJL107C & 14 & 15 & 19 & 58.24176 & 34.5679 & 19.75498 & & \\
\hline YPR076W & 14 & 15 & 20 & 39.70917 & 33.49359 & 23.46185 & & \\
\hline YJL106W & 14 & 15 & 21 & 55.11945 & 41.15942 & 75.38462 & & \\
\hline YPR075C & 14 & 15 & 22 & 67.10183 & 67.12598 & 56.8479 & & \\
\hline YJL092W & 14 & 15 & 23 & -16.2214 & -19.9593 & 26.25871 & & \\
\hline YOR202W & 14 & 15 & 24 & -14.214 & 78.82177 & 18.35273 & & \\
\hline YOR202W & 14 & 16 & 1 & 100 & 27.13755 & -7.77202 & & \\
\hline YOR202W & 14 & 16 & 2 & 80.09259 & -8.30189 & 9.962264 & & \\
\hline YOR202W & 14 & 16 & 3 & 39.63039 & -8.71261 & 10.7943 & & \\
\hline YOR202W & 14 & 16 & 4 & -3.30882 & -5.41612 & 6.517094 & & \\
\hline YOR202W & 14 & 16 & 5 & 88.5918 & -111.346 & -2.0202 & & \\
\hline YOR202W & 14 & 16 & 6 & 25.66265 & -11.442 & 26.49007 & & \\
\hline YOR202W & 14 & 16 & 7 & 30.69182 & 8.171206 & 63.38673 & & \\
\hline
\end{tabular}




$\begin{array}{lrllrrrr}\text { HIS3 } & \text { YOR202W } & 14 & 16 & 8 & 42.41417 & 28.62275 & 48.69888 \\ \text { HIS3 } & \text { YOR202W } & 14 & 16 & 9 & 53.49162 & 19.86183 & 50 \\ \text { HIS3 } & \text { YOR202W } & 14 & 16 & 10 & 55.94887 & 43.97727 & 58.01527 \\ \text { HIS3 } & \text { YOR202W } & 14 & 16 & 11 & 54.04878 & -0.14205 & 57.84173 \\ \text { HIS3 } & \text { YOR202W } & 14 & 16 & 12 & 75.89641 & 25.30949 & 96.80851 \\ \text { HIS3 } & \text { YOR202W } & 14 & 16 & 13 & 41.36657 & 27.06186 & 100 \\ \text { HIS3 } & \text { YOR202W } & 14 & 16 & 14 & 55.11163 & -10.6742 & \text { \#DIV/0! } \\ \text { HIS3 } & \text { YOR202W } & 14 & 16 & 15 & 57.25646 & 24.13793 & 55.75221 \\ \text { HIS3 } & \text { YOR202W } & 14 & 16 & 16 & 47.67096 & -14.0221 & \text { \#DIV/0! } \\ \text { HIS3 } & \text { YOR202W } & 14 & 16 & 17 & 51.54525 & 5.575326 & \text { \#DIV/0! } \\ \text { HIS3 } & \text { YOR202W } & 14 & 16 & 18 & 51.86916 & 33.54271 & \text { \#DIV/0! } \\ \text { HIS3 } & \text { YOR202W } & 14 & 16 & 19 & 42.93139 & 35.99062 & \text { \#DIV/0! } \\ \text { HIS3 } & \text { YOR202W } & 14 & 16 & 20 & 41.63347 & 34.80278 & 31.85841 \\ \text { HIS3 } & \text { YOR202W } & 14 & 16 & 21 & 57.61006 & -35.0734 & 3.680982 \\ \text { HIS3 } & \text { YOR202W } & 14 & 16 & 22 & 47.51309 & 26.16633 & -5.19952 \\ \text { HIS3 } & \text { YOR202W } & 14 & 16 & 23 & -14.0845 & 50.14837 & 15.59633 \\ \text { HIS3 } & \text { YOR202W } & 14 & 16 & 24 & 99.45355 & 3.962264 & 3.231441\end{array}$

